NAT'L INST. OF STAND \& TECH R.I.C

Al1104 в 17991

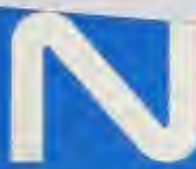

united States Department of Commerce Technology Administration

National Institute of Standards and Technology

NIST Special Publication 926

\title{
POSTER PAPERS
}

International Conference on Atomic and Molecular Data and Their Applications (ICAMDATA 97)

Wolfgang L. Wiese and Peter J. Mohr, Editors

M1111111 | | |111111\% 
$T$ he National Institute of Standards and Technology was established in 1988 by Congress to "assist industry in the development of technology ... needed to improve product quality, to modernize manufacturing processes, to ensure product reliability ... and to facilitate rapid commercialization ... of products based on new scientific discoveries."

NIST, originally founded as the National Bureau of Standards in 1901, works to strengthen U.S. industry's competitiveness; advance science and engineering; and improve public health, safety, and the environment. One of the agency's basic functions is to develop, maintain, and retain custody of the national standards of measurement, and provide the means and methods for comparing standards used in science, engineering, manufacturing, commerce, industry, and education with the standards adopted or recognized by the Federal Government.

As an agency of the U.S. Commerce Department's Technology Administration, NIST conducts basic and applied research in the physical sciences and engineering, and develops measurement techniques, test methods, standards, and related services. The Institute does generic and precompetitive work on new and advanced technologies. NIST's research facilities are located at Gaithersburg, MD 20899, and at Boulder, CO 80303. Major technical operating units and their principal activities are listed below. For more information contact the Publications and Program Inquiries Desk, 301-975-3058.

Office of the Director

- National Quality Program

- International and Academic Affairs

Technology Services

- Standards Services

- Technology Partnerships

- Measurement Services

- Technology Innovation

- Information Services

Advanced Technology Program

- Economic Assessment

- Information Technology and Applications

- Chemical and Biomedical Technology

- Materials and Manufacturing Technology

- Electronics and Photonics Technology

Manufacturing Extension Partnership Program

- Regional Programs

- National Programs

- Program Development

\section{Electronics and Electrical Engineering}

Laboratory

- Microelectronics

- Law Enforcement Standards

- Electricity

- Semiconductor Electronics

- Electromagnetic Fields ${ }^{1}$

- Electromagnetic Technology ${ }^{1}$

- Optoelectronics

\section{Chemical Science and Technology \\ Laboratory \\ - Biotechnology \\ - Physical and Chemical Properties ${ }^{2}$ \\ - Analytical Chemistry \\ - Process Measurements \\ - Surface and Microanalysis Science}

Physics Laboratory

- Electron and Optical Physics

- Atomic Physics

- Optical Technology

- Ionizing Radiation

- Time and Frequency

- Quantum Physics'

\section{Materials Science and Engineering} Laboratory

- Intelligent Processing of Materials

- Ceramics

- Materials Reliability

- Polymers

- Metallurgy

- NIST Center for Neutron Research

\section{Manufacturing Engineering} Laboratory

- Precision Engineering

- Automated Production Technology

- Intelligent Systems

- Fabrication Technology

- Manufacturing Systems Integration

\section{Building and Fire Research}

Laboratory

- Structures

- Building Materials

- Building Environment

- Fire Safety Engineering

- Fire Science

Information Technology Laboratory

- Mathematical and Computational Sciences ${ }^{2}$

- Advanced Network Technologies

- Computer Security

- Information Access and User Interfaces

- High Performance Systems and Services

- Distributed Computing and Information Services

- Software Diagnostics and Conformance Testing

\footnotetext{
'At Boulder, CO 80303.

${ }^{2}$ Some elements at Boulder, CO.
} 


\section{POSTER PAPERS}

\section{International Conference on Atomic and Molecular Data and Their Applications (ICAMDATA 97)}

at the National Institute of Standards and Technology

Gaithersburg, Maryland, USA

September 29 - October 2, 1997

Wolfgang L. Wiese and Peter J. Mohr, Editors

Atomic Physics Division

Physics Laboratory

National Institute of Standards and Technology

Gaithersburg, MD 20899

August 1998

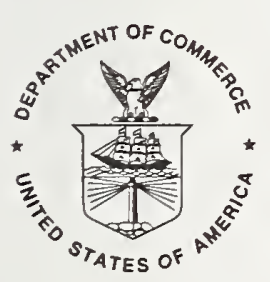

U.S. Department of Commerce

William M. Daley, Secretary

Technology Administration

Gary R. Bachula, Acting Under Secretary for Technology

National Institute of Standards and Technology

Raymond G. Kammer, Director 
National Institute of Standards and Technology

Special Publication 926

Natl. Inst. Stand. Technol.

Spec. Publ. 926

277 pages (Aug. 1998)

CODEN: NSPUE2
U.S. Government Printing Office

Washington: 1998
For sale by the Superintendent of Documents

U.S. Government Printing Office

Washington, DC 20402 


\section{Sponsor \\ National Institute of Standards and Technology: \\ Physics Laboratory \\ Atomic Physics Division \\ Standard Reference Data Program}

\section{Co-Sponsors}

Electric Power Research Institute

Office of Fusion Energy Science,

Department of Energy

Institute of Theoretical Atomic and

Molecular Physics at the Harvard-Smithsonian

Center for Astrophysics

Lawrence Livermore National Laboratory

U.S. Naval Research Laboratory

SEMATECH

Office of Space Sciences,

National Aeronautics and Space Administration 


\section{Conference Committee}

Dr. Wolfgang Wiese, NIST-Atomic Physics Division, Conference Chair

Dr. Peter Mohr, NIST-Atomic Physics Division, Conference Secretary

Dr. James Babb, Harvard-Smithsonian Center for Astrophysics

Prof. Kurt Becker, Stevens Institute of Technology

Dr. George Doschek, Naval Research Laboratory

Prof. James Lawler, University of Wisconsin

Dr. David Leckrone, NASA Goddard Space Flight Center

Prof. Michael Pindzola, Auburn Unicersity Prof. Anil Pradhan, Ohio State University

Dr. John Rumble, NIST-Standard Reference Data Program

Dr. David Schultz, Oak Ridge National Laboratory

Dr. Peter Smith, Harvard-Smithsonian Center for Astrophysics

\section{International Program}

\section{Committee}
A. Dalgarno (USA), Chair
R. Janev (Austria), Vice-Chair
D. Schultz (USA), Treasurer
K. Berrington (N. Ireland), Secretary
L. Brown (USA)
M. Capitelli (Italy)
G. Lister (USA)
H. Mason (United Kingdom)
K. Niemax (Germany)
L. Presnyakov (Russia)
E. Roueff (France)
H. Tawara (Japan)
W. Wiese (USA)

\section{International Advisory Board}

N. Bardsley (USA)

P. Burke (N. Ireland)

R. Crompton (Australia)

J. Delcroix (France)

G. Dunn (USA)

F. Gianturco (Italy)

R. Hulse (USA)

J. Li (P.R. China)

I. Martinson (Venuzuela)

J. Rumble (USA)

E. Salzborn (Germany)

M. Seaton (United Kingdom)

I. Sobelman (Russia)

K. Takayanagi (Japan) 
This Special Publication contains the poster papers presented at the first International Conference on Atomic and Molecular Data and Their Applications (ICAMDATA), which was convened at the National Institute of Standards and Technology (NIST), Gaithersburg, MD from September 29 through October 2, 1997. One hundred seventy-one registered participants from 20 countries attended the conference. In addition to the poster papers recorded here, twenty-seven invited talks were given, which are collected in a separate volume of proceedings [1].

Atomic and molecular $(\mathrm{A}+\mathrm{M})$ data are essential for the solution of a wide range of problems in diverse fields of science and technology. Major current areas of science requiring large amounts of $\mathrm{A}+\mathrm{M}$ data are astrophysics, plasma physics, atmospheric physics, and basic atomic physics. Major current technology areas in which $A+M$ data are an essential input are the plasma processing of materials, the development of lighting sources, and spectrochemistry.

The enormous variety of $\mathrm{A}+\mathrm{M}$ data and the diverse data needs have led to quite a fragmentation of this field, and a focal point has been missing. This conference, as well as a planned future conference series, is therefore intended to serve as such a focal point, with one of its main purposes to bring together data producers, compilers, and users in an open forum and provide close interactions and intensive exchanges of ideas.

The principal topics of the poster presentations were (a) the production of collision and radiation data, both atomic and molecular, including benchmark data; (b) the presentation and detailed description of available atomic and molecular databases, their management and data assessment, and (c) reviews of data needs in various user communities.

It is a pleasure to acknowledge the competent editorial assistance of C. Marlow and R. Jocson.

\section{Reference}

[1] P. J. Mohr and W. L. Wiese, Eds. Atomic and Molecular Data and Their Applications, AIP Conf. Proceed., 434, New York 1998 



\section{Contents}

\section{Databases}

A Compilation of Electron-Molecule Scattering Cross Sections

M. Brunger and S. Buckman.....

Electron Interactions with Plasma Processing Gases

L. Christophorou and J. Olthoff .

NIST Atomic Spectra Database

G. R. Dalton, R. A. Dragoset, J. R. Fuhr, D. E. Kelleher, S. A. Kotochigova,

W. C. Martin, P. J. Mohr, A. Musgrove, K. Olsen, L. Podobedova, E. B. Saloman,

J. Sugar, W. L. Wiese, C. Stern Grant, G. Eichhorn, R. L. Kelly, T. Shirai,

V. I. Azarov, A. E. Kramida, A. N. Ryabtsev, J. Blaise, J. F. Wyart

Atomic Database Development of Mid-to-High-Z Elements

A. Dasgupta and P. Kepple

GAPHYOR Data Center http://gaphyor.lpgp.u-psud.fr

J. L. Delcroix, D. Humber, and C. Leprince......

NIST Atomic and Molecular Databases on the World Wide Web

R. Dragoset, P. Mohr, K. Olsen, E. Saloman, G. Wiersma, and D. Zucker

Vibrational and Electronic Energy Levels of Polyatomic Transient Molecules M. Jacox

Database for Dielectronic Recombination Rate Coefficients to the Excited

States of the Carbon Atom and Ions

T. Kato, U. Safronova, and J. Dubau

Critical Evaluation of Transition Probabilities of Na-Ca ( $\mathrm{Z}=11-20)$

D. Kelleher and L. Podobedova

Database for Electron-Impact Total Ionization Cross Sections of Atoms and

Molecules

Y. K. Kim, D. Zucker, M. Ali, and M. Rudd

The Atomic Spectroscopy Bibliography Database of the Institute for

Spectroscopy, Troitsk

A. Kramida, A. Ryabtsev, and G. Vedeneeva

Atomic Data Center on PC

K. Lu, D. Baba, and R. Engleman

Los Alamos Opacity Web Ring

N. Magee, Jr. and R. Clark 
TOPbase/TIPbase

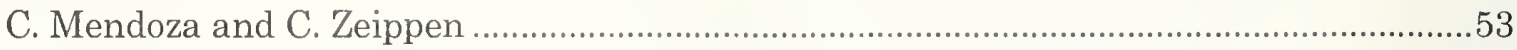

NIFS Retrieval Display System for Atomic Data Through the WWW

I. Murakami, M. Kato, T. Kato, and NEC nifs team

Wavelengths of Six Times Ionized Germanium, Ge VII

L. I. Podobedova and A. N. Ryabtsev

The ORNL Controlled Fusion Atomic Data Center

D. Schultz, P. Kristic, F. Ownby, F. Meyer, C. Havener, M. Bannister, W. Liu,

D. Jeffery, and P. Stancil

The Database <<SPECTR $>$ on Spectral Projects of Atoms and Ions

I. Skobelev, A. Faenov, A. Magunov

The Plasma Chemistry Database

W. Trail, C. Davis, and W. Morgan

Development of an Atomic Spectroscopy Reference Implemented on a PC

Platform Under Windows 95

K. Visser and G. Hattingh.

\section{Data Needs}

Absolute Infrared Intensities for: Standards and Reactive Molecules

C. Chackerian, Jr.

NASA's Laboratory Astrophysics Program

H. Hasan and H. Thronson .88

Atomic Inputs to Calculate Radiative Forces in Stellar Models

G. Massacrier, J. F. Gonzalez, M. C. Artru, G. Michaud, J. Richer, and F. LeBlanc .92

New Electron Temperature and Density Diagnostics for Photoionized Gas

D. Savin, T. Bartsch, M. Chen, S. Kahn, D. Liedahl, J. Linkemann, A. Müller,

S. Schippers, M. Schmitt, D. Schwalm, and A. Wolf. 96

\section{Data Production}

\subsection{Atomic Structure and Spectroscopy}

The Platinum Group Ion Project

V. Azarov, S. Churilov, R. Kildiyarova, A. Ryabtsev, A. Raassen, P. Uylings,

Y. Joshi, L. Tchang-Brillet, and J. F. Wyart 103

An Integrated Approach to Bound and Continuum States: Application to Beryllium-like Ions

K. Berrington and J. Pelan 106 
Efficient Method to Account for Correlation Effects when Generating Atomic Parameters for Complex Atoms

P. Bogdanovich, G. Gaigalas, and Z. Rudzikas

Relativistic Calculations in the Arsenic Sequence: Fine-Structure Transitions

E. Charro and I. Martín

Atomic Database for C I from C II

J. Dubau, T. Kato, and U. I. Safronova

A Project for Large-Scale Stark Broadening Data Production: Ca IX and Ca X Spectral Lines

M. Dimitrijević and S. Sahal-Bréchot

Accurate MCHF/MCDF Transition Rates

C. Fischer, P. Jönsson, and S. Fritzsche.....

Secondly Quantized Multi-Configurational Approach for Atomic Databases

G. Gaigalas and Z. Rudzikas

Polarization Profile Calculations for Plasma Diagnostics

M. Á. González and M. A. Gigosos

Laboratory Measurements of Resonant Contributions to Fe XXIV

Line Emission

M. Gu, P. Beiersdorfer, G. Brown, S. Kahn, D. Liedahl, K. Reed, and D. Savin

Modeling Electromagnetic Interactions in Quantized Electronic Systems

V. L. Jacobs

Relativistic Oscillator Strengths in Chlorine

I. Martín, C. Lavín, and A. Velasco.

Oscillator Strengths of Rydberg Transitions

W. Mende and M. Kock

Atomic Data for Lighting Applications

G. Nave, C. Sansonetti, and J. Reader

Investigation of the Autoionization Spectra of Samarium from

$48,800 \mathrm{~cm}^{-1}$ to $51,200 \mathrm{~cm}^{-1}$

H. Park, H. C. Kim, Y. J. Rhee, and J. Lee

156

Upgrading the Atomic and Molecular Database for Astrophysics and

Atmospheric Physics by Fourier Transform Spectroscopy

J. C. Pickering, R. Schermaul, G. Cox, J. Rufus, A. P. Thorne, and R. C. M. Learner ......159

Calculations of Oscillator Strengths for Singly Ionized Iron Group Elements

by Means of the Orthogonal Operator Approach

A. Raassen and P. Uylings....

Autoionizing Rates for Excited States of Many-Electron Ions

U. I. Safranova and W. R. Johnson

Complete Atomic Database for Autoionizing Levels of B-like Ions with $\mathrm{Z}=\mathbf{6 - 5 4}$

U. I. Safronova, A. S. Shlyaptseva, M. Cornille, and J. Dubau

Inner-shell Excitation Energies for C-, N-, O-, and F-like Ions with Z = 6-54

U. I. Safronova and A. S. Shlyaptseva. 
Accurate Lifetime Measurements of Fine-Structure States of Neutral Lithium by Beam-Gas-Laser-Spectroscopy

A. Schmitt, U. Volz, and H. Schmoranzer

Accurate Atomic Data for S I, S II, S III

S. Tayal

Precise Atomic Lifetimes Measured at a Heavy-Ion Storage Ring

E. Träbert.

Light Ion Absorption, Probed with High Spatial, Temporal, and Spectral

Resolution: The Spectrum of C II

P. Villoresi and P. Nicolosi....

Systematic Studies of the $n=2$ to $n=3,4$ Transitions in the Ions of Elements $\mathrm{Cu}$ through As Isoelectronic with Li I through Na I Produced by Laser

Irradiation

J. F. Wyart, T. Missalla, J. C. Gauthier, and C. Chenais-Popovics.

Self-Consistent Sets of Oscillator Strength for Ultraviolet Lines in C I, S I, and $\mathrm{Ni}$ II

J. Zsargó, S. Federman, and J. Cardelli

\subsection{Collisions}

Electron Impact Ionization and Surface Induced Reactions of Fusion Plasma Edge Constituents

K. Becker, F. Biasioli, G. Denifl, H. Deutsch, T. Fiegele, V. Grill, T. Märk, C. Mair,

S. Matt, D. Muigg, P. Scheier, M. Sonderegger, A. Stamatovic, and R. Wörgötter

The Role of Electron Impact Ionization Cross Sections in

Low-Temperature Plasmas

K. Becker, V. Tarnovsky, H. Deutsch, and T. Märk

Atomic Data: Energy Levels, Transition Rates, and Collision Strengths;

An Example of Mg VIII

A. Bhatia

Collisional and Spectroscopic Data Relevant to the Fundamental Processes in Plasmas Containing Si-Organic Admixtures

R. Foest, R. Basner, M. Schmidt, P. Kurunczi, and K. Becker

Measurements of the Absolute Cross Sections of Inelastic Processes for Slow Atomic Collisions

M. Gochitashvili, B. Kikiani, and R. Lomsadze.....

Ionization, Charge Transfer, and Stripping Cross Sections for Alkali Metal Ion Collisions with Inert Gas Atoms and $\mathrm{H}_{2}$ and $\mathrm{N}_{2}$ Molecules in the $\mathbf{0 . 5}-7.0 \mathrm{keV}$

Energy Range

B. I. Kikiani, R. A. Lomsadze.

Atomic Data for Recombination Calculations

R. Kisielius, P. Storey, and A. Davey..... 
Unified Electron-Ion Recombination Cross Sections and Rates

S. Nahar, H. Zhang, and A. Pradhan.

K-Matrix Correction of B, CB Cross-Sections

L. Vainshtein

The Iron Project (OSU): Large-Scale Computations of Atomic Data

H. Zhang, M. Bautista, S. Nahar, P. Romano, and A. Pradhan

\subsection{Molecules}

Ultraviolet Emission in the Dissociation of $\mathrm{N}_{2}$ and $\mathrm{O}_{2}$ by $\mathrm{K}^{+}$Impact

M. Gochitashvili, B. Kikiani, R. Kvizhinadze, and N. Jaliashvili

Electron Collision Cross Sections for the $\mathbf{C F}_{4}$ Molecule by

Electron Swarm Study

Y. Hayashi and Y. Nakamura

Inelastic Cross Sections for the $\mathrm{C}_{3} \mathrm{~F}_{8}$ Molecule from Electron Transport

Coefficients in $\mathrm{C}_{3} \mathrm{~F}_{8}$-Ar Mixtures

B. H. Jeon and Y. Nakamura.

Accurate ab initio Calculation of Molecular Constants

S. Kotochigova and I. Tupitsyn

Electron Collisions with Small Molecules

B. McLaughlin, C. Ballance, D. Thompson, K. Berrington, and P. Burke

Electron Transport Coefficients in $\mathrm{C}_{2} \mathrm{~F}_{6}$-Ar Mixtures and Inelastic Cross

Sections for the $\mathrm{C}_{2} \mathrm{~F}_{6}$ Molecule

H. Okumo and Y. Nakamura

Validation of Ozone Infrared Line Intensities

M. Smith.

Hyperfine Structure Constants for Diatomic Molecules

I. Tupitsyn and S. Kotochigova...... 


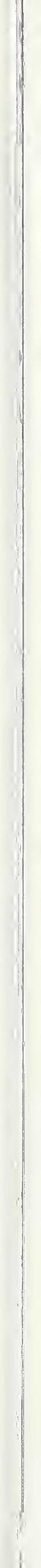


1 Databases 


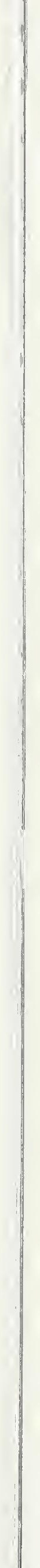




\title{
A Compilation of Electron-Molecule Scattering Cross Sections
}

\author{
M.J. Brunger ${ }^{+}$and S.J. Buckman* \\ +Department of Physics, Flinders University of South Australia \\ *AMPL, Australian National University, Canberra
}

\section{Introduction}

The intention of this project is to attempt to update the critical review and cross section data compilation of Trajmar et al. [1] to account for measurements over the past 15 years. As in the original review the emphasis will be on both differential and integral cross section measurements at low to intermediate energies (meV-1000 eV) for elastic scattering, rotational, vibrational and electronic excitation. These cross sections are of relevance in applied areas as divergent as plasma deposition and etching of semiconductors, gaseous high voltage switches and electrostatic precipitators for the processing of atmospheric pollutants. The active constituents in each of these devices are typically polyatomic molecules and as such there has been a marked increase in both experimental and theoretical studies for these complicated targets.

We will also attempt to discuss in a critical fashion the comparison between the various experimental results and between the experimental and theoretical cross sections. However it is beyond the extent of this article, and the expertise of the authors, to offer a detailed critique of the many theoretical approaches. We shall give a brief discussion of experimental techniques with particular emphasis on new developments in the measurement of absolute scattering cross sections since Trajmar et al. [1] Finally we note that whilst temporary negative ion resonances are often of great importance in low energy electron-molecule scattering the treatment of resonance dynamics is beyond the scope of this project and we will only discuss them in the context of cross section enhancement.

\section{Scope}

The extent of the review article is perhaps best illustrated by its table of contents, as given below:

1.

2.

2.1

2.2

2.3

2.4

2.5

2.6

2.7
Introduction

Experimental Techniques Recent Developments

High resolution sources

Single collision experiments

Swarm experiments

Parallel detection techniques

Sources of excited species

Normalisation techniques

Techniques for the generation

of integral cross sections
3.

3.1

3.2

3.3

3.4

3.5

3.6

3.7

3.8
Experimental Data - Diatomic Molecules

$\mathrm{H}_{2}$

$\mathrm{N}_{2}$

$\mathrm{O}_{2}$

Halogens

$\mathrm{CO}$

NO

Hydrogen Halides

Alkali Halides 
4.

\section{1}

4.2

4.3

4.4

4.5

4.6

4.7

4.8

4.9

4.10
Cross section trends amongst diatomic molecules. Experimental Data Polyatomic Molecules

$\mathrm{H}_{2} \mathrm{O}$

$\mathrm{H}_{2} \mathrm{~S}$

$\mathrm{HCN}$

$\mathrm{O}_{3}$

OCS

$\mathrm{CO}_{2}$

$\mathrm{N}_{2} \mathrm{O}$

$\mathrm{NH}_{3}$

$\mathrm{SO}_{2}$

$\mathrm{CH}_{4}$
4.11

4.12

4.13

4.14

4.15

4.16

5.

6.

7.

8.

8.1
Hydrocarbons and derivatives

Silane and derivitives

Germane and derivitives

Halocarbons and derivitives

$\mathrm{SF}_{6}$

Cross section trends amongst

polyatomic molecules

Epilogue

Summary and Suggested

Measurements

\section{References}

Appendix

Tables of cross section data

It is our intention that, subject to satisfying copyright requirements, all the cross section data tables will be placed on the Web for viewing and downloading.

\section{Status of Project}

At this time we have covered about $80-85 \%$ of the topic and hope that the final sections and editing can be completed in the next 6-9 months. The article will contain in excess of 260 tables of cross section data and over 200 figures, as well as a (hopefully) critical assessment of the cross sections for each gas. The reference list currently stands at 750 , most of which is due to work that has been generated since the original review of Trajmar et al. [1]

\section{Highlights}

One of the nice things about a review such as this is that, in having an extensive database available at your fingertips, you can start to get a picture for any trends in the cross sections for a given molecule, or across different molecular species, and for any gaps (either experimental or theoretical) in the literature. This in turn allows one to form an opinion as to where it might be profitable to conduct new experiments or perform new calculations in the future.

We now examine just two of these areas, both of which we believe are illustrative of some very interesting physics.

\section{Cross Section Trends Across Molecules}

In the graphs in Fig. 1, we show the results of some elastic differential cross section (DCS) measurements for electron scattering from $\mathrm{N}_{2}, \mathrm{CO}, \mathrm{O}_{2}, \mathrm{NO}$, and $\mathrm{CO}_{2}$. All the illustrated data were measured at the Australian National University (ANU) with a crossed beam apparatus and employed the relative flow technique [2] to set the absolute scale. 

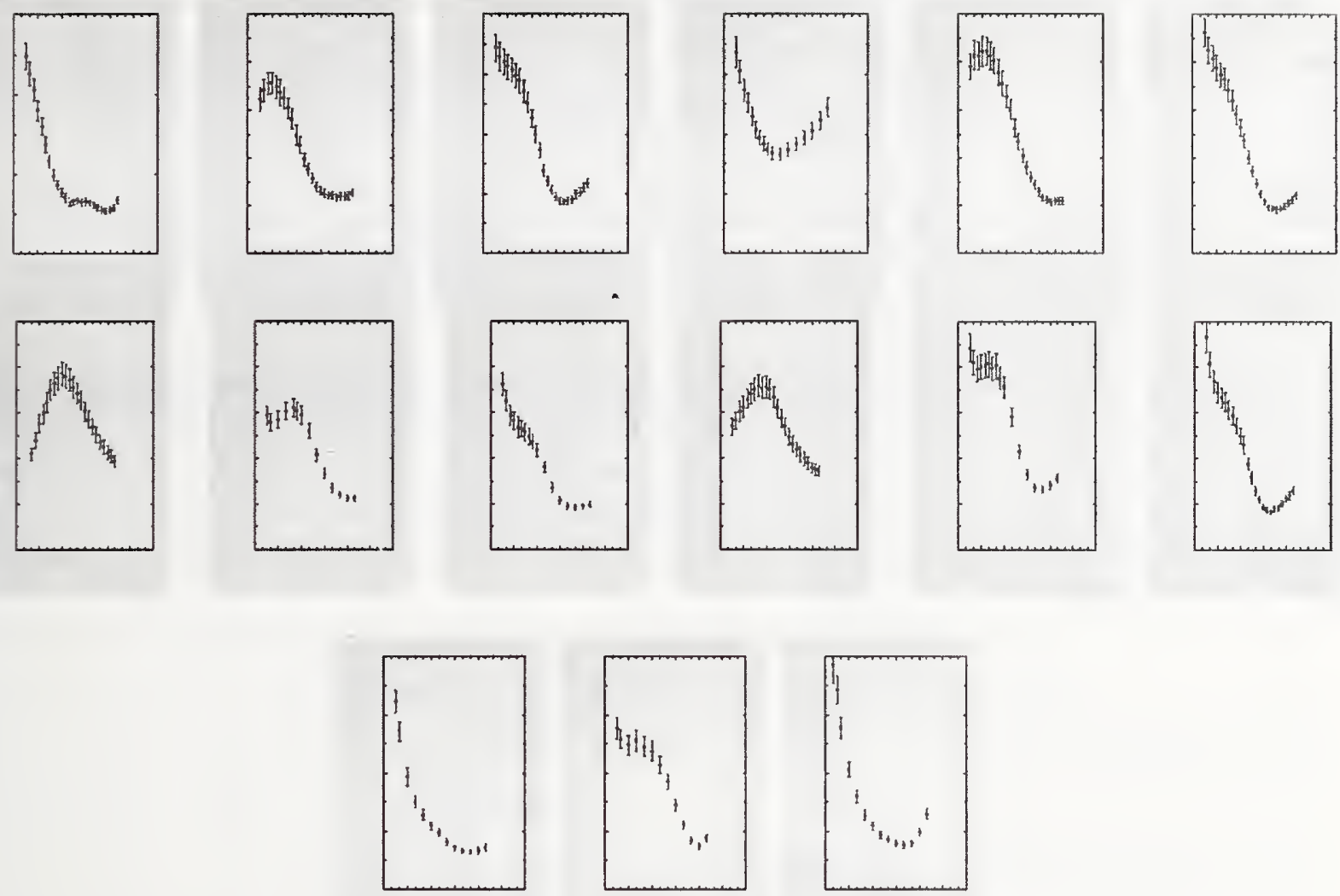

Figure 1: Elastic differential cross section (DCS) measurements for electron scattering from $\mathrm{N}_{2}$, $\mathrm{CO}, \mathrm{O}_{2}, \mathrm{NO}$ and $\mathrm{CO}_{2}$. See text for explanation.

For each of these molecules we observe an interesting behavior in the elastic DCS at forward angles. This occurs at energies above the shape resonance in each molecule but below about 10 $\mathrm{eV}$. This behavior appears to be associated with those systems that possess a strong, low energy shape resonance. Where the resonances are at relatively low energy and are "sharp", e.g. $\mathrm{O}_{2}$ and NO, the DCS at low energy $(\sim 1 \mathrm{eV})$ dips strongly at forward scattering angles. It then passes through a transition from weak forward scattering to a forward-peaked DCS in the energy region 4-7 eV. In the other systems $\left(\mathrm{N}_{2}, \mathrm{CO}, \mathrm{CO}_{2}\right)$, which all have a broad shape resonance that peaks between $2-4 \mathrm{eV}$, the DCS in the resonance region are strongly forward peaked but then decrease in magnitude at forward angles as the energy increases just above the resonance.

Sun et al. [3] noted, in their calculations of $\mathrm{N}_{2}$ elastic DCS, that above the resonance, where the $\Pi_{\mathrm{g}}$ symmetry is no longer dominant, it is essential to include higher order partial waves and additional symmetries to reproduce the observed behavior. In the case of diatomic systems it is interesting to note that each of the above molecules possess similar dipole polarisabilities and so it is tempting to speculate that it is this which may be responsible for the angular behavior of the DCS. However, as it also appears that this effect is present in a class of polyatomic molecule systems the problem is deserving of some careful theoretical attention. 


\section{Polyatomic Molecule Scattering Theory}

Most polyatomic molecule scattering calculations have concentrated on DCS and integral cross sections (ICS) for the elastic scattering process, although some calculations for excitation of the vibrational modes of molecules are available in the literature. In this latter case both DCS and ICS are also typically reported. An extensive series of calculations have been performed for elastic scattering from methane which, because of its near-spherical symmetry, has become something of a prototypical system for low energy electron-polyatomic molecule scattering calculations. A summary of the available experimental and theoretical data for this molecule can be found in the recent publication of Bundschu et al. [4]

In Fig. 2, we show the level of agreement that has been obtained amongst experimental groups and between theory and experiment for $\mathrm{CH}_{4}$. While we only show DCS at $1 \mathrm{eV}$ and $3 \mathrm{eV}$, the level of accord in these figures is indicative of that found up to about $5.5 \mathrm{eV}$. Note the very good agreement between the two most recent experimental measurements [4] [5], both of which independently applied the relative flow technique, and between these measurements and the calculation of Gianturco and Sanna [4].
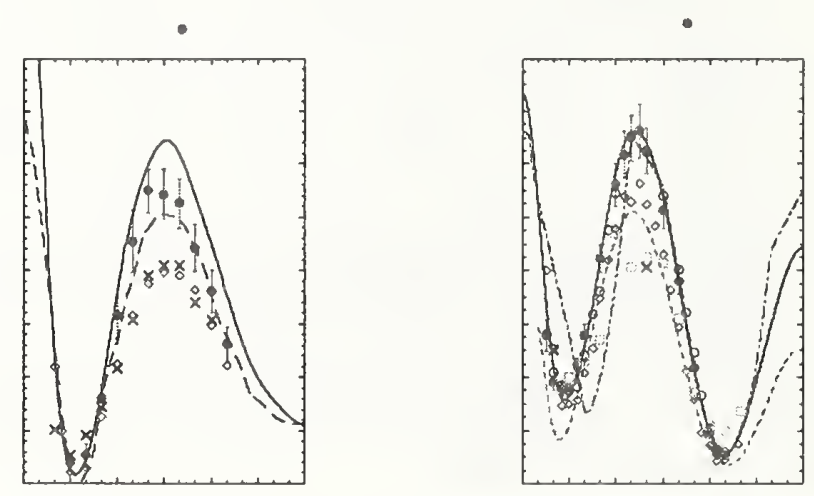

Figure 2: Comparison of theory and experiment for $\mathrm{CH}_{4}$ at $1 \mathrm{eV}$ and $3 \mathrm{eV}$. See text for explanation.

Unfortunately, this level of agreement between theory and experiment for low energy elastic polyatomic molecule scattering is quite unique. In general, for energies less than $10 \mathrm{eV}$, one usually finds quite poor agreement between the calculations and measurements, as we now specifically illustrate for $\mathrm{e}^{\cdot}+\mathrm{CO}_{2}$ scattering.

In the next figure, we show the situation in $\mathrm{CO}_{2}$ at energies of 1 and $5 \mathrm{eV}$. Whilst it is clear that the level of agreement between the various experiments is only fair, the major point that we wish to make here is the serious discrepancies between experiment and theory. Neither the model of Takekawa and Itikawa or that of Gianturco and Stoecklin adequately reproduces the shape or magnitude of these low energy DCS, even when additional symmetries were employed in the latter case. 

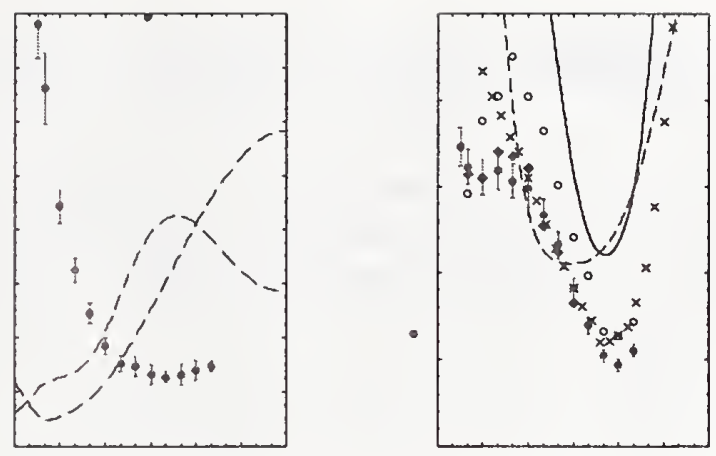

Figure 3: Comparison of theory and experiment for $\mathrm{CO}_{2}$ at $1 \mathrm{eV}$ and $5 \mathrm{eV}$. See text for explanation.

\section{References}

[1] S. Trajmar, D. F. Register, and A. Chutjian, Phys. Rep. 97, 219 (1983)

[2] J. C. Nickel, P. W. Zetner, G. Shen, and S. Trajmar, J. Phys. E 22, 730 (1989)

[3] W. Sun, M. A. Morrison, W. A. Isaacs, W. K. Trail, D. T. Alle, R. J. Gulley, M. J. Brennan, and S. J. Buckman, Phys. Rev. A 52, 1229 (1995)

[4] C. T. Bundschu, J. C. Gibson, R. J. Gulley, M. J. Brunger, S. J. Buckman, N. Sanna, and F. A. Gianturco, J. Phys. B 30, 2239 (1997)

[5] L. Boesten and H. Tanaka, J. Phys. B 24, 821 (1991)

[6] H. Tanaka, T. Ishikawa, T. Masai, T. Sagara, L. Boesten, M. Takekawa, Y. Itikawa, and M. Kimura, private communication (1997)

[7] F. A. Gianturco and T. Stoecklin, J. Phys. B 29, 3933 (1996)

[8] M. Takekawa and Y. Itikawa, J. Phys. B 29, 4222 (1996) 


\title{
Electron Interactions with Plasma Processing Gases
}

\author{
L. G. Christophorou and J. K. Olthoff \\ National Institute of Standards and Technology \\ Gaithersburg, MD 20899
}

\section{Introduction}

To assess the behavior of gases in their uses in manufacturing semiconductor devices and other applications and to promote the modeling of these processes, it is necessary to have accurate information on the fundamental interactions of low energy $(<100 \mathrm{eV})$ electrons with process gases. NIST has begun the formation of a database containing fundamental electron-interaction data for gases of interest to the plasma community. In support of this effort, we have undertaken the assessment and evaluation of the available information on cross sections and rate coefficients for collisional interactions of electrons with three groups of gases: those used in etching, deposition, or cleaning (e.g., $\mathrm{CF}_{4}, \mathrm{CHF}_{3}, \mathrm{C}_{2} \mathrm{~F}_{6}, \mathrm{C}_{3} \mathrm{~F}_{8} \mathrm{Cl}_{2}$, and $\mathrm{HBr}$ ), those used as buffer gases (e.g., Ar, $\mathrm{He}$ ), and those that are present in practical systems as impurities (e.g., $\mathrm{O}_{2}, \mathrm{~N}_{2}, \mathrm{H}_{2} \mathrm{O}$ ). In this paper, we summarize our recommended data on cross sections and rate coefficients for the gases $\mathrm{CF}_{4}, \mathrm{CHF}_{3}, \mathrm{C}_{2} \mathrm{~F}_{6}$ and $\mathrm{CCl}_{2} \mathrm{~F}_{2}$, based upon our assessment of available data, and indicate specific electron-interaction data needs for these gases. The assessed data for the four gases are available via the World Wide Web at http://www.eeel.nist.gov/811/refdata.

\section{Reference Data Sets}

Carbon Tetrafluoride $\left(\mathrm{CF}_{4}\right)$ - Figures 1 and 2 show the assessed electron scattering cross section data [1] and the assessed electron attachment, ionization, and effective ionization coefficients for $\mathrm{CF}_{4}$, respectively. The data on electron scattering cross sections for $\mathrm{CF}_{4}$ are reasonably complete, as indicated by the good agreement between the total scattering cross section and the sum of the individual cross sections shown by the dotted line in Figure 1. The coefficient data are also of good quality. The few data needs include the experimental determination of direct and indirect vibrational excitation, and investigation of the apparent discrepancy between the measured cross section for dissociation into neutrals and other related cross sections (recent unpublished measurements indicate that the experimental cross section values shown here for dissociation into neutrals are much smaller than the true values).

Trifluoromethane $\left(\mathrm{CHF}_{3}\right)$ - Figure 3 shows the meager electron scattering-cross section data [2] for this important plasma processing gas. With the possible exception of the data for total dissociation and total ionization, the rest of the data in Figure 3 are approximate. Basic measurements and calculations are needed for virtually all elastic and inelastic electron scattering processes, including momentum transfer, vibrational excitation, elastic scattering, differential scattering, and electron transport, attachment and ionization coefficients. Confirmation is needed of the cross section for total electron scattering and total ionization measurements. Resolution is also needed of the discrepancy between the measured cross section for dissociation into neutrals and other related cross section data. 
Perfluoroethane $\left(C_{2} F_{6}\right)$ - Figure 4 shows the electron-interaction cross sections for this molecule [3], although many of these are still preliminary (e.g., the cross section for momentum transfer at low energies). Only the cross sections for dissociative attachment and total dissociation are recommended, nonetheless, the cross sections in Figure 4 are the most reasonable values presently available. Figure 5 shows our recommended data for attachment, ionization and effective ionization coefficients for this molecule. Cross section data are needed especially for dissociation into neutrals and vibrational and electronic excitation. Additional data are needed for the momentum transfer, elastic integral, total ionization, and total scattering cross sections.

Dichlorodifluoromethane (Freon-12, $\mathrm{CCl}_{2} \mathrm{~F}_{2}$ ) - The available cross section data [4] for the $\mathrm{CCl}_{2} \mathrm{~F}_{2}$ molecule are shown in Figure 6. They are reasonably complete but still limited. No cross section data exist on momentum transfer and dissociation into neutrals. Further measurements are needed of the total ionization cross section, and measurements of the elastic integral cross section over a broader energy range are indicated. The attachment, ionization and effective ionization coefficients are satisfactory (Fig.7).

\section{References}

[1] L. G. Christophorou, J. K. Olthoff, and M. V. V. S. Rao, J. Phys. Chem. Ref. Data 25, 1341 (1996)

[2] L. G. Christophorou, J. K. Olthoff, and M. V. V. S. Rao, J. Phys. Chem. Ref. Data 26, 1 (1997)

[3] L. G. Christophorou and J. K. Olthoff, J. Phys. Chem. Ref. Data (in press)

[4] L. G. Christophorou, J. K. Olthoff, and Y. Wang, J. Phys. Chem. Ref. Data 26, 1205 (1997)

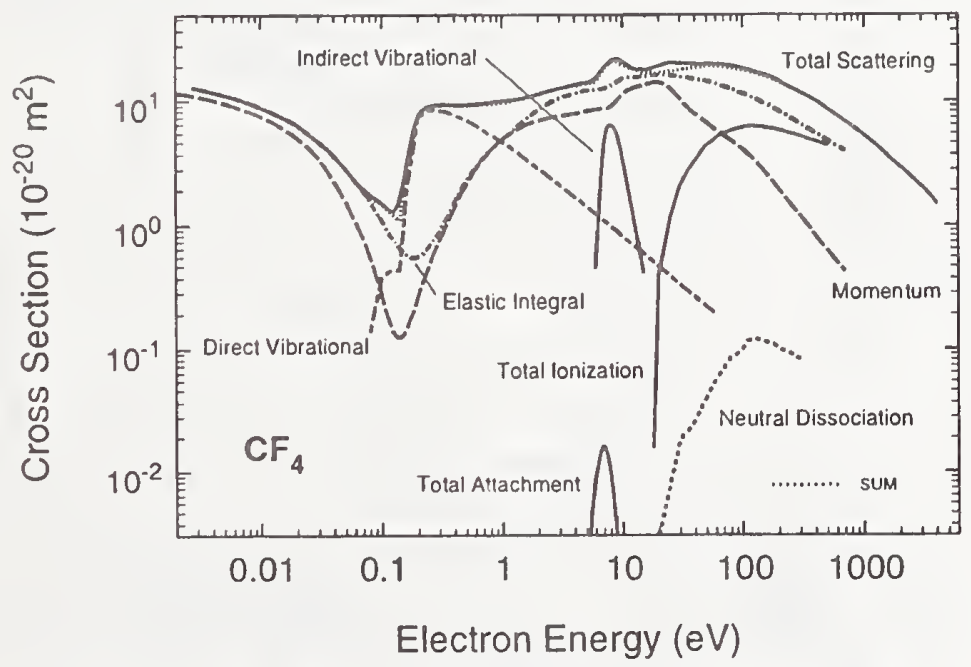

Figure 1: Electron-interaction cross sections for $\mathrm{CF}_{4}$ [1]. 


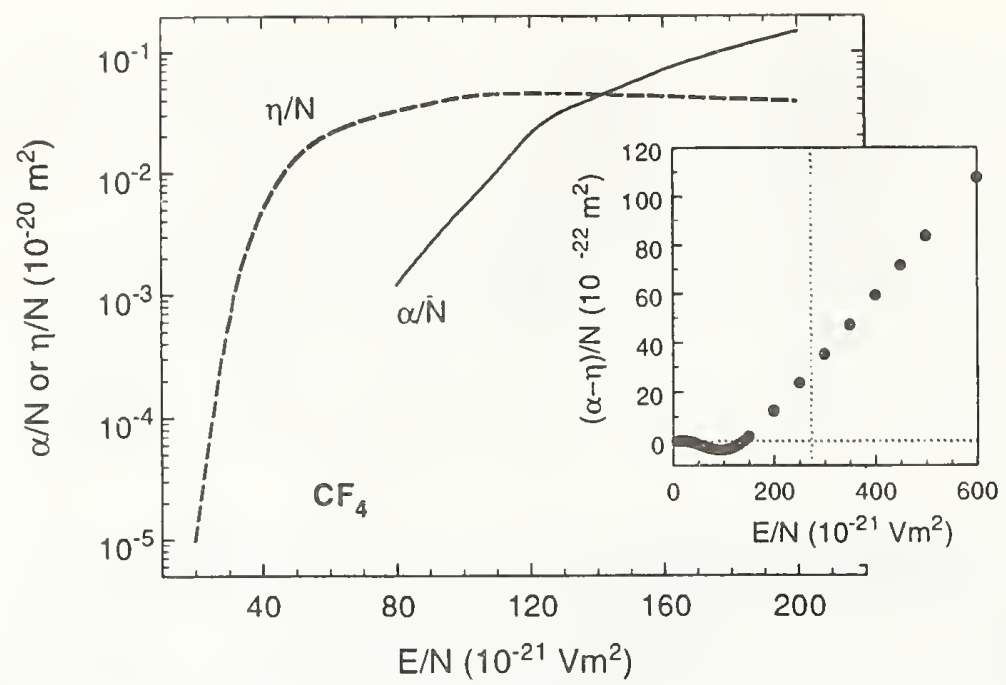

Figure 2: Electron-interaction coefficients for $\mathrm{CF}_{4}$ [1].

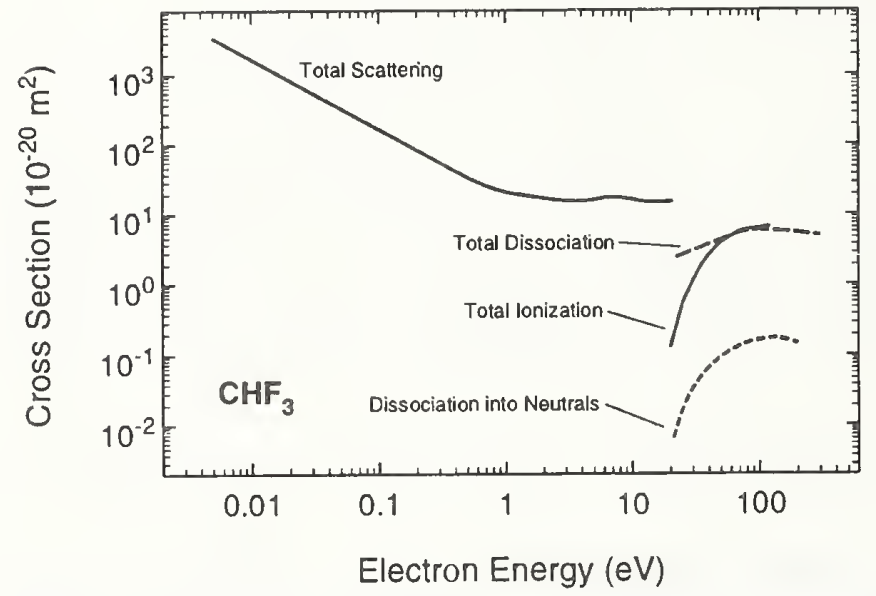

Figure 3: Electron-interaction cross sections for $\mathrm{CHF}_{3}$ [2].

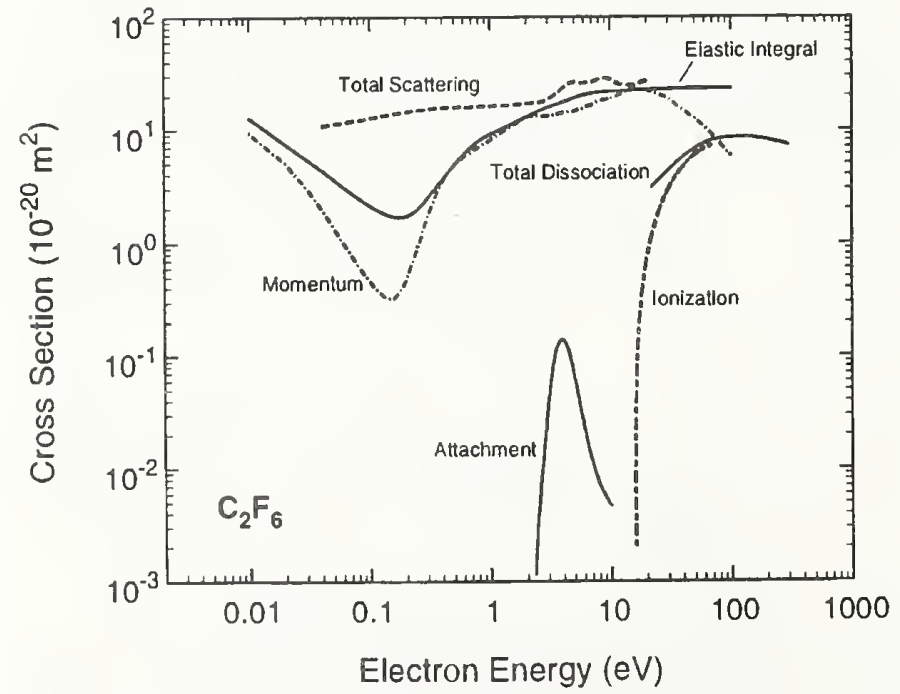

Figure 4: Electron-interaction cross sections for $\mathrm{C}_{2} \mathrm{~F}_{6}$ [3]. 


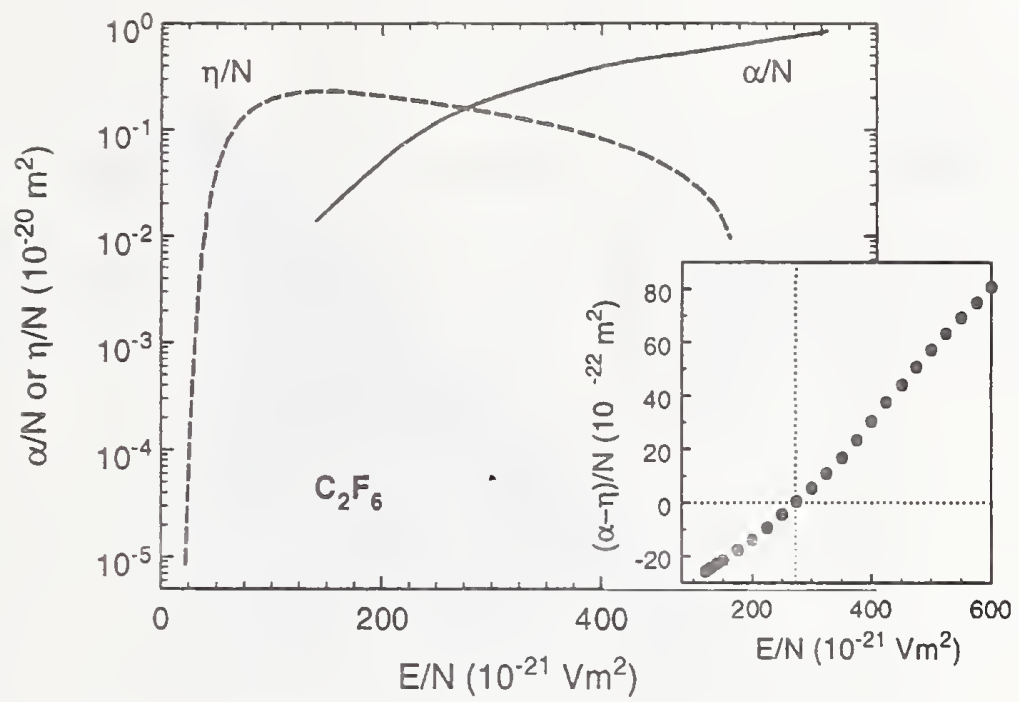

Figure 5: Electron-interaction coefficients for $\mathrm{C}_{2} \mathrm{~F}_{6}$ [3].

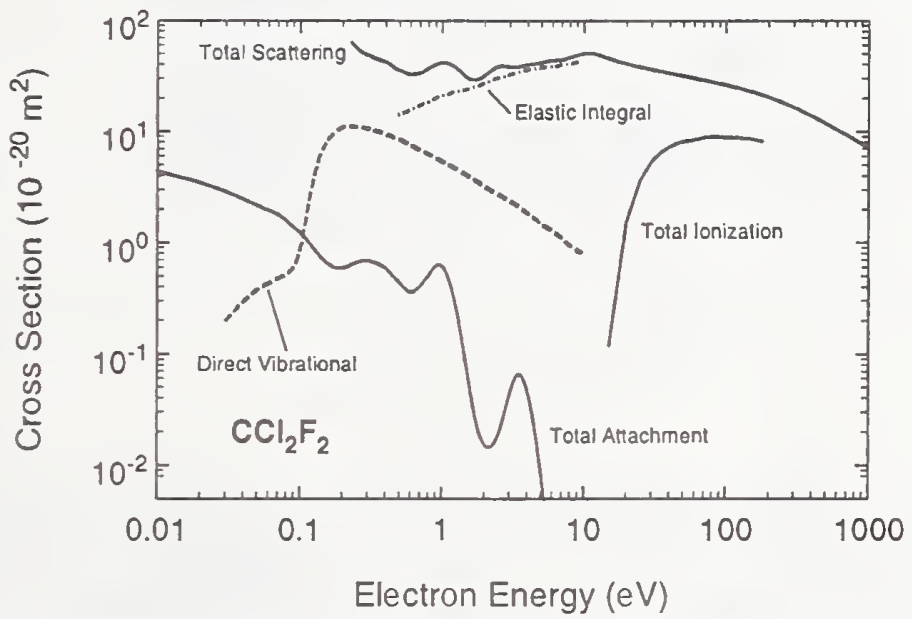

Figure 6: Electron-interaction cross sections for $\mathrm{CCl}_{2} \mathrm{~F}_{2}$ [4].

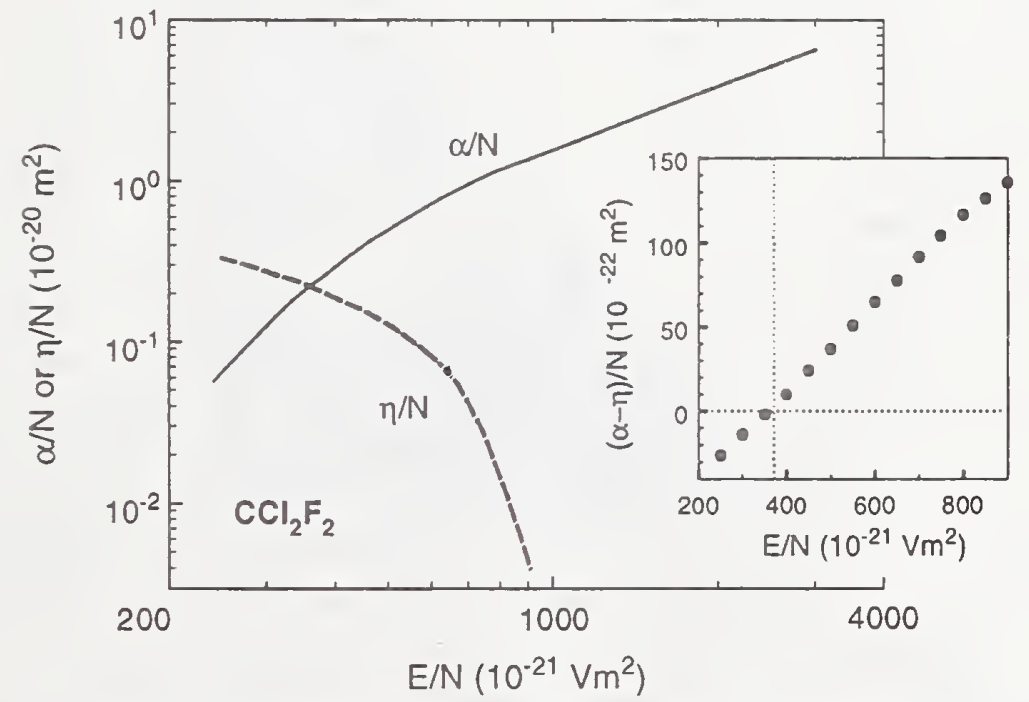

Figure 7: Electron-interaction coefficients for $\mathrm{CCl}_{2} \mathrm{~F}_{2}$ [4]. 


\title{
NIST Atomic Spectra Database
}

G. R. Dalton, R. A. Dragoset, J. R. Fuhr, D. E. Kelleher, S. A. Kotochigova, W. C. Martin, P. J. Mohr, A. Musgrove, K. Olsen, L. Podobedova,

E. B. Saloman, J. Sugar, W. L. Wiese

NIST, Gaithersburg, MD

C. Stern Grant, G. Eichhorn

Center for Astrophysics, Cambridge, $M A$

\author{
R. L. Kelly \\ Naval Postgraduate School, Monterey, CA \\ T. Shirai \\ JAERI, Tokai-mura, Japan \\ V. I. Azarov, A. E. Kramida, A. N. Ryabtsev \\ Institute for Spectroscopy, Troitsk, Russia
}

J. Blaise, J. F. Wyart
CNRS, Orsay, France

Version 1 of the NIST Atomic Spectra Database became available online as an interactive Web server in 1995. It is accessible at the NIST Physics Laboratory Web site (physics.nist.gov, select Physical Reference Data). Version 1 has data on atomic energy levels for some 500 spectra, transition probability data for spectra of the iron-group elements, and comprehensive wavelength data for spectra of several elements. It includes no data for one or more of these three types of data for many important spectra, however.

Pending new critical compilations of the most needed data, we are extending the database by editing and adding data from earlier NIST compilations, selected non-NIST compilations, and selected recent publications or unpublished material. The new Version 2 (hereafter referred to as "ASD") contains significantly more data on atomic and ionic transitions and energy levels. It should be available on the Web within a few months. ASD has data for about 950 spectra, with about 70,000 energy levels and 90,000 lines, 40,000 of which have transition probabilities.

The data available in both Versions 1 and 2 are indicated according to spectrum in Figures 1 , 2, and 3. The additional energy level data in Version 2 (Fig. 1) are largely taken from material compiled by R. L. Kelly in connection with his wavelength tables [1]. The additional transition probability data (Fig. 2) include new NIST compilations for the C, N, and O spectra [2], and data from other, mostly earlier, NIST compilations $[3,4,5,6]$. In addition to wavelengths compiled in connection with transition probability data, extensive wavelength data for the first five spectra of many elements are included from the tables of Reader et al. [7] (Fig. 3).

Version 2 thus has energy level data for most spectra of $\mathrm{H}$ through $\operatorname{Kr}(Z=1-36)$, Mo $(Z=42)$, and for 65 spectra of the rare-earth elements La through Lu $(Z=57-71)$. Wavelengths of observed 


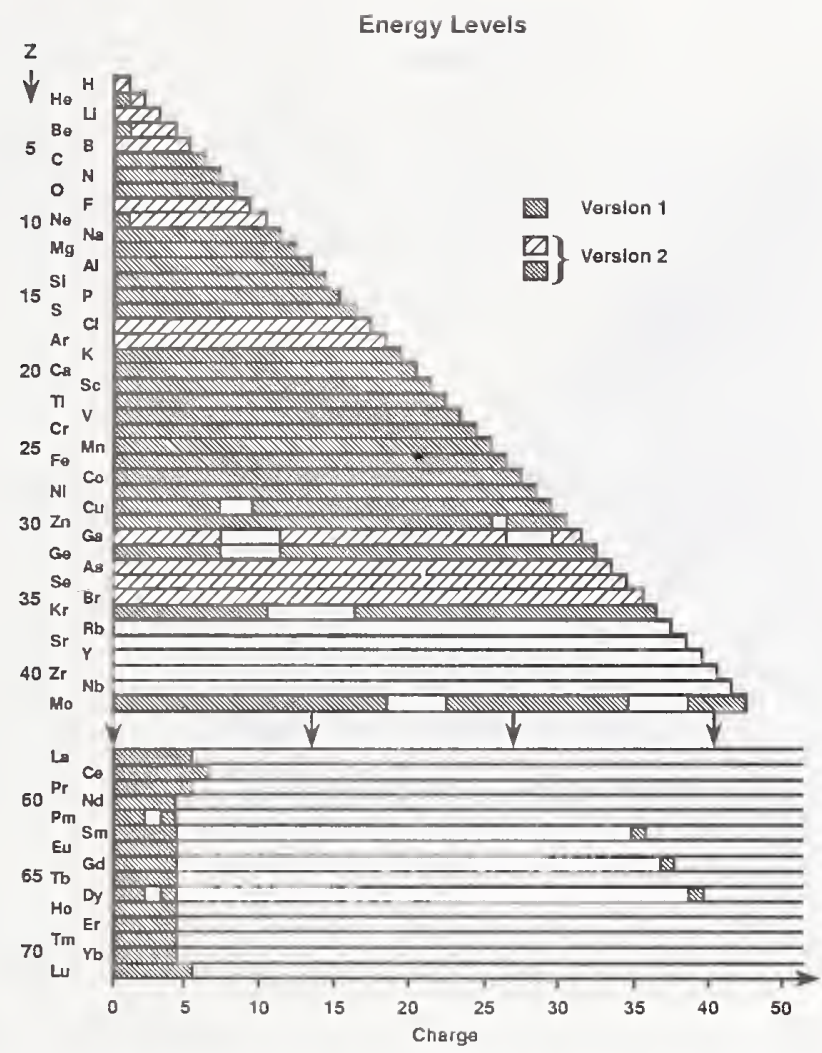

Figure 1: Holdings of energy-level data in Versions 1 and 2 of the NIST Atomic Spectra Database.

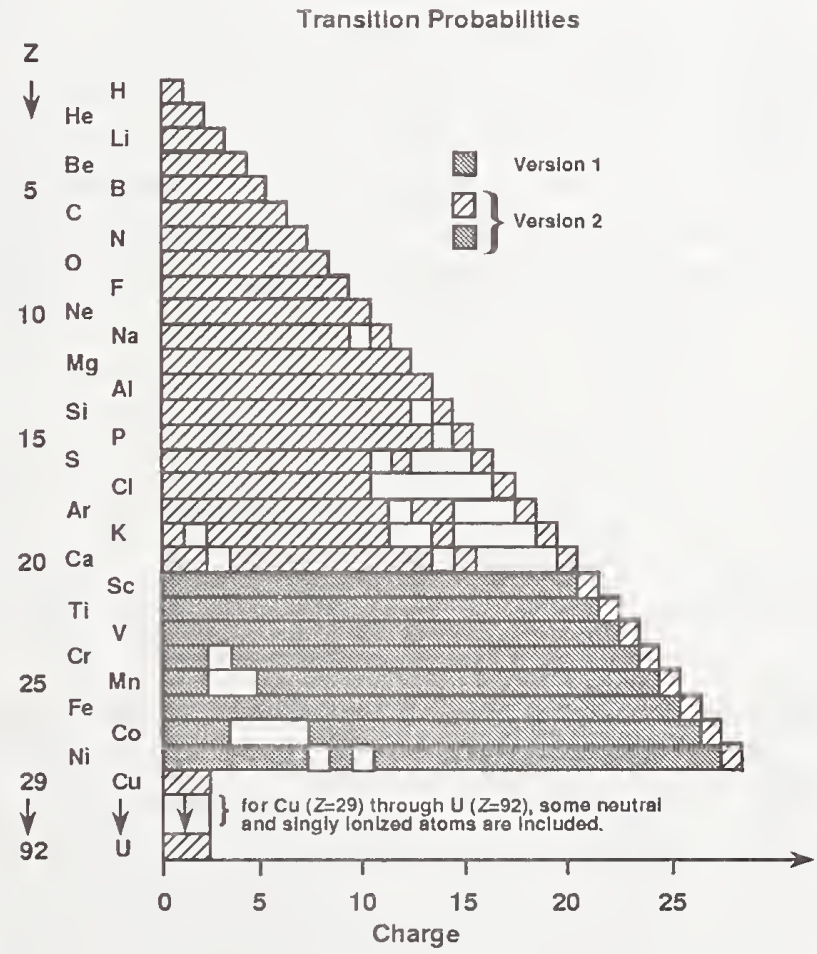

Figure 2: Holdings of transition-probability data in Versions 1 and 2 of the NIST Atomic Spectra Database. 


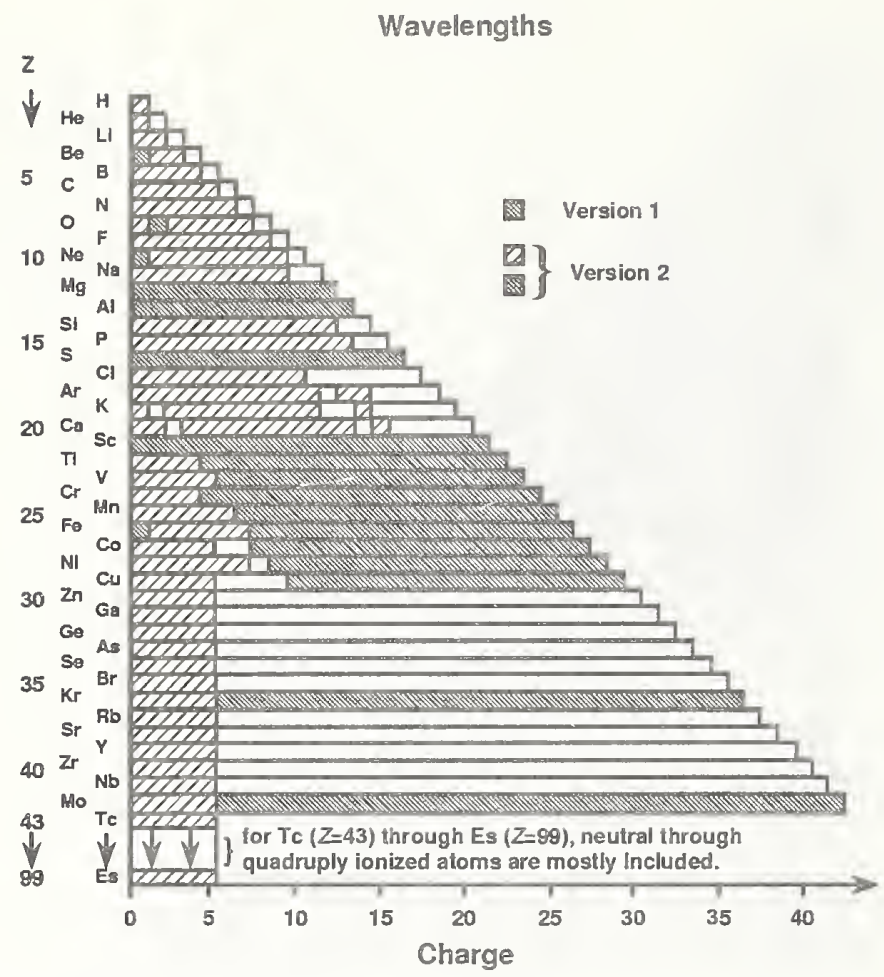

Figure 3: Holdings of wavelength data in Versions 1 and 2 of the NIST Atomic Spectra Database.

transitions are included for 99 elements. Energy-level classifications and transition probabilities are included for the lines of most spectra of $\mathrm{H}$ through $\mathrm{Ni}(Z=1-28)$. Comprehensive lists of observed wavelengths with classifications based on critically compiled level data are available for some elements, including all spectra of $\mathrm{Mg}, \mathrm{Al}, \mathrm{S}$, and Sc. Wavelengths without level classifications are included for prominent lines of up to the first five spectra of $\mathrm{Cu}$ through Es $(Z=29-99)$, with selected transition probabilities for the first two spectra. Several extensive data sets from recent NIST compilations are being prepared for inclusion in the database during the next year.

The two broad categories of queries handled by ASD are for data on energy levels and for data on spectral lines. The data are displayed in formats similar to those in NIST print publications of atomic spectroscopic data. In addition to graphical user interface format, users can choose output in an ASCII form easily read by spread-sheet software. ASD offers a range of user-specified options and selection criteria, each with its own default. For example, for transition strengths one may choose to search or display any combination of transition probability, oscillator strength, line strength, $\log (g f)$, and relative intensity. One may also choose from a variety of units and selection criteria on transition-strength accuracy and properties of energy levels. Outputs for transitions may be ordered by wavelength or by multiplets for a particular spectrum.

The database search programs for ASD are written in ANSI C and are thus portable to any environment, including PC's and UNIX-based machines. Only the graphical user interface for input/output is platform-specific. ASD comes with a built-in character-based interface, which itself is platform independent, and thus facilitates its inclusion in other character-based environments such as ALADDIN. In addition to the Web version, a PC version with a Windows interface will be made available on diskettes for purchase. This will replace the currently available DOS version. 


\section{Acknowledgements}

We gratefully acknowledge partial funding of work on this database by the U.S. Department of Energy, Office of Fusion Energy Sciences, and by the UV, Visible and Gravitational Astrophysics Division of NASA. We also appreciate support under a Collaborative Research Grant, CRG 930793, from NATO.

\section{References}

[1] R. L. Kelly, "Atomic and Ionic Spectrum Lines Below 2000 A: Hydrogen through Krypton," J. Phys. Chem. Ref. Data 16, Supplement 1 (1987)

[2] W. L. Wiese, J. R. Fuhr, and T. M. Deters, "Atomic Transition Probabilities of Carbon, Nitrogen, and Oxygen," J. Phys. Chem. Ref. Data Monograph 7 (1996)

[3] W. L. Wiese, M. W. Smith, and B. M. Glennon, "Atomic Transition Probabilities (H through Ne-A Critical Data Compilation," Natl. Stand. Ref. Data Ser., Natl. Bur. Stand. (U.S.) 4, Vol. I (1966)

[4] W. L. Wiese, M. W. Smith, and B. M. Miles, "Atomic Transition Probabilities (Na through Ca-A Critical Data Compilation," Natl. Stand. Ref. Data Ser., Natl. Bur. Stand. (U.S.) 22, Vol. II (1969)

[5] NIST Atomic Transition Probability Tables, J. R. Fuhr and W. L. Wiese, in "CRC Handbook of Chemistry and Physics," 77th Edition, D. R. Lide, ed., p. 10-128 - 10-186, CRC Press, Boca Raton, FL 1996

[6] Atomic Spectroscopy, W. C. Martin and W. L. Wiese, in "Atomic, Molecular, and Optical Physics Handbook," G. W. F. Drake, ed., Ch. 10, p. 135-153, AIP Press, New York 1996

[7] J. Reader, C. H. Corliss, W. L. Wiese, and G. A. Martin, "Wavelengths and Transition Probabilities for Atoms and Atomic Ions, Part I. Wavelengths, Part II. Transition Probabilities," Natl. Stand. Ref. Data Ser., Natl. Bur. Stand. (U.S.) 68 (1980) 


\title{
Atomic Database Development of Mid-to-High-Z Elements
}

\author{
Arati Dasgupta and Paul C. Kepple \\ Radiation Hydrodynamics Branch, Plasma Physics Division, \\ Naval Research Laboratory, Washington, DC 20375
}

\section{Introduction}

Detailed atomic databases are needed for some applications, such as in hydrodynamics codes for plasma diagnostics and analysis of x-ray spectra. For plasma diagnostics requiring data for many ionization stages with many fine-structure levels, atomic models developed using very complex calculations have limitations. Again, for accurate diagnostics, a detailed level accounting cannot be simplified by an averaging process such as used in the average atom model, which will inhibit all the important and relevant radiation dynamics. To include a large number of states with a minimum of computational effort, we have developed atomic models where many of the multiplet levels are lumped into the configuration states, but the important low-lying states are kept at fine structure levels and they are connected by the relevant atomic processes to allow detailed analysis of diagnostically important emission processes.

This model for F-like and Ne-like stages has already been used for diagnosing[1] selenium plasmas. In the present work we use this model to calculate databases for all L- and M-shell ionization stages of titanium. Currently, z-pinch experiments are being performed using titanium wires producing $\mathrm{x}$-ray emission with very high yields. Titanium is also an important element in tokamak discharges for fusion research. Our complete database will include both radiative and collisional data, including dielectronic recombination (DR) connecting all the levels for all the ion stages. However, we will present here only partial data for Ti XIX and Ti XX.

Effectiveness of this method will be evaluated by comparing our results with other published calculations, and these theoretical methods used to calculate the data for all important processes when properly benchmarked by measurements can provide adequate data.

\section{Atomic Model}

For the Ti XIX and TI XX ion stages we have kept all the $n=2$ and 3 fine structure levels while the higher lying excited levels are lumped in our model. The Ti XX model consists of 10 levels including the ground level, two $\Delta n=02 p$ excited state, 5 fine structure levels of the $n=3$ manifold, one lumped $n=4$ level and one lumped level of $n=5,6$, and 7 excited states. The Ti XIX model contains 25 levels including the ground state, fine structure levels of $9 n=2$ and $10 n=3$ excited states, 3 lumped inner-shell excited configurations of $2 p 3 l$, one lumped $n=4$ level and one lumped level of $n=5,6$, and 7 states. All the excited levels are fully coupled to the ground states and among themselves for each ion. Table 1 lists the energy level designations and energies of these levels.

The atomic structure data such as the energies and radiative transition probabilities for the finestructure levels are calculated using the DFW atomic code of Sampson et al.[2]. For the lumped $n=3$ states, these structure data are obtained using the HFR (Hartree-Fock with Relativistic corrections) method of Cowan[3]. These radiative transition rates from the excited levels to the ground states 
for Ti XX and XIX are also included in Table 1. Our level energies and transition probabilities agree very well with other state-of-the-art calculations.

For dipole allowed transitions, collisional data are obtained using the Semiclassical Impact Parameter (SCIP) method[4] and for other transitions, the Coulomb Born Distorted Wave (CBDW) method[5] is used to calculate collisional data coupling the levels in our model. For diagnostically important transitions when either the SCIP or the CBDW methods are inadequate, collisional data are obtained using a suite of more sophisticated fully relativistic atomic structure and distorted wave (RDW) codes[2, 6]. Collision strengths for some of the transitions for Ti XX and Ti XIX are shown in Tables 2 and 3 and they are compared to those obtained using another DW calculation[7]. Our collisional excitation data will be updated by including resonance excitation in the future. Electronimpact ionization cross sections are calculated by the exchange classical impact-parameter method. Again, for important transitions the ionization cross sections are replaced by those calculated using the RDW ionization codes of Ref. [8].

The ground to ground DR rates can be approximated by the semi-empirical formulas derived by Burgess and modified by Merts, Cowan, and Magee (BM)[9]. However for ions of interest, more accurate and detailed state-specific DR data are obtained using the HFR method of Cowan[3]. DR data such as autoionization rates and DR branching ratios from the ground and $\Delta n=0$ states of the recombining ion to each specific fine structure level of the recombined ions are calculated. For low lying doubly-excited states, DR branching ratios are explicitly calculated, while for higher Rydberg states, we used a $1 / n^{3}$ falloff extrapolation of the DR branching ratios. The DR rates for Ti XX and TI XIX are shown in Table 4. Our total ground-to-ground DR rates for Ti XX agree very well with those obtained by Chen using a multi-configuration Dirac-Fock calculation[10].

\section{References}

[1] A. Dasgupta, K. G. Whitney, H. L. Zhang, and D. H. Sampson, Phys. Rev. E 55, 3460 (1997)

[2] D. H. Sampson, H. L. Zhang, A. K. Mohanty, and R. E. H. Clark, Phys. Rev. A 40, 604 (1989)

[3] Atomic Structure Code of R. D. Cowan, "The Theory of Atomic Structure and Spectra", University of California Press, Berkeley, CA 1981

[4] E. Oran and J. Davis, J. Appl. Phys. 45, 2480 (1974)

[5] J. Davis, P. C. Kepple, and M. Blaha, J. Quant. Spectrosc. Radiat. Transfer 16, 1043 (1977)

[6] H. L. Zhang, D. H. Sampson, and A. K. Mohanty, Phys. Rev. A 40, 616 (1989); D. H. Sampson, H. L. Zhang, and C. J. Fontes, At. Data Nucl. Data Tables 48, 25 (1991); H. L. Zhang and D. H. Sampson, Phys. Rev. A 47, 208 (1993)

[7] R. E. H. Clark, J. Abdallah, Jr., G. Csanak, J. B. Mann, and R. D. Cowan, Theoretical Atomic Physics Code Development I. ACE: Another Collisional Excitation Code, Los Alamos National Laboratory manual (December 1988)

[8] H. L. Zhang and D. H. Sampson, Phys. Rev. A 42, 5378 (1990); D. H. Sampson and H. L. Zhang, Phys. Rev. A 45, 1657 (1992)

[9] A. Burgess, Astrophys. J. 141, 1589 (1965); A. L. Merts, R. D. Cowan, and N. M. Magee, Jr., Los Alamos Scientific Laboratory Report No. LA-6220-MS, 1976 (unpublished)

[10] M. H. Chen, Phys. Rev. A 33, 994 (1986) 
Table 1: Level identifications, state designations, energies and radiative decay rates to the ground levels for Ti XX and Ti XIX.

\begin{tabular}{|c|c|c|c|c|c|c|c|}
\hline \multicolumn{4}{|c|}{ Ti XX } & \multicolumn{4}{|c|}{ Ti XIX } \\
\hline level & state & Eng (eV) & $A^{r}\left(s^{-1}\right)$ & level & state & Eng (eV) & $A^{T}\left(s^{-1}\right)$ \\
\hline 1 & $1 s^{2} 2 s^{2} S_{1 / 2}$ & 0.0 & & 1 & $1 s^{2} 2 s^{2}{ }^{1} S_{0}$ & 0.0 & \\
\hline 2 & $1 s^{2} 2 p^{2} P_{1 / 2}^{o}$ & $4.0287[1]$ & $1.466[9]$ & 2 & $1 s^{2} 2 s 2 p{ }^{3} P_{0}^{o}$ & $3.5842[1]$ & \\
\hline 3 & $1 s^{2} 2 p^{2} P_{3 / 2}^{o}$ & $4.7966[1]$ & $2.504[9]$ & 3 & $1 s^{2} 2 s 2 p^{3} P_{1}^{o}$ & $3.7888[1]$ & $1.274[7]$ \\
\hline 4 & $1 s^{2} 3 s^{2} S_{1 / 2}$ & $8.0163[2]$ & & 4 & $1 s^{2} 2 s 2 p{ }^{3} P_{2}^{o}$ & $4.3145[1]$ & \\
\hline 5 & $1 s^{2} 3 p^{2} P_{1 / 2}^{o}$ & $8.1276[2]$ & $3.558[12]$ & 5 & $1 s^{2} 2 s 2 p{ }^{1} P_{1}^{o}$ & $7.4791[1]$ & $1.408[10]$ \\
\hline 6 & $1 s^{2} 3 p^{2} P_{3 / 2}^{o}$ & $8.1504[2]$ & $3.473[12]$ & 6 & $1 s^{2} 2 p^{2}{ }^{3} P_{0}$ & $9.6645[1]$ & \\
\hline 7 & $1 s^{2} 3 d^{2} D_{3 / 2}$ & $8.1926[2]$ & & 7 & $1 s^{2} 2 p^{2}{ }^{3} P_{1}$ & $1.0018[2]$ & \\
\hline 8 & $1 s^{2} 3 d^{2} D_{5 / 2}$ & $8.1998[2]$ & & 8 & $1 s^{2} 2 p^{2}{ }^{3} P_{2}$ & $1.0370[2]$ & \\
\hline 9 & $1 s^{2} 4 l$ & $1.0851[3]$ & $2.923[11]$ & 9 & $1 s^{2} 2 p^{2}{ }^{1} D_{2}$ & $1.1509[2]$ & \\
\hline 10 & $1 s^{2} 5 l-7 l$ & $1.2663[3]$ & $4.298[10]$ & 10 & $1 s^{2} 2 p^{2}{ }^{1} S_{0}$ & $1.3894[2]$ & \\
\hline & & & & 11 & $1 s^{2} 2 s 3 s{ }^{3} S_{1}$ & $7.6600[2]$ & \\
\hline & & & & 12 & $1 s^{2} 2 s 3 s^{1} S_{0}$ & $7.7404[2]$ & \\
\hline & & & & 13 & $1 s^{2} 2 s 3 p{ }^{3} P_{0}^{o}$ & $7.8239[2]$ & \\
\hline & & & & 14 & $1 s^{2} 2 s 3 p^{3} P_{1}^{o}$ & $7.8279[2]$ & $4.073[11]$ \\
\hline & & & & 15 & $1 s^{2} 2 s 3 p{ }^{3} P_{2}^{o}$ & $7.8447[2]$ & \\
\hline & & & & 16 & $1 s^{2} 2 s 3 p{ }^{3} P_{1}^{o}$ & $7.8683[2]$ & $5.508[12]$ \\
\hline & & & & 17 & $1 s^{2} 2 s 3 d^{3} D_{1}$ & $7.9349[2]$ & \\
\hline & & & & 18 & $1 s^{2} 2 s 3 d^{3} D_{2}$ & $7.9369[2]$ & \\
\hline & & & & 19 & $1 s^{2} 2 s 3 d^{3} D_{3}$ & $7.9407[2]$ & \\
\hline & & & & 20 & $1 s^{2} 2 s 3 d^{3} D_{2}$ & $8.0075[2]$ & \\
\hline & & & & 21 & $1 s^{2} 2 p 3 s$ & $8.0981[2]$ & $1.421[10]$ \\
\hline & & & & 22 & $1 s^{2} 2 p 3 p$ & $8.2513[2]$ & \\
\hline & & & & 23 & $1 s^{2} 2 p 3 d$ & $8.3612[2]$ & $2.604[10]$ \\
\hline & & & & 24 & $1 s^{2} 2 s 4 l$ & $1.0312[3]$ & $1.261[11]$ \\
\hline & & & & 25 & $1 s^{2} 2 s 5 l-7 l$ & $1.1967[3]$ & $1.807[10]$ \\
\hline
\end{tabular}

Table 2: Collision strengths for some of the $n=2-2$ transitions in Ti XIX.

\begin{tabular}{|c|c|c|c|c|}
\hline Transition & Transition Energy (eV) & $E / E_{t h}$ & This Work & Ref. [7] \\
\hline \multirow[t]{5}{*}{$2 s^{2}{ }^{1} S-2 s 2 p{ }^{1} P$} & $6.793[2]$ & 1.05 & $4.27[-1]$ & $4.28[-1]$ \\
\hline & & 3.47 & $4.84[-1]$ & $4.82[-1]$ \\
\hline & & 6.38 & $5.46[-1]$ & $5.22[-1]$ \\
\hline & & 9.85 & $6.04[-1]$ & $5.62[-1]$ \\
\hline & & 14.0 & $6.55[-1]$ & $6.06[-1]$ \\
\hline \multirow[t]{5}{*}{$2 s 2 p^{1} P-2 p^{2}{ }^{1} D$} & $4.413[2]$ & 1.05 & $8.47[-1]$ & $9.55[-1]$ \\
\hline & & 3.47 & $9.58[-1]$ & 1.04 \\
\hline & & 6.38 & 1.08 & 1.10 \\
\hline & & 9.85 & 1.19 & 1.18 \\
\hline & & 14.0 & 1.29 & 1.27 \\
\hline \multirow[t]{4}{*}{$2 s^{2}{ }^{1} S-2 s 2 p{ }^{3} P_{0}$} & $3.548[2]$ & 1.04 & $1.83[-3]$ & $2.07[-3]$ \\
\hline & & 1.64 & $1.76[-3]$ & $2.03[-3]$ \\
\hline & & 4.04 & $1.63[-3]$ & $1.88[-3]$ \\
\hline & & 9.04 & $1.39[-3]$ & $1.60[-3]$ \\
\hline
\end{tabular}


Table 3: Collision strengths for some of the $n=2-2,3$ transitions in Ti XX.

\begin{tabular}{|c|c|c|c|c|}
\hline Transition & Transition Energy $(\mathrm{eV})$ & $E / E_{t h}$ & This Work & Ref. [7] \\
\hline \multirow[t]{4}{*}{$2 s{ }^{2} S-2 p{ }^{2} P_{1 / 2}$} & $4.036[2]$ & 1.05 & $1.93[-1]$ & $2.16[-1]$ \\
\hline & & 3.47 & $2.17[-1]$ & $2.34[-1]$ \\
\hline & & 6.38 & $2.42[-1]$ & $2.46[-1]$ \\
\hline & & 14.0 & $2.92[-1]$ & $2.79[-1]$ \\
\hline \multirow[t]{4}{*}{$2 s^{2} S-2 p{ }^{2} P_{3 / 2}$} & $4.793[2]$ & 1.05 & $3.80[-1]$ & $4.20[-1]$ \\
\hline & 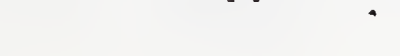 & 3.47 & $4.28[-1]$ & $4.57[-1]$ \\
\hline & & 6.38 & $4.79[-1]$ & $4.82[-1]$ \\
\hline & & 14.0 & $5.76[-1]$ & $5.50[-1]$ \\
\hline \multirow[t]{4}{*}{$2 s^{2} S-3 d^{2} D_{3 / 2}$} & $8.201[3]$ & 1.30 & $1.98[-2]$ & $2.43[-2]$ \\
\hline & & 2.20 & $2.28[-2]$ & $2.84[-2]$ \\
\hline & & 4.00 & $2.59[-2]$ & $3.37[-2]$ \\
\hline & & 9.00 & $2.84[-2]$ & $3.91[-2]$ \\
\hline
\end{tabular}

Table 4: Dielectronic recombination rate coefficients (in $10^{-11} \mathrm{~cm}^{3} / \mathrm{sec}$ ) from the ground state of Ti XXI and the ground and $\Delta n=0$ levels of Ti XX.

\begin{tabular}{|c|c|c|c|c|c|}
\hline \multirow{3}{*}{$\begin{array}{l}\mathrm{T} \\
(\mathrm{keV})\end{array}$} & \multicolumn{2}{|c|}{ Ti XXI } & \multicolumn{3}{|c|}{ Ti XX } \\
\hline & \multirow[t]{2}{*}{ This calc. } & \multirow[t]{2}{*}{ Ref. [10] } & \multicolumn{2}{|c|}{$1 s^{2} 2 s^{2} S$} & \multirow[t]{2}{*}{$1 s^{2} 2 p^{2} P$} \\
\hline & & & $\Delta n=0$ & $\Delta n=1$ & \\
\hline 0.02 & & & 14.9 & $1.44(-4)$ & $9.91(-4)$ \\
\hline 0.04 & & & 8.26 & $1.72(-2)$ & $5.00(-2)$ \\
\hline 0.06 & & & 5.33 & $6.79(-2)$ & $1.49(-1)$ \\
\hline 0.08 & & & 3.79 & $1.28(-1)$ & $2.47(-1)$ \\
\hline 0.10 & & & 2.87 & $1.92(-1)$ & $3.42(-1)$ \\
\hline 0.20 & $3.79(-7)$ & $3.57(-7)$ & 1.14 & $5.22(-1)$ & $7.91(-1)$ \\
\hline 0.30 & $5.50(-5)$ & $5.25(-5)$ & $6.43(-1)$ & $7.11(-1)$ & 1.01 \\
\hline 0.50 & $2.50(-3)$ & $2.41(-3)$ & $3.08(-1)$ & $7.43(-1)$ & $9.95(-1)$ \\
\hline 1.00 & $3.26(-2)$ & $3.20(-2)$ & $1.12(-1)$ & $4.99(-1)$ & $6.41(-1)$ \\
\hline 2.00 & $7.61(-2)$ & $7.40(-2)$ & $3.99(-2)$ & $2.46(-1)$ & $3.09(-1)$ \\
\hline 3.00 & $7.88(-2)$ & $7.71(-2)$ & & & \\
\hline 4.00 & $7.08(-2)$ & $6.91(-2)$ & $1.42(-2)$ & $1.03(-1)$ & $1.28(-1)$ \\
\hline 5.00 & $6.16(-2)$ & $6.07(-2)$ & & & \\
\hline 6.00 & $5.34(-2)$ & $5.15(-2)$ & & & \\
\hline 8.00 & $4.09(-2)$ & $3.93(-2)$ & & & \\
\hline 10.00 & $3.22(-2)$ & $3.11(-2)$ & $3.61(-3)$ & $2.88(-2)$ & $3.55(-2)$ \\
\hline
\end{tabular}




\title{
GAPHYOR Data Center http://gaphyor.lpgp.u-psud.fr
}

\author{
Jean-Loup Delcroix, Denis Humbert, Claudette Leprince \\ delcroix@supelec.fr, gaphyor@lpgp.u-psud.fr \\ Université Paris-Sud, Orsay
}

The GAPHYOR Data Center produces a Database on the properties of atoms, molecules and neutral or ionized gases, including Chemical Reactions, with about 500,000 entries.

\section{Structure of a GAPHYOR record}

The structure of a GAPHYOR entry was originally conceived to describe collisions/reactions in gases. It nów describes also simpler (energy levels, ...) or more complex (gas-surface interactions, clusters, ...) phenomena. Every GAPHYOR entry includes the following fields:

$$
\begin{gathered}
\text { /se/M1,M2,M3/pr/M4,M5,M6/ } \\
\text { /info/enva/val/rem/ } \\
\text { /an/jo,vo,page/au1,au2,...au10/nastci/ }
\end{gathered}
$$

\section{Initial and Final state: M1 M2 M3 - M5 M6 M7}

Atomic and molecular reactants: /n form ix/s/

- form Chemical formula: Example /H1C3N1O2/.

1 to 4 elements, no more than 8 atoms in the molecule.

- i Index of ionization, $x$ Index of excitation, s Index of internal structure.

- $\mathbf{n}$ Multiplicity of the Reactant (e.g. $2 \mathrm{H}$ atoms in initial state).

Electrons, photons, fields, particles.

Special Reactants:

- Iso-electronic series. Example: $\mathrm{Aa}(\mathrm{He}) \mathrm{Z}(02-12)$

- Clusters. Examples: $\mathrm{He}(\mathrm{H} 2 \mathrm{O}) 3 /+, \quad$ (C)60

- Surface reactants (solid/liquid wails, adsorbed atoms/molecules). 


\section{Sections SE and Processes PR}

GAPHYOR is a "broad band" Database organized into 5 Sections:

- 1 Properties of isolated atoms and molecules

- 2 Photonic Collisions

- 3 Electronic Collisions

- 4 Collisions between atoms or molecules

- 5 Macroscopic properties of Gases

\begin{tabular}{|l|ccccc|c|}
\hline & Struct. & $h v$ coll. & $e$ coll. & At.mol.coll. & Mac.prop. & $\Sigma$ \\
\hline Fact.Data & 267178 & 26941 & 29743 & 124753 & 32006 & 480621 \\
Num.Data & 6642 & 347 & 1650 & 9010 & 734 & 18383 \\
\hline$\Sigma$ & 272730 & 27244 & 30810 & 132107 & 32642 & 495533 \\
\hline
\end{tabular}

Table 1: Statistics of Data (June 15th, 1997)

The list of Processes (PR) included in every Section (SE) is given in Appendix 1.

\section{Additional information INFO}

- Type of Data: $\mathbf{S}$ Total and transport cross sections, $\mathbf{R}$ Reaction rate constants, ...

- Nature of Data: T Theoretical data, E Experimental data, ...

- Energy domains: L Thermal, J Medium, H High energies

- Special information: 8 Clusters, / Gas-surface interactions, ...

\section{Numerical Data}

- ENVA gives the energy, or energy range covered by the Data. Example: $10-50-100 \mathrm{eV}$

- VAL gives the values them-selves (cross-sections, ...). Example: $3.5-12.2-6.4 \mathrm{E}-16 \mathrm{~cm}^{2}$

- REM gives additional information (e.g. spectroscopic notations).

Note that the general idea is to give simple information (eg. lifetimes, reaction rates), or some orders of magnitude.

\section{Internet server}

GAPHYOR WWW server is now well developed and user-friendly. Its main features are:

- Query forms. Two query forms (simple and advanced search) are offered.

- Intermediate report. This gives a sketch of the first results of a search and offers some specific additional choices to the user. 
- Data displays. Two displays are offered: a full display of all the found entries (possibly selecting the most recent ones), or, for large Data sets, a tabular display obtained by downloading a postscript file, which can then be printed locally.

Simplified statistics on User Locations are shown below.

\begin{tabular}{|cc|cc|}
\hline na & data sets & org.na & data sets \\
\hline fr & 6098 & u-psud.fr & 1099 \\
$? ?$ & 2293 & uni-heidelberg.de & 857 \\
de & 1194 & cnrs-imn.fr & 597 \\
ru & 1151 & univ-lille1.fr & 580 \\
us & 993 & cea.fr & 535 \\
jp & 530 & onera.fr & 337 \\
$\ldots$ & $\ldots$ & $\ldots$ & $\ldots$ \\
\hline$\Sigma$ & 14885 & & 14885 \\
\hline
\end{tabular}

Table 2: Nations and Organizations using GAPHYOR

\section{GAPHYOR Handbook}

GAPHYOR is going to publish a new (fully redesigned) version of the HANDBOOK. The Volumes will be grouped in two Series:

- Chemical Volumes (17) describing the properties of a given group of Chemical systems.

- Thematic Volumes (9) describing a given group of Processes for all Chemical Systems.

The publication timing will be a function of the various orders from users for one or several specific Volumes. The sizes are small, so as to make it easy for every user to order only the Volumes covering their own field of interest.

The orders (see form in the Web) will be satisfied within a period of one month after receipt of payment.

\begin{tabular}{|c|c|c|c|c|}
\hline & Chemical Volumes & Selection & Pages & Price \$ \\
\hline $\mathrm{C} 01$ & Hydrogen,Deuterium, Tritium & $62 \%$ & 130 & 12 \\
\hline $\mathrm{C} 02$ & $\mathrm{H}+$ Noble Gases $(\mathrm{He}, \mathrm{Ne}, \mathrm{Ar}, \mathrm{Kr}, \mathrm{Xe}, \mathrm{Rn})+.$. & $72 \%$ & 130 & 12 \\
\hline $\mathrm{C} 03$ & $\mathrm{H}+$ Mono-, bi- and trivalent elements..+ & $60 \%$ & 130 & 12 \\
\hline $\mathrm{C} 04$ & $\mathrm{H}+$ Carbon/Titanium Groups..+ & $48 \%$ & 410 & 30 \\
\hline$\ldots$ & see List in the Web & $\ldots$ & $\ldots$ & $\ldots$ \\
\hline \multirow[t]{2}{*}{ C17 } & Surfaces/Clusters & $52 \%$ & 130 & 12 \\
\hline & Thematic Volumes & & & \\
\hline T00 & Cross Sections/Reaction Rates (Numerical values) & $100 \%$ & 110 & 10 \\
\hline$\ldots$ & see List in the Web & $\ldots$ & $\ldots$ & $\ldots$ \\
\hline $\mathrm{T} 30$ & Electronic Collisions (Section 3) & $62 \%$ & 210 & 18 \\
\hline \multirow[t]{2}{*}{ T50 } & Macro.Prop. of Gases (Section 5: Transport coefficients,..) & $58 \%$ & 210 & 15 \\
\hline & & $\Sigma$ & 4640 & 321 \\
\hline
\end{tabular}

Table 3: Handbook Volumes 


\section{Appendix 1}

Section 1. Structure

EN Energy levels, wave functions

EA Unstable energy levels

EI Energy of isomerization

VR Potentiel curves, structure of molecules

DP Dipolar moments

PE Electric (or magnetic) polarizability

TR Radiative transition (probability,...)

IN Autoionization

DT Autodetachment

Section 2. Photonic collisions

SN Effective absorption, total diffusion

AN True absorption

SC Angular diffusion (scattering)

EL Elastic diffusion (Thomson, Rayleigh)

DO Depolarization, Change of polarization

EX Photoexcitation

ER Emission of line

DX Photodeexcitation (stimulated emission)

FF Free-free absorption(inv. bremsstrahlung)

IN Photoionization

IM Creation of an ion pair (positive-negative)

DT Photodetachment

DS Photodissociation

EE Photoemission of electrons (ions) by solids

\section{Section 3. Electronic Collisions}

SN Total cross sections

SM Transport cross sections (momentum, ...)

EL Elastic collision

DO Depolarization, Change of polarization

EX Excitation

DX Deexcitation

BS Bremsstrahlung

IN Ionization

IM Creation of an ion pair (positive-negative)

DT Detachment

AT Attachment

RR Radiative recombination

RD Dielectronic recombination

$\mathrm{RE}$ e-e-i recombination

RO e-i-o recombination

RS Dissociative recombination

DS Dissociation

DG Desorption

EE Emission of electrons by a solid

PU Emission of neutrals or ions by solids (sputtering)

Section 4. Atom./mol. Coll. (and Reactions)

EN Energy or enthalpy of reaction

$\mathrm{KE}$ Constant of chemical equilibrium

SN Total cross sections
SM Transport cross sections (momentum, ...)

SC Angular diffusion (scattering)

SP Stopping power

EL Elastic collision

DO Depolarization, change of polarization

EX Excitation

DX Deexcitation (quenching)

TE Excitation transfer

IN Ionization

IM Creation of an ion pair (positive-negative)

DT Detachment

TI Ionizing charge transfer

RI Recombination ion-ion

CX Charge transfer

XD Dissociative charge transfer

CA Capture of electrons

SR Loss of electrons (stripping)

DS Dissociation

IR Interchange reaction (of one or several atoms)

IA Associative interchange reaction

ID Dissociative interchange reaction

AS Association

DG Desorption

AD Adsorption

EE Emission of electrons by a solid

PU Emission of neutrals or ions by solids (sputtering)

Section 5. Macroscopic properties

ST Statistics of levels

FT Thermodynamic functions

PV Compressibility, equation of state

PE Dielectric and magnetic constants

DN Diffusion

VI Viscosity

CT Thermal conductivity

LW Line broadening and shift (collisional effects)

PI First coefficient of Townsend

DT Detachment

AT Attachment

FE Distribution function of electrons

ME Mobility of electrons

CE Electrical conductivity

DE Diffusion of electrons

MD Characteristic temperature of electrons $(D / \mu)$

PC Power delivered by electron-neutral collisions

FI Distribution function of ions

MI Mobility of ions

DI Diffusion of ions

DA Ambipolar diffusion

Remark This list is slightly simplified (see the Web) 


\title{
NIST Atomic and Molecular Databases on the World Wide Web
}

\author{
R. A. Dragoset, P. J. Mohr, K. J. Olsen, E. B. Saloman, G. Wiersma, D. S. Zucker \\ National Institute of Standards and Technology \\ Office of Electronic Commerce in Scientific and Engineering Data
}

Gaithersburg, MD 20899

The Physics Laboratory of the National Institute of Standards and Technology is using the World Wide Web to disseminate the physical reference data it has been producing and/or evaluating. Each Web database developed has an interface specially designed for its user community. The databases currently available are: 1) CODATA Recommended Values of the Fundamental Physical Constants; 2) Fundamental Constants Bibliographic Database; 3) Atomic Spectroscopic Database; 4) Platinum Lamp Spectrum, $113 \mathrm{~nm}$ to $443 \mathrm{~nm}$; 5) Bibliographic Database of Atomic Transition Probabilities; 6) Infrared Spectrometer Calibration Tables; 7) Rest Frequencies for Observed Interstellar Microwave Transitions; 8) Electron-Impact Ionization Cross Section Database; 9) Tables of X-ray Mass Attenuation Coefficients; 10) Bibliographic Database of X-ray Attenuation Coefficient Measurements; 11) X-Ray Form Factor, Attenuation, and Scattering Tabulation; 12) Radionuclide Half-Life Measurements Made at NIST; and 13) Atomic Model Data for Electronic Structure Calculations. The Web versions of these databases represent the work of many people - from the compilers and evaluators of the data to the designers and programmers of the web interfaces. Work continues to add new databases and to improve those currently provided. The databases may be obtained from the Web at the URL http://physics.nist.gov/ by selecting the link Physical Reference Data.

The 13 databases currently on the Web are divided into seven categories:

\section{Physical Constants, Units, and Measurement Uncertainty}

\section{Fundamental Physical Constants}

by E. Richard Cohen and Barry N. Taylor

This database gives values of the basic constants and conversion factors of physics and chemistry resulting from the 1986 least-squares adjustment of the fundamental physical constants as published by the CODATA Task Group on Fundamental Constants and recommended for international use by CODATA.

\section{Fundamental Constants Bibliographic Database}

by Peter J. Mohr and Barry N. Taylor

This searchable database gives the citations for the most important theoretical and experimental publications relevant to the fundamental physical constants and closely related precision measurements published since the mid 1980s. Earlier papers of particular interest are also included. The database is regularly updated through the addition of papers from the current literature and made more comprehensive through the addition of earlier papers. 


\section{Atomic Spectroscopic Data}

\section{Atomic Spectroscopic Database}

by J. R. Fuhr, W. C. Martin, A. Musgrove, J. Sugar, and W. L. Wiese

This database includes most of the existing critically evaluated NIST data on atomic energy levels, transition probabilities, and wavelengths that are reasonably up-to-date. This interactive database has energy level data for over 500 spectra, transition probabilities for $\mathrm{Sc}$ through $\mathrm{Ni}$, and wavelength data for spectra of several elements.

\section{Spectrum of Platinum Lamp for Ultraviolet Spectrograph Calibration} by J. E. Sansonetti, J. Reader, C. J. Sansonetti, and N. Acquista

An atlas of the spectrum of a platinum/neon hollow-cathode reference lamp in the region $1130 \AA$ to $4330 \AA$ is given, with the spectral lines marked and their intensities, wavelengths, and classifications listed. Graphical figures of the spectrum are included.

\section{Bibliographic Database on Atomic Transition Probabilities} by J. R. Fuhr and H. R. Felrice

This interactive database contains references on atomic transition probabilities (oscillator strengths, line strengths, and radiative lifetimes). Both theoretical and experimental papers are listed.

\section{Molecular Spectroscopic Data}

\section{Wavenumber Tables for Calibration of Infrared Spectrometers by Arthur G. Maki and Joseph S. Wells}

This database is an atlas of molecular spectra and associated tables of wavenumbers from heterodyne frequency measurements for the calibration of infrared spectrometers. Five molecules are included in the atlas: CO, OCS, $\mathrm{N}_{2} \mathrm{O}, \mathrm{NO}$, and $\mathrm{CS}_{2}$. The spectra cover the $488 \mathrm{~cm}^{-1}$ to $3120 \mathrm{~cm}^{-1}$ and $4000 \mathrm{~cm}^{-1}$ to $4400 \mathrm{~cm}^{-1}$ regions.

\section{Frequencies for Interstellar Molecular Microwave Transitions}

by Frank J. Lovas

This database contains critically evaluated transition frequencies for molecular transitions detected in interstellar and circumstellar clouds recommended by NIST for reference in future astronomical observations in the microwave and millimeter wavelength regions. 


\section{Ionization Data}

\section{Electron-Impact Ionization Cross Section Database}

by Y.-K. Kim and M. E. Rudd

This is a database of total ionization cross sections of various molecules by electron impact. The cross sections were calculated using the Binary-Encounter-Bethe (BEB) model, which combines the Mott cross section with the high- $T$ behavior of the Bethe cross section. Selected experimental data are included.

\section{X-Ray and Gamma-Ray Data}

\section{X-Ray Attenuation and Absorption for Materials of Dosimetric Interest} by J. H. Hubbell and S. M. Seltzer

Tables and graphs of computed photon mass attenuation coefficients and mass energy-absorption coefficients from $1 \mathrm{keV}$ to $20 \mathrm{MeV}$ are presented for all of the elements $(Z=1$ to 92$)$ and for 48 compounds and mixtures of radiological interest. These coefficients are basic quantities used in calculations of the penetration and the energy deposition by photons (x-ray, gamma-ray, bremsstrahlung) in biological, shielding, and other materials.

\section{Bbibliography of Photon Attenuation Measurements}

by J. H. Hubbell

This bibliography contains papers (1907-1995) reporting absolute measurements of photon (XUV, x-ray, gamma-ray, bremsstrahlung) total interaction cross sections or attenuation coefficients for the elements and some compounds used in a variety of medical, industrial, defense, and scientific applications. The energy range covered is from $10 \mathrm{eV}$ to $13.5 \mathrm{GeV}$.

\section{X-Ray Form Factor, Attenuation and Scattering Tabulation}

by C. T. Chantler

The primary interactions of $\mathrm{x}$ rays with isolated atoms from $Z=1$ (hydrogen) to $Z=92$ (uranium) are described and computed within a self-consistent Dirac-Hartree-Fock framework. The results are provided over the energy range from either $1 \mathrm{eV}$ or $10 \mathrm{eV}$ to $433 \mathrm{keV}$, depending on the atom. Self-consistent values of the $f_{1}$ and $f_{2}$ components of the atomic scattering factors are tabulated, together with the photoelectric attenuation coefficient for the atom, $\mu$, and the value for the $\mathrm{K}$ shell, $\mu \mathrm{K}$, as functions of energy and wavelength. 


\section{Nuclear Physics Data}

\section{Radionuclide Half-Life Measurements Made at NIST}

by M. P. Unterweger, D. D. Hoppes, and F. J. Schima

This database is a table of the half lives of 56 important radionuclides measured with ionization chambers by the Radioactivity Group of NIST. The included half lives range from 1.8 hours to 31.5 years.

\section{Condensed Matter Physics Data}

\section{Atomic Model Data for Electronic Structure Calculations} by Svetlana Kotochigova, Zachary Levine, Eric Shirley, Mark Stiles, and Charles Clark

This database consists of evaluated data for use in total-energy calculations of electronic structure by density-functional theory. It contains total energies and orbital energy eigenvalues for all atoms from hydrogen to uranium, with micro-Hartree uncertainty in the total energy, as computed in the local-density approximation (LDA), the local-spin-density approximation (LSD), the relativistic local-density approximation (RLDA), and the scalar-relativistic local-density approximation (ScRLDA).

\section{Conclusion}

There are several new or improved databases currently being prepared for dissemination on the Web to meet the needs of the atomic and molecular data community. In particular, a description of the extended version of the Atomic Spectroscopic Database under development is included in this NIST Special Publication in the paper "NIST Atomic Spectroscopic Database," by G. R. Dalton, et al. All Physics Laboratory databases can be accessed from the Web at http://physics.nist.gov/ by selecting the link Physical Reference Data. 


\title{
Vibrational and Electronic Energy Levels of Polyatomic Transient Molecules
}

\author{
Marilyn E. Jacox \\ National Institute of Standards and Technology \\ Gaithersburg, MD 20899
}

\begin{abstract}
The database concerned with the vibrational and electronic energy of polyatomic transient molecules with from 3 to 16 atoms now contains the experimentally determined vibrational fundamentals and electronic band origins of approximately 2,140 neutral and ionic transient molecules. This database is distributed in both published and computer-searchable forms. Emphasis is placed on species with lifetimes too short for study using conventional sampling techniques. The compilation also includes many high temperature molecules and several less reactive species which have presented spectral evaluation problems and which are important in a wide variety of environmental and industrial chemical systems. Radiative lifetimes and the principal rotational constants are given. Observations in the gas phase, in molecular beams, and in rare-gas and diatomic molecule matrices are evaluated. The types of measurement surveyed include conventional and laser-based absorption and emission techniques, laser absorption with mass analysis, and photoelectron spectroscopy.
\end{abstract}

\section{Discussion}

Transient molecules are important in a wide variety of chemical reaction systems, including those typical of combustion, plasma processing, chemical vapor deposition, decomposition of energetic materials, and environmental research and monitoring. A database concerned with critically evaluated vibrational and electronic energy levels of polyatomic transient molecules with from 3 to 16 atoms now contains the experimentally determined vibrational fundamentals and electronic band origins of approximately 2,140 neutral and ionic species. Emphasis is placed on molecules with lifetimes too short for study using conventional sampling techniques. These molecules include free radicals, molecular ions, and other short-lived reaction intermediates. The database is gradually being extended to include small high-temperature molecules. At this time, it contains spectroscopic information on many polyatomic oxides. Certain other especially important species that are difficult to isolate in pure form and have presented spectral evaluation problems, including $\mathrm{OClO}, \mathrm{HNCO}, \mathrm{H}_{2} \mathrm{O}_{2}$, cis- and trans- $\mathrm{HONO}$, and $\mathrm{HONO}_{2}$, are also included.

The focus of this database is on the spectroscopic properties of the species of interest under conditions of minimal interaction with other species. Thus, the most definitive data are those obtained in low-pressure gas-phase measurements, including studies of molecular beams. Also included are data for the molecule trapped in rare-gas matrices and/or in small molecule matrices such as $\mathrm{H}_{2}, \mathrm{~N}_{2}, \mathrm{O}_{2}, \mathrm{CO}$, or $\mathrm{CO}_{2}$. Matrix shifts for uncharged molecules and ionic species trapped in Ne are generally less than $1 \%$, as are those of uncharged molecules trapped in $\mathrm{Ar} . \mathrm{H}_{2}$ is also a promising matrix material, but there are insufficient data for a satisfactory estimate of matrix shifts characteristic of molecules trapped in solid $\mathrm{H}_{2}$. More polar matrix materials such as $\mathrm{H}_{2} \mathrm{O}$ 
and the Freons are not included, nor are data for high-temperature molecules in the solid state, which generally have very broad absorptions.

Many types of measurement are included in the database. Among these are conventional and laser-based absorption and emission techniques, laser absorption with mass analysis, and photoelectron spectroscopy.

As of 1 October 1997, earlier versions of the database were available both as a monograph [1] and as a computer-searchable database, VEEL 4.0 [2]. Spectroscopic data for 1,582 molecules are included in the monograph, and data for 1,796 molecules are included in VEEL 4.0. Both forms include excited-state band origins, ground- and excited-state vibrational fundamentals, and a full bibliography. The monograph also includes radiatiye lifetimes and principal rotational constants, cited to $0.001 \mathrm{~cm}^{-1}$. The system requirements for VEEL 4.0 are an IBMa AT class or PS/2 personal computer $(80286,80386,80486$, or Pentium processor) with PC- or MS-DOS 2.1 or greater or with Windows 95, as well as 512 kilobytes of RAM and at least 4.0 megabytes of hard disk storage. VEEL 4.0 can also be used with Macintosh computers that have PC emulation software, and instructions for installing it on systems which use Windows NT are available.

Three new outputs which revise and extend the coverage of the database will soon become available. A supplement to the monograph [1] is to be published early in 1998 in the Journal of Physical and Chemical Reference Data. Version 5.0 of VEEL is to be released in late 1997. The December 1997 version of NIST Standard Reference Database 69 (http://WebBook.NLST.gov) is also scheduled to include the data contained in VEEL 5.0, searchable by vibrational and electronic energy levels, together with other molecular properties.

\section{Acknowledgments}

This work was supported by the Standard Reference Data Program of the National Institute of Standards and Technology.

\section{Footnotes and References}

[1] M. E. Jacox, "Vibrational and Electronic Energy Levels of Polyatomic Transient Molecules," Monograph No. 3, J. Phys. Chem. Ref. Data, 461 pages (1994)

[2] NIST Standard Reference Database 26, NIST Vibrational and Electronic Energy Levels of Small Polyatomic Transient Molecules (VEEL), Version 4.0

\footnotetext{
${ }^{a}$ Certain commercial instruments and software are identified in this paper in order to specify adequately the range of utility of the VEEL data package. In no case does such identification imply recommendation or endorsement by the National Institute of Standards and Technology, nor does it imply that the items identified are necessarily the best available for the purpose.
} 


\title{
Database for Dielectronic Recombination Rate Coefficients to the Excited States of the Carbon Atom and Ions
}

\author{
T. Kato, U. Safronova, and J. Dubau* \\ National Institute for Fusion Science, Nagoya, 464-01, Japan \\ *Observatory of Paris, Meudon, F-92195 MEUDON Cedex, France
}

\section{Introduction}

Dielectronic recombination processes for L-shell ions are important in plasmas such as the solar corona and divertor plasma for plasma diagnostics. We have calculated the dielectronic recombination rate coefficients to the excited states $(n=2-6)$ of L-shell carbon atom and ions (C I - C IV). These data are necessary to calculate the population density of excited states of each ion as well as for total recombination rate coefficients. Atomic data (energy levels, transition probabilities, autoionization rates) are calculated by the Cowan code and AUTOLSJ methods [1].

Scaling methods are used for highly excited states because the contribution of the highly excited states up to more than $\mathrm{n}=100$ is not negligible. We present the dielectronic recombination rate coefficients to the excited states by a parameter fit to an analytical formula. Density effects on the effective recombination rate coefficients are discussed.

\section{Method}

Dielectronic recombination rate coefficients $\alpha_{d}$ to the excited states $n l[L S]$ of an ion $\mathrm{X}^{(\mathrm{z}-\mathrm{l})+}$ from an initial state $\alpha_{0}$ of a recombining ion $\mathrm{X}^{\mathrm{z}}$ are calculated by

$$
\begin{aligned}
\alpha_{\mathrm{d}}\left(\mathrm{nl}[\mathrm{LS}] \mid \alpha_{0}\right)= & \left(\mathrm{h}^{2} / 2 \pi \mathrm{mkT}_{\mathrm{e}}\right)^{3 / 2} \sum_{\gamma} \sum_{J}\left(\mathrm{~g}_{\mathrm{z} \cdot \mathrm{l}}\left(\gamma\left[\mathrm{L}^{\prime} \mathrm{S}^{\prime} J^{\prime}\right]\right) / 2 \mathrm{~g}_{\mathrm{z}}(\mathrm{l})\right) \\
& \times\left(\mathrm{A}_{\mathrm{a}}\left(\gamma\left[\mathrm{L}^{\prime} \mathrm{S}^{\prime} J^{\prime}\right], \alpha_{0}\right) \operatorname{Ar}_{\mathrm{r}}\left(\gamma\left[\mathrm{L}^{\prime} \mathrm{S}^{\prime} J^{\prime}\right], \mathrm{nl}[\mathrm{LSJ}]\right) /\left(\sum \mathrm{A}_{\mathrm{a}}+\sum \mathrm{A}_{\mathrm{r}}\right) \exp \left(-\mathrm{E}_{\mathrm{s}} / \mathrm{kT}_{\mathrm{e}}\right)\right.
\end{aligned}
$$

for the process as follows,

$$
\mathrm{X}^{\mathrm{Z}+}\left(\alpha_{0}\right)+\mathrm{e} \rightarrow \mathrm{X}^{(\mathrm{Z}-\mathrm{l})}+* *(\gamma) \rightarrow \mathrm{X}^{(\mathrm{Z}-1)+}(\mathrm{nl}[\mathrm{LS}])+\mathrm{h} v
$$

where $\gamma$ is the autoionizing state, $n l$ the bound states, $A_{a}$ the autoionization rate, $A_{r}$ radiative transition probability, $E_{s}$ the energy of the state $\gamma$ relative to the initial state $\alpha_{0}$ which we assumed to be the ground state of a recombining ion. The values for $\mathrm{A}_{\mathrm{a}}, \mathrm{A}_{\mathrm{r}}$, and $\mathrm{E}_{\mathrm{s}}$ are calculated for fine structure levels with $n=1-6$ [1-4] and the rate coefficients are summed into LS levels by Eq. (l). 


\section{Fit Parameters for the Rate Coefficients $\alpha_{d}(n l[L S])$}

We have fitted the rate coefficients to each excited state by a fitting formula as follows,

$$
\alpha_{d}\left(\mathrm{nl}[\mathrm{LS}] \mid \alpha_{0}\right)=\sum \mathrm{iA} \mathrm{A}_{\mathrm{i}} \exp \left\{-\left(\mathrm{E}_{\mathrm{i}} / \mathrm{T}_{\mathrm{e}}\right)\right\} \mathrm{T}_{\mathrm{e}}^{-3 / 2} \mathrm{~cm}^{3} \mathrm{~s}^{-1}
$$

where $E_{i}$ and $T_{e}$ are in $\mathrm{eV}$. An example of these parameters is given in Table 1. The data for C I - C III are available upon request (takako@nifs.acjp).

\section{4 nl-Dependence of $\alpha_{d}$}

The rate coefficients have large values even if the principal quantum number is above $\mathrm{n}=100$, and their contribution is not negligible for the total recombination rate and for the population density in a recombining plasma. We obtained the values for $n>6$ by using scaling formulae [1] of $A_{r}(2 p n l, 2 s n l)=A_{r}(2 p 6 l, 2 s 6 l)$ and $A_{a}(2 p n l)=(6 / n)^{3} A_{a}(2 p 6 l)$ for each LSJ level and summed up with LSJ after scaling. In Fig.1, we show the distribution of $\alpha_{\mathrm{d}}(\mathrm{nl})$ of C I as an example.

\section{Total Recombination Rate Coefficients and the Density Effect}

In order to obtain the total recombination rate coefficients we summed the rates up to $\mathrm{n}=500$. However, a real plasma has a finite electron density, and the collisional effects for such high $\mathrm{n}$ cannot be neglected. For example, the thermal limit is estimated to be $\mathrm{n} \sim 15$ for a plasma of $\mathrm{T}_{\mathrm{e}}=30 \mathrm{eV}$ and $\mathrm{n}_{\mathrm{e}}=10^{8} \mathrm{~cm}^{-3}$. Therefore, it is necessary to include the density effect when the recombination rate coefficients are used to obtain the ion abundances in plasmas. We define the effective recombination rate coefficient as follows,

$$
\alpha_{\text {eff }} \equiv \sum_{\mathrm{i}}\left(\mathrm{A}_{\mathrm{i} 1} / \mathrm{n}_{\mathrm{e}}+\mathrm{C}_{\mathrm{i} 1}\right) \mathrm{n}(\mathrm{i})+\alpha_{\mathrm{t}}(1) \mathrm{n}_{\mathrm{e}}+\alpha_{\mathrm{r}}(1)+\alpha_{\mathrm{d}}(1)
$$

where $\alpha_{t}(1)$ and $\alpha_{r}(1)$ indicate the three body and radiative recombination rate coefficients to the ground state, respectively and $\mathrm{C}_{\mathrm{i} 1}$ is the collisional de-excitation rate coefficient from the level $\mathrm{i}$ to the ground state. The $\alpha_{\text {eff }}$ for the various electron densities as a function of electron temperature are shown in Fig. 2. However, in order to obtain $\alpha_{\text {eff, }}$ we should consider collisional and microfield l-mixing in autoionizing levels which are not included in our calculation. Further investigations are required for these processes.

\section{References}

[1] J. Dubau, T. Kato, U. I. Safronova, these proceedings (1997)

[2] U. I. Safronova and T. Kato, Physica Scripta 53, 461-472 (1996)

[3] T. Kato, U. I. Safronova and M. Ohira, Physica Scripta 55, 185 - 199 (1997)

[4] U. Safronova, T. Kato and M. Ohira, NIFS-DATA-37 (1996), J. Quant. Spectrosc. Radiat. Transfer 58, 193 (1997) 
Table 1: Fitting parameters by formula Eq. (3) for $\alpha_{d}(k)$ of C I.

\begin{tabular}{|c|c|c|c|c|c|}
\hline Excited state & & $\mathrm{A}_{1}\left(\mathrm{~cm}^{3} \mathrm{~s}^{-1}\right)$ & $\mathrm{E}_{1}(\mathrm{eV})$ & $\mathrm{A}_{2}\left(\mathrm{~cm}^{3} \mathrm{~s}^{-1}\right)$ & $\mathrm{E}_{2}(\mathrm{eV})$ \\
\hline $2 \mathrm{~s}^{2} 2 \mathrm{p}^{2}$ & ${ }^{3} \mathrm{P}$ & $1.051 \mathrm{E}-13$ & $1.723 \mathrm{E}+00$ & $1.088 \mathrm{E}-12$ & $3.887 \mathrm{E}+00$ \\
\hline $2 \mathrm{~s}^{2} 2 \mathrm{p}^{2}$ & $1 \mathrm{D}$ & $6.402 \mathrm{E}-13$ & $2.310 \mathrm{E}+00$ & $5.551 \mathrm{E}-13$ & $4.706 \mathrm{E}+00$ \\
\hline $2 \mathrm{~s} 22 \mathrm{p} 2$ & $1 \mathrm{~S}$ & $1.276 \mathrm{E}-13$ & $3.522 \mathrm{E}+00$ & $1.263 \mathrm{E}-14$ & $1.247 \mathrm{E}+01$ \\
\hline $2 \mathrm{~s} 2 \mathrm{p}^{3}$ & ${ }^{5} \mathrm{~S}$ & $1.177 \mathrm{E}-13$ & $3.664 \mathrm{E}+00$ & $3.820 \mathrm{E}-14$ & $1.017 \mathrm{E}+00$ \\
\hline $2 \mathrm{~s} 2 \mathrm{p}^{3}$ & ${ }^{3} \mathrm{D}$ & $5.296 \mathrm{E}-13$ & $4.615 \mathrm{E}+00$ & $3.368 \mathrm{E}-14$ & $1.588 \mathrm{E}+00$ \\
\hline $2 \mathrm{~s} 2 \mathrm{p}^{3}$ & ${ }^{3} \mathrm{P}$ & $7.903 \mathrm{E}-13$ & $7.606 \mathrm{E}+00$ & $4.663 \mathrm{E}-14$ & $2.488 \mathrm{E}+00$ \\
\hline $2 s^{2} 2 p^{3} s$ & ${ }^{3} \mathrm{P}$ & $4.716 \mathrm{E}-13$ & $6.671 \mathrm{E}+00$ & $4.671 \mathrm{E}-15$ & $1.584 \mathrm{E}+00$ \\
\hline $2 \mathrm{~s}^{2} 2 \mathrm{p} 3 \mathrm{~s}$ & ${ }^{1} \mathrm{P}$ & $4.263 \mathrm{E}-13$ & $9.887 \mathrm{E}+00$ & $2.828 \mathrm{E}-14$ & $5.982 \mathrm{E}+00$ \\
\hline $2 s^{2} 2 p 3 p$ & ${ }^{1} \mathrm{P}$ & $4.525 \mathrm{E}-16$ & $2.323 \mathrm{E}+00$ & $5.378 \mathrm{E}-14$ & $8.250 \mathrm{E}+00$ \\
\hline $2 s^{2} 2 p 3 p$ & ${ }^{3} \mathrm{D}$ & $3.551 \mathrm{E}-16$ & $2.820 \mathrm{E}+00$ & $5.530 \mathrm{E}-13$ & $1.015 \mathrm{E}+01$ \\
\hline $2 s^{2} 2 p 3 p$ & ${ }^{3} \mathrm{~S}$ & $8.293 \mathrm{E}-17$ & $2.835 \mathrm{E}+00$ & $1.130 \mathrm{E}-13$ & $9.877 \mathrm{E}+00$ \\
\hline $2 s^{2} 2 p 3 p$ & ${ }^{3} \mathrm{P}$ & $2.585 \mathrm{E}-13$ & $9.126 \mathrm{E}+00$ & $2.995 \mathrm{E}-14$ & $3.057 \mathrm{E}+00$ \\
\hline $2 s^{2} 2 p 3 p$ & ${ }^{1} \mathrm{D}$ & $1.394 \mathrm{E}-13$ & $7.211 \mathrm{E}+00$ & $7.422 \mathrm{E}-17$ & $2.311 \mathrm{E}+00$ \\
\hline $2 s^{2} 2 p 3 p$ & ${ }^{1} \mathrm{~S}$ & $1.917 \mathrm{E}-14$ & $9.648 \mathrm{E}+00$ & $3.491 \mathrm{E}-17$ & $3.532 \mathrm{E}+00$ \\
\hline $2 s^{2} 2 p 3 d$ & ${ }^{3} \mathrm{P}$ & $7.198 \mathrm{E}-13$ & $7.971 \mathrm{E}+00$ & $2.832 \mathrm{E}-14$ & $2.385 \mathrm{E}+00$ \\
\hline $2 \mathrm{~s}^{2} 2 \mathrm{p} 3 \mathrm{~d}$ & ${ }^{1} \mathrm{D}$ & $2.822 \mathrm{E}-13$ & $1.195 \mathrm{E}+01$ & $3.188 \mathrm{E}-14$ & $7.500 \mathrm{E}+00$ \\
\hline $2 s^{2} 2 p 3 d$ & ${ }^{3} \mathrm{~F}$ & $3.012 \mathrm{E}-12$ & $1.177 \mathrm{E}+01$ & $3.278 \mathrm{E}-13$ & $7.593 \mathrm{E}+00$ \\
\hline $2 s^{2} 2 p 3 d$ & ${ }^{3} \mathrm{D}$ & $1.420 \mathrm{E}-12$ & $1.016 \mathrm{E}+01$ & $9.464 \mathrm{E}-15$ & $2.468 \mathrm{E}+00$ \\
\hline $2 s^{2} 2 p 3 d$ & ${ }^{1} \mathrm{P}$ & $2.759 \mathrm{E}-13$ & $1.118 \mathrm{E}+01$ & $2.014 \mathrm{E}-15$ & $4.325 \mathrm{E}+00$ \\
\hline $2 s^{2} 2 p 3 d$ & ${ }^{1} \mathrm{~F}$ & $8.183 \mathrm{E}-13$ & $1.135 \mathrm{E}+01$ & $2.966 \mathrm{E}-14$ & $6.126 \mathrm{E}+00$ \\
\hline $2 \mathrm{~s}^{2} 2 \mathrm{p} 4 \mathrm{~s}$ & ${ }^{3} \mathrm{P}$ & $6.280 \mathrm{E}-13$ & $9.549 \mathrm{E}+00$ & $9.864 \mathrm{E}-16$ & $1.608 \mathrm{E}+00$ \\
\hline $2 s^{2} 2 p 4 s$ & ${ }^{1} \mathrm{D}$ & $3.266 \mathrm{E}-13$ & $1.189 \mathrm{E}+01$ & $1.628 \mathrm{E}-14$ & $7.139 \mathrm{E}+00$ \\
\hline $2 s^{2} 2 p 4 p$ & ${ }^{1 P}$ & $5.684 \mathrm{E}-14$ & $9.861 \mathrm{E}+00$ & $2.220 \mathrm{E}-16$ & $2.426 \mathrm{E}+00$ \\
\hline $2 s^{2} 2 p 4 p$ & ${ }^{3} \mathrm{D}$ & $7.955 \mathrm{E}-13$ & $1.180 \mathrm{E}+01$ & $7.628 \mathrm{E}-16$ & $2.862 \mathrm{E}+00$ \\
\hline $2 s^{2} 2 p 4 p$ & ${ }^{3} \mathrm{~S}$ & $1.508 \mathrm{E}-13$ & $1.142 \mathrm{E}+01$ & $2.162 \mathrm{E}-16$ & $2.875 \mathrm{E}+00$ \\
\hline $2 \mathrm{~s}^{2} 2 \mathrm{p} 4 \mathrm{p}$ & ${ }^{3} \mathrm{p}$ & $3.271 \mathrm{E}-13$ & $1.140 \mathrm{E}+01$ & $1.060 \mathrm{E}-14$ & $3.154 \mathrm{E}+00$ \\
\hline $2 \mathrm{~s}^{2} 2 \mathrm{p} 4 \mathrm{p}$ & ${ }^{1} \mathrm{D}$ & $1.826 \mathrm{E}-13$ & $8.673 \mathrm{E}+00$ & $4.409 \mathrm{E}-16$ & $2.944 \mathrm{E}+00$ \\
\hline $2 s^{2} 2 p 4 p$ & ${ }^{1} \mathrm{~S}$ & $2.975 \mathrm{E}-14$ & $1.080 \mathrm{E}+01$ & $2.291 \mathrm{E}-16$ & $3.524 \mathrm{E}+00$ \\
\hline $2 s^{2} 2 p 4 d$ & ${ }^{1} \mathrm{D}$ & $4.912 \mathrm{E}-13$ & $1.241 \mathrm{E}+01$ & $1.285 \mathrm{E}-14$ & $7.266 \mathrm{E}+00$ \\
\hline $2 \mathrm{~s}^{2} 2 \mathrm{p} 4 \mathrm{~d}$ & ${ }^{3} \mathrm{~F}$ & $3.024 \mathrm{E}-12$ & $1.123 \mathrm{E}+01$ & $2.781 \mathrm{E}-15$ & $3.138 \mathrm{E}+00$ \\
\hline $\operatorname{Sum}(n=7-500)$ & & $1.006 \mathrm{E}-09$ & $1.189 \mathrm{E}+01$ & $1.378 \mathrm{E}-11$ & $6.406 \mathrm{E}+00$ \\
\hline
\end{tabular}




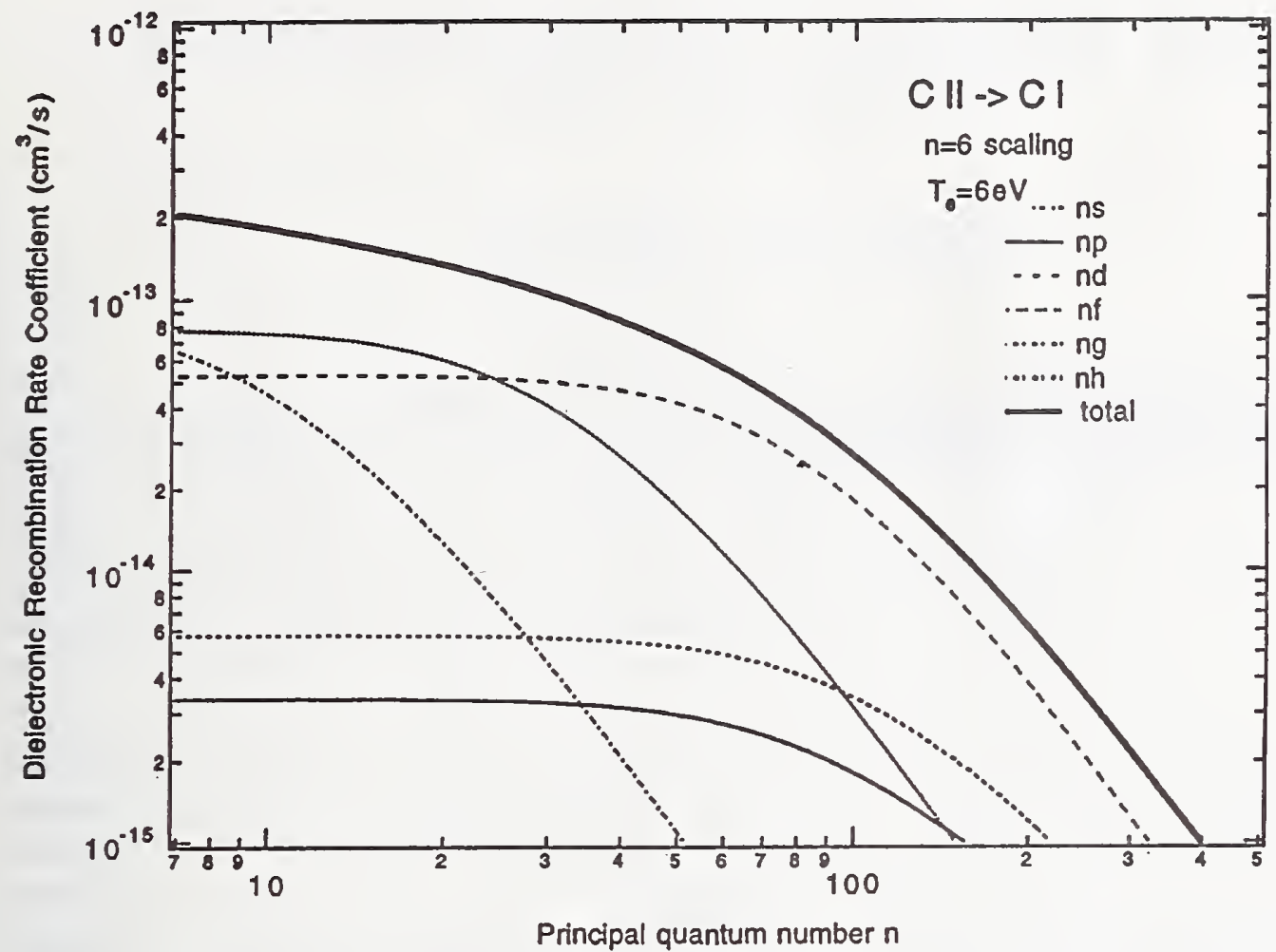

Figure 1: The dielectronic recombination rate coefficients to $\mathrm{n}-\mathrm{l}$ states from $\mathrm{C}$ II to $\mathrm{C}$ I.

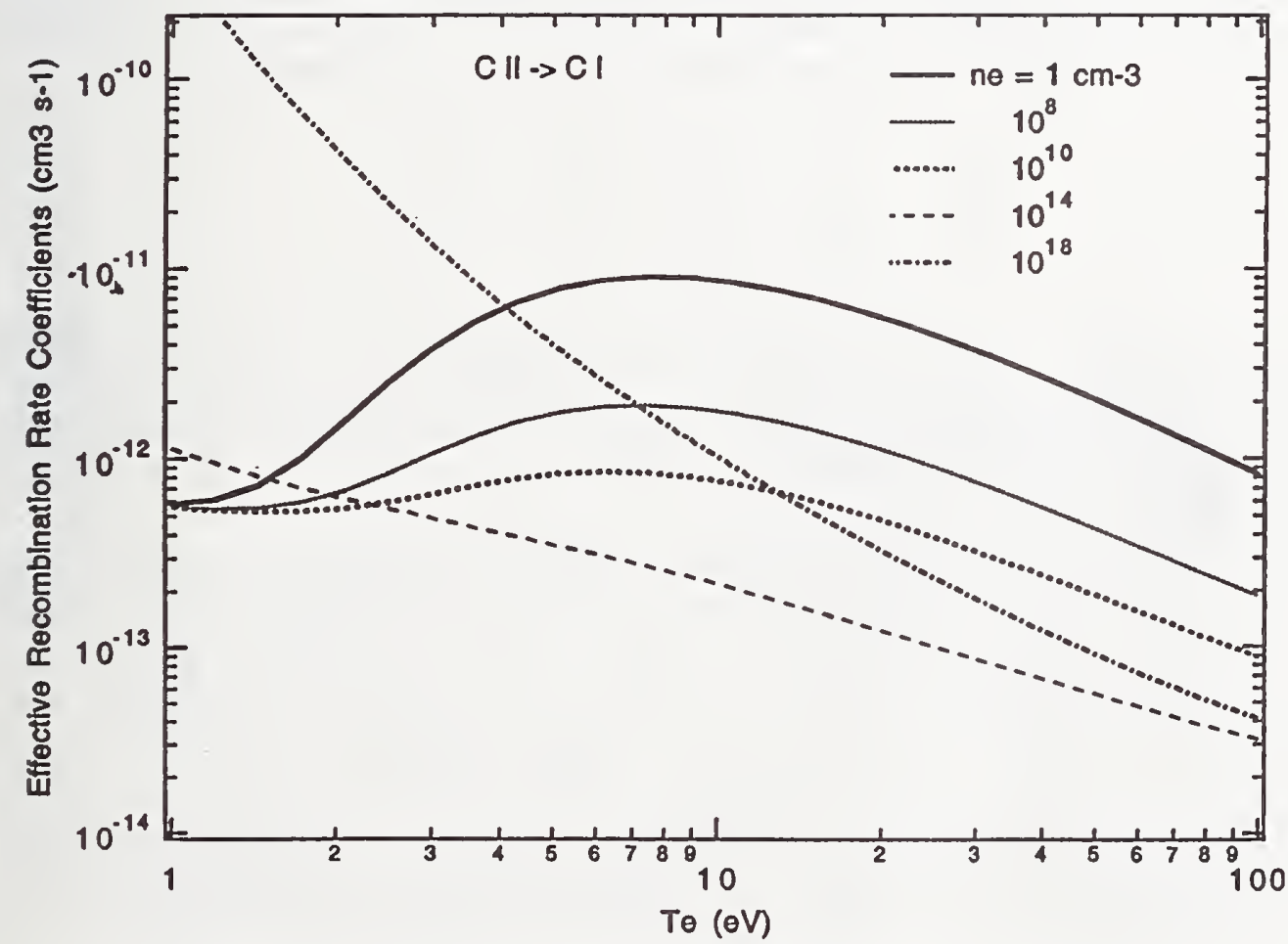

Figure 2: The effective recombination rate coefficients from C II to C I. 


\title{
Critical Evaluation of Transition Probabilities of $\mathrm{Na}-\mathrm{Ca}(\mathrm{Z}=11-20)$
}

\author{
D. E. Kelleher and L. I. Podobedova
}

NIST, Gaithersburg, MD 20899

NIST is in the process of critically evaluating and compiling transition probabilities of $\mathrm{H}$-Ca $(\mathrm{Z}=1-20)$. These elements were covered about 30 years ago in Vols. I and II of "Atomic Transition Probabilities" by W. L. Wiese et al. Much work has been done since then, mostly theoretical, with by far the most extensive results being provided by members of the "Opacity Project" (OP). In 1996, "Atomic Transition Probabilities of Carbon, Nitrogen, and Oxygen" was published by W. L. Wiese, J. R. Fuhr, and T. M. Deters [1]. Work on updating the other elements is continuing, and revision of $\mathrm{Vol}$. II is currently focused on $\mathrm{Na}, \mathrm{Mg}, \mathrm{Al}$, and $\mathrm{Si}(\mathrm{Z}=11-14)$. For many spectra, our new compilations contain about an order of magnitude more transitions than the earlier ones.

Our new compilations rely heavily on OP results because they are so extensive and because they appear to be reasonably accurate in many cases. Accurate experiments (and high precision computational results) are also essential, primarily for reference values in critical evaluation and for benchmark values in their own right. OP calculations cover an extensive range of allowed transitions, essentially comprehensive up to $n=10$ and $\ell=3,4$. OP values have been made electronically available for all stages of $\mathrm{H}-\mathrm{Ca}$, except $\mathrm{P}, \mathrm{Cl}$, and $\mathrm{K}$, which are planned for future work. Currently available OP calculations do not, however, include the effects of the spin-orbit interaction, and only average-multiplet values are reported. We decompose the LS multiplet averages into their LSJ fine structure components using the following LS coupling rule:

$$
S_{L S J \cdot L^{\prime} S^{\prime} J^{\prime}}=S_{L S} \cdot L^{\prime} S^{\prime}(2 J+1)\left(2 J^{\prime}+1\right)\left\{\begin{array}{ccc}
L & S & J \\
J^{\prime} & 1 & L^{\prime}
\end{array}\right\}^{2},
$$

where $S$ is the line strength, and the curly brackets indicate a $6-J$ symbol. We consider only those transitions for which experimental energies are tabulated in NIST energy level tables for both the lower and upper level. We take only the line strengths from the Opacity Project, using experimental energies to derive wavelengths and to convert line strengths to oscillator strengths and transition probabilities.

For the stronger transitions of most spectra, reasonably good agreement is found between the Opacity Project on the one hand, and experiments and accurate calculations which include spin-orbit effects on the other. Large spin-orbit effects such as those in noble-gas-like spectra can probably account for large differences observed in such spectra. However, the origin of large discrepancies in all but the strongest transitions in some other spectra is not yet understood. Note, for example, the comparison of line strengths for OP [2] and CIV-3 [3] in fluorine-like spectra of $\mathrm{Na}$ III and Si VI in Figures 1 and 2. Comparisons in the figures only include transitions for which the outermost electron of the upper level is in an $n=3$ principal quantum number, because only these are listed in [3]. 


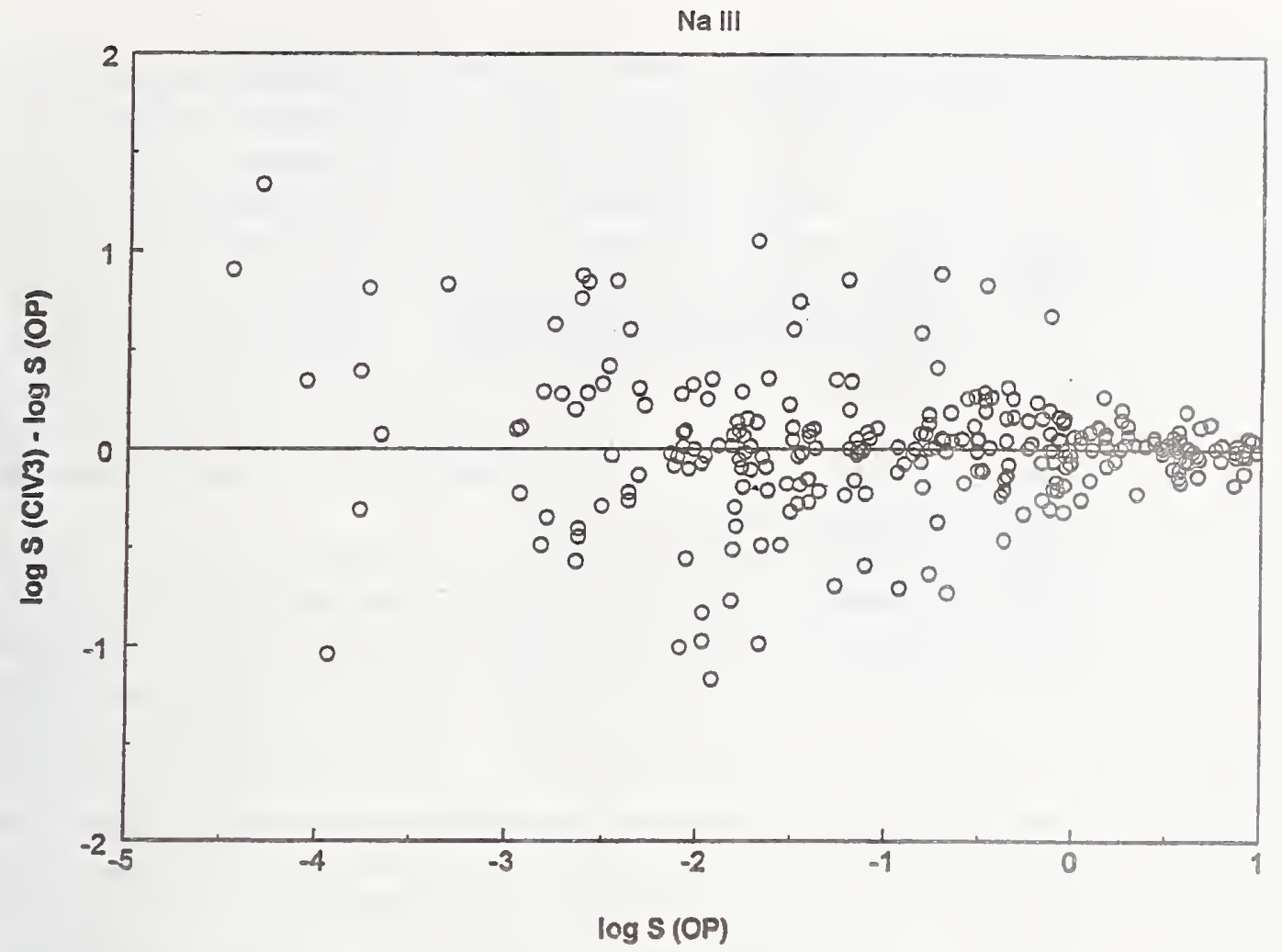

Figure 1: Logarithms of the ratios of line strengths S(CIV-3)/S(OP) are plotted vs. log S(OP) for fluorine-like $\mathrm{Na}$ III. All transitions involve $2 \mathrm{~s}^{2} 2 \mathrm{p}^{4} 3 \ell$ upper levels.

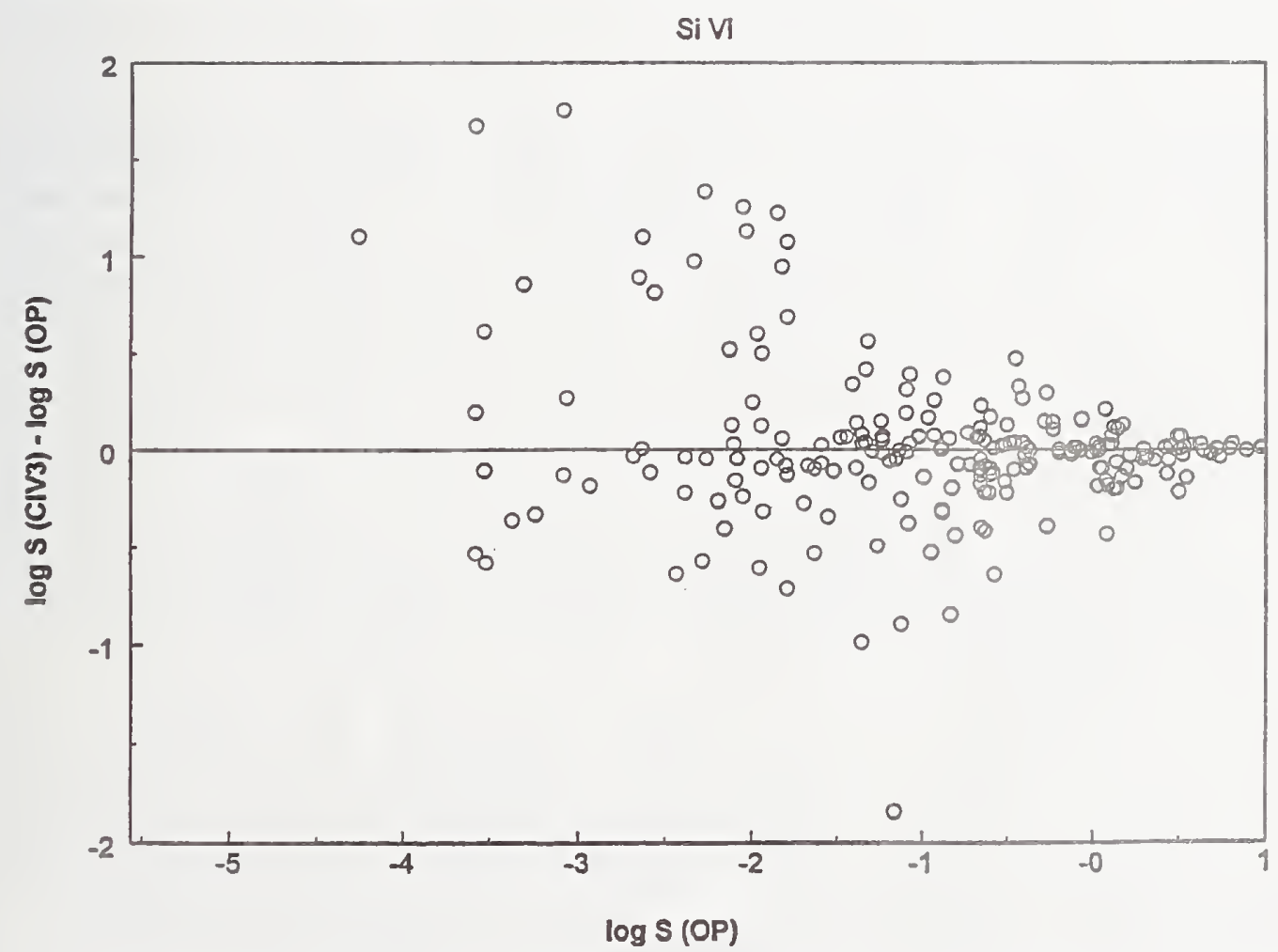

Figure 2: Logarithms of the ratios of line strengths $\mathrm{S}(\mathrm{CIV}-3) / \mathrm{S}(\mathrm{OP})$ are plotted vs. $\log \mathrm{S}(\mathrm{OP})$ for fluorine-like Si VI. All transitions involve $2 \mathrm{~s}^{2} 2 \mathrm{p}^{4} 3 \ell$ upper levels. 
The discrepancy in line-strengths for fluorine-like spectra between OP and CIV-3 results deserves special attention. These methods represent two of the most important methods to-date for large scale line strength calculations. The OP method relies on CIV-3 (or the similar "Superstructure" approach) for its core state correlations, as discussed below. All but the lowest three bound levels of $\mathrm{Na}$ III have configurations of the type $2 \mathrm{~s}^{2} 2 \mathrm{p}^{4}() \mathrm{n} \ell$, where the parentheses indicate a parent term of either ${ }^{1} \mathrm{~S},{ }^{3} \mathrm{P}$, or ${ }^{1} \mathrm{D}$. Bound interloper levels, such as the $2 \mathrm{~s} 5 \mathrm{pn} \ell$ levels, exist only for the more highly ionized members of the isoelectronic series. The three parent configurations cited above lie sufficiently close in energy that both intermediate coupling and configuration interaction occur at noticeable and frequently comparable levels throughout the bound spectrum. Relative Landé intervals of the quartet states (relative intervals for doublets cannot be derived from level energies) are typically far from those expected for LS coupling.

The OP uses a close-coupling expansion (CC) approximation that represents the total wavefunction as a superposition of ionic core and valence-electron wavefunctions. The ionic core (without the valence electron) is described by a configuration-interaction method, using either CIV-3 (Slater-type Orbitals) or superstructure (effective-charge statistical-model potentials). The $\mathrm{R}$-matrix method is used to solve the core plus valence electron problem in the inner region. It divides the problem into two regions of space: the "inner" and "outer" regions, and requires that the wavefunctions in these two regions and their radial derivatives match at an intermediate boundary. The outer-region wavefunction approaches a "Coulomb" solution asymptotically. It is usually evaluated by integrating the asymptotic solutions inward. The $a b$ initio CC expansion method is similar in spirit to, but considerably more sophisticated than, such semi-empirical methods as the Coulomb approximation, quantum defect theory, or core polarization models. Even the latter, for example, must use a short-range cut-off of the potential to simulate the effect of exchange between the excited and core electrons. The CC approach is generally more efficient than variational methods for large-sweep calculations of transitions involving more highly excited levels. In principle at least, the assumptions of the CC model become increasingly valid for more highly excited states. (One caveat is that the CC model must be built on an intermediate-coupled core, if intermediate coupling is significant.) This advantage tends to offset the intrinsic fact that binding energies are smaller for more highly excited states. Thus, a fixed absolute error yields a larger relative error, as well as the fact that more basis states often need to be included to obtain the same level of absolute accuracy. Some authors argue that the CC method becomes more accurate than variational methods for more highly excited states because it builds in the effect of highly excited states and the continuum; as a practical matter, it may prove difficult to expand the basis set sufficiently as $n$ and $\ell$ increase. The CC method, however, usually cannot practically build-in as much correlation between the core and low-excited electron as can full-blown multi-configurational variational methods. Thus the latter can be superior for calculating transitions involving the lowest-excited levels, for transitions whose strengths are sensitive to cancellations in the dipole matrix elements, and, of course, for calculating wavefunctions for the ion core used in $\mathrm{CC}$ calculations.

Recently, the Opacity Project group in Belfast has gained the capability to include intermediate coupling via inclusion of the Breit Pauli (BP) interaction in their CC calculations. This is an important new development. NIST has received the $\mathrm{Na}$ III (F-like) energy levels of and the transition strengths between the odd $J=1 / 2, J=3 / 2$, and even $J=1 / 2$ states [4]. The energies agree with experimental values to within $0.8 \%$ or better, and the agreement between the length and velocity values for the electric dipole matrix element is encouraging. The agreement between the CIV-3 and CC with BP, however, is not much improved compared to CIV-3 vs. OP results. The origin of these discrepancies is not understood and is presently under study. It will thus be 
interesting to compare CIV-3 and forthcoming CC/BP calculations of transition strengths with experimental results available for other spectra, particularly for weaker transitions.

Because it overcomes the primary limitation of OP calculations, this extended CC/BP version may offer the potential for mass production of accurate transition probabilities of all spectra, at least for $\mathrm{Z}<\sim 36$ and for transition strengths whose values are not dominated by cancellation effects. Only time will tell, however, if the extended method is capable of generating reasonably accurate line strengths for values significantly less than one.

\section{References}

[1] W. L. Wiese, J. R. Fuhr, and T. M. Deters, "Atomic Transition Probabilities of Carbon, Nitrogen, and Oxygen" J. Phys. Chem. Ref. Data, Monograph 7 (1996)

[2] K. Butler and C. J. Zeippen, Opacity Project, to be published (data is available on the internet via "topbase")

[3] H. M. S. Blackford and A. Hibbert, At. Data Nucl. Data Tables 58, 101 (1994)

[4] K. Berrington, private communication 


\title{
Database for Electron-Impact Total Ionization Cross Sections of Atoms and Molecules*
}

\author{
Y.-K. Kim and D. S. Zucker \\ National Institute of Standards and Technology, \\ Gaithersburg, MD 20899-0001 \\ M. A. Ali \\ Chemistry Department, Howard University, \\ Washington, DC 20059 \\ M. E. Rudd \\ Department of Physics and Astronomy, \\ University of Nebraska-Lincoln, Lincoln, NE 68588-0111
}

\section{Introduction}

A database for electron-impact total ionization cross sections of 43 atoms and molecules is available as part of the NIST Physics Laboratory web presentation:

\section{http://physics.nist.gov/PhysRefData/Ionization/Xsection.html}

This database presents theoretical cross sections based on the Binary-Encounter-Bethe (BEB) model for a large number of atoms, molecules, and radicals of interest in modeling low-temperature plasmas in magnetic fusion devices, plasma processing of semiconductors, radiation damages to materials, and cleaning of air pollutants by corona discharge. The database includes molecules such as $\mathrm{H}_{2}, \mathrm{H}_{2} \mathrm{O}, \mathrm{NO}_{2}, \mathrm{O}_{3}, \mathrm{SiH}_{4}$, and $\mathrm{SF}_{6}$. For each target, the database compares the $\mathrm{BEB}$ cross section to available theoretical and experimental data.

\section{Theory}

The BEB model [1]-[4] combines a modified form of the Mott cross section with the Bethe cross section for high incident energy, T. The BEB model requires three constants per orbital, viz., the binding energy $B$, the average kinetic energy $U$, and the electron occupation number $N$. Then, the ionization cross section per orbital is given by:

$$
\sigma_{B E B}=\frac{S}{t+u+1}\left[\frac{Q \ln t}{2}\left(1-\frac{1}{t^{2}}\right)+(2-Q)\left(1-\frac{1}{t}-\frac{\ln t}{t+1}\right)\right],
$$

where $\mathrm{t}=\mathrm{T} / \mathrm{B}, \mathrm{u}=\mathrm{U} / \mathrm{B}, \mathrm{S}=4 \pi \mathrm{a} 0^{2} \mathrm{~N}(\mathrm{R} / \mathrm{B})^{2}$, $\mathrm{a}_{0}=0.529 \AA, \mathrm{R}=13.61 \mathrm{eV}$ and $\mathrm{Q}$ is a weighted integral of the target $=\mathrm{s}$ continuum oscillator strength [1]. For most targets, $\mathrm{Q}=1$ is an excellent approximation. The total ionization cross section, $\sigma$, is obtained by summing $\sigma$ BEB over occupied orbitals.

The first logarithmic term in eq (1) is associated with the dipole interaction term from the

\footnotetext{
* Work at NIST was supported in part by the Office of Fusion Sciences, USDOE.
} 
Bethe theory, $1-1 /$ t comes from the direct and exchange Coulomb interaction in the Mott cross section, and the last logarithmic term results from the interference between the direct and exchange terms of the Coulomb interaction.

Equation (1) is not a fitting formula, nor does it contain any adjustable or empirical parameters. Instead, the model uses ab initio constants obtained from the ground-state wave function of the target (with $Q=1$ ). To match the experimental threshold, however, we recommend using the experimental vertical ionization potential for the outermost valence orbital, if available. Experimental ionization potentials (mostly adiabatic) of many molecules and radicals are available from another NIST database [5].

\section{Results}

The BEB model was found to be very effective in reproducing known ionization cross sections from threshold to several $\mathrm{keV}$ (within $\pm 10 \%$ of reliable experimental data at the peak) for most of more than 50 atoms, molecules, and radicals we have tested so far. As an example, the BEB cross sections for $\mathrm{CH}$ and $\mathrm{CF}_{4}$ are compared to available experimental data in Figures 1 and 2.

Using this database, a user can (a) calculate the BEB cross section on-line by typing in the desired incident energy; (b) look at graphical comparison of the BEB cross section to other theory and experiment with full references; and (c) download orbital constants and BEB cross sections in ASCII form. We found so far that the BEB model works extremely well for hydrocarbons, both stable molecules and radicals. The BEB model works better for closed-shell molecules than open shell molecules, and better for molecules than atoms.

\section{Outlook}

Ionization cross sections for more atoms and molecules will be added to the database as they become available. For instance, preliminary studies indicate that the BEB model produces reliable cross sections for closed-shell compounds of fluorine, such as $\mathrm{CF}_{4}, \mathrm{CHF}_{3}$, and $\mathrm{C}_{4} \mathrm{~F}_{8}$, while substantial disagreement with experiments (sometimes a factor of 2) is found for radicals of fluorine compounds, such as CF and NF.

\section{References}

[1] Y.-K. Kim and M. E. Rudd, Phys. Rev. A 50, 3954 (1994)

[2] W. Hwang, Y.-K. Kim, and M. E. Rudd, J. Chem. Phys. 104, 2956 (1996)

[3] Y.-K. Kim, W. Hwang, N. M. Weinberger, M. A. Ali, and M. E. Rudd, J. Chem. Phys. 106, $1026(1997)$

[4] M. A. Ali, Y.-K. Kim, W. Hwang, N. M. Weinberger, and M. E. Rudd, J. Chem. Phys. 106, 9602 (1997)

[5] S. G. Lias, J. F. Liebman, R. D. Levin, and S. A. Kafafi, "NIST Positive Ion Energetics Database" 
[6] V. Tarnovsky, A. Levin, H. Deutsch, and K. Becker, J. Phys. B 29, 139 (1996)

[7] H. U. Poll, C. Winkler, D. Margreiter, V. Grill and T. D. Märk, Int. J. Mass Spectrom. Ion Processes 112, 1 (1992)

[8] R. A. Bonham, private communication (1997)

[9] M. V. V. S. Rao and S. K. Srivastava, Abstracts of the XXth International Conference on the Physics of Electronic and Atomic Collisions, 23-24 July 1997, Vienna, Austria, p. MO150

[10] J. A. Beran and L. Kevan, J. Phys. Chem. 73, 3866 (1969) 


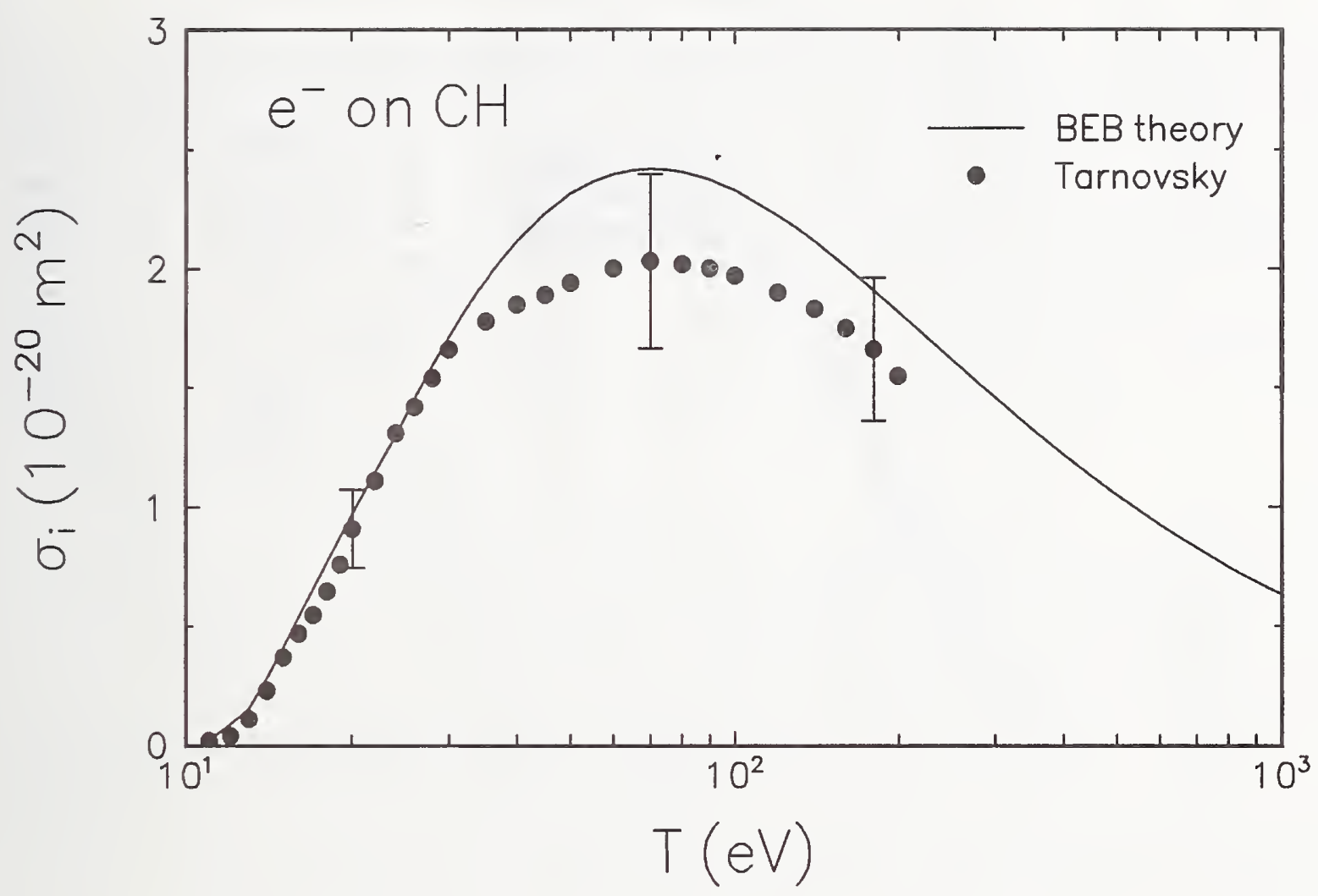

Figure 1: Comparison of ionization cross sections for $\mathrm{CH}$. $\sigma$ i, total ionization cross section; T, incident energy; solid curve, BEB cross section; circles, experimental data by Tarnovsky et al. [6]. 


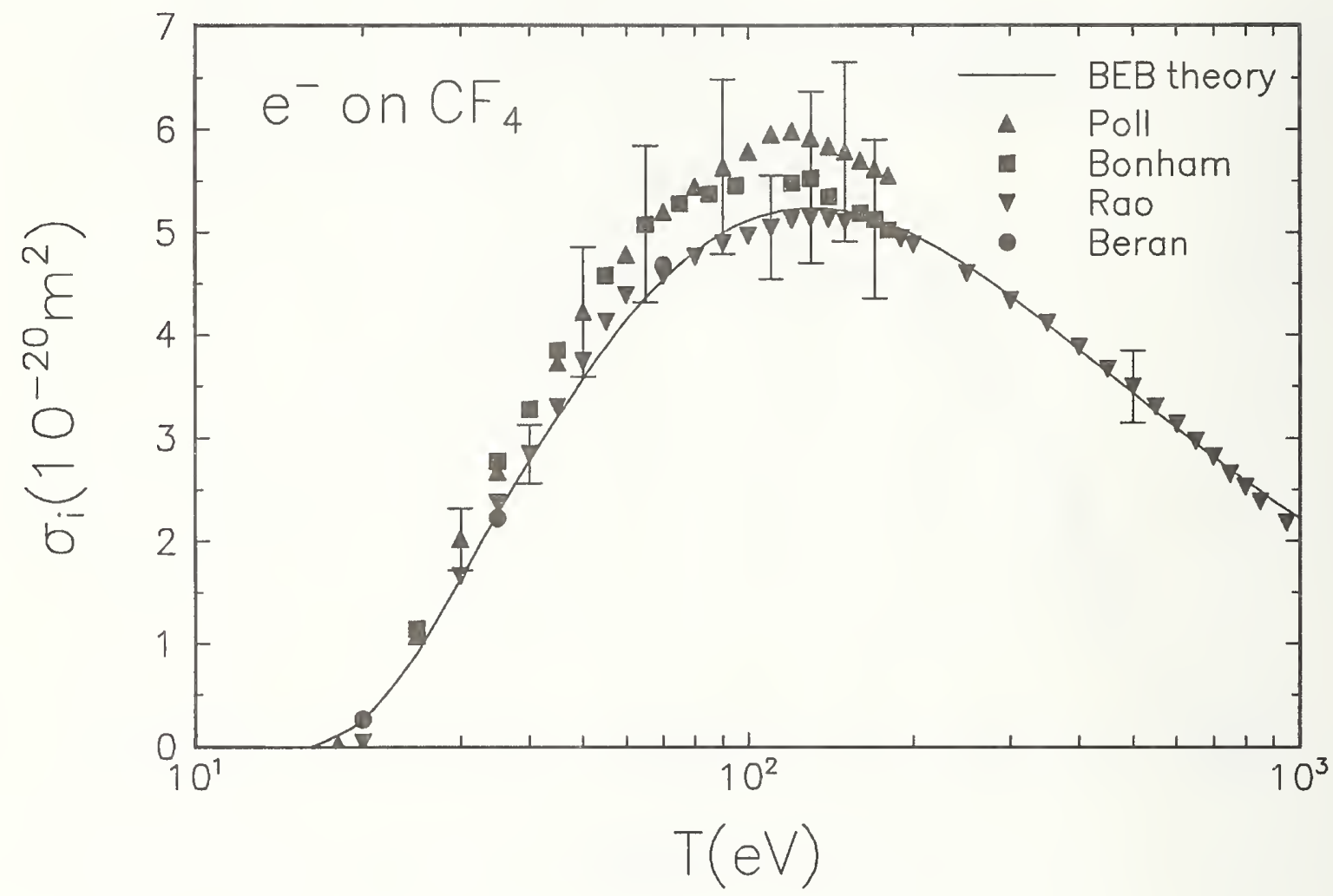

Figure 2: Comparison of ionizations cross sections for $\mathrm{CF}_{4}$, $\sigma$ i, total ionization cross section; $\mathrm{T}$, incident energy; solid curve, BEB cross section; upright triangle, experimental data by Poll et al. [7]; squares, data by Bonham [8]; inverted triangles, data by Rao and Srivastava [9]; circles, data by Beran and Kevan [10]. 


\title{
The Atomic Spectroscopy Bibliography Database of the Institute for Spectroscopy, Troitsk
}

\author{
A. E. Kramida*, A. N. Ryabtsev, and G. V. Vedeneeva \\ Institute for Spectroscopy of the Russian Academy of Sciences, \\ Troitsk, 142092, Russia.
}

A data bank BIBL containing bibliography on atomic data for plasma physics, atomic physics, astrophysics etc. is being developed at the Institute for Spectroscopy since 1987. The bibliographic information is supplemented by abstracts of papers and search keywords. A special system of spectroscopic keywords has been designed for the BIBL database. It allows very easy retrieval of information on search requests commonly required by spectroscopists, such as papers containing some specific kind of data concerning some specific ion or isoelectronic sequence. These kinds of search requests can not be executed in any other available bibliographic system. The data retrieval system is very simple and easy to use. It is self-documented and utilizes a menu system supplied with a context-sensitive help. The database works on IBM PC compatible computers with the MS DOS operating system or those that can emulate the MS DOS system.

The topics covered by BIBL are:

1. Spectra of atoms and positive ions:

ionization potentials, line classification, energy levels, wavelengths, hyperfine structure, isotopic effects, broadening and shifts of spectral lines, the Stark and Zeeman effects, plasma diagnostics, astrophysical spectra, theory of atomic spectra, radiation and autoionization rates, oscillator strengths, QED and relativistic effects in atoms and ions, atomic-spectroscopy tests of the fundamental principles, spectral sources, techniques of spectral measurements.

2. Cross sections of the collision processes:

excitation and ionization by electrons and photons, multiphoton processes, Auger decay, ion-electron recombination. To a lesser extent - charge exchange, excitation and ionization in collisions with heavy particles - if any new data related to atomic structure are obtained in these publications.

The bibliography related to experimental and theoretical papers on identification and prediction of atomic and ionic structure should be complete from 1983, the year of the last issue of NBS Special Publication "Bibliography on Atomic Energy Levels and Spectra", but the experimental spectral analysis can be traced back to about 1970. On the other topics, it is systematically maintained since 1989. For the last three years, the commercial database "Current Contents on Diskettes" has been used for this purpose, with corresponding software for transforming the articles found into the BIBL format. Presently, there are about 6000 bibliographic records stored in the BIBL system.

The BIBL database is available free of charge and can be received on the Internet via the ftp service. The link to its location can be found on ISAN home page at address http://ls.isan.troitsk.ru/. The files are supplied in compressed form and occupy about 2 Mbytes, while the size of the uncompressed database is about 10 Mbytes.

An on-line network access to the database is under development and is planned to be ready within a year. The information will appear on ISAN Internet home page.

•e-mail kramida@isan.troitsk.ru 


\title{
Atomic Data Center on PC
}

\author{
K. T. Lu, Dennis Baba, and Rolf Engleman \\ Atomic Engineering Corp.
}

\section{Introduction}

"Atomic Data Center on PC" is a long range and large scale program of Atomic Engineering Corp. This data center provides a generic format which makes spectroscopic data uniform and usable in a PC-based format. This program consists of three functions:

(1) data collection and production,

(2) database development and

(3) evaluation and analysis.

The data center consists of two types of data:

(A) Reference laboratory data of atomic and ionic data specialized on heavy elements with Z>26.

AEDB consists of data of energy levels and lifetimes and is capable of "multi-photon Grotrian Diagram". ASDB consists of data of wavelengths, transition probabilities and intensities. The source of data are from open literatures and data archive at National Solar Observatory in Tucson, AZ.

(B) Knowledge database for laser remote sensing.

This database is designed for "new fingerprints" and related parameters for laser remote sensing. This program is in collaboration with "Laser Induced Breakdown Spectroscopic (LIBS) User Facility" at Los Alamos National Lab.

\section{Database Management}

Multichannel Quantum Defect Theory (MQDT) [1,2,3] is used to organize, manage and evaluate the data in the Atomic Engineering System (AES) [4]. MQDT is an unified theory comprising the Rydberg states of spectroscopy and the autoionizing states of collisional processes in atoms and small molecules [2]. It organizes atomic structures in terms of channel sets and structural parameters. A "channel set" is defined as a set of discrete and continuum states of an electron plus ion core. Each state differs in the energy of the excited electron. Subsequently, an atomic structure is characterized by three sets of quantum defect parameters:

(1) eigen-quantum defect $M_{a}$,

(2) channel mixing angle $0_{a}$, and

(3) dipole transition matrix element $d_{a}$.

These parameters can be obtained by fitting the analytic relations to the discrete energy levels, oscillator strengths and photoionization cross sections. These parameters may also be calculated [5]. Relativistic MQDT is used to characterize high $\mathrm{Z}$ ions [6]. 
Highly efficient and powerful algorithms based on the structure parameters has been developed to organize and evaluate the data : energy levels, oscillator strengths/intensities/transition probabilities, lifetimes, photoionization cross sections and quantum defect of isoelectronic sequencies [4]. For example the whole Rydberg series and autoionizing structures as well as photoelectron angular distribution of noble gases $\mathrm{Ne}, \mathrm{Ar}, \mathrm{Kr}, \mathrm{Xe}$ and $\mathrm{Rn}$ are characterized by 5 interaction channels with 5 eigen-quantum defect parameters, 5 dipole matrix elements and 10 channel mixing angle parameters [1].

\section{Data Evaluation Procedures}

We developed the following procedures to evaluate transition probability, intensity and lifetime. The transition probability $\mathrm{Aul}_{\mathrm{u}}$, intensity $\mathrm{I}_{\mathrm{ul}}$ and lifetime tu of upper energy level Eu are related by the following expression:

$$
\mathrm{Aul}=\left(\mathrm{I}_{\mathrm{ul}} / \Sigma_{\mathrm{l}^{\mathrm{I}}} \mathrm{I}_{\mathrm{ul}}\right) / \mathrm{t}_{\mathrm{u}}
$$

where l' refers to all the lower energy levels to which the upper level u decays.

The intensity data are taken using a hollow cathode discharge and measured by high resolution Fourier Transform Spectrometer at National Solar Observatory at Kitt Peak, AZ, U.S.A. The intensity is measured by an automated AEC software MCCS system [7] that fits a line profile and the integrated area. The analysis of the spectral lines in terms of energy levels, J values and assignments is also carried out using MCCS software.

The lifetime is obtained using published data by laser-induced fluorescence from atoms and ions in a beam. We have developed an evaluation procedure for lifetime measurements based on the threshold laser induced fluorescence measurement as function of beam density and laser intensity (see the following) [8].

The transition probability is obtained from equation (1). Most of the up-to-date data on transition probabilities are obtained by this method [9].

In the absence of lifetime data, we obtain the reliable intensity by normalizing the intensity to the known data of transition probability. The normalized intensity carries the similar weight of reliability as the transition probability in terms of concentration measurement. For example, there is no reliable transition probability available for $257.601 \mathrm{~nm}$ line of $\mathrm{Mn}$-II however it was seen in the LIBS spectrum. Following the above procedure, we determined that the normalized intensity of $257.601 \mathrm{~nm}$ line to be 35 .

\section{Knowledge Database for Laser Remote Sensing}

The formation of a plasma plume and generation of emission induced by a high-power laser beam in solid material has led to the development of elemental composition analytic tools of condensedphase specimens [10]. One high power laser beam vaporizes the sample and generates a plasma plume. Emission from the plasma is collected by a detector, e.g., a laser induced breakdown spectrometer (LIBS). This method has the advantage of being developed as a powerful in-situ and remote sensing tool which requires minimally invasive sample preparation and separation.

However, there are several challenging problems of a in-situ laser induced breakdown spectroscopy. The calibration problem is more serious because of the lack of reliable library 
database for environmental matrix effects, such as soil matrix, moisture and laser induced plasma. The spectral shifts, broadening and "quenching" effects generated by environmental effects make quantitative characterization difficult. Lack of computational techniques to apply the knowledge base to the correction of in-situ measurements in real-time for quantitative characterization contributes to the challenging problems.

An intelligent software system, the Multi-spectral Chemical Characterization System (MCCS), was used to analyze the matrix effects of two of the toxic metals, $\mathrm{Be}$ and $\mathrm{Pb}$, in soil samples measured by a LIB spectrometer [11]. MCCS can convert ASCII, binary, tape and Flexible Image Transport System (FITS) which is a standard data format for Astrophysics and Astronomy into AEC's data format. The raw data collected from LIBS are downloaded into the MCCS. The spectra are measured by the powerful and efficient algorithm which is capable of measuring 100 integrated spectra in terms of width, peak height and position in less than 15 sec. The spectra are then searched, calibrated and identified in reference to the knowledge database. The search algorithm includes a "tolerance" parameter to account for the spectral shift and broadening caused by the matrix effects. The algorithm also includes "filters" in disentangling "spectral interference". These unique softwares allow user to identify quantitatively the relative concentration ratio (ppm) of the analyte in a sample. The final decision making can be displayed in terms of tables and graphics. The results indicate that the selected fingerprints used to measure $\mathrm{Be}$ at $313 \mathrm{~nm}$ survive the matrix effects. The fingerprint of $\mathrm{Pb}, 405.9 \mathrm{~nm}$, used in the calibration is quenched by the laser induced plasma. The following reports an investigation of the calibration procedure for laser induced effects. Then we use these results as guidance to search for "new fingerprints" under various environmental matrix effects.

\section{$5 \quad$ The Calibration Procedure for Laser Induced Effects}

We have developed a calibration procedure for non-LTE laser induced effects. The calibration procedure combines modeling with experimental measurement.

In a laser induced plasma the discrete line emission develops in the fringes (optical thin region) of the expanding plasma core where local thermodynamic equilibrium may not exist. The usual method based on local thermal dynamic equilibrium in measuring the intensities relatively and calibrating their relationship to the respective elemental composition, which has been analyzed by calibration standards, may not be sufficient.

This non-LTE model is based on the assumption that the quenching of the emission process of an element is caused by the competing ionization process of the same element. The emission rate can be derived from a rate equation analysis, which includes as many as four processes: ionization process, emission process, excitation process and stimulated process.

In order to test the model, we compare the results with a laboratory experimental investigation [8]. The experimental measurements were carried out at JilinUniversity, in a $\mathrm{Pb}$ atomic beam excited by a Quanta-Ray Nd:YAG dye laser system. The state $6 \mathrm{p} 7 \mathrm{~s}{ }^{3} \mathrm{P}_{1}$ at $283.3 \mathrm{~nm}$ is excited by a uv photon ( $\mathrm{w} \sim 283.3 \mathrm{~nm}$ ). The $405.9 \mathrm{~nm}$ radiates to level $3,6 \mathrm{p}^{2}{ }^{3} \mathrm{P}_{2}$. The ionization process from level 2 can be produced by a two photon process since the two photon energy is greater than the ionization energy of $\mathrm{Pb}(=7.415 \mathrm{ev})$. The photoelectrons were collected by a channeltron. The ionization signal is processed by a boxcar and displaced on a chart recorder. The fluorescence signal was simultaneously measured by a PMT with a filter. The data shows that the fluorescence process is simultaneously present and in competition with the ionization process [8]. The result of a model calculation compared with the experimental observation shows that at lower 
laser intensity, the $405.9 \mathrm{~nm}$ emission increases with increasing laser intensity [8]. This fluorescence rate decreases as the laser intensity increased beyond $10^{16}$ photons $/ \mathrm{cm}^{2}$ [8]. To involve the ionization process is essential, as given in equation (1), for a correct analysis of the measured data since a computation [12] which does not include the ionization process failed in predicting the decrease of fluorescence rate. Therefore the decreasing of the fluorescence signal is caused by the increasing of the ionization signal.

We have also carried out a laser induced fluorescence measurement of $\mathrm{Pb} 405.9 \mathrm{~nm}$ as a function of atomic beam density [8]. The result shows that there is a flat value of fluorescence yield below beam density $10^{8}$ atoms $/ \mathrm{cm}^{3}$. The fluorescence yield increases exponentially for beam density $>10^{8}$ atoms $/ \mathrm{cm}^{3}$ [8]. The laser power was of the order of sub-MW/ $\mathrm{cm}^{2}$. At low density the atomic beam is in thermal expansion. The mean free path among the atoms may be too large to generate laser induced effects. The flat fluorescence value is close to the atomic condition and is recommended for reference data whereas the higher beam density result may have laser induced effects. This investigation may explain the discrepancies of some of transition probabilities measured by lasers compared with non-laser methods.

The above modeling was based on laser induced ionization in atomic beams with a density $\sim 10^{8}$ atoms $/ \mathrm{cm}^{3}$ in vacuum. For laser induced plasmas in air with a density $>10^{12}$ atoms $/ \mathrm{cm}^{3}$, the ionization rate is at least two orders of magnitude larger $\sim 10^{-16} \mathrm{~cm}^{2}$. Consequently, the emission rate is reduced by at least two orders of magnitude. This investigation generates the key result : there is a threshold value of the laser intensity for emission process to be detected. Beyond that value, the emission process will be quenched and the relaxation process favors ionization. The low melting point and low ionization limit of $\mathrm{Pb}$ also contribute to the excessive generation of laser induced plasma. Consequently, the excited atom releases its energy via ionization and "quenched" the emission process. These results explain why certain "fingerprints" of metals based on laboratory results, e.g. $405.9 \mathrm{~nm}$ of $\mathrm{Pb}$ failed to show up in the field results using high power laser induced plasma method.

\section{Knowledge Database for Environmental Matrix Effects}

The manifestation of environmental matrix effects on the spectral lines are shifts, broadening and quenching. A knowledge database to store the calibrated data as well as the new fingerprints of matrix effects is designed. As the $\mathrm{Pb} 405.9 \mathrm{~nm}$ case shows, the calibration based on fingerprints from a reference database did not guarantee success. However, reliable and quantitative reference databases are necessary for the first step of any analytic science.

The spectral shifts and broadening of laser induced spectral lines in the presence of high pressure noble gas are available for $\mathrm{Mg}$ [13], $\mathrm{Ba}[14], \mathrm{Pb}$ [8], $\mathrm{Sn}[15]$, $\mathrm{Sr}[16]$ and Stark broadening [17].

\section{References}

[1] (a) K. T. Lu and U. Fano, Phys. Rev. A 2, 81 (1970)

(b) K. T. Lu, Phys. Rev. A 4, 579 (1971)

(c) K. T. Lu, in Photophysics and Photochemistry in the Vacuum Ultraviolet, NATO ASI Series, 217-244 (1985) 
[2] U. Fano and A. R. P. Rau, "Atomic Collisions and Spectra", Academic Press, New York (1986)

[3] (a) C. M. Lee and K. T. Lu, Phys. Rev. A 8, 1241 (1973)

(b) M. Aymar, Phys. Reports 110, 163 (1984)

[4] "Atomic Engineering System: version 1 Help Manual", U. S. Copyright No.,TXu 461487 , Feb. 1991

[5] (a) C. M. Lee, Phys. Rev. A 10, 1598 (1974)

(b) C. Greene and L. Kim, Phys. Rev. A 38, 5953 (1988)

(c) Z. W. Lu and K. T. Lu, Phys. Rev. A 31, 1515-1521 (1985)

[6] C. M. Lee and W, R. Johnson, Phys. Rèv. A 32, 979 (1980)

[7] "Multi-spectral Chemical Characterization System: Version 1.0", US Copyrights No. TXu 710-412, Aug. 16, 1995

[8] D. Ding, H. Liu and K. T. Lu, Acta Optica Sinica 8, 404 (1988)

[9] For example: M. E. Wikliffe and J. E. Lawler, J. Opt. Soc. Am. B. 15, 737 (1997)

[10] Leon J. Radziemski, "Review of Selected Analytical Applications of Laser Plasmas and Laser Ablation, 1987-1994", Microchemical Journal 50, 218-234 (1994)

[11] K. T. Lu, Dennis Baba, Mike Murray and Fang Xu, "Analysis of Matrix Effect of RCRA Metal Elements in Soil Measured by In-situ Laser Induced Breakdown Spectrometer", in "American Environmental Laboratory", March 10, 1997

[12] M. A. Boshov, A. V. Zybin, V. G. Koloshnikov and K. N. Koshelev, Spectrochimica Acta 32B, $279(1977)$

[13] J. Y. Zhang et al., J. Phys. B. At. Mol. Phys. 21, 581 (1988)

[14] D. H. Wu, U. F. Yand, and K. T. Lu, J. Phys. B. At. Mol. Opt. Phys. 23, L149 (1990)

[15] M. Jin, D. Ding, et al., in "Twelfth International Conference on Atomic Physics", Abstracts of Contributed papers VII-8, Ann Arbor, MI, 1990

[16] J. Q. Sun, E. Matthias, et al., Phys. Rev. A 43, 5956 (1991)

[17] C. Clark, K. T. Lu, and A. Starace, "Effects of Magnetic and Electric Fields on Highly Excited Atoms," in Progress in Atomic Spectroscopy, Part C, p.247-320, Ed. Beyer and H. Kleinpoppen, Plenum (1984) 


\title{
Los Alamos Opacity Web Page
}

\author{
N. H. Magee, Jr. and R. E. H. Clark \\ Atomic \& Optical Theory Group, T-4 \\ and \\ Code Integration Group, $X$-CI \\ Los Alamos National Laboratory, Los Alamos NM 87545
}

\section{Introduction}

The Los Alamos opacity database is now available on the World Wide Web at http://t4.lanl.gov. The database contains both the original Astrophysical Opacity Library [1] distributed worldwide in the 1980's (for historical reference) and the new improved opacities from the Light Element Detailed Configuration OPacity (LEDCOP) code [2]. Users can access the opacity data using the multigroup opacity code TOPS [3] to obtain Rosseland and Planck gray opacities, group mean opacities over selected energy ranges, the monchromatic absorption coefficients and the average ionization over a wide range of temperatures and densities. As described below, these quantities are available for all of the elements presently on the database and TOPS will provide the same quantities for any arbitrary mixture of these elements.

\section{Short History of the Opacity Database}

The Los Alamos opacities have been distributed world wide since the 1960's, mainly for use in astrophysical modeling. At first, the opacity tables were calculated for each individual request, but this became very time consuming. All of these tables were individually calculated from scratch for each new mixture, recalculating the opacities from the individual elements over and over again. In the late 1970 's, it was realized that the energy dependent absorption coefficients could be calculated once for each element and then combined with absorption coefficients of other elements to form as many mixtures as desired. There were only two restrictions for this method.

First, all molecular opacities and abundances had to be removed from the calculations since one must have all of the elemental data in the equation of state (EOS) to form molecular species. Second, the opacities had been calculated on a temperature-density $(T-\rho)$ grid, but the calculation had to be switched from the $(T-\rho)$ grid to a temperature-electron degeneracy $(T-\eta)$ grid. The electron degeneracy parameter characterizes the electron pressure of the plasma at the ion-plasma boundary, so two elements calculated at the same $\eta$ "see" the same plasma conditions and can be mixed together. Extensive comparisons were run in the late 1970's between the mixed opacities and the ab initio mixture calculations and differences were on the order of a few percent.

The Los Alamos Astrophysical Opacity Library was developed from this change in methodolgy. The first comprehensive set of elemental opacities was calculated in the late 1970's and distributed with software programs for mixing the elements together. Most of this distribution was done via magnetic tapes, with FTP being utilized in the early 1990's. Even with FTP, the data transfer was very slow since the library contained more than $300,000,000$ words with more being added as new opacity calculations were being done for the library. It was then decided to place all of the detailed 
absorption coefficient data files on the Web and use the upgraded mixing program TOPS to allow users to access the full database and download the data in as much detail as they wished.

\section{Brief Code Descriptions}

The Los Alamos LEDCOP opacity code uses a basis set of detailed LS terms (plus average configuration terms for complex ion stages) to calculate opacities for elements with $Z<31$. Each ion stage is considered in detail and interactions with the plasma are treated as pertubations. Calculations are done in local thermodynamic equilibrium (LTE) and only radiative processes are included. The EOS model is based on the Saha equation [4], including degeneracy, where the bound Rydberg sequences are cut off by plasma corrections [5]. The code does not consider liquid or solid phases, but uses an explicit ion model [6] to treat all of the bound electron states of every ionization stage.

A self-consistent Hartree-Fock code [7] with relativistic corrections is used to generate both single configuration LS term energies, radial dipole matrix elements and angular factors for all ion stages and unresolved transition array (UTA) energies and variances [8] in intermediate coupling for selected ion stages. Nonhydrogenic photoionization cross sections are calculated for each configuration subshell with $l<5$, using distorted wave continuum functions and the same Hartree-Fock structure calculations as for the bound-bound transitions in order to conserve oscillator strength across the photo-electric edges. Users should access the web page reference link for more details.

The Astrophysical Opacity Library consists of random access binary files. The TOPS code is the vehicle for accessing these data files and performing numerical integrations to obtain the Rosseland and Planck opacities. TOPS can provide opacities for an arbitrary mixture of pure elements. As mentioned previously the data is stored as a function of $T$ and $\eta$. The TOPS code mixes elements at similar $\eta$ 's and then interpolates on $\eta$ to obtain a material density. The user can select an arbitrary density and, for multigroup opacities, an arbitrary photon energy grid. The temperature grid is limited to subsets of the temperature grid on which the original data are tabulated; no interpolation in temperature is allowed in the TOPS code.

\section{Database Structure}

At present, the database contains monochromatic absorption coefficients for all of the elements from hydrogen to zinc. Older element calculations are being upgraded and the database may be extended to higher $\mathrm{Z}$ elements in the future. Each element in the library is calculated on a uniform $T, \eta$ and $u(u=h \nu / k T)$ grid to allow the mixing of the elements to form mixtures. This provides an almost unlimited choice for users to customize opacity tables from pure elements to two element mixtures, such as the carbon-iron mix presented in this paper, to complex astrophysical mixtures containing more than 20 elements. The older 1977 element data is included so that users can baseline the changes in the opacities over the 20 year span of the calculations. The old element data can be mixed just like the newer data, and can even be mixed with the newer data since the older photon grid is a subset of the photon grid used for the newest calculations. The TOPS code automatically chooses the correct grid when the materials are mixed.

Table 1 lists the temerature, density and $u$ ranges covered by the database. The density range is shown rather than the electron degeneracy range since the density is a more familiar quantity. Every element in the library has approximately $1500 \mathrm{~T}-\eta$ points, covering the ranges listed below. Therefore, each element has approximately 12,000,000 words of data. As disk space allows, the authors hope to expand the number of $u$ grid points from the present 3,900 to 14,900 . 
Table 1: Ranges covered by the opacity database.

\begin{tabular}{|c|l|l|}
\hline Quantity & Range & Comments \\
\hline $\begin{array}{c}\text { Temperature } \\
\text { Density }\end{array}$ & $\begin{array}{c}.5 \text { or } 1.0 \mathrm{eV} \text { to } 100,000 \mathrm{eV} \\
10^{-10} \mathrm{gm} / \mathrm{cc} \text { to } 10^{+9} \mathrm{gm} / \mathrm{cc}\end{array}$ & $\begin{array}{l}10 \text { temperatures/decade. } \\
1 \text { to } 3 \text { densities/decade. Highest } \\
\text { density is temperature dependent. } \\
1977 \text { data stops at } u=30 .\end{array}$ \\
\hline $\begin{array}{l}\text { u } \\
\text { 0. to } 30 . \text { or } 30,000 .\end{array}$ & \multicolumn{2}{l}{} \\
\hline
\end{tabular}

\section{Sample Web Page Output .}

A short sample output is shown for a carbon-iron mixture at two temperatures and two densities. The default output has been chosen, so the table displays the Rosseland and Planck gray opacities and the the average number of free electrons per atom. Note that a warning message has been printed, indicating that one of the requested densities was off the table. The TOPS code will use the values from the highest calculated density for all densities above the last density point.

Table 2: Sample opacity tables for CFe mixture.

\begin{tabular}{|c|c|c|c|c|c|}
\hline \multicolumn{6}{|c|}{ TOPS results } \\
\hline \multirow{2}{*}{\multicolumn{6}{|c|}{$\begin{array}{l}\text { Opacities in } \mathrm{cm}^{* *} 2 / \mathrm{gm}, \mathrm{T} \text { in } \mathrm{keV} \text {, density in } \mathrm{gm} / \mathrm{cc} \\
\text { WARNING - Requested densities went off table boundaries }\end{array}$}} \\
\hline & & & & & \\
\hline \multicolumn{6}{|c|}{ Temp Den Req Den used } \\
\hline $1.0000 \mathrm{E}-03$ & $1.0000 \mathrm{E}+00$ & $7.0739 \mathrm{E}-01$ & & & \\
\hline \multicolumn{6}{|c|}{ Normalized composition for requested elements } \\
\hline No. Fraction & Mass Fraction & At. No. & Chem. Sym. & Mat Id. & \\
\hline $5.0000 \mathrm{E}-01$ & $1.7699 \mathrm{E}-01$ & 6 & & 117831 & \\
\hline $5.0000 \mathrm{E}-01$ & $8.2301 \mathrm{E}-01$ & 26 & $\mathrm{Fe}$ & 112142 & \\
\hline \multicolumn{6}{|c|}{ Temperature grid used the following 2 points } \\
\hline \multicolumn{6}{|c|}{$1.0000 \mathrm{E}-03 \quad 1.2500 \mathrm{E}-03$} \\
\hline \multicolumn{6}{|c|}{ Density grid used the following 2 points } \\
\hline $1.0000 \mathrm{E}-01$ & $1.0000 \mathrm{E}+00$ & & & & \\
\hline \multicolumn{6}{|c|}{ Rosseland and Planck opacities and free electrons } \\
\hline Density & Ross opa & Planck opa & No. Free & Av Sq Free & $\mathrm{T}=1.0000 \mathrm{E}-03$ \\
\hline $1.0000 \mathrm{E}-01$ & $4.1416 \mathrm{E}+05$ & $8.4327 \mathrm{E}+05$ & $5.2602 \mathrm{E}-02$ & $5.2604 \mathrm{E}-02$ & \\
\hline $1.0000 \mathrm{E}+00$ & $4.5953 \mathrm{E}+05$ & $2.5213 \mathrm{E}+06$ & $2.3540 \mathrm{E}-02$ & $2.3541 \mathrm{E}-02$ & \\
\hline Density & Ross opa & Planck opa & No. Free & Av Sq Free & $\mathrm{T}=1.2500 \mathrm{E}-03$ \\
\hline $1.0000 \mathrm{E}-01$ & $3.3323 \mathrm{E}+05$ & $1.2938 \mathrm{E}+06$ & $1.0226 \mathrm{E}-01$ & $1.0231 \mathrm{E}-01$ & \\
\hline $1.0000 \mathrm{E}+00$ & $3.7040 \mathrm{E}+05$ & $3.8026 \mathrm{E}+06$ & $5.3035 \mathrm{E}-02$ & $5.3057 \mathrm{E}-02$ & \\
\hline
\end{tabular}

\section{Critical Evaluation}

One of the most difficult tasks in opacity calculations has been assigning error criteria to the final results. The calculations are a complex mixture of atomic physics input data and plasma modeling. 
The plasma models include the EOS occupancy determination and the broadening of the boundbound line transition profiles. Up to 1988, there was very little organized comparison between the various theoretical codes and no experimental data. Both of these situations changed in 1988 with the meeting of the first International Opacity Code Comparison Workshop and with the publication of the first opacity transmission experiment from RAL [9] in England. Since that year, there have been three more workshops [10] with more than 15 participating groups, and more than half a dozen experiments from groups around the world.

Opacities from the various codes have changed by factors of 2 to 10 over the years since 1988, especially in $T-\rho$ regions where the $\Delta n=0$ bound-bound line transitions are important. The authors feel that these large uncertainties are now resolved and that one can place an upper limit of $25 \%$ on the integrated Rosseland opacities for low and moderate densities where LTE is appropriate. At higher densities, such as a few gm/cc at temperatures of 10 to $50 \mathrm{eV}$, the agreement between the independent codes can differ by more than a factor of 2 and there is almost no reliable experimental data, so it is not possible to set an uncertainty below $50 \%$ for these regions.

\section{References}

[1] W. F. Huebner, A. L. Merts, N. H. Magee, and M. F. Argo, "Astrophysical Opacity Library", LA-6760-M, Los Alamos National Laboratory 1977

[2] N. H. Magee, Jr., J. Abdallah, Jr., R. E. H. Clark, et al., "Atomic Structure Calculations and New Los Alamos Astrophysical Opacities" Astrophysical Applications of Powerful New Databases, Astronomical Society of the Pacific Conference Series, S. J. Adelman and W. L. Wiese eds., 78, 51 (1995)

[3] J. Abdallah, Jr. and R. E. H. Clark, "TOPS: A Multigroup Opacity Code", LA-10454-M, Los Alamos National Laboratory 1985

[4] A. N. Cox, "Stellar Absorption Coefficients and Opacities" Stars and Stellar Systems, L. H. Allen and D.B. McLaughlin eds., vol. 8, p. 195, University of Chicago, Chicago 1965

[5] J. C. Stewart and K. D. Pyatt, Ap. J., vol. 144, 1203 (1966)

[6] W. F. Huebner, "Atomic and Radiative Processes in the Solar Interior" The Physics of the Sun, P. A. Sturrock ed., vol. 1, p. 33, D. Reidel Publishing Company, Dordrecht-Holland 1986

[7] J. Abdallah, Jr., R. E. H. Clark, and R. D. Cowan, "CATS: Cowan Atomic Structure Code", LA-11436-M, vol. 1, Los Alamos National Laboratory 1988

[8] D. P. Kilcrease, J. Abdallah, Jr., J. J. Keady, and R. E. H. Clark, J. Phys. B, vol. 26, L717 (1993)

[9] S. J. Davidson, J. M. Foster, C. C. Smith, K. A. Warburton, and S. J. Rose, Appl. Phys. Lett. $\mathbf{5 2}, 847$ (1988)

[10] Third International Opacity Workshop \& Code Comparison Study (WorkOp-III;94), MaxPlanck-Institut für Quantenoptik Report No. 204, A. Rickert et al. eds., (1995) 


\section{TOPbase/TIPbase}

\author{
C. Mendoza \\ Physics Center, IVIC, \\ Caracas, Venezuela
}

\author{
C. J. Zeippen \\ Observatoire de Paris \\ Meudon, France
}

\section{Introduction}

TOPbase/TIPbase is an atomic database service that has been available on the Internet since January 1993 from both the Centre de Données Astronomiques de Strasbourg (CDS), France, http://cdsweb.u-strasbg.fr/OP.html, and HEASARC, Goddard Space Flight Center, NASA, USA, http://asca.gsfc.nasa.gov/docs/topbase/home.html. Direct access to the commandbased database can be managed by telnet to cdsarc.u-strasbg.fr (IP=130.79.128.5) in France and topbase.gsfc.nasa.gov (IP=128.183.126.111) in the USA with account $\mathrm{id}=$ topbase and $\mathrm{PW}=$ Seaton+. TOPbase $[1,2]$ contains term energies, f-values and photoionization cross sections computed in LS coupling in the Opacity Project (OP) [3] for astrophysically abundant ions ( $\mathrm{Z}=1$ $14 ; Z=16, Z=18 ; Z=20 ; Z=26$ ). This database has averaged over 100 calls a month since it became operational. TIPbase (under development) will offer the data from the current IRON Project (IP) [4], namely level energies, radiative transition probabilities, electron impact excitation collision strengths and rates for fine structure transitions of ions of astrophysical interest, with emphasis on the iron group. In the present paper, we briefly discuss the atomic data, the database design, work in progress and some important points that have emerged during our five-year experience offering such a service.

\section{Atomic data}

The atomic data contained in TOPbase/TIPbase are calculated by an international group of experts from Canada, France, Germany, UK, USA and Venezuela with more than 15-year experience in large collaborative computational projects. State-of-the-art numerical methods are used such as those implemented in the atomic structure codes CIV3 [5] and SUPERSTRUCTURE [6] and collisional approaches based on the R-matrix method [7, 8, 9]. The emphasis is both on accuracy and completeness of massive datasets thus leading to series of papers where such issues are extensively discussed, namely the series "Atomic Data for Opacity Calculations (I-XXIII)" in the Journal of Physics B and the series "Atomic Data from the IRON Project (I-XXVIII)" in Astronomy \& Astrophysics. A measure on the quality of the data produced by the OP and IP is given, for instance, by the large number of f-values computed in the former that have been selected for the NIST reference compilations (e.g. [10]).

\section{Database design}

TOPbase was one of the first on-line atomic databases that became operational on the Internet. Due to a general lack of standards and having taken the practical resolution to avoid the commercial packages in order to ensure portability at low costs, the design of the TOPbase database 
management system has been entirely homemade [1]. It is closely related to the physics of the atomic data and to the most frequent search patterns that are likely to be repeated by users and applications. In this respect, a bound state $(i)$ is uniquely addressed by a key defined in terms of the following attributes: ( $\mathrm{nz}$, ne, islp, ilv), where $\mathrm{nz}$ and ne are respectively the atomic number and electron number of the ion; islp gives the quantum numbers (total spin multiplicity, orbital angular momentum and parity) of the spectroscopic series to which it belongs, defined as islp $=100(2 S+1)+10 L+P$; and $i l v$ is the level energy position within the spectroscopic series. The key for a transition $(i \rightarrow j)$ is similarly given in terms of the attributes (nz, ne, islp, jslp, ilv, jIv). The input selectors, based on numerical ranges of these keys, and the index structure have been devised to facilitate searches along isoelectronic and isonuclear series, in energy or wavelength ranges, within an ionic system or a spectroscopic series, or just for a single level or transition. As shown in Fig. 1 the data manipulation scheme has been implemented on two levels: the view where searches and time-consuming block data retrievals are performed from disk; and the table where fast and versatile functions (sorting, column and row selections, graphic display) are carried out iteratively in main storage to satisfy user requirements. Both data structures allow display, printing, disk downloading and graphic processing. The present user interface is command-based providing a fairly powerful yet simple query language. Although most regular users have not complained about this query environment, it does discourage the occasional user who is not really prepared to invest time in learning the commands. Thus, a web-based user interface has been developed for TOPbase which is to become operational by the beginning of 1998 . In spite of the reduction in interactivity of a web interface, TOPbase keeps most of its design features, e.g. the numeric access codes and the view and table data structures. Furthermore, the design of TIPbase will follow the guidelines and scheme of TOPbase.

\section{Recent developments}

We summarize here recent work that has either been finished or is currrently in progress regarding updates of the data and improvements of the software.

\subsection{Work concluded}

- Improved radiative data (e-levels, f-values, photoionization cross sections) for the ions Fe I-IV.

- Improved radiative data for the ions of oxygen.

- The TOPbase web user interface. It will become operational in January 1998.

\subsection{Work in progress}

- New radiative data for the $\mathrm{P}$ and $\mathrm{Cl}$ isonuclear sequences.

- Radiative data in intermediate coupling for the isoelectronic sequences of $\mathrm{Ne}$ and Ar.

- Electron impact excitation rates for fine structure transitions in the isonuclear sequence of Fe.

- The TIPbase database management system and user interface. 


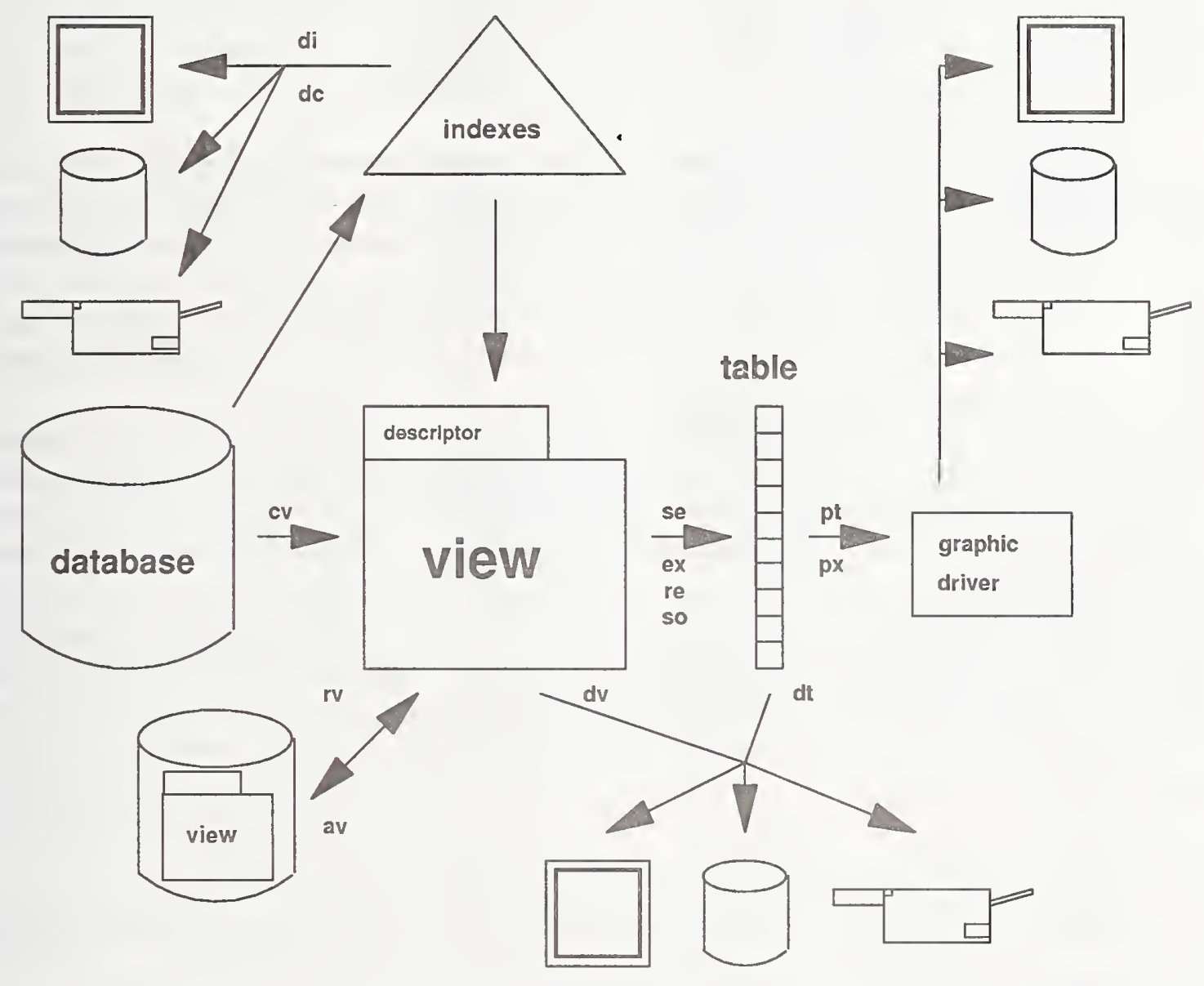

TOPBASE general structure

Figure 1: TOPbase data manipulation scheme showing the two main data structures, the view and the table, the display and graphic capabilities and query commands. 


\section{Comments}

We would like to make here some comments, intended mainly for future entrepreneurs, regarding the development and maintenance of atomic database services which have arisen in our five-year experience running such a service.

We have found a sizable need for atomic data in the world-wide scientific community although we feel that the demand would sharply go down if it is addressed on business terms. Therefore it is unlikely that many outsiders will be attracted by the opportunities offered by the new atomic database projects, and therefore their development will have to rely mainly on inhouse initiatives that would have to apply for grants to the traditional funding agencies. In other words, nobody is bound to get rich from running an atomic data business.

In our opinion it is essential to offer atomic database services within the context of a data center. In the case of TOPbase, we have worked out a good relationship and operational terms with both the CDS and NASA. Furthermore, the concept of a data center appears to be undergoing an interesting evolution. Rather than being a center for data compilation and publication, it is now becoming a contractor, promoter and evaluator of distributed database portfolios which should be as close as possible to the data producers. Moreover, the marketing of the data and the establishment of new client bases are among their new responsibilities.

The general absence of standards surely is a deterement to the proliferation of atomic databases and makes life very difficult for both users and developers. Apart from the consensus on web-based user interfaces, we feel that it is timely to sort out some of the remaining key issues: data-entry and output formats, management systems, interactivity, data transfer and compression protocols, and documentation.

In the context of electronic data dissemination, there is the implicit responsibility of ensuring data integrity. Since the latter would eventually be passed on to the data producer, the source reference must always be quoted in all database transactions.

\section{References}

[1] W. Cunto and C. Mendoza, Rev. Mex. Astron. Astrofis. 23, 107 (1992)

[2] W. Cunto, C. Mendoza, F. Ochsenbein, C. J. Zeippen, Astron. Astrophys. 275, L5 (1993)

[3] The Opacity Project Team, The Opacity Project, Vol. 1, IOPP, Bristol (1995)

[4] D. G. Hummer, K. A. Berrington, W. Eissner, et al., Astron. Astrophys. 279, 298 (1993)

[5] A. Hibbert, Comput. Phys. Commun. 9, 141 (1975)

[6] W. Eissner, M. Jones, and H. Nussbaumer, Comput. Phys. Commun. 8, 270 (1974)

[7] P. G. Burke, A. Hibbert, and W. D. Robb, J. Phys. B 4, 153 (1971)

[8] P. G. Burke and W. D. Robb, Adv. Atom. Molec. Phys. 11, 143 (1975)

[9] P. G. Burke, in "Physics of Electronic and Atomic Collisions" (S. Datz, ed.), p. 447, NorthHolland, Amsterdam 1982

[10] W. L. Wiese, J. R. Fuhr, and T. M. Deters, "Atomic Transition Probabilities of Carbon, Nitrogen, and Oxygen - A Critical Data Compilation", J. Phys. Chem. Ref. Data Monograph 7 (1996) 


\title{
NIFS Retrieval Display System for Atomic Data through the WWW
}

\author{
Izumi Murakami, Masatoshi Kato, Takako Kato, NEC nifs team \\ Data and Planning Center, National Institute for Fusion Science, \\ Oroshi-cho, Toki, Gifu 509-5292, Japan
}

\begin{abstract}
The Data and Planning Center at NIFS has developed a retrieval display system for atomic data since 1979. This year we reconstructed our atomic database to be accessible via the World Wide Web. We introduce our atomic and molecular numerical databases and describe how to use them.
\end{abstract}

\section{Introduction}

Data compilation and evaluation of atomic and molecular data for fusion research was initiated by working groups organized by Professors K. Takayanagi, H. Suzuki et al. in our former institute (Institute of Plasma Physics, Nagoya Univ.) in 1975 [1]. This work has continued in the Research Information Center in IPP, Nagoya from 1979 [2] including surface data and in the Data and Planning Center of the National Institute for Fusion Science since 1989 [3]. The retrieval and display system for four kinds of numerical data, listed below, was developed as a joint research program with Drs. Y. Kanada, K. Takasugi and R. Ogasawara et al. This system has been open for users since $1981[4,5]$ through a main frame computer (FACOM).

In 1997, we changed the database system to be accessible via the World Wide Web, and the system has become easier to access and retrieve data from.

We have four numerical databases:

AMDIS Cross sections for ionization and excitation of atoms, ions, and molecules by electron impact

CHART Cross sections for charge transfer and ionization of atoms, ions, and molecules by collisions

SPUTY Experimental sputtering yields for monatomic solids by ions

\section{BACKS Particle and energy-backscattering coefficients of light ions from solids}

Each database has numerical data tables with references and empirical formulas. The bibliographic information on numerical data contains the method of the experiment or theory, as well as the authors' names, paper title, journal name, page, and published year. Following, we describe how to access the new databases, how to use the retrieval display system, and $\lambda$ provide examples. 


\section{How to Access the Databases and Retrieve Data}

We require users to register and get an account before they access the databases. This does not mean that we charge users. We need to know who is using the databases and for what purpose.

For registration, access the URL address, http://dbshino.nifs.ac.jp/. Following the instructions, go to the brief description page on the databases and regulations, and then go to the registration page where you may submit your personal information for registration to dbmaster@dbshino.nifs.ac.jp. Do not forget to write your purpose for using the databases. We opened the databases only for scientific research, and we will determine who is acceptable as a user. You will receive your account ID and password in a week by e-mail.

At the front page, we also link to our other web site at URL= http:llamdata.nifs.ac.jp/ for information on the numerical databases. You will find tables showing how many data sets are stored for each ion species. After clicking the position of the ion you are interested in, you will find graphs of the cross section from AMDIS. Also, there are examples of data stored in ALADDIN format with graphs for rate coefficients as well as cross sections.

Here, we briefly describe how to use our numerical databases. The procedure is as follows:

1. Access the system at URL=http://dbshino.nifs.ac.jp, type in your account ID and password.

2. Choose a database name from the list.

3. Set searching conditions, such as element name, and search.

4. Select process(es) which you are interested in from the list.

5. Select either standard or custom for bibliographic information or numerical data for tables and graphs and push the display button.

6. Select units for the data to be displayed for AMDIS, CHART, and BACKS and push the display button.

7. 20 bibliographic data items are shown in one page.

8. For AMDIS, you may choose data one by one to be displayed in tables or graphs.

9. For BACKS, choose angles or energy to be plotted in a graph.

10. Before drawing graphs, you may specify how to draw axes, whether to draw an empirical curve or not, and the positions for drawing symbol descriptions. A postscript file of the graphs can be downloaded after they are drawn.

For each step in this procedure, you move on to a deeper web page. To each web page is attached a help page which pops up as a separate window. In Step 2, you will find CURVE which draws empirical formulas for specific ions.

\section{$3 \quad$ Examples}

Following are examples of searching for ionization cross sections by electron impact and excitation cross sections (AMDIS). 


\subsection{AMDIS Ionization}

To search for cross sections for a single ionization of neutral Neon, put " $N e$ " for element, " 0 " for initial ionic state, and " 1 " for final ionic state, as shown in Fig. 1. In addition, you may specify author names, published years, and some more conditions or select theoretical, experimental, or evaluated data.

You can make numerical data tables or graphs. The graph of the cross section is shown in Fig. 2 for this case. An empirical curve calculated with Lotz's formula can be drawn together with the data.

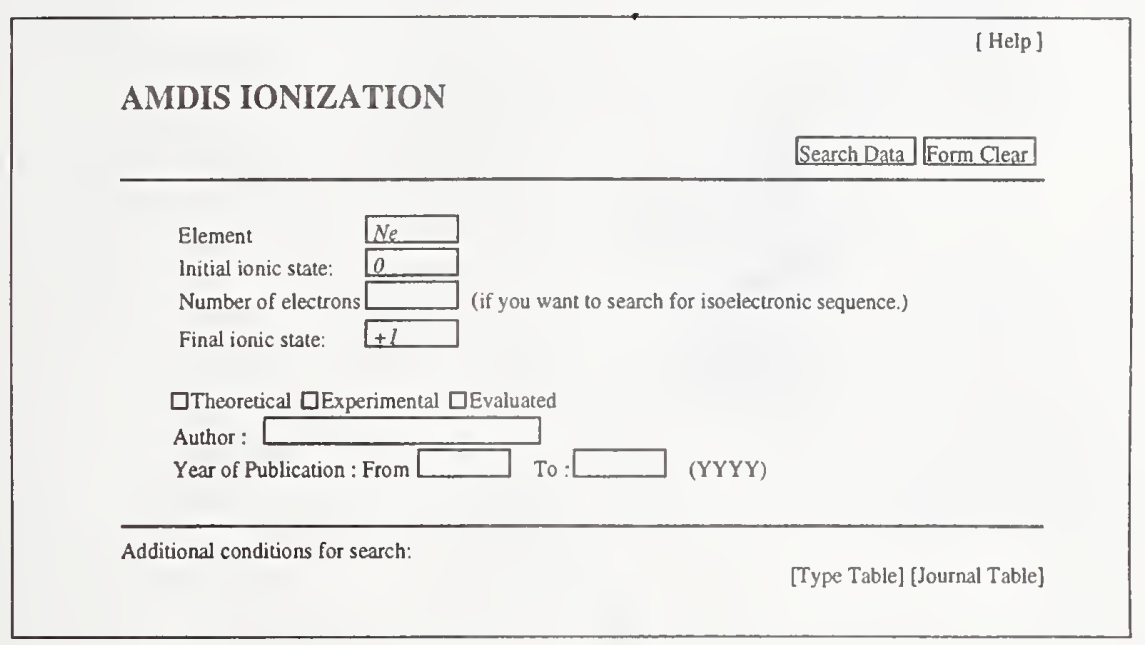

Figure 1: A sample for setting search conditions for AMDIS-IONIZATION.

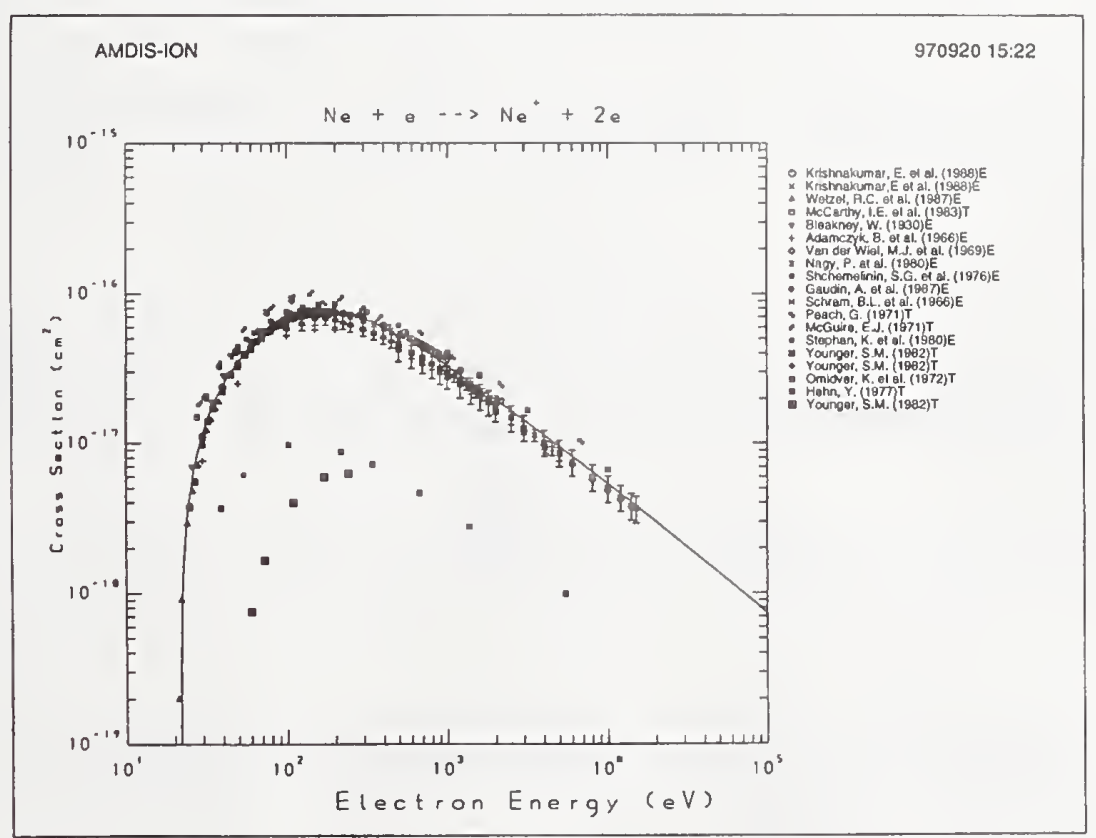

Figure 2: Cross section for the single ionization of neutral Neon, drawn in AMDIS-IONIZATION. The Lotz's empirical formula is also drawn. 


\subsection{AMDIS Excitation}

Similarly, you can search for the excitation cross section from $1 \mathrm{~s}^{2}{ }^{1} \mathrm{~S}$ to $1 \mathrm{~s} 2 \mathrm{p}{ }^{1} \mathrm{P}$ for He-like carbon, for example. Enter " $C$ " for element and " 2 " for the number of electrons. For the initial state configuration, enter " $1 s 2$ ". For the final state, enter " $1 s 2 p$ " for the configuration, " 1 " for the multiplicity, and " $P$ " for the orbital angular momentum.

When making a graph, you can select either a cross section or collision strength. An example of the latter is shown in Fig. 3.

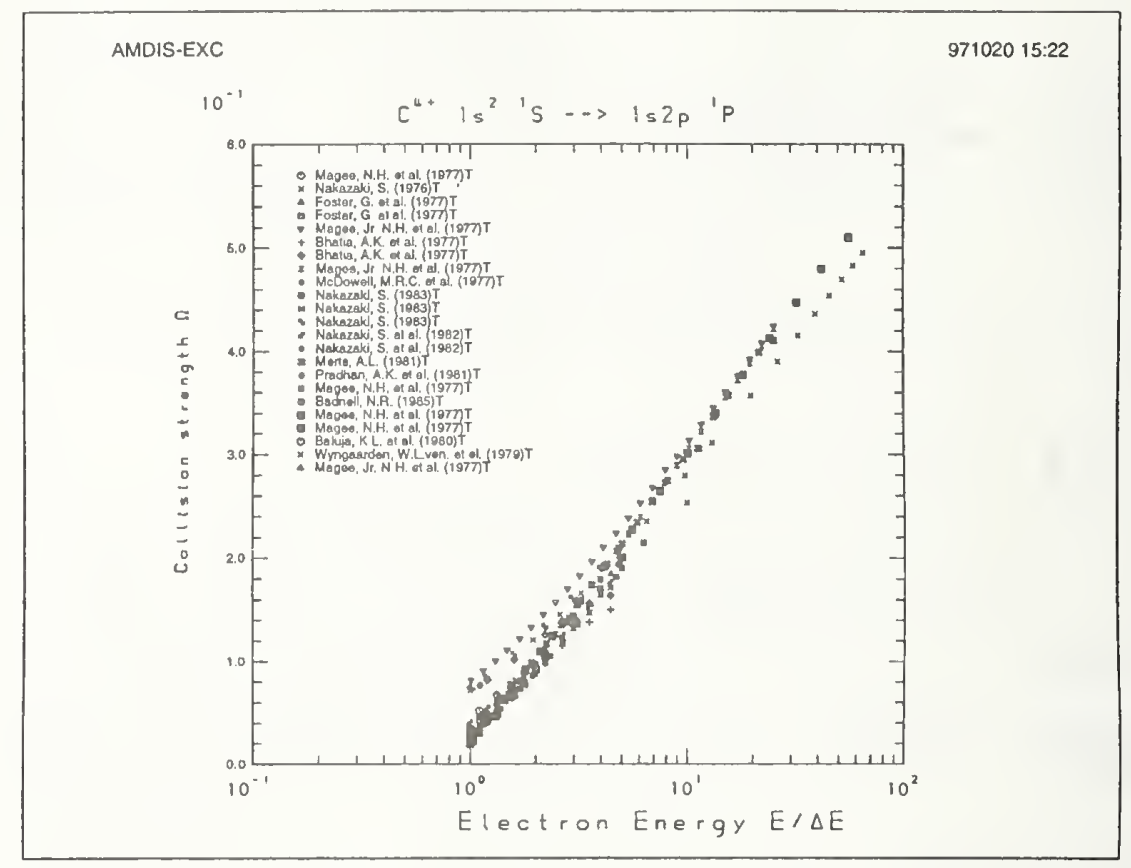

Figure 3: Collision Strength as a function of $E / \triangle E$, drawn in AMDIS-EXCITATION.

\section{Remarks}

We are interested in atomic data needs and appreciate your comments and requests. We welcome your suggestions on improving the databases. Please send your requests, questions, and your data by e-mail to dbmaster@dbshino.nifs.ac.jp. Instructions on data formats are being prepared and will appear soon.

We are grateful to Dr. Y. Kanada, Dr. K. Takasugi, and Dr. R. Ogasawara for creating the database and retrieval display system and for their help and advice on the new database system.

\section{References}

[1] Cross sections for Atomic Processes Vol.1, IPPJ-DT-48 (1975), Vol.2, IPPJ-DT-50 (1976) edited by K. Takayanagi, H. Suzuki.

[2] IPPJ-AM series, IPP, Nagoya Univ., Japan

[3] NIFS-DATA series, National Institute for Fusion Science, Japan

[4] T. Kato, Y. Itikawa, Y. Kanada, and R. Watanabe, Physica Scripta 23, 198 (1981)

[5] T. Kato et al., J. Nucl. Mater. 128 \& 129, 1006 (1984) 


\title{
Wavelengths of Six Times Ionized Germanium, Ge VII
}

\author{
L. I. Podobedova ${ }^{1,2}$ and A. N. Ryabtsev 2 \\ ${ }^{1}$ National Institute of Standards and Technology, Gaithersburg MD 20899 U.S.A. \\ 2Institute of Spectroscopy RAS, Troitsk, 142092 Russia
}

A compilation of Ge VII wavelengths has been carried out for the NIST Atomic Spectra Database on the World Wide Web. The compilation is based on our previous analyses of the germanium spectra: the $3 d^{8}-3 d^{7} 4 p$ transition array in the range $160-210 \AA$, $3 p^{6} 3 d^{8}-3 p^{5} 3 d^{9}$ in the range $123-142$ $\AA$ and $3 d^{7} 4 \mathrm{~s}-3 d^{7} 4 \mathrm{p}$ in the range $739-987 \AA$ [1-3] and the compilation of energy levels by Sugar and Musgrove [4]. A Russian publication [1] with the complete list of wavelengths of Ge VII is not well known; no new data on this spectrum were obtained since our analyses.

The Ge VII spectrum was excited in a three-electrode vacuum spark with the discharge parameters $\mathrm{C}=1-10 \mu \mathrm{F}, \mathrm{L}=500-1000 \mathrm{nH}$, and $\mathrm{U}=4-10 \mathrm{kV}$. Transitions to the ground state, lying in the region shorter than $200 \AA$, were studied from spectrograms obtained with a 3-m grazing incidence vacuum spectrograph (angle of incidence $85^{\circ}$ ) with a 3600 lines $/ \mathrm{mm}$ grating. When working in the region longer than $700 \AA$, where transitions between excited states are located, we used a 6.65-m normal incidence vacuum spectrograph with a 1200 grooves/mm grating. The standard error for the wavelengths in the short-wave region was estimated at $0.004 \AA$, and in the long-wavelength region, $0.007 \AA$.

The Ge VII spectrum belongs to the iron isoelectronic sequence, having a ground-state configuration $3 \mathrm{p}^{6} 3 \mathrm{~d}^{8}$ and the lowly excited configurations $3 \mathrm{~d}^{7} 4 \mathrm{~s}, 3 \mathrm{~d}^{7} 4 \mathrm{p}, 3 \mathrm{p}^{5} 3 \mathrm{~d}^{9}$ and $3 \mathrm{~d}^{7} 4 \mathrm{f}$. The $3 \mathrm{p}^{5} 3 \mathrm{~d}^{9}$ configuration, with the excitation of an electron from an inner shell, which is unknown at the beginning of the isoelectronic sequence, decreases in energy with respect to valence electron excitations as the ionization stage increases. As a result, a strong interaction between the $3 p^{6} 3 d^{7} 4 p$ and $3 p^{5} 3 d^{9}$ configurations is observed for a number of ions of the sequence including Ge VII (Fig. 1). The analysis of the resonance transition array $3 d^{8}-3 d^{7} 4 \mathrm{p}$, located in the region 160 $210 \AA$, has been carried out in [2]. All levels of the ground configuration $3 \mathrm{~d}^{8}$ and 85 of 110 levels of the $3 \mathrm{~d}^{7} 4 \mathrm{p}$ configuration have been found. Other levels of the latter configuration either do not have transitions to the ground configuration or the transitions are very week. These levels can be found by studying transitions to the $3 d^{7} 4 \mathrm{~s}$ configuration. The analysis of the $3 d^{7} 4 p-3 d^{7} 4 s$ and $3 p^{6} 3 d^{8}-3 p^{5} 3 d^{9}$ transition arrays and the necessary corrections of the $3 d^{7} 4 p$ configuration were made in our subsequent work [3]. Thus all levels of the $3 d^{8}, 3 d^{7} 4 s, 3 s^{7} 4 p$ and $3 p^{5} 3 d^{9}$ configurations were established. Later, these levels were compiled in [4].

The purpose of the present work is to check our previous analysis and to present a wavelength table in the format of the NIST Database. It should be noted that we interpreted the spectra on the basis of single-configuration calculations because of our calculation limitations at that time. Besides, the knowledge of other ions of the Fe-like isoelectronic sequence was very poor. In 1980, when we started to work on the Ge VII spectrum, the highest well-studied member of this sequence was $\mathrm{Cu}$ IV. The spectra $\mathrm{Zn} \mathrm{V}$ and Ga VI were under study, and only preliminary values for the levels of the term $3 \mathrm{~d}^{8} 3 \mathrm{~F}$ were known. Since that time, a great amount of work has been done for many spectra of Fe-like ions. Now, the resonance transition array $3 d^{8}-3 d^{7} 4 \mathrm{p}$ has also been studied for the Zn V-Br X, Rb XII and Sr XIII ions. The $3 \mathrm{p}^{6} 3 \mathrm{~d}^{8}-3 \mathrm{p}^{5} 3 \mathrm{~d}^{9}$ transition array is known for the Ga VI-Br X and Ag XXII-Sn XXV ions. In these latter ions, this transition array 


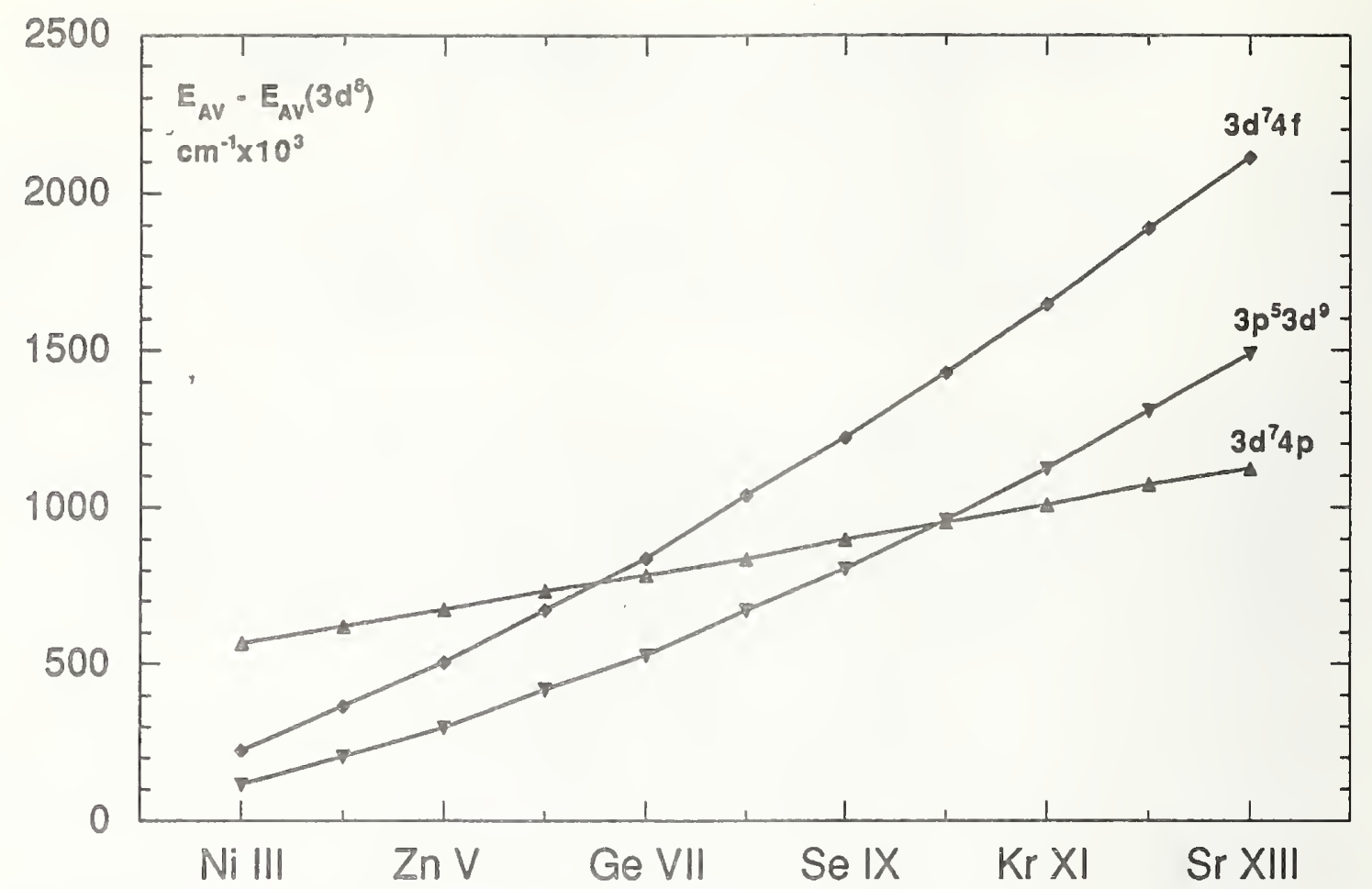

Figure 1: Average energies of the three lowest odd configurations realtive to that of the ground configuration along the Fe I sequence.

The Hartree-Fock (HF) and least-square fit (LSF) calculations were carried out for the studied configurations by means of the Cowan code. The final values of the corresponding parameters and their ratios are given in Table 1 . All 47 energy levels of the even $3 \mathrm{~d}^{8}$ and $3 \mathrm{~d}^{7} 4 \mathrm{~s}$ configurations are included in the LSF calculation, and the mean-square deviation $\sigma$ is $91 \mathrm{~cm}^{-1}$. The effective parameters $\beta$ and $T$ for the $3 \mathrm{~d}^{8}$ configuration were fixed because of the instability of the fitting process, and the values for them were taken to be the same as for the $3 d^{7} 4$ s configuration. The odd configurations $3 \mathrm{~d}^{7} 4 \mathrm{p}, 3 \mathrm{p}^{5} 3 \mathrm{~d}^{9}$ and $3 \mathrm{~d}^{7} 4 \mathrm{f}$ were included in the calculation with their interaction. As noted above, there is a strong interaction between the $3 d^{7} 4 \mathrm{p}$ and $3 \mathrm{p}^{5} 3 \mathrm{~d}^{9}$ configurations, even though the average energies of these configurations are separated by $226,640 \mathrm{~cm}^{-1}$. According to the HF calculation, the $3 \mathrm{~d}^{7} 4 \mathrm{f}$ configuration is located $83,680 \mathrm{~cm}^{-1}$ higher and also disturbs some levels of the $3 \mathrm{p}^{5} 3 \mathrm{~d}^{9}$ configuration. All 122 energy levels of the $3 \mathrm{~d}^{7} 4 \mathrm{p}$ and $3 \mathrm{p}^{5} 3 \mathrm{~d}^{9}$ configurations were included in the LSF calculation and the mean square deviation $\sigma$ is $131 \mathrm{~cm}^{-1}$. We increased the average energy of the unknown $3 \mathrm{~d}^{3} 4 \mathrm{f}$ configuration by $3005 \mathrm{~cm}^{-1}$ with respect to the $\mathrm{HF}$ value as in the $3 d^{7} 4 \mathrm{p}$ configuration. All parameters were fixed according to the usual scaling factors from the HF values: 0.85 for the electrostatic interaction and unity for the spin-orbit interaction. The configuration interaction parameters were scaled by a factor of 0.85 for both even and odd configurations. In conclusion, we note that the LSF/HF ratios for all studied configurations are close to those predicted by extrapolation along the isoelectronic series. It is also interesting to note that the $3 \mathrm{p}^{5} 3 \mathrm{~d}^{9}{ }^{1} \mathrm{~F}_{3}{ }^{0}$ level deviates from the computed value by only $-7 \mathrm{~cm}^{-1}$; in the singleconfiguration calculation [1] this deviation was $-7155 \mathrm{~cm}^{-1}$.

On the basis of this work, we confirm our previous classifications for all spectral lines and energy levels except for the $3 \mathrm{p}^{6} 3 \mathrm{~d}^{8}{ }^{3} \mathrm{P}_{1}-3 \mathrm{p}^{5} 3 \mathrm{~d}^{9}{ }^{3} \mathrm{P}_{0}{ }^{\circ}$ transition. Instead of the line at $135.018 \AA$, the correct line is at $135.008 \AA$. Correspondingly, the $3 \mathrm{p}^{5} 3 \mathrm{~d}^{9}{ }^{3} \mathrm{P}_{0}{ }^{\circ}$ level, having only one transition to the ground state, changes from $769854 \mathrm{~cm}^{-1}$ to $769908 \mathrm{~cm}^{-1}$. We also added second classifications 
for three lines, in accordance with the transition probabilities calculated with the final parameters of the LSF calculation. Besides, a few misprints in the designations of the classified lines have been corrected. After checking the analysis, we prepared a table of the classified wavelengths including more than $520 \mathrm{Ge}$ VII spectral lines. This table will appear in an upcoming new version of the NIST Atomic Spectra Database.

\section{References}

[1] L. I. Podobedova, A. N. Ryabtsev, Reprint ISAN (Institute of Spectroscopy, USSR Academy Sciences) No.11, p. 28 (1986)

[2] L. I. Podobedova, A. A. Ramonas, and A. N. Ryabtsev, Opt. Spectrosc. 49, 247 (USSR 1980)

[3] L. I. Podobedova, A. N. Ryabtsev, Opt. Spectrosc. 60, 694 (USSR 1986)

[4] J. Sugar and A. J. Musgrove, Phys. Chem. Ref. Data 22, 1213 (1993) 
Table 1: Parametric values of the energy integrals and their ratios to the Hartree-Fock values for the $3 \mathrm{~d} 8+3 \mathrm{~d} 74 \mathrm{~s}$ and $3 \mathrm{~d} 74 \mathrm{p}+3 \mathrm{p} 53 \mathrm{~d} 9+3 \mathrm{~d} 74 \mathrm{f}$ configurations in GeVII $\left(\mathrm{cm}^{-1}\right)$.

\begin{tabular}{|c|c|c|c|c|c|}
\hline Configuration & Parameter & LSF & Error & $\mathrm{HF}$ & $\mathrm{LSF} / \mathrm{HF}^{*}$ \\
\hline \multirow[t]{7}{*}{$3 p 63 d 8$} & $\operatorname{Eav}(3 d 8)$ & 19212 & 40 & 21541 & -2329 \\
\hline & $\mathrm{F} 2(3 \mathrm{~d}, 3 \mathrm{~d})$ & 130539 & 311 & 148806 & 0.877 \\
\hline & $\mathrm{F} 4(3 \mathrm{~d}, 3 \mathrm{~d})$ & 83108 & 283 & 93874 & 0.885 \\
\hline & $\alpha(3 \mathrm{~d})$ & 66 & 007 & & \\
\hline & $\beta(3 \mathrm{~d})$ & 550 & f & & \\
\hline & $\mathrm{T}(3 \mathrm{~d})$ & -8 & $\mathrm{f}$ & & \\
\hline & $\zeta(3 \mathrm{~d})$ & 1948 & 43 & 1950 & 0.999 \\
\hline \multirow[t]{10}{*}{$3 \mathrm{~d} 74 \mathrm{~s}$} & $\operatorname{Eav}(3 \mathrm{~d} 74 \mathrm{~s})$ & 436151 & 19 & 434305 & 1846 \\
\hline & $\mathrm{F} 2(3 \mathrm{~d}, 3 \mathrm{~d})$ & 134696 & 104 & 154339 & 0.873 \\
\hline & $\mathrm{F} 4(3 \mathrm{~d}, 3 \mathrm{~d})$ & 87076 & 203 & 97613 & 0.892 \\
\hline & $\alpha(3 d)$ & 68 & 7 & & \\
\hline & $\beta(3 d)$ & 550 & 121 & & \\
\hline & $\mathrm{T}(3 \mathrm{~d})$ &.-8 & 1 & & \\
\hline & $\zeta(3 \mathrm{~d})$ & 2107 & 18 & 2069 & 1.018 \\
\hline & $\mathrm{G} 2(3 \mathrm{~d}, 4 \mathrm{~s})$ & 14097 & 82 & 14281 & 0.987 \\
\hline & $\mathrm{R} 2(3 \mathrm{~d}, 3 \mathrm{~d} ; 3 \mathrm{~d}, 4 \mathrm{~s})$ & 11270 & f & 13259 & 0.850 \\
\hline & $\sigma$ & 91 & & & \\
\hline \multirow[t]{11}{*}{$3 \mathrm{~d} 74 \mathrm{p}$} & $\operatorname{Eav}(3 d 74 p)$ & 553079 & 15 & 550074 & 3005 \\
\hline & $\mathrm{F} 2(3 \mathrm{~d}, 3 \mathrm{~d})$ & 135781 & 89 & 154506 & 0.879 \\
\hline & $\mathrm{F} 4(3 \mathrm{~d}, 3 \mathrm{~d})$ & 87292 & 193 & 97730 & 0.893 \\
\hline & $\alpha$ & 57 & 6 & & \\
\hline & $\beta$ & 768 & 100 & & \\
\hline & $\mathrm{T}(3 \mathrm{~d})$ & -9 & 1 & & \\
\hline & $\zeta(3 \mathrm{~d})$ & 2092 & 16 & 2072 & 1.010 \\
\hline & $\zeta(4 \mathrm{p})$ & 3812 & 34 & 3278 & 1.163 \\
\hline & $\mathrm{F} 2(3 \mathrm{~d}, 4 \mathrm{p})$ & 35302 & 129 & 36020 & 0.980 \\
\hline & $\mathrm{G} 1(3 \mathrm{~d}, 4 \mathrm{p})$ & 11210 & 58 & 11938 & 0.939 \\
\hline & $\mathrm{G} 3(3 \mathrm{~d}, 4 \mathrm{p})$ & 11284 & 151 & 11409 & 0.989 \\
\hline \multirow[t]{8}{*}{$3 \mathrm{p} 5$ 3d9 } & $\operatorname{Eav}(3 p 53 d 9)$ & 779719 & 57 & 807033 & -27314 \\
\hline & $\zeta(3 p)$ & 23397 & 124 & 22166 & 1.056 \\
\hline & $\zeta(3 \mathrm{~d})$ & 1857 & 62 & 1946 & 0.954 \\
\hline & $\mathrm{F} 1(3 \mathrm{p}, 3 \mathrm{~d})$ & 11686 & 627 & & \\
\hline & $F 2(3 p, 3 d)$ & 148729 & 548 & 149744 & 0.993 \\
\hline & $\mathrm{G} 1(3 \mathrm{p}, 3 \mathrm{~d})$ & 148827 & 362 & 181099 & 0.822 \\
\hline & $\mathrm{G} 2(3 \mathrm{p}, 3 \mathrm{~d})$ & -5981 & 698 & & \\
\hline & $\mathrm{G} 3(3 \mathrm{p}, 3 \mathrm{~d})$ & 114830 & 594 & 112606 & 1.020 \\
\hline \multirow[t]{18}{*}{$3 \mathrm{~d} 74 \mathrm{f}$} & $\operatorname{Eav}(3 \mathrm{~d} 74 \mathrm{f})$ & 863400 & $\mathrm{f}$ & 860395 & 3005 \\
\hline & $\mathrm{F} 2(3 \mathrm{~d}, 3 \mathrm{~d})$ & 131967 & $\mathrm{f}$ & 155255 & 0.850 \\
\hline & $\mathrm{F} 4(3 \mathrm{~d}, 3 \mathrm{~d})$ & 83502 & $\mathrm{f}$ & 98238 & 0.850 \\
\hline & $\zeta(3 \mathrm{~d})$ & 2087 & $\mathrm{f}$ & 2087 & 1.000 \\
\hline & $\zeta(4 \mathrm{f})$ & 8 & $\mathrm{f}$ & 8 & 1.000 \\
\hline & $\mathrm{F} 2(3 \mathrm{~d}, 4 \mathrm{f})$ & 12868 & $\mathrm{f}$ & 15139 & 0.850 \\
\hline & $\mathrm{F} 4(3 \mathrm{~d}, 4 \mathrm{f})$ & 4711 & $\mathrm{f}$ & 5542 & 0.850 \\
\hline & $\mathrm{Gl}(3 \mathrm{~d}, 4 \mathrm{f})$ & 5841 & $\mathrm{f}$ & 6872 & 0.850 \\
\hline & $\mathrm{G} 3(3 \mathrm{~d}, 4 \mathrm{f})$ & 3365 & $\mathrm{f}$ & 3959 & 0.850 \\
\hline & $\mathrm{G} 5(3 \mathrm{~d}, 4 \mathrm{f})$ & 2309 & $\mathrm{f}$ & 2717 & 0.850 \\
\hline & $\mathrm{R} 1(3 \mathrm{~d}, 4 \mathrm{p} ; 3 \mathrm{~d}, 3 \mathrm{~d})$ & 12652 & $\mathrm{f}$ & 14885 & 0.850 \\
\hline & $\mathrm{R} 3(3 \mathrm{~d}, 4 \mathrm{p} ; 3 \mathrm{~d}, 3 \mathrm{~d})$ & 12651 & $\mathrm{f}$ & 14883 & 0.850 \\
\hline & $\mathrm{R} 2(3 \mathrm{~d}, 4 \mathrm{p} ; 3 \mathrm{~d}, 4 \mathrm{f})$ & 13408 & $\mathrm{f}$ & 15774 & 0.850 \\
\hline & $\mathrm{R} 4(3 \mathrm{~d}, 4 \mathrm{p} ; 3 \mathrm{~d}, 4 \mathrm{f})$ & 4586 & $\mathrm{f}$ & 5395 & 0.850 \\
\hline & $\mathrm{R} 1(3 \mathrm{~d}, 4 \mathrm{p} ; 4 \mathrm{f}, 3 \mathrm{~d})$ & 1655 & $\mathrm{f}$ & 1947 & 0.850 \\
\hline & $\mathrm{R} 3(3 \mathrm{~d}, 4 \mathrm{p} ; 4 \mathrm{f}, 3 \mathrm{~d})$ & 1694 & $\mathrm{f}$ & 1993 & 0.850 \\
\hline & $\mathrm{R} 1(3 \mathrm{~d}, 3 \mathrm{~d} ; 3 \mathrm{~d}, 4 \mathrm{f})$ & -25658 & $\mathrm{f}$ & -30185 & 0.850 \\
\hline & $R 3(3 d, 3 d ; 3 d, 4 f)$ & -13185 & $\mathrm{f}$ & -15512 & 0.850 \\
\hline
\end{tabular}

\footnotetext{
"Differences $\operatorname{Eav}(\mathrm{LSF})-\operatorname{Eav}(\mathrm{HF})$ are presented for Eav. f - The parameters are fixed.
} 


\title{
The ORNL Controlled Fusion Atomic Data Center
}

\author{
D. R. Schultz, P. S. Krstić, F. M. Ownby, F. W. Meyer, C. C. Havener, \\ M. E. Bannister, W. Liu, D. J. Jeffery, and P. C. Stancil \\ Physics Division, Oak Ridge National Laboratory \\ Oak Ridge, TN 37831-6373
}

\begin{abstract}
The principal mission of the Controlled Fusion Atomic Data Center is the collection, evaluation, and dissemination of atomic collision data relevant to fusion energy development. With the advent of the widespread use of the World Wide Web, the data center's resources are being placed on-line to facilitate their use by end-users (cf. http://www-cfadc.phy.ornl.gov/). As this development continues, initially disparate, individually compiled resources will be transformed into integrated tools for retrieving recommended data, or displaying and manipulating the information available. The data center's present capabilities, recent data production/evaluation efforts, and goals for future development are highlighted here.
\end{abstract}

\section{Introduction}

Since its inception at Oak Ridge National Laboratory in 1958 and formal establishment in 1963, the Controlled Fusion Atomic Data Center (CFADC) has published indexed compilations of bibliographical information and recommended numerical data regarding atomic, molecular, and particlesolid collisions of interest in fusion energy research. Rather than attempting to provide here an exhaustive history of the CFADC and its activities, we briefly summarize the resources available through the data center, primarily through the World Wide Web [1].

\section{The "Redbooks"}

In order to provide convenient recommended sets of atomic, molecular, and particle-solid collision data to the fusion energy research community, the CFADC has periodically published collections containing numerical and graphical data. Owing to their red bindings, these series of volumes have become known in the community as the "Redbooks." Usually a small group of experts has surveyed the existing experimental and theoretical works within a given range of reactions, and determined and catalogued recommended reaction cross sections. The latest series of such collections of recommended cross sections, rates, and numerical fits is entitled "Atomic Data for Fusion" [2]. Since only 500 copies of each volume were originally printed and distributed, the number of users to which these collections have been available was limited. Therefore, we have recently scanned the two most requested volumes and made them available through an indexed set of hypertext links on the data center Web site [1]. Users can view, print, or download the graphical and tabular data of interest directly from the Web.

Furthermore, the recommended data contained in the Redbooks have been recorded in the ALADDIN format, for ease of exchange within the fusion energy research community (see below). In the near future, hypertext linkages of the Redbook pages will allow the user-viewable graphs and tables to be directly linked to these separately organized ALADDIN data entries, and ultimately to 
other resources. For example, when a CFADC bibliographic search is performed through the Web interface, Redbook graphical, tabular, and fit coefficient data will be returned in addition to the bibliographic listing. The existence of the Web-based version of the Redbooks will allow them to be updated in the future without the necessity of re-distributing new editions. That is, as data of improved accuracy or for new reactions becomes available which lead to modifications or extensions of the Redbook recommendations, new pages can easily be placed on the Web site.

\section{The CFADC Bibliography}

Another principal product of the CFADC has been a series of published bibliographies. Access to such information is a crucial first step in the process of providing critically evaluated data for applications like fusion energy research. After a long series of publications beginning in the 1960's, the semiannual CFADC bibliographic updates have recently been published along with contributions from other foreign data centers through the International Atomic Energy Agency (IAEA) in volumes entitled "International Bulletin on Atomic and Molecular Data for Fusion." Convenient cumulative indices to the bibliographic entries were published in 1980 and 1987 through the IAEA as well [3].

The ever increasing number of collected entries has lead to a succession of computer-based databases for archiving and organizing this information. Presently, the CFADC makes available entries dating from 1978 to the present (about 30,000 entries) over the Web [1]. This method of dissemination is quite effective in that it allows users unlimited direct access to the full, current database. Efforts are also underway to place the CFADC archival entries (dating from c. 1950 to 1977 and numbering approximately 40,000) on the Web-based system, and to link the bibliographic search engine with other resources such as the Redbooks and ALADDIN. For the benefit of the reader, we also mention that a group lead by the Laboratoire de Physique des Gaz et des Plasmas at the Université Paris-Sud maintains a completely independent general atomic and molecular bibliographic database which can also be searched via the Web (see http://gaphyor.Ipgp.u-psud.fr/).

\section{The International ALADDIN Network}

Coordinated through the IAEA Atomic and Molecular Data Unit, the CFADC participates in an international network of atomic data centers whose missions involve the support of fusion energy research. Among this data center network's primary activities is the maintenance of a standard format of atomic and molecular data exchange called ALADDIN (A Labeled Atomic Data INterface). Originally developed by Russell Hulse of PPPL, ALADDIN was adopted in 1988 by the network. Almost two dozen collections of recommended or evaluated atomic collision data and particle-surface interaction data exist in this format, representing data for hundreds of individual reactions. This data provides a primary resource for atomic and molecular data for fusion energy research, often directly linked to plasma modeling codes. Furthermore, ALADDIN possesses a FORTRAN interface code through which searches of the data files for individual reaction data can be made, and tabulations from the fitted data coefficients can be outputted.

The data files, computer code, associated evaluation functions, and dictionaries have been distributed from the IAEA A+M Data Unit or the CFADC to interested parties, and are now available through the Web [1] for direct viewing and downloading. Preliminary linkage of the CFADC bibliographic search engine and the ALADDIN files has been recently accomplished and ALADDIN can be run directly over the Web. The IAEA maintains an Internet linkage to ALADDIN and is planning a Web interface. Web-based interfaces will also hopefully lead to flexible, updatable methods of maintaining and enhancing this valuable resource. 


\section{5}

The Atomic Data and Analysis Structure

Developed in collaboration between Strathclyde University and the Joint European Torus (JET) principally by Hugh Summers, the Atomic Data and Analysis Structure (ADAS) is a collisionalradiative modeling suite designed to aid in the interpretation of the spectra of radiating plasmas. Its primary application has been fusion plasma diagnostics for JET, but has also recently been employed to aid in interpreting observations by the Solar and Heliospheric Observatory satellite. A consortium, of which the CFADC is a member, has also recently been formed to allow access to this powerful set of computer codes and data collections for a wider user community. A detailed description of the ADAS suite can be found on-line at Strathclyde University or mirrored at the CFADC (see the "links" hyperlink on the CFADC Web-site [1]).

\section{The ORNL Multicharged Ion Research Facility}

An important aspect of the ORNL atomic physics for fusion program in addition to the CFADC is the Multicharged Ion Research Facility (MIRF). This facility has a long history of providing atomic data for electron-ion, ion-atom, and ion-surface collisions. Experiments in each of these areas are actively ongoing and utilize a state-of-the-art Caprice Electron Cyclotron Resonance ion source. Descriptions of the MIRF experiments are available through the CFADC Web site [1] as are indexed tabulations of certain portions of the data measured there. For example, the data obtained over the past three decades regarding electron impact ionization of atomic ions has been collected and organized into a hyperlinked Web resource. Similar work has been initiated for the ion-atom merged beams experiment, and linkages between these data collections and the bibliographic search engine will be made in the future.

\section{Data Projects}

Another aspect of the CFADC's mission is to provide whenever possible new calculations and/or evaluations of data which is urgently needed in fusion energy research. To this end, we have recently concentrated on the production of data for use in charge exchange recombination spectroscopy by computing large collections of state-selective charge transfer cross sections. These have been considered for a range of ions including those of Be [4] (owing to its low atomic number and consequent low induced radiation losses, it has been a primary plasma-facing component material candidate), $\mathrm{Ne}$ [5] (due to its introduction into the divertor to beneficially enhance radiation losses for energy exhaust), and Ar (due to its introduction as a diagnostic species) colliding with primary plasma components $\left(\mathrm{H}, \mathrm{H}_{2}\right.$, and $\left.\mathrm{He}\right)$.

Also, due to the need to model the momentum transport in the divertor plasma in present and next-step fusion devices, we have computed and tabulated the elastic and related transport cross sections for collisions among the isotopic variants of $\mathrm{H}^{+}+\mathrm{H}, \mathrm{H}_{2}$, and various other reactions of interest [6]. Work on production of both charge transfer and transport cross sections is ongoing. Some of these tabulations are available on the Web site under the "eprints" hyperlink, and future plans are to more systematically include them in data collections linked to the bibliographic search engine. 


\section{Towards the Future}

Even though the needs of fusion and other applications for collected, evaluated, and recommended atomic data persist and in fact intensify, the human resources available for such activities continues to dwindle. Thus, an effective leveraging of these human resources by utilization of the World Wide Web as a means for disseminating data produced or collected at the CFADC, and across other sites maintained by other data centers, will be a dominant trend in the future. Linkages will be made between the disparate, individual data collections and resources. This will improve users' ability to find the required information, and will form a powerful aid for data evaluation. For example, combining in a single graphically oriented Web tool the ability to display existing recommended data, results from well-established scaling laws, new basic data from linked data collections, and data contained in other resources even across the Web, will facilitate efforts to carry out projects to recommend data and provide centralized, updatable files for access by users. Also, recognizing that the atomic data needs of a wide range of applications are in essence similar, the CFADC has initiated a program of production and collection of data of importance in astrophysics, an another important leveraging of resources. These data efforts are complimented by ongoing state-of-theart modeling of astrophysical environments such as those present in later epochs of supernovae explosions.

\section{Acknowledgement}

The Controlled Fusion Atomic Data Center is supported by the U.S. Department of Energy's Office of Fusion Energy Sciences through Oak Ridge National Laboratory, managed by Lockheed Martin Research Corporation under grant number DE-AC05-96OR22464.

\section{References}

[1] Entry to the CFADC's on-line resources is through http://www-cfadc.phy.ornl.gov/ on the World Wide Web.

[2] "Atomic Data for Fusion", Vol. 1, Collisions of $\mathrm{H}, \mathrm{H}_{2}, \mathrm{He}$ and Li Atoms and Ions with Atoms and Molecules, C. F. Barnett, ed., ORNL-6086, 1990; "Atomic Data for Fusion", Vol. 3, Particle Interactions with Surfaces, E. W. Thomas, ed., ORNL-6088, 1985; "Atomic Data for Fusion", Vol. 4, Spectroscopic Data for Iron, W. L. Wiese, ed., ORNL-6089, 1985; "Atomic Data for Fusion", Vol. 5, Collisions of Carbon and Oxygen Ions with Electrons, $\mathrm{H}, \mathrm{H}_{2}$ and $\mathrm{He}$, R. A. Phaneuf, R. K. Janev, and M. S. Pindzola, eds., ORNL-6090, 1987

[3] "Computer Index to Atomic and Molecular Data (CIAMDA 80)", International Atomic Energy Agency, Vienna 1980; "Computer Index to Atomic and Molecular Data (CIAMDA 87)", International Atomic Energy Agency, Vienna 1987

[4] D. R. Schultz, P. S. Krstić, and C. O. Reinhold, Physica Scripta T62, 69 (1996)

[5] D. R. Schultz and P. S. Krstić, Atomic and Plasma-Material Interaction Data for Fusion 6, 173 (1995)

[6] D. R. Schultz, S. Yu. Ochinnikov, and S. V. Passovets, Elastic and Related Cross Sections for Low-Energy Collisions among Hydrogen and Helium Ions, Neutrals, and Isotopes, in "Atomic and Molecular Processes in Fusion Edge Plasmas", R. K. Janev, ed., Plenum, New York 1995; P. S. Krstić, D. R. Schultz, and G. Bent, Physica Script T62, 21 (1996) 


\title{
The Database «SPECTR» on Spectral Properties of Atoms And Ions
}

\author{
I. Yu. Skobelev, A. Ya. Faenov, and A. I. Magunov \\ Multicharged Ion Spectra Data Center (MISDC) of VNIIFTRI, \\ Mendeleevo, 141570 Russia
}

\section{Introduction}

The database "SPECTR» has been created in the Multicharged Ion Spectra Data Center (MISDC) of VNIIFTRI as a tool to allow the fast determination of information on spectra of atoms and ions. It is a database containing data on spectroscopic constants of isolated atoms and ions (energy levels, wavelength of spectral lines, radiative probabilities, ionization potentials) and. some collision data (dielectronic recombination rates, electron impact excitation cross sections and rates, electron impact ionization cross sections and rates).

Currently, the database "SPECTR» contains more than 450000 numerical records. The largest part of the data concern the UV and X-ray spectral regions, $\lambda<100$. Besides published data (experimental and theoretical), the experimental data produced at MISDC for the X-ray spectral band $\lambda<26$ are used in database «SPECTR».

\section{MISDC of VNIIFTRI and Database "SPECTR"}

The multicharged Ions Spectra Data Center of VNIIFTRI is a research group, working in the field of X-ray spectroscopy of multicharged ions and its application for the diagnostics of hightemperature plasmas.

\section{Main Fields of Investigations:}

A. creation of Bragg and Bragg-Fresnel optical elements for high-resolution X-ray spectroscopy (see, for example, [1-9])

B. high-precision X-ray spectroscopy of multicharged ions (emission spectra observations, spectral line identification, accurate wavelength measurements) (see, for example, [6, 10-22])

C. creation of X-ray spectroscopy diagnostic methods and their application to measurements of different plasma parameters (laser-produced plasmas, Z- and X-pinches, plasma focus) (see, for example, [23-28])

D. creation of a database on spectra of atoms and ions (database "SPECTR")

\section{Database "SPECTR"}

The database SPECTR (DB "SPECTR"), on one hand, contains a great number of data on characteristics of atoms and ions, and, on the other hand, represents also the software to quickly find the information needed, to browse on the screen and to make a hard copy.

The information stored in DB SPECTR may be divided into two parts. The first part (and now the larger one) is the data on properties of isolated atoms and ions, that is, the data on energy 
level structure, wavelengths of radiative transitions and its probabilities. The second part contains the data on characteristics of collision processes. The main difference between these two parts is the following: The first part deals with a set of constants, while the second one deals with a set of functions. That is, for any radiative transition its wavelength, radiative probability, energies of upper and lower levels are numerical quantities, but its collisional characteristics are functions of the relative velocity of the colliding particles. Therefore, the software used to operate on these two different types of data is also somewhat different.

\section{$D B$ "SPECTR" Software}

The database "SPECTR" software is based on FoxPro Database Management System. The information is stored in well-known DBF-format and can be transferred easily into another formats.

\begin{tabular}{|c|c|}
\hline \multicolumn{2}{|l|}{ DB "SPECTR" Data } \\
\hline Data on spectral lines & 397885 records \\
\hline Data on energy levels & 75100 records \\
\hline Data on ionization potentials & 2292 records \\
\hline Data on excitation cross sections and rates & 4932 records \\
\hline Data on ionization cross sections and rates & 276 records \\
\hline Data on references & 504 records \\
\hline The total number of records & 481134 \\
\hline
\end{tabular}

\section{Qualitative Characteristics}

DB "SPECTR" is a database on the characteristics of isolated atoms and ions, i.e., on spectral lines (wavelengths and radiative probabilities) and energy levels. The small number of collisional data were included mainly to test the software developed. Since the MISDC research team works in the field of X-ray spectroscopy, the main part of data (about $75 \%$ ) refers to the multicharged ions.

\section{Data sources}

$\checkmark$ Published experimental data. For the X-ray region, the database contains practically all published experimental data, for the UV and visible regions only some data.

$\checkmark$ Our own experimental data for multicharged ions. The MISDC research group has produced experimental data on the X-ray spectra of multicharged ions for more than 20 years. For example, during last 5 years we have produced new accurate data on satellite lines caused by radiative transitions in $\mathrm{He}-$, Li-, Be-, B-, C-, N-, O-, F-, Na-, Mg- like ions, and on high-n transitions in He- and Ne-like ions. Producing this kind of information is the main field of scientific interest of the MISDC group. For this purpose we create new types of X-ray highresolution spectrographs (with spherically bent crystals, for example) and develop new methods which improve the accuracy of wavelength measurements.

$\checkmark$ Theoretical data, both published (not all, of course) and calculated especially for DB "SPECTR" in some Russian institutes (see, for example, [29]). 
The search operations are executed fast, and some examples are presented in this table:

Database SPECTR

( 4 May 1997)

Total number of records 481134

Pentium-133, 16 MB RAM

\begin{tabular}{|l|l|c|c|}
\hline \multicolumn{1}{|c|}{ QUERY } & $\begin{array}{c}\text { SEARCH } \\
\text { TIME }\end{array}$ & $\begin{array}{c}\text { THE NUMBER OF } \\
\text { RECORDS SELECTED }\end{array}$ \\
\hline 1 & Lines of copper ions in spectral region $10.1-10.11 \AA$ & $2 \mathrm{~s}$ & 9 \\
\hline 2 & Lines of silver ions in spectral region $10-15 \AA$ & $5 \mathrm{~s}$ & 94 \\
\hline 3 & Lines of H-like and He-like silicon & $4 \mathrm{~s}$ & 3192 \\
\hline 4 & $3 \mathrm{~d}-2 \mathrm{p}$ transitions in Ne-like and Na-like silver & $2 \mathrm{~s}$ & 313 \\
\hline 5 & $1 \mathrm{~s} 2 \mathrm{p}{ }^{1} \mathrm{P}_{1} \cdot 1 \mathrm{~s}^{2}{ }^{1} \mathrm{~S}_{0}$ transition in He-like uranium & $2 \mathrm{~s}$ & 2 \\
\hline 6 & $2 \mathrm{~s}^{6} \mathrm{p}^{6} 3 \mathrm{p} \cdot 2 \mathrm{~s}^{2} 2 \mathrm{p}^{6}$ transitions in Ne-like ions & $1 \mathrm{~s}$ & 411 \\
\hline 7 & Lines of OI atom & $1 \mathrm{~s}$ & 441 \\
\hline 8 & Lines of O-like and N-like iron & $3 \mathrm{~s}$ & 998 \\
\hline
\end{tabular}

\section{References}

[1] S. A. Bel'kov, B. A. Bryunetkin, N. V. Zhidkov, I. Yu. Skobelev, N. A. Suslov, A. Ya. Faenov, Quantum Electronics 24, 253 (1994)

[2] A. Ya Faenov, S. A. Pikuz, A. I. Erko, B. A. Bryunetkin, V. M. Dyakin, G. V. Ivanenkov, A. R. Mingaleev, T. A. Pikuz, V. M. Romanova, T. A. Shelkovenko, Physica Scripta 50, 333 (1994)

[3] S. A. Pikuz, V. M. Romanova, T. A. Shelkovenko, T. A. Pikuz, A. Ya. Faenov, E. Forster, J. Vol'f, O. Verhran, Quantum Electron. 22, 21 (1995)

[4] T. A. Pikuz, A. Ya. Faenov, S. A. Pikuz, V. M. Romanova, T. A. Shelkovenko, Journal of X-ray Science and Technology 5, 323 (1995)

[5] A. I. Erko, L. A. Panchenko, S. A. Pikuz, A. R. Mingaleev, V. M. Romanova, T. A. Shelkovenko, A. Ya. Faenov, B. A. Bryunetkin, T. A. Pikuz, I. Yu. Skobelev, Rev.Sci:Instrum. 66, 1047 (1995)

[6] I. Yu. Skobelev, A. Ya. Faenov, B. A. Bryunetkin, V. M. Dyakin, T. A. Pikuz, S. A. Pikuz, T. A. Shelkovenko, V. M. Romanova, JETP 81, 692 (1995).

[7] J. P. Geindre, P. Audebert, A. Rousse, J. C. Gauthier, A. Ya. Faenov, T. A. Pikuz, S.A. Pikuz, T. A. Shelkovenko, Physica Scripta 53, 645 (1996)

[8] A. Ya. Faenov, T. A. Pikuz, A. A. Firsov, L. A. Panchenko, Yu. I. Koval, M. Fraenkel, A. Zigler, Physica Scripta 55, 167 (1997)

[9] M. Sanchez del Rio, A. Ya. Faenov, V. M. Dyakin, T. A. Pikuz, S. A. Pikuz, V. M. Romanova, T. A. Shelkovenko, Physica Scripta 55, 735 (1997)

[10] A. Ya. Faenov, S. A. Pikuz, A. S. Shlyaptseva, Physica Scripta 49, 41 (1994)

[11] S. A. Pikuz, D. A. Hammer, D. H. Kalantar, A. Faenov, I. Yu. Skobelev, Phys.Rev.A 49, 3450 (1994) 
[12] B. ABryunetkin, A. Ya. Faenov, M. P. Kalashnikov, P. V. Nickles, M. Schnurer, I. Yu. Skobelev, J. Abdallah, R. E. H. Clark, JQSRT 53, 45 (1995)

[13] S. Bollanti, P. Di Lazzaro, F. Flora, et al, Physica Scripta 51, 326 (1995)

[14] A. Ya. Faenov, D. A. Hammer, J. Nilsen, A. Osterheld, S. A. Pikuz, T. A. Pikuz, V. M. Romanova, T. Shelkovenko, I. Yu. Skobelev, Physica Scripta 51, 454 (1995)

[15] A. Ya. Faenov, I. Yu. Skobelev, S. A. Pikuz, G. A. Kyrala, J. A. Gobble, K. D. Fulton, J. Abdallah Jr., D. P. Kilcrease, Phys. Rev. A. 51, 3529 (1995)

[16] A. Faenov, B. Bryunetkin, V. Dyakin, S. Pikuz, I. Skobelev, U. Safronova, T. Pikuz, J. Nilsen, A. Osterheld, Phys.Rev.A 52, 3644 (1995)

[17] A. I. Magunov, V. M. Dyakin, A. Ya. Faenov et al, JETP 83, 267 (1996)

[18] A. I. Magunov, V. M. Dyakin, A. Ya. Faenov, et al, Phys. Rev. A 54, 3971 (1996)

[19] A. L.Osterheld, J. Nilsen, S. Ya. Khakhalin, A. Ya. Faenov, S. A. Pikuz, Physica Scripta 54, 240 (1996)

[20] I. Yu. Skobelev, A. Ya. Faenov, V. M. Dyakin et al, Phys.Rev.E 55, 3773 (1997)

[21] I. Yu. Skobelev, A. Ya. Faenov, A. I. Magunov, A. L. Osterheld, B. K. F. Young, J. Dunn, R. E. Stewart, Physica Scripta T73, 104 (1997)

[22] J. Abdallah, Jr., A. Faenov, D. Hammer, et al, Physica Scripta 53, 705 (1996)

[23] S. A. Pikuz, B. A. Brynetkin, G. V. Ivanenkov, A. R. Mingaleev, V. M. Romanova, I. Yu. Skobelev, A. Ya. Faenov, S. Ya. Khakhalin, T. A. Shelkovenko, JQSRT 51, 291 (1994)

[24] T. Pisarczyk, B. A. Brunetkin, A. Ya. Faenov, et al, Physica Scripta 50, 72 (1994)

[25] V. M. Dyakin, A. Ya. Faenov, A. I. Magunov, et al, Physica Scripta 52, 201 (1995)

[26] A. Faenov, V. Dyakin, A. Magunov, T. Pikuz, I. Skobelev, S. Pikuz, A. Kasperczyk, T. Pisarczyk, J. Wolowski, B. Zielinska, Physica Scripta 53, 591 (1996)

[27] F. B. Rosmej, B. A. Bryunetkin, A. Ya. Faenov, I. Yu. Skobelev, M. P. Kalashnikov, P. V. Nickles, M. Schnurer, J. Phys. B Lett. 29, L299 (1996)

[28] A. Bartnik, V. M. Dyakin, J. Kostecki, I. Yu. Skobelev, A. Ya. Faenov, H. Fiedorowicz, M. Szczurek, R. Jarocki, Quantum Electronics 27, 334 (1997)

[29] V. A. Boiko, V. G. Pal'chikov, I. Yu. Skobelev, A. Ya. Faenov "Spectroscopic Data of Atoms and Ions (Atom Spectra with One and Two Electrons)". CRC Press, 1994 


\title{
The Plasma Chemistry Database
}

\author{
W. K. Trail, C. W. Davis, and W. L. Morgan \\ Kinema Research \& Software, Monument, Colorado \\ http://www. kinema. com/kinema
}

We are developing a comprehensive plasma chemistry database of atomic and molecular properties and collision data in Java for use on a LAN, or over the World Wide Web. The database will serve two principal functions: first, it will make this data easily accessible to scientists on the web through a graphical user interface (GUI), and second it will make the data accessible through standard SQL database calls in Java thus enabling our plasma chemistry software tools to access appropriate input data across the web or across a LAN during execution. An example of the current version of this database is on our web site.

The database, which currently contains roughly 50 species, provides atomic and molecular properties such as permanent moments, polarizabilities, electron affinities, heats of fusion, rotational constants, etc., as well as critically selected experimental or theoretical electron collision data for a wide range of processes including elastic scattering, rotational excitation, vibrational excitation, electronic excitation, attachment, dissociative attachment, ionization, and dissociation. (We will eventually add heavy particle collision data.) The collision cross sections are in two forms: raw cross sections and coefficients for analytic fits. Because reaction rates are so useful in plasma chemistry the interface provides the ability to calculate them from cross sections over a user-selected temperature range. In addition, it automatically carries out an Arrhenius fit to the resultant reaction rate and displays the coefficients.

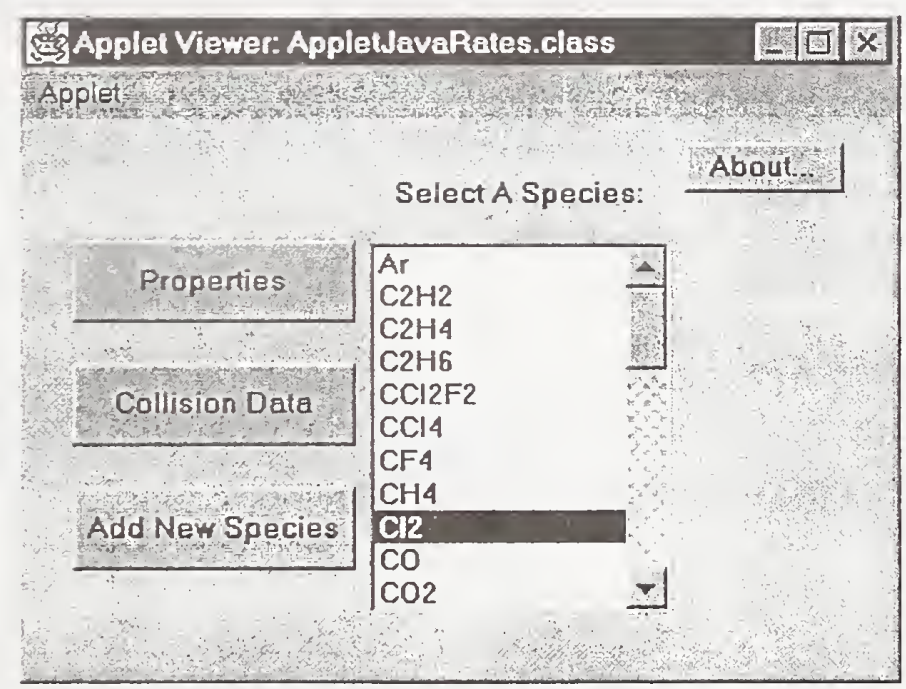

Figure 1: The main page of the graphical user interface to the database.

The first stage of this project was to build the GUI. The GUI allows the user to select an atom or molecule, and view the available raw data. All of the data are easily extracted from the interface using standard cut and paste utilities. In Fig. 1 we show the main page; the user simply selects a species and chooses to view either 'Properties' or 'Collision Data'. In Fig. 2 we show the properties screen for $\mathrm{Cl}_{2}$. In Fig. 3 we show the 'Collision Data' screen for momentum transfer in electron- $\mathrm{Cl}_{2}$ 
scattering. The user can view the collision cross sections for other processes by clicking on the 'Next' and 'Prev.' buttons. In addition to displaying a table of cross sections for this process, this screen allows the user to calculate reaction rates from the cross sections over a selected temperature range. Also on this screen is a built in $2-\mathrm{D}$ plot utility which allows the user to view a plot of the reaction rates or the cross sections. For example, in Fig. 4 we show a plot of the reaction rate for vibrational excitation of $\mathrm{Cl}_{2}$ by electron collisions.

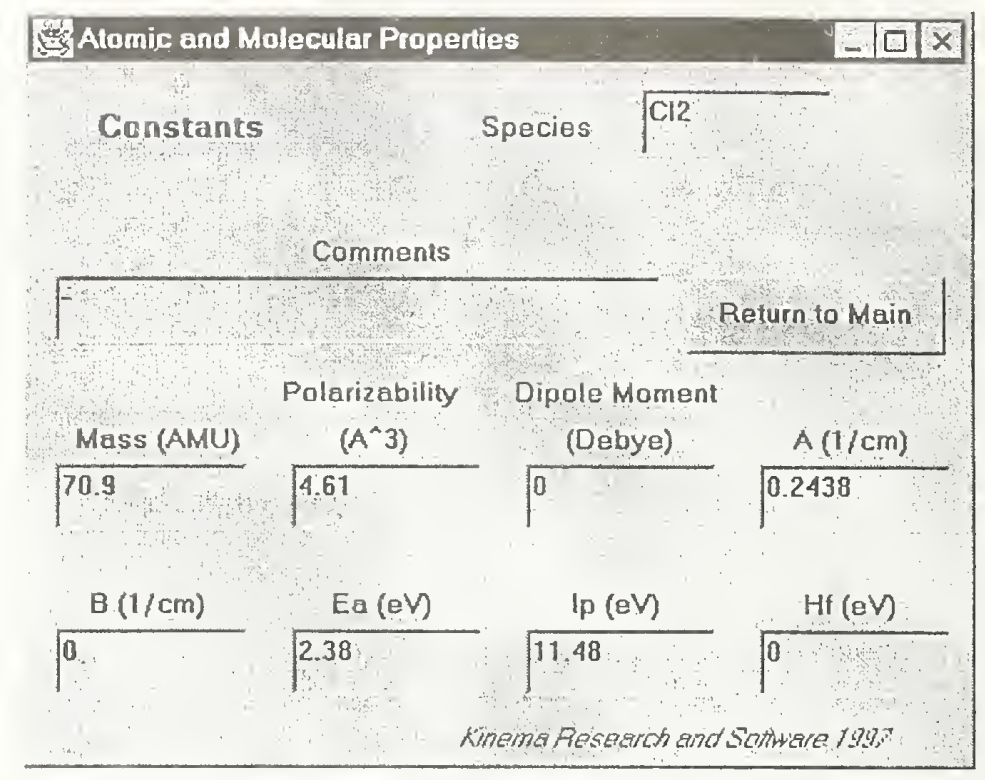

Figure 2: The 'Properties' screen for $\mathrm{Cl}_{2}$. This page displays the mass, polarizability, dipole moment, scattering length, rotational constant, electron affinity, ionization energy, and the heat of fusion.

Building the Java interface between the database and our plasma chemistry software comprises the second stage of this project. Because most of our software is written in Fortran 77 and Fortran 90, we will first develop a Java interface that essentially provides a convenient way to move data from the database to the appropriate input files. The Fortran programs will be executed from this Java interface.

For the final stage of this project we will migrate our plasma chemistry software to Java. Our programs will then be able to access the appropriate input directly from the database (over the Web) during execution. Some further advantages of a Java-only suite of programs include platform-independence, access to the massive Java class libraries, and ease of distribution (and execution) over a network. 


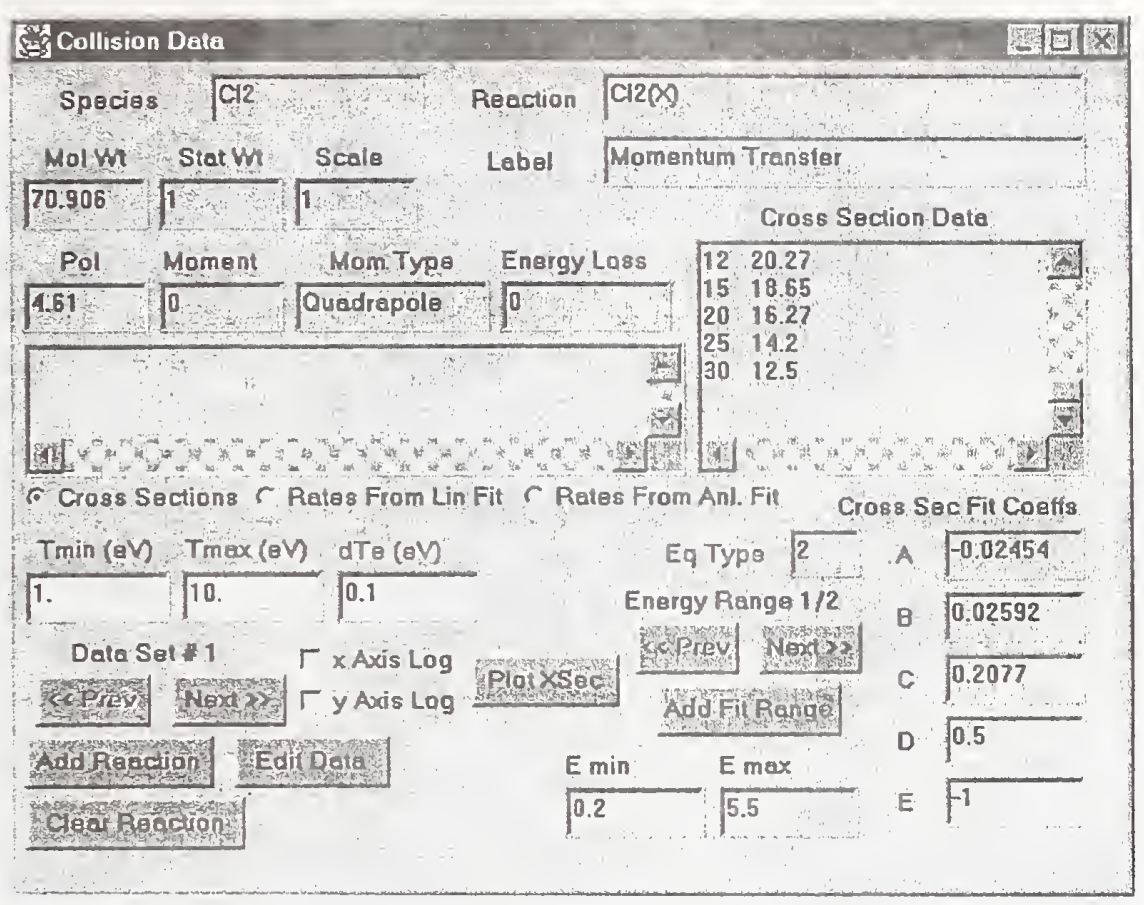

Figure 3: The 'Collision Data' screen provides information on the collision process displayed in the 'Label' field. This screen provides tools for calculating reaction rates, coefficients for fits, buttons for selecting other processes, and plotting utilities. The 'Add Reaction', 'Clear Data', and 'Edit Data' buttons in the lower left will allow users to replace, add new, or modify data in the database.

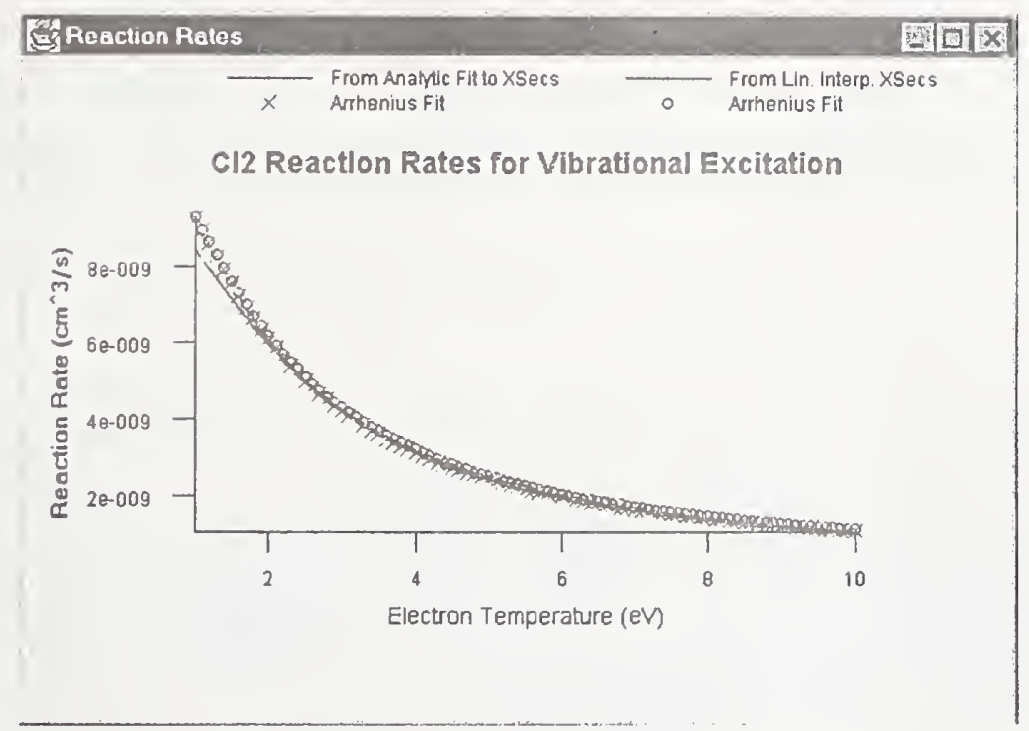

Figure 4: This is a plot of the reaction rate as calculated from the $\mathrm{v}=0-1$ vibrational excitation cross section. The curve labeled 'From Lin. Interp. XSecs' is the reaction rate as calculated from a linear interpolation of the raw cross section data. An Arrhenius fit to these reaction rates is represented by the circles. The curve labeled 'From Analytic Fit to XSecs' is the reaction rate as calculated from our analytic fit to the raw cross section data. An Arrhenius fit to these reaction rates is represented by the X's. (The plots are more easily viewed in color on a computer screen.) 


\title{
Development of an Atomic Spectroscopy Reference Implemented on a PC Platform under Windows 95
}

\author{
K. Visser and G.E. Hattingh \\ Physics Department \\ University of Stellenbosch \\ STELLENBOSCH \\ Republic of South Africa \\ e-mail:kv@maties.sun.ac.za
}

\section{Introduction}

Atomic Spectroscopic reference data comprising the characteristic energy levels and their associated eigenstate descriptions, wavelengths, and transition probabilities are available mostly in printed format. The major computerized databases implemented on the Personal Computer (PC) platform for Atomic Spectroscopy consist of the NIST Standard Reference Database 38 "Spectroscopic Properties of Atoms and Atomic Ions" and the NIST Standard Reference Database 61 "Database for Atomic Spectroscopy". On the World Wide Web the NIST Atomic Spectroscopy Database, version 1.1 , is currently available at http://aeldata.nist.gov/nist_atomic_spectra.html

Our objectives are to develop software providing

- a comprehensive database integrating the available critically evaluated Atomic Spectroscopic information,

- a search engine serving as input filter to selectively retrieve user-specified information from the database,

- user-specified custom-designed formatting of the output data both as tabular alphanumeric text and in graphical format,

- appropriate calculations using the primary data as input to derive secondary parameters.

For the initial phase of the project we decided to develop the software for the IBM and compatible PC platform under the Windows 95 operating system and used Borland's Delphi 3 compiler to generate a 32 bit application program. The program is developed in a modular way with each module representing a particular application, which can be activated by clicking on its named tab. Presently available and operational modules are:

- Energy level tables;

- Wavelength and Multiplet tables;

- Wavelengths calculated from the energy level data and applying user-selectable subsets of the selection rules for the various radiative transition types. 
Upon initiation the program searches for all associated data files (one per element) present. For each file found it uses an efficient blockread procedure to transfer the file's available data records as a single block at high speed into memory. These data records are kept memory resident to avoid repetitive reading and swapping of the database information in and out of memory during subsequent searches. Adding more elements to the program is a simple matter of making their correctly formatted data files available in the assigned directory. An array of indices referring to the first and last record for each elemental ionization stage is kept for quick searches in the appropriate subset of the memory resident list of data records.

The database files for the program use an internal encoding format to decrease their size compared to the original ASCII text files, thereby increasing the efficiency of the program in terms of speed and smaller data file size. Each variable is enumerated and mapped onto the smallest integer type which covers its range. For instance, instead of storing the name or chemical symbol of the element and its ionization stage as ASCII fields in the data records, the hundred plus elements and possible ionization stages are mapped onto a subset of the integers ranging from 0 to 127 and declared as variables of type Byte. By packing variables of lesser range bit-wise together, to optimally fit into the range of the smallest suitable integer type, memory and mass storage space are conserved. Strings, such as are used for the description of the electron configuration and parent term, the term value, the multiplet number, line codes and references to the original source of the data, are variables which occur repetitively in the database records. They are also enumerated and the 2 Byte variable, serving as referencing index into the stringlist, is stored in the appropriate record fields of the database file instead of repetitively storing the longer string of text itself. This enumeration and internal formatting of the database files led to a 7.2 times reduction of the comparative ASCII text file size for energy levels and a 5.1 times reduction for wavelength files. The ASCII text files containing the stringlists for configurations, etc., have a size of $80 \mathrm{~kb}$.

The displayed tables can be dynamically reformatted according to the user's choice with respect to which columns of data are currently visible and which are hidden from view so as to remove information which the user might consider unnecessary for his current needs. The displayed data columns/rows in the scrollable tabular list can also be dragged and dropped to change their vertical and horizontal ordering. The widths of the displayed columns are also dynamically resizable.

\section{Energy Levels Module and Wavelength \& Multiplet Module}

The database currently includes energy level data for 417 atomic/ionic species from 20 elements $(\mathrm{O}$, $\mathrm{Na}, \mathrm{Mg}, \mathrm{Al}, \mathrm{Si}, \mathrm{P}, \mathrm{S}, \mathrm{K}, \mathrm{Ca}, \mathrm{Sc}, \mathrm{Ti}, \mathrm{V}, \mathrm{Cr}, \mathrm{Mn}, \mathrm{Fe}, \mathrm{Ni}, \mathrm{Co}, \mathrm{Cu}, \mathrm{Kr}$ \& Mo) consisting of 33,004 energy level records. It also contains wavelength data for 47 atomic/ionic species from 4 elements $(\mathrm{O}, \mathrm{Mg}$, $\mathrm{Al}, \mathrm{Sc}$ ) with 11,320 wavelength records. Expansion for the energy levels of another 123 ionic species from 17 more elements and 17,785 energy level records are underway, as well as wavelength data for another 186 ionic species from 10 more elements and 23,648 wavelength records.

Single or multiple elements can be selected/deselected via mouse clicks on the element name buttons arranged to resemble the periodic table, as shown in Fig. 1, with the selected state being indicated by a change of color. Unavailable elements are grayed out.

For ease of element selection an element list is also provided which can either contain the elements arranged alphabetically by name or chemical symbol and atomic number. A command button to toggle the selected state for all available elements is also provided.

Single or multiple ionization stages can be selected/deselected by clicking on their individual buttons. Different ionization stages for each individual element or ionization stages common to all elements can be selected for all actively selected elements. A similar "Select/deselect All" command 
button is also provided for the ion selection. Additional search criteria that can be specified are the energy/wavelength range; the electron configuration; the term values; parity; total angular momentum quantum number $J$; orbital angular momentum symbol $L$ and spin multiplicity $m_{s}$.

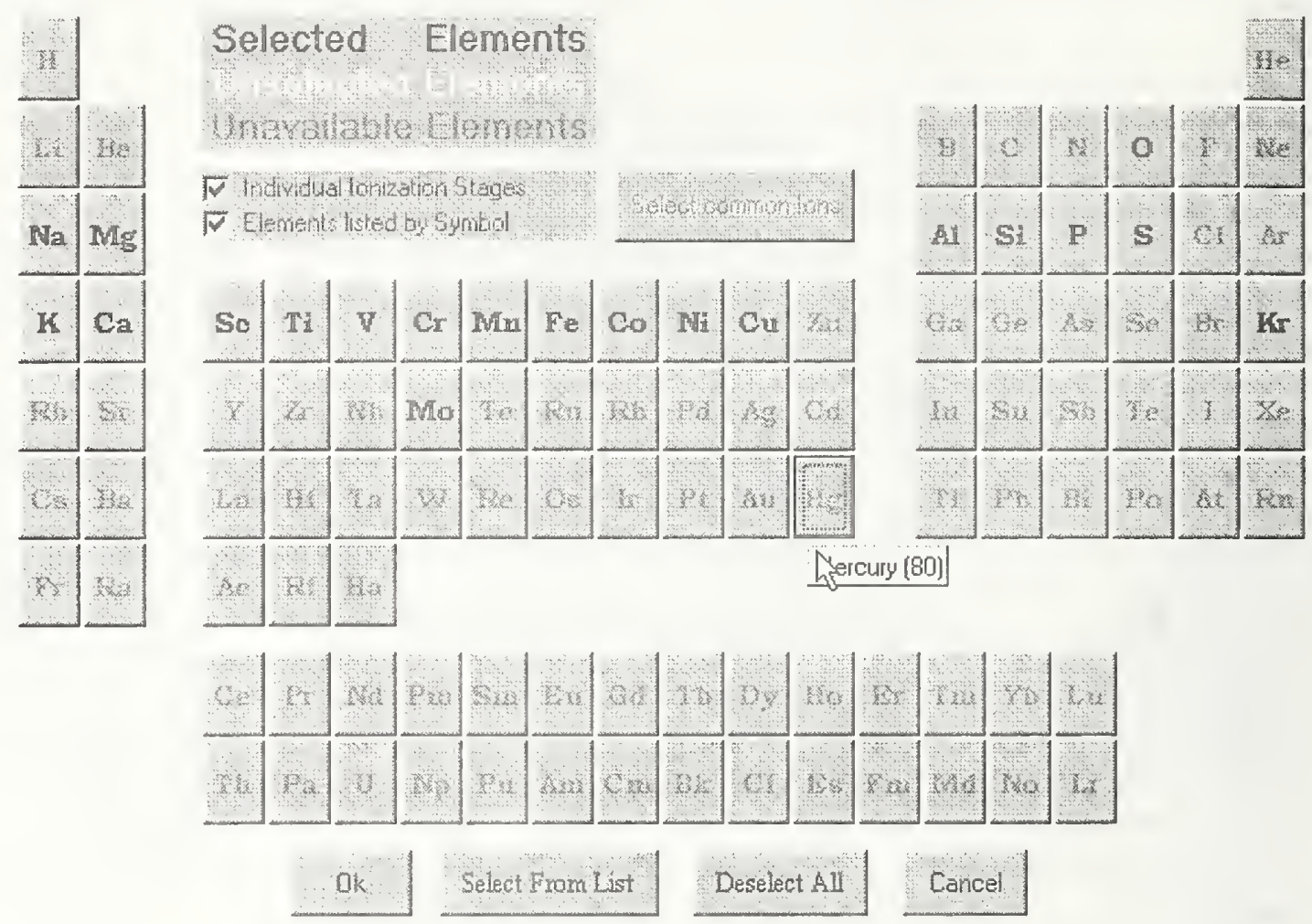

Figure 1: Selection of elements from the periodic table.

\section{Calculated Wavelength Module}

In this module theoretically expected wavelengths can be calculated from the energy levels by applying the Ritz combination principle

$$
\lambda_{v a c}=\frac{h c}{\left|E_{i}-E_{f}\right|}
$$

to pairs of levels $E_{i}$ and $E_{f}$ that might be radiatively connected. Both the energy levels of a single element and ionization stage can be selected at a time. The user can specify the following criteria that pairs of levels must meet for selection in the derived wavelength calculation:

- a transition mode from:

- absorption from a single lower state, or

- emission from a single excited state, or

- transitions involving all states; 
(For absorption and emission the initial state can be picked from the applicable energy level list of the selected atom/ion.)

- a wavelength interval in terms of its upper and lower wavelength limits;

- LS-coupling can be activated or ignored to prevent or allow intercombination lines;

- electric dipole (E1), magnetic dipole (M1) and electric quadrupole (E2) radiation types or combinations thereof can be checked to activate their respective selection rules;

- combinations of changes in the total angular momentum $\delta J$ can be checked.

For the interval $(2 \mu \mathrm{m}>\lambda>2000 \AA)$ wavelengths in air are calculated from

$$
\lambda_{\text {air }}=\frac{\lambda_{v a c}}{n(\lambda)}
$$

with the refractive index of air calculated from the Edlen formula[1]

$$
n(\lambda)=1+8.34213 \times 10^{-5}+\frac{2.406030 \times 10^{6}}{\left(1.30 \times 10^{10}-\sigma^{2}\right)}+\frac{1.5997 \times 10^{4}}{\left(3.89 \times 10^{9}-\sigma^{2}\right)}
$$

with the wavenumber $\sigma$ in $\mathrm{cm}^{-1}$. The displayed table of calculated wavelengths can sorted according to wavelength, or energy of the upper or lower state.

The module can be used for such applications as theoretical prediction of suitable transitions for a multi-step laser excitation scheme, identification of the states involved in experimentally observed wavelengths and selection of closely grouped wavelengths from largely different excitation states suitable for spectroscopic temperature determination.

\section{Future Development}

We plan to develop the program further to include modules for

- transition probability tables,

- Grotrian diagram creation from the energy levels and selection rules,

- calculation of simulated spectra,

- hyperfine structure,

- line profiles and convolution,

- Zeeman splitting.

Other future developments planned, include: expansion of the number of available elements, allowance for editing of the data fields and insertion of data records by a user which are to be saved as overlay files so as not to corrupt the original data files, saving and retrieving of the last used options and configurations. We also envision making a demonstration version of the program available on the World Wide Web.

\section{References}

[1] B. Edlén, Metrologia 2, 71 (1966) 

2 Data Needs 



\title{
Absolute Infrared Intensities for: Standards and Reactive Molecules
}

\author{
Charles Chackerian, Jr. \\ NASA Ames Research Center, Atmospheric Physics \\ Research Branch, MS 245-4, Moffett Field, \\ CA $94035-1000$
}

\section{Introduction}

In recent years laser techniques have provided us with very accurate infrared line positions and the term "secondary frequency standard" has entered the lexicon of infrared spectroscopy. For example, line positions of carbon monoxide in the $5 \mu \mathrm{m}$ spectral region have been determined with an absolute accuracy of about $30 \mathrm{KHz}$ [1]. Frequency information is prerequisite for obtaining accurate intensity information since the determination of transition dipole parameters from experimental intensity measurements requires numerical values for transition frequencies, transition initial state energies, and rovibrational partition functions. Transition frequencies are also used, along with quantum-mechanical models, to obtain the vibration-rotational state wave functions associated with the transitions of interest. The wave functions can then be used along with absolute or relative intensity information with an appropriate quantum mechanical model to determine parameters that describe the dipole surface of the molecule; some examples are for $\mathrm{CO}$ [2], $\mathrm{OH}$ [3] and $\mathrm{CH}_{3} \mathrm{Cl}$ [4].

To the best of my knowledge, there are no reports of absolute line intensities of rovibrational transitions in the literature that could serve as secondary standards to an accuracy better than $\pm 5 \%$.

I estimate the absolute accuracy that currently could probably be obtained for secondary infrared intensity standards. Following that, I discuss intensity determinations for unstable molecular species. I do not attempt to give a rational for the level of accuracy needed for any particular application or propose specific molecules that could be candidates for use as secondary intensity standards.

\section{Primary Intensity Standard}

A "perfectly performing" spectrometer does not require a primary intensity standard in order to determine absolute line intensities from absorption measurements. Such an instrument responds linearly to photons over the appropriate radiative flux range and has negligible scattered light. However, were we to test the spectrometer's performance we would ultimately be limited by the accuracy of a primary radiation standard. I will assume here that a black body can serve this purpose. Blackbodies in operation at the National Physical Laboratory and the National Institute of Standards and Technology have emissivities equal to 0.999 or better and operate at temperatures known to about 200 millikelvin. At $5 \mu \mathrm{m}$ our blackbody operating at $450 \mathrm{~K}$, with uncertainties in its temperature of $0.2 \mathrm{~K}$ and emissivity of $0.1 \%$, would have an uncertainty in its emitted differential flux (Watts/ $\mathrm{cm}^{2}$-micron-sr) of $0.39 \%$. The latter uncertainty depends on the temperature of the blackbody and the precise wavelength, but for 
the purposes of the discussion to follow we will assume that our ultimate accuracy for infrared intensities is on the order of $0.4 \%$.

In some cases it is possible to obtain absolute intensity information via relative intensity measurements over a large spectral interval, and in this situation a blackbody source is needed to establish the spectrometer's relative sensitivity with wavelength.

\section{Secondary Intensity Standards}

Secondary intensity standards have not been established for molecular infrared transitions, but in this section results obtained by our group are used to illustrate possible sources of errors in intensity determinations as well as expected accuracies which could be obtained from highquality measurements.

The best way to determine the absolute intensity of rovibrational transitions of a stable molecule is with conventional absorption spectroscopy. The equations in the Appendix below show the relationships between the transmission of an individual rovibrational line (Eq. 1) and the rotationless transition moment for a rovibrational band (Eq. 3). The equations also indicate that accuracies of transition frequencies, energy levels, gas pressure, composition, broadening cross sections, absorbing path length, and temperature all play a role in determining the absolute accuracy of the measured transition moment, and therefore, the accuracy of any of these quantities will be a factor in determining the accuracy of the individual line intensities.

Table 1 shows the most precise results we have obtained to date for the rotationless transition moment of a rovibrational band. The four spectra of the $\left(00^{0} 2\right)_{I} \bullet\left(00^{0} 0\right)_{I}$ band of ${ }^{12} \mathrm{C}^{16} \mathrm{O}^{18} \mathrm{O}$ were obtained using a natural abundance sample of carbon dioxide and the FTS at the National Solar Observatory, Kitt Peak, AZ. The double-sided interferograms produced excellent instrumental profiles in the spectral domain.

Table 1: Intensity data* for $4639.5 \mathrm{~cm}^{-1}$ band of ${ }^{12} \mathrm{C}^{16} \mathrm{O}^{18} \mathrm{O}$ determined via FTS spectroscopy (carbon dioxide in natural isotopic abundance).

$\begin{array}{cccc}\text { Spectrum } & \begin{array}{c}\text { Pressure } \\ \text { (Torr) }\end{array} & \begin{array}{c}\text { Path } \\ \text { (meters) }\end{array} & \begin{array}{c}\left|<00^{0} \mathbf{2}\right| \mathbf{M}\left|0_{0} \mathbf{0}_{0}>\right| \\ \text { (Debye) }\end{array} \\ \text { KP03 } & 65.0 & 97.04 & 0.0014312 \\ \text { KP04 } & 45.0 & 409.82 & 0.0014310 \\ \text { KP05 } & 45.0 & 145.16 & 0.0014351 \\ \text { KP13 } & 59.7 & 193.28 & 0.0014352\end{array}$

*R. Kshirsagar, L. P. Giver, and C. Chackerian, Jr.

(private communication, NASA Ames Research Center)

The transition moments obtained from the individual spectra were obtained by plotting the square root of the reduced intensity of individual lines in the band vs. the $m$ quantum number. The reduced line intensity is defined in Eq.(2) of the Appendix. The vibration-rotation interaction term is small enough to be undetermined; the precision of the transition moment squared determinations is $\pm 0.15 \%$. The individual line intensities used in the reduced intensity plots were obtained via non-linear least-squares fitting of the FTS spectra. The paper of Benner 
et al. [5] gives a discussion of this technique which requires knowledge of the instrumental function as well as in this case the pressure broadening of ${ }^{12} \mathrm{C}^{16} \mathrm{O}^{18} \mathrm{O}$ by ${ }^{12} \mathrm{C}^{16} \mathrm{O}_{2}$. The isotopic composition is obtained from a standard compilation [6], and the uncertainties for the above isotopes is negligible. At low pressures errors in pressure broadening are not as important as errors in the instrumental function and inherent errors in the pressure gauge or neglect of thermal transpiration [7] if the gas sample and capacitance manometer are at different temperatures. The pressure uncertainty of our measurements is about $0.22 \%$. Our temperature uncertainty is about $0.17 \%$ which results in a $0.34 \%$ error (the rotational partition function is proportional to the temperature). This temperature error will also add an additional $0.17 \%$ error through the Boltzmann factor. Temperature errors can also cause an error in the vibration-rotation interaction term, $\mathrm{F}$, of Eq.(7). If the compositional, path length, and data reduction errors can be assumed to be zero, the error in the absolute intensity would be about $0.75 \%$. The latter estimate is clearly conservative in the sense that it is probably too low but is still greater than the precision of $\pm 0.30 \%$ we have achieved for the above cited intensity measurements on ${ }^{12} \mathrm{C}^{16} \mathrm{O}^{18} \mathrm{O}$. It is probably reasonable to assume that we can achieve an absolute accuracy of about $2 \%$ in the determination of infrared rovibrational line intensities.

\section{Unstable/Reactive Molecules}

The determination of absolute intensities for reactive molecules is particularly difficult in the infrared because: ( $i$ ) the sources that produce the spectra are usually not in LTE, except possibly for the lowest rotational levels, and (ii) the concentration of molecules in the optical path is not known. Lifetime techniques are not possible because the infrared radiative lifetimes are longer than collisional deactivation times.

For some diatomic molecules it has been possible to exploit the effect vibration-rotation interaction has on modifying intensities. Here knowledge of the abundance of the optically active species is not required, only the ratios of emission line intensities originating in common upper levels [3,8] or the relative intensities across an absorption band [9]. Eq.(3) taken in ratios is used for the emission experiments to determine the electric dipole moment function. The vibrational transition moment squared is determined from these measurements with absolute errors on the order of $\pm 10 \%$.

\section{Conclusions}

We have demonstrated that infrared line intensities can be determined with a precision of about $0.3 \%$. For the particular experiment described the absolute error on the transition moment squared could be about $1 \%$. It is probably reasonable to assume that we can achieve an absolute accuracy of about $2 \%$ in the determination of infrared rovibrational line intensities of stable molecules.

The absolute accuracies achieved for a small number of unstable molecules is on the order of $\pm 10 \%$. 


\section{Appendix}

The equations which relate the absorption of radiation in an isolated rovibrational line (centered at the frequency $\sigma_{0}$ ) to its intensity is given by the Beer-Lambert Law,

$$
\frac{I\left(\Delta \sigma=\sigma-\sigma_{o}\right)}{I_{o}(\sigma)}=\exp \left[-\operatorname{Spl} \frac{H(\Delta \sigma, Y)}{\gamma_{D}}\left(\frac{\ln 2}{\pi}\right)^{1 / 2}\right] \text {, }
$$

where $I_{0}(\Delta \sigma)$ and $I(\Delta \sigma)$ are the intensities respectively, incident on and emergent from the absorbing gas sample. $S$ is the line intensity, $p$ the partial pressure of the species of interest, $l$ the absorbing path length, and $H$ the line shape. The Voigt line shape parameter, $Y=\left(\gamma_{L} / \gamma_{D}\right) \sqrt{\ln 2}$, is a function of the temperature and pressure. The Doppler broadening, $\gamma_{D}$, is a function of the molecular weight of the optically active species as well as the temperature. The pressure broadening coefficient, $\gamma_{L}=\sum_{i} p_{i} \gamma_{i L}^{o}(T / T)^{n} i$, is a function of the temperature, $T(K)$, as well as the collisional cross sections between the various species, $i$. Here $\gamma_{i L}^{o}$ is referenced to the standard temperature. The line intensity in Eq.(2) for

$$
\begin{aligned}
& S_{\text {if }}\left\{\left(\frac{8 \pi^{3} N_{L} T_{O}}{3 h c}\right) \sigma_{O} \frac{G_{S r}}{Z_{v r}(T) T} \exp \left(\frac{-h c E_{i}}{k T}\right)\left[1-e^{-h c \sigma_{o} / k T}\right] \cdot H L\left(r_{i}, r_{f}\right)\right\}^{-1} \\
& \quad=S_{\text {reduced }}=\left|\left\langle v_{i}\left|M\left(Q_{x}\right)\right| v_{f}\right\rangle\right|^{2} F\left(v_{i}, r_{i} ; v_{f}, r_{f}\right)
\end{aligned}
$$

a rovibrational transition between initial and final states, $(i, f)$, depends on the temperature through the rovibrational partition function $Z$ and exponential factors. $T_{O}$ is the standard temperature, $N_{L}$ Loschmidt's Number and $G_{S r}$ a statistical weight. The transition probability is factored into vibrational, rotational, $H L$, and rovibrational interaction terms, $\left|\left\langle v_{i}\left|M\left(Q_{x}\right)\right| v_{f}\right\rangle\right|^{2} H L\left(r_{i}, r_{f}\right) F\left(v_{i}, r_{i} ; v_{f}, r_{f}\right), \quad Q_{x}$ represents an appropriate vibrational coordinate(s). The reduced intensity is related to the individual line intensities, $S_{i f}$, as well as quantities which can be calculated. A plot of $\left(S_{\text {reduced }}\right)^{1 / 2}$ versus an appropriate rotational quantum number yields the vibrational transition moment as well as any dependence of the transition moment on rotational quantum numbers.

Unstable/reactive species molecular spectra are often observed in emission. The volume emission coefficient, $I$, is given by Eq.(3)

$$
I=K^{\prime} \sigma^{4} N_{i} e^{-\left(E_{i} / k T\right)}|\langle i|M| f \mid\rangle|^{2}
$$




\section{References}

[1] M. H. Wappelhorst, S. Saupe, B. Meyer, T. George, W. Urban and A. Le Floch, J. Mol. Specrosc. 181, 357-362 (1997)

[2] C. Chackerian, Jr., R. Farrenq, G. Guelachvili, C. Rossetti, and W. Urban, Can. J. Phys. 62, 1579-1585 (1984)

[3] D. Nelson, A. Schiffman and D.Nesbitt, J. Chem. Phys. 93, 7003-7019 (1990)

[4] F. Cappellani, G. Restelli and G. Tarrago, J. Mol. Spectrosc. 146, 326-333 (1991)

[5] D. C. Benner, C. P. Rinsland, V. M. Devi, M. A. H. Smith, and D. Atkins, J. Quant, Spectrosc. Rad. Transfer 53, 705-721 (1995)

[6] P. De Beaver, M. Galled, N.E. Holder, and I. L. Barrens, J. Phys. Chem. Ref. Data 13, 809892 (1984)

[7] K. F. Poulter, M.-J. Rodgers, P. J. Nash, J. T. Thompson and M. P. Perkin, Vacuum 33, 311 316 (1983)

[8] C. Chackerian, Jr., G. Guelachvili, A. Lopez-Pinero and R. H. Tipping, J. Chem. Phys. 90, $641-649$ (1989)

[9] J. B. Burkholder, P. D. Hammer, J. Howard, A. G. Maki, G. Thompson, and C. Chackerian, Jr., J. Mol. Spectrosc. 124, 139-161 (1987) 


\title{
NASA's Laboratory Astrophysics Program
}

\author{
Hashima Hasan and Harley Thronson \\ Office of Space Science, NASA Headquarters \\ Washington D.C. 20546
}

\section{Introduction}

NASA's Office of Space Science (OSS) operates an active laboratory astrophysics program conducted primarily at universties, federally funded laboratories, and research institutes. Topic areas currently supported include electron impact cross sections, photodissociation proceses, electron-ion collisons, oscillator strengths for a variety of atomic transitions, polycyclic aromatic hydrocarbons, interstellar grains, and compilation of atomic databases. Related theoretical work is also supported to complement the laboratory research. The objective of the program is to enable and maximize the scientific return from the data of NASA's past, present and future space science missions. A brief description of the scientific program and NASA's needs for laboratory astrophysics is given in this paper. A workshop encompassing laboratory needs for OSS in the fields of astrophysics, planetary atmospheres, and space physics is planned on April 1 - 3, 1998 at Harvard University.

\section{Program Description}

Laboratory Astrophysics needs for NASA are driven by the data from its ongoing and planned missions, as well as from archival data (Table 1). Currently, the program is split along wavelength lines into two subprograms. Specific research projects are proposed by the scientific community in response to solicitations by NASA in the form of NASA Research Announcements (NRA). An annual solicitation for the Ultraviolet, Visible and Gravitational Astrophysics Research \& Analysis program provides the community an opportunity for a timely response to perform laboratory research for enabling the interpretation of new data from UV/Visible missions. The Infrared, Sub-mm and Radio Astrophysics program solicits proposals every three years. With the operation of the Near Infrared Camera and Imaging Spectrograph (NICMOS) and proposed launch of the Space Infrared Telescope Facility (SIRTF) and the Stratospheric Observatory for Infrared Astronomy (SOFIA), there may be need for more frequent solicitation in this area. Currently, only one program is funded in High Energy Astrophysics, though with the launch of the Advanced X-ray Facility (AXAF), there will perhaps be a need to expand this program.

A wide variety of projects, the focus of each of which is to enable the understanding of specific astrophysical phenomena, such as those within diffuse interstellar couds, stellar atmospheres, dust grains, etc. are under study. Names of principal investigators, institutions, and project titles are listed in Tables 2 and 3. In a broad sense, the program supports the fundamental science questions defined by NASA's Structure and Evolution of the Universe and Astronomical Search for Orgins science themes. 
Table 1

NASA's Astrophysics Missions

(partial list)

\section{Currently in operation}

Extreme Ultraviolet Explorer (EUVE)

Hubble Space Telescope (HST)

Wide Field and Planetary Camera 2

Space Telescope Imaging Spectrograph (STIS)

Near-Infrared Camera \& Multi-Object Spectrograph (NICMOS)

\section{Archival phase}

International Ultraviolet Explorer (IUE)

Orbiting \& Retrievable Far \& Extreme Ultraviolet Telescope (ORFEUS and ORFEUS2)

Interstellar Medium Absorption Profile Spectrograph (IMAPS)

Hubble Space Telescope (HST)

Wide Field and Planetary Camera (WFPC)

Goddard High Resolution Spectrograph (GHRS)

Faint Object Spectrograph (FOS)

High Speed Photometer (HSP)

ASTRO and ASTRO2

Ultraviolet Imaging Telescope (UIT)

Hopkins Ultraviolet Telescope (HUT)

Wisconsin Ultraviolet Photo-Polarimeter Experiment (WUPPE)

\section{Missions in development}

Sub-millimeter Wave Astronomy Satellite (SWAS)

Stratospheric Observatory for Infrared Astronomy (SOFIA)

Space Infrared Telescope Facility (SIRTF)

Wide Field Infrared Explorer (WIRE)

Space Interferometry Mission (SIM)

Far Ultraviolet Spectroscopic Explorer (FUSE)

Hubble Space Telescope (HST)

Advanced Camera for Surveys (ACS)

Cosmic Origins Spectrograph (COS)

Galaxy Evolution Explorer (GALEX) 


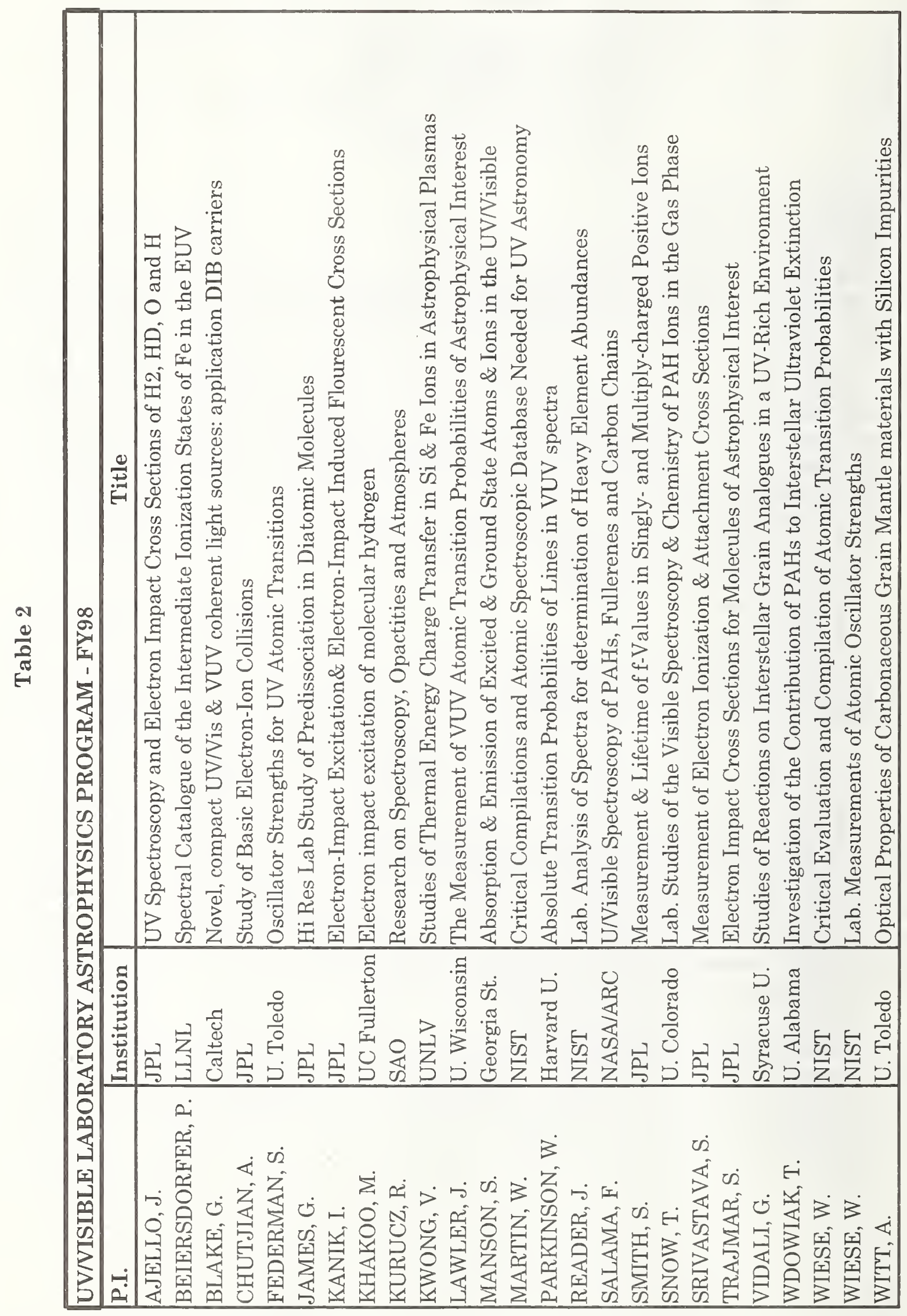




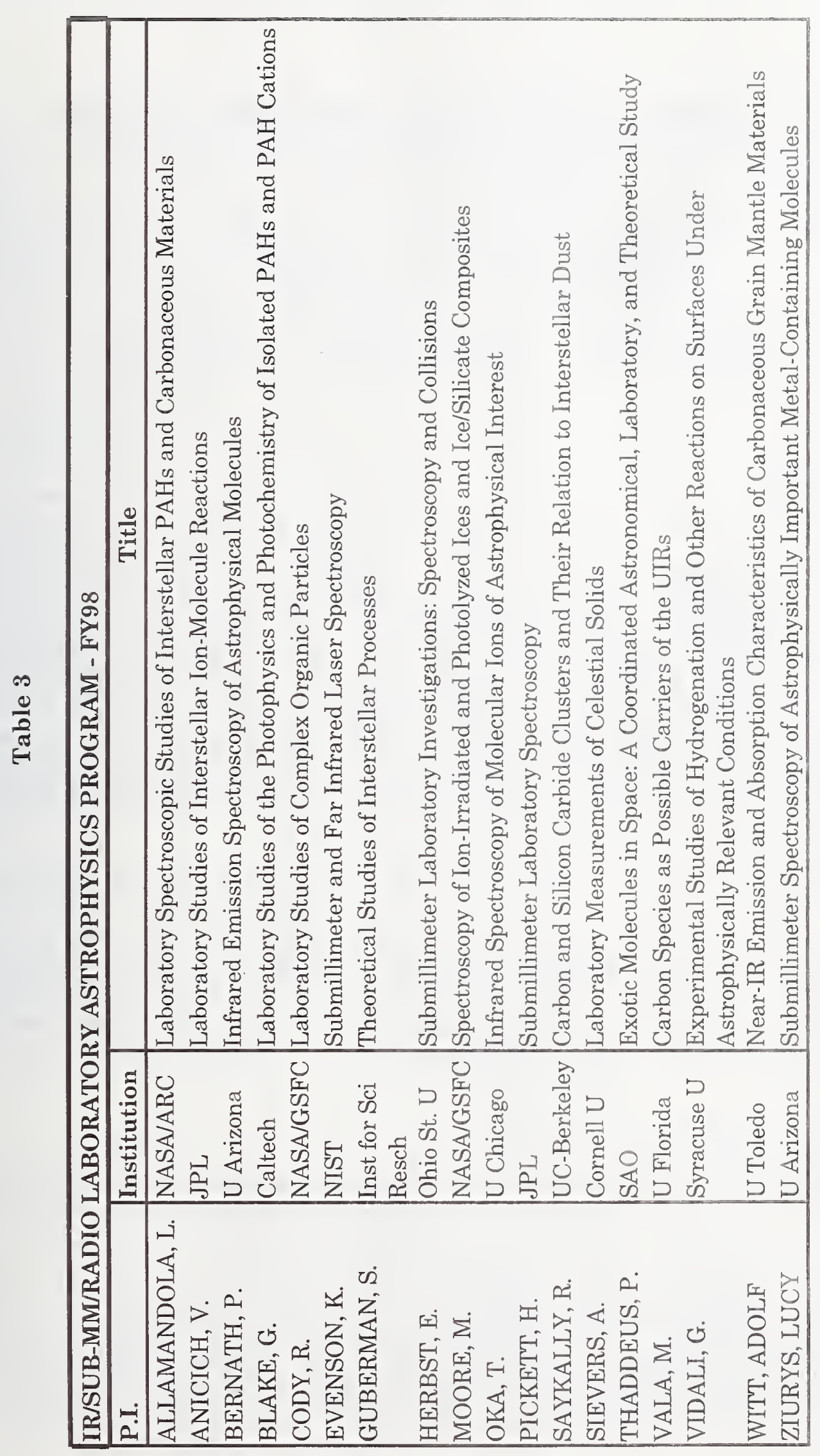




\title{
Atomic Inputs to Calculate Radiative Forces in Stellar Models
}

\author{
G. Massacrier ${ }^{1}$, J.-F. Gonzalez ${ }^{1}$, M.-C. Artru ${ }^{1}$ \\ G. Michaud ${ }^{2,3}$, J. Richer ${ }^{2,3}$, F. LeBlanc ${ }^{4}$ \\ 1 CRAL, 46 allée d'Italie F-69364 Lyon Cédex 07, France \\ ${ }^{2}$ CERCA, 5160 boul. Décarie, Montréal, Québec, H3X 2H9, Canada \\ ${ }^{3}$ Dép. de physique, Univ. de Montréal, Montréal, H3C 3J7, Canada \\ ${ }^{4}$ Dép. de physique, Univ. de Moncton, Moncton, E1A 3E9, Canada
}

\section{Introduction}

Photoabsorption processes imply momentum transfer from the radiation flux to the atomic absorbers in a plasma. The resulting radiative forces act selectively on different chemical species. According to the local imbalance between gravitational $\mathrm{g}$ and radiative accelerations $\mathrm{g}_{\mathrm{rad}}$ in a star, a given element can diffuse up or down in layers where mixing hydrodynamical processes are negligible. Effects of chemical stratification occurring during stellar lifetimes are observed as peculiar surface abundances. The diffusion of strongly absorbing species can ultimately influence the evolutionary path of the star and might affect its pulsational stability.

Standard stellar evolution codes assume homogeneity of chemical composition throughout the star (except in nuclear reaction zones). Rosseland opacities $\kappa_{\mathrm{R}}$ are interpolated from a grid of plasma conditions in $T$ (temperature), $\rho$ (density) and $X, Y, Z$ (mass fractions of hydrogen, helium and 'metals'). The relative abundances of metals are held fixed in $Z$.

When including atomic diffusion in stellar models, local under- or over-abundances may develop. Because of their influence on radiative accelerations but also on the Rosseland opacity these local variations of the metallic relative abundances in $Z$ have to be taken into account. This increases the number of free parameters and precludes the use of precalculated data. One has to turn to in-line computations. Very efficient and finely tuned methods must be found as the Rosseland opacity and the radiative accelerations for many elements must be computed at each layer and each evolutionary step of the star for several iterative steps.

\section{Radiative Accelerations on an Ion}

The radiative acceleration on an element $A$ in the ionization state $k$ at radius $r$ in a star is given by:

$$
g_{\mathrm{rad}}^{k}(A)=\frac{L_{\mathrm{rad}}(r)}{4 \pi r^{2} c} \frac{\kappa_{\mathrm{R}}}{X_{A, k}} \int_{0}^{\infty} \frac{\tilde{\kappa}_{A, k}(u)}{\kappa(u)} \mathcal{P}(u) \mathrm{d} u
$$

where $L_{\mathrm{rad}}(r) / 4 \pi r^{2} c$ is the total radiative momentum flux at $r, \kappa_{\mathrm{R}}$ is the Rosseland opacity, $X_{A, k}$ is the mass fraction of the ionization state, $u=\frac{h \nu}{k T}$ is the dimensionless frequency variable, $\kappa(u)$ is the total opacity at frequency $u$, and $\tilde{\kappa}_{A, k}(u)$ is the opacity for the ion under consideration (corrected for the fact that only that part of the photon momentum absorbed and transfered to the final ion 
is needed; see below). $\mathcal{P}(u)$ is the normalized black body flux:

$$
\mathcal{P}(u)=\frac{15}{4 \pi^{2}} \frac{u^{4} \mathrm{e}^{u}}{\left(\mathrm{e}^{u}-1\right)^{2}}
$$

The frequency dependent factor $\mathcal{P}(u) / \kappa(u)$ appears because the 'diffusion' approximation for the radiative flux is assumed. Hence Eq. (1) is relevant only for stellar envelopes. In atmospheres the actual flux should be used. The value of $g_{\mathrm{rad}}^{k}(A)$ depends on the competition for the photons between the ion and the other elements. Increasing the abundance of the element leads to saturation and may also modify the radiative acceleration on the other elements.

Total monochromatic opacities must first be computed:

$$
\kappa(\nu)=\sum_{A} \kappa_{A}(\nu), \quad \kappa_{A}(\nu)=\sum_{k} \kappa_{A, k}(\nu), \quad \rho \kappa_{A, k}(\nu)=\sum_{\substack{\text { transitions } \\ i \rightarrow j}} N_{i} \sigma_{i j}(\nu) .
$$

where $N_{i}$ is the population density of the absorbing state $i$ (of the ion $A, k$ ), and $\sigma_{i j}(\nu)$ the cross section for the transition. This requires data for energy levels, oscillator strengths and line profiles for bound-bound absorptions, as well as photoionization cross sections for bound-free absorptions. All these data for all main contributors to the Rosseland opacity are now available or can be derived from recent large atomic data banks.

In the modified opacity $\tilde{\kappa}_{A, k}(\nu)$ of Eq. (1), bound-bound contributions are summed as in Eq. (3). But for bound-free absorptions two modifications occur. First, two ionization states are involved in the process. The state $k$ for which we want the acceleration is the final ion. The photoionization cross sections one has to use are those of the previous $(k-1)$ state. Second, in such a process, only a part of the absorbed photon momentum is transmitted to the final ion (the rest goes away with the photoelectron). This implies the use of modified cross sections, usually written $\tilde{\sigma}_{i j}(\nu)=f_{\text {ion }, i j}(\nu) \sigma_{i j}(\nu)$. This second point applies as well to free-free absorptions. $f_{\text {ion }}$ is an atomic parameter almost absent in the literature (more details in an accompanying contribution [1]).

\section{Diffusion of an Element}

For reasons of rapidity and efficiency the diffusion of an element $A$ is treated as a whole, and not through its individual ions. The effective radiative acceleration to be used to study the diffusion of $A$ is some average of its ion accelerations. The approach is to weight $g_{\mathrm{rad}}^{k}(A)$ with the ion population times its diffusion coefficient:

$$
g_{\mathrm{rad}}(A)=\frac{\sum_{k} N_{k} D_{k} g_{\mathrm{rad}}^{k}(A)}{\sum_{k} N_{k} D_{k}}
$$

For an ion diffusing in a bath of protons, one roughly has $D_{k} \propto 1 / z_{k}^{2}$, where $z_{k}$ is the net charge of the ion (an estimate for neutrals $\left(z_{k}=0\right.$ ) leads to an effective approximate value $z_{k} \simeq 0.1$ ). The process of averaging enhances the weight of less ionized states.

Redistribution processes have to be taken into account too. Following a bound-bound absorption, an ion may be ionized through (electronic) collisions before being deflected (by protons). Thus the ionization state in which the ion diffuses before being deffected may differ from the one in which the photon was absorbed. As the diffusion coefficients depend on the charge of the ion, the previous average has to be modified to take this effect into account. Only rough approaches are used at the moment. Precise computations would require large sets of collisional data. 


\section{Calculations of Radiative Accelerations}

\subsection{Smoothed Background Procedure}

Systematic attempts ([2], [3], [4]) to calculate radiative accelerations were performed using the TOPbase data bank [5] calculated by the Opacity Project group ([6], [7]). The method is that explained in [8]. The radiative acceleration from each transition is calculated separately and then summed. The total opacity $\kappa(u)$ is divided into two parts: the opacity of the transition under consideration and a background opacity. The latter is a constant in the frequency interval $\Delta u$ that contains the natural frequency of the line. It is computed by evenly distributing other bound-bound cross sections in the interval.

The method is motivated by the fact that the background opacity has to be computed only once to get the acceleration of all the ions. It allows a good treatment of saturation. Electron recoil, averages with diffusion coefficients and redistribution can be included. However evenly distributing in an interval all lines falling in that interval artificially fills the valleys in the background opacity. Thus at low temperatures when line widths are small this method underestimates exact accelerations. It is also too time consuming to be used in-line in present evolution codes.

\subsection{Opacity Sampling Procedure}

In [9] and [10] another approach was used to calculate radiative accelerations. At a given temperature and density an opacity spectrum is pre-computed for each element at given points on a certain frequency grid (opacity sampling). Then Eq. (1) is directly integrated. The method is accurate in as much as the sampling is sufficient not to miss important lines in the elements. Hence the lower the temperature, the finer the grid mesh should be. Optimization of the number of grid points and use of an non-evenly spaced grid are discussed in [11].

Actual computations ([9]) were done based on the OPAL opacities for the 21 most abundant elements ([12], [13], [14], [15]). Opacities obtained from OPAL are already summed on the ionization states. Radiative acceleration on an element $A$ is then calculated according to:

$$
g_{\mathrm{rad}}(A)=\frac{L_{\mathrm{rad}}(r)}{4 \pi r^{2} c} \frac{\kappa_{\mathrm{R}}}{X_{A}} \int_{0}^{\infty} \frac{\kappa_{A}(u)}{\kappa(u)} \mathcal{P}(u) \mathrm{d} u
$$

This expression is simply the momemtum absorbed from the radiation. It assumes that this momentum is entirely transfered to the element $A$. Though the effects of electron recoil, averaging over the ions or redistribution are not included with this use of elemental opacities, the gain in computation time obtained by not treating ions individually allows one to calculate radiative accelerations in-line in stellar evolution codes for any given chemical composition ([16], [17]). A correction factor can also be pre-computed ([9]) wich is simply the ratio of the radiative accelerations of an element calculated with and without these corrections.

\section{References}

[1] G. Massacrier and K. El-Murr, this volume

[2] G. Alecian, G. Michaud, and J. Tully, Astrophys. J. 411, 882 (1993)

[3] J.-F. Gonzalez, M.-C. Artru, and G. Michaud, Astron. Astrophys. 302, 788 (1995)

[4] F. LeBlanc and G. Michaud, Astron. Astrophys. 303, 166 (1995) 
[5] W. Cunto, C. Mendoza, F. Ochsenbein, and C. J. Zeippen, Astron. Astrophys. 275, L5 (1993)

[6] M. J. Seaton, J. Phys. B: At. Mol. Opt. Phys. 20, 6363 (1987)

[7] M. J. Seaton, Y. Yan, D. Mihalas, A. K. Pradhan, Mon. Not. Roy. Astr. Soc. 266, 805 (1994)

[8] J.-F. Gonzalez, F. LeBlanc, M.-C. Artru, and G. Michaud, Astron. Astrophys. 297, 223 (1995)

[9] J. Richer, G. Michaud, F. J. Rogers, C. A. Iglesias, S. Turcotte, and F. LeBlanc, Astrophys. $J .$, in press (1998)

[10] M. J. Seaton, Monthly Not. Roy. Astr. Soc. 289, 700

[11] F. LeBlanc, G. Michaud, and J. Richer, in preparation

[12] F. J. Rogers and C. A. Iglesias, Astrophys. J. Suppl. 79, 507 (1992)

[13] F. J. Rogers and C. A. Iglesias, Astrophys. J. 401, 361 (1992)

[14] C. A. Iglesias and F. J. Rogers, Astrophys. J. 443, 460 (1995)

[15] C. A. Iglesias and F. J. Rogers, Astrophys. J. 464, 943 (1996)

[16] S. Turcotte, J. Richer, and G. Michaud, Astrophys. J., submitted

[17] S. Turcotte, J. Richer, G. Michaud, C. A. Iglesias, and F. J. Rogers, Astrophys. J., submitted 


\title{
New Electron Temperature and Density Diagnostics for Photoionized Gas
}

\author{
D. W. Savin ${ }^{1}$, T. Bartsch², M. H. Chen³, S. M. Kahn¹, D. A. Liedahl3 , J. Linkemann, \\ A. Müller ${ }^{2}$, S. Schippers ${ }^{4}$, M. Schmitt ${ }^{4}$ D. Schwalm4, and A. Wolf ${ }^{4}$ \\ Department of Physics and Columbia Astrophysics Laboratory, Columbia University, \\ New York, NY 10027 USA \\ 2Institut für Kernphysik, Strahlenzentrum der Justus-Liebig-Universität, \\ D-35392 Giessen, Germany \\ ${ }^{3}$ Lawrence Livermore National Laboratory, Livermore, CA 94550 USA \\ ${ }^{4}$ Max-Planck-Institut für Kernphysik and Physikalisches Institut der Universität \\ Heidelberg, D-69117 Heidelberg, Germany
}

The ionization and heating of the media surrounding accretion-powered compact sources, such as cataclysmic variables, X-ray binaries, and active galactic nuclei, is dominated by photoionization. Theoretical models of photoionized gases show that the ionization structure is determined by photoionization balanced by radiative recombination (RR) and dielectronic recombination (DR). The electron temperature at which the fractional abundance of a given ion peaks [1] is far below the temperature where the ion would exist in coronal equilibrium [2,3]. As a result, X-ray line emission is produced by $\mathrm{RR}$ and $\mathrm{DR}$ and not by electron impact excitation $[1,4]$. Also, the radiative recombination continuum ( $\mathrm{RRC}$ ) of an ion is predicted to appear as a distinct narrow feature just above the ionization threshold of the ion [5].

With the improved spectral resolution offered by the SIS detectors of ASCA [6] it has become possible to observe some of these unique properties of photoionized gases. From ASCA observations, the RRC for Ne X has been identified in SIS spectra of the low-mass X-ray pulsar 4U 1626-67 [7] and the Mg XII, Si XIV, and S XVI RRC have been identified in spectra of the X-ray binary Cygnus X-3 [8]. From the widths of the RRC features, electron temperatures of $\sim 5-100 \mathrm{eV}$ are inferred for the $\mathrm{X}$ ray emission line regions. As predicted, these temperatures are far below the temperatures where these ions are formed in coronal equilibrium.

The upcoming launches of the X-ray astronomy satellites AXAF, XMM, Spectrum-Röntgen Gamma, and Astro- $E$ are expected to open a new era in the spectroscopy of extrasolar X-ray sources. The combination of the large collecting area telescopes and high resolution spectrometers on these satellites will produce high quality spectra to which a wide range of plasma diagnostic techniques will be applied. Of particular interest will be the $0.7-2.0 \mathrm{keV}(6-18 \AA$ ) spectral band which is dominated by the $L$-shell transitions (i.e. $n \geq 3 \rightarrow n=2$ ) of Fe XVII to Fe XXIV (the iron $L$-shell ions). These ions exist over a wide range of conditions and are expected to provide many valuable plasma diagnostics.

$L$-shell iron forms at the same conditions where Ne X, Mg XII, Si XIV, and S XVI form. Thus, the temperatures measured in $4 \mathrm{U}$ 1626-67 and Cyg X-3 largely validate the predicted temperature range for $L$-shell iron. However, the exact temperature at which a given ion forms depends on many variables, such as the metallicity of the gas, the shape of the ionizing spectrum, the presence of additional heating and/or cooling mechanisms, and radiative transfer effects. These can be expected to vary for different sources. For most iron $L$-shell ions at these low temperatures, DR via $\Delta n=0$ core excitations dominates recombination. It is therefore important 


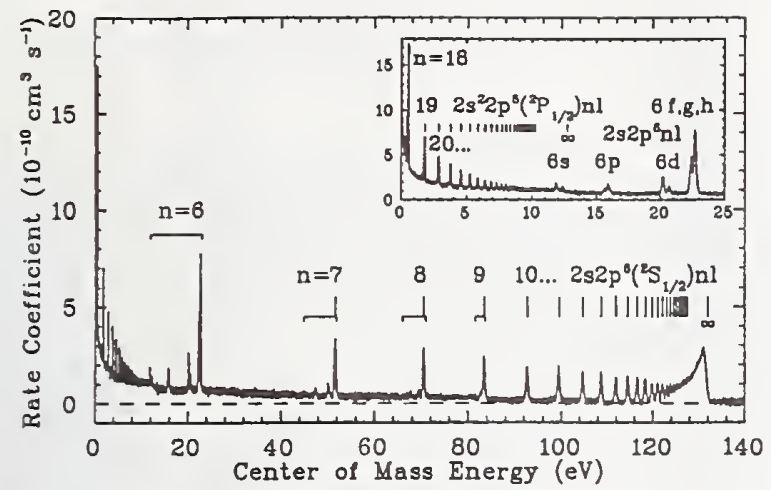

Figure 1: Measured Fe XVIII to Fe XVII recombination rate coefficient versus collision energy. The nonresonant "background" is due to RR.

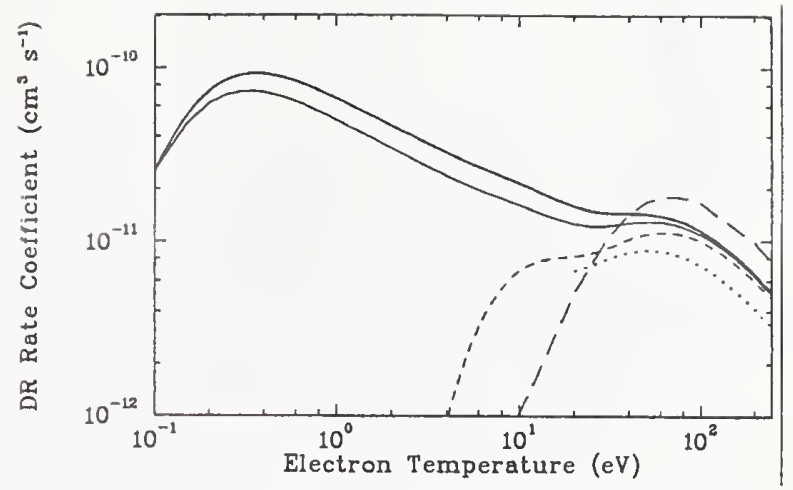

Figure 2: Fe XVIII to Fe XVII Maxwellian averaged $\Delta n=0 \mathrm{DR}$ rate coefficients. See text for explanation.

that the iron $L$-shell $\mathrm{DR}$ rates be benchmarked over a range which encompasses the predicted temperature range.

To address the needs for modeling photoionized gases, we have initiated a series of experiments to measure the $\Delta n=0 \mathrm{DR}$ rates for the iron $L$-shell ions. Measurements are carried out using the heavy-ion Test Storage Ring (TSR) at the Max-Planck-Institut für Kernphysik in Heidelberg, Germany [9]. Here we present our results for $\Delta n=0$ DR of Fe XVIII [10]. In particular we have investigated the capture channels

$$
\mathrm{Fe}^{17+}\left(2 s^{2} 2 p^{5}\left[{ }^{2} P_{3 / 2}\right]\right)+e^{-} \rightarrow \begin{cases}\mathrm{Fe}^{16+}\left(2 s^{2} 2 p^{5}\left[{ }^{2} P_{1 / 2}\right] n l\right) & (n=18, \ldots, \infty) \\ \mathrm{Fe}^{16+}\left(2 s 2 p^{6}\left[{ }^{2} S_{1 / 2}\right] n l\right) & (n=6, \ldots, \infty) .\end{cases}
$$

The radiative stabilization of these autoionizing states to bound configurations of Fe XVII leads to DR resonances for collision energies between 0 and $132 \mathrm{eV}$.

Fig. 1 shows the measured Fe XVIII DR resonances. We have integrated the measured DR resonance strengths and energies with a Maxwellian electron velocity distribution to yield a total Fe XVIII $\Delta n=0$ DR rate coefficient as a function of electron temperature (Fig. 2, upper solid line). Various theoretical DR rates are also shown in Fig. 2. At $k_{B} T_{e} \sim 15 \mathrm{eV}$, near where Fe XVIII is predicted to peak in fractional abundance in photoionized gases, our inferred DR rate is a factor of 2 larger than the calculations of Ref. [11] (long dashed line), Ref. [12] (dotted line), and Ref. [13] (short dashed line). These theoretical rates all tend rapidly to zero at $k_{B} T_{e}<20 \mathrm{eV}$ because they have not included DR via $2 p_{1 / 2} \rightarrow 2 p_{3 / 2}$ core excitations. The calculations of Ref. [11] and Ref. [13] used LS-coupling and thus do not include fine-structure core excitations. The calculations of Ref. [12] used intermediate-coupling which can account for fine structure; but that work did not include the $2 p_{1 / 2} \rightarrow 2 p_{3 / 2}$ channel because the calculations were carried out for the high temperatures of collisionally ionized plasmas (where this channel is unimportant). Also shown in Fig. 2 are our new calculations [10] which include the $2 p_{1 / 2} \rightarrow 2 p_{3 / 2}$ channel (lower solid line). Our new calculations agree to within $\sim 30 \%$ with our measurements.

The commonly used models of photoionized gas incorporate DR rates which have been calculated using either the Burgess formula [14] or LS-coupling. The Burgess formula is known to be inappropriated for low temperatures [15]. And as we have shown, LS-coupling calculations do not properly account for all possible DR channels at low temperatures where DR can proceed 


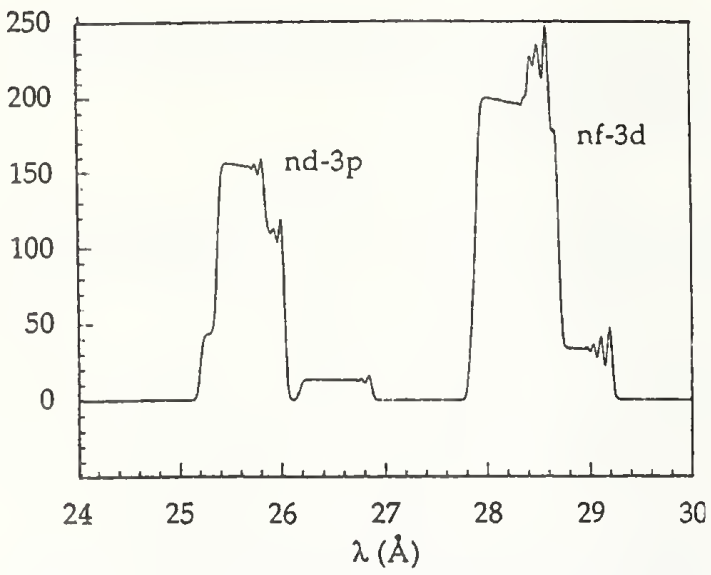

Figure 3: Preliminary calculations of the Fe XVII soft X-ray spectrum produced by Fe XVIII DR via $2 p_{1 / 2} \rightarrow 2 p_{3 / 2}$ core excitations. The intensity (ordinate) is in arbitrary units. The $n d \rightarrow 3 p$ and $n f \rightarrow 3 d$ transition arrays are shown for $18 \leq n \leq 100$.

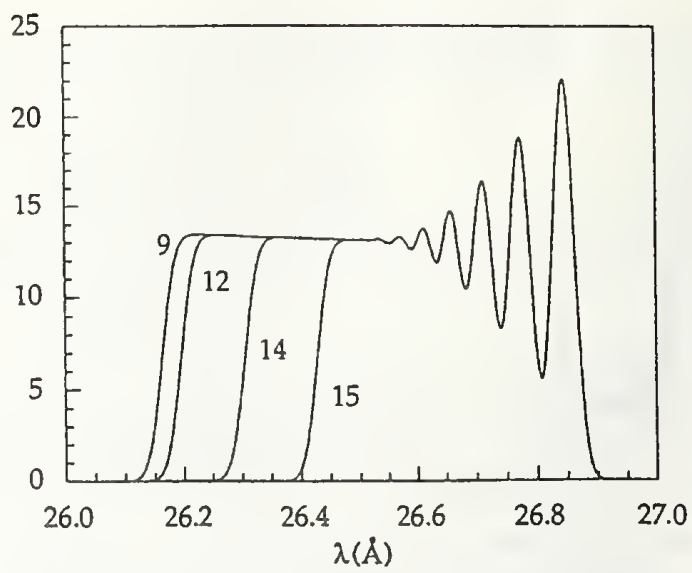

Figure 4: Preliminary calculations of the electron density dependence of the Fe XVII nd $\rightarrow 3 p$ transition array produced by Fe XVIII DR via $2 p_{1 / 2} \rightarrow 2 p_{3 / 2}$ core excitations. The transition array is shown for $\log n_{e}=9,12,14$, and $15 \mathrm{~cm}^{-3}$. The intensity (ordinate) is in arbitrary units.

primarily via $n l_{j} \rightarrow n l_{j^{\prime}}^{\prime}$ excitations of core electrons. A re-evaluation of the recommended low temperature DR rates is needed to assess for what $L$-shell and $M$-shell ions new rates must be determined.

$L$-shell iron $\Delta n=0 \mathrm{DR}$ via $2 s \rightarrow 2 p$ core excitations has been proposed as a possible electron temperature diagnostic for photoionized gas $[16,17]$. Here dielectronic capture proceeds via a $\left[2 s^{2} 2 p^{k}+\mathrm{e}^{-} \rightarrow 2 s 2 p^{k+1} n l\right]$ channel. Stabilization typically proceeds via a $2 p \rightarrow 2 s$ radiative decay. However, for low enough $n$, the $2 s^{-1}$ core can "freeze", and the outer electron radiatively cascade to the $L$-shell, filling the $2 s^{-1}$ hole. These cascades occur in the presence of an excited $2 p$ electron. In the low density limit, the resulting spectrum is unique to the DR process. Thus, as the temperature dependence of $\mathrm{RR}$ and $\mathrm{DR}$ is different, the ratio of lines produced by $\mathrm{RR}$ and $\mathrm{DR}$ provide the makings of a temperature diagnostic. Developing these diagnostics requires accurate knowledge of the DR resonance energies and strengths, which can be provided by measurements such as those presented here.

Here we propose a new electron temperature and density diagnostic for photoionized gas which is based on DR via a $2 p_{1 / 2} \rightarrow 2 p_{3 / 2}$ core excitation. Because the $2 p_{3 / 2} \rightarrow 2 p_{1 / 2}$ radiative transition is electric-dipole forbidden, DR via this channel stabilizes by a radiative decay of the captured electron. The energy of the $2 p_{1 / 2} \rightarrow 2 p_{3 / 2}$ transition is $\sim 15 \mathrm{eV}$, and the recombining electrons are captured into high $n$ levels. For Fe XVIII, we find $n \geq 18$. These high-lying electrons have a high branching ratio for undergoing an $n \rightarrow 3$ transition and producing soft X-rays. Fig. 3 shows a preliminary calculated soft X-ray spectrum resulting from Fe XVIII $\Delta n=0$ DR via $2 p_{1 / 2} \rightarrow 2 p_{3 / 2}$ core excitations. The resolution is $\Delta \lambda=0.07 \AA$ which is comparable to that planned for the spectrometers of AXAF and XMM. The broad features are due to unresolved $n \rightarrow 3$ transitions. The high energy sides of these transition arrays are due to very high $n$ levels. These weakly-bound levels can be collisionally ionized before they radiatively decay. The highest bound $n$ level is a function of the electron density $n_{e}$. Thus the widths of these transition arrays are sensitive to $n_{e}$ and can be used to infer the density of the radiating gas. Fig. 4 shows the density sensitivity for one of the $n d \rightarrow 3 p$ transition arrays shown in Fig. 3. Further experimental and theoretical work is in progress to explore this new class of temperature diagnostics. 


\section{Acknowledgments}

We thank M. Grieser, R. Repnow, and the TSR group for their expert technical support during the beam time. The authors thank T. R. Kallman, F. Paerels, J. C. Raymond, and D. A. Verner for stimulating conversations. This work was supported in part by NASA High Energy Astrophysics $\mathrm{X}$-Ray Astronomy Research and Analysis grant NAGW-4185. Travel to Heidelberg and living expenses for D. W. S. were supported by NATO Collaborative Research Grant CRG-950911. The experimental work has been supported in part by the German Federal Minister for Education, Science, Research, and Technology (BMBF) under Contract Nos. 06 GI 475, 06 GI 848, and 06 HD 854I. Work performed at Lawrence Livermore National Laboratory was performed under the auspices of the US Department of Energy (contract number W-7405-ENG-48).

\section{References}

[1] T. R. Kallman et al., Astrophys. J. 465, 994 (1996)

[2] M. Arnaud and R. Rothenflug, Astron. Astrophys. Suppl. Ser. 60, 425 (1985)

[3] M. Arnaud and J. Raymond, Astrophys. J. 398, 394 (1992)

[4] D. A. Liedahl et al., Astrophys. J. 350, L37 (1990)

[5] S. Hatchett, J. Buff, and R. McCray, Astrophys. J. 206, 847 (1976)

[6] Y. Tanaka, H. Inoue, and S. M. Holt, Publ. Astron. Soc. Japan 46, L37 (1994)

[7] L. Angelini et al., Astrophys. J.1 449, L41 (1995)

[8] D. A. Liedahl. and F. Paerels, Astrophys. J.1 468, L33 (1996)

[9] D. Habs et al., Nucl. Instrum. Methods B 43, 390 (1989)

[10] D. W. Savin et al., Astrophys. J. 489, L115 (1997)

[11] L. J. Roszman, Phys. Rev. A 35, 2138 (1987)

[12] M. H. Chen, Phys. Rev. A 38, 2332 (1988)

[13] Dasgupta and K. G. Whitney, Phys. Rev. A 42, 2640 (1990)

[14] Burgess, Astrophys. J. 141, 1588 (1965)

[15] P. J. Storey, in "Planetary Nebulae", ed. D. R. Flower, p. 199, D. Reidel Publishing Company, Dordrecht 1983

[16] D. A. Liedahl, Ph. D. Thesis, University of California at Berkeley 1992

[17] S. M. Kahn and D. A. Liedahl, in "Physics of Multiply Charged Ions", ed. D. Liesen, p. 169, Plenum Press: New York 1995 



\section{Data Production}

3.1 Atomic Structure and Spectroscopy 



\title{
The Platinum Group Ion Project
}

\author{
V. I. Azarov, S. S. Churilov, R. R. Kildiyarova, A. N. Ryabtsev ${ }^{1}$ \\ A. J. J. Raassen, P. H. M. Uylings ${ }^{2}$, Y. N. Joshi ${ }^{3}$, L. Tchang-Brillet ${ }^{4}$, J.-F. Wyart ${ }^{5}$ \\ ${ }^{1}$ Institute for Spectroscopy, Russian Academy of Sciences, \\ Troitsk, Moscow region 142092, Russia \\ ${ }^{2}$ Van der Waals-Zeeman Instituut, Universiteit van Amsterdam, \\ Valckenierstraat 65-67, 1018 XE Amsterdam, The Netherlands \\ ${ }^{3}$ Physics Department, St.Francis Xavier University, \\ Antigonish, NS, B2G 2W5, Canada \\ ${ }^{4}$ Departement Atomes et Molecules en Astrophysique, \\ Observatoire de Meudon, 92190 Meudon, France \\ ${ }^{5}$ Laboratoire Aime Cotton, CNRS II, bat. 505, \\ Centre Universitaire, 91405 Orsay, France
}

\section{Introduction}

Elements of the 5d-sequence show complex spectra and large relativistic effects, reflected in a strong mixing of levels and high values of the spin orbit interactions. Over the last decade many investigations focusing on the resonance transitions of $5 \mathrm{~d}$-elements were published [1]. One reason for these investigations is the increase of interest in 5d-elements in astrophysics after the observation of spectral lines of $5 \mathrm{~d}$-elements in chemically peculiar stars, such as $\chi$ Lupi and $\kappa$ Cancri [2]. Another reason is the development of sophisticated computational methods, such as the orthogonal operator approach [3], [4], [5] and the Multi Configuration Dirac Fock code [6]. These new computational methods allow the successful investigation of the complex systems of the $5 \mathrm{~d}$-sequence, thanks to far better values for initial estimates of the energy level values and transition probabilities. A third reason for exploring the 5d-elements is interest in relativistic effects that appear to be strong for these heavy elements. The orthogonal operator approach provides the tools to investigate these small, thus far neglected higher order correlation and relativistic effects quantitatively, as was recently showed for the $3 \mathrm{~d}$-elements [7]. To study these effects properly, however, many data from different elements in the $5 \mathrm{~d}$-sequence and from different stages of ionization are necessary.

Efforts of our team have resulted in many investigations of elements in the platinum group. The state of these analyses is shown in table 1. These analyses have resulted in experimental wavelengths and energy level values, as well as calculated transition probabilities. In most of the spectra indicated in table 1 about 400 lines have been identified. The high dispersive 10m normal incidence vacuum spectrograph in Meudon was used to obtain the spectrograms. This spectrograph is equipped with a $3600 \mathrm{l} / \mathrm{mm}$ grating, resulting in a plate factor of $0.26 \AA / \mathrm{mm}$. Other observational instruments are the $6.6 \mathrm{~m}$ normal incidence vacuum spectrograph at the Institute for Spectroscopy in Troitsk and the $3 \mathrm{~m}$ normal incidence spectrograph of the St. Francis Xavier University in Antigonish. Spectrograms have been measured with semi-automatic and fully-automatic comparators in Antigonish and Troitsk, respectively. The analyses were supported by the "package IDEN" [8], [9] developed in Troitsk. 


\section{Present Investigations}

The main objective of the project presented here is the extension of the analyses, focussed in the past on spectra of doubly ionized elements of the platinum group. These lower stages of ionization are more interesting from the astrophysical point of view, but they are also a challenge due to the complexity of these systems. The lower stages of ionization are dominated by strong correlation effects. For this reason the energy matrix has to be extended to $\left(5 \mathrm{~d}^{N}+5 \mathrm{~d}^{N-1} 6 \mathrm{~s}\right.$ $\left.+5 \mathrm{~d}^{N-2} 6 \mathrm{~s}^{2}\right)$ for the even system and to $\left(5 \mathrm{~d}^{N-1} 6 \mathrm{p}+5 \mathrm{~d}^{N-2} 6 \mathrm{~s} 6 \mathrm{p}+5 \mathrm{~d}^{N-3} 6 \mathrm{~s}^{2} 6 \mathrm{p}\right)$ for the odd system. This extension of the basis will result in complicated calculations of energy level values and transition probabilities using orthogonal operators along the lines presented in [5]. The transition probabilities, in combination with the wavelengths calculated from the experimental energy level values, provide a large amount of valuable data.

Another result from these efforts is the possibility to quantize higher order magnetic and electrostatic effects as was done in the 3d-elements [7]. To undertake these studies a large amount of data is necessary together with a good knowledge of interacting configurations. For the higher stages of ionization the latter is not that relevant. We show an example of the consistency of small, thus far neglected 3-particle electrostatic higher order effects, observed in Os V and Au V [10] (see table 2). Here we have chosen a comparison between two iso-ionic spectra. Iso-ionic comparisons are far more relevant since they are less sensitive to the reproduction of errors, as is the case for iso-electronic comparisons. The example given for four times ionized platinurn group elements shows the stability of our computational method in providing reliable estimates for starting the analyses of unknown systems of doubly ionized 5d-elements. For the strong 2-particle electrostatic interactions and the 1-particle magnetic interactions this stability was already known for years, but the stability of the small higher order effects in the orthogonal operator approach offers the possibility to extrapolate even these small effects to unknown spectra and to obtain good predictions, necessary to investigate the complex systems successfully.

\section{References}

[1] A. J. J. Raassen, V. I. Azarov, P. H. M. Uylings, Y. N. Joshi, L. Tchang-Brillet, and A. B. Ryabtsev, Phys. Scr. 54, 56 (1996)

[2] D. S. Leckrone, G. M. Wahlgren, S. G. and Johansson, Astrophys. J. 377, L37 (1991)

[3] A. J. J. Raassen and P. H. M. Uylings, Phys. Scr. T65, 84 (1996)

[4] P. H. M. Uylings and A. J. J. Raassen, Phys. Scr. 54, 505 (1996)

[5] P. H. M. Uylings, A. J. J. Raassen, and J.-F. Wyart, J. Phys. B.: At. Mol. Opt. Phys. 26, 4683 (1993)

[6] F. A. Parpia, C. Froese-Fischer, and I. P. Grant, Comput.Phys.Commun. 94, 249 (1996)

[7] J. E. Hansen, B. R. Judd, A. J. J. Raassen, and P. H. M. Uylings, Phys. Rev. Lett. 78, 3078 (1997)

[8] V. I. Azarov, Phys. Scr. 44, 528 (1991)

[9] V. I. Azarov, Phys. Scr. 48, 656 (1993)

[10] A. J. J. Raassen, R. Gayasov, and Y. N. Joshi, Phys. Scr., submitted 
Table 1: State of knowledge of platinum group ion spectra.

\begin{tabular}{|c|c|c|c|c|c|c|}
\hline & $\begin{array}{l}W \\
74\end{array}$ & $\mathbb{R}_{5}$ & $\begin{array}{c}\text { Os } \\
76\end{array}$ & $\frac{\operatorname{Ir}}{77}$ & $\begin{array}{l}\mathrm{Pt} \\
78\end{array}$ & $\underset{79}{A \mathbf{u}}$ \\
\hline & $5 d^{6}$ & $5 d^{7}$ & $5 d^{8}$ & $5 d^{9}$ & $5 d^{10}$ & \\
\hline & $5 d^{5}$ & $5 d^{6}$ & $5 d^{7}$ & $5 d^{8}$ & $5 \mathrm{~d}^{9}$ & $5 d^{10}$ \\
\hline & $5 d^{4}$ & $5 d^{5}$ & $5 d^{6}$ & $5 d^{7}$ & $5 d^{8}$ & $5 d^{9}$ \\
\hline & $5 d^{3}$ & $5 d^{4}$ & $5 d^{5}$ & $5 d^{6}$ & $5 d^{7}$ & $5 d^{8}$ \\
\hline & $5 d^{2}$ & $5 \mathrm{~d}^{3}$ & $5 d^{4}$ & $5 d^{5}$ & $5 d^{6}$ & $5 \mathrm{~d}^{7}$ \\
\hline & $5 d^{1}$ & $5 d^{2}$ & $5 d^{3}$ & $5 d^{4}$ & $5 d^{5}$ & $5 d^{6}$ \\
\hline & & $5 \mathrm{~d}^{1}$ & $5 d^{2}$ & $5 d^{3}$ & $5 \mathrm{~d}^{4}$ & $5 d^{5}$ \\
\hline II & & & $5 d^{1}$ & $5 d^{2}$ & $5 \mathrm{~d}^{3}$ & $5 d^{4}$ \\
\hline $\mathbf{I X}$ & & & & $5 d^{1}$ & $5 d^{2}$ & $5 d^{3}$ \\
\hline X & & & & & $5 d^{1}$ & $5 d^{2}$ \\
\hline
\end{tabular}

$5 \mathrm{~d}^{N}$ : unknown

$5 \mathrm{~d}^{N}$ : known in past and from others

$5 \mathrm{~d}^{N}$ : in progress in our team

$5 \mathrm{~d}^{N}$ : finished in our team

Table 2: Comparison between the 3-particle electrostatic ddp-type parameters in Os V and Au V

\begin{tabular}{lrr} 
Parameter & Os $\mathrm{V}$ & $\mathrm{Au} \mathrm{V}$ \\
\hline $\mathrm{T}_{16}$ & -25.4 & -25.2 \\
$\mathrm{~T}_{17}$ & 14.5 & 10.5 \\
$\mathrm{~T}_{18}$ & -18.2 & -16.1 \\
$\mathrm{~T}_{19}$ & -15.6 & -10.0 \\
$\mathrm{~T}_{20}$ & -38.2 & -47.1 \\
$\mathrm{~T}_{21}$ & -11.5 & -19.4 \\
$\mathrm{~T}_{22}$ & -48.9 & -15.1 \\
$\mathrm{~T}_{23}$ & -12.7 & -3.2 \\
$\mathrm{~T}_{24}$ & -15.1 & -17.7 \\
$\mathrm{~T}_{25}$ & 7.9 & 5.1 \\
$\mathrm{~T}_{26}$ & -41.6 & -33.5 \\
$\mathrm{~T}_{27}$ & 14.2 & 1.7 \\
$\mathrm{~T}_{28}$ & 59.0 & 44.6 \\
$\mathrm{~T}_{29}$ & -30.1 & -20.5 \\
$\mathrm{~T}_{30}$ & 8.0 & 29.6 \\
$\mathrm{~T}_{31}$ & -5.9 & -17.7 \\
$\mathrm{~T}_{32}$ & -11.0 & 2.6 \\
$\mathrm{~T}_{33}$ & 14.5 & 7.2 \\
$\mathrm{~T}_{34}$ & -40.8 & -46.1 \\
$\mathrm{~T}_{35}$ & -64.0 & -65.5
\end{tabular}




\title{
An Integrated Approach to Bound and Continuum States: Application to Beryllium-like Ions
}

\author{
Keith A. Berrington and John C. Pelan \\ Department of Applied Mathematics and Theoretical Physics, \\ Queen's University, Belfast BT7 1NN, U.K.
}

Modern research in the theory of atomic physics is characterised by a desire to understand the collective behavior of charged particles and their response to added energy or radiation, from quantum-mechanical first principles ( $a b$ initio); the concomitant aim being the quantitative prediction of atomic properties and the establishment of standards for areas of science and technology where atoms play a role - whether in laboratory plasmas or in environments such as astrophysics.

Because of the implied $a b$ initio approach, the theme of much recent research is the incorporation of more effects into the wavefunction: the unification of processes formerly treated as independent. So atomic properties associated with bound and continuum states, electron impact excitation and ionization cross sections, photon excitation and ionization, and fine-structure levels, can be calculated in an integrated way. [1]

Resonances are perhaps the most obvious manifestation of interference effects in quantum mechanics. One of the most widely used, and perhaps most fully developed, of the sophisticated models available to atomic physics is the R-matrix approach [2]. This model was first introduced in nuclear physics specifically to study resonance reactions[3], the idea being that a resonance could be described in terms of the colliding particles forming a compound state, the total wavefunction being expanded as a complete set of these states. The complicated interaction zone, the internal region of configuration space, is delineated by a sphere of radius $r=a$ around the centre of mass, with the external region reducing to a simpler two-body problem. These ideas were developed for atomic physics by Burke and others and encapsulated in a computer program package for electron scattering and photoionization from atoms and ions [4].

It may seem suprising for a method originating in the study of resonances and continuum processes, but R-matrix techniques - and indeed any multiconfigurational close-coupling approach - can be applied to the bound state problem with great success.

This was exploited in the drive for quality data for stellar opacity applications by Seaton and others [5], who extended the R-matrix method and programs to photoabsorption processes relevant to stellar envelope opacities, in particular for bound-bound and photoionization transitions. The Opacity Project (OP) provided an important data base for atomic physics, the TOPBASE facility at the Centre de Donnèes Astronomiques de Strasbourg [6].

Within this paradigm, we are now concerned to explore the potential of close-coupling ab initio methods for high precision atomic structure calculations. Particularly for excited and continuum states, such methods should eventually be superior to atomic structure methods which use the same functional basis from $r=0$ to $r=\infty$. Recent developments are again based on the R-matrix model, within the high optimization offered by close-coupling. These include exploitation of the 'QB' method[7] for systematic resonance analysis, use of target 'core base states' and pseudo-states for accurate representation of inner-shell and continuum processes, and incorporation of radiative and relativistic effects via the Opacity Project codes together with terms of the Breit-Pauli Hamiltonian. 
Table 1: Comparison of $2 s^{2}-2 s 2 p$ gf-values for $\mathrm{C}^{++} J=0^{\mathrm{e}}-1^{\circ}$, taken from the recent R-matrix calculation of all bound and autoionizing fine-structure energy levels to $n=10$ [10], with other theories (OP [5], CIV3 [12] and MCHF [13]) and experiments.

\begin{tabular}{|llll|}
\hline Transition & Present[10] & NIST[8] & Other \\
\hline $2 s^{2}-2 s 2 p^{3} P_{1}^{\circ}$ & $1.93 \mathrm{E}-7$ & $1.87 \mathrm{E}-7$ & $1.89 \pm 0.07 \mathrm{E}-7[12], 1.87 \pm 0.002 \mathrm{E}-7[9]$ \\
$2 s^{2}-2 s 2 p^{1} P_{1}^{\circ}$ & 0.761 & 0.759 & $0.780[5], 0.757[13], 0.754 \pm 0.014[11]$ \\
\hline
\end{tabular}

Oscillator strengths for all ions of $\mathrm{C}, \mathrm{N}$ and $\mathrm{O}$ were subject to a review and assessment by Wiese et al in 1996 [8]. This review, together with a more recent lifetime measurement on $\mathrm{C}^{++}$ using the heavy ion storage ring at Heidelberg [9], provide a sensitive test of theory. Using the theoretical techniques described above, a complete set of data has just been calculated [10] for finestructure energy levels for bound and autoionizing states of the form $1 s^{2} 2 s n l$ and $1 s^{2} 2 p n l\left(J=0^{\mathrm{e}}\right.$ and $J=1^{\circ}$ ) up to $n=10$ for the Be-like ion $\mathrm{C}^{++}$, together with electric dipole oscillator strengths whose accuracy is estimate to be within $\sim 3 \%$ by comparison with experiment (table 1 ). Work is progressing on other ions, for example $\mathrm{Ne}, \mathrm{Ar}, \mathrm{P}$ and $\mathrm{Cl}$, to upgrade the $\mathrm{OP}$ database and to extend it to fine-structure transitions.

In a higher energy regime, similar theoretical techniques can be used to quantify so-called 'hollow atom' effects. Although the methods are developed for complex atoms, consider the four-body problem in atomic physics, which is the simplest system to show hollow atom interference between direct and indirect ionization involving excitation-autoionization and triply excited intermediate states. In the particular case of hollow lithium, this occurs when both $\mathrm{K}$ electrons from the ground state configuration become excited into the L shell during a collision, and there has been much interest in the properties of this system. In a recent application of the R-matrix and QB method (described above), hollow atom interference effects and resonances in Li have been examined and quantified. Outer-shell and inner-shell effects, excitation and ionization channels, direct and indirect ionization processes, resonant and non-resonant behavior are combined for photon and electron impact processes. This includes resonance analysis (table 2), electron impact ionization of ground state $\mathrm{Li}^{+}$and photoionization of $\mathrm{Li}$ (table 3 ), electron excitation and ionization of metastable states and double photoionization. Good agreement with experiment where available is obtained, with a tabulation of all $4802 l 2 l^{\prime} n l^{\prime \prime}$ states for $n \leq 9, l^{\prime \prime} \leq 3$, and a demonstration of the chaotic behavior of overlapping series [14].

These bound and continuum state techniques are in progress to being extended to more complex atoms, particularly for atoms (eg. C, O, Ne, Mg etc.) where there is current interest in the dynamics of atoms and ions at X-ray energies in astrophysical and technology applications.

\section{References}

[1] K. A. Berrington, J. C. Pelan, and L. Quigley, J. Phys. B 30, 4973 (1997)

[2] P. G. Burke and K. A. Berrington (eds), "Atomic and Molecular Processes: An R-matrix Approach", Institute of Physics Publishing 1993, ISBN 0-7503-0199-6

[3] Wigner, Phys. Rev. 70 15, 606 (1946)

[4] K. A. Berrington, W. B. Eissner, and P. H. Norrington (1995)

[5] The Opacity Project, Institute of Physics Publishing 1995, ISBN 0750302887 
Table 2: Li resonances coupled to $2 s^{2}{ }^{1} S$ : effective $n$ and width ( $\Gamma$ meV). Identifications (ID) assume a $2 s^{2}$ core, except those labelled ${ }^{3} P(2 s 2 p)$ and $Q \equiv 2 s 2 p^{2}$.

\begin{tabular}{|c|c|c|c|c|c|c|c|c|c|c|c|}
\hline ID & $n_{1} S^{\mathrm{e}}$ & $\Gamma$ & ID & $n_{1_{S}}$ & $\Gamma$ & ID & $n_{1} S^{e}$ & $\Gamma$ & ID & $n_{1} S^{\mathrm{e}}$ & $\Gamma$ \\
\hline & ${ }^{2} \mathbf{S}^{\mathrm{e}}$ & & & ${ }^{2} \mathbf{P}^{0}$ & & \multicolumn{3}{|c|}{${ }^{2} \mathrm{D}^{\mathrm{e}}$} & \multicolumn{3}{|c|}{${ }^{2} \mathrm{~F}^{\mathrm{O}}$} \\
\hline$Q$ & 1.664 & 82 & $2 p$ & 1.215 & 123 & $Q$ & 1.425 & 98 & $4 f$ & 3.985 & 0.9 \\
\hline $3 s$ & 2.243 & 141 & $2 p^{3}$ & 2.239 & 55 & $3 d$ & 2.968 & 97 & ${ }^{3} P 3 d$ & 4.490 & 4.8 \\
\hline $4 s$ & 3.181 & 22 & $3 p$ & 2.430 & 65 & ${ }^{3} P 3 p$ & 3.244 & 17 & $5 f$ & 4.981 & 0.5 \\
\hline${ }^{3} P 3 p$ & 3.677 & 8.5 & ${ }^{3} P 3 s$ & 2.860 & 19 & $4 d$ & 3.944 & 23 & $6 f$ & 5.980 & 0.4 \\
\hline $5 s$ & 4.228 & 8.2 & $4 p$ & 3.471 & 18 & $5 d$ & 4.924 & 8.1 & $7 f$ & 6.979 & 0.2 \\
\hline $6 s$ & 5.212 & 5.0 & $5 p$ & 4.440 & 6.9 & $6 d$ & 5.911 & 3.8 & $8 f$ & 7.978 & 0.2 \\
\hline $7 s$ & 6.205 & 3.1 & ${ }^{3} P 3 d$ & 4.798 & 5.7 & $7 d$ & 6.888 & 2.1 & $9 f$ & 8.977 & 0.1 \\
\hline $8 s$ & 7.198 & 2.1 & $6 p$ & 5.387 & 3.2 & ${ }^{3} P 4 p$ & 7.561 & 4.9 & & & \\
\hline $9 s$ & 8.187 & 1.5 & ${ }^{3} P 4 s$ & 5.958 & 3.9 & $8 d$ & 7.976 & 2.3 & & & \\
\hline${ }^{3} P 4 p$ & 9.971 & 1.9 & $7 p$ & 6.560 & 2.8 & $9 d$ & 8.933 & 1.1 & & & \\
\hline & & & $8 p$ & 7.506 & 1.5 & & & & & & \\
\hline & & & $9 p$ & 8.490 & 1.0 & & & & & & \\
\hline
\end{tabular}

Table 3: Total ionization cross sections $\left(10^{-18} \mathrm{~cm}^{2}\right)$ for $\mathrm{e}^{-}+\mathrm{Li}^{+} 1 s^{2}$ and $\gamma+\mathrm{Li} 1 s^{2} 2 s$, as a function of $\mathrm{e}^{-}$energy $(\mathrm{eV})$ relative to $\mathrm{Li} 1 \mathrm{~s}^{2}$.

\begin{tabular}{|l|ccccccccc|}
\hline $\mathrm{e}^{-}(\mathrm{eV}):$ & 146.13 & 147.00 & 147.02 & 147.14 & 147.20 & 147.33 & 147.43 & 150.33 & 153.93 \\
\hline $\mathrm{e}^{-}+\mathrm{Li}^{+}$ & 2.918 & 2.962 & 2.962 & 2.973 & 2.977 & 2.975 & 2.980 & 3.097 & 3.244 \\
$\gamma+\mathrm{Li}$ & 0.603 & 0.928 & 0.962 & 0.508 & 0.523 & 0.606 & 0.533 & 0.540 & 0.512 \\
\hline
\end{tabular}

[6] W. Cunto, C. Mendoza, F. Ochsenbein, and C. J. Zeippen, A\&SA 275 L5 (1993)

[7] L. Quigley and K. A. Berrington, J. Phys. B 29, 4529 (1996)

[8] W. L. Wiese, J. R. Fuhr, and T. M. Deters, "Atomic Transition Probabilites of C, N and O", J. Phys. Chem. Ref. Data Monograph No. 7, (AIP) ISBN 1-56396-602-6 (1996)

[9] J. Doerfert, E. Träbert, A. Wolf, D. Schwalm, and O. Uwira, Phys. Rev. Lett. 78, 4355 (1997)

[10] K. A. Berrington, J. C. Pelan, and L. Quigley, Phys. Scr., in press (1998)

[11] N. Reistad, R. Hutton, A. E. Nilsson, I. Martinson, and S. Mannervik, Phys. Scr. 34, 151 (1986)

[12] J. Fleming, A. Hibbert, and R. P. Stafford, Phys. Scr. 49, 316 (1994)

[13] J. Fleming, N. Vaeck, A. Hibbert, K. L. Bell, and M. R. Godefroid, Phys. Scr. 53, 446 (1996)

[14] K. A. Berrington and S. Nakazaki, J. Phys. B 30, in press (1997) 


\title{
Efficient Method to Account for Correlation Effects when Generating Atomic Parameters for Complex Atoms
}

\author{
P. Bogdanovich, G. Gaigalas and Z. Rudzikas \\ State Institute of Theoretical Physics and Astronomy, \\ A. Gostauto 12, 2600 Vilnius, Lithuania
}

\section{Introduction}

The potential of a simplified method of superposition of configurations for studying the energy spectra of atoms and ions has been discussed in some earlier papers[1-4]. In this method a part of admixed configurations, obtained mainly by one-electron excitations, is taken into account directly when calculating the multi-configurational energy matrix, whereas the correlation corrections, connected with the majority of configurations, obtained by virtual two-electron excitations, are accounted for in second-order perturbation theory as corrections to Slater integrals and similar quantities [4]. Such an approach allows us to take into consideration a fairly large number of admixed configurations, preserving a comparatively small order of the matrix to be diagonalized. This is of extreme importance for calculations of energy spectra of complex configurations.

The possibility to approximately account for a large number of admixed configurations (in the second order of perturbation theory) has allowed us to avoid the use of solutions of multiconfigurational equations and to employ the so-called transformed radial orbitals, obtained from Hartree-Fock functions of the configuration considered, with the help of simple algebraic multipliers of the type of various positive degrees of a radial variable. The main advantage of this approach is the exceptional simplicity of the generation of the basis combined with its fairly high efficiency. Recently a similar approach has been also applied to the relativistic Dirac-Hartree-Fock approximation [5,6].

This paper is aimed at exploring the possibility to apply such a method for calculations of electronic transitions in atoms and ions. We expect a fairly high efficiency because of the following facts:

1) The transformed radial orbitals are fairly useful for calculations of matrix elements of the operators of electronic transitions [7].

2) It follows from the above-mentioned publications that the approach under consideration allows the determination of fairly accurate wavelengths. This is rather important when calculating the majority of characteristics of electronic transitions.

3) The high accuracy of the energy levels of a configuration leads to sufficiently accurate weights of the wave functions of a pure coupling scheme, obtained after diagonalization of the energy matrix. This also contributes to an increase in the accuracy of the matrix elements of the electronic transitions.

4) The approach used allows us to include in the multi-configurational wave function practically all necessary configurations - both giving the principal contributions to the expansion as well as the most important ones for the electronic transition considered. 


\section{Results of the Calculation of the Transition $2 \mathrm{~s}^{2} 2 \mathrm{p}^{2}-2 \mathrm{~s} 2 \mathrm{p}^{3}$ in OIII}

Let us consider the electronic transitions $2 \mathrm{~s}^{2} 2 \mathrm{p}^{2}-2 \mathrm{~s} 2 \mathrm{p}^{3}$ in OIII. These transitions were studied by many authors, therefore we can compare our results with fairly accurate theoretical and experimental data. Moreover, the role of correlation effects is very important for these configurations. Therefore, it will be possible to estimate how accurately we are taking them into account.

The transformed radial orbitals were defined as follows:

$$
P_{T R}\left(n^{\prime} l^{\prime} \mid r\right)=N \cdot r^{k} \cdot P_{H F}(n l \mid r),
$$

( $N$ is the normalizing factor). They are orthogonalized to all Hartree-Fock and transformed functions already included in the basis used. The values of $n^{\prime} l^{\prime}, k$ and $n l$ are presented in Table 1.

Table 1: Parameters of the transformed radial orbitals used.

\begin{tabular}{|r|r|r|r|r|r|r|r|r|r|r|r|r|r|r|}
\hline$n^{\prime} \boldsymbol{l}^{\prime}$ & $\boldsymbol{k}$ & $\boldsymbol{n} \boldsymbol{l}$ & $\boldsymbol{n}^{\prime} \boldsymbol{l}^{\prime}$ & $\boldsymbol{k}$ & $\boldsymbol{n} \boldsymbol{l}$ & $\boldsymbol{n}^{\prime} \boldsymbol{l}^{\prime}$ & $\boldsymbol{k}$ & $\boldsymbol{n} \boldsymbol{l}$ & $\boldsymbol{n}^{\prime} \boldsymbol{l}^{\prime}$ & $\boldsymbol{k}$ & $\boldsymbol{n} \boldsymbol{l}$ & $\boldsymbol{n}^{\prime} \boldsymbol{l}^{\prime}$ & $\boldsymbol{k}$ & $\boldsymbol{n} \boldsymbol{l}$ \\
\hline$\underline{5 \mathrm{~s}}$ & 1 & $2 \mathrm{~s}$ & $5 \mathrm{p}$ & 1 & $2 \mathrm{~s}$ & $5 \underline{5 \mathrm{~d}}$ & 2 & $2 \mathrm{~s}$ & $5 \mathrm{f}$ & 3 & $2 \mathrm{~s}$ & $5 \mathrm{~g}$ & 4 & $2 \mathrm{~s}$ \\
\hline$\underline{6 \mathrm{~s}}$ & 1 & $2 \mathrm{p}$ & $6 \mathrm{p}$ & 1 & $2 \mathrm{p}$ & $\underline{6 \mathrm{~d}}$ & 1 & $2 \mathrm{p}$ & $6 \mathrm{f}$ & 2 & $2 \mathrm{p}$ & $6 \mathrm{~g}$ & 3 & $2 \mathrm{p}$ \\
\hline $6 \mathrm{~h}$ & 3 & $2 \mathrm{p}$ & $7 \mathrm{~s}$ & 2 & $2 \mathrm{~s}$ & $7 \mathrm{p}$ & 2 & $2 \mathrm{~s}$ & $7 \mathrm{~d}$ & 3 & $2 \mathrm{~s}$ & $7 \mathrm{f}$ & 4 & $2 \mathrm{~s}$ \\
\hline $7 \mathrm{~g}$ & 5 & $2 \mathrm{~s}$ & $8 \mathrm{~s}$ & 2 & $2 \mathrm{p}$ & $8 \mathrm{p}$ & 2 & $2 \mathrm{p}$ & $8 \mathrm{~d}$ & 2 & $2 \mathrm{p}$ & $8 \mathrm{f}$ & 3 & $2 \mathrm{p}$ \\
\hline $8 \mathrm{~g}$ & 4 & $2 \mathrm{p}$ & $8 \mathrm{~h}$ & 5 & $2 \mathrm{p}$ & $9 \mathrm{~s}$ & 1 & $1 \mathrm{~s}$ & $9 \mathrm{p}$ & 1 & $1 \mathrm{~s}$ & $9 \mathrm{~d}$ & 2 & $1 \mathrm{~s}$ \\
\hline
\end{tabular}

The quantum numbers of the radial orbitals which according to [7] play the main role while refining the matrix elements of the transition operator are underlined. In this approach the principal quantum numbers $n$ have no physical meanings and they have nothing to do with the numbers of nodes of the radial orbital considered. Their values starting with $n=5$ were used.

While calculating the energy matrix and, correspondingly, multi-configurational wave function (as in [4] for calculations of the energy spectra), the fairly important quasidegenerate configuration $2 \mathrm{p}^{4}$ was admixed to $2 \mathrm{~s}^{2} 2 \mathrm{p}^{2}$, as well as all configurations obtained from these two by one-electron excitations. Moreover, all configurations necessary to improve the matrix element of the transition operator and obtained from the configuration $2 \mathrm{~s} 2 \mathrm{p}^{3}$ by one electron excitations in states of opposite parity [7] were automatically included in the matrix. The contributions of the remaining possible admixed configurations were taken into consideration in second order perturbation theory in terms of the corrections to the radial integrals of electrostatic interactions and similar quantities. The configuration $2 s 2 p^{3}$ has no configurations of the same parity which are quasidegenerate to it. When improving it, all possible one-electron admixed configurations were taken into account to form the energy matrix as well as the configurations obtained from $2 \mathrm{~s}^{2} 2 \mathrm{p}^{2}$ and, due to strong mixing, from $2 \mathrm{p}^{4}$ by one-electron excitations in the states of the opposite parity [7]: $2 \mathrm{~s}^{2} 2 \mathrm{pnis} ; 2 \mathrm{~s}^{2} 2 \mathrm{pnid}$. It is necessary to underline that according to the above-mentioned list, configurations of the kind $2 p^{3} n_{i}$ and $2 p^{3} n_{i d}$ must be included. However, they already appeared earlier as the result of one-electron excitation of the configuration considered.

The results obtained for some transitions are presented in Table 2. It contains the experimental wavelengths and the oscillator strengths ( $g f$ ) of electric dipole transitions, calculated in the single-configuration Hartree-Fock (HF) approximation and with the method of simplified superposition of configurations (SSC) as well as calculated with the usual superposition of configurations approach with the same radial orbitals accounting for a complete set of possible admixed configurations ( $\mathrm{SC}$ ). In the latter case the order of the matrix to be calculated and 
diagonalized increases many times. The table also contains recommended data on oscillator strengths [8] as well as the results of multi-configuration calculations [9]. Our results are presented for both the "length" (L) and "velocity" (V) forms of the transition operator. Moreover, the upper lines contain ab initio values whereas the lower ones contain data obtained using experimental energy values.

As seen from Table 2, the results obtained in the SSC and SC approaches differ insignificantly, especially for the case of the "length" form of transition operator. They are very close to the data found using more elaborate methods [8,9] and are not significantly improved when using experimental energy values. As was expected, the largest discrepancies occur when calculating intercombination transitions. The SSC data, obtained for the "velocity" form of the transition operator, are slightly less accurate. This may be an indication that the basis of radial orbitals used is not sufficiently complete while using this operator.

\section{Conclusion}

The numerical data presented indicate that the method of simplified superposition of configurations (SSC), in which only part of the admixed configurations is directly taken into account when calculating the energy matrix, whereas the majority of admixed configurations, obtained by two-electron virtual excitations, are accounted for in second-order perturbation theory, is fairly efficient not only for calculations of the energy spectra, but also for studies of electronic transitions. This method may be easily applied to study complex electronic configurations, having several open shells, and, thus, may efficiently contribute to the generation of accurate atomic data for relevant databases..

\section{References}

[1] P. Bogdanovich, G. Zukauskas, Sov. Phys. Collection 23, No. 3, 13-26 (1983)

[2] P. Bogdanovich, G. Zukauskas, S. Sadziuviene, Sov. Phys. Collection 24, No. 3, $27-37$ (1984)

[3] P. O . Bogdanovich et all, Izvestija vuzov SSSR. Fizika 33, 6-17 (1990)

[4] P. Bogdanovich,G. Gaigalas,A. Momkauskaite, Z. Rudzikas, Physica Scripta 56, 230-239 (1997)

[5] V. A. Dziuba, V. V. Flambaum, M. G. Kozlov, 28th EGAS, Abstracts, 62, 1996

[6] V. A. Dziuba, V. V. Flambaum, M. G. Kozlov, Phys. Rev. A 54, 3948-3959 (1996)

[7] P. Bogdanovich, Lithuanian Physics Journal 31, 79-83 (1991)

[8] W. L. Wiese, J. R. Fuhr, T. M. Deters, J. Phys. Chem. Ref. Data, Monograph 7, (1996)

[9] H. Nussbaumer, P. J. Storey, Astron. Astrophys. 99, 177-181 (1981) 
Table 2: Oscillator strengths ( $g$ f) for some electric dipole transitions in OIII.

\begin{tabular}{|c|c|c|c|c|c|c|c|}
\hline LSJ-L'S'J' J' & $\lambda_{\exp }(\mathrm{A})$ & & $\mathrm{HF}$ & $\mathrm{SSC}$ & $\mathrm{SC}$ & [8] & [9] \\
\hline \multirow[t]{3}{*}{${ }^{3} \mathrm{P}_{2}-{ }^{3} \mathrm{D}_{3}$} & \multirow[t]{3}{*}{835.289} & $\mathrm{~L}$ & 0.841 & 0.431 & 0.456 & \multirow[t]{3}{*}{0.438} & \multirow[t]{3}{*}{0.44} \\
\hline & & & 0.856 & 0.433 & 0.451 & & \\
\hline & & V & & $\begin{array}{l}0.492 \\
0.489\end{array}$ & $\begin{array}{l}0.464 \\
0.469\end{array}$ & & \\
\hline \multirow[t]{4}{*}{${ }^{3} \mathrm{P}_{0^{-}}{ }^{3} \mathrm{D}_{1}$} & \multirow[t]{4}{*}{832.929} & $\mathrm{~L}$ & 0.203 & 0.104 & 0.110 & \multirow[t]{4}{*}{0.106} & \multirow[t]{4}{*}{11.160} \\
\hline & & & 0.207 & 0.105 & 0.109 & & \\
\hline & & V & & 0.118 & 0.112 & & \\
\hline & & & & 0.118 & 0.114 & & \\
\hline \multirow{4}{*}{${ }^{3} \mathrm{P}_{2-}{ }^{3} \mathrm{P}_{2}$} & \multirow[t]{4}{*}{703.854} & $\mathrm{~L}$ & 0.541 & 0.499 & 0.524 & \multirow{4}{*}{0.510} & \multirow{4}{*}{0.505} \\
\hline & & & 0.550 & 0.499 & 0.516 & & \\
\hline & & V & & 0.515 & 0.522 & & \\
\hline & & & & 0.516 & 0.530 & & \\
\hline \multirow[t]{4}{*}{${ }^{3} \mathrm{P}_{2}{ }^{-3} \mathrm{~S}_{1}$} & \multirow[t]{4}{*}{508.178} & $\mathrm{~L}$ & 1.392 & 0.906 & 0.945 & \multirow[t]{4}{*}{0.935} & \multirow[t]{4}{*}{0.925} \\
\hline & & & 1.343 & 0.918 & 0.932 & & \\
\hline & & V & & 1.014 & 0.976 & & \\
\hline & & & & 1.002 & 0.990 & & \\
\hline \multirow[t]{4}{*}{${ }^{3} \mathrm{P}_{0}{ }^{-3} \mathrm{~S}_{1}$} & \multirow[t]{4}{*}{597.838} & $\mathrm{~L}$ & 0.277 & 0.180 & 0.188 & \multirow[t]{4}{*}{0.186} & \multirow[t]{4}{*}{0.185} \\
\hline & & & 0.268 & 0.183 & 0.186 & & \\
\hline & & V & & 0.202 & 0.194 & & \\
\hline & & & & 0.199 & 0.197 & & \\
\hline \multirow[t]{4}{*}{${ }^{1} \mathrm{D}_{2-}{ }^{1} \mathrm{D}_{2}$} & \multirow[t]{4}{*}{599.590} & $\mathrm{~L}$ & 2.682 & 1.414 & 1.485 & \multirow[t]{4}{*}{1.455} & \multirow[t]{4}{*}{1.460} \\
\hline & & & 2.557 & 1.438 & 1.469 & & \\
\hline & & V & & 1.674 & 1.557 & & \\
\hline & & & & 1.647 & 1.574 & & \\
\hline \multirow[t]{4}{*}{${ }^{1} \mathrm{D}_{2}{ }^{-1} \mathrm{P}_{1}$} & 525.794 & $\mathrm{~L}$ & 1.008 & 1.158 & 1.209 & 1.195 & 1.160 \\
\hline & & & 0.972 & 1.163 & 1.188 & & \\
\hline & & $\mathrm{V}$ & & 1.199 & 1.225 & & \\
\hline & & & & 1.194 & 1.246 & & \\
\hline${ }^{1} \mathrm{~S}_{0-}{ }^{1} \mathrm{P}_{1}$ & 597.814 & $\mathrm{~L}$ & 0.668 & 0.241 & 0.246 & 0.240 & 0.250 \\
\hline & & & 0.684 & 0.242 & 0.244 & & \\
\hline & & V & & 0.312 & 0.269 & & \\
\hline & & & & 0.311 & 0.272 & & \\
\hline${ }^{3} \mathrm{P}_{2}-{ }^{1} \mathrm{D}_{2}$ & 535.481 & $\mathrm{~L}$ & $1.01-4$ & $.440-4$ & $.525-4$ & $.555-4$ & $.585-4$ \\
\hline & & & $.959-4$ & $.444-4$ & $517-4$ & & \\
\hline & & V & & $.428-4$ & $.553-4$ & & \\
\hline & & & & $.424-4$ & $.561-4$ & & \\
\hline${ }^{1} \mathrm{D}_{2}{ }^{3} \mathrm{D}_{3}$ & 1002.49 & $\mathrm{~L}$ & $.347-4$ & $.179-4$ & $.190-4$ & $.190-4$ & $.200-4$ \\
\hline & & & $.364-4$ & $.181-4$ & $.190-4$ & & \\
\hline & & V & & $.343-4$ & $.319-4$ & & \\
\hline & & & & $.338-4$ & $.319-4$ & & \\
\hline
\end{tabular}




\title{
Relativistic Calculations in the Arsenic Sequence: Fine-Structure Transitions
}

\author{
E. Charro and I. Martín \\ Departamento de Química Física, \\ Universidad de Valladolid, \\ 47011 Valladolid, Spain
}

\section{Introduction}

One of the properties of highly ionized atoms that is a subject of considerable interest for many fields, such astrophysics and fusion plasma research, is the transition probability between different electronic levels or the oscillator strength. One of the isoelectronic series of the heavier species which has not been studied so far, to our knowledge, is that of the arsenic atom. As I has itself been the object of great interest in the last few years ([4]), given the detection of its $4 p^{2} 5 s^{4} P_{3 / 2}-4 p^{3}{ }^{4} S_{3 / 2}$ line in the spectrum of Chi Lupi, a HgMn type star, with a spectrometer on board the Hubble Space Telescope. Other atomic systems that have been found to exist in different ionization degrees in Chi Lupi are Sr, Y, Zr, Ru and Pd [5], which are potencially members of the As I isoelectronic sequence, although there are so far no reports on the existence of these elements in As-like form.

For many years we have applied the Quantum Defect Orbital method, both in its non-relativistic (QDO) [1] and its relativistic (RQDO) [2] versions, to the calculation of oscillator strengths and photoionization cross sections of a rather large number of atomic species, including several isoelectronic sequences ([3]). Nevertheless, for the reasons given above, we have undertaken the study of the intensities of all the allowed lines of the $4 s^{2} 4 p^{3}-4 s^{2} 4 p^{2} 5 s$ transition array in a number of As-like ions ranging from $\mathrm{Br}$ III $(\mathrm{Z}=35)$ to $\mathrm{La} \mathrm{XXV}(\mathrm{Z}=57)$. Two methodologies have been followed: the aforementioned RQDO method and the multiconfiguration Dirac-Fock (MCDF) formalism with the GRASP code written by Grant et al. [6] .

\section{Relativistic Quantum Defect Orbital Method}

The RQDO method has been described in detail [2]. We shall thus only mention here those aspects of the formalism which are relevant to this study.

The relativistic quantum defect orbitals are the analytical solutions of a quasirelativistic secondorder Dirac-like equation with a model Hamiltonian that contains the quantum defect as a parameter. This model Hamiltonian allows for an effective variation of the screening effects with radial distance and, as a consequence, the radial solutions behave approximately correctly in the core region of space, and display a correct behaviour at large radial distances. These have been found to be, in many cases, the most relevant regions contributing to the transition integral. The RQDO's lead to closed-form analytical expressions for the transition integrals, which allows us to calculate transition probabilities with simple algebra and little computational effort.

The RQDO Hamiltonian accounts for core-valence polarization only implicitly, through the inclusion of the quantum defect. However, it is our experience that the explicit inclusion of the above effect is often important. As in some previous works (see, e.g., [3] ) we have performed 
two types of RQDO $f$-value calculations: one with the standard dipole-length transition operator, $Q(r)=r$, and another with a core-polarization corrected form of the former [7]:

$$
Q(r)=r\left[1-\frac{\alpha}{r^{3}}\left[1-\exp \left(-r / r_{c}\right)\right]^{3}\right]
$$

where $\alpha$ is the core polarizability and $r_{c}$ is a cut-off radius, chosen to be in these calculations the mean radius of the outermost orbital of the core, calculated in accord with an expression given by Chichkov and Shevelko [8]. The core has been considered in all cases to be $\operatorname{Ar} 3 d^{10} 4 s^{2}$. For the core polarizabilities we have taken the values supplied by Fraga et al [9], which do not go beyond $\mathrm{Nb}$ IX $(\mathrm{Z}=41)$. For the higher ions in the sequence we have found the core polarizabilities in atomic units from the following expression

$$
\ln \alpha=38.838-10.615 \ln Z,
$$

which has been obtained through a fitting of the $\alpha$-values of Fraga et al. [9] from As I ( $\mathrm{Z}=33$ ) to Nb IX $(Z=41)$, with a correlation factor of 0.9967 . $\mathrm{Z}$ is the atomic number.

In the RQDO context, energy level data are required in order to obtain the quantum defects. For Br III we have been employed the data provided from a compilation by Kelly [10]. For the quartet levels of the $4 s^{2} 4 p^{2} 5 s$ configuration we have taken the experimental energy values of O'Sullivan [11] for Rb V and O'Sullivan and Maher [12] for Sr VI. No experimental energies have been found for the remaining levels of these two ions and we have employed the energies obtained with our MCDF calculations. For the ions Y VII to Mo X we have used experimental energies by Reader and Acquista [13] . Early energy data measured by Rahimullah et al.[14] for these ions are in good agreement with those of Reader and Acquista [13] .

All the above experimental data have relied on theoretical predictions and observed intensities for the identification of the lines, as well as an analysis of the systematic trends followed by the level energies along the isoelectronic sequence [11, 12, 13, 14]. O'Sullivan [11] and O'Sullivan and Maher [12] rely on MCDF calculations. Reader and Acquista [13] employ Hartree-Fock (HF) calculations. These authors estimate uncertainties in the range from $0.6 \mathrm{~cm}^{-1}$ to $15 \mathrm{~cm}^{-1}$ for the levels of the $4 s^{2} 4 p^{3}$ and $4 s^{2} 4 p^{2} 5 s$ configurations, the uncertainties increasing from the ground to the excited configurations, and also from Y VII to Mo X.

In order to have a basis on to which test the quality of the energies employed, we have plotted the energies of the ground and excited configurations, respectively. They present a regular behaviour, in all cases, along the isoelectronic sequence, and the connection between the present MCDF and the experimental energies [13] is smooth.

Other input data that are also needed in the RQDO calculations were the ionization energies of the atomic systems. The corresponding values from As I to Kr IV are supplied by Kelly [10] and a few more by Fraga et al. [9]. For the remaining ions we have employed an extrapolation formula obtained by fitting the ionization energies in $\mathrm{cm}^{-1}$ of the previous ions. This formula is

$$
E_{i}=86.96 Z^{3}-2806.66 Z^{2}
$$

where, again, $Z$ is the atomic number. The correlation factor was 0.9998 .

\section{Multiconfiguration Dirac-Fock Method}

The MCDF method, as implemented in the GRASP code [6], is a generalization of the Dirac-Fock formalism. For details of the atomic MCDF model we refer to a review by Grant [15]. 
The extended average level (EAL) mode with the Breit and QED corrections [6] has been employed. The following configurations have been introduced: $4 s^{2} 4 p^{3}+4 p^{5}$ to describe the ground configuration, and $4 s^{2} 4 p^{2} 5 s+4 s^{1} 4 p^{4}+4 s^{2} 4 p^{2} 4 d^{1}$ to describe the excited configuration. The choice of these configurations has been made on the grounds of the comments by Bieron and Migdalek [16] and O'Sullivan [11] and by observing that this configuration mixing leads to a good accord between dipole-length (Babushkin gauge) and dipole velocity (Coulomb gauge) MCDF oscillator strengths for most of the transitions studied.

\section{Results and Analysis}

For each transition, two RQDO sets of $f$-values are given, one obtained with the standard dipolelength transition operator, $Q(r)=r$, and the other with the core-polarization corrected transition operator, Eq. (2.1). The two sets of MCDF oscillator strengths correspond to calculations in the dipole-length and dipole-velocity forms, respectively. The experimental $f$-values reported by O'Sullivan [11] and O'Sullivan and Maher [12] up to Mo X have been considered for comparative purposes. In all the transitions, our $\mathrm{MCDF}$ calculations did not reach convergence both for $\mathrm{Br}$ III and Kr IV. For Rb V, all theoretical as well as experimental $f$-values are anomalously low in magnitude. This feature is explained by O'Sullivan [11] in terms of a large configuration mixing taking place in this particular ion that leads to a major re-distribution of intensity between the $4 p-5 s$ and $4 p-4 d$ transitions. In all studied transitions both our RQDO and MCDF $f$-values are in good accord with experiment. As we go down in the sequence, a greater similarity between the dipole-length and dipole velocity MCDF oscillator strengths is observed. In most transitions, the effects of correcting the RQDO $f$-values for core polarization are sizable and bring them closer to the MCDF oscillator strengths, in particular to those corresponding to the dipole-length calculation. A sharp increase that occurs in most transitions of Sr VI is apparent both in our calculations and experiment. O'Sullivan [11] refers to it as "array quenching" and explains it in terms of a sudden change in eigenvector composition of the $4 p^{2} 5 s$ term.

In all transitions, the RQDO $f$-values are generally greater in magnitude than the MCDF results, the differences increasing with atomic number, except for the ${ }^{2} D_{3 / 2}-{ }^{2} P_{3 / 2}$ transition. Nevertheless, the RQDO-MCDF relative difference of $96 \%$ occurring at La XXV (Z=57) for the ${ }^{4} S_{3 / 2}-{ }^{4} P_{5 / 2}$ transition is certainly an exception. For the other transitions, the largest RQDOMCDF relative differences in the heaviest of the ions studied range from $15 \%$ in Sn XVIII ( $\mathrm{Z}=50)$ for the ${ }^{2} P_{3 / 2}-{ }^{2} S_{1 / 2}$ transition and Cs XXIII (Z $\left.=55\right)$ for the ${ }^{2} D_{3 / 2}-{ }^{2} P_{3 / 2}$ transition to $33,5 \%$ for $\operatorname{LaXXV}(\mathrm{Z}=57)$ for the ${ }^{4} S_{3 / 2}-{ }^{4} P_{5 / 2}$ transition. Apart from the more correct wavefunctions supplied by the MCDF approach, as compared to the RQDO wavefunctions, a possible reason for the discrepancies might be the fact that the LS coupling scheme has been adopted for all the ions in the RQDO procedure, unlike Biémont and Hansen [17] who adopted an intermediate coupling scheme in their calculations of magnetic dipole and electric quadrupole transitions in the ground state of the germanium and arsenic isoelectronic sequences.

In summary, RQDO and MCDF oscillator strengths for the $4 s^{2} 4 p^{3}-4 s^{2} 4 p^{2} 5 s$ transition array of a number of As-like ions are presented. When experimental data are available, our results are in good accord with them. Some discrepancies between the RQDO and MCDF $f$-values are apparent, particularly for the heavier ions, for which we consider the MCDF results to be superior in quality. Nevertheless, the RQDO procedure has once more proved to be a very useful tool for estimating transition probabilities. It is particularly adequate in the cases where mass-production of data is needed, given its cost-efficiency and reasonable correctness. It offers also the advantage of its analyticity and lack of convergence problems from which elaborate ab initio calculations often suffer. 
One drawback of the RQDO formalism is that it needs quantum defects from other sources as input. But this can be, in many cases, circumvented by making use of the regularities presented by the quantum defects both along spectral series and isoelectronic sequences, which allow for interor extrapolations.

\section{Acknowledgements}

This work has been supported by the DGICYT of the Spanish Ministry of Education within Project No. PB94-1314-C03-03 and the J.C.L. within Project No. VA 21/97.

\section{References}

[1] G. Simons, J. Chem. Phys. 60, 645 (1974); I. Martín and G. Simons, J. Chem. Phys. 62, 4799 (1975); Mol. Phys. 32, 1017 (1976)

[2] I. Martín and J. Karwowski, J. Phys. B: At. Mol. Opt. Phys. 24, 1538 (1991); J. Karwowski and I. Martín, Phys. Rev. A43, 4832 (1991)

[3] E. Charro, I. Martín, and C. Lavín, J. Quant. Spectrosc. Radiat. Transfer 56, 241 (1996); Astron. Astrophys. Suppl. Series. 124, 1 (1997), and references therein

[4] G. J. Bengston, U. Berzinsh, J. Larsson, and S. Svanberg, Astron. Astrophys. 263, 440 (1992)

[5] D. S. Leckrone, S. Johansson, G. M. Wahlgren, C. R. Proffitt, and T. Brage, Phys. Scr. T65, $110(1996)$

[6] I. P. Grant, B. J. McKenzie, P. H. Norrington, D. F. Mayers, and N. C. Pyper, Comput. Phys. Commun. 21, 207 (1980); K. G. Dyall, I. P. Grant, C. T. Johnson, F. A. Parpia, and E. P. Plummer, Comput. Phys. Commun. 55, 425 (1989)

[7] D. Bielińska-Waz, M. Sc. Thesis, University Nicholas Copernicus, Toruń Poland 1992

[8] B. N. Chichkov and V. P. Shevelko, Phys. Scr. 23, 1055 (1981)

[9] S. Fraga, J. Karwowski, and K. M. S. Saxena, "Handbook of Atomic Data", Elsevier, New York 1976

[10] R. L. Kelly, J. Chem. Ref. Data 16, Supp.1 (1987)

[11] G. O'Sullivan, J. Phys. B: At. Mol. Opt. Phys. 22, 978 (1989)

[12] G. O’Sullivan and M. Maher, J. Phys. B: At. Mol. Opt. Phys. 22, 377 (1989)

[13] J. Reader and N. Acquista, J. Opt. Soc. Am. 71, 434 (1981)

[14] K. Rahimullah, M.S.Z. Chagtai, and S. Khatoon, Phys. Scr. 14, 221 (1976); Phys. Scr. 18, 96 (1978)

[15] I. P. Grant, in "Methods in Computational Chemistry", Vol. 2., p. 1, edited by S.Wilson, Plenum Press, New York 1988

[16] J. R. Bieroń and J. Migdalek, J. Phys. B: At. Mol. Opt. Phys. 25, 4099 (1980)

[17] E. Biémont and J. E. Hansen, Phys. Scr. 33, 117 (1986)

Tables and figures are available upon request to the authors. 


\title{
Atomic Database for C I from C II
}

\author{
J. Dubau ${ }^{+}$, T.Kato*, and U. I. Safronova * \\ + Observatoire of Paris, Meudon, F-92195 MEUDON Cedex, France \\ ${ }^{*}$ National Institute for Fusion Science, Oroshi-cho, Toki, Gifu, 509-52, Japan
}

\section{Introduction}

Carbon is the main constituent of the graphite tiles which cover the inner side of the vacuum chamber of the latest tokamaks (JET, TFTR, JET-60). Carbon is also one of the most abundant elements of the Universe. The tokamak spectra are very rich in $C$ lines emitted in the outer region of the plasma, the coolest part, where $C$ is not completely ionized and can still emit a line spectrum. The recombination rates will play an important role in the plasma cooling when more ionized species recombine progressively.

The dielectronic recombination $(D R)$ process for $C$ ( $C$ I from $C$ II) can be schematically represented by $1 s^{2} 2 s^{2} 2 p+e \Rightarrow 1 s^{2} 2 s 2 p^{2} n l, 1 s^{2} 2 s 2 p^{3}(L S), 1 s^{2} 2 p^{3} n l, 1 s^{2} 2 p^{4}$

$$
1 s^{2} 2 s^{2} 2 p, 1 s^{2} 2 s 2 p^{2}+\text { e or } 1 s^{2} 2 s^{2} 2 p^{2}, 1 s^{2} 2 s^{2} 2 p n l, 1 s^{2} 2 s 2 p^{3}\left(L^{\prime} S^{\prime}\right)+h \nu,
$$

where $L S={ }^{3} S,{ }^{1} D,{ }^{1} P$ for autoionizing states and $L^{\prime} S^{\prime}={ }^{5} S,{ }^{3} D,{ }^{3} P$ for bound states. An intensity factor $Q_{d}$ for transitions from autoionizing levels $\gamma=1 s^{2} 2 s 2 p^{2} n l, 1 s^{2} 2 s 2 p^{3}(L S), 1 s^{2} 2 p^{3} n l, 1 s^{2} 2 p^{4}$ to excited states $\gamma^{\prime}=1 s^{2} 2 s^{2} 2 p^{2}, 1 s^{2} 2 s^{2} 2 p n l, 1 s^{2} 2 s 2 p^{3}\left(L^{\prime} S^{\prime}\right)$ can be defined as follows:

$$
Q_{d}\left(\gamma, \gamma^{\prime} \mid \alpha_{0}\right)=g_{\gamma} A_{r}\left(\gamma, \gamma^{\prime}\right) \frac{A_{a}\left(\gamma, \alpha_{0}\right)}{A_{r}(\gamma)+A_{a}(\gamma)}, A_{r}(\gamma)=\sum_{\gamma^{\prime \prime}} A_{r}\left(\gamma, \gamma^{\prime \prime}\right), A_{a}(\gamma)=\sum_{\alpha^{\prime}} A_{a}\left(\gamma, \alpha^{\prime}\right)
$$

where $A_{\tau}\left(\gamma, \gamma^{\prime}\right)$ are radiative transition probabilities and $A_{a}\left(\gamma, \alpha^{\prime}\right)$ is the autoionization rate, with $\alpha^{\prime}=1 s^{2} 2 s^{2} 2 p, 1 s^{2} 2 s 2 p^{2}$ and $\alpha_{0}=1 s^{2} 2 s^{2} 2 p$.

We used the Cowan [1] and SUPERSTRUCTURE codes taking into account 28 even and 29 odd parity configurations $1 s^{2} 2 l_{1} 2 l_{2} 2 l_{3} n l$ with up to $n=6$ and $0 \leq l \leq(\mathrm{n}-1)$. The contributions of the configurations with $6 \leq n \leq 500$ are taken into account in the calculation of all $1 s^{2} 2 l_{1} 2 l_{2} 2 l_{3} n l[L S J]$ states up to $n=6$. In the present paper we can present only a small amount of our numerical data due to the limited space.

\section{Energy Levels, Radiative Transition Probabilities}

We carried out detailed calculations of radiative transition probabilities and autoionization rates for the intermediate states $1 s^{2} 2 s^{2} 2 p n l, 1 s^{2} 2 s 2 p^{3}, 1 s^{2} 2 p^{4}, 1 s^{2} 2 s 2 p^{2} n l$ and $1 s^{2} 2 p^{3} n l$ with $n=2-6$

The atomic energy levels, radiative transition probabilities and autoionization rates were obtained by using the atomic structure code of Cowan [1]. Table 1 lists energies calculated by the Cowan (a) and SUPERSTRUCTURE (b) codes, together with theoretical data obtained by Nahar and Pradhan (c) [2] and recommended data (d) by Wiese et al. [3] for $C$ I with the $1 s^{2} 2 s^{2} 2 p^{2}, 1 s^{2} 2 s 2 p^{3}, 1 s^{2} 2 s^{2} 2 p n l$ intermediate states. The differences in the theoretical data (a) and (b) can be explained by different refinements of the Hartree-Fock approximation. The Cowan 
code allows the use of scaled factors for radial integrals. We used a scaled factor equal to 0.85 . The SUPERSTRUCTURE code is based on a scaled Thomas-Fermi-Dirac-Amadi potential. The scaling parameter is different for each angular momentum $l$. These parameters are iterated to give the minimum energy of a term or a group of terms. It should be noted that both methods did not take into account correlation effects properly which explains the disagreement of these data (columns $a$ and $b$ in Table 1 ) with the recommended data (column $c$ ) for some states.

\section{Total Dielectronic Recombination Rate Coefficients}

The total dielectronic recombination rate coefficient is obtained by the sum over all the levels,

$$
\alpha_{d}^{t}\left(\alpha_{0}\right)=3.3 \times 10^{-24}\left(\frac{I_{H}}{T_{e}}\right)^{3 / 2} \sum_{\gamma, \gamma^{\prime}} e^{-\frac{E_{S}}{T_{e}}} Q_{d}\left(\gamma, \gamma^{\prime} \mid \alpha_{0}\right) / g\left(\alpha_{0}\right) .
$$

The sum over $\gamma$ means sum over all autoionization levels. As we already mentioned before we calculated numerically the $Q_{d}\left(\gamma, \gamma^{\prime} \mid \alpha_{0}\right)$ values with $\gamma^{\prime}=1 s^{2} 2 s^{2} 2 p n l, 1 s^{2} 2 s 2 p^{3}\left({ }^{5} S,{ }^{3} D,{ }^{3} P\right)$ and $\gamma=1 s^{2} 2 s 2 p^{3}\left({ }^{3} S,{ }^{1} D,{ }^{1} P\right), 1 s^{2} 2 p^{4}, 1 s^{2} 2 s 2 p^{2} n l, 1 s^{2} 2 p^{3} n l$ up to $n=6$. We take into account the states with $n \geq 6$ by scaling $Q_{d}$. It was shown in Ref.[4] that the largest contribution to $Q_{d}$ for large $n$ are due to the $2 s-2 p$ transitions. In our case it is transitions as $2 s^{2} 2 p n l[L S]-2 s 2 p^{2}(L S) n l\left[L^{\prime} S^{\prime}\right]$. Radiative transition probabilities for these transitions are almost constant for large $n$ and nonradiative transition probabilities (autoionizing rates) are proportional to $\frac{1}{n^{3}}$ :

$$
\begin{aligned}
& A_{r}\left(2 s^{2} 2 p n l[L S], 2 s 2 p^{2}\left(L_{12} S_{12}\right) n l\left[L^{\prime} S^{\prime} J^{\prime}\right]\right)=A_{r}\left(2 s^{2} 2 p n_{0} l[L S], 2 s 2 p^{2}\left(L_{12} S_{12}\right) n_{0} l\left[L^{\prime} S^{\prime} J^{\prime}\right]\right), \\
& A_{a}\left(2 s 2 p^{2}\left(L_{12} S_{12}\right) n l\left[L^{\prime} S^{\prime} J^{\prime}\right] \mid 2 s^{2} 2 p^{2} P\right)=\left(\frac{n_{0}}{n}\right)^{3} A_{a}\left(2 s 2 p^{2}\left(L_{12} S_{12}\right) n_{0} l\left[L^{\prime} S^{\prime} J^{\prime}\right] \mid 2 s^{2} 2 p^{2} P\right) .
\end{aligned}
$$

Table 2 gives these data for $\mathrm{n}=4,5,6$ with $l=\mathrm{s}, \mathrm{p}, \mathrm{d}, \mathrm{f}, \mathrm{g}$ and all kinds of $L^{\prime} S^{\prime} J^{\prime}$. We chose for illustration the data with the largest values of $Q_{d}$. We can see from this Table that Eqs. $(4,5)$ are correct for transitions with large values of $A_{r}$ and $A_{a}$.

\section{Acknowledgments}

U.I.Safronova would like to thank the members of the Data and Planning Center, the National Institute for Fusion Science; the Director of the Center, Prof.H.Momota and the Director of NIFS, Prof.A.Iiyoshi, for their hospitality and financial support.

\section{References}

[1] R. D. Cowan, "The Theory of Atomic Structure and Spectra", University of California Press, Berkeley 1981

[2] S. N. Nahar and A. K. Pradhan, Phys. Rev. A 44, 2935 (1991)

[3] W. L. Wiese, M. W. Smith, and B. M. Glennon, Atomic Transition Probabilities v. 1, NSRDS-4 Washington, DC (1966)

[4] T. Kato, U. I. Safronova, and M. Ohira, Physica Scripta 54 (1996) 
Table 1: Energy $\left(10^{3} \mathrm{~cm}^{-1}\right)$ and sum of weighted radiative transition probabilities $\left(\sum\left(\mathrm{g} A_{r}\right)\right.$ in $\sec ^{-1}$ ) of carbon (C I) for $1 s^{2} 2 s 2 l_{1} 2 l_{2}\left(L_{12} S_{12}\right) n l[L S J]$ states. Comparison of different methods and recommended data from Ref.[3]: $a$-Cowan code, $b$-SUPERSTRUCTURE code, $c$-Ref.[2], $d$-Ref.[3]

\begin{tabular}{|c|c|c|c|c|c|c|c|c|}
\hline \multirow[t]{2}{*}{$2 s 2 l_{1} n l_{2}$} & \multirow[t]{2}{*}{$L_{12} S_{12}$} & \multirow[t]{2}{*}{$L S$} & \multirow[t]{2}{*}{$\mathrm{J}$} & \multicolumn{4}{|c|}{$E$ in $10^{3} \mathrm{~cm}^{-1}$} & \multirow{2}{*}{$\begin{array}{c}\sum\left(g A_{r}\right) \\
a\end{array}$} \\
\hline & & & & $a$ & $b$ & $c$ & $d$ & \\
\hline $2 s^{2} 2 p^{2}$ & $\left({ }^{3} P\right)$ & ${ }^{3} P$ & 0 & 0.000 & 0.000 & 0.000 & 0.000 & $0.0000+00$ \\
\hline $2 s^{2} 2 p^{2}$ & $\left({ }^{1} D\right)$ & ${ }^{1} D$ & 2 & 10.651 & 13.398 & 10.502 & 10.194 & $0.0000+00$ \\
\hline $2 s^{2} 2 p^{2}$ & $\left({ }^{1} S\right)$ & ${ }^{1} S$ & 0 & 18.953 & 20.411 & 23.023 & 21.648 & $0.0000+00$ \\
\hline $2 s^{2} 2 p 3 p$ & $\left({ }^{2} P\right)$ & ${ }^{1} P$ & 1 & 66.496 & 66.608 & 69.661 & 68.858 & $0.2661+08$ \\
\hline $2 s^{2} 2 p 3 p$ & $\left({ }^{2} P\right)$ & ${ }^{3} D$ & 2 & 67.069 & 67.403 & 69.650 & 69.711 & $0.8172+08$ \\
\hline $2 s^{2} 2 p 3 p$ & $\left({ }^{2} P\right)$ & ${ }^{3} S$ & 1 & 67.776 & 68.401 & 71.855 & 70.744 & $0.5717+08$ \\
\hline $2 s^{2} 2 p 3 p$ & $\left({ }^{2} P\right)$ & ${ }^{3} P$ & 1 & 70.429 & 70.876 & 72.722 & 71.365 & $0.1337+09$ \\
\hline $2 s^{2} 2 p 3 p$ & $\left({ }^{2} P\right)$ & ${ }^{1} D$ & 2 & 71.863 & 73.077 & 74.248 & 72.611 & $0.2293+09$ \\
\hline $2 s^{2} 2 p 3 p$ & $\left({ }^{2} P\right)$ & ${ }^{1} S$ & 0 & 73.420 & 76.223 & 75.992 & 73.976 & $0.5352+08$ \\
\hline $2 s 2 p^{3}$ & $\left({ }^{4} S\right)$ & ${ }^{5} S$ & 2 & 31.866 & 24.432 & 32.515 & 33.735 & $0.0000+00$ \\
\hline $2 s 2 p^{3}$ & $\left({ }^{2} D\right)$ & ${ }^{3} D$ & 2 & 67.675 & 66.919 & 63.943 & 64.093 & $0.1710+10$ \\
\hline $2 s 2 p^{3}$ & $\left({ }^{2} P\right)$ & ${ }^{3} P$ & 1 & 78.763 & 76.186 & 76.014 & 75.256 & $0.2419+10$ \\
\hline $2 s^{2} 2 p 3 s$ & $\left({ }^{2} P\right)$ & ${ }^{3} P$ & 1 & 58.402 & 58.689 & 60.585 & 60.353 & $0.9067+09$ \\
\hline $2 s^{2} 2 p 3 s$ & $\left({ }^{2} P\right)$ & ${ }^{1} P$ & 1 & 59.686 & 61.388 & 62.385 & 61.982 & $0.1219+10$ \\
\hline $2 s^{2} 2 p 3 d$ & $\left({ }^{2} P\right)$ & ${ }^{3} P$ & 1 & 75.570 & 80.375 & 80.996 & 79.319 & $0.4165+09$ \\
\hline $2 s^{2} 2 p 3 d$ & $\left({ }^{2} P\right)$ & ${ }^{1} D$ & 2 & 76.069 & 76.288 & 79.350 & 77.681 & $0.3626+09$ \\
\hline $2 s^{2} 2 p 3 d$ & $\left({ }^{2} P\right)$ & ${ }^{3} D$ & 2 & 76.606 & 77.050 & 80.119 & 78.307 & $0.1836+10$ \\
\hline $2 s^{2} 2 p 3 d$ & $\left({ }^{2} P\right)$ & ${ }^{1} F$ & 3 & 76.682 & 77.433 & 80.327 & 78.531 & $0.1767+10$ \\
\hline $2 s^{2} 2 p 3 d$ & $\left({ }^{2} P\right)$ & ${ }^{3} F$ & 3 & 76.429 & 76.739 & 79.965 & 78.216 & $0.3085+09$ \\
\hline $2 s^{2} 2 p 3 d$ & $\left({ }^{2} P\right)$ & ${ }^{1} P$ & 1 & 76.644 & 77.653 & 79.877 & 78.728 & $0.2985+09$ \\
\hline $2 s^{2} 2 p 4 s$ & $\left({ }^{2} P\right)$ & ${ }^{3} P$ & 0 & 76.383 & & 79.680 & 78.105 & $1.453+08$ \\
\hline $2 s^{2} 2 p 4 s$ & $\left({ }^{2} P\right)$ & ${ }^{1} P$ & 1 & 76.932 & & 79.877 & 78.338 & $7.806+08$ \\
\hline $2 s^{2} 2 p 4 p$ & $\left({ }^{2} P\right)$ & ${ }^{1} D$ & 2 & 80.914 & & 83.168 & 81.770 & $1.012+08$ \\
\hline $2 s^{2} 2 p 4 p$ & $\left({ }^{2} P\right)$ & ${ }^{1} S$ & 0 & 82.434 & & 83.794 & 82.252 & $5.231+07$ \\
\hline $2 s^{2} 2 p 4 d$ & $\left({ }^{2} P\right)$ & ${ }^{3} D$ & 1 & 82.147 & & 85.681 & 83.830 & $6.559+08$ \\
\hline $2 s^{2} 2 p 4 d$ & $\left({ }^{2} P\right)$ & ${ }^{1} F$ & 3 & 82.241 & & 85.780 & 83.949 & $1.989+09$ \\
\hline $2 s^{2} 2 p 5 s$ & $\left({ }^{2} P\right)$ & ${ }^{1} P$ & 1 & 82.443 & & 85.661 & 83.882 & $3.972+08$ \\
\hline $2 s^{2} 2 p 5 p$ & $\left({ }^{2} P\right)$ & ${ }^{1} D$ & 2 & 84.186 & & 87.062 & 85.400 & $4.708+07$ \\
\hline $2 s^{2} 2 p 5 p$ & $\left({ }^{2} P\right)$ & ${ }^{1} S$ & 0 & 84.613 & & 87.325 & 85.626 & $1.312+07$ \\
\hline $2 s^{2} 2 p 5 d$ & $\left({ }^{2} P\right)$ & ${ }^{1} F$ & 3 & 84.868 & & 88.300 & 86.450 & $7.044+08$ \\
\hline $2 s^{2} 2 p 5 d$ & $\left({ }^{2} P\right)$ & ${ }^{1} P$ & 1 & 84.860 & & 88.358 & 86.491 & $1.335+08$ \\
\hline $2 s^{2} 2 p 6 s$ & $\left({ }^{2} P\right)$ & ${ }^{1} P$ & 1 & 84.940 & & 88.253 & 86.414 & $2.338+08$ \\
\hline $2 s^{2} 2 p 6 p$ & $\left({ }^{2} P\right)$ & ${ }^{3} P$ & 0 & 85.625 & & 88.877 & 87.077 & $4.477+06$ \\
\hline $2 s^{2} 2 p 6 d$ & $\left({ }^{2} P\right)$ & ${ }^{1} D$ & 2 & 86.127 & & 89.489 & 87.632 & $1.786+08$ \\
\hline $2 s^{2} 2 p 6 d$ & $\left({ }^{2} P\right)$ & ${ }^{3} F$ & 2 & 86.160 & & 89.599 & 87.706 & $2.013+08$ \\
\hline
\end{tabular}


Table 2: Energy excitation $\left(E_{S}\right.$ in $\left.\mathrm{eV}\right)$, weighted radiative transition probabilities $\left(\left(g A_{r}\right)\right.$ in $\left.\sec ^{-1}\right)$, autoionization rate $\left(A_{a}\right.$ in $\left.s e c^{-1}\right)$ and factor intensities $\left(Q_{d}\right.$ in $\left.\sec ^{-1}\right)$ for $2 s^{2} 2 p n l$ $2 s 2 p^{2}\left(L_{12} S_{12}\right) n l\left(L^{\prime} S^{\prime} J^{\prime}\right)$ transitions with $n=4,5$, and 6 .

\begin{tabular}{|c|c|c|c|c|c|c|c|c|c|}
\hline \multirow[b]{2}{*}{$n$} & \multicolumn{3}{|c|}{ Upper level } & \multirow[t]{2}{*}{$A_{a}$} & \multirow[t]{2}{*}{$\sum\left(A_{a}\right)$} & \multirow[t]{2}{*}{$g A_{r}$} & \multirow[t]{2}{*}{$\sum\left(g A_{r}\right)$} & \multirow[t]{2}{*}{$Q_{d}$} & \multirow[t]{2}{*}{$E_{S}$} \\
\hline & $L_{12} S_{12}$ & $L^{\prime} S^{\prime}$ & $J^{\prime}$ & & & & & & \\
\hline $2 s^{2} 2 p m s$ & $2 s 2 p^{2}(1$ & $\left.{ }_{2} S_{12}\right)$ & $\left.{ }^{\prime} S^{\prime} J^{\prime}\right)$ & & & & & & \\
\hline 4 & $\left({ }^{1} D\right)$ & ${ }^{1} D$ & 2 & $7.702+13$ & $1.502+14$ & $2.712+09$ & $1.027+09$ & $8.914+07$ & 7.948 \\
\hline 5 & $\left({ }^{1} D\right)$ & ${ }^{1} D$ & 2 & $3.574+13$ & $7.562+13$ & $3.327+09$ & $1.338+09$ & $1.104+08$ & 8.599 \\
\hline 6 & $\left({ }^{1} D\right)$ & ${ }^{1} D$ & 2 & $2.426+13$ & $5.127+13$ & $2.577+09$ & $1.398+09$ & $1.293+08$ & 8.897 \\
\hline $2 s^{2} 2 p n p$ & $2 s 2 p^{2}(I$ & $\left.{ }_{2} S_{12}\right)$ & $\left(S^{\prime} J^{\prime}\right)$ & & & & & & \\
\hline 4 & $\left({ }^{1} D\right)$ & ${ }^{1} F$ & 3 & $3.047+13$ & $3.489+13$ & $5.592+09$ & $4.254+09$ & $6.193+08$ & 8.187 \\
\hline 5 & $\left({ }^{1} D\right)$ & ${ }^{1} F$ & 3 & $4.854+12$ & $7.879+12$ & $4.192+09$ & $3.281+09$ & $3.368+08$ & 8.710 \\
\hline 6 & $\left({ }^{1} D\right)$ & ${ }^{1} F$ & 3 & $2.633+12$ & $4.188+12$ & $5.308+09$ & $3.802+09$ & $3.984+08$ & 8.954 \\
\hline 4 & $\left({ }^{3} P\right)$ & ${ }^{3} \mathrm{~Pa}$ & 2 & $2.630+13$ & $6.458+13$ & $2.379+10$ & $2.339+10$ & $1.587+09$ & 12.49 \\
\hline 5 & $\left({ }^{3} P\right)$ & ${ }^{3} \mathrm{~Pa}$ & 2 & $2.884+12$ & $2.456+13$ & $2.290+10$ & $2.246+10$ & $4.393+08$ & 13.03 \\
\hline 6 & $\left({ }^{3} P\right)$ & ${ }^{3} \mathrm{~Pa}$ & 2 & $1.454+12$ & $1.178+13$ & $2.328+10$ & $2.297+10$ & $4.725+08$ & 13.28 \\
\hline $2 s^{2} 2 p m d$ & $2 s 2 p^{2}(1$ & $\left.{ }_{2} S_{12}\right)$ & $\left.S^{\prime} J^{\prime}\right)$ & & & & & & \\
\hline 4 & $\left({ }^{1} S\right)$ & ${ }^{3} D$ & 3 & $1.329+13$ & $1.602+13$ & $1.495+10$ & $1.435+10$ & $1.984+09$ & 10.88 \\
\hline 5 & $\left({ }^{1} S\right)$ & ${ }^{3} D$ & 3 & $3.851+12$ & $7.046+12$ & $1.460+10$ & $1.420+10$ & $1.293+09$ & 11.22 \\
\hline 6 & $\left({ }^{1} S\right)$ & ${ }^{3} D$ & 3 & $2.323+12$ & $4.198+12$ & $1.501+10$ & $1.417+10$ & $1.306+10$ & 11.39 \\
\hline 4 & $\left({ }^{3} P\right)$ & ${ }^{3} \mathrm{Fa}$ & 2 & $4.384+13$ & $4.580+13$ & $2.100+10$ & $2.075+10$ & $3.310+09$ & 12.84 \\
\hline 5 & $\left({ }^{3} P\right)$ & ${ }^{3} \mathrm{Fa}$ & 2 & $1.278+13$ & $1.482+13$ & $2.183+10$ & $2.017+10$ & $1.881+09$ & 13.18 \\
\hline 6 & $\left({ }^{3} P\right)$ & ${ }^{3} \mathrm{Fa}$ & 2 & $6.991+12$ & $8.113+12$ & $2.189+10$ & $2.139+10$ & $3.071+09$ & 13.36 \\
\hline 4 & $\left({ }^{3} P\right)$ & ${ }^{1} F$ & 3 & $4.895+13$ & $5.632+13$ & $3.084+10$ & $2.895+10$ & $4.194+09$ & 12.86 \\
\hline 5 & $\left({ }^{3} P\right)$ & ${ }^{1} F$ & 3 & $1.418+13$ & $1.831+13$ & $3.114+10$ & $3.006+10$ & $3.879+09$ & 13.19 \\
\hline 6 & $\left({ }^{3} P\right)$ & ${ }^{1} F$ & 3 & $8.314+12$ & $1.050+13$ & $3.144+10$ & $3.009+10$ & $3.968+09$ & 13.37 \\
\hline $2 s^{2} 2 p n f$ & $2 s 2 p^{2}(I$ & $\left.{ }_{2} S_{12}\right)$ & $\left.S^{\prime} S^{\prime}\right)$ & & & & & & \\
\hline 4 & $\left({ }^{3} P\right)$ & ${ }^{1} D$ & 2 & $3.140+11$ & $3.740+11$ & $2.362+10$ & $2.351+10$ & $3.429+09$ & 12.87 \\
\hline 5 & $\left({ }^{3} P\right)$ & ${ }^{1} D$ & 2 & $1.970+11$ & $1.460+11$ & $2.273+10$ & $2.266+10$ & $2.405+09$ & 13.20 \\
\hline 6 & $\left({ }^{3} P\right)$ & ${ }^{1} D$ & 2 & $1.170+11$ & $1.670+11$ & $2.286+10$ & $2.271+10$ & $2.581+09$ & 13.37 \\
\hline 4 & $\left({ }^{3} P\right)$ & ${ }^{3} G a$ & 3 & $4.201+12$ & $4.233+12$ & $3.003+10$ & $2.990+10$ & $4.940+09$ & 12.87 \\
\hline 5 & $\left({ }^{3} P\right)$ & ${ }^{3} G a$ & 3 & $7.880+11$ & $9.260+11$ & $3.089+10$ & $3.079+10$ & $4.347+09$ & 13.20 \\
\hline 6 & $\left({ }^{3} P\right)$ & ${ }^{3} G a$ & 3 & $5.450+11$ & $6.320+11$ & $3.082+10$ & $3.074+10$ & $4.387+09$ & 13.37 \\
\hline 4 & $\left({ }^{3} P\right)$ & ${ }^{1} G$ & 4 & $4.257+12$ & $4.288+12$ & $3.850+10$ & $3.839+10$ & $6.337+09$ & 12.87 \\
\hline 5 & $\left({ }^{3} P\right)$ & ${ }^{1} G$ & 4 & $8.150+11$ & $9.620+11$ & $3.964+10$ & $3.953+10$ & $5.556+09$ & 13.21 \\
\hline 6 & $\left({ }^{3} P\right)$ & ${ }^{1} G$ & 4 & $5.670+11$ & $6.620+11$ & $3.945+10$ & $3.941+10$ & $5.589+09$ & 13.38 \\
\hline $2 s^{2} 2 p n g$ & $2 s 2 p^{2}$ & $\left.{ }_{2} S_{12}\right)$ & $\left.S^{\prime} J^{\prime}\right)$ & & & & & & \\
\hline 5 & $\left({ }^{3} P\right)$ & ${ }^{1} G$ & 4 & $1.6+10$ & $1.8+10$ & $4.021+10$ & $4.015+10$ & $4.766+09$ & 13.21 \\
\hline 6 & $\left({ }^{3} P\right)$ & ${ }^{1} G$ & 4 & $1.8+10$ & $2.0+10$ & $4.020+10$ & $4.016+10$ & $4.923+09$ & 13.38 \\
\hline 5 & $\left({ }^{3} P\right)$ & ${ }^{1} H$ & 5 & $1.9+10$ & $2.5+10$ & $4.870+10$ & $4.862+10$ & $5.231+09$ & 13.21 \\
\hline 6 & $\left({ }^{3} P\right)$ & ${ }^{1} H$ & 5 & $2.1+10$ & $2.7+10$ & $4.813+10$ & $4.810+10$ & $5.366+09$ & 13.38 \\
\hline
\end{tabular}




\title{
A Project for Large-Scale Stark Broadening Data Production: Ca IX and Ca X Spectral Lines
}

\author{
Milan S. Dimitrijević ${ }^{1}$ and Sylvie Sahal-Bréchot ${ }^{2}$ \\ ${ }^{1}$ Astronomical Observatory, Volgina 7, 11050 Belgrade, Yugoslavia \\ ${ }^{2}$ Observatoire de Paris-Meudon, 92190 Meudon, France
}

\section{Introduction}

In a series of papers we have performed large scale calculations of Stark broadening parameters for a number of spectral lines of various emitters [1], [2], [3], [4]. Such data are of interest for a number of problems in astrophysics, physics and plasma technology. One may mention as examples calculation of stellar opacities, stellar atmospheres modelling and investigations, abundance determinations, interpretation and modelling of stellar spectra, laboratory plasma diagnostics, research and modelling, radiative transfer calculations and investigation of laser produced plasmas and plasmas created in fusion research. In order to satisfy the numerous Stark broadening data needs for astrophysical and laboratory plasma research and stellar opacities calculations, we are making a continuing effort to provide Stark broadening data for a large set of atoms and ions. Our calculations are performed within the semiclassical - perturbation formalism [5,6], for transitions where a sufficiently complete set of reliable atomic data exists and good accuracy for the obtained results is expected.

Extensive calculations have been performed up to now [4] for a number of radiators, and consequently, Stark broadening parameters for $79 \mathrm{He} \mathrm{I}, 62 \mathrm{Na}, 51 \mathrm{~K}, 61 \mathrm{Li}, 25 \mathrm{Al}, 24 \mathrm{Rb}, 3 \mathrm{Pd}, 19 \mathrm{Be}$, $270 \mathrm{Mg}, 31 \mathrm{Se}, 33 \mathrm{Sr}, 14 \mathrm{Ba}, 28 \mathrm{Ca}$ II, $30 \mathrm{Be}$ II, $29 \mathrm{Li}$ II, $66 \mathrm{Mg}$ II, $64 \mathrm{Ba}$ II, 19 Si II, $3 \mathrm{Fe} \mathrm{II,} 2 \mathrm{Ni}$ II, 12 B III, 23 Al III, 10 Sc III, 27 Ba III, 32 Y III, 10 Ti IV, 39 Si IV, 90 C IV, 5 O IV, 114 P IV, 19 O V, $30 \mathrm{~N} \mathrm{~V}, 25 \mathrm{C} \mathrm{V}, 51 \mathrm{P} \mathrm{V}, 33 \mathrm{~V} \mathrm{~V}, 30 \mathrm{O}$ VI, $21 \mathrm{~S}$ VI, $10 \mathrm{O}$ VII, $10 \mathrm{~F}$ VII, $20 \mathrm{Ne}$ VIII, $4 \mathrm{Ca}$ IX, $8 \mathrm{Na}$ IX, $48 \mathrm{Ca}$ X, 7 Al XI, 4 Si XI, Si XII, and 26 V XIII multiplets become available.

Data for particular lines of F I, Ga II, Ga III, Cl I, Br I, I I, Cu I, Hg II, N III, F V and S IV also exist.

In continuation of our project, we have calculated within the semiclassical-perturbation formalism the electron-, proton-, and He III-impact line widths and shifts for $4 \mathrm{Ca}$ IX and $48 \mathrm{Ca} \mathrm{X}$ multiplets, as a function of temperature and perturber density.

\section{Results and Discussion}

A summary of the formalism has been published several times [7], and will not be repeated here. The energy levels for the Ca IX and Ca X lines have been taken from Bashkin and Stoner [8]. In addition to electron-impact full halfwidths and shifts, Stark-broadening parameters due to proton-, and $\mathrm{He}$ III- impacts have been calculated. Our results for $4 \mathrm{Ca}$ IX and $48 \mathrm{Ca} \mathrm{X}$ multiplets for perturber densities from $10^{18}$ to $10^{22} \mathrm{~cm}^{-3}$ and temperatures from 200,000 to $2,000,000 \mathrm{~K}$ will be published elsewhere $[10,11]$.

In Tables 1 and 2, we present a sample of results obtained for Ca IX and $\mathrm{Ca} X$, respectively. We also specify a parameter C [9], which gives an estimate for the maximum perturber density for 
which the line may be treated as isolated when it is divided by the corresponding electron-impact full width at half maximum. For each value given in Tables 1 and 2, the collision volume (V) multiplied by the perturber density $(\mathrm{N})$ is much less than one and the impact approximation is valid $[5,6]$. When the impact approximation is not valid, the ion broadening contribution may be estimated by using quasistatic estimates $[12,13]$. The accuracy of the results obtained decreases when broadening by ion interactions becomes important.

We hope that the present results will be of interest for stellar, laboratory, fusion and laserproduced plasma investigations and modeling, as well as for the testing and developing of Stark broadening theory.

\section{References}

[1] M. S. Dimitrijević and S. Sahal-Bréchot, Astron. Astrophys. Suppl. Series 109, 551 (1995)

[2] M. S. Dimitrijević and S. Sahal-Bréchot, Astron. Astrophys. Suppl. Series 115, 351 (1996)

[3] M. S. Dimitrijević, and S. Sahal-Bréchot, Astron. Astrophys. Suppl. Series 119, 369 (1996)

[4] M. S. Dimitrijević, Zh. Prikl. Spektrosk. 63, 810 (1996)

[5] S. Sahal-Bréchot, Astron. Astrophys. 1, 91 (1969)

[6] S. Sahal-Bréchot, Astron. Astrophys. 2, 322 (1969)

[7] M. S. Dimitrijević, S. Sahal-Bréchot, and V. Bommier, Astron. Astrophys. Suppl. Series 89, $581(1991)$

[8] S. Bashkin, J. O. Stoner, Jr., "Atomic Energy Levels and Grotrian Diagrams", Vol. 1, North Holland, Amsterdam 1975

[9] M. S. Dimitrijević and S. Sahal-Bréchot, JQSRT 31, 301 (1984)

[10] M. S. Dimitrijević and S. Sahal-Bréchot, Astron. Astrophys. Suppl. Series, submitted 1997

[11] M. S. Dimitrijević and S. Sahal-Bréchot, Bull. Astron. Belgrade 156, in press (1997)

[12] H. R. Griem, "Spectral Line Broadening by Plasmas", Academic Press, New York 1974

[13] S. Sahal-Bréchot, Astron. Astrophys. 245, 322 (1991) 
Table 1: Electron- and proton-impact broadening full half-widths (FWHM) and shifts for Ca IX for a perturber density of $10^{18} \mathrm{~cm}^{-3}$ and temperatures from 200,000 to 2,000,000 K. By dividing $\mathrm{C}$ with the full linewidth, we obtain an estimate for the maximum perturber density for which the line may be treated as isolated and tabulated data may be used.

\begin{tabular}{llllll}
\hline \hline Perturber & Density & $=1 . \mathrm{E}+18 \mathrm{~cm}^{-3}$ & & & \\
Perturbers & are: & Electrons & & \\
Transition & $\mathrm{T}(\mathrm{K})$ & Width $(\AA)$ & Shift $(\AA)$ & Width $(\AA)$ & Shift $(\AA)$ \\
\hline Ca IX 3P 5S & 200000. & $0.768 \mathrm{E}-03$ & $0.953 \mathrm{E}-04$ & $0.640 \mathrm{E}-04$ & $0.103 \mathrm{E}-03$ \\
$116.1 \mathrm{~A}$ & 500000. & $0.551 \mathrm{E}-03$ & $0.932 \mathrm{E}-04$ & $0.133 \mathrm{E}-03$ & $0.147 \mathrm{E}-03$ \\
$\mathrm{C}=0.29 \mathrm{E}+19$ & 1000000. & $0.439 \mathrm{E}-03$ & $0.893 \mathrm{E}-04$ & $0.178 \mathrm{E}-03$ & $0.178 \mathrm{E}-03$ \\
& 2000000. & $0.355 \mathrm{E}-03$ & $0.766 \mathrm{E}-04$ & $0.231 \mathrm{E}-03$ & $0.211 \mathrm{E}-03$ \\
& 3000000. & $0.314 \mathrm{E}-03$ & $0.671 \mathrm{E}-04$ & $0.262 \mathrm{E}-03$ & $0.228 \mathrm{E}-03$ \\
& 5000000. & $0.269 \mathrm{E}-03$ & $0.562 \mathrm{E}-04$ & $0.318 \mathrm{E}-03$ & $0.254 \mathrm{E}-03$ \\
\hline \hline
\end{tabular}

Table 2: Electron- and proton-impact broadening full half-widths (FWHM) and shifts for Ca X for a perturber density of $10^{18} \mathrm{~cm}^{-3}$ and temperatures from 200,000 to 2,000,000 K. By dividing $\mathrm{C}$ with the full linewidth, we obtain an estimate for the maximum perturber density for which the line may be treated as isolated and tabulated data may be used.

\begin{tabular}{|c|c|c|c|c|c|}
\hline $\begin{array}{l}\text { Perturber } \\
\text { Perturbers } \\
\text { Transition } \\
\end{array}$ & $\begin{array}{l}\text { Density } \\
\text { are: } \\
\mathrm{T}(\mathrm{K}) \\
\end{array}$ & $\begin{array}{l}=1 . \mathrm{E}+18 \mathrm{~cm}^{-3} \\
\text { Electrons } \\
\text { Width }(\AA)\end{array}$ & $\operatorname{Shift}(\AA)$ & $\begin{array}{l}\text { Protons } \\
\text { Width }(\AA)\end{array}$ & $\operatorname{Shift}(\AA)$ \\
\hline Ca X 3S 3P & 200000 . & $0.498 \mathrm{E}-02$ & $-0.601 \mathrm{E}-04$ & $0.302 \mathrm{E}-04$ & $-0.282 \mathrm{E}-04$ \\
\hline $563.1 \mathrm{~A}$ & 500000 & $0.322 \mathrm{E}-02$ & $-0.532 \mathrm{E}-04$ & $0.983 \mathrm{E}-04$ & $-0.676 \mathrm{E}-04$ \\
\hline \multirow[t]{4}{*}{$\mathrm{C}=0.56 \mathrm{E}+21$} & 1000000. & $0.238 \mathrm{E}-02$ & $-0.679 \mathrm{E}-04$ & $0.179 \mathrm{E}-03$ & $-0.113 \mathrm{E}-03$ \\
\hline & 2000000. & $0.180 \mathrm{E}-02$ & $-0.605 \mathrm{E}-04$ & $0.264 \mathrm{E}-03$ & $-0.160 \mathrm{E}-03$ \\
\hline & 3000000 . & $0.155 \mathrm{E}-02$ & $-0.581 \mathrm{E}-04$ & $0.303 \mathrm{E}-03$ & $-0.188 \mathrm{E}-03$ \\
\hline & 5000000. & $0.130 \mathrm{E}-02$ & $-0.561 \mathrm{E}-04$ & $0.351 \mathrm{E}-03$ & $-0.214 \mathrm{E}-03$ \\
\hline Ca X 3S 4P & 200000 & $0.481 \mathrm{E}-03$ & $0.314 \mathrm{E}-05$ & $0.138 \mathrm{E}-04$ & \\
\hline $111.0 \mathrm{~A}$ & 500000 . & $0.321 \mathrm{E}-03$ & $0.343 \mathrm{E}-05$ & $0.279 \mathrm{E}-04$ & $0.438 \mathrm{E}-05$ \\
\hline \multirow[t]{4}{*}{$\mathrm{C}=0.84 \mathrm{E}+19$} & 1000000 . & $0.244 \mathrm{E}-03$ & $0.303 \mathrm{E}-05$ & $0.383 \mathrm{E}-04$ & $0.691 \mathrm{E}-05$ \\
\hline & 2000000. & $0.191 \mathrm{E}-03$ & $\mathrm{E}-05$ & $0.441 \mathrm{E}-04$ & $E-05$ \\
\hline & 3000000 . & $0.168 \mathrm{E}-03$ & E-05 & $\mathrm{E}-04$ & $0.107 \mathrm{E}-04$ \\
\hline & 5000000. & $0.145 \mathrm{E}-03$ & $\mathrm{E}-05$ & E-04 & $0.123 \mathrm{E}-04$ \\
\hline Ca X 3S 5P & 200000 & $0.571 \mathrm{E}-03$ & -05 & -04 & -05 \\
\hline $82.8 \mathrm{~A}$ & 500000 & $0.395 \mathrm{E}-03$ & -04 & E-04 & $\mathrm{E}-04$ \\
\hline \multirow[t]{4}{*}{$\mathrm{C}=0.22 \mathrm{E}+19$} & 1000000. & $0.310 \mathrm{E}-03$ & -04 & $0.666 \mathrm{E}-04$ & $0.208 \mathrm{E}-04$ \\
\hline & 2000 & $0.250 \mathrm{E}-03$ & 0.99 & -04 & 0.249 \\
\hline & 3000000 . & $0.224 \mathrm{E}-03$ & 0.910 & $E-04$ & $0.275 \mathrm{E}-04$ \\
\hline & 5000000. & $0.196 \mathrm{E}-03$ & $0.773 \mathrm{E}-05$ & $0.890 \mathrm{E}-04$ & $0.310 \mathrm{E}-04$ \\
\hline Ca X 3S 6P & 200000 & $0.858 \mathrm{E}-03$ & $0.227 \mathrm{E}-04$ & $0.830 \mathrm{E}-04$ & $0.230 \mathrm{E}-04$ \\
\hline $73.2 \mathrm{~A}$ & 500000 & $0.614 \mathrm{E}-03$ & $0.238 \mathrm{E}-04$ & $0.111 \mathrm{E}-03$ & $0.358 \mathrm{E}-04$ \\
\hline \multirow[t]{4}{*}{$\mathrm{C}=0.97 \mathrm{E}+18$} & 1000000. & $0.494 \mathrm{E}-03$ & $0.221 \mathrm{E}-04$ & $0.126 \mathrm{E}-03$ & $0.432 \mathrm{E}-04$ \\
\hline & 2000000. & $0.408 \mathrm{E}-03$ & $0.206 \mathrm{E}-04$ & $0.142 \mathrm{E}-03$ & $0.515 \mathrm{E}-04$ \\
\hline & 3000000 . & $0.369 \mathrm{E}-03$ & $0.185 \mathrm{E}-04$ & $0.151 \mathrm{E}-03$ & $0.573 \mathrm{E}-04$ \\
\hline & 5000000. & $0.327 \mathrm{E}-03$ & $0.151 \mathrm{E}-04$ & $0.164 \mathrm{E}-03$ & $0.632 \mathrm{E}-04$ \\
\hline $\mathrm{Ca} \mathrm{X} 4 \mathrm{~S} 4 \mathrm{P}$ & 200000 & 0.103 & $-0.161 \mathrm{E}-02$ & $0.259 \mathrm{E}-02$ & $-0.175 \mathrm{E}-02$ \\
\hline $1475.2 \mathrm{~A}$ & 500000 . & $0.698 \mathrm{E}-01$ & $-0.234 \mathrm{E}-02$ & $0.534 \mathrm{E}-02$ & $-0.336 \mathrm{E}-02$ \\
\hline
\end{tabular}




\title{
Accurate MCHF/MCDF Transition Rates
}

\author{
Charlotte Froese Fischer ${ }^{a}$, Per Jönsson ${ }^{a}$, and Stephan Fritzsche ${ }^{b}$ \\ ${ }^{a}$ Vanderbilt University, Nashville, TN 37235, USA \\ ${ }^{b}$ Universität Kassel, Kassel, Germany
}

\section{Introduction}

With today's powerful workstations and high performance architectures, it has been possible to improve considerably the accuracy to which atomic properties can be predicted. This is done by using systematic methods, where wave functions for a series of models including more and more physical effects are obtained from expansions defined in terms of an orbital basis of increasing size. Typically, the first model is one that includes only valence correlation. But often core-polarization is also important in which case a core-valence calculation is need. In some instances, even core-core correlation produces a small correction. By monitoring convergence within the different models and comparing the converged results themselves, an uncertainty estimate can be obtained. These methods can be used both for non-relativistic MCHF calculations where Breit-Pauli corrections are added in configuration interaction (CI) calculations, or for MCDF calculations starting with the Dirac-Coulomb Hamiltonian, adding Breit and leading QED corrections in relativistic CI.

\section{Iso-electronic Sequences}

A number of iso-electronic sequences have been explored using MCDF. In fact, the recently implemented non-orthogonal methodology allowing for separately optimized initial and final state wave functions was first tested on the $\mathrm{Mg}$-like sequence [1].

The same MCDF procedures have been applied to the study of the Be-like E1 transitions $2 s^{2}{ }^{1} S-2 s 2 p{ }^{1,3} P_{1}$. For low- $Z$, the transition rates in Babushkin (length) and Coulomb (velocity) gauges do not show good agreement $[2,3]$, though in C III the former is in excellent agreement with Breit-Pauli [4] and recent experiment [5]. Table 1 shows some results for the sequence. For the intercombination line (IC) the difference in gauges is decreasing with $Z$ whereas for the allowed resonance transition, the difference is only one or two units in the fourth decimal place for all $Z$. A detailed analysis (in preparation) also shows that the frequency dependent Breit correction is important for high- $Z$.

Other sequences investigated recently using MCHF with Breit-Pauli are the $2 s^{2}{ }^{1} S-2 s 3 p{ }^{1,3} P_{1}$ allowed and intercombination lines for $Z \leq 10[6,7]$. The experimental data for these transition rates have been analyzed semi-empirically [8] and values predicted for the sequence. Except for $\mathrm{N}$ IV, our computed values are in closer agreement to the semi-empirical predictions than experiment itself.

\section{Spectrum Calculations}

Many variational studies have concentrated on accurate, single line, benchmark calculations. The $\mathrm{MCHF}+\mathrm{BP}$ and MCDF programs also permit the study of portions of a spectrum. In the latter, 
Table 1: Transition energies (in $\mathrm{cm}^{-1}$ ) and rates (in s ${ }^{-1}$ ) for the $2 s^{2}{ }^{1} S_{0}-2 s 2 p{ }^{3} P_{1}$ intercombination (IC) and $2 s^{2}{ }^{1} S_{0}-2 s 2 p^{1} P_{1}$ allowed dipole (E1) lines in the Be isoelectronic sequence. In the table $[\mathrm{x}]$ denotes powers of ten.

\begin{tabular}{llcccccc}
\hline \hline$Z$ & & $\Delta E_{I C}$ & $A_{I C}^{l}$ & $A_{I C}^{v}$ & $\Delta E_{E 1}$ & $g f_{l}$ & $g f_{v}$ \\
\hline \hline 7 & MCDF & 67301 & $5.586[2]$ & $8.597[2]$ & 131029 & 0.6113 & 0.6118 \\
& Exp & 67272 & & & 130694 & & \\
8 & MCDF & 82104 & $2.212[3]$ & $3.074[3]$ & 159154 & 0.5122 & 0.5124 \\
& Exp & 82079 & & & 158798 & & \\
9 & MCDF & 96899 & $6.961[3]$ & $9.041[3]$ & 187224 & 0.4414 & 0.4416 \\
& Exp & 96861 & & & 186841 & & \\
10 & MCDF & 111730 & $1.862[4]$ & $2.305[4]$ & 215350 & 0.3882 & 0.3884 \\
& Exp & 111705 & & & 214952 & & \\
11 & MCDF & 126640 & $4.410[4]$ & $5.275[4]$ & 243625 & 0.3470 & 0.3471 \\
& Exp & 126612 & & & 243208 & & \\
12 & MCDF & 141662 & $9.508[4]$ & $1.118[5]$ & 272133 & 0.3141 & 0.3142 \\
& Exp & 141631 & & & 271687 & & \\
$\vdots$ & & & & & & & \\
24 & MCDF & 341252 & $2.772[7]$ & $2.824[7]$ & 667962 & 0.1623 & 0.1623 \\
& Exp & 341120 & & & 667150 & & \\
25 & MCDF & 360178 & $3.773[7]$ & $3.834[7]$ & 709557 & 0.1578 & 0.1578 \\
& Exp & 359970 & & & 708770 & & \\
26 & MCDF & 379363 & $5.041[7]$ & $5.118[7]$ & 753366 & 0.1539 & 0.1540 \\
& Exp & 379130 & & & 752502 & & \\
27 & MCDF & 398983 & $6.631[7]$ & $6.721[7]$ & 799649 & 0.1505 & 0.1505 \\
& Exp & 398720 & & & 799040 & & \\
28 & MCDF & 418964 & $8.583[7]$ & $8.689[7]$ & 848677 & 0.1474 & 0.1476 \\
& Exp & 418720 & & & 847494 & & \\
\hline \hline
\end{tabular}

orbitals may be optimized for a series of levels. Using this option, the $3 s^{2} 3 p^{3}, 3 s 3 p^{4}, 3 s^{2} 3 p^{2} 3 d$ levels of selected elements of the iso-electronic sequence in the range $22 \leq Z \leq 32$ were computed along with E1 transitions between these levels [9]. Some lifetimes for levels of Fe XII, which all show remarkable agreement in length and velocity forms, are reported in Table 2.

In Table 3 we report excitation energies of selected levels from Fe XII ions, comparing them with previous theory and experiment. When both are available, the present MCDF results are generally in better agreement with experiment.

\section{Complex Systems}

The MCHF+BP and MCDF programs have also been applied to the study of forbidden transitions among the twelve (12) lowest levels of $3 d^{5}$ of Fe IV. A re-examination of the data for these levels was suggested by Rubin et al [11] as a result of an analysis of the the flux of Fe IV $\left(3 d^{5}{ }^{4} P_{5 / 2}-3 d^{5}{ }^{6} S_{5 / 2}\right)$ in the Orion Nebula, using the Goddard High-Resolution Spectrograph. From a theoretical point of view, an accurate $a b$ initio calculation is extremely complex. The MCHF+BP approach was restricted to the mixing of the four lowest terms, ${ }^{6} S,{ }^{4} G,{ }^{4} P$, and ${ }^{4} D$, each with a correlated expansion. The resulting Breit-Pauli calculations required almost four hours on a Cray T3E computer with 32 nodes. Still missing, was the mixing with other quartets and the doublets. In the MCDF approach, the expansions grow in size rapidly with the orbital set (there being more relativistic 
Table 2: Excitation Energies and Lifetimes of levels of $3 s 3 p^{4}$

\begin{tabular}{|c|c|c|c|c|c|}
\hline \multicolumn{2}{|c|}{ Fe XII Levels } & \multicolumn{2}{|c|}{ Energy $\left(\mathrm{cm}^{-1}\right)$} & \multicolumn{2}{|c|}{ Lifetime (s) } \\
\hline No. & $J^{P}$ & Calc. & Exp. & Length & Velocity \\
\hline 6 & $5 / 2+$ & 274620 & 274373 & $6.11(-10)$ & $6.17(-10)$ \\
\hline 7 & $3 / 2+$ & 284131 & 284005 & $5.70(-10)$ & $5.78(-10)$ \\
\hline 8 & $1 / 2+$ & 288431 & 288307 & $5.40(-10)$ & $5.49(-10)$ \\
\hline 9 & $3 / 2+$ & 341076 & 340020 & $2.75(-10)$ & $2.78(-10)$ \\
\hline 10 & $5 / 2+$ & 342949 & 341703 & $3.02(-10)$ & $3.05(-10)$ \\
\hline
\end{tabular}

Table 3: Excitation energies for a few selected levels of the $3 s^{2} 3 p^{3}, 3 s 3 p^{4}$, and $3 s^{2} 3 p^{2} 3 d$ configurations for $\mathrm{Fe}$ XII ions. All energies $\left(\right.$ in $\mathrm{cm}^{-1}$ ) are displayed with respect to the $3 s^{2} 3 p^{3}{ }^{4} S_{3 / 2}$ ground-state level.

\begin{tabular}{rrrrrrr}
\hline Level & $\begin{array}{c}\text { Designation } \\
\text { of states }\end{array}$ & $J^{P}$ & $\begin{array}{c}\text { Huang et al. } \\
\text { Ref. 9 }\end{array}$ & $\begin{array}{r}\text { Fawcett } \\
\text { Ref. 10 }\end{array}$ & This work & Experiment \\
\hline 1 & $3 s^{2} 3 p^{3}{ }^{4} S$ & $3 / 2-$ & 0 & 0 & 0 & 0 \\
2 & ${ }^{2} D$ & $3 / 2-$ & 45375 & & 42667 & $41555 \pm 1$ \\
3 & ${ }^{2} D$ & $5 / 2-$ & 49932 & & 47130 & $46088 \pm 1$ \\
4 & ${ }^{2} P$ & $1 / 2-$ & 78297 & & 75532 & $74108 \pm 1$ \\
5 & ${ }^{2} P$ & $3 / 2-$ & 84503 & & 81792 & \\
6 & $3 s 3 p^{4}{ }^{4} P$ & $5 / 2+$ & 274625 & 274344 & 274620 & \\
7 & ${ }^{4} P$ & $3 / 2+$ & 284157 & 283556 & 284131 & \\
8 & ${ }^{4} P$ & $1 / 2+$ & 288345 & 287737 & 288431 & \\
9 & ${ }^{2} D$ & $3 / 2+$ & 345263 & & 341076 & $339761 \pm 10$ \\
10 & ${ }^{2} D$ & $5 / 2+$ & 347176 & & 342949 & \\
21 & $3 s^{2} 3 p^{2} 3 d^{4} D$ & $5 / 2+$ & 460430 & 453305 & 454327 & \\
22 & ${ }^{4} D$ & $7 / 2+$ & 470196 & & 463593 & \\
23 & ${ }^{2} G$ & $7 / 2+$ & 509637 & & 496836 & \\
24 & $3 s 3 p^{4} P$ & $3 / 2+$ & 516954 & & 507969 & \\
25 & $3 s^{2} 3 p^{2} 3 d^{4} P$ & $5 / 2+$ & 528771 & 512530 & 517607 & $513708 \pm 10$ \\
\hline \hline
\end{tabular}


orbitals) and so the correlation effects had to be restricted. Only after the inclusion of the Breit correction were computed levels in the observed order. Thus spin-orbit alone cannot predict the energy structure. The transition rates for transitions within these levels, vary over orders of magnitude. There appears to be good agreement between our theories for some transitions, particularly for the M1 transitions where the $\Delta S=0$ and $\Delta L=0$ selection rules are obeyed.

Heavy atoms also are of great interest. They differ from light atoms in that a fully relativistic Dirac-Fock-Breit formalism may be necessary and correlation exhibited by the mixing of configuration states is very strong. We are currently extending the MCDF codes for larger, more efficient correlation studies. At the same time, open $f$-shells appear. Correlation studies of such states in MCDF will be extremely difficult because of the size of the expansions that occur in $j j$-coupling. In fact, only the core electrons are "relativistic": it may be possible to describe the outer electrons in the Breit-Pauli scheme. This approach is being investigated. The MCHF package has been extended to include an arbitrary number of $f$ electrons. For this purpose, an efficient method is used for finding algebraic expressions for matrix elements. It is based on second quantization in the coupled tensorial form, angular momentum theory in three spaces (orbital, spin and quasispin), and a generalized graphical technique [13]. Coefficients of fractional parentage are replaced by the far fewer "reduced" coefficients of fractional parentage. Codes based on this formalism have been found to be, on average, 2-6 times faster.

This research was supported by the Division of Chemical Sciences, Office of Basic Energy Scinces, Office of Energy Science, U.S. Department of Energy.

\section{References}

[1] P. Jönsson and C. Froese Fischer, J. Phys. B (accepted)

[2] P. Jönsson and C. Froese Fischer, Phys. Rev. A (submitted)

[3] S. Fritzsche, A.-M. Mårtensson-Pendrill, and B. Fricke, in Proceedings of the 5th Int. Colloq. on Atomic Spectra and Oscillator Strengths, Meudon, France, 1995, ed. by W.-Ü.L. TchangBrillet, J.F. Wyart, and C.J. Zeippen, p. 70

[4] C. Froese Fischer and G. Gaigalas, Phys. Scripta 56, 436 (1997)

[5] J. Doerfert, E. Träbert, A. Wolf, D. Schwalm, and O. Uwira, Phys. Rev. Lett. 78, 4355 (1997)

[6] C. Froese Fischer, M. R. Godefroid, and J. Olsen, J. Phys. B 30, 1163 (1997)

[7] C. Froese Fischer, G. Gaigalas, and M. R. Godefroid, J. Phys. B 30, 3333 (1997)

[8] L. J. Curtis, S. T. Maniak, R. W. Ghrist, R. E. Irving, D. G. Ellis, M. Henderson, M. H. Kacher, E. Träbert, J. Granzow, P. Bengtsson, and L. Engström, Phys. Rev. A 51, 4575 (1995)

[9] S. Fritzsche, C. Froese Fischer, and B. Fricke, Atomic and Nuclear Data Tables (accepted)

[10] K.-N. Huang, At. Data Nucl. Data Tables 30, 313 (1984)

[11] B. C. Fawcett, At. Data Nucl. Data Tables 35, 203 (1986)

[12] R. H. Rubin et al., Astrophys. J. 474, L131 (1997)

[13] G. Gaigalas, Z. Rudzikas, C. Froese Fischer, J. Phys. B 30, 3747 (1997) 


\title{
Secondly Quantized Multi-Configurational Approach for Atomic Databases
}

\author{
Gediminas Gaigalas and Zenonas Rudzikas \\ Institute of Theoretical Physics and Astronomy, \\ A. Goštauto 12, Vilnius 2600, Lithuania
}

\section{Introduction}

Studies of the structure of atoms and ions (ultracold to relativistic included) as well as their twobody interactions with photons, electrons and other particles require accurate methods for the description of such objects.

In order to obtain accurate values of atomic quantities it is necessary to account for relativistic and correlation effects. Relativistic effects may be taken into account as Breit-Pauli corrections or in a fully relativistic approach. In both cases for complex atoms and ions, a considerable part of the effort must be devoted to integrations over spin-angular variables, occurring in the matrix elements of the operators under consideration.

Many existing codes for integrating are based on a scheme by Fano [1]. The integrations over spin-angular variables in this case constitute a considerable part of the problem, especially when atoms with many open shells are treated, and the operators are not trivial. In the papers of Gaigalas et al [2,3], an efficient approach for finding matrix elements of any one- and two-particle atomic operator between complex configurations is suggested. It is free of shortcomings of previous approaches. This approach allows one to generate fairly accurate databases of atomic parameters (Froese Fischer et al $[4,5]$ ).

Further development of the approaches by Gaigalas et al $[2,3]$ for the spin-spin and spin-otherorbit relativistic corrections in the Breit-Pauli approximation is presented in this paper.

\section{Matrix Elements Between Complex Configurations}

According to the approach of Gaigalas et al [3], a general expression of the submatrix element for any two-particle operator between functions with $u$ open shells can be written as follows:

$$
\begin{gathered}
\left(\psi_{u}^{b r a}(L S)\|G\| \psi_{u}^{k e t}\left(L^{\prime} S^{\prime}\right)\right)= \\
=\sum_{n_{i} l_{i}, n_{j} l_{j}, n_{i}^{\prime} l_{i}^{\prime}, n_{j}^{\prime} l_{j}^{\prime}}\left(\psi_{u}^{b r a}(L S)\left\|\hat{G}\left(n_{i} l_{i}, n_{j} l_{j}, n_{i}^{\prime} l_{i}^{\prime}, n_{j}^{\prime} l_{j}^{\prime}\right)\right\| \psi_{u}^{k e t}\left(L^{\prime} S^{\prime}\right)\right)= \\
\sum_{n_{i} l_{i}, n_{j} l_{j}, n_{i}^{\prime} l_{i}^{\prime}, n_{j}^{\prime} l_{j}^{\prime} \kappa_{12}, \sigma_{12}, \kappa_{12}^{\prime}, \sigma_{12}^{\prime}} \sum(-1)^{\Delta} \Theta^{\prime}\left(n_{i} \lambda_{i}, n_{j} \lambda_{j}, n_{i}^{\prime} \lambda_{i}^{\prime}, n_{j}^{\prime} \lambda_{j}^{\prime}, \Xi\right) \times T\left(n_{i} \lambda_{i}, n_{j} \lambda_{j}, n_{i}^{\prime} \lambda_{i}^{\prime}, n_{j}^{\prime} \lambda_{j}^{\prime}, \Lambda^{\text {bra }}, \Lambda^{k e t}, \Xi, \Gamma\right) \times \\
\times R\left(\lambda_{i}, \lambda_{j}, \lambda_{i}^{\prime}, \lambda_{j}^{\prime}, \Lambda^{\text {bra }}, \Lambda^{k e t}, \Gamma\right), \\
\times \\
\times
\end{gathered}
$$

where $\Lambda^{b r a} \equiv\left(L_{i} S_{i}, L_{j} S_{j}, L_{i}^{\prime} S_{i}^{\prime}, L_{j}^{\prime} S_{j}^{\prime}\right)^{b r a}$ is the array of bra function shells' terms, and similarly for $\Lambda^{k e t}$ and $\lambda \equiv l s$. 
Thus, to calculate the spin-angular part of a submatrix element of this type, one has to obtain:

1. The recoupling matrix $R\left(\lambda_{i}, \lambda_{j}, \lambda_{i}^{\prime}, \lambda_{j}^{\prime}, \Lambda^{b r a}, \Lambda^{k e t}, \Gamma\right)$, which has an analytical expression in terms of just $6 \mathrm{j}$ - and $9 \mathrm{j}$-coefficients.

2. Submatrix elements $T\left(n_{i} \lambda_{i}, n_{j} \lambda_{j}, n_{i}^{\prime} \lambda_{i}^{\prime}, n_{j}^{\prime} \lambda_{j}^{\prime}, \Lambda^{b r a}, \Lambda^{k e t}, \Xi, \Gamma\right)$ for tensorial products of creation/annihilation operators that act upon a particular electron shell. So, all the advantages of tensorial algebra and quasispin formalism (Rudzikas [6]) may be efficiently exploited in the process of their calculation.

3. The phase factor $\Delta$.

4. $\Theta^{\prime}\left(n_{i} \lambda_{i}, n_{j} \lambda_{j}, n_{i}^{\prime} \lambda_{i}^{\prime}, n_{j}^{\prime} \lambda_{j}^{\prime}, \Xi\right)$ which is proportional to the two-electron submatrix element of operator $\widehat{G}$.

Further development of this approach for the spin-spin and spin-other-orbit relativistic corrections in the Breit-Pauli approximation is presented in the following section.

\section{The Spin-Spin and Spin-Other-Orbit Operators}

The spin-spin operator $H^{s s}$ itself contains tensorial structure of two different types, summed over $k$ :

$$
H^{s s} \equiv \sum_{k}\left[H_{s s}^{(k+1 k-12,112)}+H_{s s}^{(k-1 k+12,112)}\right] .
$$

Their submatrix elements are:

$$
\begin{aligned}
& \left(n_{i} \lambda_{i} n_{j} \lambda_{j}\left\|H_{s s}^{(k+1 k-12,112)}\right\| n_{i^{\prime}} \lambda_{i^{\prime}} n_{j^{\prime}} \lambda_{j^{\prime}}\right)=\frac{3}{\sqrt{5}} \sqrt{(2 k+3)^{(5)}} \times \\
& \times\left(l_{i}\left\|C^{(k+1)}\right\| l_{i^{\prime}}\right)\left(l_{j}\left\|C^{(k-1)}\right\| l_{j^{\prime}}\right) N^{k-1}\left(n_{i} l_{i} n_{j} l_{j}, n_{i^{\prime}} l_{i^{\prime}} n_{j^{\prime}} l_{j^{\prime}}\right) \text {, } \\
& \left(n_{i} \lambda_{i} n_{j} \lambda_{j}\left\|H_{s s}^{(k-1 k+12,112)}\right\| n_{i^{\prime}} \lambda_{i^{\prime}} n_{j^{\prime}} \lambda_{j^{\prime}}\right)=\frac{3}{\sqrt{5}} \sqrt{(2 k+3)^{(5)}} \times \\
& \times\left(l_{i}\left\|C^{(k-1)}\right\| l_{i^{\prime}}\right)\left(l_{j}\left\|C^{(k+1)}\right\| l_{j^{\prime}}\right) N^{k-1}\left(n_{j} l_{j} n_{i} l_{i}, n_{j^{\prime}} l_{j^{\prime}} n_{i^{\prime}} l_{i^{\prime}}\right),
\end{aligned}
$$

where we use a shorthand notation $(2 k+3)^{(5)} \equiv(2 k+3)(2 k+2)(2 k+1)(2 k)(2 k-1)$ and radial integral in (3), (4) is defined as in Glass and Hibbert [7]:

$$
\begin{aligned}
& N^{k}\left(n_{i} l_{i} n_{j} l_{j}, n_{i^{\prime}} l_{i^{\prime}} n_{j^{\prime}} l_{j^{\prime}}\right)= \\
& =\frac{\alpha^{2}}{4} \int_{0}^{\infty} \int_{0}^{\infty} P_{i}\left(r_{1}\right) P_{j}\left(r_{2}\right) \frac{r_{2}^{k}}{r_{1}^{k+3}} \epsilon\left(r_{1}-r_{2}\right) P_{i^{\prime}}\left(r_{1}\right) P_{j^{\prime}}\left(r_{2}\right) d r_{1} d r_{2,},
\end{aligned}
$$

where $\epsilon(x)$ is a Heaviside step-function, 


$$
\epsilon(x)= \begin{cases}1 ; & \text { for } x>0 \\ 0 ; & \text { for } x \leq 0 .\end{cases}
$$

The spin-other-orbit operator $H^{\text {soo }}$ itself contains tensorial structure of six different types, summed over $k$ :

$$
H^{s s o} \equiv \sum_{k}\left[H_{\text {soo }}^{(k-1 k 1,101)}+H_{\text {soo }}^{(k-1 k 1,011)}+H_{\text {soo }}^{(k k 1,101)}+H_{\text {soo }}^{(k k 1,011)}+H_{\text {soo }}^{(k+1 k 1,101)}+H_{\text {soo }}^{(k+1 k 1,011)}\right]
$$

Their submatrix elements are:

$$
\begin{aligned}
& \left(n_{i} \lambda_{i} n_{j} \lambda_{j}\left\|H_{\text {soo }}^{\left(k-1 k 1, \sigma_{1} \sigma_{2} 1\right)}\right\| n_{i^{\prime}} \lambda_{i^{\prime}} n_{j^{\prime}} \lambda_{j^{\prime}}\right)=2 \cdot\left(\sigma_{1}+2 \sigma_{2}\right)\{(2 k-1)(2 k+1) \times \\
& \left.\times\left(l_{i}+l_{i^{\prime}}-k+1\right)\left(k-l_{i}+l_{i^{\prime}}\right)\left(k+l_{i}-l_{i^{\prime}}\right)\left(k+l_{i}+l_{i^{\prime}}+1\right)\right\}^{1 / 2} \times \\
& \times(k)^{-1 / 2}\left(l_{i}\left\|C^{(k)}\right\| l_{i^{\prime}}\right)\left(l_{j}\left\|C^{(k)}\right\| l_{j^{\prime}}\right) N^{k-2}\left(n_{j} l_{j} n_{i} l_{i}, n_{j^{\prime}} l_{j^{\prime}} n_{i^{\prime}} l_{i^{\prime}}\right) \text {, } \\
& \left(n_{i} \lambda_{i} n_{j} \lambda_{j}\left\|H_{s o o}^{\left(k k 1, \sigma_{1} \sigma_{2} 1\right)}\right\| n_{i^{\prime}} \lambda_{i^{\prime}} n_{j^{\prime}} \lambda_{j^{\prime}}\right)=-2 \cdot\left(\sigma_{1}+2 \sigma_{2}\right)(2 k+1)^{1 / 2}\left(l_{i}\left\|C^{(k)}\right\| l_{i^{\prime}}\right) \times \\
& \times\left(l_{j}\left\|C^{(k)}\right\| l_{j^{\prime}}\right)\left\{(k(k+1))^{-1 / 2}\left(l_{i}\left(l_{i}+1\right)-k(k+1)-l_{i^{\prime}}\left(l_{i^{\prime}}+1\right)\right) \times\right. \\
& \times\left\{(k+1) N^{k-2}\left(n_{j} l_{j} n_{i} l_{i}, n_{j^{\prime}} l_{j^{\prime}} n_{i^{\prime}} l_{i^{\prime}}\right)-k N^{k}\left(n_{i} l_{i} n_{j} l_{j}, n_{i^{\prime}} l_{i^{\prime}} n_{j^{\prime}} l_{j^{\prime}}\right)\right\}- \\
& \left.-2(k(k+1))^{1 / 2} V^{k-1}\left(n_{i} l_{i} n_{j} l_{j}, n_{i^{\prime}} l_{i^{\prime}} n_{j^{\prime}} l_{j^{\prime}}\right)\right\} \text {, } \\
& \left(n_{i} \lambda_{i} n_{j} \lambda_{j}\left\|H_{s o o}^{\left(k+1 k 1, \sigma_{1} \sigma_{2} 1\right)}\right\| n_{i^{\prime}} \lambda_{i^{\prime}} n_{j^{\prime}} \lambda_{j^{\prime}}\right)=2 \cdot\left(\sigma_{1}+2 \sigma_{2}\right)\{(2 k+1)(2 k+3) \times \\
& \left.\times\left(l_{i}+l_{i^{\prime}}-k\right)\left(k-l_{i}+l_{i^{\prime}}+1\right)\left(k+l_{i}-l_{i^{\prime}}+1\right)\left(k+l_{i}+l_{i^{\prime}}+2\right)\right\}^{1 / 2} \times \\
& \times(k+1)^{-1 / 2}\left(l_{i}\left\|C^{(k)}\right\| l_{i^{\prime}}\right)\left(l_{j}\left\|C^{(k)}\right\| l_{j^{\prime}}\right) N^{k}\left(n_{i} l_{i} n_{j} l_{j}, n_{i^{\prime}} l_{i^{\prime}} n_{j^{\prime}} l_{j^{\prime}}\right) \text {. }
\end{aligned}
$$

The radial integrals in (8)-(10) are (see Glass and Hibbert [7]):

$$
\begin{aligned}
& V^{k}\left(n_{i} l_{i} n_{j} l_{j}, n_{i^{\prime}} l_{i^{\prime}} n_{j^{\prime}} l_{j^{\prime}}\right)= \\
& =\frac{\alpha^{2}}{4} \int_{0}^{\infty} \int_{0}^{\infty} P_{i}\left(r_{1}\right) P_{j}\left(r_{2}\right) \frac{r_{<}^{k-1}}{r_{>}^{k+2}} r_{2} \frac{\partial}{\partial r_{1}} P_{i^{\prime}}\left(r_{1}\right) P_{j^{\prime}}\left(r_{2}\right) d r_{1} d r_{2} \text {. }
\end{aligned}
$$

Now we have all we need (the operators for tensorial structure and their submatrix elements) for obtaining the value of a matrix element of these operators for any number of open shells in bra and ket functions. This lets us exploit all advantages of the approach by Gaigalas et al [3].

The spin-spin and spin-other-orbit operators itself generally contain tensorial structure of several different types. Therefore the expression (1) must be used separately for each possible tensorial structure for performing spin-angular integrations according to [3]. Each type of tensorial structure is associated with a different type of recoupling matrix $R\left(\lambda_{i}, \lambda_{j}, \lambda_{i}^{\prime}, \lambda_{j}^{\prime}, \Lambda^{\text {bra }}, \Lambda^{k e t}, \Gamma\right)$ and with different matrix elements of standard tensorial quantities $T\left(n_{i} \lambda_{i}, n_{j} \lambda_{j}, n_{i}^{\prime} \lambda_{i}^{\prime}, n_{j}^{\prime} \lambda_{j}^{\prime}, \Lambda^{b r a}, \Lambda^{k e t}, \Xi, \Gamma\right)$. 


\section{Conclusions}

The tensorial forms of the general secondly quantized spin-spin interaction operator (2) and spinother-orbit interaction operator (7) and its submatrix elements ( for spin-spin interaction expressions (3), (4) and for spin-other-orbit expressions (8), (9) and (10)) are presented. In calculating its matrix elements between functions with $u$ open shells this allows to exploit all the advantages of method by Gaigalas et al [3]:

1. to obtain both diagonal and off-diagonal elements with respect to the configuration matrix elements in a unified approach,

2. to use in practical applications the tables of submatrix elements of standard quantities, which here are both the coordinate representation and the occupation number representation tensorial operators,

3. to apply the quasispin formalism for the occupation numbers parts and make use of it,

4. to make use of having recoupling matrices simpler than in other known approaches.

\section{References}

[1] U. Fano, Phys. Rev. A 67, 140 (1965)

[2] G. A. Gaigalas and Z. B. Rudzikas, J. Phys. B 29, 3303 (1996)

[3] G. A. Gaigalas, Z. B. Rudzikas, and C. Froese-Fischer, J. Phys. B 30, 3747 (1997)

[4] C. Froese-Fischer, A. Ynnerman, and G. Gaigalas, Physical Review A 51, 4611 (1995)

[5] C. Froese-Fischer and G. Gaigalas, J. Phys. B 29, 1169 (1996)

[6] Z. B. Rudzikas, "Theoretical Atomic Spectroscopy", p. 410, Cambridge University Press, Cambridge 1997

[7] R. Glass and A. Hibbert, Comput. Phys. Commun. 16, 19 (1978) 


\title{
Polarization Profile Calculations for Plasma Diagnostics
}

\author{
Manuel Ángel González and Marco Antonio Gigosos \\ Departamento de Óptica, Universidad de Valladolid, \\ 47071 Valladolid, Spain
}

\section{Introduction}

Computer simulation techniques have been used to calculate Stark broadened profiles that can be measured by polarization spectroscopy for the two-photon transition $n=2 \rightarrow n=1$ (Lyman- $\alpha$ ) of hydrogen. The plasma model and the calculation techniques used in the simulation are described in Ref.[1]. The fine structure of the level $n=2$ has been taken into account in order to study plasmas at very low electron densities (lower than $10^{21} \mathrm{~m}^{-3}$ ). The calculations include plasmas of pure hydrogen, pure deuterium, as well as each of these with heavier perturbers. Also considered has been the -academic- case of static perturber ions. This allows us to evaluate the effects of ion dynamics, which, for this spectral line, are of fundamental importance. The plasma conditions considered cover the range for electron densities between $4.6 \times 10^{19}$ and $1.0 \times 10^{23} \mathrm{~m}^{-3}$ and for temperatures between 2,000 and $100,000 \mathrm{~K}$.

The computer simulation allows us to directly obtain the emission and absorption profiles for the two-photon transition between the levels $n=2$ and $n=1$ of hydrogen. The polarization profiles can be obtained from these profiles using the Kramers-Kronig relations (see p.459 of refs.[2] and [3]). The calculational technique used allows us to obtain the emission and polarization spectra simultaneously for the diagnosis of different plasma configurations. All in all, spectra have been obtained for more than 400 conditions of density, temperature and perturber mass.

\section{Polarization Spectroscopy}

Polarization spectroscopy is based on the measurement of the change of the polarization state of a probe wave when it passes through a studied medium [2]. Such a change is induced by a polarized pumping wave. This pumping wave changes the optical behavior of the substance. That is, it changes its refractive index and its absorption coefficient in a non-isotropic way. Up to a point, polarization spectroscopy is similar to saturation spectroscopy, which is based on the measurement of the absorption of a probe wave that passes through a substance whose optical response is changed by a pumping beam.

In the experiment studied in this work, the probe beam detects the induced susceptibility by a two-photon transition $[3,4,5,6]$ between the hydrogen atom levels of the Lyman- $\alpha$ transition. The pumping beam produces a transition from the state $1 s$ to the virtual state $l=1, m=1$ ( $p$ state) because it is a $\sigma^{+}$polarized beam, which gives a different saturation of the three possible states of that virtual level: only the $m=1$ state is saturated. This is equivalent to an anisotropy in the medium, because there is no uniform population between the different $m$ sublevels. In the same process, the probe beam is absorbed, producing a transition between the virtual level and the $n=$ 2 level. The selection rule $|\Delta l|=1$ forces the arriving level to be the $2 s$. one, because there is no level with $l=2$ in that energy interval. 


\section{Calculation process of the polarization profiles}

The profile $A(\omega)$ of a spectral line can be readily obtained from the autocorrelatian function of the atomic dipole moment by means of a Fourier transform of its average \{\} over a certain statistical ensemble [7]

$$
A(\omega)=\frac{1}{\pi} \operatorname{Re} \int_{0}^{\infty} e^{i \omega t}\{C(t)\}, \quad C(t)=\operatorname{tr}\left(\mathbb{D} \cdot U^{+}(t) \mathbf{D} U(t)\right)
$$

The time development operator $U(t)$ obeys the following form of the Schrödinger equation under the no-quenching approximation:

$$
\begin{aligned}
\operatorname{ih} \frac{d}{d t} U(t) & =\left(H_{0}+V(t)\right) U(t), \\
V(t) & =q \mathbf{E}(t) \cdot \mathbf{R},
\end{aligned}
$$

where $H_{0}$ includes the fine structure of the level $n=2 . q \mathbf{R}$ is the dipole momentum operator of the emitter, and $\mathbf{E}(t)$ is the electric microfield generated by ions and electrons surrounding it.

In this work, we are interested in the polarization profiles obtained by a two-photon absorption process. Such a spectrum is given by the addition of the squares of the absorption and the dispersion profiles [2]:

$$
P(\omega)=A(\omega)^{2}+D(\omega)^{2}
$$

The Kramers-Kronig relations allow us to obtain the dispersion profile from the absorption one:

$$
D(\omega)=\frac{1}{\pi} \text { V.P. } \int_{-\infty}^{+\infty} \frac{A(z)}{z-\omega} \mathrm{d} z
$$

Operating on it, it can be shown that

$$
\begin{aligned}
& A(\omega)=\frac{1}{\pi} \int_{0}^{\infty}\left[\cos (\omega t) C_{R}(t)-\sin (\omega t) C_{I}(t)\right] \\
& D(\omega)=\frac{1}{\pi} \int_{0}^{\infty}\left[\cos (\omega t) C_{I}(t)+\sin (\omega t) C_{R}(t)\right]
\end{aligned}
$$

where $C_{R}(t)=\operatorname{Re} C(t)$ and $C_{I}(t)=\operatorname{Im} C(t)$.

In a computer simulation process, a microfield time sequence is obtained for some selected plasma conditions, and the numerical integration of equations (2) is a previous step to the calculation of $\{C(t)\}$.

The simulated plasma is made up of a set of $N_{p}$ independent ions and $N_{p}$ independent electrons placed at random inside a spherical volume in such a way that the homogeneity of the 
spatial distribution of particles is guaranteed. Both electrons and ions move along straight line trajectories with a Maxwellian velocity distribution, as established in the $\mu$-ion model [8]. The emitting atom is located at the center of the simulation sphere. Details of the particle substitution technique can be found in Ref. [1]. The electric field of the set of simulated ions and electrons is determined at the center of the sphere and readily carried to the system of differential equations on which the calculation of the evolution operator of the emitter finally depends. Debye screening has been included in the calculation of the electric field to account for the correlation effects between charged particles of different sign.

The results obtained for low densities yield a dependence of the width and shape of the spectrum with density and temperature that fits very well with an impact model of the broadening, both electronic and ionic (see Fig. 1).

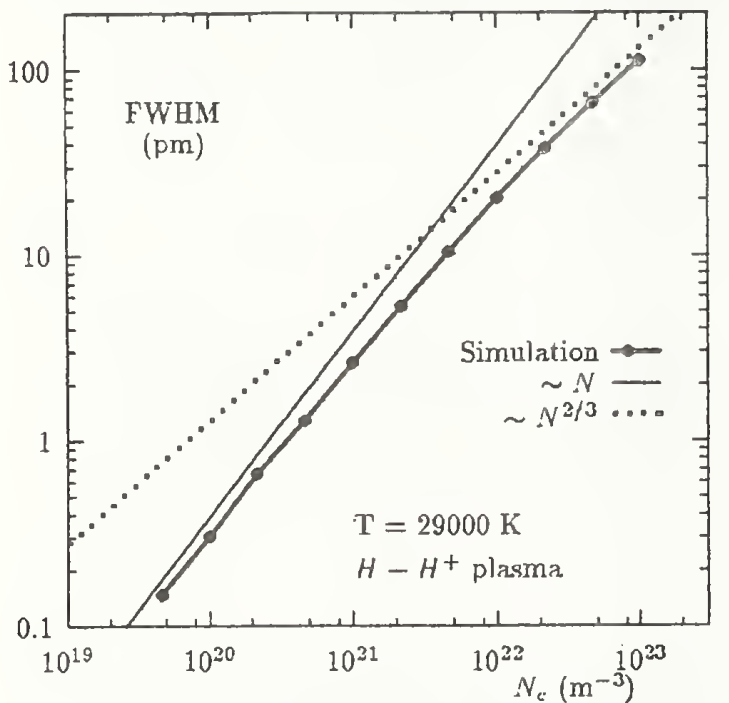

Figure 1: Dependence of the full width at half maximum (FWHM) with the electron density. For low densities, the trend is linear with $\mathrm{N}_{\mathrm{e}}$, which is characteristic of impact broadening.

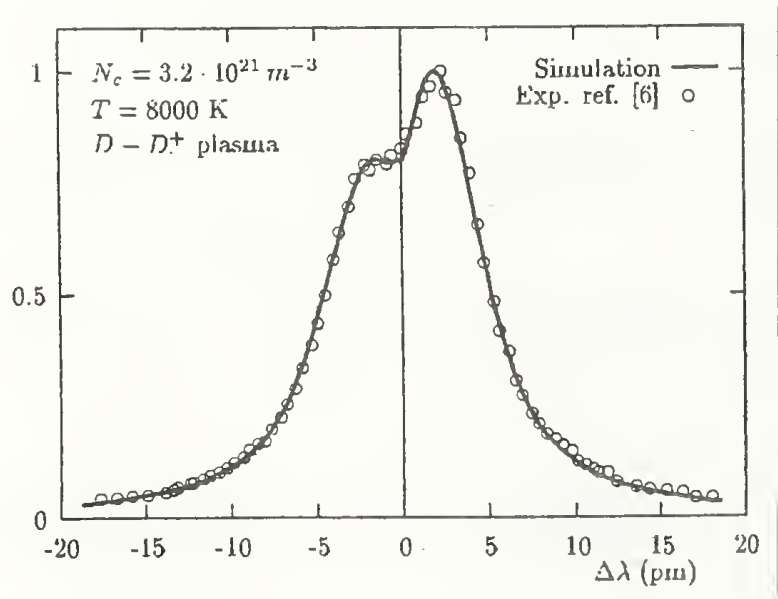

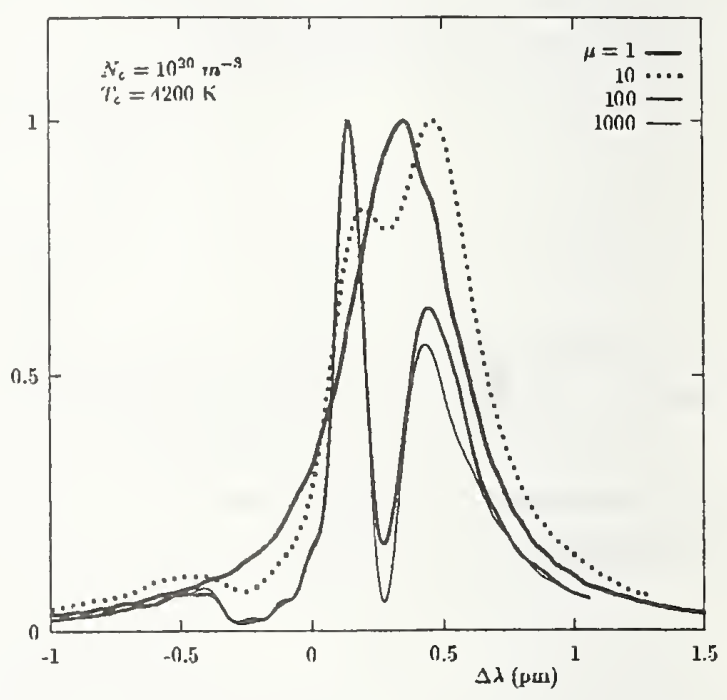

Figure 2: Polarization spectra by two-photon absorption. The effect of the reduced mass $\mu$ of the emitter-perturber pair is shown.

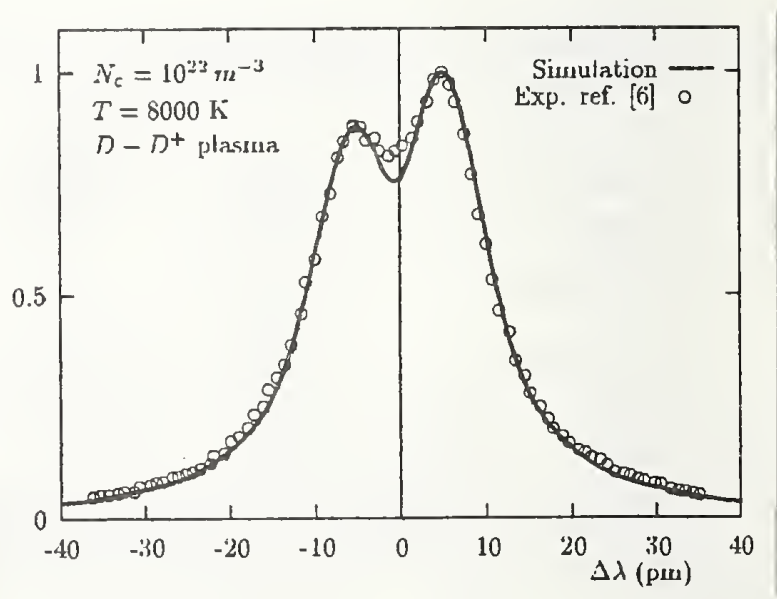

Figure 3: Two-photon polarization spectrum of the $1 \mathrm{~s} \rightarrow 2 \mathrm{~s}$ Lyman- $\alpha$ transition. Comparison of experiment and simulation. 
The obtained results have allowed us to make tables of complete line profiles for plasma diagnosis. In Fig. 4 the dependence of the full width at half maximum (FWHM) of the studied transition is shown as a function of the electron density and the temperature of the plasma. The smooth dependence of $\Delta \lambda_{1 / 2}$ with temperature for the emission profiles allows us to obtain the density of perturbers from direct measurements of the line width.

\section{Acknowledgments}

The authors of this work thank the Departamento de Informática of Valladolid University for the use of its calculation resources, Dr. Cardeñoso for his close collaboration in the development of the work, and professors González Vizmanos and Fuentes García for their collaboration in the maintenance of the calculations. The attendance of this conference has been partially financed by the spanish DIGICYT under grant PB94-0216.

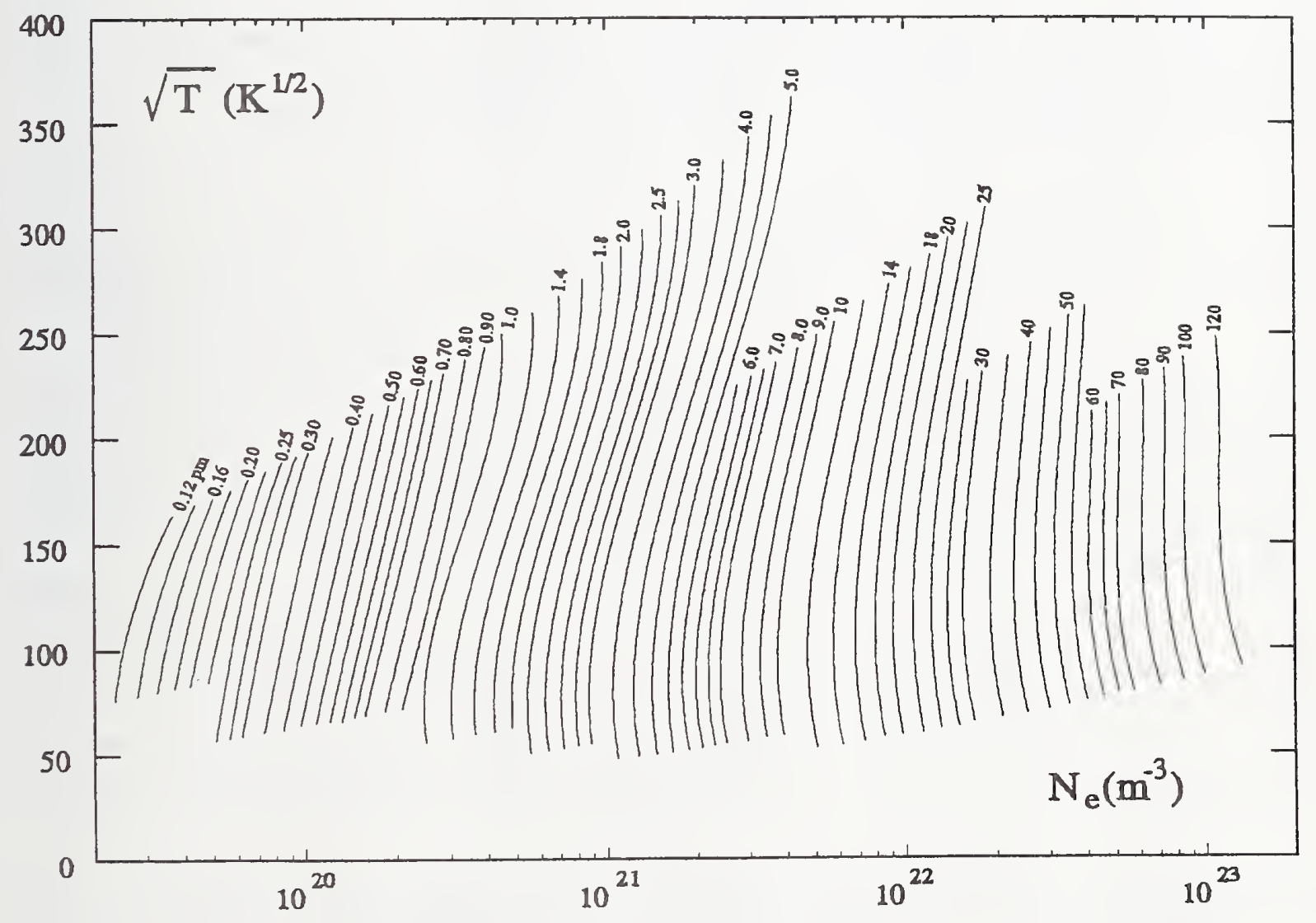

Figure 4: Curves of constant Stark width in a $\mathrm{H}-\mathrm{H}+$ plasma $(\mu=0.5)$. The numbers close to the lines show the full width at half maximum (in pm) for the transition $n=1 \rightarrow n=2$ that can be measured with polarization spectroscopy by two photon absorption. 


\section{References}

[1] M. A. Gigosos and V. Cardeñoso, J. Phys. B: At. Mol. Opt. Phys. 29, 4795 (1996)

[2] W. Demtröder, Laser Spectroscopy, (Springer-Verlag, Berlin Heidelberg 1996)

[3] J. Seidel, Phys. Rev. Letters 57, 2514 (1986)

[4] K. Danzmann, K. Grützmacher and B. Wende, Phys. Rev. Letters 57, 2151 (1986)

[5] Andreas Steiger, Starkverbreiterung des 2S-Zustands von Wasserstoff und Deuterium in Bogenplasmen niedriger Dichte, Ph. D. Thesis, Berlin (1993)

[6] J. Seidel, A. Steiger, and K. Grützmacher, Spectral Line Shapes, Vol. VIII, eds. A. D. May, J. R. Drummond, E. Oks, p. 32. (AIP Press, Toronto 1994)

[7] H.R. Griem, Spectral line Broadening by Plasmas, (Academic Press, New York 1974)

[8] J. Seidel and R. Stamm, J. Quant. Spectrosc. Radiat. Transfer 27, 499 (1982) 


\title{
Laboratory Measurements of Resonant Contributions to Fe XXIV Line Emission
}

\author{
M. F. Gu${ }^{1}$, P. Beiersdorfer², G. V. Brown², S. M. Kahn, \\ D. A. Liedahl'2, K. J. Reed ${ }^{2}$, and D.W. Savin ${ }^{1}$ \\ ${ }^{1}$ Columbia Astrophysics Laboratory, Columbia University, New York, NY 10027 \\ ${ }^{2}$ Lawrence Livermore National Laboratory, Livermore, CA 94550
}

\section{Introduction}

Recent Advanced Satellite for Cosmology and Astrophysics (ASCA) observations of the Centaurus cluster of galaxies [1] exhibit discrepancies with the relative line intensities of various Fe XXIII and XXIV $L$-shell emission lines predicted by standard plasma codes. This discrepancy is seen with all standard emission codes and is attributed to problems with the atomic physics used in the emission codes. New distorted wave (DW) calculations of Fe XXIII and Fe XXIV electron impact excitation (EIE) rate coefficients [2] appear to provide better agreement with the ASCA observations. Using the Lawrence-Livermore electron beam ion trap (EBIT), Savin et al. [3] measured the relative line emission of several Fe XXIV $3 \rightarrow 2$ and $4 \rightarrow 2$ lines and found good agreement with the calculations of Liedahl et al. These measurements, however, were carried out at electron energies significantly greater than the excitation threshold energies of the lines observed. The calculations remain to be experimentally verified for energies at which resonant processes may be important and for energies near threshold where DW calculations may overpredict the EIE cross section.

A number of X-ray astronomy satellites are scheduled for launch in the next few years. The Advanced X-ray Astrophysics Facility $(A X A F)$ is scheduled for launch in 1998, and the X-Ray Multi-mirror Mission (XMM) and Astro-E in 1999. These satellites will carry spectrometers with resolving powers in the Fe $L$-shell emission region over an order of magnitude greater than the spectrometers aboard $A S C A$. Interpreting $A X A F, X M M$, and Astro- $E$ spectra will require atomic data at an accuracy significantly greater than the data presently used in the standard emission codes.

To address some of the existing and upcoming needs of X-ray astrophysics, we have continued our studies of Fe XXIV line emission. In this work, we measured Fe XXIV $3 \rightarrow 2$ line emission at energies around threshold, using EBIT to examine the resonance contributions to the line emissivity. Here, we present relative cross sections, at electron energies between 700 and $1500 \mathrm{eV}$, for producing line emission at wavelength $\lambda=11.18 \AA$ of the Fe XXIV $3 d_{5 / 2} \rightarrow 2 p_{3 / 2}$ transition.

Various processes can contribute to line emission observed from a collisional plasma. Direct excitation (DE) is the most important one at energies above the EIE threshold. Below threshold, dielectronic recombination (DR) produces high $n$ satellites which cannot be resolved from the EIE line. Resonant excitation (RE) can populate the same levels as DE via dielectronic capture followed by autoionization to the level of interest. In this work, we have measured the following processes:

$$
\begin{aligned}
& \mathrm{DE} \\
& F e^{23+}(2 s)+e^{-} \rightarrow F e^{23+}\left(3 d_{5 / 2}\right)+e^{-} \rightarrow F e^{23+}\left(2 p_{3 / 2}\right)+h v_{1}+e^{-}
\end{aligned}
$$


- $\mathrm{RE}$

$$
\mathrm{Fe}^{23+}(2 s)+e^{--} \rightarrow \mathrm{Fe}^{22+}\left(4 \mathrm{l}^{\prime} \mathrm{nl} \mathrm{l}^{\prime}\right) \rightarrow \mathrm{Fe}^{23+}\left(3 d_{5 / 2}\right)+e^{-} \rightarrow \mathrm{Fe}^{23+}\left(2 \mathrm{p}_{3 / 2}\right)+h \mathrm{n}_{1}+e^{-}
$$

- DR

$\mathrm{Fe}^{23+}(2 s)+e^{-} \rightarrow \mathrm{Fe}^{22+}\left(3 d_{5 / 2} n l^{\prime}\right) \rightarrow \mathrm{Fe}^{22+}\left(2 p_{3 / 2} n l^{\prime}\right)+h v_{2}+e^{-}$

Quantum mechanically, there is no way to distinguish between $\mathrm{DE}$ and RE, and we measure the coherent sum of the two processes.

\section{Experimental Technique}

EBIT [4] uses a magnetically confined electron beam to produce a potential well which traps the ions in the radial direction. In the axial direction, ions are trapped by voltages applied to the top and bottom drift tubes. The electron beam is also used to ionize and excite the trapped ions. Radiative decay of the excited ions results in X-ray emission which is observed by a flat crystal spectrometer (FCS) through one of the X-ray ports. A thallium hydrogen phthalate (001) crystal is used. The dispersion plane of the FCS is perpendicular to the electron beam. Between the EBIT and the FCS is a $4 \mu \mathrm{m}$ polypropylene window. X-rays are detected by a flowing gas (90\% $\mathrm{Ar}$ and $10 \% \mathrm{CH}_{4}$ at 1 atmosphere) position sensitive proportional counter with a $4 \mu \mathrm{m}$ polypropylene window.

After injecting iron into the EBIT, the electron beam energy is kept at $4 \mathrm{keV}$ for $200 \mathrm{~ms}$. Then every $10 \mathrm{~ms}$ the beam energy is swept between 700 and $1500 \mathrm{eV}$ using a sawtooth pattern $2 \mathrm{~ms}$ in length. The intensity of a particular line is $\mathrm{I} \propto \int d \mathrm{r} n_{e}(\mathrm{r}) n_{q}(\mathbf{r}) \sigma v$, where $n_{e}(\mathrm{r})$ is the electron density, $n_{q}(\mathrm{r})$ is the ion density, $\sigma$ is the cross section for producing the observed line emission, and $v$ is the electron velocity. During the sweeping of the beam energy, the charge balance changes insignificantly because the ionization and recombination time scales at these energies are $\geq 100 \mathrm{~ms}$. We maintain a nearly constant $n_{e}(\mathrm{r})$ by varying the anode voltage of the electron gun. We record the intensity of line emission at $\lambda=11.18 \AA$ as a function of beam energy. Because $\int d \mathbf{r} n_{e}(\mathbf{r}) n_{q}(\mathbf{r})$ is kept constant versus $E$, we can derive cross sections for producing these lines by normalizing our measurements to theory.

\section{Uncertainty Analysis}

Uncertainties are listed in Table 1 at the $1 \sigma$ confidence level.

Table 1: Uncertainties for observed line emission measurements.

\begin{tabular}{|r|c|}
\hline Source & $\begin{array}{c}\lambda=11.18 \AA \\
\text { (percent) }\end{array}$ \\
\hline Statistics & $8-14$ \\
Background Subtraction & 5 \\
Polarization Effects & 5 \\
Electron Density & 2 \\
Normalization & 10 \\
\hline Quadrature Sum & $15-19$ \\
\hline
\end{tabular}


There are about 200 counts per beam energy bin ( $\sim 12 \mathrm{eV}$ per bin) for the line emission at $\lambda=11.18 \AA$ above the DE threshold. The DR satellites have peak counts of between 50 and 100 . The $1 \sigma$ statistical uncertainty varies between $8 \%$ and $14 \%$.

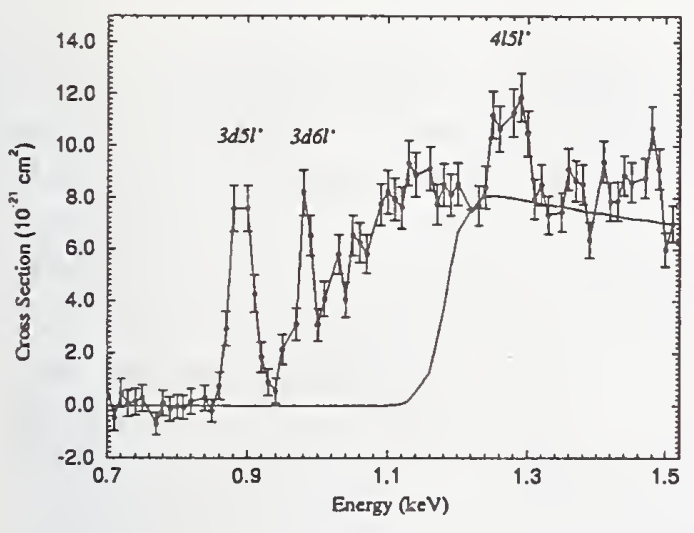

Figure 1: Normalized cross sections for producing line emission at $\lambda=11.18 \AA$.

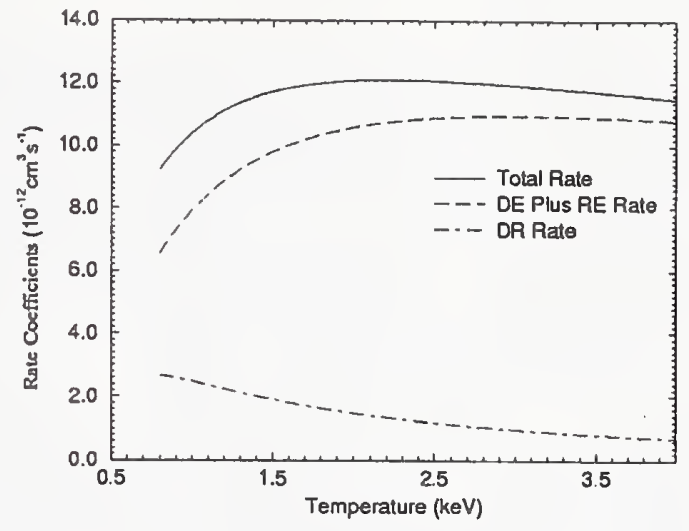

Figure 2: Rate coefficients for producing line emission at $\lambda=11.18 \AA$.

We observed line emission due to charge transfer (CT) with neutral gas in the EBIT. This signal is independent of beam energy. At beam energies between 700 and $800 \mathrm{eV}$, the CT signal is clearly resolved from DR resonances and seen to be constant. We subtract this background from the total intensity. The relative uncertainty introduced by this subtraction is about $5 \%$.

Line emission produced by a unidirectional electron beam is linearly polarized and anisotropic [5]. Crystals have different integrated reflectivities for X-rays polarized parallel to and perpendicular to the dispersion plane [6]. The observed line intensities depend on the polarization of the emitted lines. This is a function of collision energy. In the energy range covered here, polarization due to $\mathrm{DE}$ does not change significantly. Polarization of the DR satellites should approach that of $\mathrm{DE}$ as the beam energy approaches the DE threshold [7]. RE is expected to be less polarized than DE emission. We assume the line emission at all energies has the same polarization. Polarization effects need to be further investigated but are estimated to introduce $<5 \%$ uncertainty.

While sweeping the electron beam energy, we try to keep the electron density constant by ramping the extraction voltage of the electron gun in sync with the beam energy. The measured beam current indicates that the electron density varies by less than $10 \%$ between 700 and $1500 \mathrm{eV}$. We have corrected the observed line intensities for this variation. The electrons in the EBIT have a transverse energy of $\sim 150 \mathrm{eV}$ [8]. The resulting change of the pitch angle between the electron velocity and the beam direction results in a small variation of electron density versus beam energy. Using the normalization energy as our reference point, $n_{e}$ varies by $\pm 2 \%$ across the measured energy range due to the changing pitch angle.

Our normalization point lies $\sim 40 \mathrm{eV}$ above the $\mathrm{DE}$ threshold and $\geq 40 \mathrm{eV}$ away from all $\mathrm{RE}$ resonances. Given the electron beam energy spread of $50 \mathrm{eV}$ [9], we estimate that emission at the normalization point is not significantly affected by either DR just below the DE threshold or the $\mathrm{RE}$ resonances. Thus we assume the line emission is completely due to $\mathrm{DE}$ at this energy and use HULLAC (Hebrew University/Lawrence Livermore Atomic Code) [2] calculated cross sections. 
Statistical uncertainties at the normalization energy introduce uncertainties of $\sim 10 \%$ in the cross sections for line emission at $\lambda=11.18 \AA$.

\section{Results}

Fig. 1 shows the normalized cross section for producing $\lambda=11.18 \AA$ line emission. The error bars on the data points show the $1 \sigma$ statistical uncertainties. The normalization point is presented without an error bar. The $3 l 5 l^{\prime}$ and $3 l 6 l^{\prime}$ DR resonances are well separated. All other DR resonances between $1.0 \mathrm{keV}$ and the $\mathrm{DE}$ threshold are unresolved and appear as a continuous increase in the cross sections. The $\mathrm{RE}$ resonance at a beam energy of about $1.25 \mathrm{keV}$ is produced by dielectronic capture into the Fe XXIV $4 l 5 l^{\prime}$ level followed by autoionizing to the $3 d_{5 / 2}$ level.

Using the relative cross sections obtained above, we calculate the rate coefficients of each process. The DE rate is calculated by using HULLAC [2] cross sections. For experimentally inferred rates, only line emission within the measured energy range is included. Fig. 2 shows the experimentally inferred rates for $\mathrm{DR}, \mathrm{DE}$ plus $\mathrm{RE}$, and the total rates. The total rate includes the experimentally inferred DR, DE plus RE, and the theoretical DE rates at energies outside of our measured range. The theoretical $\mathrm{DE}$ rates do not include the cascade contributions. At a temperature of $1.7 \mathrm{keV}$, near where Fe XXIV emissivity peaks, DR contributions amount to $20 \%$ for the $\lambda=11.18 \AA$ line emission. $\mathrm{RE}$ enhances the rates by $\sim 5 \%$. However, the unmeasured $\mathrm{RE}$ contributions at higher energies can be expected to increase the rates further. Thus, plasma emission codes which do not include the effect of DR and RE on line emissivities can be expected to underestimate the line intensity by at least $\sim 25 \%$.

\section{Acknowledgements}

The authors wish to thank S. T. Manson for stimulating conversations and P. A. D'Antonio, E. W. Magee, and D. H. Nelson for their expert technical support. Work at Lawrence Livermore National Laboratory was performed under the auspices of the U.S. Department of Energy (contract W-7405-ENG-48). This program is supported by a NASA High Energy Astrophysics X-Ray Astronomy Research and Analysis grant NAGW-4185 (Columbia University) and work order W-19127 (LLNL).

\section{References}

[1] Fabian, A. C. et al., ApJ 436, L63 (1994)

[2] Liedahl, D. A. et al., ApJ 438, L115 (1995)

[3] Savin, D. W. et al., ApJ 470, L73 (1996)

[4] Levine, M. A. et al., Phys. Scr. T22, 157 (1988)

[5] Percival, I. C., and Seaton, M. J., Philos. Trans. R. Soc. London A 251, 113 (1958)

[6] Henke, B. L., Gullikson, E. M., and Davis, J. C., At. Data Nucl. Data Tables 54, 181 (1993)

[7] Inal, M. K. and Dubau, J., J. Phys B. 22, 3329 (1989)

[8] Marrs, R. E., (private communication)

[9] Beiersdorfer, P. et al., Phys. Rev. A 46, 3812 (1992) 


\title{
Modeling Electromagnetic Interactions in Quantized Electronic Systems
}

\author{
Verne L. Jacobs \\ Complex Systems Theory Branch, \\ Condensed Matter and Radiation Sciences Division, \\ Naval Research Laboratory, \\ Washington, D. C. 20375-5000
}

\section{Introduction}

We have been developing a density-matrix approach [1] that provides a convenient framework for the systematic computer simulation of the electromagnetic spectra emitted or absorbed by a quantized electronic system. A quantized electronic system is characterized by discrete energy-level structures, which correspond to bound or quasi-bound states, and by radiative transitions that produce line-like spectral features, which are advantageous in the development of sources of coherent electromagnetic radiation.

Extensive applications of our density-matrix approach have been made to radiative transitions of many-electron atomic systems in strong fields [2, 3], and detailed spectral simulations have been provided for $\mathrm{K}$-alpha dielectronic satellite spectra of highly charged atomic ions in electron-ion beam interactions [4] and in high-temperature plasmas [5]. In these simulations, it has been necessary to assemble a very large data base of atomic energy levels and transition probabilities. We have also been interested in applications to radiation processes involving energetic electron beams propagating in strong electric and magnetic fields, as in free-electron lasers and electron cyclotron masers or gyrotrons, and in crystal fields, giving rise to electron channeling radiation and coherent bremsstrahlung [6]. Currently, we are adapting our density-matrix approach to coherent electromagnetic interactions in low-dimensional semiconductor microstructures [7], such as quantum wells, quantum wires, and quantum dots. These structures have been referred to as quantum-confinement systems, because they can give rise to discrete or quasi-discrete levels and to radiative transitions that are closely analogous to the spectral-line transitions in atomic systems. In contrast to natural atomic systems, which may be treated as identical, the quantum dots that are often referred to as "artificial atoms" may occur in arrays, and their geometric and physical properties may vary in a random manner. Consequently, our microscopic description is expected to be most appropriate for the investigation of the properties of a single microstructure, where the homogeneous line-broadening mechanisms and elementary relaxation processes may be examined.

The numerous device applications of the electromagnetic processes of interest include laser systems, electro-optical components, optical communications technologies, and radiation detectors. New quantum-optics applications include quantum computing, coherent control, and quantum cryptography. In addition, this fundamental study forms the basis for the spectroscopic investigation of basic material properties and interactions. 


\section{Density-Matrix Organization of the Spectral Simulation}

The starting point for a detailed spectral simulation should be a realistic determination of the quantized electronic structure for the single-electron or many-electron subsystem of interest. The complete spectrum of electronic states may include discrete bound states, continuum states, and quasi-bound states or autoionization resonances. These electronic states may be taken to be eigenstates of a Hamiltonian in the presence of an external (classical) electric or magnetic field or an intemal crystal field that can be represented by a local potential function. The correct treatment of coherent electromagnetic interactions usually involves the self-consistent determination of the radiation fields, as solutions of the coupled dynamical equations governing the interacting quantized electronic subsystem and the electromagnetic field.

The effects of many-particle interactions can be treated as self-energy corrections to the single-particle energy levels. In the description of many-electron atomic systems, the starting point is usually a many-electron approximation, which must be systematically improved. In the treatment of electrons (or holes) in ideal crystals or in semiconductor microstructures, the initial single-particle description must also be systematically improved to take into account the effects of the electrostatic interaction between charged particles, which are referred to as correlation effects. The inclusion of these effects leads to dielectric screening and to band-gap renormalization. The effects of lattice vibrations may be described, within the framework of many-body quantum field theory, in terms of electron-phonon collisional interactions.

The total probabilities for the various radiative transitions can be evaluated using the familiar Fermi Golden-Rule formula, which is obtained from the lowest-order perturbation theory describing the interaction of the electronic subsystem of interest with the quantized electromagnetic field. The familiar radiation parameters include the (Einstein) spontaneous emission rates and the cross sections for the induced emission and absorption processes. In order to describe the spectral line shapes, it is necessary to employ a high-order, non-perturbative description of the interaction of the electronic system with the quantized electromagnetic field and with the multitude of perturbing particles or quasi-particles. In order to accomplish this objective, it is necessary to adopt a quantum statistical description of the interaction of electromagnetic radiation with the charged particles.

A complete non-perturbative statistical description of the interaction between the quantized electronic subsystem of interest and the quantized electromagnetic field, as well as its interaction (or entanglement) with a much larger quantum system representing the surrounding environment, is provided by the density-matrix approach. In the time domain, the equation of motion for the density operator representing the combined, enlarged system provides the starting point for the general quantum dynamical description. It is often desirable to introduce a transformation to the frequency domain, in which the basic quantity is the Liouville-space resolvent operator. In order to obtain a practical formulation, it is necessary to introduce reduced density-matrix descriptions, in which the interactions of the subsystem of interest with the environment are treated stochastically, as collisional and radiative relaxation processes. In this manner, reduced density operators are defined for the electronic subsystem and for the electromagnetic radiation field. If coherent interactions are of interest, the reduced system must be defined to include the coherently coupled subsystems.

Our density-matrix description [1] may be viewed as a comprehensive framework for the systematic organization of the basic collisional-interaction and radiation-transition data. In order to obtain the electronic-level populations from the traditional data set of transition probabilities and cross sections, it is necessary to simplify the equations of motion for the reduced density 
operators. The Markov or short-memory-time approximation leads to the conventional rate or Master equations for the electronic-level populations, together with the electronic-state coherences. In the case of free or quasi-free particles, non-equilibrium distribution functions can be determined. A separate reduced density-matrix equation for the radiation field must be included in order to provide a self-constant treatment of the non-linear behavior that is characteristic of coherent electromagnetic processes.

In the frequency-domain formulation of the density-matrix description, it is convenient to introduce a generalized Fermi Golden-Rule formula for the evaluation of the spontaneous radiative emission rate, as well as for the various cross sections of interest. This formula is expressed in terms of the initial-state density operator for the electronic subsystem and the final-state projection operator appropriate to the relevant photon detection process. The Liouville-space reduced transition operator occurring in this formula is defined in terms of a Liouville-space resolvent operator, which may be evaluated to give the spectral-line shape produced by an array of radiative transitions. This is a standard procedure in the isolated-line approximation, but it may be necessary to employ an overlapping-line description. The Liouville-space self-energy operator provides the open-system description of the collisional and radiative relaxation processes. In order to include Stark and Zeeman broadening, it is necessary to evaluate the line-shape formula using the "atomic" data that has been determined from electronic eigenstates in the presence of static or dynamic external electric and magnetic fields. In order to make comparisons with experimental observations, it is usually necessary to include either thermal or turbulent Doppler broadening.

\section{Spectral Simulations}

Spectral simulations have been carried out for many-electron atomic systems in electron-ion beam interactions [4] and in high-temperature plasmas [2, 3, and 5]. An ultimate goal in these simulations has been to employ the same set of atomic data consistently in the description of the non-equilibrium atomic-population kinetics and in the determination of the spectral-line shapes. These atomic spectral simulations have been found to be useful in determinations of electric-field and magnetic-field distributions and for diagnostics of plasma densities and temperatures. Spectral simulations for coherent radiation processes of energetic electrons in crystal lattices, such as coherent bremsstrahling and channeling radiation [6], can provide information on the elementary microscopic interactions and on the local crystal environment. Our density-matrix approach [1] may provide a comprehensive framework for detailed and systematic spectral simulations of electromagnetic interactions in low-dimensional semiconductor electronic systems [7], such as quantum wells, quantum wires, and quantum dots.

\section{Acknowledgments}

This work has been supported by the Department of Energy and by the Office of Naval Research. 


\section{References}

[1] V. L. Jacobs, J. Cooper, and S. L. Haan, Phys. Rev. A 50, 3005 (1994).

[2] L. A. Woltz, V. L. Jacobs, C. F. Hooper, and R. C. Mancini, Phys. Rev. A 44, 1281 (1991).

[3] V. L. Jacobs, J. Quant. Spectrosc. Radiat. Transfer 54, 195 (1995).

[4] V. Decaux, P. Beiersdorfer, S. M. Kahn, and V. L. Jacobs, Astrophys. J. 482, 1076 (1997).

[5] V. L. Jacobs, G. A. Doschek, J. F. Seely, and R. D. Cowan, Phys. Rev. A 39, 2411 (1989).

[6] A. W. Sáenz, A. Nagl, and H. Überall, Phys. Rev. B 37, 7238 (1988).

[7] H. Haug and S. W. Koch, Quantum Theory of the Optical and Electronic Properties of Semiconductors, Second Edition, (World Scientific, Singapore, 1993). 


\title{
Relativistic Oscillator Strengths in Chlorine
}

\author{
I. Martin, C. Lavín, A.M. Velasco \\ Departamento de Química Física, Facultad de Ciencias, \\ 47005 Valladolid, Spain
}

\section{Introduction}

Models of interstellar chlorine chemistry can be used to constrain models of interstellar clouds. One difficulty in confirming the basic ideas of this model is that the analyses have been based on rather poorly known oscillator strengths for Cl I, as has been emphasized by Keenan \& Dufton [1] and Morton [2]. We have followed the Relativistic Quantum Defect Orbital (RQDO) procedure [3] with and without explicit inclusion of core-valence correlation. The observations [4] of the ClI spectrum show that LS coupling is valid for the deepest $3 \mathrm{p}^{4} \mathrm{~ns}, \mathrm{np}$ and nd configurations whereas pair coupling is progressively better satisfied for higher $l$ and $n$ values. In our calculations of oscillator strengths, we have followed the LS coupling scheme, except for the $3 \mathrm{p}^{4}\left({ }^{3} \mathrm{P}\right) 4 \mathrm{f}-3 \mathrm{p}^{4}\left({ }^{3} \mathrm{P}\right) 5 \mathrm{~g}$ lines, for which the $J_{c} l$ coupling scheme seems clearly to be more appropriate.

\section{Method of Calculation}

The absorption oscillator strength for an electric dipole transition between an initial state, $|\mathrm{i}\rangle$, and a final state, $|j\rangle$, is given by

$$
f_{i j}=\frac{2}{3} \frac{\left(E_{j}-E_{i}\right)}{2 J+1} S
$$

where $\left(E_{j}-E_{i}\right)$ is the transition energy (in atomic units), $2 J+1$ is the degeneracy of the initial level and $S$ is the so-called line strength (in atomic units). In a one-configuration treatment, such as ours, the angular and radial parts of the line strength can be separated. Within LS coupling, the line strength is written as follows:

$$
S\left(\gamma S L J, \gamma^{\prime} S^{\prime} L^{\prime} J^{\prime}\right)=\left|\delta S S^{\prime} \delta_{c c^{\prime}} R_{\text {line }}\left(S L J, S^{\prime} L^{\prime} J^{\prime}\right) R_{m u l t}\left(\gamma L, \gamma^{\prime} L^{\prime}\right)(-1)^{l-l>} \sqrt{l>} \int R_{n l}(r) R_{n^{\prime} l^{\prime}}(r) r d r\right|^{2}
$$

(2)

where $J$ and $J^{\prime}, L$ and $L^{\prime}, S$ and $S^{\prime}$, and $\gamma$ and $\gamma^{\prime}$ represent the quantum numbers required to complete the specification of the states $|i\rangle$ and $|j\rangle$, respectively. The subscripts $c$ and $c^{\prime}$ refer to the core. The integral in (2) contains the radial parts of the initial and final wavefunctions only. The line factor, $R_{\text {line, }}$ 


$$
R_{\text {line }}=\sqrt{2 J+1} \sqrt{2 J^{\prime}+1}(-1)^{S+J+L^{\prime}+1}\left\{\begin{array}{ccc}
S & J & L \\
1 & L^{\prime} & J^{\prime}
\end{array}\right\},
$$

is given by the above expression, where the symbol in braces is a $6-j$ symbol. $\left(R_{\text {line }}\right)^{2}$ gives the relative strength of the lines within a multiplet. In (2), $n l$ and $n^{\prime} l^{\prime}$ are, respectively, the principal and orbital angular momentum quantum numbers of the jumping electron, and $l>$ indicates the larger of the two orbital quantum numbers, $l$ and $l^{\prime}$, involved in the transition.

The multiplet factor, $R_{\text {mult }}$, depends upon the particular configurations involved in the transition:

- for $3 s^{2} 3 p^{4} n l-3 s^{2} 3 p^{4} n^{\prime} l^{\prime}$ the multiplet factor adopts the form

$$
R_{m u l t}=\sqrt{2 L+1} \sqrt{2 J^{\prime}+1}(-1)^{L_{c}+L+l^{\prime}+1}\left\{\begin{array}{lll}
L_{c} l & L \\
1 & L^{\prime} & l^{\prime}
\end{array}\right\}
$$

-and for $3 s^{2} 3 p^{5}-3 s^{2} 3 p^{4} n^{\prime} l^{\prime}$ the multiplet factor is

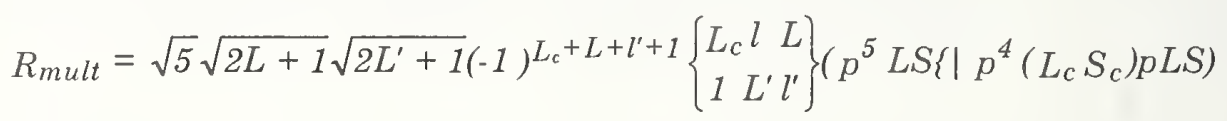

where $\left(p^{5} L S\left\{/ p^{4}\left(L_{c} S_{c}\right) p L S\right)\right.$ is the coefficient of fractional parentage, CFP.

The expression for the line strength in the $J_{c} l$ coupling scheme is

$$
S=(2 J+1)\left(2 J^{\prime}+1\right)\left\{\begin{array}{c}
K \frac{1}{2} J \\
J^{\prime} 1 K^{\prime}
\end{array}\right\}^{2}(2 K+1)\left(2 K^{\prime}+1\right)\left\{\begin{array}{c}
J_{c} l K \\
1 K^{\prime} l^{\prime}
\end{array}\right\}^{2} l>\left|\int R_{n l} R_{n^{\prime} l^{\prime}} r d r\right|^{2}
$$

where $J_{c}$ is the total angular momentum quantum number of the electron core $3 \mathrm{p}^{4}{ }^{3} \mathrm{P}$ level; $K$ results from the coupling of the orbital angular momentum, $l$, of the valence electron with $J_{c}$, and $J$ is obtained through the addition of the spin of the valence electron to $K$.

\section{Results}

\section{$3 p^{5} P^{0}-3 p^{4}\left({ }^{3} P\right) 4 s^{2} P$ Transitions}

Some lines of the $3 \mathrm{p}^{5}{ }^{2} \mathrm{P}^{0}-3 \mathrm{p}^{4}\left({ }^{3} \mathrm{P}\right) 4 \mathrm{~s}^{2} \mathrm{P}$ multiplet of neutral chlorine have been the object of several theoretical studies, as well as measurements, because of their importance in astrophysics. Table 1 contains fine structure oscillator strengths for the $3 \mathrm{p}^{5}{ }^{2} \mathrm{P}^{0}-3 \mathrm{p}^{4}\left({ }^{3} \mathrm{P}\right) 4 \mathrm{~s}{ }^{2} \mathrm{P}$ transitions in $\mathrm{Cl} \mathrm{I}$. In this table we display, together with the RQDO results [5], the f-values calculated by Biemont et al. [6] with the SUPERSTRUCTURE (SST) computer program, taking the most important configuration interaction and relativistic effects into account, as well as the results of Ojha \& Hibbert [7], who used large multiconfiguration expansions in the atomic structure code CIV3. Biémont et al. and Ojha \& Hibbert report f-values calculated in dipole length and velocity approaches. 
The most accurate laboratory data are the measurements of Schectman et al. [8] using beam-foil spectroscopy. These authors estimated that their results should be accurate to about $7 \%$. The present results, without explicit account for polarization, are in good accordance with the most accurate theoretical and experimental results. Our f-values for the $3 \mathrm{p}^{5}{ }^{2} \mathrm{P}^{0}{ }_{3 / 2}-3 \mathrm{p}^{4}\left({ }^{3} \mathrm{P}\right) 4 \mathrm{~s}^{2} \mathrm{P}_{3 / 2}$ and $3 \mathrm{p}^{5}{ }^{2} \mathrm{P}^{0}{ }_{1 / 2}$ $3 \mathrm{p}^{4}\left({ }^{3} \mathrm{P}\right) 4 \mathrm{~s}^{2} \mathrm{P}_{3 / 2}$ lines are larger than the earlier measurements for these transitions and confirm the recent experimental data of Schectman et al. For the remaining transitions, our f-values are in good accord with the measurements of Clyne \& Nipp [9].

\section{$3 p^{4}\left({ }^{3} P\right) 4 f-3 p^{4}\left({ }^{3} P\right) 5 g$ Transitions}

In Table 2 , we display RQDO f-values for $3 \mathrm{p}^{4}\left({ }^{3} \mathrm{P}\right) 4 \mathrm{f}-3 \mathrm{p}^{4}\left({ }^{3} \mathrm{P}\right) 5 \mathrm{~g}$ transitions calculated in the $J_{c} l$ coupling scheme [5]. No comparison data have been found in the literature. The level designations in this table are given according to the notation $3 \mathrm{p}^{4}\left({ }^{2 \mathrm{~S}+1} \mathrm{~L}_{\mathrm{J}}\right) \mathrm{n} l[\mathrm{~K}] \mathrm{J}$ where $J_{c}$ is the total angular momentum quantum number of the electron core $3 \mathrm{p}^{4}{ }^{3} \mathrm{P}$ level; $K$ results from the coupling of the orbital angular momentum, $l$, of the valence electron with $J_{c}$, and $J$ is obtained through the addition of the spin of the valence electron to $K$. According to Quinet et al. [10], in pure $J_{c} l$ coupling, the energies of a pair of levels for a given $J_{c}$ should fall on a parabola when plotted against $h=1 / 2\left[K(K+1)-J_{c}\left(J_{c}+1\right)-l(l+1)\right]$. This approximation is verified in the cases of $4 \mathrm{f}$ and $5 \mathrm{~g}$ electrons.

\section{Acknowledgements}

This work has been supported by the D.G.I.C.Y.T. of the Spanish Ministry of Education and Science, under Project No.PB94-1314-C03-03

\section{References}

[1] Keenan F.P., Dufton P.L., MNRAS 242, 52p (1990)

[2] Morton D.C., ApJS 77, 119 (1991)

[3] Martin I., Karwowski J., J. Phys. B 24, 1539 (1991); Karwowski J., Martin I., Phys. Rev. A 43, 4832 (1991)

[4] Radziemski L.J.Jr., Kaufman V., J. Opt. Soc. Am. 59, 424 (1969)

[5] Lavín C., Velasco A.M., Martín I., Astronomy and Astrophysics, in press

[6] Biémont E., Gebarowski R., Zeippen C.J., A\&A 287, 290 (1994)

[7] Ojha P.C., Hibbert A., Phys. Scr. 42, 424 (1990)

[8] Schectman R.M., Federman S.R., Beideck D.J., Ellis D.G., ApJ 406, 735 (1993)

[9] Clyne M.A.A., Nip W.S., J. Chem. Soc. Faraday Trans. II 73, 161 (1977)

[10] Quinet P., Palmeri P., Biémont E., Brault J.W., Phys. Rev. A 49, 2446 (1994)

[11] Wiese W.L., Smith M.W., Miles B.M., Atomic Transition Probabilities, Vol.II, U.S. Govt. Print Off., Washington D.C., NSRDS-NBS 22 (1969)

[12] Schwab J.J., Anderson J.G., J. Quant. Spectr. Rad. Transfer 27, 445 (1982) 
Table 1: Oscillator strengths for the $3 \mathrm{p}^{5}-3 \mathrm{p}^{4}\left({ }^{3} \mathrm{P}\right) 4$ s fine structure transitions in $C l I$.

\begin{tabular}{|c|c|c|c|c|c|c|}
\hline & RQDOa & $\mathrm{RQDO}^{\mathrm{b}}$ & SSTc & CIV3d $^{d}$ & $\mathrm{CCe}$ & Experiment \\
\hline $3 p^{2} \mathrm{Po}_{3 / 2}-4 s^{2} \mathrm{P}_{3 / 2}$ & 0.1368 & 0.1281 & 0.147 & 0.1324 & 0.114 & $\begin{array}{l}0.100 \pm 0.030^{\mathrm{f}} \\
0.109 \pm 0.010^{\mathrm{g}} \\
0.153 \pm 0.011^{\mathrm{h}}\end{array}$ \\
\hline $3 p^{2} \mathrm{Po}_{3 / 2}-4 \mathrm{~s}^{2} \mathrm{P}_{1 / 2}$ & 0.0249 & 0.0240 & 0.0299 & 0.0264 & 0.0233 & $0.028 \pm 0.006^{f}$ \\
\hline $3 p^{2} \mathrm{Po}_{1 / 2}-4 \mathrm{~s}^{2} \mathrm{P}_{3 / 2}$ & 0.0561 & 0.0523 & 0.0530 & 0.0474 & 0.0418 & $\begin{array}{l}0.038 \pm 0.006^{f} \\
0.055 \pm 0.004^{h}\end{array}$ \\
\hline $3 p^{2} \mathrm{P}^{0}{ }_{1 / 2}-4 \mathrm{~s}^{2} \mathrm{P}_{1 / 2}$ & 0.1017 & 0.0980 & 0.116 & 0.1051 & 0.088 & $0.093 \pm 0.016^{f}$ \\
\hline
\end{tabular}

aRQDO without explicit polarization correction [5]; ${ }^{b} R Q D O$ with explicit polarization correction [5]; 'Biémont et al. [6],

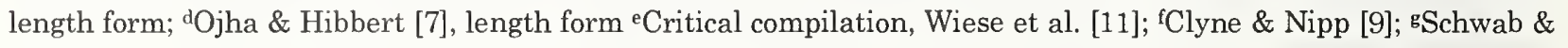
Anderson [12]; hSchectman et al. [8].

Table 2: Oscillator strengths for the $3 \mathrm{p}^{4}\left({ }^{3} \mathrm{P}_{2}\right) 4 \mathrm{f}[\mathrm{K}]_{J}-3 \mathrm{p}^{4}\left({ }^{3} \mathrm{P}_{2}\right) 5 \mathrm{~g}[\mathrm{~K}]_{3}$ transitions in $C l I$.

\begin{tabular}{|c|c|c|c|c|c|}
\hline & $\mathrm{RQDO}^{\mathrm{a}}$ & $\mathrm{RQDO}^{\mathrm{b}}$ & & $\mathrm{RQDO}^{\mathrm{a}}$ & $\mathrm{RQDO}^{\mathrm{b}}$ \\
\hline$[5]_{11 / 2}-[6]_{13 / 2}$ & 1.2217 & 1.2211 & {$[3]_{7 / 2}-[4]_{9 / 2}$} & 1.0697 & 1.0691 \\
\hline$[5]_{1 / 2}-[6]_{11 / 2}$ & 0.1031 & 0.1030 & {$[3]_{7 / 2}-[4]_{7 / 2}$} & 0.0307 & 0.0306 \\
\hline$[5]_{9 / 2}-[6]_{11 / 2}$ & 1.2354 & 1.2348 & {$[3]_{5 / 2}-[4]_{7 / 2}$} & 1.0989 & 1.0983 \\
\hline$[5]_{11 / 2}-[5]_{11 / 2}$ & 0.1031 & 0.1030 & {$[3]_{7 / 2}-[3]_{7 / 2}$} & 0.2247 & 0.2246 \\
\hline$[5]_{11 / 2}-[5]_{9 / 2}$ & 0.0016 & 0.0016 & {$[3]_{7 / 2} \cdot[3]_{5 / 2}$} & 0.0083 & 0.0083 \\
\hline$[5]_{9 / 2}-[5]_{11 / 2}$ & 0.0019 & 0.0019 & {$[3]_{5 / 2}-[3]_{7 / 2}$} & 0.0111 & 0.0111 \\
\hline$[5]_{9 / 2}-[5]_{9 / 2}$ & 0.1028 & 0.1027 & {$[3]_{5 / 2}-[3]_{5 / 2}$} & 0.2220 & 0.2219 \\
\hline$[5]_{11 / 2}-[4]_{9 / 2}$ & 0.0040 & 0.0040 & {$[2]_{5 / 2} \cdot[3]_{7 / 2}$} & 1.0668 & 1.0662 \\
\hline$[5]_{9 / 2} \cdot[4]_{9 / 2}$ & 0.0001 & 0.0001 & {$[2]_{5 / 2}-[3]_{5 / 2}$} & 0.0532 & 0.0531 \\
\hline$[5]_{9 / 2}-[4]_{7 / 2}$ & 0.0040 & 0.0039 & {$[2]_{3 / 2}-[3]_{5 / 2}$} & 1.1201 & 1.1201 \\
\hline$[4]_{9 / 2}-[5]_{11 / 2}$ & 1.1263 & 1.1257 & {$[1]_{3 / 2}-[2]_{5 / 2}$} & 1.1216 & 1.1209 \\
\hline$[4]_{9 / 2}-[5]_{9 / 2}$ & 0.0209 & 0.0208 & {$[1]_{3 / 2}-[2]_{5 / 2}$} & 0.2691 & 0.2690 \\
\hline$[4]_{7 / 2}-[5]_{9 / 2}$ & 1.1481 & 1.1475 & {$[1]_{1 / 2} \cdot[2]_{3 / 2}$} & 1.3466 & 1.3459 \\
\hline$[4]_{9 / 2}-[4]_{9 / 2}$ & 0.1802 & 0.1801 & {$[2]_{5 / 2}-[2]_{5 / 2}$} & 0.2051 & 0.2050 \\
\hline$[4]_{9 / 2}-[4]_{7 / 2}$ & 0.0041 & 0.0041 & {$[2]_{5 / 2} \cdot[2]_{3 / 2}$} & 0.0149 & 0.0149 \\
\hline$[4]_{7 / 2}-[4]_{9 / 2}$ & 0.0052 & 0.0052 & {$[2]_{3 / 2}-[2]_{5 / 2}$} & 0.0224 & 0.0224 \\
\hline$[4]_{7 / 2}-[4]_{7 / 2}$ & 0.1794 & 0.1793 & {$[2]_{3 / 2}-[2]_{3 / 2}$} & 0.0897 & 0.0897 \\
\hline$[4]_{9 / 2}-[3]_{7 / 2}$ & 0.0093 & 0.0093 & & & \\
\hline$[4]_{7 / 2}-[3]_{5 / 2}$ & 0.0089 & 0.0089 & & & \\
\hline$[4]_{7 / 2}-[3]_{7 / 2}$ & 0.0004 & 0.0004 & & & \\
\hline
\end{tabular}

$\overline{\mathrm{aRQDO} \text { without explicit polarization correction; }{ }^{\mathrm{R} Q D D O} \text { with explicit polarization correction [3] }}$. 


\title{
Oscillator Strengths of Rydberg Transitions
}

\author{
W Mende and M Kock \\ Institut für Atom und Molekülphysik, Abteilung Plasmaphysik, \\ Universität Hannover, Callinstrasse 38, \\ D-30167 Hannover, Germany
}

\section{Introduction}

In the present extended abstract we will describe a novel experimental technique which allows the determination of absolute oscillator strengths of transitions into high-lying Rydberg states where in current experiments the main quantum number $n$ varies from 30 to 60 . The largest attainable upper quantum number is limited by the spectral width and the incremental scanning step size of the laser system. The lower limit of $n$ results from the requirement that the ionization probability must be very nearly equal to unity for atoms being in a high Rydberg state. Absolute f-values are obtained from a measured photoionization cross section exploiting the fact that the discrete f-values are continuously merging into the differential ones.

\section{Procedure}

By means of laser spectroscopic techniques we investigate transitions from the $\mathrm{Sr}$ I $5 s 5 p^{1} P_{1}^{o}$ resonance state into Rydberg states as well as into the continuum near the the first ionization threshold. The first laser is set to the resonance wavelength of $460.7 \mathrm{~nm}$ while the second laser is tuned over the Rydberg states into the continuum. Our experimental arrangement to study Rydberg lines has been described elsewhere (Willke and Kock 1991, Mende and Kock 1996). The detector for the laser-produced ions is our metal vapour oven designed as a thermionic diode. A cross section of our oven is given in Fig.1.

The oven was typically operated at a temperature of $520^{\circ} \mathrm{C}$ with argon as a buffer gas at pressures between 10 and $15 \mathrm{hPa}$. At such pressures the ionization probability for Rydberg states with $n \geq 20$ is $p_{n}=1$. For $n<20$ it is no longer guaranteed that the ionization probability is still unity, thus setting a lower limit for our measuring procedure. As long as the condition, $p_{n} \simeq 1$, is fulfilled there is a simple relationship between $f$-value and photoionization cross section (see Mende and Kock 1996):

$$
\frac{1}{4 \pi \epsilon_{0}} \frac{\pi e^{2}}{m c} f_{1 n}=\frac{S^{1 n}}{S^{1+}} \frac{\nu_{1 n}}{\nu_{1+}} \sigma^{1+}
$$

The equation holds under the additional assumption that the absorption of the lines occurs in an optically thin layer. The $f$-value $f_{1 n}$ is then directly related to the photoionization cross section $\sigma^{1+}$ measured at frequencies $\nu_{1+}$ and $\nu_{1 n}$, respectively, the ratio of the ion signals $S^{1 n}$ due to absorption in the line, and the direct absorption into the continuum, $S^{1+}$.

The necessary absolute photoionization cross section $\sigma^{1+}$ at the first ionization threshold was determined with the saturation method (see e.g. Burkhardt et al 1988, He et al 1991, Mende et al 1995). Within the excited vapour column the second laser pulse, when tuned to a fixed wavelength 

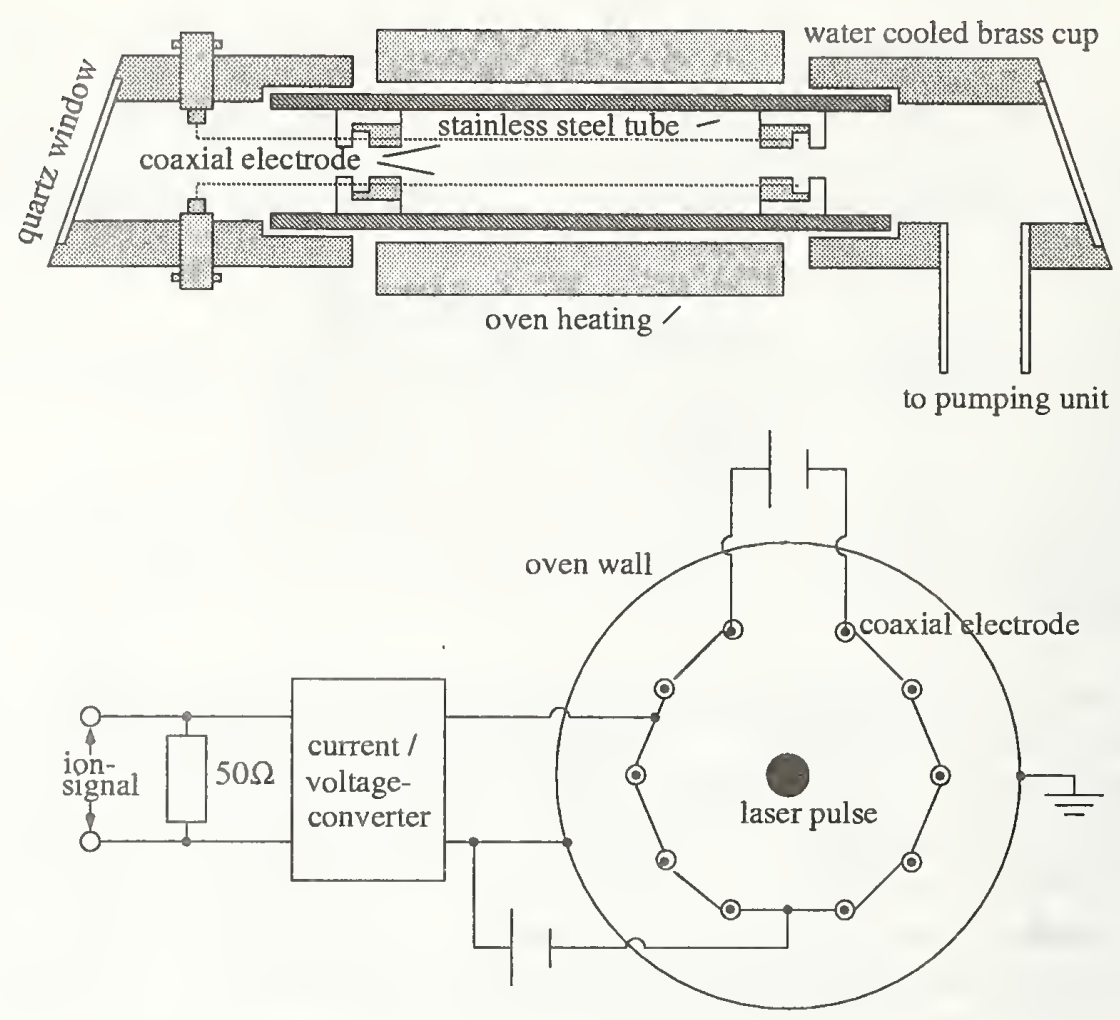

Figure 1: Cross sectional drawing of thermionic diode.

in the ion spectrum, produces the following number of photoions:

$$
Z=\int_{V} N_{o}\left(1-\exp \left(-\sigma^{1+} g(\rho) \phi\right)\right) d V
$$

Here $g(\rho)$ is the spatial distribution of the laser pulse while $\phi$ is the time-integrated number of photons of the ionizing laser pulse. By fitting this expression to a saturation curve, $N_{o}$ and $\sigma^{1+}$ can be obtained on absolute scales provided the spatial distribution $g(\rho)$ is known. The influence of different spatial laser profiles $g(\rho)$ on saturation behaviour can be seen in Fig.2. A top-hat, a Gaussian and a modified Lorentzian function $g(\rho)$ are used with the saturation function given in equation (2):

$$
\begin{aligned}
g_{\text {top-hat }}(\rho) & =\frac{1}{\pi \Delta \rho^{2}} \text { for } \rho<\Delta \rho, \text { else } 0 . \\
g_{\text {Gauss }}(\rho) & =\frac{1}{\pi \Delta \rho^{2}} \exp \left(-\left(\frac{\rho}{\Delta \rho}\right)^{2}\right) . \\
g_{\text {Lorentz }}(\rho) & =\frac{1}{\pi \Delta \rho^{2}} \frac{\Delta \rho^{4}}{\left(\rho^{2}+\Delta \rho^{2}\right)^{2}} .
\end{aligned}
$$

Only with a top-hat shaped laser pulse can complete saturation be achieved. Incidently, the wings in the Gaussian and Lorentzian curve are responsible for the fact that the curves in Fig. 2 do not reach complete saturation.

Fig. 3 displays a measured saturation curve together with a least-squares fit of equation (2). Note that it is not sufficient to describe the spatial distribution by a top-hat function. The actual distribution must be taken instead. We monitored the distribution with a CDD camera and fitted 

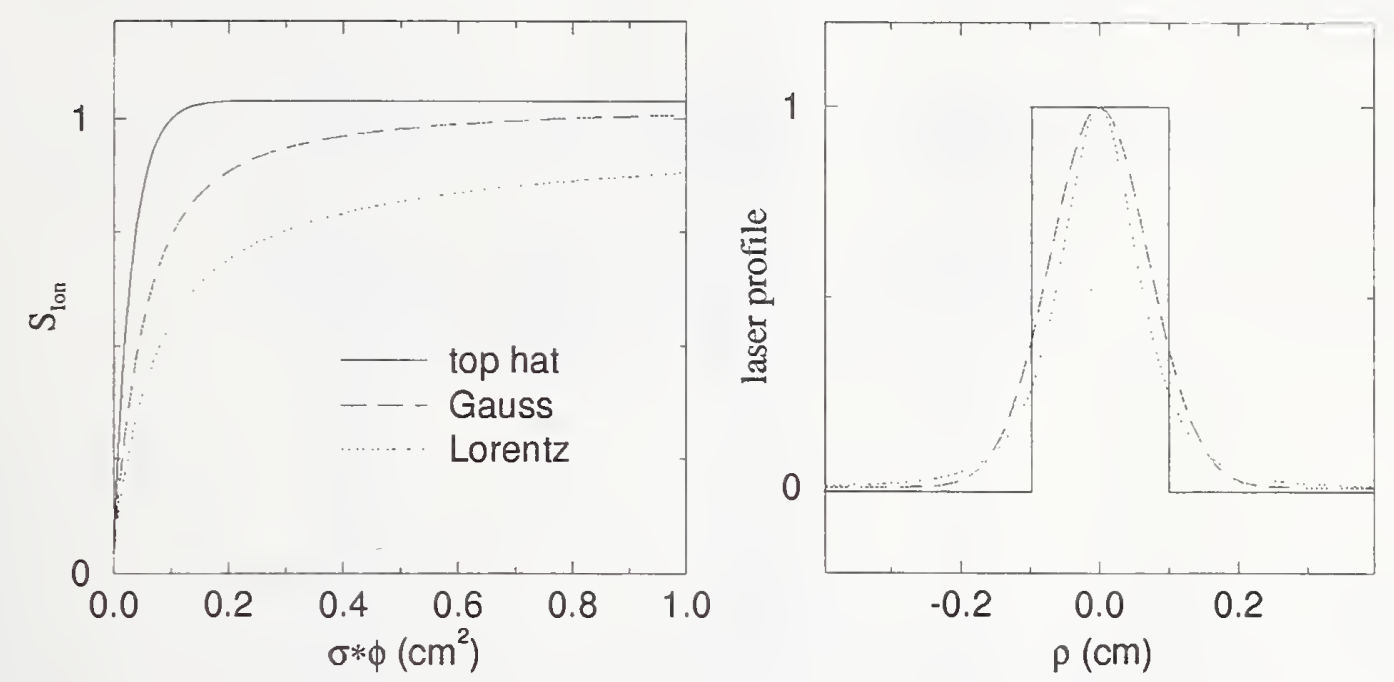

Figure 2: Saturation function with different spatial laser profiles.

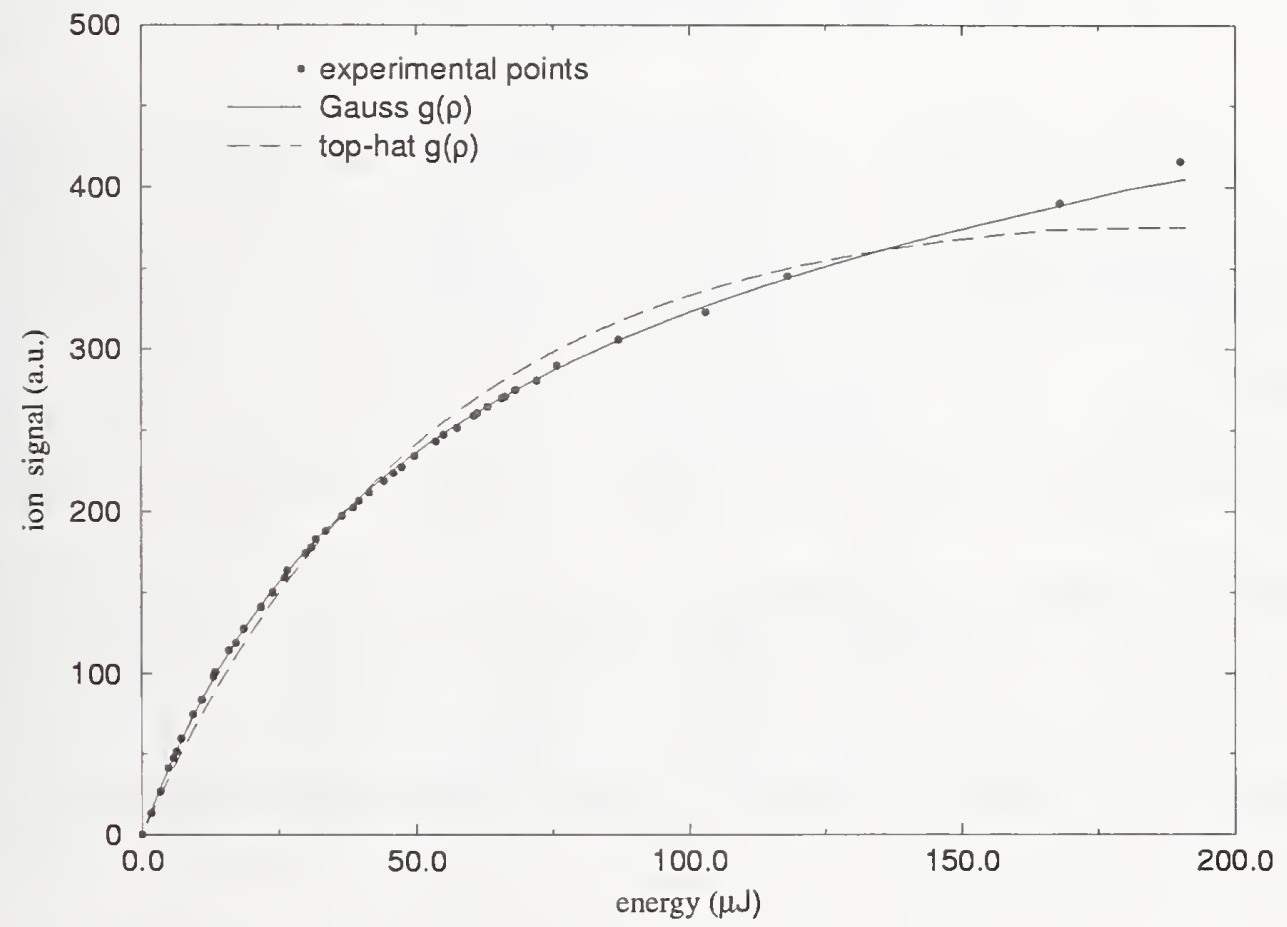

Figure 3: Saturation behaviour of the ion signal versus energy variation of the ionizing laser pulse at $412 \mathrm{~nm}$. The full curve is a least-squares fit of the data points with the saturation function given in Eq. (2). Due to the wings of the Gaussian shaped laser pulse, complete saturation was not achieved.

it to a bell-shape curve. We have calibrated our f-values with the $176 \mathrm{Mb} \pm 20 \%$ cross section we found with the saturation technique. The accuracy to which we can determine absolute f-values is mainly limited by the saturation technique. Since only a small saturation could be obtained 
Table1. Absolute oscillator strengths of the Sr I $5 s 5 p{ }^{1} P_{1}^{o} \rightarrow 5 s n d{ }^{1} D_{2}$ transitions.

\begin{tabular}{|c|c|c|c|}
\hline $\mathrm{n}$ & f-value & $\mathrm{n}$ & f-value \\
\hline 30 & $6.76 \mathrm{E}-4$ & 46 & $2.44 \mathrm{E}-4$ \\
31 & $5.96 \mathrm{E}-4$ & 47 & $2.46 \mathrm{E}-4$ \\
32 & $5.56 \mathrm{E}-4$ & 48 & $2.50 \mathrm{E}-4$ \\
33 & $4.92 \mathrm{E}-4$ & 49 & $2.19 \mathrm{E}-4$ \\
34 & $4.38 \mathrm{E}-4$ & 50 & $2.02 \mathrm{E}-4$ \\
35 & $4.79 \mathrm{E}-4$ & 51 & $2.13 \mathrm{E}-4$ \\
36 & $4.19 \mathrm{E}-4$ & 52 & $1.88 \mathrm{E}-4$ \\
37 & $4.07 \mathrm{E}-4$ & 53 & $1.77 \mathrm{E}-4$ \\
38 & $3.97 \mathrm{E}-4$ & 54 & $1.65 \mathrm{E}-4$ \\
39 & $3.55 \mathrm{E}-4$ & 55 & $1.57 \mathrm{E}-4$ \\
40 & $3.50 \mathrm{E}-4$ & 56 & $1.55 \mathrm{E}-4$ \\
41 & $3.33 \mathrm{E}-4$ & 57 & $1.32 \mathrm{E}-4$ \\
42 & $3.35 \mathrm{E}-4$ & 58 & $1.28 \mathrm{E}-4$ \\
43 & $3.01 \mathrm{E}-4$ & 59 & $1.23 \mathrm{E}-4$ \\
44 & $2.84 \mathrm{E}-4$ & 60 & $1.01 \mathrm{E}-4$ \\
45 & $2.66 \mathrm{E}-4$ & & \\
\hline
\end{tabular}

with our laser system, the uncertainty of $20 \%$ is relatively large. With higher laser energy available the uncertainty could be easily reduced to $10 \%$. Nevertheless, in the present experiment it is the dominant contribution to the total uncertainty of $(22-24) \%$ in the f-values given in table 1 for the Rydberg transitions $5 s 5 p{ }^{1} P_{1}^{o} \rightarrow 5 s n d{ }^{1} D_{2}$ with $n=30-60$.

\section{References}

Burkhardt C.E., Libbert J.L., Xu J., Leventhal J.J., and Kelly J.D., Phys. Rev A 38, 5949-52 (1988)

He L.W., Burkhardt C.E., Ciocca M., and Leventhal J.J., Phys. Rev. Lett. 67, 2131-4 (1991)

Mende W., Bartschart K., and Kock M., J. Phys. B: At. Mol. Opt. Phys. 28, 2385-93 (1995)

Mende W. and Kock M., J. Phys. B: At. Mol. Opt. Phys. 29, 655-663 (1996)

Willke B. and Kock M., Phys. Rev A 43, 6433-5 (1991) 


\title{
Atomic Data for Lighting Applications
}

\author{
Gillian Nave, Craig Sansonetti, Joseph Reader \\ National Institute of Standards and Technology, \\ Gaithersburg, MD 20899, U.S.A.
}

\section{Introduction}

Although lighting accounts for about $20 \%$ of U.S. electric power consumption, even the best white light sources convert only about one third of the power they consume to usable light. Improved discharge sources are a promising avenue for development of more efficient lighting, but lack of basic atomic data places fundamental limitations on this research. NIST is currently establishing a new experimental program aimed at determining atomic wavelengths, energy levels, and branching ratios that are needed for lighting applications. The centerpiece of this effort is a high resolution Fourier transform spectrometer recently installed at NIST. Its broad spectral coverage, high resolution, and linear intensity response will permit observation of almost all spectroscopic data of interest.

\section{Atomic Data of Interest to Lighting}

Rare earth metals are commonly used in metal halide lamps to provide a white light spectrum similar to that of natural light. Three elements of interest are thullium, holmium and dysprosium. These metals have very rich atomic spectra, with many thousands of lines throughout the visible region, but relatively little radiation in the ultraviolet and infrared regions. In many cases the atomic data for these elements are missing. Many energy levels remain to be found and many lines are unclassified. Transition probability data are needed, even for the strongest lines in the spectrum.

Fourier transform (FT) spectrometry offers many advantages for measuring these atomic data. Accurate measurements of line intensities are possible with the large dynamic range $\left(\sim 10^{5}\right)$ and linear intensity response. A FT spectrometer measures all of the lines in the spectrum simultaneously, minimizing the effects of source drift, and its wide wavenumber coverage permits the measurement of all of the decay channels from a particular upper level simultaneously. The high resolution and signal-to-noise ratio allow blended lines to be easily distinguished and minimize the errors due to mis-identification of spectral lines.

\section{The NIST Fourier Transform Spectrometer}

Three years ago, NIST acquired a high-resolution FT spectrometer from the Los Alamos National Laboratory [1, 2]. The instrument has a spectral resolution of $0.0025 \mathrm{~cm}^{-1}$ and we currently have optics and detectors to cover the wavelength range from $200 \mathrm{~nm}$ to around $8 \mu \mathrm{m}$.

Two major improvements have been made since we acquired the spectrometer. First, the instrument has been installed in a new vacuum chamber. A vacuum chamber is needed both to eliminate the effects of air turbulence in the optical path, and to allow operation in the infra-red, where there are molecular bands from water vapor and carbon dioxide. 


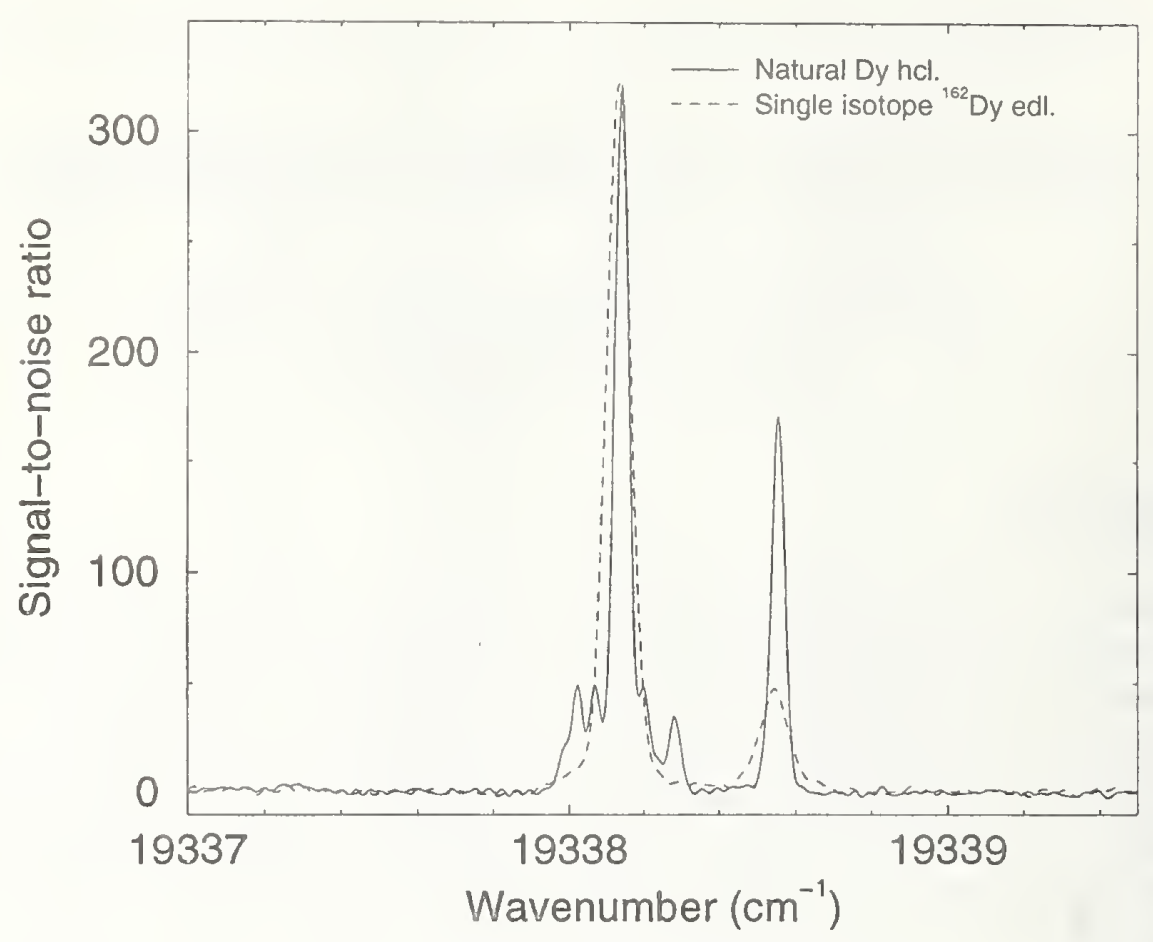

Figure 1: Comparison of the Dy line at $19338 \mathrm{~cm}^{-1}\left(4 \mathrm{f}^{10}\left({ }^{5} \mathrm{I}\right) 6 \mathrm{~s}{ }^{6} \mathrm{I}_{17 / 2}-4 \mathrm{f}^{9}\left({ }^{6} \mathrm{H}^{\mathrm{o}}\right) 5 \mathrm{~d} 6 \mathrm{~s}{ }^{6} \mathrm{H}_{15 / 2}^{\mathrm{o}}\right)$ in the hollow cathode and electrodeless discharge lamp.

The second change has been a complete replacement of the data acquisition system. The new system, based on the method of Brault [3], uses two 20 bit A/D converters to sample the interferogram at a fixed sampling rate of $39.9 \mathrm{kHz}$. Laser fringes are recovered from the servo system used to control the catseye retroreflectors. Both the fringes and the A/D samples are referenced to the same $40 \mathrm{MHz}$ clock, and the interferogram can then be recovered by using an interpolating digital filter. The digital filter increases the signal-to-noise ratio as it greatly reduces noise from outside the spectral region of interest. The new system is also much less sensitive to vibrations than the previous system, and hence ghosts from non-uniform sampling of the interferogram are reduced.

The instrument has been operating in the region from $250 \mathrm{~nm}$ to $2.5 \mu \mathrm{m}$ since March, 1997. In addition to our observations of spectra of rare earth elements of interest to the lighting industry, we have been studying spectra of mercury, bismuth and krypton, which are of astrophysical interest.

\section{The Spectrum of Dysprosium}

The first spectra we are investigating in connection with our program of atomic data for lighting are those of neutral and singly-ionized dysprosium. Dysprosium is used in metal halide lamps, and accurate transition probability data are needed for modelling the lamp discharge. We are collaborating with Wickliffe and Lawler, of the University of Wisconsin, to measure branching ratios with our FT spectrometer, which will be combined with the Wisconsin lifetime data [4] to provide transition probabilities.

We have recorded spectra of high current hollow cathode lamps from $8500 \mathrm{~cm}^{-1}$ to 30000 $\mathrm{cm}^{-1}(1.2 \mu \mathrm{m}$ to $330 \mathrm{~nm})$ using our FT spectrometer. The cathode was a $60 \mathrm{~mm}$ long cylinder 
of natural dysprosium with an $8 \mathrm{~mm}$ diameter bore. Currents ranged from $100 \mathrm{~mA}-500 \mathrm{~mA}$ and the carrier gas was $200 \mathrm{~Pa}$ of Ar. Radiometric calibration was done with a tungsten strip lamp. Dysprosium spectra were also recorded with the Chelsea FT 500 vacuum ultraviolet Fourier transform spectrometer at Lund University, Sweden. These covered the range $25000 \mathrm{~cm}^{-1}$ to 50000 $\mathrm{cm}^{-1}$ and were radiometrically calibrated with a deuterium lamp.

Natural dysprosium has four isotopes, and the spectral lines from the hollow cathode lamps are thus complicated by isotope structure. To assist in the analysis of these spectra we also recorded the spectrum of an electrodeless discharge lamp containing the single isotope ${ }^{162} \mathrm{Dy}$. However the electrodeless discharge lamp is not as useful as the hollow cathode lamp for measuring branching ratios, as many spectral lines are strongly self-absorbed. Radiometric calibration is also less reliable as it is not possible to measure the transmission of the lamp envelope. Figure 1 compares a line in the hollow cathode lamp and the electrodeless discharge lamp. Although the line in the electrodeless discharge lamp is wider than the line in the hollow cathode lamp, it is not complicated by isotope structure.

So far we have obtained wavelengths for roughly 4000 Dy I and Dy II lines observed in the ${ }^{162}$ Dy electrodeless discharge lamp. These have been used to revise the energy level values of 376 energy levels in ${ }^{162} \mathrm{Dy}$ I and 228 energy levels in ${ }^{162} \mathrm{Dy}$ II. Initial branching ratios have been obtained for some of the lower Dy II levels and even parity Dy I levels. These show good agreement with the preliminary branching ratios of Wickliffe and Lawler[5], who have used a different calibration technique, giving increased confidence in the radiometric calibration.

\section{Acknowledgments}

We are grateful to U. Griesmann for his assistance with the FTS data acquisition system and the hollow cathode. We thank U. Litzèn of the University of Lund for assisting in the use of the FTS by one of us (G.N.). We thank E. Worden for providing the electrodeless discharge lamp. This work is partially supported by the Electric Power Research Institute.

The identification of commercial products in this paper does not imply recommendation or endorsement by the National Institute of Standards and Technology, nor does it imply that the items identified are necessarily the best available for the purpose.

\section{References}

[1] D. Hof, B. A. Palmer, Rep. LALP-88-39, Los Alamos National Laboratory, Los Alamos, NM, Oct. 1988

[2] J. Simons, B. A. Palmer, D. E. Hof, R. C. Oldenborg, J. Opt. Soc. Am. B 6, 1097 (1989)

[3] J. W. Brault, it Appl. Opt. 35, 2891 (1996)

[4] J. J. Curry, E. A. Den Hartog, J. E. Lawler, J. Opt. Soc. Am. B, in press

[5] M. E. Wickliffe, J. E. Lawler, private communication 


\title{
Investigation of the Autoionization Spectra of Samarium from $48800 \mathrm{~cm}^{-1}$ to $51200 \mathrm{~cm}^{-1}$
}

\author{
Hyunmin Park, Hyun-chae Kim, Yong-joo Rhee, and Jongmin Lee \\ Laboratory for Quantum Optics, Korea Atomic Energy Research Institute, \\ P.O. Box 105, Yusong, Taejon 305-600, Korea
}

\section{Introduction}

The autoionization state is a quasi-bound state of an atom which lies above the ionization threshold of the outer valence electrons. This state plays a great role in the photoionization of atoms in plasmas and astrophysics. Hence, the investigation of the autoionization states has been of interest and a number of experimental and theoretical results have been reported recently.

The spectrum of the rare earth metal Sm is complex because of its partially filled $4 f$ shell and the structures of the $\left[4 f^{6} 6 s^{2}\right]{ }^{7} F$ ground term. To our knowledge, there are only few results reported on the autoionization state of the Sm [1], [2]. In this paper, we report the autoionization spectra of atomic samarium(Sm) in the region from $48800 \mathrm{~cm}^{-1}-51200 \mathrm{~cm}^{-1}$, obtained from the three-step excitation. We measured the energy levels of the odd autoionization states.

\section{Experiments and Results}

Three-step excitation was used to investigate the autoionization state of $\mathrm{Sm}$. The first excitation step was fixed at a transition from the ground state, $\left[4 f^{6} 6 s^{2}\right]{ }^{7} F_{1}$, to the first excited state $\left[4 f^{6} 6 s 6 p\right]$ ${ }^{7} F_{2}^{0}$, the energy of which is $17190 \mathrm{~cm}^{-1}$. Then the even states with the energies $34522 \mathrm{~cm}^{-1}$, $34420 \mathrm{~cm}^{-1}$, and $34399 \mathrm{~cm}^{-1}$ were chosen as the intermediate levels. Finally, the energies of the autoionization state were obtained by scanning the wavelengths of the ionization laser from $600 \mathrm{~nm}$ to $700 \mathrm{~nm}$.

The experimental setup for three-step excitation is well described in Ref.[3]. The setup consists of a laser system, a time of flight mass spectrometer, and a detection system. For the first and the second excitation step, we used two single-mode dye lasers pumped by a frequency-doubled Nd:YAG laser. The transition to an autoionization state from the second excited state was made by using another broadband dye laser pumped by the same Nd:YAG laser. Two commercial wavemeters having resolutions of $200 \mathrm{MHz}$ were used to measure the wavelengths of the two single-mode dye lasers. Additionally, to calibrate the ionization laser, a small portion of the dye laser beam was used to observe the optogalvanic spectrum of a Sm-Ar hollow-cathode discharge lamp. The frequencies of the single-mode pulse lasers were stabilized within $100 \mathrm{MHz}$ during an experiment by a feedback system which consisted of two fabry-perot etalons and a piezoelectric transducer(PZT) attached to the oscillator of the laser. For step-wise excitation, the lasers were made to interact with the Sm atoms with a time delay of $10 \mathrm{~ns}$, respectively.

The atomic beam of samarium was produced by a resistively heated tantalum boat containing the natural solid Sm up to a temperature of about $1000 \mathrm{~K}$ in a vacuum chamber. The generated $\mathrm{Sm}$ atomic beam was collimated by two slits and directed perpendicularly to the laser beam path 
Table 1: List of the investigated autoionization states

\begin{tabular}{|c|c|c|c|c|c|}
\hline $\begin{array}{l}\begin{array}{l}\text { Intermediate } \\
\text { state }\left(\mathrm{cm}^{-1}\right)\end{array} \\
\end{array}$ & $\begin{array}{c}\text { Ionizing Laser } \\
\text { wavelength }(\mathrm{nm})\end{array}$ & $\begin{array}{c}\text { Autoionization } \\
\text { state }\left(\mathrm{cm}^{-1}\right)\end{array}$ & $\begin{array}{c}\text { Fano } \\
\text { q-parameter }\end{array}$ & $\begin{array}{l}\text { Width } \\
\left(\mathrm{cm}^{-1}\right)\end{array}$ & $\begin{array}{c}\text { signal } \\
\text { strength }\end{array}$ \\
\hline \multirow{28}{*}{$\begin{array}{l}34399 \\
(J=1)\end{array}$} & 601.367 & 51022.9 & \multirow{17}{*}{32.7} & \multirow{17}{*}{23.7} & $\mathrm{~m}$ \\
\hline & 604.574 & 50934.8 & & & $\mathrm{~s}$ \\
\hline & 604.995 & 50923.3 & & & w \\
\hline & 607.195 & 50863.4 & & & $\mathrm{~m}$ \\
\hline & 611.276 & 50753.5 & & & $\mathrm{w}$ \\
\hline & 612.171 & 50729.5 & & & $\mathrm{w}$ \\
\hline & 613.285 & 50699.9 & & & $\mathrm{w}$ \\
\hline & 621.263 & 50490.6 & & & $\mathrm{w}$ \\
\hline & 624.497 & 50407.3 & & & w \\
\hline & 625.611 & 50378.8 & & & $\mathrm{w}$ \\
\hline & 627.732 & 50324.8 & & & w \\
\hline & 629.152 & 50288.9 & & & $\mathrm{~m}$ \\
\hline & 632.072 & 50215.5 & & & $\mathrm{w}$ \\
\hline & 633.200 & 50187.3 & & & w \\
\hline & 634.378 & 50158.0 & & & $\mathrm{w}$ \\
\hline & 635.273 & 50135.8 & & & $\mathrm{w}$ \\
\hline & 643.565 & 49932.9 & & & w \\
\hline & 647.099 & 49848.1 & \multirow[t]{8}{*}{52.9} & \multirow[t]{8}{*}{5.1} & $\mathrm{~s}$ \\
\hline & 654.862 & 49664.9 & & & $\mathrm{w}$ \\
\hline & 656.428 & 49628.5 & & & $\mathrm{w}$ \\
\hline & 663.415 & 49468.1 & & & $\mathrm{~m}$ \\
\hline & 666.181 & 49405.6 & & & $\mathrm{~m}$ \\
\hline & 669.106 & 49340.0 & & & $\mathrm{~m}$ \\
\hline & 670.169 & 49338.5 & & & $\mathrm{~m}$ \\
\hline & 683.314 & 49029.3 & & & $\mathrm{~m}$ \\
\hline & 685.258 & 48987.8 & 48.6 & 5.0 & $\mathrm{~s}$ \\
\hline & 685.437 & 48984.0 & 56.2 & 6.4 & s \\
\hline & 698.932 & 48702.4 & & & $\mathrm{w}$ \\
\hline \multirow{8}{*}{$\begin{array}{l}34420 \\
(J=2)\end{array}$} & 602.733 & 51007.0 & \multirow{8}{*}{$\begin{array}{l}41.1 \\
64.6\end{array}$} & \multirow{8}{*}{$\begin{array}{l}6.0 \\
7.8\end{array}$} & $\mathrm{~m}$ \\
\hline & 603.408 & 50988.5 & & & w \\
\hline & 615.171 & 50671.6 & & & $\mathrm{~s}$ \\
\hline & 615.967 & 50650.9 & & & $\mathrm{~s}$ \\
\hline & 624.580 & 50426.8 & & & $\mathrm{w}$ \\
\hline & 626.324 & 50382.3 & & & $\mathrm{w}$ \\
\hline & 635.038 & 50163.2 & & & $\mathrm{w}$ \\
\hline & 642.951 & 49947.8 & & & $\mathrm{w}$ \\
\hline \multirow{6}{*}{$\begin{array}{l}34544 \\
(J=3)\end{array}$} & 624.082 & 50541.3 & \multirow{6}{*}{$\begin{array}{c}109.5 \\
22.4 \\
-8.0 \\
-87.8\end{array}$} & \multirow{6}{*}{$\begin{array}{c}8.1 \\
6.0 \\
22.4 \\
9.1\end{array}$} & $\mathrm{w}$ \\
\hline & 628.314 & 50433.4 & & & $\mathrm{~m}$ \\
\hline & 630.376 & 50381.4 & & & $\mathrm{~s}$ \\
\hline & 630.774 & 50371.3 & & & s \\
\hline & 639.332 & 50159.2 & & & s \\
\hline & 652.319 & 49728.5 & & & $\mathrm{~s}$ \\
\hline
\end{tabular}


in order to reduce the Doppler broadening to $30 \mathrm{MHz}$. The photo ions of $\mathrm{Sm}$ were detected at the end of the flight tube by a microchannel plate(MCP). Finally, the ion signals were averaged by a boxcar averager and the mass spectrum was recorded by a digitizing oscilloscope.

The experimental results showed a large number of autoionization lines in the region 48800 $\mathrm{cm}^{-1}-51200 \mathrm{~cm}^{-1}$. Neglecting the small lines, all experimental results are summarized in Table 1. In Table 1, s, m, and w represent strong, medium, and weak transition strengths, respectively.

By fitting the experimental data against Fano's formula [4], we obtained the resonance energy $\left(E_{r}\right)$ and the linewidth $(\Gamma)$ of the autoionizing states. Fano's formula is expressed as follows:

$$
\sigma_{\text {total }}(\varepsilon)=\sigma_{a} \frac{(\varepsilon+q)^{2}}{\varepsilon^{2}+1}+\sigma_{b}
$$

with $\varepsilon=\left(E-E_{\tau}\right) /(\Gamma / 2)$, where $\sigma_{\text {total }}$ represents the transition cross section, $q$ is the Fano parameter, $E$ is the incident photon energy, and $\sigma_{a}$ and $\sigma_{b}$ are the fitting parameters. Most $q$ parameters recorded in Table 1 are much larger than 1 because the measured autoionization spectra exhibit nearly symmetric line profiles. In the energy range investigated in this work, we can observe ten relatively strong transition lines. Those lines can be used for efficient photoionization of Sm.

We presume that the measured autoionization lines are from $\left[4 f^{6} 6 s 5 d\right]$ configurations. However, to identify the measured lines, we need more experimental and theoretical efforts.

\section{Summary}

We investigated the autoionization spectra of atomic samarium by three-step excitation. In this study, we measured the energy levels of the odd autoionization states located in the region from $48800 \mathrm{~cm}^{-1}-51200 \mathrm{~cm}^{-1}$.

The first resonant step was fixed at a transition from the ground state, $\left[4 f^{6} 6 s^{2}\right]{ }^{7} F_{1}$, to the first excited state $\left[4 f^{6} 6 s 6 p\right]^{7} F_{2}^{0}$, the energy of which is $17190 \mathrm{~cm}^{-1}$. The even states with energies of $34522 \mathrm{~cm}^{-1}, 34420 \mathrm{~cm}^{-1}$, and $34399 \mathrm{~cm}^{-1}$ were then chosen as the intermediate levels. Finally, the energies of the autoionization states were obtained by scanning the wavelengths of an ionization laser from $600 \mathrm{~nm}$ to $700 \mathrm{~nm}$.

As a result of this work, forty-two autoionization states were found in the investigated range from $48800 \mathrm{~cm}^{-1}$ to $51200 \mathrm{~cm}^{-1}$. Among the observed lines, Some lines are analysed by Fano's formula and $q$ parameters are determined.

\section{References}

[1] V. E. Dobryshin, N. A. Karpov, S. A. Kotochigova, B. B. Krynetskii, V. A. Mishin, O. M. Stelmakh, and V. M. Shustryakov, Opt. Spektrosk. 54, 415 (1983)

[2] Hu Sufen, MEI Shimin, ZHANG Sen, CHEN Xing, and XU Yunfei, Chinese Phys. Lett. Vol. 6, No. 2 (1989)

[3] Hyunmin PARK, Hyun-chae KIM, Jong-hoon YI, Jae-Min HAN, and Jongmin LEE, J. of Korean Physical Society. Vol. 30, 453 (1997)

[4] U. Fano, Phys. Rev. 124, 1866 (1961) 


\title{
Upgrading the Atomic and Molecular Database for Astrophysics and Atmospheric Physics by Fourier Transform Spectroscopy
}

\author{
J. C. Pickering, R. Schermaul, G. Cox, J. Rufus, A. P. Thorne, and R. C. M. Learner \\ Spectroscopy Group, Blackett Laboratory, Imperial College, London, SW7 2BZ, UK \\ email: j.pickering@ic.ac.uk \\ P. L. Smith \\ Harvard-Smithsonian CfA, Cambridge, MA 02138, USA
}

\begin{abstract}
There is currently a great demand for improvements to the atomic and molecular databases for astrophysics, atmospheric physics, and theoretical physics. At Imperial College (IC), the VUV Fourier Transform (FT) Spectrometer, with its high resolving power (up to 2 million at $2000 \AA$ ), broad spectral range and unique wavelength range (down to $140 \mathrm{~nm}$ ), is used in a continuing program to improve the accuracy and completeness of both atomic and molecular data of importance for astrophysics and atmospheric physics.
\end{abstract}

\section{Atomic Spectroscopy}

Until recently it was generally assumed that the atomic spectra of elements such as the transition group were well known; the existing tables of data were thought to be adequate by users such as astronomers. But now, with a new generation of telescopes equipped with spectrometers of high resolving power, the situation is very different. Many of the atomic spectra of relevance to astrophysics were last investigated back in the 1930s and 1940s using grating spectrographs and photographic plates. Accuracies of the resulting wavelengths, and line intensities estimated by eye, simply cannot meet the needs of either the astronomers, who wish, for example, to interpret the high resolution stellar spectra now accessible with the Hubble Space Telescope's (HST) spectrographs, or meet the needs of the modern atomic theorists, who with greater computing power can now carry out calculations of unprecedented sophistication. Improvements of at least an order-of-magnitude in accuracy of wavelengths and energy levels are urgently required.

Accurate transition wavelengths are of vital importance in the identification of new atomic energy levels and the correction of known energy levels. These wavelengths and energy levels are used in the identification of stellar spectral line features, and they also feed into semi-empirical calculations in atomic physics which rely heavily on experimental data of high quality. High accuracy of wavelengths, energy levels and transition probabilities, as well as knowledge of hyperfine structure and isotope shifts, is essential in generating reliable synthetic stellar spectra and in disentangling blended features and weak lines of abundant elements in astrophysical spectra of objects ranging from chemically peculiar stars to supernovae remnants. In particular, the dramatic improvements in the quality of UV stellar spectra, observed using the GHRS and STIS spectrographs of the HST, have highlighted the inadequacy of the laboratory atomic data base needed to interpret these observations, especially in the VUV region (below $2000 \AA$ ), where stellar spectral lines from doubly 
and singly ionized species predominate. The IC FT spectrometer, with its unique short wavelength cut-off, is ideal for recording these spectra.

In recent years, high resolution FT visible and UV spectra of the line rich, astrophysically important neutral and singly ionized transition group elements ( $\mathrm{Fe}, \mathrm{Ni}, \mathrm{Cr}, \mathrm{Co}, \mathrm{Ti}$, and $\mathrm{V}$ ) have been recorded at IC using water-cooled, hollow cathode lamps as sources. In all cases, these data have been supplemented by IR observations at the NSO National Solar Observatory, USA, typically out to $5 \mu \mathrm{m}$. Analyses of the spectra of Fe I, Ni I, Co I and Co II, led to improvements of at least an order of magnitude in accuracy of wavelengths and atomic energy levels and the identification of hundreds of new levels [1], [2], [3], [4], and, for Co I, the determination of hyperfine structure (hfs) constants [5]. The analyses of the spectra of Cr I, Cr II, V I and V II await completion at IC. With the FT spectrometer and suitably reliable intensity calibration, accurate branching ratios can be measured, which, when combined with lifetime measurements, yield oscillator strengths. Isotope shifts can also be observed, and the VUV platinum spectrum was recorded at IC so that accurate studies of isotope shifts could be made. A new project studying doubly ionized spectra is underway, initially beginning with Fe III, excited using a Penning discharge source.

\subsection{Term Analysis}

The dramatic improvement that can be achieved in the database is illustrated by the cobalt spectrum. Visible-VUV (140-600 nm) spectra recorded at IC were used together with FT IR (600$3000 \mathrm{~nm}$ ) spectra recorded at Kitt Peak NSO. For strong transitions the wavenumber accuracy is within 1-2 $\mathrm{mK},\left(1 \mathrm{mK}=0.001 \mathrm{~cm}^{-1}\right)$. In the Co I spectrum analysis [3], over 60 new energy levels were found and several hundred lines identified for the first time. Hfs splitting factors for Co I were found for over $82 \%$ of the known energy levels (see Section 1.2). For Co II, the improvement is even more dramatic; over 170 new energy levels were found, and the number of identified transitions doubled [4],[6] . The new experimental data was used in theoretical calculations to give calculated transition probabilities [7]. The vast improvements in knowledge of these spectra were not limited to the visible-VUV spectral regions, -most of the IR transitions were reported for the first time.

\subsection{Measurements of Hyperfine Structure}

Knowledge of hfs in iron-group elements is important not only for theoretical atomic physics but also for determinations of elemental abundances in stellar atmospheres. It has been noticeable in work reporting on stellar abundances that elements with hfs have often been neglected because of the lack of good laboratory atomic data. For the iron-group elements with odd atomic number, such as $\mathrm{Co}$, the effect of hfs as a line broadening mechanism must be taken into account in abundance studies when using both curve-of-growth methods and spectrum synthesis methods. It is also important to correctly allow for any hfs in the resolution of blended lines in stellar spectra.

Although hfs has been studied for many years, the methods used, such as laser spectroscopy, atomic-beam magnetic resonance and Fabry-Perot interferometry, allowed observations of only a few selected lines, and so the hyperfine interaction constants A (magnetic dipole) and B (electric quadrupole) were known for relatively few levels. However, with the FT spectrometer, combining high resolution and a wide spectral range, thousands of lines may be observed. Co I was investigated [5] in the first use of high resolution FT spectrometry for comprehensive measurements of hfs over a wide spectral range, $3.8 \mu \mathrm{m}$ to $180 \mathrm{~nm}$. Computer fits of line profiles of over 1000 Co I transitions yielded A factors for 297 energy levels with an uncertainty of between 0.2 and $3 \%$. For 208 of these A factors no previous measurements are known. Approximate values of the B factors were also found. Currently, studies of hfs in Co II and V II are also underway at IC. 


\subsection{The Measurement of Branching Ratios and Oscillator Strengths}

The FT spectrometer is an ideal instrument for measuring accurate branching ratios because of its broad spectral range and smoothly varying response. Branching ratios can be reliably obtained for lines widely differing in wavelength. The limit in accuracy is determined by the uncertainty in the calibration of the VUV radiometric standard lamp, and we estimate the limit in uncertainty in relative intensities for strong lines to be about $4 \%$. Measurements of Ti II branching ratios have been made and have yielded oscillator strengths when combined either with experimental lifetime measurements where these exist, or with calculated lifetimes. More branching ratio measurements will be made in the future depending on current interests in the astronomical community.

\subsection{Doubly Ionized Spectra}

The defects in the database for neutral and singly ionized species apply even more strongly for the third spectra. The accuracy of the current wavelength data is about $10 \mathrm{~m} \AA$ whereas wavelengths are now needed to an accuracy of 1 part in $10^{7}$ - or $0.2 \mathrm{~m} \AA$ at $2000 \AA$. Two recent advances in laboratory techniques now make it possible to obtain vast improvements in wavelength and oscillator strength measurements of third spectra:

i The extension of the wavelength range of the FT spectrometer into the VUV region (down to about $140 \mathrm{~nm}$ ) with the development of a $\mathrm{MgF}_{2}$ beamsplitter at IC [8].

ii The development of a light source, a dc Penning discharge, that is compatible with the FT spectrometer and that excites third spectra and lines from high-lying levels of second spectra that cannot be produced in dc hollow cathode discharges. These have traditionally been excited using pulsed hollow cathode lamps and sliding sparks, but the poor "shot-to-shot" reproducibility of these sources makes them unsuitable for use with an FT spectrometer. The Penning discharge source used was originally developed as a VUV radiometric standard by Heise et al. [9].

Preliminary measurements were made at IC using this source, and results in the region 150$220 \mathrm{~nm}$ using iron cathodes have yielded Fe II and Fe III spectra with signal-to-noise ratios (SNRs) of several hundred for the stronger Fe III lines. The improvement in wavelength accuracy is at least an order of magnitude over the existing data. Ni III will also be recorded. Analyses of these spectra should yield dramatic improvements in accuracy of energy levels and wavelengths, as well as many new energy levels. The spectra will be intensity calibrated so that branching ratio measurements can be obtained, and these may be combined with either theoretical or experimental lifetimes to give oscillator strengths. In the future, other transition group elements will also be measured.

\section{Molecular Spectroscopy}

FT spectrometers are also used to record absorption spectra of species important in studies of the earth's atmosphere and other planetary atmospheres. Recent projects include:

\subsection{Measurements of UV Absorption Cross Sections of $\mathrm{SO}_{2}$}

In order to avoid underestimating absorption cross sections, the sharpest features in the absorption spectrum need to be fully resolved. With support from ESA, the absorption spectrum of $\mathrm{SO}_{2}$ was measured at high resolution using the IC FT spectrometer between 280-330 nm. These 
measurements are of direct application to pollution measurements by downward-looking satellites such as GOME (Global Ozone Monitoring Experiment) and SCIAMACHY (Scanning Imaging Absorption Spectrometer for Atmospheric Chartography). Work is also in progress, in collaboration with the Harvard-Smithsonian CfA and Wellesley College, USA, on measurements of the far UV (190-220 nm) bands of $\mathrm{SO}_{2}$. The resulting accurate absorption cross sections will enable planetary astronomers to reliably interpret $\mathrm{SO}_{2}$ in HST spectra of the atmosphere of Io and Venus.

\subsection{Measurements of VUV Absorption Cross Sections of $\mathrm{O}_{2}$ and NO}

For accurate modeling of the penetration of solar radiation into the upper atmosphere in the wavelength region 220-175 nm, computations involving $\mathrm{O}_{2}$ and minor species, notably $\mathrm{NO}$, must be carried out on a line-to-line basis. This requires both accurate wavenumbers and high resolution in order to obtain true line profiles and absolute cross sections. Good SNRs for absorption measurements require a background continuum of high photon flux, and in the VUV the best such source is synchrotron radiation. For this purpose, the IC VUV FT spectrometer was taken to the Photon Factory (KEK) in Japan, and spectra of $\mathrm{O}_{2}$ and $\mathrm{NO}$ were recorded from 195-160 nm at resolution matched to the Doppler widths of about $0.12 \mathrm{~cm}^{-1}$. Analysis of these spectra is in progress.

\subsection{Precise Line Parameter Measurements in the Visible $A$ Band Spectrum of $\mathrm{O}_{2}$}

The oxygen $A$ band shows a weak absorption in a relatively empty portion of the atmospheric spectrum and is well suited for atmospheric remote sensing. Accurate $\mathrm{O}_{2}$ data is crucially important for remote sensing of cloud-top height and coverage for satellite retrieval of atmospheric ozone, which is utilized by the GOME and the SCIAMACHY. They are also needed for the measurement of middle atmosphere ozone from $\mathrm{O}_{2} \mathrm{~A}$ band airglow emission measurements proposed for the High Resolution Doppler Instrument.

The spectrum of the oxygen A band, $b^{1} \Sigma_{g}^{+}(v=0) \leftarrow X^{3} \Sigma_{g}^{-}(v=0)$, has recently been measured and analysed in order to improve the currently available data on this band. The measurements were carried out at the Rutherford Appleton Laboratory (UK) using a high-resolution Bruker FT spectrometer, together with a long pathlength multireflection absorption cell. Spectra were recorded at two different temperatures, various $\mathrm{O}_{2}$ pressures, and absorption pathlengths in order to retrieve information on temperature and pressure dependent effects. Spectra recorded using the FT spectrometer at the NSO were also included in the analysis. Precise line intensities with an accuracy of approximately $0.3 \%$ and pressure-broadening coefficients (accuracy approx. 2-3\%) were retrieved including their temperature dependence. This data enabled us to determine the $A$ band transition moment and the Einstein transition probability with standard errors of approximately $0.1 \%$. The spectra were of sufficient quality that the line intensities, broadening coefficients, and the transition probability for the corresponding band in the isotopomer ${ }^{16} \mathrm{O}^{18} \mathrm{O}$ could be determined.

\section{Acknowledgements}

The work on doubly ionized spectra was supported in part by the Smithsonian Astrophysical Observatory IR\&D Grant, and by NASA grant NAG5-4348 to Harvard University. JCP and PLS thank D. S. Leckrone and W. Parkinson for their support, and R. Kling, W. Mende and M. Kock for their assistance. PPARC of the UK provides financial support for much of the laboratory astrophysics program at IC. RS and RCML thank J. Ballard for making the Molecular Spectroscopy Facility at RAL available on several occasions. The scientific support by D. Newnham and the 
technical support by M. Page and P. O'Brien is most gratefully acknowledged. RS and RCML also thank J. Brault for his help in making the observations at the NSO. We also apreciate the financial support of the oxygen project by NERC of the UK.

\section{References}

[1] G. Nave, S. Johansson, R. C. M. Learner, A. P. Thorne, and J. W. Brault, ApJS 94, 221 (1994)

[2] U. Litzen, J. W. Brault, and A. P. Thorne, Physica Scripta 47, 628 (1993)

[3] J. C. Pickering and A. P. Thorne, ApJS 107, 761 (1996)

[4] J. C. Pickering, A. J. J. Raassen, P. H. M. Uylings, and S. Johansson, submitted ApJ (1997)

[5] J. C. Pickering, ApJS 107, 811 (1996)

[6] J. C. Pickering, Physica Scripta, in press (1997)

[7] A. J. J. Raassen, J. C. Pickering, and P. H. M. Uylings, submitted Astron. \& Astrophys. (1997)

[8] A. P. Thorne, Physica Scripta T65, 31 (1996)

[9] C. Heise, J. Hollandt, R. Kling, M. Kock, and M. Kühne, Applied Optics 33, 5111 (1994) 


\title{
Calculations of Oscillator Strengths for Singly Ionized Iron Group Elements by Means of the Orthogonal Operator Approach
}

\author{
A. J. J. Raassen and P. H. M. Uylings \\ Van der Waals-Zeeman Instituut, Universiteit van Amsterdam, \\ Valckenierstraat 65-67, 1018 XE Amsterdam, The Netherlands
}

\section{Introduction}

The relatively high abundances of iron group elements in stellar objects stimulates interest in these elements. The need for accurate laboratory data is further increased due to the launch of high dispersive space laboratories such as the Goddard High Resolution Spectrograph on board the Hubble Space Telescope [1].

Being an odd- $\mathrm{Z}$ number element cobalt is far less abundant than the even- $\mathrm{Z}$ iron group elements, $\mathrm{Cr}, \mathrm{Fe}$ and $\mathrm{Ni}$. However, Co II lines appear up to OB-type stars and are observed in absorption in the interstellar medium (ISM). This requires accurate transition probabilities (oscillator strengths) for transitions from the low lying levels in the $\left(3 d^{8}+3 d^{7} 4 s\right)$ system, which is responsible for the absorption lines.

State of the art experimental values from radiative lifetimes and branching fractions are accurate, but restricted to a limited number of transitions [2].

Advanced computer codes exist to calculate energy level values and wave functions ab initio. These codes allow the introduction of very many interacting configurations. Ab-initio calculations are powerful in relatively simple systems with one term outside the closed shell only. In that case the system is dominated by electrostatic interactions, mixing levels of different configurations having the same term names.

In iron group and other transition group elements, in which we deal with several d-electrons the systems show many terms of different multiplicity and total angular orbital moment (L) within a relatively small energy range. Including more and more correlation is not the point in calculations of transition elements. These systems are dominated by magnetic interactions, of which the SpinOrbit interaction is strongest. These magnetic interactions are responsible for the mixing of levels with the same total angular momentum $\mathrm{J}$, but with different multiplicity $(2 \mathrm{~S}+1)$ or different orbital angular momentum (L). This effect strongly affects transition probabilities or oscillator strengths and is responsible for the appearance of intercombination lines. Therefore, for the iron group elements, it is far more important to describe well the magnetic mixing that appears from the spacing between levels of the same J-value and the non-diagonal magnetic matrix element between them, than to include more configurations.

The orthogonal operator approach, developed over the last years [3], provides the possibility to describe spacings between levels far more accurate by than other computational methods. The orthogonal operator approach is a semi-empirical method, in which the eigenvalues of the Hamiltonian are fitted to experimental energy values by adjusting the electrostatic and magnetic interactions of the Hamiltonian. The method is basically founded on the Slater-Condon Theory. However, their interactions, as used by the Cowan method, are orthogonalized in our method. This stabilizes the 
fitting, makes the operators as independent as possible and offers the possibility of adding higher order electrostatic and magnetic effects; thus far, these interactions had to be left out as they corrupted the least squares fitting procedure. The extension of the Hamiltonian results in a far smaller average deviation. This implies a better description of the level structure, i.e. the spacings between the levels, resulting in optimal eigenvector compositions. These intermediate eigenvectors are used to sandwich the pure LS-transition probabilities. Afterwards these quantities are multiplied by the electric dipole transition integrals. The latter (see Table 1) are obtained by means of the MCDF code of Parpia et al. [4] and corrected for core-polarization.

\section{Results}

As input for the fitting procedure, level values obtained by Pickering et al. [5] were used. The levels included covered $\left(3 d^{8}+3 d^{7} 4 s+3 d^{6} 4 s^{2}+3 d^{7} 4 d+3 d^{7} 5 s+3 d^{7} 5 d+3 d^{7} 6 s\right)$ for the even system and $\left(3 d^{7} 4 p+3 d^{6} 4 s 4 p+3 d^{5} 4 s^{2} 4 p+3 d^{7} 5 p+3 d^{7} 4 f\right)$ for the odd system. For studying the resonance transitions in the $\left(3 \mathrm{~d}^{8}+3 \mathrm{~d}^{7} 4 \mathrm{~s}\right)-3 \mathrm{~d}^{7} 4 \mathrm{p}$ transition array only the three lowest configurations in both systems are relevant. In the even system the mean deviation, the average deviation between the calculated eigenvalues and the experimental energy values, is $39 \mathrm{~cm}^{-1}$, mainly due to some larger deviations in the $4 \mathrm{~d}$ - and $5 \mathrm{~d}$ configuration. For most of the levels in the the lowest even configurations the deviation is only a few $\mathrm{cm}^{-1}$. In the odd system the mean deviation is 19 $\mathrm{cm}^{-1}$. Using the level values and eigenvector compositions of the two lowest even and the lowest odd configuration results in a large number of oscillator strengths and wavelengths, which will be published elsewhere [6]. Here we restrict ourselves to giving the oscillator strengths for lines from the lowest even multiplets recently measured by Mullman et al. [2] and kindly provided to us by Lawler, present at the ICAMDATA conference. In Table 2 our values are given together with the experimental values of Mullman et al. and values from the Kurucz database [7]. Our results agree very well with those of Mullman et al., solving some large discrepancies between Mullman and Kurucz data. These discrepancies not only concern weak intercombination lines but also the strong resonance transitions between the lowest even term $3 \mathrm{~d}^{8}{ }^{3} \mathrm{~F}$ levels and $3 \mathrm{~d}^{7}\left({ }^{4} \mathrm{~F}\right) 4 \mathrm{p}{ }^{3} \mathrm{~F}$ and ${ }^{3} \mathrm{G}$ levels (see lines at $2027.040 \AA$ and $2025.760 \AA$ ). For sixteen of the twenty-eight lines, given by Mullman et al. [2], our values are within Mullmans error bars, and nineteen are within $10 \%$. Only a few weaker lines with $\log (\mathrm{gf})$ values around -2.0 show larger deviations.

\section{Acknowledgements}

It is a pleasure to thank Dr J. E. Lawler for sharing his results with us in advance of the publication.

Table 1: Values for the electric dipole transition integrals in Co II calculated by means of MCDF including core polarization

\begin{tabular}{c|ccccccc} 
& $3 \mathrm{~d}^{8}$ & $3 \mathrm{~d}^{7} 4 \mathrm{~s}$ & $3 \mathrm{~d}^{6} 4 \mathrm{~s}^{2}$ & $3 \mathrm{~d}^{7} 4 \mathrm{~d}$ & $3 \mathrm{~d}^{7} 5 \mathrm{~s}$ & $3 \mathrm{~d}^{7} 5 \mathrm{~d}$ & $3 \mathrm{~d}^{7} 6 \mathrm{~s}$ \\
\hline $3 \mathrm{~d}^{7} 4 \mathrm{p}$ & .79 & -2.65 & - & -3.20 & 2.13 & -0.79 & 0.57 \\
$3 \mathrm{~d}^{6} 4 \mathrm{~s} 4 \mathrm{p}$ & - & 0.65 & -2.44 & - & - & - & - \\
$3 \mathrm{~d}^{5} 4 \mathrm{~s}^{2} 4 \mathrm{p}$ & - & - & 0.55 & - & - & - & - \\
$3 \mathrm{~d}^{7} 5 \mathrm{p}$ & 0.22 & 0.03 & - & 4.88 & -6.41 & -6.00 & 4.89 \\
$3 \mathrm{~d}^{7} 4 \mathrm{f}$ & 0.19 & - & - & -5.56 & - & 7.32 & -
\end{tabular}


Table 2: Comparison between theory and experiment ${ }^{a}$ of $\log (\mathrm{gf})$-values for the $\left(3 \mathrm{~d}^{8}+3 \mathrm{~d}^{7} 4 \mathrm{~s}\right) \rightarrow$ $3 \mathrm{~d}^{7} 4 \mathrm{p}$ transitions involving the lowest even multiplets.

\begin{tabular}{|c|c|c|c|c|c|c|c|c|c|}
\hline$\lambda(\AA)$ & experiment $^{a}$ & present & Kurucz $^{b}$ & $\mathrm{~J}_{f}$ & $\mathrm{E}_{f}\left(\mathrm{~cm}^{-1}\right)$ & even & $\mathrm{J}_{i}$ & $\mathrm{E}_{i}\left(\mathrm{~cm}^{-1}\right)$ & odd \\
\hline 2693.091 & -2.20 & -2.09 & -1.94 & 2.0 & $13260.69^{*}$ & $\left.\left.1\right|_{2} ^{3} \mathrm{P}\right)$ & 3.0 & $50381.72^{*}$ & $\left.\left.1\right|_{3} ^{4} \mathrm{~F}\right)^{3} \mathrm{~F}$ \\
\hline 2587.220 & 0.037 & 0.028 & -0.49 & 3.0 & $10708.33^{*}$ & $\left.\left.2\right|_{3} ^{4} \mathrm{~F}\right)^{3} \mathrm{~F}$ & 4.0 & $49348.30^{*}$ & $\left.1{ }_{3}^{4} \mathrm{~F}\right)^{3} \mathrm{G}$ \\
\hline 2580.326 & 0.36 & 0.39 & 0.43 & 4.0 & $9812.86^{*}$ & $\left.\left.2\right|_{3} ^{4} \mathrm{~F}\right)^{3} \mathrm{~F}$ & 5.0 & $48556.05^{*}$ & $\left.\left.1\right|_{3} ^{4} \mathrm{~F}\right)^{3} \mathrm{G}$ \\
\hline 2564.034 & 0.07 & 0.072 & 0.31 & 3.0 & $10708.33^{*}$ & $\left.\left.2\right|_{3} ^{4} \mathrm{~F}\right)^{3} \mathrm{~F}$ & 4.0 & $49697.68^{*}$ & $\left.\left.1\right|_{3} ^{4} \mathrm{~F}\right)^{3} \mathrm{~F}$ \\
\hline 2559.405 & -0.21 & -0.17 & 0.17 & 2.0 & $11321.86^{*}$ & $\left.\left.2\right|_{3} ^{4} \mathrm{~F}\right)^{3} \mathrm{~F}$ & 3.0 & $50381.72^{*}$ & $\left.\left.1\right|_{3} ^{4} \mathrm{~F}\right)^{3} \mathrm{~F}$ \\
\hline 2546.160 & -1.61 & -1.64 & -1.61 & 2.0 & $11651.28^{*}$ & $\left.\left.1\right|_{2} ^{1} \mathrm{D}\right)$ & 2.0 & $50914.32^{*}$ & $\left.\left.1\right|_{3} ^{4} \mathrm{~F}\right)^{3} \mathrm{~F}$ \\
\hline 2528.616 & 0.06 & 0.10 & 0.32 & 4.0 & $9812.86^{*}$ & $\left.\left.2\right|_{3} ^{4} \mathrm{~F}\right)^{3} \mathrm{~F}$ & 4.0 & $49348.30^{*}$ & $\left.\left.1\right|_{3} ^{4} \mathrm{~F}\right)^{3} \mathrm{G}$ \\
\hline 2524.974 & -0.06 & 0.003 & 0.005 & 2.0 & $11321.86^{*}$ & $\left.\left.2\right|_{3} ^{4} \mathrm{~F}\right)^{3} \mathrm{~F}$ & 2.0 & $50914.32^{*}$ & $\left.\left.1\right|_{3} ^{4} \mathrm{~F}\right)^{3} \mathrm{~F}$ \\
\hline 2519.823 & -0.14 & -0.11 & -0.76 & 3.0 & $10708.33^{*}$ & $\left.\left.2\right|_{3} ^{4} \mathrm{~F}\right)^{3} \mathrm{~F}$ & 3.0 & $50381.72^{*}$ & $\left.\left.1\right|_{3} ^{4} \mathrm{~F}\right)^{3} \mathrm{~F}$ \\
\hline 2506.464 & 0.05 & 0.062 & -0.47 & 4.0 & $9812.86^{*}$ & $\left.\left.2\right|_{3} ^{4} \mathrm{~F}\right)^{3} \mathrm{~F}$ & 4.0 & $49697.68^{*}$ & $\left.\left.1\right|_{3} ^{4} \mathrm{~F}\right)^{3} \mathrm{~F}$ \\
\hline 2486.441 & -0.54 & -0.51 & -0.48 & 3.0 & $10708.33^{*}$ & $\left.\left.2\right|_{3} ^{4} \mathrm{~F}\right)^{3} \mathrm{~F}$ & 2.0 & $50914.32^{*}$ & $\left.\left.1\right|_{3} ^{4} \mathrm{~F}\right)^{3} \mathrm{~F}$ \\
\hline 2464.199 & -0.42 & -0.39 & -0.66 & 4.0 & $9812.86^{*}$ & $\left.\left.2\right|_{3} ^{4} \mathrm{~F}\right)^{3} \mathrm{~F}$ & 3.0 & $50381.72 *$ & $\left.\left.1\right|_{3} ^{4} \mathrm{~F}\right)^{3} \mathrm{~F}$ \\
\hline 2245.129 & -0.35 & -0.29 & -0.51 & 4.0 & $4028.99^{*}$ & $\left.\left.2\right|_{3} ^{4} \mathrm{~F}\right)^{5} \mathrm{~F}$ & 5.0 & $48556.05^{*}$ & $\left.\left.1\right|_{3} ^{4} \mathrm{~F}\right)^{3} \mathrm{G}$ \\
\hline 2232.072 & -1.08 & -1.06 & -1.89 & 3.0 & $4560.79 *$ & $\left.\left.2\right|_{3} ^{4} F\right)^{5} \mathrm{~F}$ & 4.0 & $49348.30^{*}$ & $\left.\left.1\right|_{3} ^{4} F\right)^{3} G$ \\
\hline 2214.793 & -1.02 & -1.03 & -0.97 & 3.0 & $4560.79 *$ & $\left.\left.2\right|_{3} ^{4} \mathrm{~F}\right)^{5} \mathrm{~F}$ & 4.0 & $49697.68^{*}$ & $\left.\left.1\right|_{3} ^{4} \mathrm{~F}\right)^{3} \mathrm{~F}$ \\
\hline 2211.428 & -1.21 & -1.22 & -1.38 & 5.0 & $3350.49 *$ & $\left.\left.2\right|_{3} ^{4} F\right)^{5} \mathrm{~F}$ & 5.0 & $48556.05^{*}$ & $\left.\left.1\right|_{3} ^{4} \mathrm{~F}\right)^{3} \mathrm{G}$ \\
\hline 2200.421 & -1.45 & -1.45 & -1.39 & 2.0 & $4950.06^{*}$ & $\left.\left.2\right|_{3} ^{4} \mathrm{~F}\right)^{5} \mathrm{~F}$ & 3.0 & $50381.72^{*}$ & $\left.\left.1\right|_{3} ^{4} \mathrm{~F}\right)^{3} \mathrm{~F}$ \\
\hline 2188.999 & -2.08 & -1.96 & -1.80 & 4.0 & $4028.99 *$ & $\left.\left.2\right|_{3} ^{4} \mathrm{~F}\right)^{5} \mathrm{~F}$ & 4.0 & $49697.68^{*}$ & $\left.\left.1\right|_{3} ^{4} \mathrm{~F}\right)^{3} \mathrm{~F}$ \\
\hline 2187.039 & -2.03 & -1.91 & -1.97 & 1.0 & $5204.70^{*}$ & $\left.\left.2\right|_{3} ^{4} \mathrm{~F}\right)^{5} \mathrm{~F}$ & 2.0 & $50914.32 *$ & $\left.\left.1\right|_{3} ^{4} \mathrm{~F}\right)^{3} \mathrm{~F}$ \\
\hline 2173.335 & -1.81 & -1.98 & -1.85 & 5.0 & $3350.49 *$ & $\left.\left.2\right|_{3} ^{4} F\right)^{5} \mathrm{~F}$ & 4.0 & $49348.30^{*}$ & $\left.\left.1\right|_{3} ^{4} F\right)^{3} G$ \\
\hline 2156.950 & -2.09 & -2.08 & -2.67 & 5.0 & $3350.49^{*}$ & $\left.\left.2\right|_{3} ^{4} \mathrm{~F}\right)^{5} \mathrm{~F}$ & 4.0 & $49697.68^{*}$ & $\left.\left.1\right|_{3} ^{4} \mathrm{~F}\right)^{3} \mathrm{~F}$ \\
\hline 2065.542 & -1.07 & -1.11 & -0.85 & 3.0 & $950.32^{*}$ & $\left.\left.1\right|_{2} ^{3} \mathrm{~F}\right)$ & 4.0 & $49348.30^{*}$ & $\left.\left.1\right|_{3} ^{4} F\right)^{3} G$ \\
\hline 2058.817 & -1.17 & -1.24 & -0.93 & 4.0 & $.00^{*}$ & $\left.\left.1\right|_{2} ^{3} \mathrm{~F}\right)$ & 5.0 & $48556.05^{*}$ & $\left.\left.1\right|_{3} ^{4} \mathrm{~F}\right)^{3} \mathrm{G}$ \\
\hline 2027.040 & -0.57 & -0.57 & -0.29 & 2.0 & $1597.20^{*}$ & $\left.\left.1\right|_{2} ^{3} \mathrm{~F}\right)$ & 2.0 & $50914.32^{*}$ & $\left.\left.1\right|_{3} ^{4} F\right)^{3} F$ \\
\hline 2025.760 & -0.95 & -0.98 & -0.26 & 4.0 & $.00^{*}$ & $\left.\left.1\right|_{2} ^{3} \mathrm{~F}\right)$ & 4.0 & $49348.30 *$ & $\left.\left.1\right|_{3} ^{4} \mathrm{~F}\right)^{3} \mathrm{G}$ \\
\hline 2022.354 & -0.49 & -0.48 & -0.44 & 3.0 & $950.32^{*}$ & $\left.\left.1\right|_{2} ^{3} \mathrm{~F}\right)$ & 3.0 & $50381.72^{*}$ & $\left.\left.1\right|_{3} ^{4} \mathrm{~F}\right)^{3} \mathrm{~F}$ \\
\hline 2011.516 & -0.48 & -0.40 & -0.38 & 4.0 & $.00^{*}$ & $\left.1 \mid{ }_{2}^{3} \mathrm{~F}\right)$ & 4.0 & $49697.68^{*}$ & $\left.\left.1\right|_{3} ^{4} \mathrm{~F}\right)^{3} \mathrm{~F}$ \\
\hline 2000.793 & -2.15 & -1.92 & -1.68 & 3.0 & $950.32^{*}$ & $\left.\left.1\right|_{2} ^{3} \mathrm{~F}\right)$ & 2.0 & $50914.32^{*}$ & $\left.\left.1\right|_{3} ^{4} \mathrm{~F}\right)^{3} \mathrm{~F}$ \\
\hline
\end{tabular}

${ }^{a}$ Mullman et al. [2] ${ }^{b}$ Kurucz [7]

\section{References}

[1] D. S. Leckrone, S. Johansson, G. M. Wahlgren, and S. J. Adelman, Phys. Scr. T47, 149 (1993)

[2] K. L. Mullman, J. C. Cooper, and J. E. Lawler, Ap.J (accepted)

[3] A. J. J. Raassen and P. H. M. Uylings, Phys. Scr. T65, 84 (1996)

[4] F. A. Parpia, C. Froese-Fischer, and I. P. Grant, Comput. Phys. Commun. 94, 249 (1996)

[5] J. C. Pickering, A. J. J. Raassen, P. H. M. Uylings, and S. Johansson, Ap.J., to be published

[6] A. J. J. Raassen, J. C. Pickering, and P. H. M. Uylings, A\& A suppl. series, to be published

[7] R. L. Kurucz, Phys. Scr. T47, 110 (1993)

URL address: http://cfa-www.harvard.edu./amp/data/stats/kurucz.html 


\title{
Autoionizing Rates for Excited States of Many-Electron Ions
}

\author{
U.I. Safronova and W.R. Johnson \\ Department of Physics, University of Notre Dame, Notre Dame, IN 46556
}

\section{Introduction}

The most important difference between autoionization states and other excited states is an additional broadening of levels caused by the decay of these states (e.g. $2 \ell n \ell^{\prime} \Rightarrow 1 s k l^{\prime \prime}$ ). One can express the autoionizing widths of a level as the square of the decay amplitude:

$$
\Gamma^{N}\left(a L S, a^{\prime} L S\right)=\sum_{a_{0} k l} \gamma\left(a L S, a_{0} k l L S\right) \gamma\left(a^{\prime} L S, a_{0} k l L S\right)
$$

where

$$
\gamma\left(a L S, a_{0} k l L S\right)=\sqrt{\frac{2 \pi}{k}}\left\langle a L S\left|\sum_{i>j} 1 / r_{i j}\right| a_{0} k l L S\right\rangle .
$$

Here $k$ is the momentum of the ejected electron in the continuum state $\left(a_{0} k l L S\right)$, and $a L S$ describes the autoionizing state. When a nonrelativistic basis is used, relativistic corrections must be taken into account perturbatively. A nonrelativistic basis was used for calculations of autoionization rates in the MZ code [1]-[2], in the model-potential method (SUPERSTRUCTURE code) [3], and in the multiconfiguration Hartree-Fock method (Cowan code [4]).

The purpose of this paper is to obtain simple formulas for autoionization rates for any atomic system by averaging over $(L S J)$. These calculations will be carried out using screened hydrogenic wave functions. The purpose of these calculations is to provide accurate, but easily used, atomic data.

\section{Results and Discussion}

Autoionization changes the state of two atomic electrons which initially can belong to either two different groups or to the same group of equivalent electrons. One of the electrons makes a transition to the continuous spectrum, and the other changes its state in the atom. Therefore, to calculate the probability of an autoionizing decay in a general multi-electron ion, it is sufficient to study the change of state in two systems: $\left(n_{1} l_{1}\right)^{p_{1}}\left(n_{2} l_{2}\right)^{p_{2}}\left(n_{3} l_{3}\right)^{p_{3}}$ and $\left(n_{1} l_{1}\right)^{p_{1}}\left(n_{2} l_{2}\right)^{p_{2}}$. We must study two types of transitions:

$$
\begin{array}{rcc}
\left(n_{1} l_{1}\right)^{p_{1}}\left(n_{2} l_{2}\right)^{p_{2}}\left(n_{3} l_{3}\right)^{p_{3}} & \Rightarrow & \left(n_{1} l_{1}\right)^{p_{1}-1}\left(n_{2} l_{2}\right)^{p_{2}-1}\left(n_{3} l_{3}\right)^{p_{3}+1} k \ell \\
\left(n_{1} l_{1}\right)^{p_{1}}\left(n_{2} l_{2}\right)^{p_{2}} & \Rightarrow \quad & \left(n_{1} l_{1}\right)^{p_{1}-2}\left(n_{2} l_{2}\right)^{p_{2}+1} k \ell .
\end{array}
$$

For $L S$ averaged autoionization decay probabilities, one obtains in each case:

$$
\begin{array}{r}
\Gamma^{N}\left[\left(n_{1} l_{1}\right)^{p_{1}}\left(n_{2} l_{2}\right)^{p_{2}}\left(n_{3} l_{3}\right)^{p_{3}} \Rightarrow\left(n_{1} l_{1}\right)^{p_{1}-1}\left(n_{2} l_{2}\right)^{p_{2}-1}\left(n_{3} l_{3}\right)^{p_{3}+1} k \ell\right]= \\
p_{1} p_{2}\left(1-\frac{p_{3}}{N_{3}}\right) A\left(n_{1} l_{1} n_{2} l_{2} ; n_{3} l_{3} k \ell\right),
\end{array}
$$


and

$$
\begin{aligned}
\Gamma^{N}\left[\left(n_{1} l_{1}\right)^{p_{1}}\left(n_{2} l_{2}\right)^{p_{2}} \Rightarrow\left(n_{1} l_{1}\right)^{p_{1}-2}\left(n_{2} l_{2}\right)^{p_{2}+1} k \ell\right]= \\
\\
\quad \frac{1}{2} p_{1}\left(p_{1}-1\right)\left(1-\frac{p_{2}}{N_{2}}\right) A\left(n_{1} l_{1} n_{1} l_{1} ; n_{2} l_{2} k \ell\right)
\end{aligned}
$$

where $N_{i}=2\left(2 l_{i}+1\right)$ and where $A\left(n_{1} l_{1} n_{2} l_{2} ; n_{3} l_{3} k \ell\right)$ is the two-electron decay probability.

$$
\begin{aligned}
& A\left(n_{1} l_{1} n_{2} l_{2} ; n_{3} l_{3} k l_{4}\right) \\
& =\frac{2 \pi}{k}\left(2 l_{3}+1\right)\left(2 l_{4}+1\right) \sum_{l}\left[\frac{1}{(2 l+1)} P_{l}^{2}\left(n_{1} l_{1} n_{2} l_{2} ; n_{3} l_{3} k l_{4}\right)+\frac{1}{(2 l+1)} P_{l}^{2}\left(n_{1} l_{1} n_{2} l_{2} ; k l_{4} n_{3} l_{3}\right)\right. \\
& \left.-\sum_{l^{\prime}}\left\{\begin{array}{lll}
l_{1} & l_{4} & l^{\prime} \\
l_{2} & l_{3} & l
\end{array}\right\} P_{l}\left(n_{1} l_{1} n_{2} l_{2} ; n_{3} l_{3} k l_{4}\right) P_{l^{\prime}}\left(n_{1} l_{1} n_{2} l_{2} ; k l_{4} n_{3} l_{3}\right)\right] \\
& A\left(n_{1} l_{1} n_{1} l_{1} ; n_{3} l_{3} k l_{4}\right)=\frac{2 \pi}{k} \frac{\left(4 l_{1}+1\right)}{\left(2 l_{1}+1\right)}\left(2 l_{3}+1\right)\left(2 l_{4}+1\right) \sum_{l}\left[\frac{2}{(2 l+1)} P_{l}^{2}\left(n_{1} l_{1} n_{2} l_{2} ; n_{3} l_{3} k l_{4}\right)\right. \\
& \left.-\sum_{l^{\prime}}\left\{\begin{array}{lll}
l_{1} & l_{4} & l^{\prime} \\
l_{1} & l_{3} & l
\end{array}\right\} P_{l}\left(n_{1} l_{1} n_{1} l_{1} ; n_{3} l_{3} k l_{4}\right) P_{l^{\prime}}\left(n_{1} l_{1} n_{1} l_{1} ; k l_{4} n_{3} l_{3}\right)\right]
\end{aligned}
$$

Here $\mathrm{P}_{l}$ can be described as a product of a radial integral and $3 \mathrm{j}$-Wigner coefficients:

$$
P_{l}\left(n_{1} l_{1} n_{1} l_{1} ; n_{3} l_{3} k l_{4}\right)=R_{l}\left(n_{1} l_{1} n_{1} l_{1} ; n_{3} l_{3} k l_{4}\right)(-1)^{l+\left(l_{1}+l_{2}+l_{3}+l_{4}\right) / 2}\left(\begin{array}{ccc}
l_{1} & l_{4} & l \\
0 & 0 & 0
\end{array}\right)\left(\begin{array}{ccc}
l_{2} & l_{3} & l \\
0 & 0 & 0
\end{array}\right)
$$

Results of our calculations of $A\left(n_{1} l_{1} n_{2} l_{2} ; n_{3} l_{3} k l_{4}\right)$ and $A\left(n_{1} l_{1} n_{1} l_{2} ; n_{3} l_{3} k l_{4}\right)$ are given in Tables 1 and 2 for $n_{1} l_{1}, n_{2} l_{2}$ with $7 \geq n_{1} \geq 1,\left(n_{1}-1\right) \geq l_{1} \geq 0,7 \geq n_{2} \geq 1,\left(n_{2}-1\right) \geq l_{2} \geq 0$. The value of $n_{3}$ was chosen such that $\sqrt{\frac{1}{n_{3}^{2}}-\frac{1}{n_{1}^{2}}-\frac{1}{n_{2}^{2}}}>0$ and $\left(n_{3}-1\right) \geq l_{3} \geq 0$. The value of $l_{4}$ was defined by the triangle rule for the $3 \mathrm{j}$-Wigner coefficient in Eq. (9). The value of $\mathrm{k}$ was given by $k=\frac{Z}{Z-\sigma} \sqrt{\frac{1}{n_{3}^{2}}-\frac{1}{n_{1}^{2}}-\frac{1}{n_{2}^{2}}}$, where $Z$ is the nuclear charge and $\sigma$ is a screening constant.

In total, 8116 kinds of $A\left(n_{1} l_{1} n_{2} l_{2} ; n_{3} l_{3} k l_{4}\right)$ and $A\left(n_{1} l_{1} n_{1} l_{2} ; n_{3} l_{3} k l_{4}\right)$ were calculated. We have selected the results with the largest values of $A$ for illustration.

\section{Acknowledgements}

The work was supported by Grant No. B336454 from the Lawrence Livermore National Laboratory.

\section{References}

[1] L. A. Vainshtein and U. I. Safronova, At. Data Nucl. Data Tables 21, 49 (1978); ibid. 25, 311 (1980)

[2] U. I. Safronova, Physica Scripta T26, 59 (1989)

[3] F. Bely-Dubau, A. N. Gabriel and S. Volonte, Mon. Not. R. Astron. Soc. 186, 405 (1979), ibid. 189, 801 (1979)

[4] R. D. Cowan, The Theory of Atomic Structure and Spectra (Berkeley, 1981) 
Table 1: Autoionizing rates $A\left(n_{1} l_{1} n_{2} l_{2} ; n_{3} l_{3} k l_{4}\right)$ in $\mathrm{s}^{-1}$ as function of $Z$ and $\sigma$

\begin{tabular}{|c|c|c|c|c|c|c|c|}
\hline$n_{1} l_{1} n_{2} l_{2}$ & $n_{3} l_{3} k l_{4}$ & $\begin{array}{c}Z=26 \\
\sigma=0\end{array}$ & $\begin{array}{c}Z=26 \\
\sigma=1\end{array}$ & $\begin{aligned} Z & =16 \\
\sigma & =1\end{aligned}$ & $\begin{array}{c}Z=10 \\
\sigma=1\end{array}$ & $\begin{array}{l}Z=6 \\
\sigma=1\end{array}$ & $\begin{array}{l}Z=6 \\
\sigma=2\end{array}$ \\
\hline $2 s 2 s$ & $1 s k s$ & $2.057 \mathrm{E}+14$ & $2.004 \mathrm{E}+14$ & $1.968 \mathrm{E}+14$ & $1.908 \mathrm{E}+14$ & $1.785 \mathrm{E}+14$ & $1.364 \mathrm{E}+14$ \\
\hline $2 s 2 p$ & $1 s k p$ & $6.088 \mathrm{E}+13$ & $5.886 \mathrm{E}+13$ & $5.753 \mathrm{E}+13$ & $5.533 \mathrm{E}+13$ & $5.098 \mathrm{E}+13$ & $3.691 \mathrm{E}+13$ \\
\hline $2 p 2 p$ & $1 s k s$ & $1.039 \mathrm{E}+13$ & $9.544 \mathrm{E}+12$ & $9.014 \mathrm{E}+12$ & $8.184 \mathrm{E}+12$ & $6.716 \mathrm{E}+12$ & $3.316 \mathrm{E}+12$ \\
\hline $2 p 2 p$ & $1 s k d$ & $1.245 \mathrm{E}+14$ & $1.160 \mathrm{E}+14$ & $1.104 \mathrm{E}+14$ & $1.016 \mathrm{E}+14$ & $8.548 \mathrm{E}+13$ & $4.511 \mathrm{E}+13$ \\
\hline $2 s 3 s$ & $1 s k s$ & $2.604 \mathrm{E}+13$ & $2.528 \mathrm{E}+13$ & $2.477 \mathrm{E}+13$ & $2.390 \mathrm{E}+13$ & $2.212 \mathrm{E}+13$ & $1.613 \mathrm{E}+13$ \\
\hline $2 s 3 p$ & $1 s k p$ & $1.736 \mathrm{E}+13$ & $1.667 \mathrm{E}+13$ & $1.621 \mathrm{E}+13$ & $1.545 \mathrm{E}+13$ & $1.396 \mathrm{E}+13$ & $9.349 \mathrm{E}+12$ \\
\hline $2 p 3 s$ & $1 s k p$ & $1.252 \mathrm{E}+13$ & $1.220 \mathrm{E}+13$ & $1.199 \mathrm{E}+13$ & $1.162 \mathrm{E}+13$ & $1.084 \mathrm{E}+13$ & $7.853 \mathrm{E}+12$ \\
\hline $2 p 3 p$ & $1 s k d$ & $2.381 \mathrm{E}+13$ & $2.208 \mathrm{E}+13$ & $2.097 \mathrm{E}+13$ & $1.918 \mathrm{E}+13$ & $1.591 \mathrm{E}+13$ & $7.845 \mathrm{E}+12$ \\
\hline $2 s 4 s$ & $1 s k s$ & $1.050 \mathrm{E}+13$ & $1.018 \mathrm{E}+13$ & $9.964 \mathrm{E}+12$ & $9.599 \mathrm{E}+12$ & $8.852 \mathrm{E}+12$ & $6.346 \mathrm{E}+12$ \\
\hline $3 s 3 s$ & $1 s k s$ & $1.284 \mathrm{E}+13$ & $1.245 \mathrm{E}+13$ & $1.218 \mathrm{E}+13$ & $1.172 \mathrm{E}+13$ & $1.078 \mathrm{E}+13$ & $7.601 \mathrm{E}+12$ \\
\hline $3 p 3 p$ & $2 p k p$ & $2.805 \mathrm{E}+14$ & $2.776 \mathrm{E}+14$ & $2.757 \mathrm{E}+14$ & $2.723 \mathrm{E}+14$ & $2.654 \mathrm{E}+14$ & $2.394 \mathrm{E}+14$ \\
\hline $3 p 3 d$ & $2 p k d$ & $1.576 \mathrm{E}+14$ & $1.565 \mathrm{E}+14$ & $1.558 \mathrm{E}+14$ & $1.545 \mathrm{E}+14$ & $1.517 \mathrm{E}+14$ & $1.402 \mathrm{E}+14$ \\
\hline $3 d 3 d$ & $2 p k f$ & 4.147E+14 & $4.057 \mathrm{E}+14$ & $3.997 \mathrm{E}+14$ & $3.895 \mathrm{E}+14$ & $3.688 \mathrm{E}+14$ & $2.981 \mathrm{E}+14$ \\
\hline $3 s 3 s$ & $2 p k p$ & $3.169 \mathrm{E}+13$ & $3.106 \mathrm{E}+13$ & $3.063 \mathrm{E}+13$ & $2.988 \mathrm{E}+13$ & $2.828 \mathrm{E}+13$ & $2.212 \mathrm{E}+13$ \\
\hline $3 s 3 p$ & $2 p k s$ & $8.273 \mathrm{E}+13$ & $8.207 \mathrm{E}+13$ & $8.162 E+13$ & $8.084 \mathrm{E}+13$ & $7.922 \mathrm{E}+13$ & $7.306 \mathrm{E}+13$ \\
\hline $3 s 3 d$ & $2 p k f$ & $1.811 \mathrm{E}+13$ & $1.823 \mathrm{E}+13$ & $1.831 \mathrm{E}+13$ & $1.846 \mathrm{E}+13$ & $1.878 \mathrm{E}+13$ & $2.019 \mathrm{E}+13$ \\
\hline $3 p 3 d$ & $2 p k s$ & $1.839 \mathrm{E}+13$ & $1.791 \mathrm{E}+13$ & $1.758 \mathrm{E}+13$ & $1.704 \mathrm{E}+13$ & $1.595 \mathrm{E}+13$ & $1.239 \mathrm{E}+13$ \\
\hline $3 p 3 d$ & $2 p k g$ & $138 \mathrm{E}+13$ & $1.130 \mathrm{E}+13$ & $1.125 \mathrm{E}+13$ & $1.115 \mathrm{E}+13$ & $1.090 \mathrm{E}+13$ & $9.623 \mathrm{E}+12$ \\
\hline $3 d 3 d$ & $2 p k p$ & $3.333 \mathrm{E}+13$ & $3.251 \mathrm{E}+13$ & $3.196 \mathrm{E}+13$ & $3.104 \mathrm{E}+13$ & $2.918 \mathrm{E}+13$ & $2.303 \mathrm{E}+13$ \\
\hline $3 s 4 p$ & $2 p k s$ & $1.794 \mathrm{E}+13$ & $1.768 \mathrm{E}+13$ & $1.750 \mathrm{E}+13$ & $1.719 \mathrm{E}+13$ & $1.655 \mathrm{E}+13$ & $1.411 \mathrm{E}+13$ \\
\hline $3 p 4 s$ & $2 p k s$ & $3.325 \mathrm{E}+13$ & $3.261 \mathrm{E}+13$ & $3.216 \mathrm{E}+13$ & $3.141 \mathrm{E}+13$ & $2.985 \mathrm{E}+13$ & $2.418 \mathrm{E}+13$ \\
\hline $3 p 4 p$ & $2 p k p$ & $7.195 \mathrm{E}+13$ & $7.028 \mathrm{E}+13$ & $6.915 \mathrm{E}+13$ & $6.723 \mathrm{E}+13$ & $6.328 \mathrm{E}+13$ & $4.941 E+13$ \\
\hline $3 p 4 d$ & $2 p k d$ & $5.530 \mathrm{E}+13$ & $5.411 \mathrm{E}+13$ & $5.330 \mathrm{E}+13$ & $5.190 \mathrm{E}+13$ & $4.896 \mathrm{E}+13$ & $3.810 \mathrm{E}+13$ \\
\hline $3 d 4 p$ & $2 p k d$ & $4.024 \mathrm{E}+13$ & $4.021 \mathrm{E}+13$ & $4.015 \mathrm{E}+13$ & $3.994 \mathrm{E}+13$ & $3.919 \mathrm{E}+13$ & $3.367 \mathrm{E}+13$ \\
\hline $3 d 4 d$ & $2 p k p$ & $1.042 \mathrm{E}+13$ & $9.845 \mathrm{E}+12$ & $9.470 \mathrm{E}+12$ & $8.863 \mathrm{E}+12$ & $7.716 \mathrm{E}+12$ & $4.557 \mathrm{E}+12$ \\
\hline $3 d 4 d$ & $2 p k f$ & $9.831 \mathrm{E}+13$ & $9.423 \mathrm{E}+13$ & $9.150 \mathrm{E}+13$ & $8.692 \mathrm{E}+13$ & $7.781 \mathrm{E}+13$ & $4.963 \mathrm{E}+13$ \\
\hline $3 d 4 f$ & $2 p k g$ & $4.169 \mathrm{E}+13$ & $3.854 \mathrm{E}+13$ & $3.653 \mathrm{E}+13$ & $3.336 \mathrm{E}+13$ & $2.765 \mathrm{E}+13$ & $1.390 \mathrm{E}+13$ \\
\hline $3 p 5 s$ & $2 p k s$ & $1.665 \mathrm{E}+13$ & $1.625 \mathrm{E}+13$ & $1.598 \mathrm{E}+13$ & $1.552 \mathrm{E}+13$ & $1.456 \mathrm{E}+13$ & $1.114 \mathrm{E}+13$ \\
\hline $3 p 5 p$ & $2 p k p$ & $3.318 \mathrm{E}+13$ & $3.225 \mathrm{E}+13$ & $3.161 \mathrm{E}+13$ & $3.054 \mathrm{E}+13$ & $2.835 \mathrm{E}+13$ & $2.081 \mathrm{E}+13$ \\
\hline $3 p 5 d$ & $2 p k d$ & $2.643 \mathrm{E}+13$ & $2.570 \mathrm{E}+13$ & $2.520 \mathrm{E}+13$ & $2.434 \mathrm{E}+13$ & $2.256 \mathrm{E}+13$ & $1.627 \mathrm{E}+13$ \\
\hline $3 d 5 p$ & $2 p k d$ & $1.731 \mathrm{E}+13$ & $1.742 \mathrm{E}+13$ & $1.746 \mathrm{E}+13$ & $1.746 \mathrm{E}+13$ & $1.719 \mathrm{E}+13$ & $1.422 \mathrm{E}+13$ \\
\hline $3 d 5 d$ & $2 p k f$ & $4.263 \mathrm{E}+13$ & $4.067 \mathrm{E}+13$ & $3.934 \mathrm{E}+13$ & $3.711 \mathrm{E}+13$ & $3.264 \mathrm{E}+13$ & $1.909 \mathrm{E}+13$ \\
\hline $3 d 5 f$ & $2 p k g$ & $2.425 \mathrm{E}+13$ & $2.221 \mathrm{E}+13$ & $2.091 \mathrm{E}+13$ & $1.887 \mathrm{E}+13$ & $1.524 \mathrm{E}+13$ & $6.866 \mathrm{E}+12$ \\
\hline $3 p 6 p$ & $2 p k p$ & $1.828 \mathrm{E}+13$ & $1.772 \mathrm{E}+13$ & $1.734 \mathrm{E}+13$ & $1.669 \mathrm{E}+13$ & $1.538 \mathrm{E}+13$ & $1.092 \mathrm{E}+13$ \\
\hline $3 p 6 d$ & $2 p k d$ & $1.479 \mathrm{E}+13$ & $1.433 \mathrm{E}+13$ & $1.402 \mathrm{E}+13$ & $1.349 \mathrm{E}+13$ & $1.239 \mathrm{E}+13$ & $8.578 \mathrm{E}+12$ \\
\hline $3 d 6 d$ & $2 p k f$ & $2.276 \mathrm{E}+13$ & $2.168 \mathrm{E}+13$ & $2.094 \mathrm{E}+13$ & $1.969 \mathrm{E}+13$ & $1.718 \mathrm{E}+13$ & $9.609 \mathrm{E}+12$ \\
\hline $3 d 6 f$ & $2 p k g$ & $1.450 \mathrm{E}+13$ & $1.323 \mathrm{E}+13$ & $1.242 \mathrm{E}+13$ & $1.115 \mathrm{E}+13$ & $8.892 \mathrm{E}+12$ & $3.793 \mathrm{E}+12$ \\
\hline $3 p 7 p$ & $2 p k p$ & $1.120 \mathrm{E}+13$ & $1.084 \mathrm{E}+13$ & $1.059 \mathrm{E}+13$ & $1.018 \mathrm{E}+13$ & $9.340 \mathrm{E}+12$ & $6.502 \mathrm{E}+12$ \\
\hline $3 d 7 d$ & $2 p k f$ & $1.369 \mathrm{E}+13$ & $1.303 \mathrm{E}+13$ & $1.258 \mathrm{E}+13$ & $1.181 \mathrm{E}+13$ & $1.025 \mathrm{E}+13$ & $5.587 \mathrm{E}+12$ \\
\hline $4 p 4 p$ & $2 p k p$ & $2.440 \mathrm{E}+13$ & $2.366 \mathrm{E}+13$ & $2.316 \mathrm{E}+13$ & $2.231 \mathrm{E}+13$ & $2.057 \mathrm{E}+13$ & $1.454 \mathrm{E}+13$ \\
\hline $4 p 4 d$ & $2 p k d$ & $1.351 \mathrm{E}+13$ & $1.332 \mathrm{E}+13$ & $1.316 \mathrm{E}+13$ & $1.288 \mathrm{E}+13$ & $1.218 \mathrm{E}+13$ & $8.990 \mathrm{E}+12$ \\
\hline $4 d 4 d$ & $2 p k f$ & $2.875 \mathrm{E}+13$ & $2.722 \mathrm{E}+13$ & $2.619 \mathrm{E}+13$ & $2.449 \mathrm{E}+13$ & $2.116 \mathrm{E}+13$ & $1.153 \mathrm{E}+13$ \\
\hline $4 d 5 d$ & $2 p k f$ & $1.117 \mathrm{E}+13$ & $1.054 \mathrm{E}+13$ & $1.012 \mathrm{E}+13$ & $9.419 \mathrm{E}+12$ & $8.040 \mathrm{E}+12$ & $4.119 \mathrm{E}+12$ \\
\hline
\end{tabular}


Table 2: Autoionizing rates $A\left(n_{1} l_{1} n_{2} l_{2} ; n_{3} l_{3} k l_{4}\right)$ in $\mathrm{s}^{-1}$ as function of $Z$ and $\sigma$

\begin{tabular}{|c|c|c|c|c|c|c|c|}
\hline$\overline{n_{1} l_{1} n_{2} l_{2}}$ & $n_{3} l_{3} k l_{4}$ & $\begin{array}{c}Z=26 \\
\sigma=0\end{array}$ & $\begin{array}{c}Z=26 \\
\sigma=1\end{array}$ & $\begin{array}{c}Z=16 \\
\sigma=1\end{array}$ & $\begin{array}{c}Z=10 \\
\sigma=1\end{array}$ & $\begin{array}{l}Z=6 \\
\sigma=1\end{array}$ & $\begin{array}{l}Z=6 \\
\sigma=2\end{array}$ \\
\hline $4 f 5 f$ & $3 d k g$ & $1.647 \mathrm{E}+14$ & $1.626 \mathrm{E}+14$ & $1.612 \mathrm{E}+14$ & $1.587 \mathrm{E}+14$ & $1.534 \mathrm{E}+14$ & $1.338 \mathrm{E}+14$ \\
\hline $4 s 5 d$ & $3 d k s$ & $1.572 \mathrm{E}+13$ & $1.565 \mathrm{E}+13$ & $1.560 \mathrm{E}+13$ & $1.551 \mathrm{E}+13$ & $1.533 \mathrm{E}+13$ & $1.464 \mathrm{E}+13$ \\
\hline $4 p 5 d$ & $d k p$ & $.090 \mathrm{E}+13$ & $2.081 \mathrm{E}+13$ & $2.075 \mathrm{E}+13$ & $2.064 \mathrm{E}+13$ & $2.040 \mathrm{E}+13$ & $1.947 \mathrm{E}+13$ \\
\hline $4 d 5 s$ & $d k s$ & $719 \mathrm{E}+13$ & $3.697 \mathrm{E}+13$ & $3.682 \mathrm{E}+13$ & $3.656 \mathrm{E}+13$ & $3.600 \mathrm{E}+13$ & $3.385 \mathrm{E}+13$ \\
\hline $4 d 5 p$ & $d k p$ & $649 \mathrm{E}+13$ & $4.619 \mathrm{E}+13$ & $4.599 \mathrm{E}+13$ & $4.564 \mathrm{E}+13$ & $4.491 \mathrm{E}+13$ & $4.205 \mathrm{E}+13$ \\
\hline $4 d 5 d$ & $d k d$ & $276 \mathrm{E}+13$ & $8.211 \mathrm{E}+13$ & $8.166 \mathrm{E}+13$ & $8.090 \mathrm{E}+13$ & $7.928 \mathrm{E}+13$ & $7.309 \mathrm{E}+13$ \\
\hline $4 d 5 f$ & $d k f$ & $.039 \mathrm{E}+13$ & $7.983 \mathrm{E}+13$ & $7.944 \mathrm{E}+13$ & $7.878 \mathrm{E}+13$ & $7.735 \mathrm{E}+13$ & $7.172 \mathrm{E}+13$ \\
\hline $4 d 5 \mathrm{~g}$ & $d k g$ & $309 E+13$ & $1.291 \mathrm{E}+13$ & $1.279 \mathrm{E}+13$ & $1.259 \mathrm{E}+13$ & $1.217 \mathrm{E}+13$ & $1.076 \mathrm{E}+13$ \\
\hline $4 f 5 p$ & lks & $263 \mathrm{E}+13$ & $1.232 \mathrm{E}+13$ & $1.212 \mathrm{E}+13$ & $1.178 \mathrm{E}+13$ & $1.108 \mathrm{E}+13$ & $8.755 \mathrm{E}+12$ \\
\hline $4 f 5 p$ & $k d$ & $60 \mathrm{E}+12$ & $7.362 \mathrm{E}+12$ & $7.569 \mathrm{E}+12$ & $7.922 \mathrm{E}+12$ & $8.659 \mathrm{E}+12$ & $1.133 \mathrm{E}+13$ \\
\hline $4 f 5 d$ & $d k p$ & $789 E+13$ & $1.752 \mathrm{E}+13$ & $1.728 \mathrm{E}+13$ & $1.686 \mathrm{E}+13$ & $1.602 \mathrm{E}+13$ & $1.312 \mathrm{E}+13$ \\
\hline $4 f 5 d$ & $d k f$ & +13 & $6.990 \mathrm{E}+13$ & $6.992 \mathrm{E}+13$ & $6.993 \mathrm{E}+13$ & $6.987 \mathrm{E}+13$ & $6.858 \mathrm{E}+13$ \\
\hline $4 f 5 f$ & $d k d$ & $575 \mathrm{E}+13$ & $1.548 \mathrm{E}+13$ & $1.529 \mathrm{E}+13$ & $1.497 \mathrm{E}+13$ & $1.432 \mathrm{E}+13$ & $1.203 \mathrm{E}+13$ \\
\hline $4 f 5 g$ & $k h$ & $714 \mathrm{E}+13$ & $9.476 \mathrm{E}+13$ & $9.317 \mathrm{E}+13$ & $9.049 \mathrm{E}+13$ & $8.509 \mathrm{E}+13$ & $6.713 \mathrm{E}+13$ \\
\hline $4 d 6 s$ & $d k s$ & $084 \mathrm{E}+13$ & $2.057 \mathrm{E}+13$ & $2.037 \mathrm{E}+13$ & $2.005 \mathrm{E}+13$ & $1.935 \mathrm{E}+13$ & $1.669 \mathrm{E}+13$ \\
\hline $4 d 6 p$ & $k p$ & +13 & +13 & $2.527 \mathrm{E}+13$ & $2.483 \mathrm{E}+13$ & $2.391 \mathrm{E}+13$ & $2.038 \mathrm{E}+13$ \\
\hline $4 d 6 d$ & $k d$ & +13 & 4.085 & $4.035 \mathrm{E}+13$ & $3.951 \mathrm{E}+13$ & $3.773 \mathrm{E}+13$ & $3.111 \mathrm{E}+13$ \\
\hline $4 d 6 f$ & $d k f$ & +13 & +13 & $923 \mathrm{E}+13$ & $3.842 \mathrm{E}+13$ & $3.671 \mathrm{E}+13$ & $3.020 \mathrm{E}+13$ \\
\hline $4 f 6 d$ & $3 d k p$ & +13 & +13 & $9.847 \mathrm{E}+12$ & $9.316 \mathrm{E}+12$ & $8.292 \mathrm{E}+12$ & $5.290 \mathrm{E}+12$ \\
\hline $4 f 6 d$ & $d k f$ & +13 & +13 & $79 \mathrm{E}+13$ & +13 & $3.391 \mathrm{E}+13$ & $3.146 \mathrm{E}+13$ \\
\hline $4 f 6 f$ & $d k g$ & $623 \mathrm{E}+13$ & $7.435 \mathrm{E}+13$ & $305 \mathrm{E}+13$ & $7.080 \mathrm{E}+13$ & $6.607 \mathrm{E}+13$ & $4.889 \mathrm{E}+13$ \\
\hline & & & & & & & $2.856 \mathrm{E}+13$ \\
\hline $4 f 6 h$ & $l k k$ & $726 \mathrm{E}+13$ & +13 & $1.512 \mathrm{E}+13$ & $1.380 \mathrm{E}+13$ & $1.142 \mathrm{E}+13$ & $5.689 \mathrm{E}+12$ \\
\hline $4 d 7 s$ & & & & & & & \\
\hline $4 d 7 p$ & & +13 & +13 & $545 \mathrm{E}+13$ & $1.510 \mathrm{E}+13$ & $1.436 \mathrm{E}+13$ & $1.156 \mathrm{E}+13$ \\
\hline $4 d 7 d$ & & & & & & & \\
\hline $4 d 7 f$ & $3 d k f$ & $368 \mathrm{E}+13$ & & $2.278 \mathrm{E}+13$ & $2.215 \mathrm{E}+13$ & +13 & $1.591 \mathrm{E}+13$ \\
\hline $4 f 7 d$ & & & & & & & \\
\hline $4 f 7 f$ & $d k g$ & $87 \mathrm{E}+13$ & $4.162 \mathrm{~F}$ & $4.075 E+13$ & $3.922 \mathrm{E}+13$ & & $2.430 \mathrm{E}+13$ \\
\hline $4 f 7 g$ & $d k h$ & $44 \mathrm{E}+13$ & $3.531 \mathrm{E}+13$ & $3.390 \mathrm{E}+13$ & $3.160 \mathrm{E}+13$ & & \\
\hline $4 f 7 h$ & & +13 & 1.390 & $1.304 \mathrm{E}+13$ & $1.169 \mathrm{E}+13$ & +12 & $4.040 \mathrm{E}+12$ \\
\hline $5 d 5 d$ & & +13 & 2.5811 & $2.543 \mathrm{E}+13$ & $2.478 \mathrm{E}+13$ & $2.342 \mathrm{E}+13$ & $1.828 \mathrm{E}+13$ \\
\hline $5 d 5 f$ & $d k f$ & $233 \mathrm{E}+13$ & $2.212 \mathrm{I}$ & $2.196 \mathrm{E}+13$ & $2.166 \mathrm{E}+13$ & $2.091 \mathrm{E}+13$ & $1.717 \mathrm{E}+13$ \\
\hline $5 f 5 f$ & & $754 \mathrm{E}+13$ & $4.584 \mathrm{~F}$ & $4.468 \mathrm{E}+13$ & $4.273 \mathrm{E}+13$ & $3.876 \mathrm{E}+13$ & $2.564 \mathrm{E}+13$ \\
\hline & $d k h$ & $310 \mathrm{E}+13$ & $1.246 \mathrm{E}+13$ & $1.204 \mathrm{E}+13$ & $1.135 \mathrm{E}+13$ & $1.004 \mathrm{E}+13$ & $6.215 \mathrm{E}+12$ \\
\hline & $d k d$ & & & $1.067 \mathrm{E}+13$ & $1.033 \mathrm{E}+13$ & $9.598 \mathrm{E}+12$ & $6.887 \mathrm{E}+12$ \\
\hline $5 d 6 f$ & $d k f$ & $1.066 \mathrm{E}+13$ & $1.049 \mathrm{E}+13$ & $1.036 \mathrm{E}+13$ & $1.012 \mathrm{E}+13$ & $9.566 \mathrm{E}+12$ & $7.094 \mathrm{E}+12$ \\
\hline & $d k f$ & & & $1.024 \mathrm{E}+13$ & $1.010 \mathrm{E}+13$ & $9.723 \mathrm{E}+12$ & $7.484 \mathrm{E}+12$ \\
\hline $5 f 6 f$ & $3 d k g$ & $1.995 \mathrm{E}+13$ & $1.909 \mathrm{E}+13$ & $1.851 \mathrm{E}+13$ & $1.752 \mathrm{E}+13$ & $1.552 \mathrm{E}+13$ & $9.117 \mathrm{E}+12$ \\
\hline $5 f 7 f$ & $3 d k g$ & & $1.058 \mathrm{E}+13$ & $1.024 \mathrm{E}+13$ & $9.646 \mathrm{E}+12$ & $8.439 \mathrm{E}+12$ & $4.635 \mathrm{E}+12$ \\
\hline $5 f 7 s$ & & & & & & & \\
\hline $5 f 7 p$ & $4 f k p$ & $2.444 \mathrm{E}+13$ & $2.437 \mathrm{E}+13$ & $2.433 \mathrm{E}+13$ & $2.425 \mathrm{E}+13$ & $2.408 \mathrm{E}+13$ & $2.341 \mathrm{E}+13$ \\
\hline $5 f 7 d$ & $4 f k d$ & $3.005 \mathrm{E}+13$ & $2.995 \mathrm{E}+13$ & $2.989 \mathrm{E}+13$ & $2.977 \mathrm{E}+13$ & $2.953 \mathrm{E}+13$ & $2.857 \mathrm{E}+13$ \\
\hline $5 f 7 f$ & $4 f k f$ & $4.241 \mathrm{E}+13$ & $4.225 \mathrm{E}+13$ & $4.214 \mathrm{E}+13$ & $4.195 \mathrm{E}+13$ & $4.154 \mathrm{E}+13$ & $3.993 \mathrm{E}+13$ \\
\hline
\end{tabular}




\title{
Complete Atomic Data Base for Autoionizing Levels of B-like ions with $Z=6-54$
}

\author{
U. I. Safronova and A. S. Shlyaptseva \\ Department of Physics, University of Nevada, Reno \\ Reno, NV 89557 \\ M. Cornille and J. Dubau \\ UPR 176 CNRS, DARC Observatoire de Paris \\ 92195 Meudon Cedex, France
}

\section{Introduction}

In this paper, we calculate the autoionization decay of doubly excited $1 s 2 s^{2} 2 p^{2}, 1 s 2 s 2 p^{3}$, and1s $2 p^{4}$ states via channels $1 s^{2} 2 s^{21} S ; 1 s^{2} 2 s 2 p^{1} P,{ }^{3} P$; and $1 s^{2} 2 p^{2}{ }^{3} P,{ }^{1} D,{ }^{1} S$ of B-like ions for a broad range of nuclear charge $Z=6-54$. Recently, absolute energies of the autoionizing and non-autoionizing (ground and singly excited) levels and the total autoionization rates have been calculated for Li-, Be- and B-like ions with $Z=6-54$ using the MZ method [1]. Not only the total autoionization rates, but also the amplitudes and rates of autoionization decay via channels, so-called partial rates, are very important in atomic kinetic calculations, for the modeling of dielectronic satellite spectra and, in particular, for polarization spectroscopy of astrophysical and laboratory high-temperature plasmas.

\section{Calculational Methods}

In the present paper we use two different calculational methods, namely MZ (the perturbation theory method) and AUTOLSJ (SUPERSTRUCTURE including configuration interaction). Earlier, we compared non-relativistic and relativistic energies calculated by these two methods for 4 to 10 electron systems of Ar and Fe ions [2].

\subsection{MZ Code}

The perturbation theory method is the basis of the MZ code developed by Vainshtein and Safronova $[3,4]$. This method uses Z-expansions to calculate various atomic characteristics. The energy matrix is constructed in the $L S$-coupling scheme, including non-relativistic and relativistic parts. The nonrelativistic part takes into account three or four terms of the Z-expansion. The relativistic part is constructed using Breit-Pauli operators. In the first step all these terms are calculated for diagonal and nondiagonal elements. In the second step, the energy matrix is diagonalized. The energies (eigen values) and the mixing coefficients (eigen vectors, $C^{J}$ ) are obtained. In the third step these mixing coefficients are used to obtain all the matrix elements in the intermediate coupling scheme. In the intermediate coupling scheme the partial autoionization rate $A^{\text {aut }}$ in decay channel $i$ can be expressed through amplitudes $\gamma[1]$ (in a.u.): 


$$
\begin{array}{r}
A_{i}^{a u t}\left(Q L S J, Q_{i}\right)=2 \sqrt{2} \pi \sum_{Q_{1} Q_{2} L_{1} S_{1}} C^{J}\left(Q L S, Q_{1} L_{1} S_{1}\right) \gamma\left(Q_{1} L_{1} S_{1}, Q_{i} L_{1} S_{1}\right) \times \\
\times \gamma\left(Q_{i} L_{1} S_{1}, Q_{2} L_{1} S_{1}\right) C^{J}\left(Q_{2} L_{1} S_{1}, Q L S\right)
\end{array}
$$

Here $Q L S J$ are the quantum numbers characterizing the autoionizing level, $Q_{i}$ is relevant to the channel $i, C^{J}\left(Q L S, Q_{1} L_{1} S_{1}\right)$ are eigenvectors mentioned above, and 1 a.u. $=4.134 \times 10^{16} \mathrm{~s}^{-1}$. To calculate the total autoionization rates $A_{\text {tot }}^{\text {aut }}$ we must sum up the partial rates $A_{i}^{\text {aut }}$ over all decay channels.

\subsection{AUTOLSJ Code}

The AUTOLSJ package coordinates the calculation of energy levels, as well as radiative and autoionization rates. In the first step, the code determines non-relativistic wavefunctions by diagonalization of the non-relativistic hamiltonian on a set of chosen configurations. The single-electron wavefunctions are calculated in "scaled" Thomas-Fermi-Dirac-Amaldi potentials which are different for each angular momentum $l$. The scaling parameters are iterated to give the minimum energy of a term or of a group of terms. These potentials depend closely on the configurations. In our case two complexes $1 s 2 s^{2} 2 p^{k}+1 s 2 p^{k+2}$ and $1 s 2 s 2 p^{k+1}$ are considered. To derive the non-relativistic and relativistic energies, the program diagonalizes the matrices relevant to the corresponding operators. In the second step (part of the AUTOLSJ package developed by Dubau et al 1981 [5]) we use the level mixing coefficients obtained by the energy matrix diagonalization to calculate the data.

\section{Results}

In Tables 1 and 2 the results of our calculations of partial rates are presented for six decay channels, namely $1 s^{2} 2 s^{2}{ }^{1} S ; 1 s^{2} 2 s 2 p{ }^{1} P,{ }^{3} P$, and $1 s^{2} 2 p^{2}{ }^{1} S,{ }^{3} P,{ }^{1} D$, respectively. They are computed by the MZ (a) and AUTOLSJ (b) codes. Also, for comparison we use the Cowan program [6] and the data by Chen et al [7]. Thus, we compare the data (both partial and total autoionization rates) from four different codes. The comparison is, in general, good. Nevertheless, some disagreements appear due to levels with relatively weak autoionization rates $\left(<10^{13} s^{-1}\right)$. Moreover, we study the Z-dependences of partial rates for each complex of autoionizing levels with $J=1 / 2,3 / 2$ and $5 / 2$. In general, they show smooth trends. But the Z-dependences for levels with $J=3 / 2$ of the configuration $1 s 2 s 2 p^{3}$ (with a maximum value of eight levels in one complex) have a non-smooth form with maxima and minima.

\section{References}

[1] U. I. Safronova and A. S. Shlyaptseva, Physica Scripta 54, 254 (1996)

[2] M. Cornille, J. Dubau, and U. I. Safronova, Physica Scripta 49, 69 (1994)

[3] V. L. Vainshtein and U. I. Safronova, Atomic Data Nuclear Data Tables 21, 44 (1978)

[4] V. L. Vainshtein and U. I. Safronova, Atomic Data Nuclear Data Tables 25, 311 (1980)

[5] J. Dubau and M. Loulergue, Physica Scripta 23, 136 (1981)

[6] R. D. Cowan, "The Theory of Atomic Spectra", University of California Press 1981

[7] M. H. Chen and B. Crasemann, Atomic Data Nuclear Data Tables 38, 381 (1988) 
Table 1: Partial autoionization rates of B-like Fe decaying via the channels $1 s^{2} 2 s^{2}{ }^{1} S, 1 s^{2} 2 s 2 p$ ${ }^{1} P$ and $1 s^{2} 2 s 2 p{ }^{3} P$, calculated by MZ (a) and AUTOLSJ (b) codes. Designations: $I=1 s 2 s^{2} 2 p^{2}$, $J=1 s 2 p^{4}, K=2 p^{3}\left[{ }^{4} S\right] 2 s\left[{ }^{5} S\right] 1 s, T=2 p^{3}\left[{ }^{4} S\right] 2 s\left[{ }^{3} S\right] 1 s, K=2 p^{3}\left[{ }^{2} P\right] 2 s\left[{ }^{3} P\right] 1 s, Q=2 p^{3}\left[{ }^{2} P\right] 2 s\left[{ }^{1} P\right] 1 s$, $K=2 p^{3}\left[{ }^{2} D\right] 2 s\left[{ }^{3} D\right] 1 s, Q=2 p^{3}\left[{ }^{2} D\right] 2 s\left[{ }^{1} D\right] 1 s$

\begin{tabular}{|c|c|c|c|c|c|c|c|}
\hline \multirow[b]{2}{*}{ Level } & \multirow[b]{2}{*}{$\mathbf{J}$} & \multicolumn{2}{|c|}{$1 s^{2} 2 s^{2}{ }^{1} S$} & \multicolumn{2}{|c|}{$1 s^{2} 2 s 2 p{ }^{1} P$} & \multicolumn{2}{|c|}{$1 s^{2} 2 s 2 p^{3} P$} \\
\hline & & $\mathrm{a}$ & $\mathrm{b}$ & $\mathrm{a}$ & $\mathrm{b}$ & $\mathrm{a}$ & $\mathrm{b}$ \\
\hline $1 I$ & $1 / 2$ & 0.691 & 1.112 & 2.63 & 2.43 & 2.93 & 2.33 \\
\hline $2 I$ & $1 / 2$ & 0.520 & 0.7190 & 0.715 & 0.609 & 14.6 & 12.3 \\
\hline $4 I$ & $1 / 2$ & 6.14 & 8.213 & 8.22 & 6.73 & 2.55 & 2.20 \\
\hline $5 J$ & $1 / 2$ & 0.0679 & 0.0025 & 0.110 & 0.103 & 0.189 & 0.0417 \\
\hline $6 J$ & $1 / 2$ & 0.0422 & 0.0041 & 0.0549 & 0.0513 & 0.187 & 0.160 \\
\hline $8 J$ & $1 / 2$ & 0.329 & 0.0026 & 0.432 & 0.3424 & 0.109 & 0.0929 \\
\hline $11 K$ & $1 / 2$ & 0 & 0 & 0.512 & 0.409 & 21.6 & 21.7 \\
\hline $13 K$ & $1 / 2$ & 0 & 0.0002 & 0.0422 & 0.0029 & 20.8 & 17.0 \\
\hline $14 K$ & $1 / 2$ & 0 & 0.0844 & 1.25 & 1.061 & 19.2 & 14.0 \\
\hline $17 T$ & $1 / 2$ & 0 & 0.0996 & 0.949 & 6.33 & 0.181 & 0.409 \\
\hline $18 Q$ & $1 / 2$ & 0 & 0.2667 & 18.0 & 9.05 & 2.07 & 3.09 \\
\hline $1 I$ & $3 / 2$ & 4.15 & 3.112 & 3.77 & 3.17 & 2.44 & 1.92 \\
\hline $2 I$ & $3 / 2$ & 0.0917 & 0.0648 & 0.0723 & 0.058 & 15.6 & 13.2 \\
\hline $3 I$ & $3 / 2$ & 14.1 & 11.31 & 8.12 & 6.86 & 2.09 & 1.80 \\
\hline $5 J$ & $3 / 2$ & 0.101 & 0.0038 & 0.0719 & 0.0569 & 0.0312 & 0.0253 \\
\hline $6 J$ & $3 / 2$ & 0.0098 & 0.00059 & 0.0079 & 0.0135 & 0.225 & 0.191 \\
\hline $7 J$ & $3 / 2$ & 0.187 & 0.0002 & 0.117 & 0.101 & 0.0463 & 0.0427 \\
\hline $10 K$ & $3 / 2$ & 0 & 0.0038 & 1.35 & 0.0429 & 6.36 & 5.05 \\
\hline $11 K$ & $3 / 2$ & 0 & 0 & 0.414 & 0.228 & 18.9 & 18.6 \\
\hline $12 K$ & $3 / 2$ & 0 & 0.0447 & 4.40 & 0.920 & 13.8 & 19.06 \\
\hline $13 K$ & $3 / 2$ & 0 & 0.0017 & 3.41 & 0.0778 & 14.1 & 15.8 \\
\hline $14 K$ & $3 / 2$ & 0 & 0.0019 & 10.6 & 11.3 & 10.5 & 7.74 \\
\hline $15 Q$ & $3 / 2$ & 0 & 0.0014 & 17.6 & 13.2 & 15.1 & 6.13 \\
\hline $16 T$ & $3 / 2$ & 0 & 0.0190 & 0.828 & 1.55 & 6.36 & 3.15 \\
\hline $18 Q$ & $3 / 2$ & 0 & 0.3050 & 17.6 & 12.5 & 1.43 & 3.42 \\
\hline $2 I$ & $5 / 2$ & 2.29 & 1.660 & 1.24 & 0.955 & 14.1 & 12.0 \\
\hline $3 I$ & $5 / 2$ & 16.1 & 12.82 & 8.71 & 7.30 & 3.70 & 3.19 \\
\hline $6 J$ & $5 / 2$ & 0.0082 & 0.00228 & 0.0045 & 0.0176 & 0.285 & 0.247 \\
\hline $7 J$ & $5 / 2$ & 0.286 & 0.0040 & 0.115 & 0.117 & 0.0316 & 0.0322 \\
\hline $9 K$ & $5 / 2$ & 0 & 0 & 0.005 & 0.005 & 0.708 & 0.549 \\
\hline $11 K$ & $5 / 2$ & 0 & 0 & 0.095 & 0.095 & 21.7 & 21.3 \\
\hline $12 K$ & $5 / 2$ & 0 & 0 & 0.433 & 0.125 & 21.7 & 21.2 \\
\hline $13 K$ & $5 / 2$ & 0 & 0 & 1.19 & 1.06 & 19.6 & 17.1 \\
\hline $15 K$ & $5 / 2$ & 0 & 0 & 20.5 & 21.7 & 1.66 & 1.03 \\
\hline $11 K$ & $7 / 2$ & 0 & 0 & 0 & 0 & 22.3 & 23.4 \\
\hline
\end{tabular}


Table 2: Partial autoionization rates of B-like Fe decaying via the channels $1 s^{2} 2 p^{2}{ }^{1} S,{ }^{3} P$ and ${ }^{1} D$, calculated by $\mathrm{MZ}$ (a) and AUTOLSJ (b) codes. Designations: $I=1 s 2 s^{2} 2 p^{2}, J=1 s 2 p^{4}$, $K=2 p^{3}\left[{ }^{4} S\right] 2 s\left[{ }^{5} S\right] 1 s, T=2 p^{3}\left[{ }^{4} S\right] 2 s\left[{ }^{3} S\right] 1 s, K=2 p^{3}\left[{ }^{2} P\right] 2 s\left[{ }^{3} P\right] 1 s, Q=2 p^{3}\left[{ }^{2} P\right] 2 s\left[{ }^{1} P\right] 1 s, K=$ $2 p^{3}\left[{ }^{2} D\right] 2 s\left[{ }^{3} D\right] 1 s, Q=2 p^{3}\left[{ }^{2} D\right] 2 s\left[{ }^{1} D\right] 1 s$

\begin{tabular}{cccccccc}
\hline & & $1 s^{2} 2 p^{2}{ }^{1} S$ & \multicolumn{2}{c}{$1 s^{2} 2 p^{2}{ }^{3} P$} & \multicolumn{2}{c}{$1 s^{2} 2 p^{2}{ }^{1} D$} \\
Level & $\mathrm{J}$ & $\mathrm{a}$ & $\mathrm{b}$ & $\mathrm{a}$ & $\mathrm{b}$ & $\mathrm{a}$ & $\mathrm{b}$ \\
\hline $1 I$ & $1 / 2$ & 1.38 & 0.934 & 8.52 & 9.15 & 0.146 & 0.145 \\
$2 I$ & $1 / 2$ & 1.01 & 0.588 & 8.76 & 12.1 & 0.093 & 0.080 \\
$4 I$ & $1 / 2$ & 12.6 & 7.07 & 1.57 & 1.80 & 1.51 & 1.22 \\
$5 J$ & $1 / 2$ & 0.749 & 0.735 & 29.7 & 22.3 & 4.07 & 3.86 \\
$6 J$ & $1 / 2$ & 0.338 & 0.318 & 32.1 & 24.5 & 2.00 & 1.81 \\
$8 J$ & $1 / 2$ & 4.54 & 3.71 & 7.24 & 6.24 & 23.3 & 18.6 \\
$11 K$ & $1 / 2$ & 0.071 & 0.051 & 0.228 & 2.19 & 0.088 & 0.068 \\
$13 K$ & $1 / 2$ & 0.051 & 0.003 & 2.63 & 2.23 & 0.006 & 0.004 \\
$14 K$ & $1 / 2$ & 0.218 & 0.166 & 9.09 & 7.76 & 0.272 & 0.214 \\
$17 T$ & $1 / 2$ & 0.214 & 1.69 & 10.5 & 5.45 & 0.268 & 2.23 \\
$18 Q$ & $1 / 2$ & 7.60 & 4.60 & 4.11 & 6.66 & 9.50 & 6.07 \\
\hline $1 I$ & $3 / 2$ & 0.031 & 0.072 & 7.19 & 8.33 & 2.17 & 2.37 \\
$2 I$ & $3 / 2$ & $3.0-4$ & 0.002 & 9.35 & 10.3 & 0.048 & 0.044 \\
$3 I$ & $3 / 2$ & 0.068 & 0.336 & 2.19 & 2.24 & 7.34 & 8.19 \\
$5 J$ & $3 / 2$ & 2.60 & 1.96 & 20.0 & 16.6 & 19.8 & 13.6 \\
$6 J$ & $3 / 2$ & 0.107 & 0.243 & 34.0 & 25.2 & 0.826 & 1.59 \\
$7 J$ & $3 / 2$ & 3.43 & 3.14 & 15.2 & 10.9 & 26.3 & 21.3 \\
$10 K$ & $3 / 2$ & 0.234 & 0.067 & 4.56 & 3.10 & 0.829 & 0.118 \\
$11 K$ & $3 / 2$ & 0.003 & 0.01 & 0.866 & 2.06 & 0.115 & 0.029 \\
$12 K$ & $3 / 2$ & 0.455 & 0.778 & 5.18 & 9.63 & 6.54 & 3.27 \\
$13 K$ & $3 / 2$ & 0.257 & 0.031 & 4.50 & 4.38 & 1.42 & 0.044 \\
$14 K$ & $3 / 2$ & 1.86 & 0.025 & 7.33 & 4.19 & 8.09 & 6.50 \\
$15 Q$ & $3 / 2$ & 0.227 & 0.029 & 3.28 & 4.10 & 1.90 & 5.80 \\
$16 T$ & $3 / 2$ & 0.311 & 0.325 & 18.3 & 18.8 & 1.93 & 0.432 \\
$18 Q$ & $3 / 2$ & 4.76 & 5.24 & 2.51 & 6.63 & 7.55 & 7.85 \\
\hline $2 I$ & $5 / 2$ & 0.009 & 0.059 & 8.19 & 9.25 & 1.19 & 1.16 \\
$3 I$ & $5 / 2$ & 0.089 & 0.356 & 1.15 & 1.28 & 8.36 & 9.42 \\
$6 J$ & $5 / 2$ & 0.046 & 0.325 & 34.4 & 24.6 & 0.363 & 2.09 \\
$7 J$ & $5 / 2$ & 6.10 & 5.01 & 0.265 & 1.58 & 46.5 & 34.4 \\
$9 K$ & $5 / 2$ & 0 & 0 & 0.091 & 0.072 & 0.002 & 0.001 \\
$11 K$ & $5 / 2$ & 0 & 0 & 0.468 & 2.19 & 0.025 & 0.019 \\
$12 K$ & $5 / 2$ & 0 & 0 & 0.225 & 9.05 & 1.24 & 1.86 \\
$13 K$ & $5 / 2$ & 0 & 0 & 1.89 & 2.22 & 0.212 & 0.137 \\
$15 Q$ & $5 / 2$ & 0 & 0 & 0.098 & 0.583 & 16.8 & 13.4 \\
\hline $11 K$ & $7 / 2$ & 0 & 0 & 0 & 2.21 & 0 & 0 \\
\hline & & & & & & &
\end{tabular}




\title{
Inner-Shell Excitation Energies for C-, N-, O-, and F-like Ions with $\mathrm{Z}=6-54$
}

\author{
U. I. Safronova and A. S. Shlyaptseva \\ Department of Physics, University of Nevada, Reno
}

Reno, NV 89557

\section{Introduction}

In this paper we continue to study the atomic characteristics for doubly-excited states. In our previous paper [1] we developed a method for the calculation of energies for systems with three, four and five electrons such as $1 s 2 s^{n} 2 p^{n^{\prime}}\left(n+n^{\prime}=2-4\right)$. This method was based on a perturbation theory starting with nonrelativistic hydrogenic basis functions. Moreover, the diagram technique was used. We treated these systems as states with an empty core and three, four and five electrons interacting with the nucleus and with each other. For such states only diagrams without loops give contributions to the nonrelativistic part of the energy. Here we study $1 s 2 s^{n} 2 p^{n^{\prime}}\left(n+n^{\prime}=5,6,7,8\right)$ states. Following the same approach, we must consider the states with an empty core and 6, 7, 8 and 9 electrons interacting with the nucleus and with each other. Obviously, it is more convenient to use hole representation, which allows to consider these states as systems with $8-n-n^{\prime}$ holes and a non-empty core with two closed shells. In this approach we can treat these systems as states with a $1 s^{2} 2 s^{2} 2 p^{6}$ core and 4, 3, 2 and 1 electrons interacting with the nucleus, the core and with each other. To describe the interaction of electrons with the core we have to take into account also diagrams with loops. For singly-excited states, these diagrams were studied in detail for systems with one electron above the core in many papers by Johnson et al (see, for example, [2]), and with two and three electrons above the core in papers $[3,4]$. In these papers $[2,3,4]$ relativistic basis functions were used, and all angular reductions for diagram contributions were given using the jj-coupling scheme. For two electrons above the core, the LS-coupling scheme was implemented in the paper by Safronova et al [5]. The first study of doubly-excited states using perturbation theory with including the first and second order diagram contributions (relevant to nonrelativistic energy) was presented in papers $[6,7]$.

\section{Energies of $1 s 2 s^{n} 2 p^{n^{\prime}}$ States with $n+n^{\prime}=5,6,7,8$ in the Hole Representation}

The field formulation of perturbation theory allows to construct the function of the $n$-hole system similar to the function of a n-electron system, replacing an operator of creation $a_{\beta_{k}}^{+}$with an operator of annihilation $a_{\beta_{k}}$ :

$$
\Phi_{n}^{Q}=\frac{1}{n !} \sum_{\beta_{1} \ldots \beta_{n}} C_{\beta_{1} \ldots \beta_{n}}^{Q} a_{\beta_{1}} \ldots a_{\beta_{n}} \Phi_{0}
$$

Here $\Phi_{0}$ and $Q$ relate to the core and an atomic system with quantum numbers $\mathrm{L}, \mathrm{S}, \mathrm{M}, \mathrm{M}_{S}$, respectively. Index $\beta_{i}$ describes a one-hole system with quantum numbers $n_{i} l_{i} m_{i} m_{S_{i}}$, and $C_{\beta_{1} \ldots \beta_{n}}^{Q}$ 
coefficients provide the antisymmetry in all indexes of the wave function $\Phi_{n}^{Q}$ (for detail see [5]). Using this determination of a hole-function we can carry out all calculations of diagram contributions. The functions of doubly-excited states $1 s 2 s^{n} 2 p^{n^{\prime}}[L S J]$ with $n+n^{\prime}=5,6,7,8$ are written as follows

$$
\begin{array}{cl}
\Phi_{1}^{Q}=\frac{1}{1 !} \sum_{\beta_{1}} C_{\beta_{1}}^{Q} a_{\beta_{1}} \Phi_{0}, & \Phi_{2}^{Q}=\frac{1}{2 !} \sum_{\beta_{1} \beta_{2}} C_{\beta_{1} \beta_{2}}^{Q} a_{\beta_{1}} a_{\beta_{2}} \Phi_{0}, \\
\Phi_{3}^{Q}=\frac{1}{3 !} \sum_{\beta_{1} \beta_{2} \beta_{3}} C_{\beta_{1} \beta_{2} \beta_{3}}^{Q} a_{\beta_{1}} a_{\beta_{2}} a_{\beta_{3}} \Phi_{0}, & \Phi_{4}^{Q}=\frac{1}{4 !} \sum_{\beta_{1} \beta_{2} \beta_{3} \beta_{4}} C_{\beta_{1} \beta_{2} \beta_{3} \beta_{4}}^{Q} a_{\beta_{1}} a_{\beta_{2}} a_{\beta_{3} a_{\beta_{4}} \Phi_{0}}
\end{array}
$$

The $n l$ quantum numbers for $\Phi_{1}^{Q}, \Phi_{2}^{Q}, \Phi_{3}^{Q}$ and $\Phi_{1}^{Q}$ should be equal to $1 s, 1 s 2 l, 1 s 2 l 2 l^{\prime}$ and $1 s 2 l 2 l^{\prime} 2 l^{\prime \prime}$ accordingly. Briefly, we can represent our states as

$$
\begin{gathered}
1 s 2 s^{2} 2 p^{6} \rightarrow 1 s^{-1} \Phi_{0}, \\
1 s 2 s^{2} 2 p^{5} \rightarrow 1 s^{-1} 2 p^{-1} \Phi_{0}, 1 s 22 p^{6} \rightarrow 1 s^{-1} 2 s^{-1} \Phi_{0}, \\
1 s 2 s^{2} 2 p^{4} \rightarrow 1 s^{-1} 2 p^{-2} \Phi_{0}, 1 s 2 s 2 p^{5} \rightarrow 1 s^{-1} 2 s^{-1} 2 p^{-1} \Phi_{0}, 1 s 2 p^{6} \rightarrow 1 s^{-1} 2 s^{-2} \Phi_{0}, \\
1 s 2 s^{2} 2 p^{3} \rightarrow 1 s^{-1} 2 p^{-3} \Phi_{0}, 1 s 2 s 2 p^{4} \rightarrow 1 s^{-1} 2 s^{-1} 2 p^{-2} \Phi_{0}, 1 s 2 p^{3} \rightarrow 1 s^{-1} 2 s^{-2} 2 p^{-1} \Phi_{0} .
\end{gathered}
$$

\section{Results and Discussions}

Tables 1,2,3 illustrate our results of calculations of the energies of $\mathrm{N}$-, $\mathrm{O}$ - and $\mathrm{F}$-like ions, respectively. The intermediate LS energies are shown only in the case of two identical terms belonging to one complex ( the states with one parity and the same $J$ but different $Q L S$ ), for example, for $1 s 2 s 2 p^{n}$ with $n=4,5$ ( ${ }^{3} P$ terms for $C$-like ions; ${ }^{2} P$ terms for $\mathrm{N}$-like ions). The same inter-electron interactions mix terms belonging to different configurations: $1 s 2 s^{2} 2 p^{n}+1 s 2 p^{n+2}\left({ }^{3} P,{ }^{1} P\right.$ terms for $n=4$ and ${ }^{2} S$ term for $\left.n=5\right)$ and $1 s^{2} 2 s^{2} 2 p^{n}+1 s^{2} 2 p^{n+2}\left({ }^{1} S,{ }^{1} D,{ }^{3} P\right.$ terms for $n=4,{ }^{2} P$ term for $n=$ 5 and ${ }^{1} S$ term for $n=5$ ). This mixing can be described by a first order perturbation theory diagram for the correlation part of the energy [5] and can be calculated by any computer method. The next part of the correlation energy can be obtained by using virtual configurations, both discrete and continuous. The advantage of the $\mathrm{MZ}$ program is to take into account this part of the correlation energy in the framework of second order perturbation theory. As a result, we believe that data obtained by this method for 2 - 10 electron systems are more precise than other methods.

We conclude that the absolute energy data given in Tables 1 - 3 can be used for many applications, particuarly in electron and photon emission spectroscopy.

\section{References}

[1] U. I. Safronova and A. S. Shlyaptseva, Physica Scripta 54, 254 (1996)

[2] W. R. Johnson, M. Idrees, and J. Sapirstein, Phys. Rev. A 35, 3218 (1987)

[3] M. S. Safronova, W. R. Johnson, and U. I. Safronova, Phys. Rev. A53, 4036 (1996)

[4] M. S. Safronova, W. R. Johnson, and U. I. Safronova, Phys. Rev. A 54, 2850 (1996)

[5] M. S. Safronova, U. I. Safronova, N. Nakamura, and S. Ohtani, Physica Scripta 53, 689 (1996)

[6] U. I. Safronova and V. N. Kharitonova, Optic and Spectroscopy 27, 550 (1969)

[7] U. I. Safronova, A. N. Ivanova, N. V. Rabinkina, and V. N. Kharitonova, Optic and Spectroscopy 28, 841 (1970) 
Table 1: Absolute values of the energy $\left(E\right.$ in $\left.10^{4} \mathrm{~cm}^{-1}\right)$ for $\mathrm{N}$-like systems. Designations: $E=1 s^{2} 2 s^{2} 2 p^{3}, F=1 s^{2} 2 p^{5}, C=1 s^{2} 2 s 2 p^{4}, I=1 s 2 s^{2} 2 p^{4}, J=1 s 2 p^{6}, Q=2 s 2 p^{5}\left[{ }^{1} P\right] 1 s, K=2 s 2 p^{5}\left[{ }^{3} P\right] 1 s$

\begin{tabular}{|c|c|c|c|c|c|c|c|c|c|}
\hline $\bar{N}$ & LSJ & $Z=12$ & $Z=14$ & $Z=16$ & $Z=18$ & $Z=20$ & $Z=22$ & $Z=24$ & $Z=26$ \\
\hline$E$ & ${ }^{4} S_{3 / 2}$ & 4111.650 & 5778.113 & 7737.435 & 9984.808 & 12523.56 & 15355.35 & 18482.17 & 21906.01 \\
\hline$E$ & ${ }^{2} D_{3 / 2}$ & 4106.136 & 5771.217 & 7729.207 & 9975.336 & 12512.96 & 15343.73 & 18469.54 & 21892.12 \\
\hline$E$ & ${ }^{2} D_{5 / 2}$ & 4106.137 & 5771.190 & 7729.087 & 9974.994 & 12512.19 & 15342.26 & 18467.07 & 21888.39 \\
\hline$E$ & ${ }^{2} P_{1 / 2}$ & 4103.307 & 5767.632 & 7724.794 & 9969.953 & 12506.38 & 15335.66 & 18459.65 & 21880.12 \\
\hline$E$ & ${ }^{2} P_{3 / 2}$ & 4103.297 & 5767.577 & 7724.608 & 9969.462 & 12505.28 & 15333.52 & 18455.87 & 21873.95 \\
\hline$C$ & ${ }^{4} P_{1 / 2}$ & 4086.832 & 5746.795 & 7699.505 & 9940.093 & 12471.81 & 15296.22 & 18415.18 & 21828.46 \\
\hline$C$ & ${ }^{4} P_{3 / 2}$ & 4086.659 & 5746.424 & 7698.798 & 9938.859 & 12469.79 & 15293.08 & 18410.50 & 21821.73 \\
\hline C & ${ }^{4} P_{5 / 2}$ & 4086.567 & 5746.230 & 7698.439 & 9938.258 & 12468.87 & 15291.76 & 18408.74 & 21819.55 \\
\hline$C$ & ${ }^{2} D_{3 / 2}$ & 4077.487 & 5735.064 & 7685.314 & 9923.337 & 12452.34 & 15273.86 & 18389.68 & 21801.54 \\
\hline$C$ & ${ }^{2} D_{5 / 2}$ & 4077.490 & 5735.062 & 7685.295 & 9923.266 & 12452.15 & 15273.43 & 18388.83 & 21799.87 \\
\hline$C$ & ${ }^{2} S_{1 / 2}$ & 4071.480 & 5727.631 & 7676.482 & 9913.147 & 12440.85 & 15261.09 & 18375.65 & 21786.14 \\
\hline$C$ & ${ }^{2} P_{1 / 2}$ & 4069.223 & 5725.140 & 7673.769 & 9910.201 & 12437.62 & 15257.52 & 18371.62 & 21781.53 \\
\hline$C$ & ${ }^{2} P_{3 / 2}$ & 4069.019 & 5724.687 & 7672.873 & 9908.574 & 12434.87 & 15253.13 & 18364.97 & 21771.88 \\
\hline$F$ & ${ }^{2} P_{1 / 2}$ & 4046.439 & 5695.997 & 7638.127 & 9867.868 & 8.37 & 15201.08 & 18307.71 & 21709.88 \\
\hline$F$ & ${ }^{2} P_{3 / 2}$ & 4046.170 & 5695.411 & 7637.003 & 9865.900 & 12385.15 & 15196.09 & 18300.32 & 21698.21 \\
\hline$I$ & ${ }^{2} S_{1 / 2}$ & 3071.879 & 4335. & 5825. & 753 & .301 & 1162 & 14010.93 & 16621.10 \\
\hline$I$ & ${ }^{2} P_{1 / 2}$ & 3074.837 & 4339.087 & 5829.303 & 7540.382 & 9475.299 & 11635.28 & 14021.71 & 16635.77 \\
\hline$I$ & ${ }^{2} P_{3 / 2}$ & 3075.046 & 4339.475 & 5829.901 & 7541.141 & 9476.049 & 11635.73 & 14021.52 & 16634.63 \\
\hline$I$ & ${ }^{4} P_{1 / 2}$ & 3082.650 & 4348.944 & 5841.125 & 7554.032 & 9490.582 & 11651.98 & 14039.72 & 16655.21 \\
\hline I & ${ }^{4} P_{3 / 2}$ & 3082.763 & 4349.172 & 5841.529 & 7554.678 & 9491.529 & 11653.27 & 14041.34 & 16657.11 \\
\hline$I$ & ${ }^{4} P_{5 / 2}$ & 3083.028 & 4349.705 & 5842.496 & 7556.308 & 9494.115 & 11657.17 & 14046.97 & 16664.89 \\
\hline$I$ & ${ }^{2} D_{3 / 2}$ & 3076.258 & 4341.136 & 5832.071 & 7543.975 & 9479.830 & 11640.86 & 14028.50 & 16643.94 \\
\hline$I$ & ${ }^{2} D_{5 / 2}$ & 3076.328 & 4341.222 & 5832.121 & 7543.869 & 9479.355 & 11639.72 & 14026.31 & 16640.33 \\
\hline$J$ & ${ }^{2} S_{1 / 2}$ & 3018.043 & 4268.472 & 5744.511 & 7440.884 & 9360.351 & 11503.91 & 13872.78 & 16468.02 \\
\hline$Q$ & ${ }^{2} P_{1 / 2}$ & 3039.916 & 4296.820 & 5779.363 & 7482.305 & 9408.425 & 11558.72 & 13934.39 & 16536.46 \\
\hline$Q$ & ${ }^{2} P_{3 / 2}$ & 3040.060 & 4297.113 & 5779.906 & 7483.251 & 9409.990 & 11561.20 & 13938.15 & 16541.95 \\
\hline$K$ & ${ }^{2} P_{1 / 2}$ & 3046.697 & 4304.596 & 5788.186 & 7492.281 & 9419.742 & 11571.68 & 13949.44 & 16554.16 \\
\hline K & ${ }^{2} P_{3 / 2}$ & 3046.837 & 4304.877 & 5788.681 & 7493.072 & 9420.909 & 11573.30 & 13951.57 & 16556.91 \\
\hline K & ${ }^{4} P_{1 / 2}$ & 3055.555 & 4315.850 & 5801.737 & 7507.982 & 9437.387 & 11591.00 & 13970.09 & 16575.81 \\
\hline K & ${ }^{4} P_{3 / 2}$ & 3055.724 & 4316.188 & 5802.348 & 7509.007 & 9439.014 & 11593.47 & 13973.71 & 16580.94 \\
\hline$K$ & ${ }^{4} P_{5 / 2}$ & 3055.942 & 4316.630 & 5803.151 & 7510.349 & 9441.110 & 11596.57 & 13978.10 & 16586.89 \\
\hline
\end{tabular}


Table 2: Absolute values of the energy $\left(E\right.$ in $\left.10^{4} \mathrm{~cm}^{-1}\right)$ for O-like systems. Designations: $E=1 s^{2} 2 s^{2} 2 p^{4}, F=1 s^{2} 2 p^{6}, C=1 s^{2} 2 s 2 p^{5}, I=1 s 2 s^{2} 2 p^{5}, K=1 s 2 s 2 p^{6}$

\begin{tabular}{cccccccccc}
\hline$N$ & LSJ & $Z=12$ & $Z=14$ & $Z=16$ & $Z=18$ & $Z=20$ & $Z=22$ & $Z=24$ & $Z=26$ \\
\hline$E$ & ${ }^{3} P_{2}$ & 4225.646 & 5976.973 & 8043.323 & 10419.92 & 13110.13 & 16115.68 & 19438.56 & 23080.73 \\
$E$ & ${ }^{3} P_{1}$ & 4225.456 & 5976.554 & 8042.504 & 10418.45 & 13107.67 & 16111.75 & 19432.56 & 23071.88 \\
$E$ & ${ }^{3} P_{0}$ & 4225.378 & 5976.394 & 8042.232 & 10418.06 & 13107.22 & 16111.42 & 19432.73 & 23073.16 \\
$E$ & ${ }^{1} D_{2}$ & 4222.031 & 5972.297 & 8037.476 & 10412.72 & 13101.30 & 16104.81 & 19425.10 & 23063.94 \\
$E$ & ${ }^{1} S_{0}$ & 4217.892 & 5967.002 & 8030.995 & 10404.98 & 13092.16 & 16094.00 & 19412.17 & 23048.21 \\
$C$ & ${ }^{3} P_{2}$ & 4197.247 & 5940.518 & 7998.601 & 10366.63 & 13047.87 & 16043.88 & 19356.54 & 22987.61 \\
$C$ & ${ }^{3} P_{1}$ & 4197.078 & 5940.145 & 7997.886 & 10365.38 & 13045.85 & 16040.81 & 19352.08 & 22981.41 \\
$C$ & ${ }^{3} P_{0}$ & 4196.986 & 5939.944 & 7997.494 & 10364.68 & 13044.67 & 16038.92 & 19349.17 & 22977.03 \\
$C$ & ${ }^{1} P_{1}$ & 4185.870 & 5926.373 & 7981.640 & 10346.75 & 13024.92 & 16017.64 & 19326.69 & 22953.72 \\
$F$ & ${ }^{1} S_{0}$ & 4158.585 & 5891.295 & 7938.581 & 10295.47 & 12965.10 & 15948.94 & 19248.71 & 22866.02 \\
$I$ & ${ }^{3} P_{0}$ & 3207.524 & 4561.043 & 6162.524 & 8007.970 & 10098.58 & 12436.31 & 15022.67 & 17859.08 \\
$I$ & ${ }^{3} P_{1}$ & 3207.718 & 4561.432 & 6163.234 & 8007.970 & 10098.58 & 12436.31 & 15022.67 & 17859.08 \\
$I$ & ${ }^{3} P_{2}$ & 3208.004 & 4562.005 & 6164.260 & 8009.653 & 10101.16 & 12440.02 & 15027.74 & 17865.68 \\
$I$ & ${ }^{1} P_{1}$ & 3203.409 & 4555.788 & 6156.323 & 7999.842 & 10089.24 & 12425.65 & 15010.42 & 17844.70 \\
$K$ & ${ }^{1} S_{0}$ & 3171.617 & 4515.824 & 6107.959 & 7942.803 & 10023.21 & 12350.30 & 14925.43 & 17749.79 \\
$K$ & ${ }^{3} S_{1}$ & 3178.780 & 4525.024 & 6119.232 & 7956.190 & 10038.76 & 12368.06 & 14945.46 & 17772.16 \\
\hline
\end{tabular}

Table 3: Absolute values of the energy $\left(E\right.$ in $\left.10^{4} \mathrm{~cm}^{-1}\right)$ for F-like systems. Designations: $E=1 s^{2} 2 s^{2} 2 p^{5}, C=1 s^{2} 2 s 2 p^{6}, I=1 s 2 s^{2} 2 p^{6}$

\begin{tabular}{cccccccccc}
\hline$N$ & LSJ & $Z=12$ & $Z=14$ & $Z=16$ & $Z=18$ & $Z=20$ & $Z=22$ & $Z=24$ & $Z=26$ \\
\hline$E$ & ${ }^{2} P_{3 / 2}$ & 4313.938 & 6142.588 & 8308.343 & 10806.40 & 13640.12 & 16811.20 & 20321.64 & 24173.43 \\
$E$ & ${ }^{2} P_{1 / 2}$ & 4313.703 & 6142.062 & 8307.314 & 10804.58 & 13637.10 & 16806.49 & 20314.60 & 24163.28 \\
$C$ & ${ }^{2} S_{1 / 2}$ & 4282.778 & 6102.162 & 8257.820 & 10745.74 & 13568.86 & 16728.78 & 20227.29 & 24065.98 \\
$I$ & ${ }^{2} S_{1 / 2}$ & 3296.441 & 4734.311 & 6438.257 & 8408.919 & 10648.28 & 13156.65 & 15935.70 & 18978.92 \\
\hline
\end{tabular}




\title{
Accurate Lifetime Measurements of Fine-Structure States of Neutral Lithium by Beam-Gas-Laser-Spectroscopy
}

\author{
A. Schmitt, U. Volz, and H. Schmoranzer \\ Fachbereich Physik, Universität Kaiserslautern \\ 67653 Kaiserslautern, Germany
}

\begin{abstract}
The radiative lifetimes of the $2 p^{2} P, 3 s^{2} S$, and $3 d^{2} D$ terms of neutral lithium were measured at improved precision $( \pm 0.14 \%, \pm 0.23 \%$, and $\pm 0.1 \%$, respectively) by means of beam-gas-laserspectroscopy. We also give the line strengths for the corresponding transitions $2 s-2 p, 2 p-3 s$, and $2 p-3 d$. For all three transitions we find an excellent agreement with recent large-scale $a b$ initio calculations.
\end{abstract}

\section{Introduction}

The three-electron atom lithium has always been a testing ground for atomic-structure calculations [1]-[11]. Its comparatively simple structure allows for very accurate calculations of line strengths by $a b$ initio methods. State-of-the-art $a b$ initio calculations using methods like CI-Hylleraas expansions (Yan and Drake [9]), multi-configuration Hartree-Fock (Froese Fischer et al. [11]) or all-orders manybody perturbation theory (Blundell et al. [2]) now have the potential to determine line strengths for the lithium atom with relative uncertainties at the $10^{-4}$ level and better. The results obtained by the various methods are generally in very good mutual agreement.

The longstanding disagreement between $a b$ initio theories and the experimental result of Gaupp et al. [12] for the $2 p$ lifetime was resolved recently in favor of theory by a new beam-gas-laserspectroscopy lifetime measurement performed in our group in 1995 (Volz and Schmoranzer [13]). This result, however, as well as the lifetime result for the $3 \mathrm{~s}$ level of lithium reported in the same paper, were of preliminary character and left some room for improvement. In the present work we give the final results of a set of new, improved lifetime measurements on the lithium levels $2 p, 3 s$, and $3 d$. The uncertainties have been narrowed down to $\pm 0.14 \%, \pm 0.23 \%$, and $\pm 0.1 \%$, respectively.

Due to the limited space, only a brief description of the experiment and the improvements made will be given here. A full report including more experimental details and a more comprehensive discussion will be published in the near future [14].

\section{Experimental method}

In beam-gas-laser-spectroscopy (see e.g. $[15,16]$ ) a monoisotopic ion beam with an energy of about $160 \mathrm{keV}$ is partially neutralized and unselectively excited by collisions in a gas cell. The beam then passes perpendicularly through the resonator of a linearly polarized $\mathrm{cw}$ dye laser in which the atomic level of interest is excited. The fluorescence photons emitted by the excited atoms are collected by a movable fiber-optics detection system, transmitted to the entrance slit of a 
monochromator, and finally detected by a photomultiplier. A similar fixed fiber-optics detection system serves as a monitor to normalize the signal of the movable system. Laser- and ion-beam choppers are used together with digital lock-in counting techniques to obtain a purely laser-induced, background free and cascade free signal. A decay curve consists of 30 data points at equidistant positions of the movable detector along the beam and covers at least three lifetimes. For a lifetime measurement, about 30 decay curves are recorded and evaluated by means of a least-squares fit. Zero-field quantum beats due to unresolved hyperfine-structure are included in the fit function if needed. Finally, the lifetime (before corrections) of the excited level is obtained from the averaged individual decay constants, using the precisely measured beam velocity as conversion factor.

\section{Corrections and uncertainties}

The largest systematical effect in beam-gas-laser-spectroscopy arises from the divergence of the atom beam which causes a slight decrease in the total detection efficiency for the fluorescence photons as the fibre-optics detector is moved downstream along the beam. As a result the decay of the excited state fluorescence appears to be faster and the lifetime appears to be shortened by, typically, a few $0.1 \%$. The correction procedure for this effect was a major contributor to the uncertainties in our previous measurements [13]. The procedure used there was based on an approximation of the atom beam profiles and of the spatial detection efficiency of the photon collection system by Gaussian-shaped model functions [15]. This resulted in a simple analytic expression for the beam divergence correction in which the measured parameters of the atom beam (widths, divergence angles) and of the fluorescence detector (width of the spatial detection efficiency) were inserted. In the present work the measured profiles of the atom beam and of the photon detection efficiency were fed into a computer program which calculated the beam divergence correction by numerical integration. This reduces the uncertainty in the correction by a factor of 2. The corrections applied are $(+0.62 \pm 0.07) \%$ for the $2 p$ level, $(+0.94 \pm 0.10) \%$ for $3 s$, and $(+0.33 \pm 0.04) \%$ for $3 d$.

Another important error source in lifetime measurements is the occurence of quantum beats. The geometry of the detection system in our experiment is designed to suppress quantum beats by a magic angle geometry. Nevertheless, residual quantum beat modulations may appear in the decay curves due to the finite acceptance angle $\left( \pm 5^{\circ}\right)$ of the detection system. A small modulation with an amplitude of $\pm 1 \%$ of the total signal found on the decay curve of the $2 p$ level could be assigned to the hyperfine-structure of the $2 p^{2} P_{3 / 2}$ state. In this case the proper theoretical expression for the quantum beat was included into the fit function. An evaluation of the decay curve without accounting for quantum beats would have yielded a lifetime about $0.3 \%$ shorter than the true value. In the case of the $3 d$ level quantum beats cannot be ruled out. However, the measured decay curve displayed no significant departure from an exact exponential behavior. For the $3 s$ level, finally, quantum beats are absent due to the low $J$ of $1 / 2$.

Other error sources included in the uncertainty estimates are the beam velocity, adjustment and linearity of the detector drive, changes in the light-guide transmission, detector saturation, quenching by residual gas, Zeeman quantum beats, and finally, statistical uncertainties. The total uncertainties of $\pm 0.14 \%(2 p), \pm 0.23 \%(3 s)$ and $\pm 0.10 \%(3 d)$ result from a quadratic addition of the individual contributions and should be interpreted as single standard deviations $(1 \sigma)$. Compared to our previous results for the levels $2 p$ and $3 s$ [13], the uncertainties could be narrowed down further, in particular for the $3 s$ lifetime. This reduction in uncertainty is for the most part due to the improved correction procedure for the atom beam divergence effect. In the case of the $3 \mathrm{~s}$ level, increased count rates and therefore lower statistical uncertainties also contributed to the improvement. 


\section{Results and discussion}

The results of our lifetime measurements and the corresponding line strengths are shown in Table 1 together with a selection of other experimental results with quoted uncertainties below $\pm 1 \%$. For the $2 s-2 p$ transition our result matches two recent more accurate results obtained by Martin et al. [19] and

Table 1: A selection of experimental line strengths $S_{i f}$ and upper state lifetimes $\tau_{i}$ for transitions in neutral lithium (uncertainties in parentheses).

\begin{tabular}{|l|l|r|l|l|l|}
\hline Transition & Authors(year) & Ref. & Method & $\tau_{i}$ (ns) & $S_{i f}$ (a.u.) \\
\hline $2 s-2 p$ & Gaupp, Kuske, and Andrä (82) & {$[12]$} & BGLS & $27.29(4)$ & $32.78(5)$ \\
& Carlsson and Sturesson (89) & {$[17]$} & pulsed laser & $27.22(20)$ & $32.86(24)$ \\
& Volz and Schmoranzer (96) & {$[13]$} & BGLS & $27.11(6)$ & $33.00(7)$ \\
& McAlexander et al. (96) & {$[18]$} & mol. spectr. & $27.102(9)$ & $33.008(11)$ \\
& Martin et al. (97) & {$[19]$} & mol. spectr. & $27.13(2)$ & $32.97(2)$ \\
& this work & & BGLS & $\mathbf{2 7 . 0 9 ( 4 )}$ & $\mathbf{3 3 . 0 2 ( 5 )}$ \\
\hline $2 p-3 s$ & Volz and Schmoranzer (96) & {$[13]$} & BGLS & $29.72(17)$ & $17.84(10)$ \\
& this work & & BGLS & $\mathbf{2 9 . 8 4 ( 7 )}$ & $\mathbf{1 7 . 7 7 ( 4 )}$ \\
\hline $2 p-3 d$ & Schulze-Hagenest et al. (77) & {$[20]$} & BGLS & $14.60(13)$ & $76.9(7)$ \\
& this work & & BGLS & $\mathbf{1 4 . 5 8 9 ( 1 4 )}$ & $\mathbf{7 6 . 9 8 ( 8 )}$ \\
\hline
\end{tabular}

McAlexander et al. [18], the latter reporting an uncertainty of only $\pm 0.033 \%$. Molecular spectroscopy was used in these works to determine the $2 s-2 p$ line strength from the long-range interaction potential of the molecular $A^{1} \Sigma_{u}^{+}$state. The earlier lifetime measurement by Gaupp et al. [12] differs from all the new measurements by more than four of its standard deviations and can thus be safely ruled out. For the levels $3 s$ and $3 d$ our new results improve the most accurate previous measurements by Volz and Schmoranzer [13] and by Schulze-Hagenest et al. [20] by factors of 2.5 and 9 , respectively.

Among the theoretical work listed in Table 2, only two approaches, namely the quantum Monte Carlo simulation by Barnett et al. [8] and the Brueckner approximation by Liaw and Chiou [7], fail to agree with experiment. The other theoretical line strengths for the three transitions investigated are in excellent agreement with the experimental results which confirms the high quality level reached by $a b$ initio calculations for neutral lithium.

\section{References}

[1] G. Peach, H. E. Saraph, and M. J. Seaton, J. Phys. B 21, 3669 (1988)

[2] S. A. Blundell, W. R. Johnson, Z. W. Liu, and J. Sapirstein, Phys. Rev. A 40, 2233 (1989)

[3] A.-M. Mårtensson-Pendrill and A. Ynnerman, Physica Scripta 41, 329 (1990)

[4] A. W. Weiss, Can. J. Chem. 70, 456 (1992)

[5] J. Pipin and D. M. Bishop, Phys. Rev. A 45, 2736 (1992)

[6] K. T. Chung, AIP Conf. Proc. 274, 381 (1993)

[7] S.-S. Liaw and F.-Y. Chiou, Phys. Rev. A 49, 2435 (1994) 
Table 2: Large-scale $a b$ initio calculations of line strengths $S_{i f}$ for transitions in neutral lithium (pair entries L/V denote length and velocity form).

\begin{tabular}{|l|l|r|l|l|}
\hline Transition & Authors(year) & Ref. & Method & $S_{\text {if }}$ (a.u.) \\
\hline $2 s-2 p$ & Peach et al. (88) & {$[1]$} & Close coupling & 33.03 \\
& Blundell et al. (89) & {$[2]$} & RMBPT all orders & $32.99 / 33.02$ \\
& Mårtensson-Pendrill et al. $(90)$ & {$[3]$} & Coupled clusters & 33.01 \\
& Weiss (92) & {$[4]$} & CI & $33.03 / 33.12$ \\
& Pipin and Bishop (92) & {$[5]$} & CI-Hylleraas & 33.00 \\
& Chung (93) & {$[6]$} & FCPC & $33.00 / 33.00$ \\
& Liaw and Chiou (94) & {$[7]$} & Brueckner approx. & $33.11 / 33.08$ \\
& Barnett et al. (95) & {$[8]$} & Monte Carlo sim. & $32.83(3)$ \\
& Yan and Drake (95) & {$[9]$} & CI-Hylleraas & 33.0056 \\
& Godefroid et al. (96) & {$[10]$} & MCHF & $33.00 / 33.00$ \\
& Froese Fischer et al. (97) & {$[11]$} & MCHF+Breit-Pauli & 33.0024 \\
\hline $2 p-3 s$ & Peach et al. (88) & {$[1]$} & Close coupling & 17.77 \\
& Blundell et al. (89) & {$[2]$} & MBPT all orders & $17.76 / 17.75$ \\
& Chung (93) & {$[6]$} & FCPC & 17.75 \\
& Liaw and Chiou (94) & {$[7]$} & Brueckner approx. & $17.89 / 17.89$ \\
& Froese Fischer et al. (97) & {$[11]$} & MCHF+Breit-Pauli & 17.7505 \\
\hline $2 p-3 d$ & Pipin and Bishop (92) & {$[5]$} & CI-Hylleraas & 76.97 \\
& Chung (93) & {$[6]$} & FCPC & $77.00 / 77.01$ \\
& Yan and Drake (95) & {$[9]$} & CI-Hylleraas & 77.0098 \\
& Froese Fischer et al. (97) & {$[11]$} & MCHF+Breit-Pauli & 77.0133 \\
\hline
\end{tabular}

[8] R. N. Barnett, E. M. Johnson, and W. A. Lester Jr., Phys. Rev. A 51, 2049 (1995)

[9] Z. C. Yan and G. W. F. Drake, Phys. Rev. A 52, R4316 (1995)

[10] M. R. Godefroid, C. Froese-Fischer, and P. Jönsson, Physica Scripta T 65, 70 (1996).

[11] C. Froese-Fischer, M. Saparov, G. Gaigalas, and M. Godefroid, At. Data Nucl. Data Tables, to be published

[12] A. Gaupp, P. Kuske, and H. J. Andrä, Phys. Rev. A 26, 3351 (1982)

[13] U. Volz and H. Schmoranzer, Physica Scripta T 65, 48 (1996)

[14] A. Schmitt, U. Volz, H. Liebel, M. Kohl, R. Henkel, H. Schmoranzer, to be published

[15] U. Volz, D. Marger, H. Roth, and H. Schmoranzer, J. Phys. B 28, 579 (1995)

[16] U. Volz, M. Majerus, H. Liebel, A. Schmitt, and H. Schmoranzer, Phys. Rev. Lett. 76, 2862 (1996)

[17] J. Carlsson and L. Sturesson, Z. Phys. D 14, 281 (1989)

[18] W. I. McAlexander, E. R. I. Abraham, and R. G. Hulet, Phys. Rev. A 54, R5 (1996)

[19] F. Martin, M. Aubert-Frécon, R. Bacis, P. Crozet, C. Linton, S. Magnier, A. J. Ross, and I. Russier, Phys. Rev. A 55, 3458 (1997)

[20] D. Schulze-Hagenest, H. Harde, W. Brand, and W. Demtröder, Z. Phys. A 282, 149 (1977) 


\title{
Accurate Atomic Data for S I, S II, and S III
}

\author{
S. S. Tayal \\ Department of Physics \\ Clark Atlanta University, Atlanta, GA 30314
}

\section{Introduction}

Prominent S I emission lines have been observed from Jupiter's satellite Io and the Io torus [1]. Absorption lines from neutral sulfur have been reported in the interstellar gas towards Oph [2]. S I emission features are also observed in the far-ultraviolet spectra of $\alpha$ Orionis [3] by the Goddard High Resolution Spectrograph (GHRS) on board the Hubble Space Telescope. Several S II and S III emission lines have been detected in ultraviolet (UV) spectra of the Io plasma torus in the magnetosphere of Jupiter by the Voyager ultraviolet spectrometers, the International Ultraviolet Explorer, and more recently by the high-resolution Hopkins Ultraviolet Telescope aboard Astro-1 and by the GHRS on board Hubble Space Telescope. The UV S III lines are important in temperature diagnostics of solar and stellar transition regions. Comprehensive laboratory analysis of S II [4] and S III [5] spectra are available. New S III lines from the $3 p 3 d$ ${ }^{3} F^{0}, 3 p 4 d{ }^{3} P^{0}, 3 p 4 p{ }^{1} S,{ }^{1} P,{ }^{1} D, 3 p 3 d^{1} P^{0},{ }^{1} D^{0},{ }^{1} F^{0}$, and $3 p 5 d^{1} P^{0}$ terms have been identified in the laboratory spectrum.

In order to give a correct interpretation of the observational data from the plasma, accurate values of oscillator strengths and electron excitation collision strengths of the constituent ions are needed. In recent years, we have carried out a program to calculate accurate atomic data for S I, S II, and S III. These studies are motivated not only because of the importance of atomic data in Io torus and stellar plasma modeling, but also to resolve existing discrepancies between the available calculations and between calculations and measurements. For example, our calculation resolved long-standing large discrepancies in f-values between theory and experiment for the $3 p^{4} 3 P-3 p 34 s^{\prime}{ }^{3} D^{0}$ transition in S I at $1479 \AA$ [6], and most of the existing discrepancies between available calculations for f-values of S III were resolved [7]. Theoretical calculations of cross sections for electron impact excitation of S II [8] and S III [9] have been carried out in concert with the experimental measurements by A. Chutjian and his co-workers at the Jet Propulsion Laboratory [10]. The measurements are made using the electron energy-loss, merged-beams method. Good agreement between theory and experiment for available low-lying transitions provides us confidence in the accuracy of our calculations and the theoretical models used in these calculations.

\section{Oscillator Strengths and Transition Probabilities}

Oscillator strengths and transition probabilities of electric-dipole-allowed and intercombination transitions from fine-structure levels of the terms belonging to the $3 s^{2} 3 p^{4}, 3 s^{2} 3 p^{3} 4 s, 3 s^{2} 3 p^{3} 5 s$, $3 s^{2} 3 p^{3} 3 d$, $3 s^{2} 3 p^{3} 4 d$ configurations of $\mathrm{S} \mathrm{I}, 3 s^{2} 3 p^{3}, 3 s 3 p^{4}, 3 s^{2} 3 p^{2} 3 d, 3 s^{2} 3 p^{2} 4 s$, and $3 s^{2} 3 p^{2} 4 p$ configurations of S II, and $3 s^{2} 3 p^{2}, 3 s 3 p^{3}, 3 s^{2} 3 p 3 d, 3 s^{2} 3 p 4 s, 3 s^{2} 3 p 4 p$, and $3 s^{2} 3 p 4 d$ configurations of S III are calculated using extensive configuration-interaction wave functions. Relativistic effects in intermediate coupling are incorporated by means of the Breit-Pauli Hamiltonian. Small 
adjustments to diagonal elements of the Hamiltonian matrices have been made so that the energy splittings are as close as possible to the experimental values. Our results of excitation energies, oscillator strengths, and transition probabilities are compared with other available calculations and with measurements to assess the quality of atomic data.

The atomic state wave functions are represented by the J-dependent CI expansions of the form

$$
\Psi_{i}\left(J M_{J}\right)=\sum_{j=1}^{M} b_{i j} \phi_{j}\left(\alpha_{j} L_{j} S_{j} J M_{J}\right),
$$

where each of the $M$ single-configuration functions $\phi_{j}$ is constructed from one-electron functions, and $\alpha_{j}$ defines the coupling of angular momenta of the electrons. The sum over $\mathrm{j}$ includes all configurations in which the orbital $L_{j}$ and spin $S_{j}$ angular momenta couple to give the total angular momentum

$$
J=L j+S j
$$

Each one-electron function is the product of a radial function, a spherical harmonic, and a spin function. The radial part of each orbital is expressed in analytic form as a sum of Slater-type-orbitals

$$
P_{n l}=\sum_{j=1}^{k} c_{j n l} r^{{ }^{j} n l} \exp \left(-\xi_{j n l} r\right),
$$

where $\mathrm{n}$ and 1 are, respectively, the principal and orbital quantum numbers and $c_{j n l}$, $\xi_{j n l}$, and $I_{j n l}$ are the expansion coefficients, exponents, and powers of $r$, respectively.

The $1 s, 2 s, 2 p, 3 s$, and $3 p$ radial functions are chosen as the Hartree-Fock functions of the $3 s^{2} 3 p^{4}{ }^{3} P, 3 s^{2} 3 p^{3}{ }^{4} S^{0}$, and $3 s^{2} 3 p^{2}{ }^{3} P$ ground states of S I, S II, and S III, respectively, and other radial functions have been obtained with the computer code CIV3 [11]. We used flexible radial functions and included a large number of configurations in the configuration-interaction (CI) expansions to ensure convergence. The calculated energy levels are in close agreement with laboratory measurements. There is usually good agreement between the length and velocity forms of oscillator strength except for weak and a few other transitions. In cases of discrepancies between the length and velocity values, the length value is recommended because it normally remains stable with respect to the addition of more configurations.

\section{Collision Strengths and Collision Rates}

Electron collisional excitation strengths for inelastic transitions in S II and S III have been calculated using the R-matrix method in a 19 -state $\left(3 s^{2} 3 p^{3}{ }^{4} S^{0},{ }^{2} D^{o},{ }^{2} P^{0}, 3 s 3 p^{4}{ }^{4} P,{ }^{2} D,{ }^{2} S, 3 s^{2} 3 p^{2} 3 d\right.$ $\left.{ }^{2} P,{ }^{4} F,{ }^{4} D,{ }^{2} F,{ }^{4} P,{ }^{3} s^{2} 3 p^{2} 4 s{ }^{4} P,{ }^{2} P, 3 s^{2} 3 p^{2} 4 p{ }^{2} S^{0},{ }^{4} D^{0},{ }^{4} P^{0},{ }^{2} D^{0},{ }^{4} S^{0},{ }^{2} P^{0}\right)$ and a 17 -state $\left(3 s^{2} 3 p^{2}{ }^{3} P,{ }^{1} D\right.$, $\left.{ }^{1} S, 3 s 3 p^{3}{ }^{5} S^{0},{ }^{3} D^{0},{ }^{3} P^{0},{ }^{1} P^{0},{ }^{3} S^{0},{ }^{1} D^{0}, 3 s^{2} 3 p 3 d{ }^{1} D^{0},{ }^{3} F^{0},{ }^{3} P^{0},{ }^{3} D^{0},{ }^{1} F^{0},{ }^{1} F^{0},{ }^{1} P^{0}, 3 s^{2} 3 p 4 s{ }^{3} P^{0},{ }^{1} P^{0}\right)$ close-coupling approximations, respectively. Rydberg series of resonances converging to the excited state thresholds are explicitly included in the scattering calculations. Resonances are found to make substantial enhancements in collision strengths at low energies for several transitions. Cross sections in our 19-state calculation for S II [8] show very good agreement with the recent merged-beams energy loss experiment [10] for the forbidden $3 s^{2} 3 p^{3}{ }^{4} S^{0}-3 s^{2} 3 p^{3}{ }^{2} D^{0}$, $3 s^{2} 3 p^{3}{ }^{4} S^{0}-3 s^{2} 3 p^{3}{ }^{2} P^{0}$, and resonance $3 s^{2} 3 p^{3}{ }^{4} S^{0}-3 s 3 p^{4}{ }^{4} P$ transitions and also with the 18-state 
R-matrix calculation [12]. A similar multistate R-matrix calculation for electron impact excitation of inelastic transitions in neutral sulfur is planned.

Collision rates are obtained from the total collision strengths by integrating over a Maxwellian velocity distribution. Effective collision strengths are presented for electron temperatures from $5,000 \mathrm{~K}$ to $100,000 \mathrm{~K}$, suitable for astrophysical applications, for all 136 inelastic transitions among 17-LS states of S III [9] and for all 171 inelastic transition transitions among 19-LS states of S II [8]. Our results are believed to be accurate to about $10 \%$ for transitions among low-lying excited states but may be less accurate for transitions involving higher excited states.

\section{Acknowledgments}

This research was supported by the National Aeronautics and Space Administration.

\section{References}

[1] S. T. Durrance, P. D. Feldman, and H. A. Weaver, Astrophys. J. (Letters) 267, L125 (1983)

[2] S. R. Federman and J. A. Cardelli, Astrophys. J. 452, 269 (1995)

[3] K. G. Carpenter, R. D. Robinson, G. M. Wahlgren, J. L. Linsky, and A. Brown, Astrophys. J. 428, 329 (1994)

[4] J. E. Pettersson, Phys. Scr. 28, 421 (1983)

[5] E. Johansson, C. E. Magnusson, I. Joelsson, and P. O. Zetterberg, Phys. Scr. 46, 221 (1992)

[6] S. S. Tayal, J. Phys. B 30, L551 (1997); S. S. Tayal, Ap. J., in press (1997)

[7] S. S. Tayal, J. Phys. B 28, 5193 (1995); S. S. Tayal At. Data and Nuclear Data Tables, in press (1997)

[8] S. S. Tayal, Ap. J. Suppl. 111, 459 (1997)

[9] S. S. Tayal, Ap. J. 481, 550 (1997)

[10] C. Liao, S. J. Smith, D. Hitz, A. Chutjian, S. S. Tayal, Ap. J. 484, 979 (1997)

[11] A. Hibbert, Comput. Phys. Commun. 9, 141 (1975)

[12] A. Ramsbottom, K. L. Bell, and R. P. Stafford, At. Data and Nuclear Data Tables 63, 57 (1996) 


\title{
Precise Atomic Lifetimes Measured at a Heavy-Ion Storage Ring
}

\author{
E. Träbert \\ Ruhr-Universität Bochum \\ D-44780 Bochum, Germany
}

\section{Technique}

A new experimental technique, derived from beam-foil spectroscopy, has been developed for the measurement of long atomic lifetimes in ions. The ions are produced in a standard ion source and then, after a first acceleration step, collisionally excited in the gas or foil stripper of an accelerator. A beam of charge-state selected $\mathrm{MeV}$-ions is transported to and then stored in the clean ultra-high vacuum conditions of a heavy-ion storage ring. This storage has been combined with straightforward optical detection (Fig. 1) to make precise and accurate atomic lifetime measurements in the millisecond range. As any element can be collisionally excited, the method is very versatile and may be used to study a number of cases which are of fundamental atomic structure or astrophysical and terrestrial plasma diagnostic interest.

A major advantage of the new technique is that the ion excitation on one hand and the trapping and detection on the other are spatially separated and can be individually optimized. The experiment works in fixed geometry. Thus there are no mechanically moving parts and no explicit dependence of the decay curves on the ion beam velocity. All that is needed to reach high precision are a reliable clock frequency, a measurement of the ion beam storage time (to correct the photon signal for ion beam losses during the measurement cycle), and a photon data rate somewhat higher than the detector dark rate. We employed a solar-blind photomultiplier and an interference filter to suppress possible stray light from ion getter pumps in the vacuum vessel. There is no need for higher spectral selectivity, as the transition of interest is often the only one of extreme longevity of the upper level in a given ionization stage of the element under study. Thanks to this longevity, measurements may begin after higher-lying levels have decayed to the ground state or to the metastable level of interest, and consequently there is practically no cascade problem. No ion-beam related background was detected. Further improvements would be possible by increasing the detection efficiency, for example by using several photomultipliers in parallel.

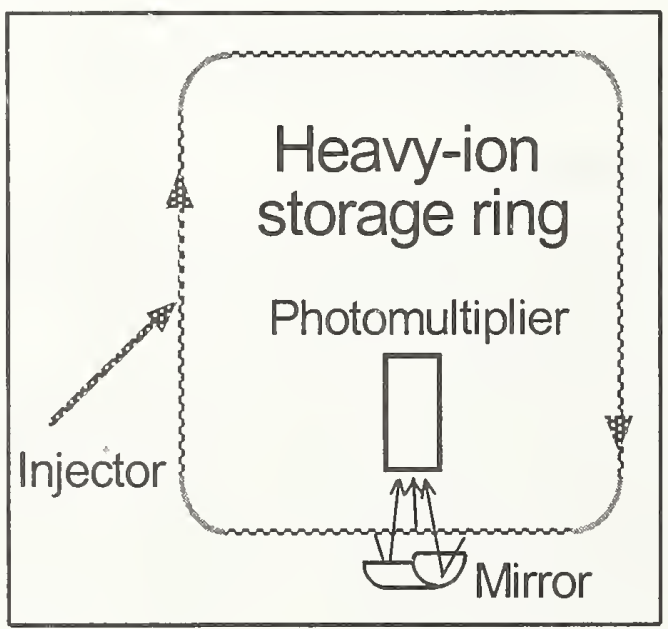

Figure 1: Schematics of the experimental arrangement 
The new technique has been applied to several intercombination $(E 1, \Delta S=1)$ transitions and to a few forbidden (M1) transitions. The selection of test cases matches the wavelength range ( $\lambda 190$ to $270 \mathrm{~nm}$ ) of our low-noise photomultiplier (with a dark rate of about 1 count/s) and the vacuum vessel window material and wavelength filter ranges.

\section{Cases Studied}

\subsection{Intercombination Transitions}

Among the systems studied are the $2 \mathrm{~s}^{2}{ }^{1} \mathrm{~S}_{0}-2 \mathrm{~s} 2 \mathrm{p}{ }^{3} \mathrm{P}^{0}{ }_{1}$ intercombination transition in the Be-like ions $\mathrm{B}^{+}$and $\mathrm{C}^{2+}$, the $3 \mathrm{~s}^{2}{ }^{1} \mathrm{~S}_{0}-2 \mathrm{~s} 2 \mathrm{p}^{3} \mathrm{P}^{0}{ }_{1}$ transition in the $\mathrm{Mg}$-like ion $\mathrm{Al}^{+}$, and the $2 \mathrm{~s}^{2} 2 \mathrm{p}^{2}$ ${ }^{3} \mathrm{P}_{1,2}-2 \mathrm{~s} 2 \mathrm{p}^{3} \mathrm{~S}_{2}^{0}$ transitions in C-like $\mathrm{N}^{+}$.

The $2 \mathrm{~s}^{2} \mathrm{~S}_{0}-2 \mathrm{~s} 2 \mathrm{p}^{3} \mathrm{P}^{0}{ }_{1}$ intercombination transition in the doubly charged carbon ion (Be-like) is of particular interest as a test case for fundamental atomic structure calculations and for some astrophysical applications (see Fig. 2). After the notable scatter of the early theoretical data, we note that some later calculational results seemingly followed experiment, even as early data from radiofrequency ion trap experiments varied considerably and appear mutually contradictive. These earlier lifetime results are now being surpassed in accuracy and precision because of the much cleaner experimental trapping conditions available at the heavy-ion storage ring, by the new experimental result of ( $\tau=9.714 \pm 0.013 \mathrm{~ms})$ [1].

In the most recent calculations by the groups at Belfast and Nashville, however, very different theoretical approaches yielded rather similar results and were quoted with intrinsic error estimates in the few-percent range. These similarities, however, were furthered by semiempirical adjustments to experimental energy level and fine structure data. No fully $a b$ initio calculation of this system so far comes close to the experimental precision and accuracy. An example is set by the case of $\mathrm{B}^{+}$: Two approaches (configuration interaction [2] and MCHF/MCDF [3] calculations) led to lifetime results for the $2 \mathrm{~s} 2 \mathrm{p}{ }^{3} \mathrm{P}^{0}{ }_{1}$ level which were claimed to have $2 \%$ uncertainties but differed from each other by about $6 \%$. This discrepancy boils down to the use of different experimental values for the $2 \mathrm{~s} 2 \mathrm{p}{ }^{3} \mathrm{P}^{0}$ fine structure intervals. With the same (right) choice of atomic structure reference data, the lifetime calculations perfectly agree with each other and with experiment ( $\tau=97.7 \pm 1 \mathrm{~ms}$ ). Assuming the validity of the theoretical treatment, our lifetime measurement with its poor spectral resolution can thus be seen as an indirect, but sensitive test on difficult to obtain experimental fine structure data.

The intercombination decay of the $3 \mathrm{~s} 3 \mathrm{p}^{3} \mathrm{P}^{0}{ }_{1}$ level in $\mathrm{Mg}$-like $\mathrm{Al}^{+}$may be seen as an analog to the above two ions in the Be sequence. In this case, our experiment ( $\tau=305 \pm 10 \mu \mathrm{s}$ ) [4] confirms the lifetime result of an earlier radiofrequency ion trap study [5] (which used laser ablation of $\mathrm{Al}$ and thus operated at a lower ambient pressure than is possible with gases). However, the latest calculations do not match the experimental data, and the results of earlier calculations have been stated with considerable uncertainties.

For the $2 \mathrm{~s}^{2} 2 \mathrm{p}^{2} \mathrm{P}_{1,2}-2 \mathrm{~s} 2 \mathrm{p}^{35} \mathrm{~S}^{0}{ }_{2}$ transition in C-like $\mathrm{N}^{+}$, which appears in auroral spectra of the upper atmosphere, a series of calculations and ion trap measurements finally led to lifetime data which agreed with each other and were quoted with about $6 \%$ uncertainty. Our storage ring data $(\tau=5.87 \pm 0.03 \mathrm{~ms}$ ) [6] reach $0.5 \%$ uncertainty and are just outside the previously given $5 \%(1 \sigma)$ error ranges of both the latest ion trap data and the most advanced theoretical treatment [7]. 


\section{Transition probability / s}

\begin{tabular}{|c|c|}
\hline $\mathrm{T}$ & Garstang \& Shamey 1967 \\
\hline$T$ & Nussbaumer 1972 \\
\hline$T$ & Mühletaler \& Nussbaumer 1976 \\
\hline$T$ & Lin \& Johnson 1977 \\
\hline $\mathrm{T}$ & Nussbaumer \& Storey 1978 \\
\hline $\mathrm{T}$ & Glass \& Hibbert 1978 \\
\hline $\mathrm{T}$ & $\begin{array}{l}\text { Laughlin, Constantinides \& } \\
\qquad \begin{array}{l}\text { Victor } 1978\end{array}\end{array}$ \\
\hline T & Cheng, Kim \& Desclaux 1979 \\
\hline T & Glass 1982 \\
\hline $\mathrm{T}$ & Cowan, Hobbs \& York 1982 \\
\hline$E$ & Kwong et al. 1983 \\
\hline E & Smith et al. 1984 \\
\hline $\mathbf{T}$ & Curtis 1991 \\
\hline $\mathbf{E}$ & Kwong et al. 1993 \\
\hline $\mathrm{T}$ & Chou, Chi \& Huang 1994 \\
\hline $\mathbf{T}$ & Fleming, Hibbert \& Stafford 1994 \\
\hline $\mathrm{T}$ & Froese Fischer 1994 \\
\hline$T$ & Ral'chenko \& Vainshtein 1995 \\
\hline $\mathbf{T}$ & Ynnerman \& Froese Fischer 1995 \\
\hline $\mathrm{T}$ & Brage, Fleming \& Hutton 1996 \\
\hline E & Doerfert et al. 1997 \\
\hline T & Jönsson \& Froese Fischer 1997 \\
\hline T & Froese Fischer \& Gaigalas 1997 \\
\hline
\end{tabular}

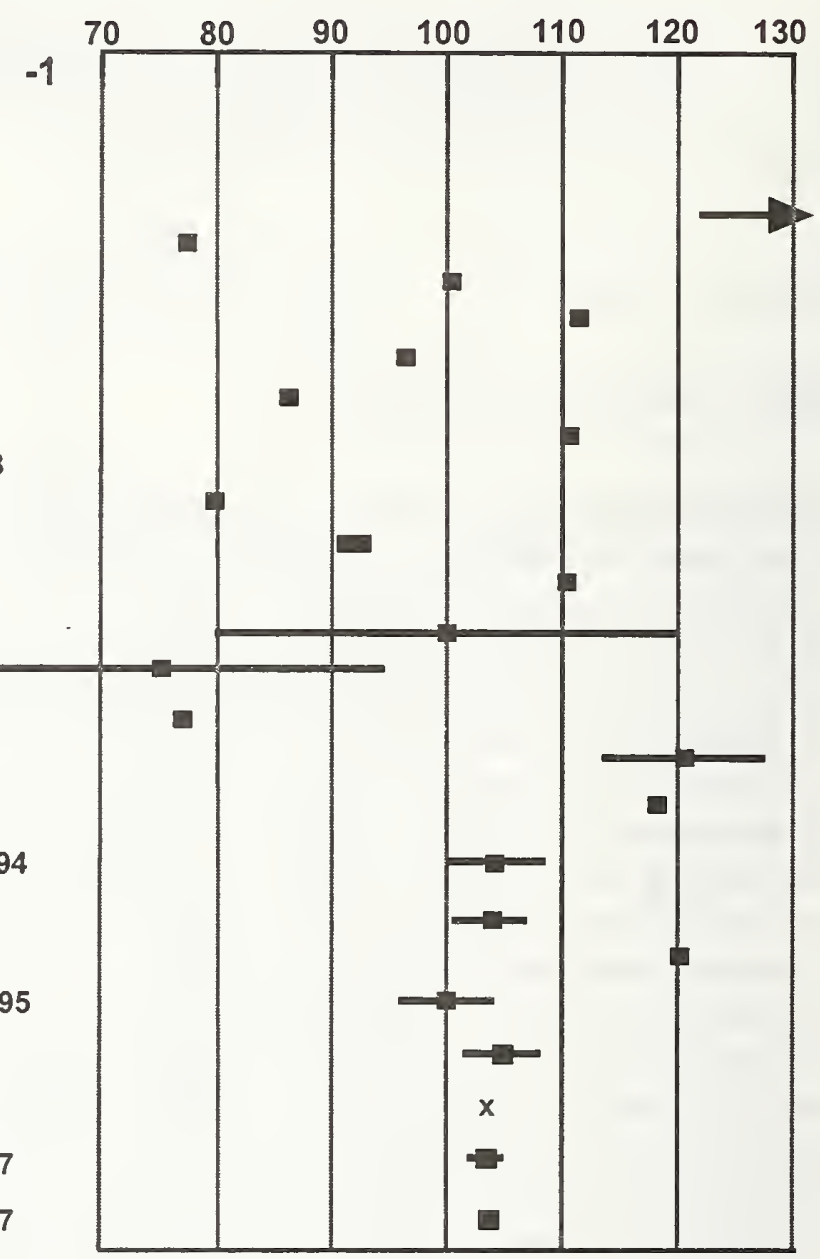

Figure 2: Intercombination transition probability in the $\mathrm{C}^{2+}$ ion. $\mathrm{T}$ Theory, E Experimental data. Our experiment is denoted "Doerfert et al. 1997" [1].

\subsection{Forbidden Transitions}

Of forbidden (M1) transitions we have studied test cases of interconnects within the ground state complex of several ions. In F-like $\mathrm{Sc}^{12+}$ our experiment aimed at the forbidden transition which connects the $2 \mathrm{~s}^{2} 2 \mathrm{p}^{5}{ }^{2} \mathrm{P}_{3 / 2,1 / 2}^{0}$ fine structure levels. The signal statistics was limited by the achievable ion beam current and thus the result limited to $3 \%$ precision. The available calculation [8] matches the experimental transition rate result of $(1000 \pm 30) \mathrm{s}^{-1}$ [4] only after a $2 \%$ correction for experimental fine structure data. For the much longer lived $2 s^{2} 2 p^{2}$ and $2 s^{2} 2 p^{4} D_{2}$ levels in C-like $\mathrm{Si}^{8+}$ and O-like $\mathrm{Si}^{6+}$ our storage ring data corroborate theory on the forbidden transition rates $[9,10]$ at the $3 \%$ level, yielding lifetimes of $38.8 \pm 0.5 \mathrm{~ms}$ and $65 \pm 3 \mathrm{~ms}$, respectively [6]. The experimental precision on these long atomic lifetimes is limited by the ion beam dynamics after injection into the storage ring: Although the long-term beam storage time constant may be as high as $40 \mathrm{~s}$ (which would cause little trouble), the initial transitory behavior of the ion beam necessitates larger corrections with notable uncertainties. 


\section{Conclusion}

The lifetimes already measured with the new technique range from $0.3 \mathrm{~ms}$ to $100 \mathrm{~ms}$. Depending on the experimental conditions (it is mostly the available ion beam current which determines the signal rate), the results have errors in the range $3 \%$ to $0.13 \%$. At such a level of precision, several of the new benchmark data test theory. Concerning intercombination transition rates, our data are found to corroborate only the latest, most extensive calculations. Even these, however, have not been obtained fully $a b$ initio, but use various adjustments to experimental atomic structure reference data. Concerning forbidden transition probabilities, theory seems much better off. Here, however, the predicted transition rates strongly depend on the assumed level splittings, and theories falling short by a few percent on this account easily yield $10 \%$ errors of the lifetime results. Such errors are clearly resolvable by the new experimental lifetime measurement technique.

Judging from our test cases, the forbidden transition rates apparently are under better theoretical control than the intercombination transition rates (after managing the term differences), probably because rather similar wavefunctions of initial and final states are involved and not the more dissimilar ones of the intercombination transition problem. However, we note that earlier on there were order-of-magnitude differences between differently calculated forbidden transition rates.

The project has been financially supported by German BMFT/BMBF and DFG research grants and by the Alexander von Humboldt Foundation.

\section{References}

[1] J. Doerfert, E. Träbert, A. Wolf, D. Schwalm, O. Uwira, Phys. Rev. Lett. 78, 4355 (1997)

[2] J. Fleming, K.L. Bell, A. Hibbert, N. Vaeck, M. Godefroid, MNRAS 279, 1289 (1996)

[3] C. Froese Fischer, G. Gaigalas, M. Godefroid, J. Phys. B 30 (1997). (in print)

[4] E. Träbert, A. Wolf, J. Linkemann, X. Tordoir (work in progress)

[5] B. C. Johnson, P. L. Smith, W. H. Parkinson, Ap. J. 308, 1013 (1986)

[6] E. Träbert, A. Wolf, J. Linkemann, E. H. Pinnington, E. J. Knystautas, A. Curtis, N. Bhattacharya, H. G. Berry (work in progress)

[7] T. Brage, A. Hibbert, D. S. Leckrone, Ap. J. 478, 423 (1997)

[8] K. T. Cheng, Y. -K. Kim, J. P. Desclaux, At. Data Nucl. Data Tables 24, 111 (1979)

[9] C. Froese Fischer, H. P. Saha, Phys. Rev. A 28, 3169 (1983)

[10] K. L. Baluja, J. Phys. B 18, L413 (1985) 


\title{
Light Ion Absorption, Probed with High Spatial, Temporal, and Spectral Resolution: The Spectrum of C II
}

\author{
Paolo Villoresi and Piergiorgio Nicolosi \\ Laboratorio di Elettronica Quantistica - D.E.I. \\ Università degli Studi di Padova - Padova, Italy \\ e-mail:pavil@dei.unipd.it
}

\begin{abstract}
We present a facility for the spectroscopic measurement of the photoabsorption of light ions in the region 30-100 $\mathrm{nm}$. The optical design of the experiment and the characteristics of the backlight continuum source allow a spatial resolution of the absorbing medium of about 0.3-0.5 $\mathrm{mm}$ and a duration of its irradiation of about $20 \mathrm{~ns}$. Therefore the absorption of a selected ionization stage of an element can be measured and, in addition, by the use of a high resolution spectrometer, its photoabsorption and photoionization spectra can be recorded with spectral resolution of 5000-8000. The facility is composed of a laser produced plasma acting as backlight continuous source, a stigmatic optical system with toroidal mirror and grating, and a UV CCD in the focal plane. The spectrum of C II is reported as example. The ground state has been effectively populated and both discrete series and continuous spectra with several Fano profiles were measured. From the calculated f-values, it will be possible to estimate the absolute photoionization cross section.
\end{abstract}

\section{Introduction}

Despite the vast amount of data available for neutrals, little photon absorption spectroscopy has been done for the elements in ionic stages, especially for the multiply charged ones $[1,2,3]$. The difficulty is that of generating a suitable column of ions for the energy level to be probed. In this context, the twin laser produced plasmas are a well established technique for almost two decades.

The laser produced plasma (LPP) characteristics as absorbing medium are the following:

- the generation of the ion is obtained by focusing a pulsed laser onto a plane target of the element under study,

- by varying the laser power density on the target, almost all the ionic stages can be produced for a large number of elements,

- in the LPP, the various ion species show different temporal and spatial evolution.

As continuum source, the LPP also shows relevant characteristics:

- the duration of the emission is comparable with that of the laser pulse: consequently, measurements with time resolution of order of $20 \mathrm{~ns}$ are possible,

- the emission in the spectral regions from VUV to the XUV may be either discrete or continuous, according to the choice of the target element and the laser power density focused onto the target,

- as a continuous backlighter, it has a well defined and small physical size, corresponding to the laser focal spot in transverse direction and a longitudinal expansion of a few tenths of a $\mathrm{mm}$, 


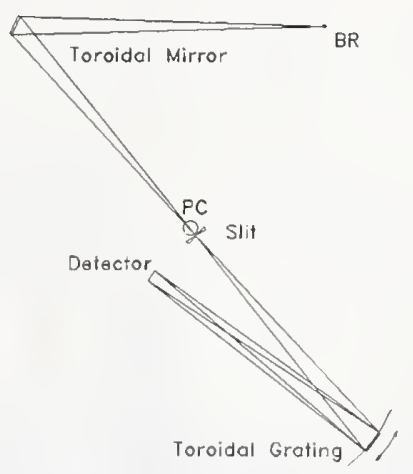

Figure 1: Optical setup, BR: background radiation, PC: plasma column

- it has very high brightness, due to the high plasma temperature and density,

- the emission is usually characterized by high reproducibility and stability,

- there is no requirement for ultra-high vacuum and there is no thermal load on the optics.

To exploit conveniently the LPP as backlighter in the EUV spectral range, an optical design with high optical aperture and a spectral resolution in the range 5000-10000 is needed.

\section{Aims of the present experiment}

In this contribution we present the measurement of the discrete and continuous spectra originating from the ground state $2 \mathrm{~s}^{2}\left({ }^{1} \mathrm{~S}\right) 2 \mathrm{p}^{2} \mathrm{P}^{0}$ of the first ion of carbon. The experimental technique is based on the two laser produced plasmas, applied in the normal incidence spectral region. The spectral range spans from $38 \mathrm{~nm}$ to $80 \mathrm{~nm}$, where the complete $2 \mathrm{p}^{2} \mathrm{P}^{0}-\mathrm{ns}{ }^{2} \mathrm{~S}$ and $2 \mathrm{p}^{2} \mathrm{P}^{0}$-nd ${ }^{2} \mathrm{D}$ series, as well as the series $2 \mathrm{~s}^{2} 2 \mathrm{p}^{2} \mathrm{P}^{0}-2 \mathrm{~s} 2 \mathrm{p} n \mathrm{p}^{2} \mathrm{P}$ appears. The first ionization limit is the $2 \mathrm{~s}^{2}$ ${ }^{1} \mathrm{~S}$ state at $50.8 \mathrm{~nm}$. The following photoionization spectrum has several resonances, giving rise to Fano profiles. The photoionization cross section has been calculated by the London group [4].

As already mentioned, the "knobs" that can be used to optimize the parameters of the experiment are the laser power density on the backlighter target and on the absorbing medium, the graphite target: for these, we have used the values of $2 \times 10^{12}$ and $1 \times 10^{10} \mathrm{~W} / \mathrm{cm}^{2}$ respectively. The focal spot on the absorbing medium target was chosen in order to increase the ion column density, and is obtained by a spherocylindrical lens, to produce a line focus of $0.1 \times 7 \mathrm{~mm}^{2}$ area. The delay between absorbing medium generation and backlight plasma generation has been varied between 50 and $120 \mathrm{~ns}$, by retarding the laser pulse in an optical delay line up to about $40 \mathrm{~m}$ long. The distance from the graphite target to the spot where the backlight beam was probing the plasma has been varied between 0.3 and $2 \mathrm{~mm}$, corresponding to noticeably different absorbing plasma densities and temperatures. In particular, with a delay of $58 \mathrm{~ns}$ between the formation of the two plasmas and at $1.7 \mathrm{~mm}$ from the $\mathrm{C}$ target, the ground state of C II was selectively populated and correspondingly the spectrum showed negligible contributions from neutral carbon absorption and only some isolated contributions from excited states of CII and from CIII.

\section{Description of the setup}

The optical setup, shown in Fig. 1, is composed of two toroidal optics setups: a mirror and a grating. The mirror, with meridional and sagittal radii of $1.26 \mathrm{~m}$ and $1.04 \mathrm{~m}$, respectively, operates at $24.6^{0}$. This is used as a condenser optics, gathering the backlight emission with 


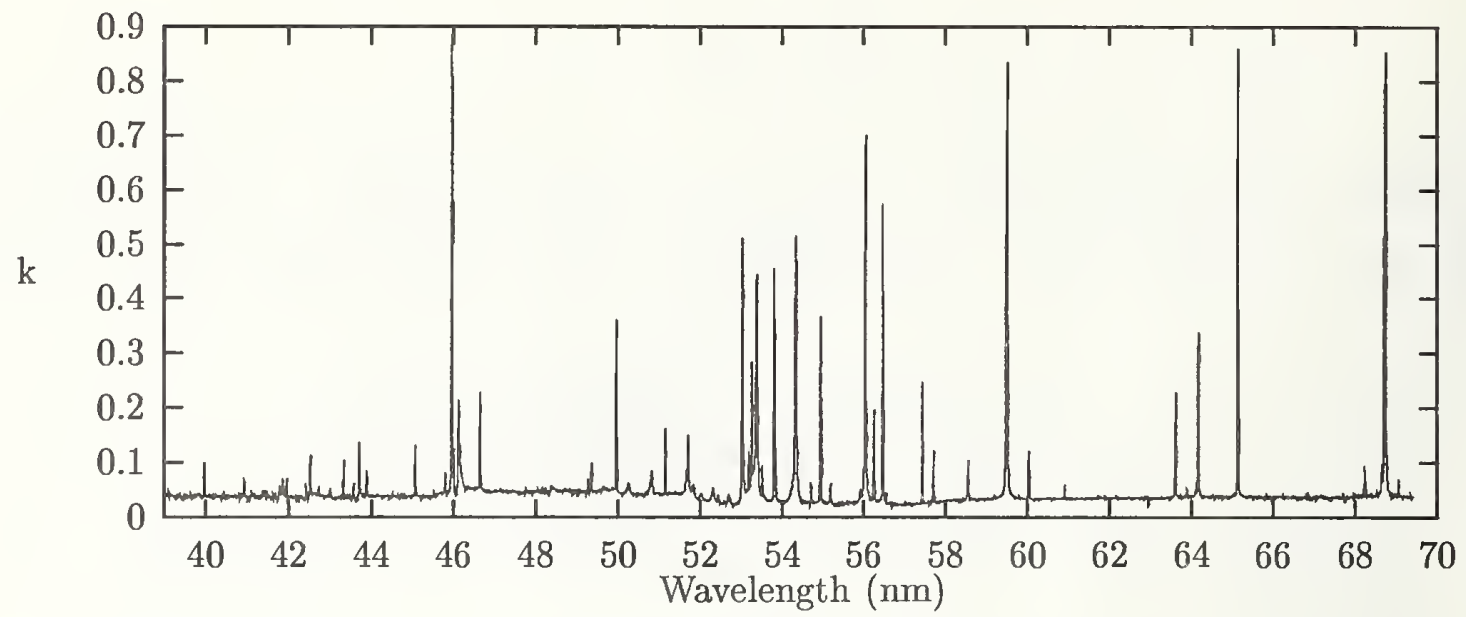

Figure 2: Absorption spectrum of C II: the discrete part begins at $50.8 \mathrm{~nm}$

about $\mathrm{f} / 10$ and focusing it onto the spectrograph entrance slit. The grating with 3600 lines/mm of radii $1.011 \mathrm{~m}$ and $0.992 \mathrm{~m}$ operates between $10^{\circ}$ and $14^{\circ}$. It provides stigmatic imaging of the absorption region with a dispersion of $6.7 \mathrm{pm}$ per detector pixel. The latter is a cooled UV CCD made by Princeton Instruments, with 16 bit of conversion, and $512 \times 512$ square pixel of $24 \mu \mathrm{m}$ of size. The laser is a Q-switched Nd:YAG, and the typical energies used for the plasma generations are $3.8 \mathrm{~J}$ on the backlight radiator and $1.2 \mathrm{~J}$ on the absorbing medium. The high $\mathrm{S} / \mathrm{N}$ ratio and the very low residual optical aberrations of the acquired spectra allowed the use of deconvolution techniques to improve the limit of resolution up to $5 \mathrm{pm}$.

\section{Results and discussion}

The C II spectrum was taken in contiguous spectral intervals, each of about $3.8 \mathrm{~nm}$ and averaged over 20 shots. The complete reconstruction of the spectrum, the evaluation of the relative oscillator strengths of the series and the photoionization cross-section is in progress, and the preliminary results are shown in Fig 2. The spectrum has been taken at $2.3 \mathrm{~mm}$ from the target surface and with a delay of $58 \mathrm{~ns}$ after the plasma generation. The discrete series are converging to the limit at $50.8 \mathrm{~nm}$. The transitions of the type $2 \mathrm{~s}^{2} 2 \mathrm{p}^{2} \mathrm{P}^{0}-2 \mathrm{~s}^{2}$ nd ${ }^{2} \mathrm{D}$ and $\mathrm{ns}{ }^{2} \mathrm{~S}$ can be observed for $\mathrm{n}$ larger than 10 , and show the effect of a strong perturbation about the $7 \mathrm{~d}$ state.

For these experimental conditions, the ground state absorption of the next ionization stage, C III, is noticeable. For instance, the strong $45.9 \mathrm{~nm}$ multiplet appears in the middle of the Fano profile originating from the interaction of $2 s^{2} 2 p^{2} \mathrm{P}^{0}-2 \mathrm{~s} 2 \mathrm{p} 4 \mathrm{p}^{2} \mathrm{D}$ and $2 \mathrm{~s} 2 \mathrm{p}\left({ }^{1} \mathrm{P}\right) 3 \mathrm{p}{ }^{2} \mathrm{D}$ with the $\left(2 \mathrm{~s}^{2}{ }^{1} \mathrm{~S}\right) k \mathrm{~d}{ }^{2} \mathrm{D}$ open channel.

The strong variation of the plasma density with the distance from the target and how it affects the observed spectra is clearly shown in Fig. 3, where the transition $2 \mathrm{~s}^{2} 2 \mathrm{p}^{2} \mathrm{P}^{0}-2 \mathrm{~s}^{2} 6 \mathrm{~d}$ ${ }^{2} \mathrm{D}$ at $54.338 \mathrm{~nm}$ for different distances from the target and with the same delay of $58 \mathrm{~ns}$ is presented. The profiles show the large variation, that occurred in less than a millimeter of displacement, of the absorption coefficient, whose integral changes by more than one order of magnitude, and of the linewidths.

For the assessment of the data quality, many efforts are made both during the acquisition and the data analysis. Particular attention is paid, during the measurements, to the absorbing plasma and the backlight continuum shot-to-shot reproducibility. Consequently, we have verified the conservation of the ratios between lines in a single spectral segment within a few percent. In addition, we are adopting an acquisition scheme in order to average over the target condition, in its shot-to-shot ageing, for all the quantitative measurements. In the profile integration, particular attention is paid to the extended wings and possible external contributions. 


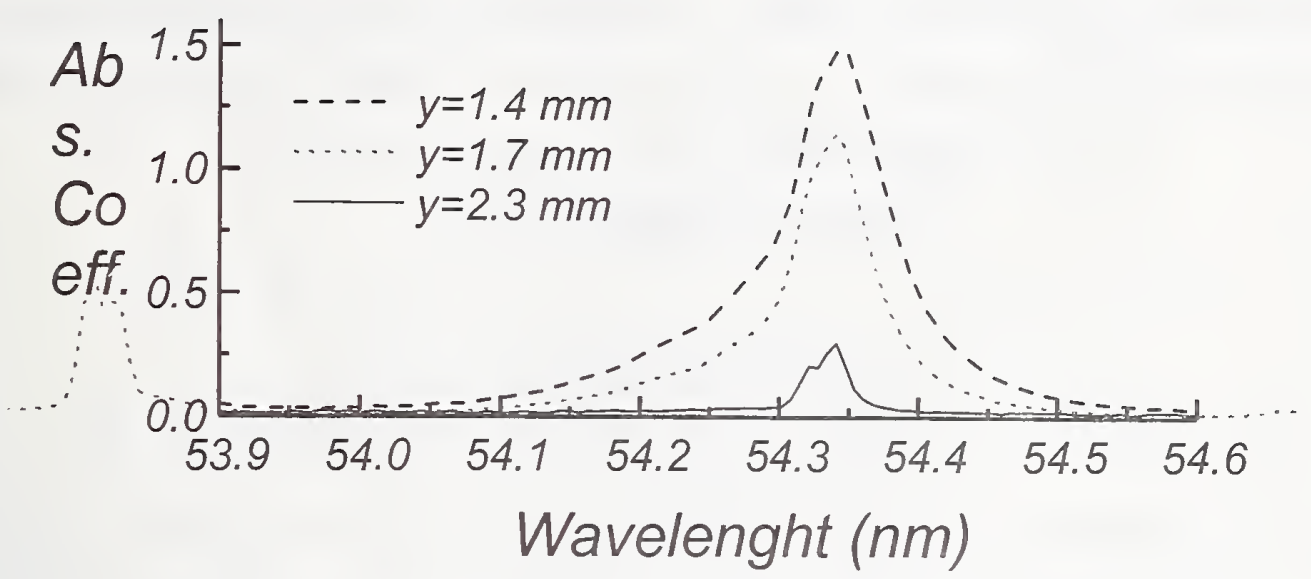

Figure 3: 2p-6d absorption at different distances from the target

\section{Conclusions}

A new experiment for absorption spectroscopy of ionized species with the dual LPP technique has been realized in the VUV spectral range.

The optical setup is stigmatic in an extended region, in order to achieve a fine spatial probing of the absorbing medium. This characteristic has shown a noticeable improvement of the species selection and then of the data quality.

Preliminary measurements of the CII photoabsorption and photoionization spectra has been performed. The analysis of the experimental data is in progress, in particular an estimate of the absolute photoionization cross-section. This is obtained through the evaluation of the column density. The latter is derived from the photoabsorption coefficients of strong suitable discrete transitions, whose oscillator strengths have been calculated $[5,6]$. Moreover, the oscillator strengths for high $n$ terms of the p-s and p-d transitions will be estimated with respect to known terms.

Finally, we are working on the identification of the perturbations in the photoionization cross section, in order to extract information on the level energies and the matrix element of the interaction.

The authors wish to express their gratitude to Prof. G. Tondello for the support and the useful suggestions as well as to Dr. E. Jannitti and to ing. R. Tesser for help in the data acquisition. This work was performed within the framework of the EC-HCM program: Investigation of the Structure and Dynamics of Atoms and Ions by Inner-Shell Excitation.

\section{References}

[1] P. Nicolosi et al., J. de Physique IV C1 suppl. 1, 89 (1991)

[2] J. T. Costello et al., Physica Scripta T 34, 77 (1991)

[3] F. J. Wuilleumier et al., N.I.M. B 87, 190 (1994)

[4] Y. Yan and M. J. Seaton, J. Phys. B 20, 6409 (1987)

[5] W. L. Wiese et al., J. Phys. Chem. Ref. Data Monograph 7 (1995)

[6] Y. Yan et al., J. Phys B 20, 116 (1987) 


\title{
Systematic Studies of the $n=2$ to $n=3,4$ Transitions in the Ions of Elements Cu through As Isoelectronic with Li I through Na I Produced by Laser Irradiation
}

\author{
J.F. Wyart'2,1, T. Missallaํ, J.C. Gauthier ${ }^{1}$, \\ and C. Chenais-Popovics ${ }^{1}$ \\ ${ }^{1}$ Laboratoire pour l'Utilisation des Lasers Intenses, Ecole Polytechnique, \\ 91128 - Palaiseau, France \\ ${ }^{2}$ Laboratoire Aimé Cotton, CNRS II, bâtiment 505, centre universitaire, \\ 91405 - Orsay, France
}

\section{Introduction}

Laser produced plasmas provide rich X-ray spectra. Their identification is of importance for the physics of ICF and X-ray lasers, of laboratory X-ray sources and of hot astrophysical objects. X-ray spectra of 5 elements ( $Z=29$ through 33) have been measured at the LULI laser facility as part of a systematic investigation of the medium-Z elements. The laser pulse conditions were 600 ps duration, frequency-doubled and 8 to $35 \mathrm{~J}$ focused on $80 \mu \mathrm{m}$ focal spots, leading to irradiations of $2 \times 10^{14}$ to $10^{15} \mathrm{Wcm}^{-2}$. Spectra were recorded in the ranges $6-8 \AA$ and $7.55-9.55 \AA$ by means of two flat crystal spectrographs (respectively PET and ADP crystals). The size of the plasma limits the linewidths to about $0.004 \AA$. The laser beam was focused at normal incidence onto the target surface. The spectra were recorded on films with an observation direction perpendicular to the laser axis and to the main plasma expansion.

Magnesium has been used as a tracer element for wavelength calibration, some targets being a mixture of $\mathrm{Mg}$ and the element being investigated. Only the $\mathrm{Ly}_{\alpha} \operatorname{doublet}(\lambda 8.4194 / 8.4253 \AA$ ) and $\mathrm{Mg} \mathrm{XI} \mathrm{He} e_{\alpha}(\lambda 9.1682 \AA)$ were obtained, and they were supplemented by theoretical wavelengths of well-resolved lithium-like lines at the short wavelength end of our observations. The wavelength uncertainty is $0.003 \AA$ for well-resolved lines but most of the emission peaks are blends of several transitions whose relative intensities depend on plasma conditions.

\section{Atomic Structure Calculations and Classification of the Lines}

In these conditions of irradiation, the ions have charge states beyond $20+$, and their emission spectrum below $10 \AA$ contains characteristic transitions between upper configurations having one electron with principal quantum numbers larger than 2 and lower levels of the ground complex $(2 s+2 p)^{N}$. Since Kelly's compilation [2], the status of the spectra being investigated has remained almost unchanged. For all ions involved, the levels with principal quantum numbers $n=2$ have been accurately described by Edlén from comparisons along isoelectronic sequences between observed and ab initio MCDF transition energies $(n=2, \Delta n=0)$ in the vuv region [1]. These "recommended" level values are available for checking the accuracy of theoretical models and approximations. We have used the atomic structure codes of HULLAC [3] based on the relativistic 
parametric potential method [4] for deriving transition energies and probabilities between full complexes

$(2)^{\mathrm{N}}$, i.e. $2 \mathrm{~s}^{2} 2 \mathrm{p}^{\mathrm{N}-2}+2 \mathrm{p}^{\mathrm{N}}$

and in opposite parity $2 \mathrm{~s}^{1} 2 \mathrm{p}^{\mathrm{N}-1}$

$(2)^{\mathrm{N} \cdot 1} 3^{1}$, i.e.

$$
\begin{gathered}
2 \mathrm{~s}^{2} 2 \mathrm{p}^{\mathrm{N}-3} 3 \mathrm{p}^{1}+2 \mathrm{~s} 2 \mathrm{p}^{\mathrm{N}-3} 3 \mathrm{~d}^{1}+2 \mathrm{~s}^{1} 2 \mathrm{p}^{\mathrm{N}-2} 3 \mathrm{p}^{1}+2 \mathrm{p}^{\mathrm{N} \cdot 1} 3 \mathrm{~s}^{1}+2 \mathrm{p}^{\mathrm{N}-1} 3 \mathrm{~d}^{1} \\
\text { and in opposite parity } \\
2 \mathrm{~s}^{2} 2 \mathrm{p}^{\mathrm{N}-3} 3 \mathrm{p}^{1}+2 \mathrm{~s}^{1} 2 \mathrm{p}^{\mathrm{N}-2} 3 \mathrm{~s}^{1}+2 \mathrm{~s}^{1} 2 \mathrm{p}^{\mathrm{N}-2} 3 \mathrm{~d}^{1}+2 \mathrm{p}^{\mathrm{N}-1} 3 \mathrm{p}^{1}
\end{gathered}
$$

$(2)^{N \cdot 1} 4^{1}$ being the same as $(2)^{N-1} 3^{1}$ with quantum number 4 instead of 3 .

The processing of full complexes is needed to take into account the most important configuration mixing effects. In Table 1, wavelengths, transition probabilities, and combining levels are reported for the prominent lines of the spectrum of carbon-like copper $\mathrm{Cu}$ XXIV which had not been reported earlier in the wavelength range 6.9-7.7 $\AA$.

Table 1: Theoretical and observed transitions between complexes of configurations $(1)^{2}(2)^{4}$ and $(1)^{2}(2)^{3} 4^{1}$ in carbon-like copper Cu XXIV.

\begin{tabular}{|c|c|c|c|c|c|c|c|}
\hline $\begin{array}{c}\lambda_{t h} \\
(\mathrm{~A})\end{array}$ & $\begin{array}{c}\mathrm{gA} \\
\left(10^{12} \mathrm{~s}^{-1}\right)\end{array}$ & $\begin{array}{c}\mathrm{E}_{t h}^{o} \\
\left(1000 \mathrm{~cm}^{-1}\right)\end{array}$ & $\mathrm{J}^{0}$ & $\begin{array}{c}\mathrm{E}_{t h}^{e} \\
\left(1000 \mathrm{~cm}^{-1}\right)\end{array}$ & $\begin{array}{c}\mathrm{J}^{e} \\
\lambda_{\text {exp }} \\
(\AA)\end{array}$ & $\begin{array}{c}\text { Int } \\
(\text { arb.units })\end{array}$ \\
\hline 6.9578 & 23.39 & 634.6 & 2.0 & 15007.0 & 3.0 & 6.959 & 5 \\
7.0341 & 30.60 & 1027.6 & 3.0 & 15243.9 & 4.0 & 7.031 & $30 \mathrm{Bl} \mathrm{XXV}$ \\
& & & & & & & \\
7.1099 & 23.76 & 14439.2 & 3.0 & 374.4 & 2.0 & 7.115 & $45 \mathrm{Bl} \mathrm{XXV}$ \\
7.1532 & 21.58 & 14169.5 & 2.0 & 189.7 & 2.0 & 7.148 & $30 \mathrm{Bl} \mathrm{XXV}$ \\
7.1533 & 27.16 & 14169.3 & 3.0 & 189.7 & 2.0 & & \\
& & & & & & & \\
7.1647 & 21.14 & 14086.0 & 2.0 & 128.6 & 1.0 & 7.166 & $35 \mathrm{Bl} \mathrm{XXV}$ \\
7.3333 & 21.58 & 964.9 & 1.0 & 14601.3 & 2.0 & 7.325 & $20 \mathrm{~W}$ \\
7.3346 & 20.24 & 973.5 & 2.0 & 14607.4 & 3.0 & & \\
7.3353 & 22.63 & 1170.0 & 1.0 & 14802.7 & 2.0 & 7.332 & $15 \mathrm{p}$ \\
& & & & & & & \\
7.3481 & 44.33 & 634.6 & 2.0 & 14243.5 & 1.0 & 7.349 & $25 \mathrm{p}$ \\
7.3501 & 53.83 & 634.6 & 2.0 & 14240.0 & 2.0 & & \\
7.3529 & 35.89 & 634.6 & 2.0 & 14234.6 & 3.0 & & \\
7.3571 & 33.06 & 13592.2 & 1.0 & 0.0 & 0.0 & 7.354 & $20 \mathrm{Bl}$ \\
7.3598 & 21.17 & 13777.1 & 2.0 & 189.7 & 2.0 & & \\
& & & & & & & \\
7.3630 & 21.52 & 973.5 & 2.0 & 14554.8 & 1.0 & 7.359 & $20 \mathrm{Bl}$ \\
7.3640 & 60.58 & 13769.2 & 3.0 & 189.7 & 2.0 & & \\
7.3673 & 28.14 & 973.5 & 2.0 & 14547.0 & 2.0 & & \\
& & & & & & & \\
7.3947 & 26.77 & 1027.6 & 3.0 & 14550.8 & 4.0 & 7.394 & 40 \\
7.3947 & 33.10 & 964.9 & 1.0 & 14488.1 & 2.0 & & \\
7.3954 & 25.10 & 973.5 & 2.0 & 14495.4 & 3.0 & & \\
7.3971 & 46.76 & 634.6 & 2.0 & 14153.4 & 3.0 & & \\
7.4000 & 58.38 & 1027.6 & 3.0 & 14541.1 & 3.0 & & \\
7.4032 & 63.46 & 1027.6 & 3.0 & 14535.3 & 4.0 & & \\
& & & & & & & \\
\hline
\end{tabular}




\begin{tabular}{|c|c|c|c|c|c|c|c|}
\hline (cont.) & & & & & & & \\
\hline 7.4299 & 37.31 & 13587.7 & 2.0 & 128.6 & 1.0 & 7.427 & 20 \\
\hline 7.4384 & 20.79 & 15605.8 & 1.0 & 2162.0 & 1.0 & & \\
\hline 7.4460 & 30.14 & 634.6 & 2.0 & 14064.7 & 3.0 & 7.446 & 30 \\
\hline 7.4499 & 116.48 & 13797.5 & 3.0 & 374.4 & 2.0 & & \\
\hline 7.4559 & 96.39 & 1422.9 & 2.0 & 14835.1 & 3.0 & 7.459 & 18 \\
\hline 7.4612 & 25.00 & 13777.1 & 2.0 & 374.4 & 2.0 & & \\
\hline 7.4632 & 51.52 & 13588.7 & 3.0 & 189.7 & 2.0 & & \\
\hline 7.4735 & 32.12 & 15512.8 & 1.0 & 2132.2 & 0.0 & 7.471 & 6 \\
\hline 7.4822 & 24.21 & 15621.5 & 2.0 & 2256.5 & 2.0 & & \\
\hline 7.4848 & 75.11 & 15617.0 & 3.0 & 2256.5 & 2.0 & 7.488 & 6 \\
\hline 7.4887 & 26.99 & 15362.0 & 1.0 & 2008.6 & 2.0 & & \\
\hline 7.4888 & 20.69 & 1204.1 & 2.0 & 14557.4 & 3.0 & & \\
\hline 7.4977 & 23.69 & 15499.5 & 2.0 & 2162.0 & 1.0 & & \\
\hline 7.4979 & 55.99 & 15345.6 & 2.0 & 2008.6 & 2.0 & 7.504 & 5 \\
\hline 7.4993 & 25.85 & 1170.0 & 1.0 & 14504.6 & 2.0 & & \\
\hline 7.5029 & 53.32 & 15336.8 & 3.0 & 2008.6 & 2.0 & & \\
\hline 7.5238 & 36.12 & 1204.1 & 2.0 & 14495.4 & 3.0 & 7.520 & 15 \\
\hline 7.5275 & 32.69 & 13800.9 & 1.0 & 516.2 & 0.0 & & \\
\hline 7.5343 & 32.56 & 15281.1 & 3.0 & 2008.6 & 2.0 & 7.53 & $\mathrm{Bl}$ \\
\hline 7.5365 & 76.01 & 1591.0 & 1.0 & 14859.8 & 2.0 & & \\
\hline 7.5403 & 29.83 & 1352.0 & 1.0 & 14614.1 & 2.0 & & \\
\hline 7.5488 & 36.41 & 1027.6 & 3.0 & 14274.6 & 3.0 & 7.540 & 25 \\
\hline 7.5503 & 104.59 & 1027.6 & 3.0 & 14272.1 & 4.0 & & \\
\hline 7.5505 & 25.45 & 15500.7 & 3.0 & 2256.5 & 2.0 & & \\
\hline 7.5517 & 23.66 & 1591.0 & 1.0 & 14833.0 & 1.0 & & \\
\hline 7.5662 & 79.44 & 973.5 & 2.0 & 14190.1 & 3.0 & 7.556 & 8 \\
\hline 7.6055 & 38.44 & 1422.9 & 2.0 & 14571.2 & 2.0 & 7.600 & $30 \mathrm{Bl}$ \\
\hline 7.6105 & 48.55 & 15301.9 & 2.0 & 2162.0 & 1.0 & & \\
\hline 7.6135 & 38.96 & 1422.9 & 2.0 & 14557.4 & 3.0 & 7.61 & $\mathrm{Bl}$ \\
\hline 7.6165 & 46.00 & 964.9 & 1.0 & 14094.3 & 2.0 & & \\
\hline 7.6207 & 74.37 & 15130.7 & 3.0 & 2008.6 & 2.0 & & \\
\hline 7.6253 & 91.18 & 15370.8 & 3.0 & 2256.5 & 2.0 & & \\
\hline 7.6281 & 60.89 & 15365.9 & 2.0 & 2256.5 & 2.0 & 7.62 & $\mathrm{Bl}$ \\
\hline 7.6308 & 59.03 & 15654.3 & 1.0 & 2549.6 & 0.0 & & \\
\hline 7.6508 & 33.89 & 1204.1 & 2.0 & 14274.6 & 3.0 & & \\
\hline 7.6528 & 26.94 & 1204.1 & 2.0 & 14271.3 & 2.0 & & \\
\hline 7.6900 & 20.91 & 15136.1 & 1.0 & 2132.2 & 0.0 & & \\
\hline
\end{tabular}



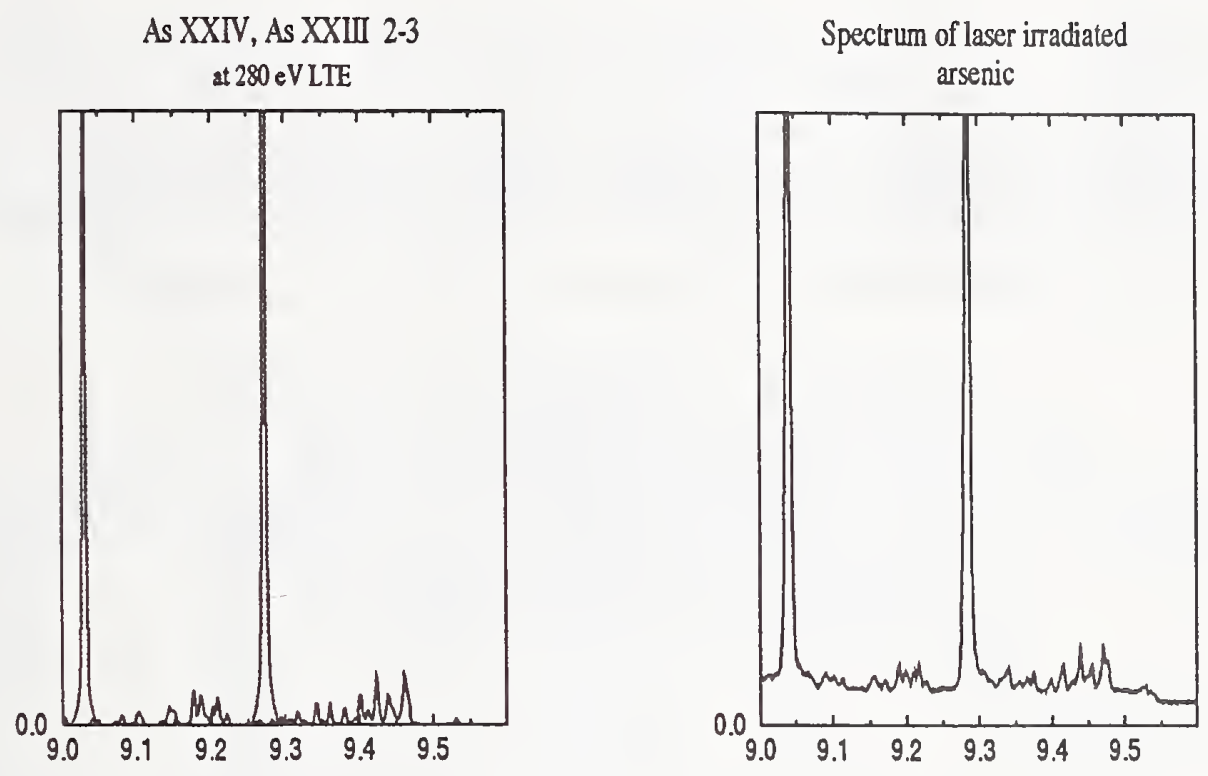

Figure 1: Comparison of observed spectrum of laser-irradiated arsenic (right) with theoretical 2-3 resonance transitions of Ne-like As XXIV and Na-like As XXIII satellites (left). The levels of As XXIII are assumed to be populated according to LTE at $280 \mathrm{eV}$ and not to decay via autoionization. The relative population of As XXIV and As XXIII is an adjustable parameter.

\section{Conclusion}

Owing to the narrow linewidths and the high signal-to-noise ratio displayed in Fig. 1, the present observations add valuable data to many earlier observations of medium-Z elements. The detailed analysis of these spectra should help in studying ionic charge distribution in laser-produced plasmas. A database with classified lines is under preparation. The ICAMDATA community will be informed when it is available.

\section{References}

[1] B. Edlen, Physica Scripta 28, 51 (1983); 28, 483 (1983); 30 ,135 (1984); 31, 345 (1985); 32, 86 (1985)

[2] R.L. Kelly, J. Phys. Chem. Ref. Data, 16, supp.1 (1987)

[3] M. Klapisch, A. Bar-Shalom and W.H. Goldstein, HULLAC computer codes, unpublished

[4] E. Koenig, Physica 62, 393 (1972); M.Klapisch, J.-L. Schwob, B.S. Fraenkel and J. Oreg, J. Opt. Soc. Am. 67, 148 (1977) 


\title{
Self-Consistent Sets of Oscillator Strength for Ultraviolet Lines in $\mathrm{C}$ I, S I, and Ni II
}

\author{
J. Zsargó*, S. R. Federman* and J. Cardelli ${ }^{\dagger}$ \\ *Department of Physics and Astronomy \\ University of Toledo, Toledo, $\mathrm{OH}$ \\ ${ }^{\dagger}$ Department of Astronomy and Astrophysics \\ Villanova University, Villanova, PA
}

\section{Introduction}

High quality spectra of interstellar absorption from C I, S I and Ni II toward several lines of sight $^{1}$ were obtained with the Goddard High Resolution Spectrograph on HST. A curve-of-growth analysis yields adjusted sets of oscillator strengths (Federman \& Cardelli 1995; Zsargó, Federman, \& Cardelli 1997; Zsargó \& Federman 1998) if the uncertainties are dominated by the errors in the atomic properties. Our GHRS spectra with signal-to-noise ratios greater than 100-200 were used to perform such an analysis. Our astronomically derived oscillator strengths were compared with available information in our papers noted above. Here comparisons with the compilations of Morton (1991) and Verner et al. (1996) are presented.

\section{Analysis}

We assumed simple Maxwellian line profiles to find the theoretical curves of growth, and the values for column densities and Doppler parameters were obtained by least-squares fit where the data for each line was weighted by the relative uncertainty in $W_{\lambda}\left[\sigma\left(W_{\lambda}\right) / W_{\lambda}\right]$ and in the $f$-value. The oscillator strengths for the lines of a given species then were adjusted to have the best overall fit to the corresponding curves of growth. For C I and S I, precise laboratory data for several multiplets allowed us to place the results on an absolute scale; such was not the case for Ni II.

\section{Discussion}

\subsection{Neutral Carbon}

As revealed in Table 1, our analysis yields significant revisions to $f$-values for 24 lines when comparison is made with previous work. Above $1200 \AA$ only forbidden lines needed significant adjustments to their oscillator strengths. This result is not surprising since most multiplets in this range had well defined $f$-values and most of the weak lines were forbidden. Below $1200 \AA$ few reliable measures of $f$-value exist. Therefore significant changes in multiplet oscillator strengths could be expected. Comparison with the compilation of Morton (1991) shows reasonable agreement (within $3 \sigma$ ) for about half the lines. Many of the differences involving dipole-allowed transitions arise because

\footnotetext{
${ }^{1} \beta^{1} \mathrm{Sco}, \rho$ Oph A, and $\chi$ Oph for C I; $\zeta$ Oph for S I; $\chi$ Oph, $\zeta$ Oph and $\rho$ Oph A for Ni II
} 
LS coupling may not apply. Our astronomical observations indicate that LS coupling is not applicable to the lines at $\lambda \lambda 1194,1156$, and possibly $\lambda 1189$. Verner et al. (1996) corrected the tabulated results from the Opacity Project by using laboratory wavelengths in converting $A$-values into $f$-values. Their $f$-values are similar to those listed by Morton (1991), except for $\lambda 1156$.

Table 1

Comparison of Oscillator Strengths for Lines of Neutral Carbon

\begin{tabular}{|c|c|c|c|c|}
\hline Wavelength $(\AA)$ & Transition & $f(\mathrm{ZsFC})^{a}$ & $f(\mathrm{M})^{b}$ & $f\left(V^{\prime} F\right)^{c}$ \\
\hline 1287.608 & $2 \mathrm{p}^{2}{ }^{3} \mathrm{P}_{1}-3 \mathrm{~d}^{1} \mathrm{D}_{2}^{\circ}$ & $1.66 \times 10^{-4}$ & $6.38 \times 10^{-5}$ & \\
\hline 1279.498 & $2 \mathrm{p}^{2}{ }^{3} \mathrm{P}_{2}-3 \mathrm{~d}^{3} \mathrm{~F}_{2}^{\mathrm{o}}$ & $9.03 \times 10^{-4}$ & $(1.98 \pm 0.50) \times 10^{-4}$ & $\ldots$ \\
\hline 1279.229 & $2 p^{2}{ }^{3} P_{2}-3 d^{3} F_{3}^{o}$ & $3.24 \times 10^{-3}$ & $(3.60 \pm 0.90) \times 10^{-3}$ & $\cdots$ \\
\hline 1279.056 & $2 \mathrm{p}^{2}{ }^{3} \mathrm{P}_{1}-3 \mathrm{~d}^{3} \mathrm{~F}_{2}^{\mathrm{o}}$ & $2.02 \times 10^{-3}$ & $(7.33 \pm 1.83) \times 10^{-4}$ & ... \\
\hline 1276.750 & $2 \mathrm{p}^{2}{ }^{3} \mathrm{P}_{1}-4 \mathrm{~s}^{1} \mathrm{P}_{1}^{\mathrm{o}}$ & $2.39 \times 10^{-3}$ & $2.87 \times 10^{-3}$ & $\cdots$ \\
\hline 1276.483 & $2 p^{2}{ }^{3} P_{0}-4 s^{1} P_{1}^{o}$ & $1.68 \times 10^{-3}$ & $4.50 \times 10^{-3}$ & \\
\hline 1274.109 & $2 \mathrm{p}^{2}{ }^{3} \mathrm{P}_{2}-3 \mathrm{~d}^{1} \mathrm{~F}_{3}^{\mathrm{o}}$ & $5.39 \times 10^{-4}$ & $4.90 \times 10^{-4}$ & \\
\hline 1270.408 & $2 \mathrm{p}^{2}{ }^{3} \mathrm{P}_{1}-3 \mathrm{~d}{ }^{1} \mathrm{P}_{1}^{\mathrm{o}}$ & $2.06 \times 10^{-4}$ & $6.54 \times 10^{-5}$ & \\
\hline 1270.143 & $2 \mathrm{p}^{2}{ }^{3} \mathrm{P}_{0}-3 \mathrm{~d}^{1} \mathrm{P}_{1}^{\mathrm{o}}$ & $4.28 \times 10^{-4}$ & $3.88 \times 10^{-4}$ & \\
\hline 1193.996 & $2 \mathrm{p}^{2}{ }^{3} \mathrm{P}_{0}-5 \mathrm{~s}{ }^{3} \mathrm{P}_{1}^{0}$ & $7.50 \times 10^{-3}$ & $(9.41 \pm 0.81) \times 10^{-3}$ & $1.05 \times 10^{-2}$ \\
\hline 1193.679 & $2 \mathrm{p}^{2}{ }^{3} \mathrm{P}_{1}-5 \mathrm{~s}^{3} \mathrm{P}_{2}^{\mathrm{o}}$ & $9.00 \times 10^{-3}$ & $(3.92 \pm 0.34) \times 10^{-3}$ & $4.40 \times 10^{-3}$ \\
\hline $1193.031^{d}$ & $2 \mathrm{p}^{2}{ }^{3} \mathrm{P}_{0}-4 \mathrm{~d}^{3} \mathrm{D}_{1}^{\mathrm{o}}$ & $6.23 \times 10^{-2}$ & $4.45 \times 10^{-2}$ & $4.76 \times 10^{-2}$ \\
\hline $1193.009^{d}$ & $2 \mathrm{p}^{2}{ }^{3} \mathrm{P}_{1}-4 \mathrm{~d}^{3} \mathrm{D}_{2}^{\mathrm{o}}$ & $4.68 \times 10^{-2}$ & $3.34 \times 10^{-2}$ & $3.56 \times 10^{-2}$ \\
\hline 1192.218 & $2 \mathrm{p}^{2}{ }^{3} \mathrm{P}_{0}-5 \mathrm{~s}{ }^{1} \mathrm{P}_{1}^{\mathrm{o}}$ & $8.77 \times 10^{-4}$ & $(2.63 \pm 0.51) \times 10^{-3}$ & 年 \\
\hline 1189.631 & $2 \mathrm{p}^{2}{ }^{3} \mathrm{P}_{2}-4 \mathrm{~d}^{3} \mathrm{P}_{2}^{\mathrm{o}}$ & $1.38 \times 10^{-2}$ & $(1.26 \pm 0.11) \times 10^{-2}$ & $9.69 \times 10^{-3}$ \\
\hline 1189.447 & $2 \mathrm{p}^{2}{ }^{3} \mathrm{P}_{2}-4 \mathrm{~d}^{3} \mathrm{P}_{1}^{\mathrm{o}}$ & $4.59 \times 10^{-3}$ & $(4.19 \pm 0.36) \times 10^{-3}$ & $3.23 \times 10^{-3}$ \\
\hline 1158.324 & $2 \mathrm{p}^{2}{ }^{3} \mathrm{P}_{0}-6 \mathrm{~s}^{3} \mathrm{P}_{1}^{0}$ & $5.83 \times 10^{-3}$ & $(3.42 \pm 0.30) \times 10^{-3}$ & $5.57 \times 10^{-3}$ \\
\hline $1158.130^{d}$ & $2 \mathrm{p}^{2}{ }^{3} \mathrm{P}_{1}-5 \mathrm{~d}^{3} \mathrm{D}_{1}^{\mathrm{o}}$ & $1.90 \times 10^{-3}$ & $(5.44 \pm 0.47) \times 10^{-3}$ & $6.10 \times 10^{-3}$ \\
\hline $1158.019^{d}$ & $2 \mathrm{p}^{2}{ }^{3} \mathrm{P}_{2}-5 \mathrm{~d}^{3} \mathrm{D}_{3}^{\circ}$ & $6.41 \times 10^{-3}$ & $(1.83 \pm 0.16) \times 10^{-2}$ & $2.05 \times 10^{-2}$ \\
\hline $1157.910^{d}$ & $2 \mathrm{p}^{2}{ }^{3} \mathrm{P}_{0}-5 \mathrm{~d}^{3} \mathrm{D}_{1}^{\circ}$ & $7.63 \times 10^{-3}$ & $(2.18 \pm 0.19) \times 10^{-2}$ & $2.44 \times 10^{-2}$ \\
\hline $1157.770^{d}$ & $2 \mathrm{p}^{2}{ }^{3} \mathrm{P}_{1}-5 \mathrm{~d}{ }^{3} \mathrm{D}_{2}^{\circ}$ & $5.71 \times 10^{-3}$ & $(1.63 \pm 0.14) \times 10^{-2}$ & $1.83 \times 10^{-2}$ \\
\hline 1156.028 & $2 \mathrm{p}^{2}{ }^{3} \mathrm{P}_{1}-5 \mathrm{~d}{ }^{3} \mathrm{P}_{1}^{\circ}$ & $3.45 \times 10^{-3}$ & $(4.31 \pm 0.37) \times 10^{-3}$ & $1.52 \times 10^{-3}$ \\
\hline 1155.979 & $2 \mathrm{p}^{2}{ }^{3} \mathrm{P}_{1}-5 \mathrm{~d}{ }^{3} \mathrm{P}_{0}^{\circ}$ & $4.60 \times 10^{-3}$ & $(5.75 \pm 0.50) \times 10^{-3}$ & $2.01 \times 10^{-3}$ \\
\hline 1155.809 & $2 \mathrm{p}^{2}{ }^{3} \mathrm{P}_{0}-5 \mathrm{~d}{ }^{3} \mathrm{P}_{1}^{\circ}$ & $3.04 \times 10^{-3}$ & $(1.73 \pm 0.15) \times 10^{-2}$ & $6.05 \times 10^{-3}$ \\
\hline
\end{tabular}

Notes:

${ }^{a}$ Our values (Zsargó et al. 1997). Typical uncertainties range from $10 \%$ to $20 \%$; the weakest lines have the greatest uncertainty.

${ }^{b}$ Morton (1991) compilation.

$c$ Verner et al. (1996).

${ }^{d}$ LS coupling seems to apply.

\subsection{Neutral Sulfur}

The comparison for the $25 \mathrm{~S}$ I lines appears in Table 2. Unlike the situation for C I, LS coupling adequately describes line strengths within a multiplet. In about $1 / 3$ of the cases, substantial differences exist with the values quoted by Morton (1991). There is good agreement with most of 
the results of Verner et al. (1996), except for several multiplets with ${ }^{3} \mathrm{D}^{\circ}$ upper states $(\lambda \lambda 1479$, $1274,1256)$.

Table 2

Comparison of Oscillator Strengths for Lines of Neutral Sulfur

\begin{tabular}{|c|c|c|c|c|}
\hline Wavelength $(\AA)$ & Transition & $f(\mathrm{FC})^{a}$ & $f(\mathrm{M})^{b}$ & $f(\mathrm{VVF})^{c}$ \\
\hline 1807.311 & $3 p^{4}{ }^{3} \mathrm{P}_{2}-4 \mathrm{~s}^{3} \mathrm{~S}_{1}^{\mathrm{o}}$ & $9.6 \times 10^{-2} d$ & $(1.11 \pm 0.10) \times 10^{-1}$ & $8.45 \times 10^{-2}$ \\
\hline 1474.571 & $3 p^{4}{ }^{3} \mathrm{P}_{2}-4 \mathrm{~s}^{\prime}{ }^{3} \mathrm{D}_{1}^{\circ}$ & $1.33 \times 10^{-3}$ & $(1.21 \pm 0.24) \times 10^{-3}$ & $4.09 \times 10^{-4}$ \\
\hline 1474.379 & $3 p^{4}{ }^{3} P_{2}-4 s^{\prime}{ }^{3} D_{2}^{o}$ & $1.79 \times 10^{-2}$ & $(1.63 \pm 0.32) \times 10^{-2}$ & $6.14 \times 10^{-3}$ \\
\hline 1473.994 & $3 p^{4}{ }^{3} P_{2}-4 s^{\prime}{ }^{3} D_{3}^{o}$ & $8.03 \times 10^{-2}$ & $(7.30 \pm 1.42) \times 10^{-2}$ & $3.44 \times 10^{-2}$ \\
\hline 1472.971 & $3 p^{4}{ }^{3} \mathrm{P}_{2}-3 \mathrm{~d}^{5} \mathrm{D}_{3}^{\circ}$ & $2.18 \times 10^{-2}$ & $(1.91 \pm 0.37) \times 10^{-2}$ & $\ldots$ \\
\hline 1444.296 & $3 \mathrm{p}^{4}{ }^{3} \mathrm{P}_{2}-4 \mathrm{~s}^{\prime}{ }^{1} \mathrm{D}_{2}^{\mathrm{o}}$ & $1.24 \times 10^{-3}$ & $(8.13 \pm 1.59) \times 10^{-4}$ & $\ldots$ \\
\hline 1425.219 & $3 p^{4}{ }^{3} P_{2}-3 d^{3} D_{1}^{\circ}$ & $1.75 \times 10^{-3}$ & $(2.38 \pm 0.46) \times 10^{-3}$ & $1.44 \times 10^{-3}$ \\
\hline 1425.188 & $3 p^{4}{ }^{3} P_{2}-3 d^{3} D_{2}^{\circ}$ & $2.69 \times 10^{-2}$ & $(3.65 \pm 0.71) \times 10^{-2}$ & $2.16 \times 10^{-2}$ \\
\hline 1425.030 & $3 p^{4}{ }^{3} \mathrm{P}_{2}-3 \mathrm{~d}^{3} \mathrm{D}_{3}^{\mathrm{o}}$ & $1.41 \times 10^{-1}$ & $(1.92 \pm 0.37) \times 10^{-1}$ & $1.21 \times 10^{-1}$ \\
\hline 1401.514 & $3 p^{4}{ }^{3} P_{2}-5 s^{3} S_{1}^{o}$ & $1.49 \times 10^{-2}$ & $(1.61 \pm 0.31) \times 10^{-2}$ & $1.42 \times 10^{-2}$ \\
\hline 1316.622 & $3 \mathrm{p}^{4}{ }^{3} \mathrm{P}_{2}-4 \mathrm{~d}^{3} \mathrm{D}_{1}^{\circ}$ & $4.54 \times 10^{-4}$ & $(4.11 \pm 0.80) \times 10^{-4}$ & $2.69 \times 10^{-4}$ \\
\hline 1316.615 & $3 \mathrm{p}^{4}{ }^{3} \mathrm{P}_{2}-4 \mathrm{~d}^{3} \mathrm{D}_{2}^{\circ}$ & $6.79 \times 10^{-3}$ & $(6.15 \pm 1.20) \times 10^{-3}$ & $4.03 \times 10^{-3}$ \\
\hline 1316.543 & $3 p^{4}{ }^{3} P_{2}-4 d^{3} D_{3}^{o}$ & $3.81 \times 10^{-2}$ & $(3.45 \pm 0.67) \times 10^{-2}$ & $2.26 \times 10^{-2}$ \\
\hline 1303.430 & $3 p^{4}{ }^{3} P_{2}-6 s^{3} S_{1}^{o}$ & $5.06 \times 10^{-3}$ & $(2.91 \pm 0.57) \times 10^{-2}$ & $4.60 \times 10^{-3}$ \\
\hline 1296.174 & $3 \mathrm{p}^{4}{ }^{3} \mathrm{P}_{2}-4 \mathrm{~s}^{\prime \prime}{ }^{3} \mathrm{P}_{1}^{0}$ & $2.2 \times 10^{-2} d$ & $(4.08 \pm 0.80) \times 10^{-2}$ & $2.78 \times 10^{-2}$ \\
\hline 1295.653 & $3 \mathrm{p}^{4}{ }^{3} \mathrm{P}_{2}-4 \mathrm{~s}^{\prime \prime}{ }^{3} \mathrm{P}_{2}^{\mathrm{o}}$ & $8.7 \times 10^{-2} d$ & $(1.23 \pm 0.24) \times 10^{-1}$ & $8.36 \times 10^{-2}$ \\
\hline 1270.787 & $3 \mathrm{p}^{4}{ }^{3} \mathrm{P}_{2}-5 \mathrm{~d}^{3} \mathrm{D}_{2}^{\circ}$ & $1.64 \times 10^{-3}$ & $1.00 \times 10^{-2}$ & $6.21 \times 10^{-4}$ \\
\hline 1270.780 & $3 \mathrm{p}^{4}{ }^{3} \mathrm{P}_{2}-5 \mathrm{~d}^{3} \mathrm{D}_{3}^{\circ}$ & $9.02 \times 10^{-3}$ & $5.51 \times 10^{-2}$ & $3.47 \times 10^{-3}$ \\
\hline 1270.769 & $3 p^{4}{ }^{3} P_{2}-5 d^{3} D_{1}^{\circ}$ & $1.09 \times 10^{-4}$ & $6.62 \times 10^{-4}$ & $4.14 \times 10^{-5}$ \\
\hline 1262.860 & $3 p^{4}{ }^{3} P_{2}-7 s^{3} S_{1}^{o}$ & $2.59 \times 10^{-3}$ & & $2.00 \times 10^{-3}$ \\
\hline 1247.160 & $3 p^{4}{ }^{3} P_{2}-6 d^{3} D_{3}^{\circ}$ & $1.64 \times 10^{-3}$ & $3.24 \times 10^{-2}$ & $7.44 \times 10^{-5}$ \\
\hline 1247.134 & $3 \mathrm{p}^{4}{ }^{3} \mathrm{P}_{2}-6 \mathrm{~d}^{3} \mathrm{D}_{2}^{0}$ & $2.90 \times 10^{-4}$ & $5.77 \times 10^{-3}$ & $1.33 \times 10^{-5}$ \\
\hline 1247.107 & $3 p^{4}{ }^{3} P_{2}-6 d^{3} D_{1}^{o}$ & $1.92 \times 10^{-5}$ & $3.81 \times 10^{-4}$ & $8.86 \times 10^{-7}$ \\
\hline 1241.905 & $3 p^{4}{ }^{3} P_{2}-8 s^{3} S_{1}^{0}$ & $1.06 \times 10^{-3}$ & & $1.02 \times 10^{-3}$ \\
\hline 1224.544 & $3 p^{4}{ }^{3} P_{2}-8 d^{3} D_{3}^{o}$ & $\leq 9.6 \times 10^{-4}$ & $1.23 \times 10^{-2}$ & $1.07 \times 10^{-3}$ \\
\hline
\end{tabular}

Notes:

${ }^{a}$ Our values (Federman \& Cardelli 1995). Typical uncertainties range from $10 \%$ to $20 \%$; the weakest lines have the greatest uncertainty.

${ }^{b}$ Morton (1991) compilation.

${ }^{c}$ Verner et al. (1996).

${ }^{d}$ Experimental results of Beideck et al. (1994).

\subsection{Singly-ionized Nickel}

Table 3 shows the adjusted oscillator strengths together with Morton's (1991) values. The adjusted and the original $f$-values differ by less than $30 \%$ in most cases, implying that the theoretical calculations of Kurucz (1989) are reliable (in a relative sense). There are several lines with considerable differences, those for $\lambda \lambda 1477,1415$ and 1345. Since we measured these lines in only one direction, 
higher than average uncertainties could arise. These three lines are among the weakest observed by us, and it is not unreasonable to find the largest discrepancies here between our results and the predictions of Kurucz (1989) from intermediate coupling calculations. Work is in progress to place these results on an absolute scale.

Table 3

Comparison of Oscillator Strengths for Lines of Singly-ionized Nickel

\begin{tabular}{llll}
\hline \hline Wavelength $(\AA)$ & Transition & $f(\text { ZsF })^{a}$ & $f(\mathrm{M})^{b}$ \\
\hline 1741.549 & $3 \mathrm{~d}^{9}{ }^{2} \mathrm{D}_{5 / 2}-4 \mathrm{p}^{2} \mathrm{D}_{5 / 2}^{\circ}$ & $7.76 \times 10^{-2}$ & $1.04 \times 10^{-1}$ \\
1709.600 & $3 \mathrm{~d}^{9}{ }^{2} \mathrm{D}_{5 / 2}-4 \mathrm{p}^{2} \mathrm{~F}_{5 / 2}^{\circ}$ & $6.66 \times 10^{-2}$ & $6.88 \times 10^{-2}$ \\
1477.222 & $3 \mathrm{~d}^{9}{ }^{2} \mathrm{D}_{5 / 2}-4 \mathrm{p}^{\prime \prime}{ }^{2} \mathrm{~F}_{5 / 2}^{\circ}$ & $1.82 \times 10^{-3}$ & $1.06 \times 10^{-3}$ \\
1467.756 & $3 \mathrm{~d}^{9}{ }^{2} \mathrm{D}_{5 / 2}-4 \mathrm{p}^{\prime \prime}{ }^{2} \mathrm{~F}_{7 / 2}^{\circ}$ & $1.81 \times 10^{-2}$ & $2.27 \times 10^{-2}$ \\
1467.259 & $3 \mathrm{~d}^{9}{ }^{2} \mathrm{D}_{5 / 2}-4 \mathrm{p}^{\prime \prime}{ }^{2} \mathrm{D}_{3 / 2}^{\circ}$ & $1.13 \times 10^{-2}$ & $1.11 \times 10^{-2}$ \\
1454.842 & $3 \mathrm{~d}^{9}{ }^{2} \mathrm{D}_{5 / 2}-4 \mathrm{p}^{\prime \prime}{ }^{2} \mathrm{D}_{5 / 2}^{\circ}$ & $5.16 \times 10^{-2}$ & $5.95 \times 10^{-2}$ \\
1449.997 & $3 \mathrm{~d}^{9}{ }^{2} \mathrm{D}_{5 / 2}-4 \mathrm{p}^{\prime \prime}{ }^{2} \mathrm{P}_{3 / 2}^{\circ}$ & $3.27 \times 10^{-3}$ & $3.53 \times 10^{-3}$ \\
1415.720 & $3 \mathrm{~d}^{9}{ }^{2} \mathrm{D}_{5 / 2}-4 \mathrm{p}^{\prime}{ }^{4} \mathrm{D}_{5 / 2}^{\circ}$ & $5.97 \times 10^{-3}$ & $4.13 \times 10^{-3}$ \\
1412.886 & $3 \mathrm{~d}^{9}{ }^{2} \mathrm{D}_{5 / 2}-4 \mathrm{p}^{\prime}{ }^{4} \mathrm{D}_{7 / 2}^{\circ}$ & $6.65 \times 10^{-3}$ & $6.65 \times 10^{-3}$ \\
1393.324 & $3 \mathrm{~d}^{9}{ }^{2} \mathrm{D}_{5 / 2}-4 \mathrm{p}^{\prime}{ }^{2} \mathrm{D}_{5 / 2}^{\circ}$ & $1.89 \times 10^{-2}$ & $2.22 \times 10^{-2}$ \\
1370.132 & $3 \mathrm{~d}^{9}{ }^{2} \mathrm{D}_{5 / 2}-4 \mathrm{p}^{\prime}{ }^{2} \mathrm{P}_{3 / 2}^{\circ}$ & $1.44 \times 10^{-1}$ & $1.31 \times 10^{-1}$ \\
1345.878 & $3 \mathrm{~d}^{9}{ }^{2} \mathrm{D}_{5 / 2}-4 \mathrm{p}^{\prime}{ }^{4} \mathrm{~S}_{3 / 2}^{o}$ & $1.44 \times 10^{-2}$ & $6.43 \times 10^{-3}$ \\
\hline
\end{tabular}

Notes:

${ }^{a}$ Our values (Zsargó and Federman 1998). Typical uncertainties 30\%.

${ }^{b}$ Morton (1991) compilation.

\section{References}

[1] D. J. Beideck, R. M. Schectman, S. R. Federman, and D. G. Ellis, ApJ 428, 393 (1994)

[2] S. R. Federman, and J. A. Cardelli, ApJ 452, 269 (1995)

[3] R. L. Kurucz (1989) - see Morton (1991)

[4] D. C. Morton, ApJS 77, 119 (1991)

[5] J. Zsargó, and S. R. Federman, $A p J$, in press (1998)

[6] J. Zsargó, S. R. Federman, and J. A. Cardelli, ApJ 484, 820 (1997)

[7] D. A. Verner, E. M. Verner, and G. J. Ferland, Atomic Data Nucl. Data Tables 64, 1 (1996) 

$3 \mid \begin{aligned} & \text { Data } \\ & \text { Production }\end{aligned}$

3.2 Collisions 



\title{
Electron Impact Ionization and Surface Induced Reactions of Fusion Plasma Edge Constituents
}

\author{
K.Becker*, F.Biasioli, G.Denifl, H.Deutsch**, T.Fiegele, V.Grill, T.D.Märk, C.Mair, \\ S.Matt, D.Muigg, P.Scheier, M.Sonderegger, A.Stamatovic, R.Wörgötter \\ Institut für Ionenphysik, Technikerstr.25, A-6020 Innsbruck, Austria \\ *Dept.Physics, Stevens Institute of Technology, Hoboken, NJ 07030, USA \\ ${ }^{* *}$ Fachbereich Physik, Universität Greifswald, D-17487 Greifswald, Germany
}

\section{Introduction}

Recent studies in the field of thermonuclear fusion based on the magnetic confinement of high temperature plasmas have demonstrated that the conditions at the plasma periphery ("plasma edge") play an important role for achieving, sustaining and controlling the thermonuclear fusion plasma [1]. In order to understand and elucidate the role of the radiative and collisional processes in the plasma edge region, in particular their influence on the plasma properties and dynamics and their use for controlling the plasma conditions, it is essential to have available a detailed and quantitative knowledge on these elementary processes such as cross sections, reaction rate coefficients etc. Because of the relatively low temperature in the plasma edge the plasma contains - besides electrons and atomic ions - also a significant number of neutral hydrogen atoms, lowcharged atomic and molecular impurities (produced and introduced for instance via plasma/wall interactions [2], via diagnostics or via cooling). According to Janev [1] the most important collision processes (from a standpoint of their effects on plasma edge properties and behavior such as ionization balance, plasma energy, plasma transport etc.) are electron impact excitation/ionization reactions with plasma edge atoms, ions and molecules [3-5]. The impurities present in such an edge plasma obviously will depend on the materials used for the plasma facing components (first wall, protective tiles, divertor plates, antennas for $\mathrm{rf}$ heating etc.) and on the gases introduced for cooling and diagnostic purposes. Janev [1] has given a list of atomic and molecular impurities to be considered in such studies including the rare gases, $\mathrm{Li}, \mathrm{Be}, \mathrm{B}, \mathrm{C}, \mathrm{Al}, \mathrm{Si}, \mathrm{Mg}, \mathrm{Ti}, \mathrm{Cr}, \mathrm{Fe}, \mathrm{Ni}, \mathrm{Cu}$, $\mathrm{Mo}, \mathrm{Nb}, \mathrm{Ta}, \mathrm{W}$, and $\mathrm{CO}, \mathrm{CO}_{2}, \mathrm{CH}_{4}, \mathrm{C}_{2} \mathrm{H}_{2}, \mathrm{C}_{\mathrm{n}} \mathrm{H}_{\mathrm{m}}, \mathrm{H}_{2} \mathrm{O}$. Some of these impurities may reach levels of about 1 to several percent of the plasma density.

Whereas electron impact ionization of neutral and ionized atomic targets is a well studied and documented subject $[3,4]$ (save for a few targets and for some cases involving multiple and stepwise ionization ), the situation in case of molecular targets is far from satisfying. According to Tawara [5], who reviewed electron impact ionization of hydrocarbon molecules many data (concerning total and partial ionization cross sections) appear to be of relatively low accuracy or have not yet been measured. The latter applies in particular to hydrocarbon molecular ions, where almost no experimental (and theoretical) studies exist (see also Ref [6]). Moreover, the subject of electron impact induced ionization of molecular ions has so far received very little attention (in contrast to the field of electron impact ionization of atomic ions [7]). A similar situation exists for the area of plasma/wall interactions [2], where an understanding of the individual "surface erosion processes" for the various target materials, in particular under typical reactor edge plasma conditions, is a crucial prerequisite for designing the thermonuclear fusion reactor. 
The present long term data study is dedicated to provide this quantitative information for the various plasma edge constituents including, (i) experimental and theoretical studies about the ionization of neutrals and ions, and (ii) reactive interaction of molecular ions with surfaces. The present studies constitute on the one hand a continuation of previous research in our laboratories (i.e., on the experimental determination of accurate ionization cross sections for neutral molecules and on theoretical concepts for the prediction of ionization cross sections for various targets, i.e., atoms, molecules and clusters [8]) and on the other hand an extension of recently started experiments on the inelastic interaction of electrons with molecular ions [9] and on reactive scattering of molecular ions on surfaces. These previous studies (and the available techniques) and also these preliminary and exploratory studies will be summarized and based on this background an outline of the present research will be given.

\section{Electron Impact Ionization of Neutral Molecules: Experimental Determination of Cross Section, Kinetic Energy Release and Ionization Energy}

A detailed description of the apparatus and the experimental procedure used in our laboratory for the accurate measurement of partial ionization cross sections of atoms and of molecules has been given previously [10]. Briefly, the experimental set-up consists of a modified Nier-type electron impact ion source, a molecular beam source (either a Knudsen-type oven or a nozzle expansion source) and a high resolution double focussing Nier-Johnson sector field mass spectrometer. The performance and operating conditions of this apparatus have been continuously improved over the past 15 years. Today it is possible to measure (absolute) partial ionization cross section functions for atomic and molecular parent ions as well as partial ionization cross section functions for fragment ions formed with excess kinetic energy with high accuracy up to electron energies of $1000 \mathrm{eV}$ [10]. This was tested recently in detail with $\mathrm{CF}_{4}$ [10] and used recently for the study of $\mathrm{C}_{2} \mathrm{H}_{6}[11], \mathrm{C}_{3} \mathrm{H}_{8}$ [12] and $\mathrm{C}_{60}$ [13].

As shown and discussed in [10] it is necessary (in order to account quantitatively for discrimination effects in the case of fragment ions produced with excess kinetic energy in the ion source) and possible to measure the kinetic energy release distributions (KERD) of the fragment ions formed by electron impact ionization in the ion source using a method developed over the years in our laboratory. This ion beam deflection method is based on the fact that the extracted ion beam shape contains information on the original ion kinetic distribution [10-12]. Detailed studies about KERDs and the average total kinetic energy release (KER) deduced from these distributions have been performed recently for $\mathrm{C}_{3} \mathrm{H}_{8}$ [14] and $\mathrm{C}_{60}$ [15] and are presently also carried out employing a new experimental method using the apparatus described in section 5 .

Moreover, using monochromatized electrons in a newly constructed high resolution ion source/mass spectrometer apparatus we were able to (i) measure in great detail the ionization threshold region and thus to (ii) deduce ionization energies; first examples of this include ionization energies of singly-and multiply-charged parent [16] and fragment [17] fullerene ions and ionization energies of $\mathrm{CO}$ clusters [18]. 


\section{Electron Impact Ionization/Dissociation of Mass Selected Molecular Ions}

In order to study electron induced ionization and dissociation of mass selected molecular ions, we have recently modified [19] our two-sector-field mass spectrometer system in such a way as to allow with help of a newly constructed high performance electrun gun the study of inelastic interactions between electrons and ions in the ion beam focus of the second field free region (half way between the magnetic and the electrostatic field). Ions passing this second field free region have already been mass selected by the magnetic sector field. Because ions are produced in this set-up by an ordinary electron-impact ionization Nier type ion source, the primary ion currents available after mass selection are in the order of about $10 \mathrm{pA}$. With the present electron gun (with electron currents of about $10 \mathrm{~mA}$ ) mounted between the two sectors it became possible to investigate electron induced ionization and fragmentation of mass selected ions. In particular we used the MIKE-scan (mass analysed ion kinetic energy scan) technique [20] (which can be used also to study the spontaneous decay of molecular ions in the metstable time regime) in order to identify and analyse fragment ions in terms of their mass and charge state and their kinetic energy release. The position of the respective MIKE peaks allows an unambiguous identification of the parent and fragment ion peaks produced and from the shape of a MIKE peak it is possible to derive the total kinetic energy release distribution (KERD) of a specific decay reaction. In a further step the average total kinetic energy release (KER value) of the decay reaction can be determined by calculating the first momentum of the KERD. So far we have used this technique to study the properties of singly-and multiply-charged fullerene ions [19].

\section{Calculation of Ionization Cross Sections for Multiple Ionization of Atoms and Atomic Ions}

Scaling laws and semiempirical methods can be powerful tools to predict electron impact ionization cross sections for neutrals and ions for modelling purposes in particular for those targets where experimental data do not exist and/or rigorous theoretical calculations are inconvenient or impossible. Many practical applications require either a quick estimate of a large number of ionization cross sections or ionization cross section functions in analytical form [21]. Such needs can often be satisfied best (i.e., most economically and least time consuming to the practitioner) through the use of scaling laws and other simple methods. Because classical, semiclassical and semi-empirical formulae fail in certain even simple cases [22], we have suggested in 1987 [23] the use of a semi-classical approach based on a combination of the binary encounter approximation and the Born-Bethe approximation. Although originally devised for the single ionization of ground state atoms $[22,23]$, this concept has been successfully extended to the single ionization of excited atoms, free radicals, molecules, clusters and fullerenes and atomic ions and to the ionization of specific shells of atoms (for more details see accompanying paper in this conference). Very recently, we have also started to apply this concept to the case of multiple ionization of atoms, so far including double and triple ionization [24] and quadruple and quintuple ionization [25], and very recently even to charge states higher than 5 of neutral atoms [26]. 


\section{$5 \quad$ Reactive Interactions of Molecular Ions with Surfaces}

Recently we have constructed in our laboratory a new tandem mass spectrometer [27] consisting of an ion source, a two sector field mass spectrometer, deceleration ion optics, a surface collision chamber and a time of flight mass spectrometer. This apparatus allows us to study the various surface induced reactions such as surface induced dissociation, ion surface reactions and chemical sputtering as a function of collision energy (from threshold up to about $3 \mathrm{kV}$ ). Thus we are able to obtain information about the mechanisms, the kinetics and the energetics of the reactions occurring. After first tests we have so far studied reactions of acetone and benzene ions and their respective cluster ions [27-30], fullerene ions and various other fusion relevant ions with hydrocarbon covered stainless steel surfaces.

This work was supported by OEAW/EURATOM, FWF, OENB, BMWFK, Wien, Austria and, in part by the Division of Chemical Sciences, Office of Basic Energy Science, Office of Energy Research, US Department of Energy and the US National Science Foundation.

\section{References}

[1] R. K. Janev, Ed., "Atomic and Molecular Processes in Fusion Edge Plasmas", Plenum, New York 1995

[2] W. O. Hofer and J. Roth, Eds., "Physical Processes of the Interaction of Fusion Plasmas with Solids", Academic Press, San Diego 1996

[3] T. D. Märk, Chapter 4 in Ref. [1], p.59

[4] P. Defrance, M. Duponchelle, and D. L. Moores, Chapter 7 in Ref. [1], p.153

[5] H. Tawara, Chapter 16 in Ref. [1], p.461

[6] D. Gregory and H. Tawara, "Book of Abstracts, 15th ICPEAC", New York 1989, p.352

[7] G. H. Dunn, Chapter 8 in "Electron Impact Ionization", T. D. Märk and G. H. Dunn, Eds., Springer, Wien 1985, p.277

[8] T. D. Märk, Plasma Phys. and Controlled Fusion 34, 2083 (1992)

[9] T. Rauth, O. Echt, P. Scheier, and T. D. Märk, Chem. Phys. Lett. 247, 515 (1995)

[10] H. U. Poll, C. Winkler, D. Margreiter, V. Grill, and T. D. Märk, Int.J.Mass Spectrom. Ion Proc. 11, 1 (1992)

[11] V. Grill, G. Walder, P. Scheier, M. Kurdel, and T. D. Märk, Int.J.Mass Spectrom.Ion Proc. 129, 31 (1993)

[12] V. Grill, G. Walder, D. Margreiter, T. Rauth, H. U. Poll, P. Scheier, and T. D. Märk, Z. Phys. D 25, 217 (1993)

[13] S. Matt, B. Dünser, M. Lezius, H. Deutsch, K. Becker, A. Stamatovic, P. Scheier, and T. D. Märk, J.Chem.Phys. 105, 1880 (1996)

[14] V. Grill, S. Matt, H. U. Poll, N. Abramzon, K. Becker, P. Scheier, and T. D. Märk, Int.J.Mass Spectrom.Ion Proc., submitted (1997)

[15] D. Muigg, G. Denifl, P. Scheier, K. Becker, and T. D. Märk, J.Chem.Phys., in print (1997)

[16] D. Muigg, P. Scheier, K. Becker, and T. D. Märk, J.Phys. B29, 5193 (1996)

[17] S. Matt, O. Echt, R. Wörgötter, V. Grill, P. Scheier, C. Lifshitz, and T. D. Märk, Chem.Phys.Lett. 264, 149 (1997)

[18] G. Denifl, D. Muigg, A. Stamatovic, and T. D. Märk, to be published (1998) 
[19] S. Matt, O. Echt, T. Rauth, B. Dünser, M. Lezius, A. Stamatovic, P. Scheier, and T. D. Märk, Z.Phys. D40, 389 (1997)

[20] R. G. Cooks, J. H. Beynon, R. M. Caprioli, and G. R. Lester, "Metsatble Ions", Elsevier, Amsterdam 1973

[21] S. M. Younger and T. D. Märk, Chapter 2 in "Electron Impact Ionization", T. D. Märk and G. H. Dunn, Eds., Springer 1985, p.24

[22] D. Margreiter, H. Deutsch, and T. D. Märk, Int.J.Mass Spectrom.Ion Proc. 139, 127 (1994)

[23] H. Deutsch and T. D. Märk, Int.J.Mass Spectrom.Ion Proc. 79, R1 (1987)

[24] H. Deutsch, K. Becker, and T. D. Märk, Contr.Plasma Phys. 35, 421 (1995)

[25] H. Deutsch, K. Becker, and T. D. Märk, J.Phys. B29, L497(1996)

[26] H. Deutsch, K. Becker and D. P. Almeida, and T. D. Märk, Int.J.Mass Spectrom.Ion Proc., in press (1997)

[27] V. Grill, R. Wörgötter, J. H. Futrell, T. D. Märk, Z.Phys. D, in press (1997)

[28] R. Wörgötter, V. Grill, Z. Herman, H. Schwarz, and T. D. Märk, Chem.Phys.Lett. 270, 333 (1997)

[29] R. Wörgötter, C. Mair, T. Fiegele, V. Grill, T. D. Märk, and H. Schwarz, Int.J.Mass Spectrom.Ion Proc. 164, L1(1997)

[30] R. Wörgötter, J. Kubista, J. Zabka, Z. Dolejsek, T. D. Märk, and Z. Herman, Int.J.Mass Spectrom.Ion Proc., in press (1997) 


\title{
The Role of Electron Impact Ionization Cross Sections in Low-Temperature Plasmas
}

\author{
K.Becker, V. Tarnovsky, H. Deutsch*, and T.D. Märk ${ }^{* *}$ \\ Department of Physics, Stevens Institute of Technology, Hoboken, NJ 07030, USA \\ ${ }^{*}$ Fachbereich Physik, Universität Greifswald, D-17487 Greifswald, Germany \\ ${ }^{* *}$ Institut für Ionenphysik, Technikerstr.25, A-6020 Innsbruck, Austria
}

\section{Introduction}

The use of non-equilibrium, low-temperature plasmas for materials processing is the key to the advancement of many rapidly developing technologies. The selective and highly anisotropic etching of materials and the controlled deposition of thin films in the fabrication of microelectronic structures is one important area of plasma-assisted material processing. Plasma polymerization and plasma-assisted surface modification are other areas of great technological importance. Plasma-based processes are used in about $35 \%$ of the steps involved in the manufacture of semiconductor chips and "the more sophisticated the chip, the larger the number of steps relying on plasma technology" [1]. The past five years have seen an increased level of activity in efforts to provide a more scientific underpinning of low-temperature plasma processing, a development stimulated by the 1991 National Research Council (NRC) report on "Plasma Processing of Materials: Scientific Opportunities and Technological Challenges" [1] and several related reports. Despite these efforts, low-temperature plasma technology (LTPT) today remains a discipline where technological advances routinely outpace the fundamental understanding of the key processes at a microscopic level. Even though process and reactor modeling and plasma diagnostics techniques made very promising advances, a serious lack of sufficient and sufficiently reliable collisional and spectroscopic data often prevents the exploitation of the full potential of modeling codes, CAD tools, and plasma diagnostics techniques. This has been noted in a recent follow-up NRC report on "Modeling, Simulation, and database Needs in Plasma Processing" [2]. The report cites three main reasons for the poorly developed status of the database, (i) the continued comparatively low level of research efforts in this area, (ii) little coordination between the activities of the few groups working in this field, particularly poor coordination between experimental efforts and theoretical calculations, and (iii) a very serious lack of communication between the community that generates the data and the community that uses the data.

\section{Background}

A low-temperature plasma is a system far from thermodynamical equilibrium. The electron temperature is much higher than the gas temperature (which is often close to the ambient temperature) and can drive "high temperature" chemistry without the adverse effects of high ambient temperatures on the processed materials. The reactive species in a processing plasma, primarily chemically active neutral and ionic radicals and molecules and atomic ions, result from the collisionally induced break-up of the parent feedstock molecules. A quantitative and detailed understanding of the processes leading to the formation of the reactive plasma constituents and of 
their structure is important for three reasons, (i) to determine the composition of the plasma and thus the plasma properties and the key plasma chemical reaction pathways, (ii) to determine the flux of reactive plasma species to the wafer or substrate and thus the utility of a particular plasma for a specific processing application, and (iii) to provide the basis for optical and mass spectrometric diagnostics of the relevant gas phase and surface processes as well as for state-ofthe-art computer simulations and $\mathrm{CAD}$ tools for process modeling and reactor design.

Electron and photon interactions with reactive plasma constituents under single collision conditions are a versatile probe of the structure and dynamics of the parent plasma constituents as well as of the dominant secondary plasma species such as chemically reactive radicals which are produced by collision-induced dissociation and ionization of the parent molecules. With average electron energies of $0.5 \mathrm{eV}$ to $5 \mathrm{eV}$ for typical technological low-temperature plasmas, the high energy tail of the electron energy distribution function (eedf) extends well into the impact energy regime above the thresholds of the cross sections for the electron-impact dissociation and ionization of the parent plasma constituents. The overlap of the eedf and the near-threshold regions of these cross sections determines the efficiency of the formation of the various neutral and ionic plasma species. The experimental determination of electron-impact cross sections for the dissociation and ionization of the parent molecules, particularly the low-energy, nearthreshold regions of the cross sections and measurements of all types of cross sections for electron collisions with chemically reactive neutral and ionic plasma radicals are of crucial importance in any effort to understand, describe, model, and characterize the properties of a particular processing plasma. The gas phase processes, in turn, determine the flux of ions and neutrals to the wafer or substrate and, therefore, indirectly determine the surface processes as well.

\section{Current Activities}

Our group at the City College of New York, which has recently been relocated to the Stevens Institute of Technology in Hoboken, NJ, in collaboration with the group of Prof. Märk in Innsbruck, Austria and Prof. Deutsch in Greifswald, Germany has been engaged for several years in a comprehensive effort aimed at the experimental and theoretical study of elementary processes and the generation of basic collisional and spectroscopic data relevant to lowtemperature processing plasmas

\subsection{Experimental Activities}

The main focus of the experimental part of the research project is the study of the collisionally induced ionization, dissociation and dissociative ionization of selected molecules and free radicals. The choice of target molecules to be investigated is based on two considerations which are given roughly equal weight, (1) the relevance of the particular species in low-temperature plasmas and (2) common features among the targets from a more basic collision physics viewpoint (e.g. interesting and/or similar molecular or electronic structure, members of a particular molecular/radical sequence, etc.). The project up to now has studied primarily molecular and radical targets of the form $A_{B}$ with $A=C, N, S i, B=F, H$, and $x=1-4$ (see tables below). The work for the fluorine-containing targets has been completed except for $\mathrm{SiF}_{4}$ which will be studied in the near future employing a mass spectrometric technique (see below). All other experiments 
were carried out in our well-characterized fast-beam apparatus [3,4]. Comparisons between the ionization behavior of the various targets have been made from several viewpoints:

1. along each row in the above table

2. along each column in the above table

3. between the various rows in the above table

4. between the various columns in the above table

Table 1: Fluorine-containing Species

\begin{tabular}{|c|c|c|c|}
\hline $\mathrm{CF}_{4}{ }^{1)}$ & $\mathrm{CF}_{3}$ & $\mathrm{CF}_{2}$ & $\mathrm{CF}$ \\
\hline$\ldots$ & $\mathrm{NF}_{3}$ & $\mathrm{NF}_{2}$ & $\mathrm{NF}$ \\
\hline $\mathrm{SiF}_{4}{ }^{2)}$ & $\mathrm{SiF}_{3}$ & $\mathrm{SiF}_{2}$ & $\mathrm{SiF}$ \\
\hline
\end{tabular}

1) This molecule was studied by several other groups, but not as part of this project 2)This molecule will be investigated in the near future

It was the objective to correlate the ionization properties (magnitude of total and partial ionization cross sections, peak ionization cross section values, cross section shapes, ratio of parent to fragment ionization cross sections, mechanisms of fragment ion formation, etc.) with the molecular and electronic structure

of the targets. We subsequently investigated the corresponding hydrides.

Table 2: Hydrogen-containing Species

\begin{tabular}{|c|c|c|c|}
\hline $\mathrm{CH}_{4}$ & $\mathrm{CH}_{3}$ & $\mathrm{CH}_{2}$ & $\mathrm{CH}$ \\
\hline$\cdots$ & $\mathrm{NH}_{3}$ & $\mathrm{NH}_{2}$ & $\mathrm{NH}$ \\
\hline $\mathrm{SiH}_{4}$ & $\mathrm{SiH}_{3}$ & $\mathrm{SiH}_{2}$ & $\mathrm{SiH}$ \\
\hline
\end{tabular}

(For reasons of experimental convenience we frequently substituted the deuterated compounds in our experiments. This is of no consequence for the results and their interpretation, since ionization cross sections are insensitive to isotope effects to a very high degree of approximation.)

By replacing the $\mathrm{F}$ atoms by the lighter $\mathrm{H}$ atoms, we essentially go from a multi-center to a quasi single-center molecule. Furthermore, by replacing the very electronegative fluorine atoms by hydrogen, the molecular and electronic properties of the targets were modified significantly.

The most recent experimental work focused on $\mathrm{ND}_{\mathrm{x}}(\mathrm{x}=1-3)$ and silane, $\mathrm{SiH}_{4}$. There are some notable similarities between the $\mathrm{ND}_{\mathrm{x}}$ ionization data and the previously obtained $\mathrm{SiD}_{\mathrm{x}}$ and $\mathrm{CD}_{\mathrm{x}}$ data. Similar to the case of $\mathrm{SiD}_{\mathrm{x}}$ and $\mathrm{CD}_{\mathrm{x}}$, the ionization of the $\mathrm{ND}_{\mathrm{x}}$ targets is dominated by the formation of the respective parent ions and the parent ionization cross section has essentially the same value for all three targets. Furthermore, dissociative ionization, although a less important process, is, in turn, dominated by a single channel, viz. the removal of a single D atom for all three targets. Lastly, all fragment ions (except for $\mathrm{D}^{+}$) are formed with little excess kinetic energy. There is also two notable differences between the $\mathrm{CD}_{\mathrm{x}}$ and $\mathrm{ND}_{\mathrm{x}}$ data on one side and the $\mathrm{SiD}_{\mathrm{x}}$ data on the other side. For one, the $\mathrm{SiD}_{\mathrm{x}}$ ionization cross sections are significantly larger (more than a 
factor of 2) than the $\mathrm{ND}_{\mathrm{x}}$ and $\mathrm{CD}_{\mathrm{x}}$ ionization cross sections, which, in turn, are of very similar magnitude. Secondly, in the case of $\mathrm{SiD}_{\mathrm{x}}$, the cross section for the dominant dissociative ionization channel had the same value for all three $\mathrm{SiD}_{\mathrm{x}}$ species, while there is a systematic decrease in the dominant disociative ionization cross section for $\mathrm{ND}_{\mathrm{x}}$ and $\mathrm{CD}_{\mathrm{x}}$ with decreasing value of $x$, i.e. the $\mathrm{ND}^{+} / \mathrm{ND}_{2}$ and $\mathrm{CD}^{+} / \mathrm{CD}_{2}$ cross sections are smaller than the $\mathrm{ND}_{2}{ }^{+} / \mathrm{ND}_{3}$ and $\mathrm{CD}_{2}{ }^{+} / \mathrm{CD}_{3}$ cross sections, while the $\mathrm{N}^{+} / \mathrm{ND}$ and $\mathrm{C}^{+} / \mathrm{CD}$ cross sections are the smallest.

Silane, $\mathrm{SiH}_{4}$, is in the same class of molecule as e.g. $\mathrm{SiF}_{4}$ and $\mathrm{CF}_{4}$ in the sense that it does have a stable parent ion. As a consequence, the fast-beam method is not suitable to study $\mathrm{SiH}_{4}$. A mass spectrometric technique had to be employed (in collaboration with the group of Dr. M. Schmidt at the INP Greifswald, Germany) to investigate this molecule. There had been three earlier studies of the ionization of silane carried out by three different groups using three different techniques, but the agreement was very poor (more than a factor 3 difference in some partial and in the total ionization cross section). Great care was exercised in the characterization of our mass spectrometer and in the analysis and minimization of possible sources of systematic errors. As a result, we believe that the apparatus is capable of measuring absolute molecular ionization cross sections at the 15-20\% level of accuracy. As expected, our experiments revealed that dissociative ionization processes are the dominant ion formation mechanisms and all molecular fragment ions (except for $\mathrm{H}_{2}{ }^{+}$) are formed with little excess kinetic energy. By contrast, the $\mathrm{H}^{+}$fragment ion is formed with a broad distribution of kinetic energies peaking at several electronvolts per fragment ion.

\subsection{Cross Section Calculations}

We have also developed and refined various semi-empirical and semi-classical methods for the calculation of total single ionization cross sections of molecules and radicals. The main effort is on the development and refinement of a modified additivity rule which allows predictions of total single ionization cross sections of complex molecules for which more rigorous calculations are difficult. This model expresses the molecular ionization cross section in terms of the ionization cross sections of the constituent atoms and empirically determined weighting factors which account for molecular bonding. Up to now, our efforts focused on refining the additivity concept for molecules of the form $A B_{n}$. We are also working on the extension of this concept to more complex molecules of the form $\mathrm{A}_{x} \mathrm{By}$ and $\mathrm{A}_{\mathrm{x}} \mathrm{B}_{\mathrm{y}} \mathrm{C}_{\mathrm{z}}$.

This work was supported in part by the Division of Chemical Sciences, Office of Basic Energy Science, Office of Energy Research, US Department of Energy, by the US National Science Foundation, and by the US National Aeronautics and Space Administration (NASA).

\section{References}

[1] "Plasma Processing of Materials: Scientific Opportunities and Technological Challenges", National Academy Press 1991

[2] "Modeling, Simulation, and Database Needs in Plasma Processing", National Academy Press 1996

[3] R. C. Wetzel, F. A. Biaocchi, T. R. Hayes, and R. S. Freund, Phys. Rev. A 35559 (1987); R. S. Freund, R. C. Wetzel, R. S. Shul, and T. R. Hayes, Phys. Rev. A 413575 (1990)

[4] V. Tarnovsky and K. Becker, Z. Phys. D 22603 (1992); V. Tarnovsky, P. Kurunczi, D. Rogoshnikov, and K. Becker, Int. J. Mass Spectrom. Ion Proc. 128181 (1993) 


\title{
Atomic Data: Energy Levels, Transition Rates, and Collision Strengths; An Example of Mg VIII
}

\author{
A. K. Bhatia \\ Goddard Space Flight Center, Greenbelt Maryland 20771
}

A number of satellites have been launched to observe radiation from various astrophysical sources. Line radiation is emitted when the levels in ions/atoms excited by electron or proton impact decay to lower levels by photon emission. From this radiation, the physical parameters, such as electron temperature and density of astrophysical plasmas, can be inferred. Electron impact excitation is, in general, more important than proton impact excitation except for fine structure levels in the ground levels. A number of methods have been used for calculating excitation cross sections: most commonly used are distorted wave (DW) approximation and the R-matrix approach. In DW approximation, channel coupling and resonances are neglected, and therefore the method is considerably simpler than the R-matrix approach. Rate coefficients and collision strengths, averaged over a Maxwellian distribution, are accurate to nearly 20\% in DW approximation and $10 \%$ in R-matrix approach. A comparison of collision strengths in Ca XIII calculated in these two approximations is given in Ref. [1]. Proton excitation rate coefficients are calculated by semi-classical approximation [2]. With a number of collaborators, I have carried out DW calculations for a number of ions. In addition to excitation cross sections, energy levels, oscillator strengths, and transition rates are required. A number of papers have been published on atomic data for various ions. Cumulative indexes of Atomic Data And Nuclear Data Tables, 64, 321 (1996) alone list 15 publications. Recent publications [3,4] are on Fe XV. The atomic data in Ref. [3] have been used to investigate the rocket spectrum of a solar flare in the 10 - $100 \AA$ range and to identify the strong line at $69.65 \AA$. The atomic data in Ref. [4] have been used to calculate intensity ratios in the EUV range and compare with those observed by SERTS in an active region of sun. The calculated results should also be useful in analyzing observations from SOHO.

The atomic data are required not only to study intensities of emitted lines but also to study elemental abundances and opacity. Atomic data have also been used to study the Bowen fluorescence mechanism (e.g. in O III [5] and N III [6]). The atomic data are also of importance in planetary atmospheric studies, laser physics, and for diagnostics of Tokomak plasmas.

To calculate the atomic data, a set of programs developed at University College has been used extensively: the Superstructure and DW programs. In the Superstructure program, configuration interaction can be taken into account, and the radial functions are calculated in a modified Thomas-Fermi potential. Spin-orbit interactions, which give fine structure splitting, and relativistic corrections are treated as a perturbation to the nonrelativistic Hamiltonian. It is important to choose target configurations carefully in order to obtain accurate energy levels, oscillator strengths, and transition rates. The same radial functions are used in the DW calculation to calculate the excitation cross sections.

As an example, our most recent calculation is on the Mg VIII ion carried out in collaboration with Dr. R. J. Thomas [7]. EUV spectral lines of Mg VIII have been observed from the solar corona by Skylab, by SERTS, and more recently by SOHO. SERTS flights [8] were in 1989, 1991, and in 1993, and lines for which intensities were measured are 311.78, 313.74, 315.02, 317.01, 339.00, 430.44 , and $436.73 \AA$. All these lines, with the exception of 430.44 and $436.73 \AA$, have been 
measured by SOHO [9] flown in 1995. The figure in Ref. [7] shows a comparison of active region spectra taken by SERTS-89 and by CDS on SOHO.

Twenty levels are included by using the configurations $2 s^{2} 2 p, 2 s 2 p^{2}, 2 p^{3}, 2 s^{2} 3 s, 2 s^{2} 3 p$, and $2 \mathrm{~s}^{2} 3 \mathrm{~d}$ in the Superstructure program described by Eissner et al. [10]. Optimum Thomas-Fermi potential is obtained by minimizing the sum of term energies. The energy levels, oscillator strengths, and radiative rates have been calculated in intermediate coupling. These are given in Tables 1 and 2 of Ref. [7]. The potential obtained in the calculation of energy levels, oscillator strengths, and transition rates is used in the DW program described by Eissner and Seaton [11]. Reactance matrices are calculated in LS coupling and transformed to intermediate coupling by using term-coupling coefficients [12] obtained from the Superstructure program. It is not practical to include a very large number of incident partial waves. Therefore, contribution from higher incident partial waves to dipole-allowed collision strengths is included using Coulomb-Bethe approximation [13]. Collision strengths are calculated at five incident energies (15.0, 22.5, 30.0, 37.5, and $45 \mathrm{Ry}$ ), and they are given in Table 2 of Ref. [7].

The temperature of maximum abundance for this ion is $\log \mathrm{T}_{\mathrm{e}}=5.9 \mathrm{~K}$. The rate coefficients are calculated at this temperature by averaging the collision strengths over a Maxwellian distribution. Level populations are calculated by the solving statistical equilibrium equations at a particular electron density, and intensity ratios are calculated using

$$
\mathrm{I}_{\mathrm{ij}}=\mathrm{n}_{\mathrm{j}} \mathrm{A}_{\mathrm{ji}} \Delta \mathrm{E}_{\mathrm{ij}} \quad \text { (energy units) }
$$

where $n_{j}$ is the fractional level population, $A_{j i}$ is the transition rate, and $\Delta \mathrm{E}_{\mathrm{ij}}$ is the energy difference between the levels $j$ and $i$.

A comparison with relative intensities observed by SERTS-89 in an active region is given in Table 1. The calculated intensity ratio $\mathrm{I}(313.74) / \mathrm{I}(317.01)=1.69$ agrees reasonably well with the observed value 1.40. Inclusion of three more configurations, $2 \mathrm{~s} 3 \mathrm{p} 3 \mathrm{~s}, 2 \mathrm{~s} 2 \mathrm{p} 3 \mathrm{p}$, and $2 \mathrm{~s} 3 \mathrm{p} 3 \mathrm{~d}$ does not change the above calculated ratio appreciably. These lines originate from the same upper level, and therefore the ratio depends only on transition rates. This indicates that the calculated transition rates are reliable. The relative intensities of 436.73 and $430.44 \AA$ remain in some disagreement with the calculated values. However, the intensity ratio $\mathrm{I}(436.73) / \mathrm{I}(430.44)$ is found to be density sensitive, and at $\log N_{e}=9.2$ (interpolated), its value is 1.68 which is the observed value from SERTS:89 (Thomas and Neupert). This density is comparable to the value of $\log \mathrm{N}_{\mathrm{e}}=9.6$ derived by Brickhouse, Raymond, and Smith [14] from the same SERTS-89 spectrum, except using Fe IX line ratios formed at a similar temperature to those of Mg VIII.

This work was supported by NASA-ATOP grant 432-38-53-14. 
Table 1: Relative Intensities Observed by SERTS-89 compared to Calculated Values at $\mathrm{Te}=8 \times 105 \mathrm{~K}$

\begin{tabular}{ccccccccc}
\hline \multicolumn{2}{c}{ Transition } & $\lambda(\AA)$ & \multicolumn{5}{c}{$\mathbf{I}_{\text {obs }}$} \\
\hline \hline & & & \multicolumn{5}{c}{$\log \left(N_{e}\right)$} \\
\cline { 4 - 8 } & & & 6 & 7 & 8 & 9 & $10^{\text {c }}$ \\
1 & 10 & 311.778 & $0.313^{\mathrm{a}} \pm 0.056$ & 0.199 & 0.199 & 0.199 & 0.199 & 0.199 \\
1 & 9 & 313.736 & $0.317 \pm 0.049$ & 1.380 & 1.062 & 0.540 & 0.395 & 0.378 \\
2 & 10 & 315.024 & $1.000 \pm 0.123$ & 1.000 & 1.000 & 1.000 & 1.000 & 1.000 \\
2 & 9 & 317.008 & $0.227 \pm 0.052$ & 0.816 & 0.628 & 0.319 & 0.233 & 0.223 \\
1 & 8 & 335.230 & $\ldots-. \mathrm{b}$ & 0.437 & 0.357 & 0.225 & 0.188 & 0.184 \\
2 & 8 & 339.000 & $0.213 \pm 0.033$ & 0.627 & 0.511 & 0.322 & 0.269 & 0.263 \\
1 & 6 & 430.445 & $0.159 \pm 0.019$ & 1.614 & 1.208 & 0.542 & 0.356 & 0.334 \\
2 & 7 & 436.726 & $0.267 \pm 0.032$ & 0.108 & 0.255 & 0.498 & 0.566 & 0.574 \\
\hline
\end{tabular}

a Blended with Ni XV, see Thomas \& Neupert (1994)

b Masked by Fe XVI $335.40 \AA$

c Above $\log \left(\mathrm{N}_{\mathrm{e}}\right)=10$, ratios do not change

\section{References}

[1] S. O. Kastner and A. K. Bhatia, Astron. Astrophys. 71, 211 (1979)

[2] K. S. Baliyan and A. K. Bhatia, J. Phys. B 27, 4281 (1994)

[3] A. K. Bhatia, H. E. Mason, and C. Blancard, Atomic Data and Nuclear Data Tables 66, 83 (1997)

[4] A. K. Bhatia and H. E. Mason, Atomic Data and Nuclear Data Tables 66, 119 (1997)

[5] A. K. Bhatia, S. O. Kastner, and W. E. Behring, ApJ 257, 887 (1982)

[6] S. O. Kastner and A. K. Bhatia, ApJ 287, 945 (1984)

[7] A. K. Bhatia and R. J. Thomas, ApJ 497, 483 (1998)

[8] R. J. Thomas and W. M. Neupert, ApJS 91, 461 (1994)

[9] R. A. Harrison et al., Solar Phys. 162, 233 (1995)

[10] W. Eissner, M. Jones, and H. Nussbaumer, Comput. Phys. Commun. 8, 270 (1974)

[11] W. Eissner and M. J. Seaton, J. Phys. B 5, 2187 (1972)

[12] H. E. Saraph, Comput. Phys. 15, 247 (1978)

[13] A. Burgess and V. B. Sheorey, J. Phys. B 7, 2403 (1974)

[14] N. S. Brickhouse, J. C. Raymond, and B. W. Smith, APJS 97, 551 (1995) 


\title{
Collisional and Spectroscopic Data Relevant to the Fundamental Processes in Plasmas Containing Si-Organic Admixtures
}

\author{
R. Foest, R. Basner, M. Schmidt, P. Kurunczi ${ }^{*}$ and K.Becker* \\ Institut für Niedertemperatur-Plasmaphysik, \\ Robert-Blum Str. 8-10, D-17489 Greifswald, Germany \\ *Department of Physics, Stevens Institute of Technology, \\ Hoboken, NJ 07030, USA
}

\section{Introduction}

Silicon-organic molecules are used in various plasma-assisted deposition and polymerization applications [1,2]. The most commonly employed monomers [3-7] include tetraethoxysilane (TEOS), $\mathrm{Si}\left(\mathrm{O}-\mathrm{CH}_{2}-\mathrm{CH}_{3}\right)_{4}$, tetramethylsilane (TMS), $\mathrm{Si}\left(\mathrm{CH}_{3}\right)_{4}$, and hexamethyldisiloxane (HMDSO), $\left(\mathrm{CH}_{3}\right)_{3}$-Si-O-Si- $\left(\mathrm{CH}_{3}\right)_{3}$. TMS is among the simplest Si-organic compounds, whereas HMDSO is the simplest of the siloxane compounds, which are characterized by a -Si-O-Si- bridge in their molecular structure. In spite of the practical importance of plasma-assisted material processing using discharge mixtures containing Si-organic admixtures, little is known about the fundamental physical and chemical processes in these plasmas and about the collision-induced decomposition of these molecules in the plasma. Ionization studies under single collision conditions combined with mass spectrometric studies in the actual plasma have shed some light on the processes in TMS-containing plasmas [8,9] and similar studies are currently underway for TEOS and HMDSO [10]. In addition to mass spectrometry, optical plasma diagnostics methods can also shed light on the fundamental plasma processes [11]. Optical emissions from plasmas containing Si-organic molecules have been studied to some extent [12]. Optical emissions in the visible and near-ultraviolet region of the electromagnetic spectrum produced by controlled electron-impact under single collision conditions on TMS, TEOS, and HMDSO have been studied recently in our group $[13,14]$.

Researchers at the Institut für Niedertemperatur-Plasmaphysik (INP) in Greifswald, Germany have been engaged in a comprehensive series of experimental studies aimed at (i) the measurement of absolute partial ionization cross sections for various Si-organics under controlled single collision conditions, (ii) in-situ studies of the behavior and properties of the neutral and ionic components in realistic deposition plasmas containing Si-organic admixtures, and (iii) correlating the single collision observations with the plasma observations. This effort is complemented by studies of the visible, near-ultraviolet, and vacuum ultraviolet emissions produced by controlled electron impact on these Si-organic cmpounds carried out at the Stevens Institute of Technology in Hoboken, NJ, USA.

\section{Experimental Details}

The experimental apparatus and the data acquisition and analysis procedures for the photoemission studies employs a crossed beam arrangement where the interaction region is defined by the intersection of the electron beam and the gas beam. A five-element electron gun 
produces a spatially well defined electron beam with a current of typically $80 \mu \mathrm{A}$ at $100 \mathrm{eV}$. The energy of the electron beam can be varied over the range of $5-200 \mathrm{eV}$ with an energy spread of about $0.8 \mathrm{eV}$ (full width at half maximum) at an energy of $100 \mathrm{eV}$. A multicapillary array produces the gas beam which crosses the electron beam at right angles. The estimated gas density in the interaction region is of the order of $10^{12}$ molecules $/ \mathrm{cm}^{3}$. A precision leak valve controls the backing pressure in the gas line to the multicapillary array. This pressure was monitored by a Baratron capacitance manometer. The analysis/detection system for the vacuum ultraviolet (VUV) emissions is positioned mutually perpendicular to both the gas beam and the electron beam. The VUV emissions are spectrally analyzed using a Minuteman model 302-VM 0.2m vacuum monochromator of the Seya-Namioka design and the VUV photons are subsequently detected with by a channel electron multiplier (CEM). The output signal of the CEM is fed into a standard circuit for single photon counting. The monochromator is equipped with a holographic grating for the wavelength range from 50 to $250 \mathrm{~nm}$. The monochromator and detector are evacuated to a pressure of $5 \times 10^{-7}$ Torr under operating conditions. The main vacuum chamber which houses the crossed-beam set-up is evacuated to a pressure in the low $10^{-7}$ Torr range without gas load which rises to about $2-5 \times 10^{-5}$ Torr under operating conditions.

At the INP, Dr. Schmidt and his co-workers have a high-resolution $(\mathrm{m} / \Delta \mathrm{m}=40000)$ double focusing sector field mass spectrometer MCH 1310 with a Nier-type electron-impact ion source $[15,16]$. The target gas pressure in the ion source, which is typically in the range 0.1 to $1 \mathrm{mPa}$ compared to a background pressure of $0.001 \mathrm{mPa}$, is measured with a spinning rotor viscosity gauge. The electron gun is operated with a stabilized electron beam current of $10 \mathrm{DA}$ emitted from a directly heated tungsten band cathode. The impact energy can varied from $5 \mathrm{eV}$ to $100 \mathrm{eV}$. The energy spread of the electron beam, which is collimated by a weak longitudinal magnetic field of $200 \mathrm{G}$, is about $0.5 \mathrm{eV}$ (FWHM). The ions are extracted from the ionization region by a penetrating electric field. The acceleration voltage between the ion source and the entrance slit of the mass spectrometer is $5 \mathrm{kV}$. Argon, which is used as reference gas, is always added to the target gas under study for calibration purposes. The ion efficiency curves (relative ionization cross sections) are measured simultaneously for Ar and the target gas in a well-defined mixture in an effort to ensure equal operating conditions for the detection of all ions. The measured relative partial ionization cross sections are put on an absolute scale by normalizing the total single ionization cross section for a given species relative to the total Argon ionization cross section of $2.77 \times 10^{-16} \mathrm{~cm}^{2}$ at $70 \mathrm{eV}$.

Recently, the ion optics for the extraction, acceleration and deflection of the product ions was rebuilt based on ion trajectory simulations [16] in conjunction with in-situ experimental studies in an effort to minimize and/or quantify the discrimination of energetic fragment ions. The modified mass spectrometer can now be operated either in a high mass resolution mode (with significant discrimination effects present) or in a high extraction efficiency mode (by partially sacrificing the high mass resolution capability). All measurements routinely employ a sweep of the ion beam across the entrance slit of the mass spectrometer and absolute cross sections are obtained by integrating the ion signal over the complete ion beam profile. The advantage of the INP instrument is its superior mass resolution and a very large dynamic range which allows very small ionization cross sections (down to $1 \times 10^{-20} \mathrm{~cm}^{2}$ ) to be measured with high precision. On the other hand, even with the new ion extraction optics, the instrument has a limited capability of extracting and detecting energetic fragment ions and, of course, it can only investigate stable targets.

Very recently, a second apparatus has become available at the INP, a time-of-flight mass spectrometer, which will be operational by the end of this year. The new instrument combines the 
capability to extract and detect very efficiently energetic fragment ions with a large dynamic range, but its use is still limited to stable targets and has a much reduced mass resolution compared to the high-resolution instrument.

The in-situ plasma measurements are carried out in a $13.56 \mathrm{MHz}$ rf capacitively coupled discharge reactor. The two electrodes, which have a diameter of $12.8 \mathrm{~cm}$ each, are separated by a gap of $4 \mathrm{~cm}$. Typical rf powers are from 15 to $150 \mathrm{~W}$. The pressure in the reactor ranges from 1-20 $\mathrm{Pa}$ and typical gas flow rates are $10 \mathrm{sccm}$ of the carrier gas (Ar) and $0.1-0.2 \mathrm{sccm}$ of the Si-organic admixture. The reactor is coupled to a VG SXP $300 \mathrm{H}$ plasma monitor (mass spectrometer) which is also equipped with an ion energy analyzer in order to determine the kinetic energy distribution of the various ions in the plasma. The plasma monitor, whose mass dependent detection sensitivity has been carefully calibrated, can be used to probe either the neutral or the ionic plasma component.

\section{Some Selected Results}

The ionization cross section measurements for TMS have been completed. It was found that by far the most dominant channel is the formation of the $\mathrm{Si}\left(\mathrm{CH}_{3}\right)_{3}{ }^{+}$fragment ion with a maximum cross section value of about $1 \times 10^{-15} \mathrm{~cm}^{2}$ which accounts for about $50 \%$ of the total TMS ionization cross section. The maximum TMS parent ionization cross section is less than $2 \%$ of that value. Based on our measured partial ionization cross sections for more than 20 fragment ions and on their measured appearance energies we proposed a collision-induced decomposition scheme for the TMS molecule in low-temperature plasmas. Three major decomposition routes were identified which all involve the total or partial removal of a $\mathrm{CH}_{3}$ methyl group.

Ionization cross section studies for the other two Si-organics have also been completed and the results are in many respects similar to what was found for TMS, viz. very small parent ionization cross sections, a dominance of dissociative ionization processes, a small number of fragment ions with comparatively large ionization cross sections. In contrast to TMS, the ionization data for TEOS and HMDSO are more difficult to interpret on account of the much more complicated cracking pattern and the much larger number of fragment ions with appreciable cross sections for their formation.

Detailed in-situ studies in realistic deposition plasmas containing Ar as the carrier gas and small (1-2\%) admixtures of TEOS and HMDSO revealed a variety of noteworthy findings: whereas the ionic plasma component in both cases still reflected to some extent the cracking pattern of the parent molecule, there was little evidence of the parent cracking pattern in the neutral component. A common feature was the significant increase (in some cases by 2.4 orders of magnitude) of certain small fragment ions in the plasma compared to calculated ion abundances using the single collision ionization cross sections. Furthermore, we found ample evidence of the formation of "new" ions in the plasma by various plasma chemical reactions. This indicates that secondary processes such as dissociation into neutral ground-state fragments followed by subsequent electron collisions and ion-molecule reactions play a very important role in the plasma chemistry of deposition plasma containing Si-organic admixtures.

The comparatively large values of the ionization cross sections for TMS, TEOS, and HMDSO (relative to e.g. Ar) and the relatively low appearance energies of many fragment ions (7-10 eV compared to $15 \mathrm{eV}$ for Ar) explain why a small TEOS admixture of $2 \%$ to an Ar plasma leads to a situation where the ionization balance in the plasma is completely determined by the Si-organic admixture. 
The photoemission studies involving TMS, HMDSO, and TEOS in the various spectral regions (vacuum ultraviolet, near-ultraviolet, and visible) revealed several common features:

(1) the vacuum ultraviolet spectra are completely dominated by the H Lyman series

(2) the Lyman- $\alpha$ and Lyman- $\beta$ lines are heavily influenced by cascading from higher $\mathrm{H}$ states

(3) the measured appearance energies indicate that partial fragmentation processes are the dominant break-up mechanisms leading to the formation of excited $\mathrm{H}$ fragment atoms

(4) the near-ultraviolet and visible spectra contain mostly the hydrogen Balmer series and emissions from the excited $\mathrm{CH}$ radical

(5) the TMS and HMDSO emission spectra show also the presence of atomic Si emissions

(6) in general, the measured emission cross sections are comparatively small

Future work will focus on a continuation of the work in TEOS and HMDSO and will eventually also involve other Si-organics, e.g. dimethylsilane, $\left(\mathrm{CH}_{3}\right)_{2} \mathrm{SiH}_{2}$, which is used in processing applications which require environmentally "green" processing gases.

This work was supported in part by the US National Science Foundation.

\section{References}

[1] "Plasma Deposition, Treatment, and Etching of Polymers", R. D'Agostino, ed., Academic Press, San Diego 1990

[2] S. Peter, R. Pintaske, G. Hecht, and F. Richter, Surf. Coat. Technol. 59, 97 (1993)

[3] H. Yasuda, "Plasma Polymerization", Academic Press, London 1985

[4] D.A. Dougherty and A. Gallagher, J. Appl. Phys. 67, 139 (1990)

[5] A.W. Luft and S. Tsuo, Appl. Phys. Comm. 8, 1 (1988)

[6] I. Tajama and M.Y. Yamamoto, J. Polym. Sci. A: Polym. Chem. 25, 1737 (1987)

[7] P. Favia, R. Lamendola, and R. D’Agostino, Plasma Sources, Sci. Technol. 1, 59 (1992)

[8] R. Basner, R. Foest, M. Schmidt, F. Sigenegger, P. Kurunczi, and K. Becker, Int. J. Mass Spectrom. Ion Proc. 153, 65 (1996)

[9] R. Basner, R. Foest, M. Schmidt, P. Kurunczi, K. Becker, and H. Deutsch, "Contributed Papers of the XXII. International Conference on Phenomena in Ionized Gases", Hoboken, NJ, USA 1995, editors: K. Becker, W.Carr, and E. Kunhardt, Stevens Institute of Technology Press, Hoboken 1995, p. IV-31

[10] R. Basner and M. Schmidt, private communications (1996, 1997); see also R. Basner, R. Foest, M. Schmidt, F. Hempel, and K. Becker, "Contributed Papers of the XXIII. International Conference on Phenomena in Ionized Gases", Toulouse, France 1997, editors: M. C. Bordage and A. Gleizes, University of Toulouse Press, Toulouse 1997, p. IV-196

[11] see e.g. J. E. Lawler, M. A. Childs, K. L. Menningen, L. W. Anderson, S. D. Bergeson, and K. L. Mullman, in "Phenomena in Ionized Gases", editors: K. Becker, W.E. Carr, and E. Kunhardt, American Institute of Physics Press, New York 1996, p. 1

[12] M. Schmidt, R. Foest, R. Basner, and M. Hannemann, Acta Phys. Comen. 35, 217 (1994)

[13] M. Ducrepin, J. Dike, R. Siegel, V. Tarnovsky, and K. Becker, J. Appl. Phys. 73, 7203 (1993)

[14] P. Kurunczi, A. Koharian, K. Becker, and K. E. Martus, Contr. Plasma Phys. 36, 723 (1996)

[15] R. Basner, M. Schmidt, H. Deutsch, V. Tarnovsky, A. Levin, and K. Becker, J. Chem. Phys. 103, 211 (1995)

[16] R. Basner, R. Foest, M. Schmidt, F. Sigeneger, P. Kurunczi, K. Becker, and H. Deutsch, Int. J. Mass Spectrom. Ion Proc. 153, 65 (1996) 


\title{
Measurements of the Absolute Cross Sections of Inelastic Processes for Slow Atomic Collisions
}

\author{
M. R. Gochitashvili, B. I. Kikiani, R. A. Lomsadze \\ Tbilisi State University, Tbilisi, 380028 Georgia
}

Measurements of the absolute cross-sections of inelastic processes in ion-atom and ion-molecule collisions in the energy range from several hundred to a thousand electron volts are connected with experimental difficulties (low values of cross sections, scattering of the primary ion at large angles, problems with the total collection of the formed energetic products of the collisions). This accounts for discrepancies between the data of different authors. On the other hand, these data are necessary for solution of many fundamental and applied problems (plasma and upper atmosphere physics, gas discharge physics and so on).

Below are described the experimental methods and arrangements that have been developed and applied in our laboratory to measure absolute total and differential cross sections of excitation $\sigma_{\mathrm{ex}}$, ionization $\sigma_{\mathrm{i}}$, charge transfer $\sigma_{\mathrm{C}}$ and electron loss (stripping) $\sigma_{\mathrm{s}}$ processes.

The main parts of the our mass-spectrometer arrangement are (Fig. 1): an ion source $\mathrm{H}$, an accelerating and focusing system $\mathrm{L}$ (quadrupole lenses), a magnetic mass-analyzer $\mathrm{M}$, collimating slits $\left(\mathrm{S}_{0}-\mathrm{S}_{3}\right)$, a collision chamber $\mathrm{C}$ and a detector of ions $\mathrm{D}$. The target gas pressure in chamber $\mathrm{C}$ (about $10^{-4}$ torr) could be varied according to the single collision condition. The differential pumping system $\mathrm{V}$ achieved a vacuum of about $10^{-6}$ torr in another part of the arrangement. When measuring cross-sections of excitation processes, the radiation emitted at the collision was observed under a $90^{\circ}$ angle to the direction of the primary ion beam. The spectral analysis of this radiation was performed in the visible (by a MDR monochromator) and vacuum ultraviolet spectral regions (by a Seya-Namioka monochromator). The intensity of the radiation was detected by photo-(PEM) and secondary electron (SEM) multipliers. A system of polaroid and quarter-wave plates $\mathrm{P}$ was used for checking the polarization of the radiation in the visible region. The investigations were carried out by different types of ion sources: alkali metal ions were generated in a surface ionization ion source; and $\mathrm{H}^{+}, \mathrm{H}_{2}+\mathrm{H}_{\mathrm{g}}+\mathrm{He}^{+}, \mathrm{O}^{+}, \mathrm{N}^{+}$ions by gas discharges and electron bombardment ion sources.

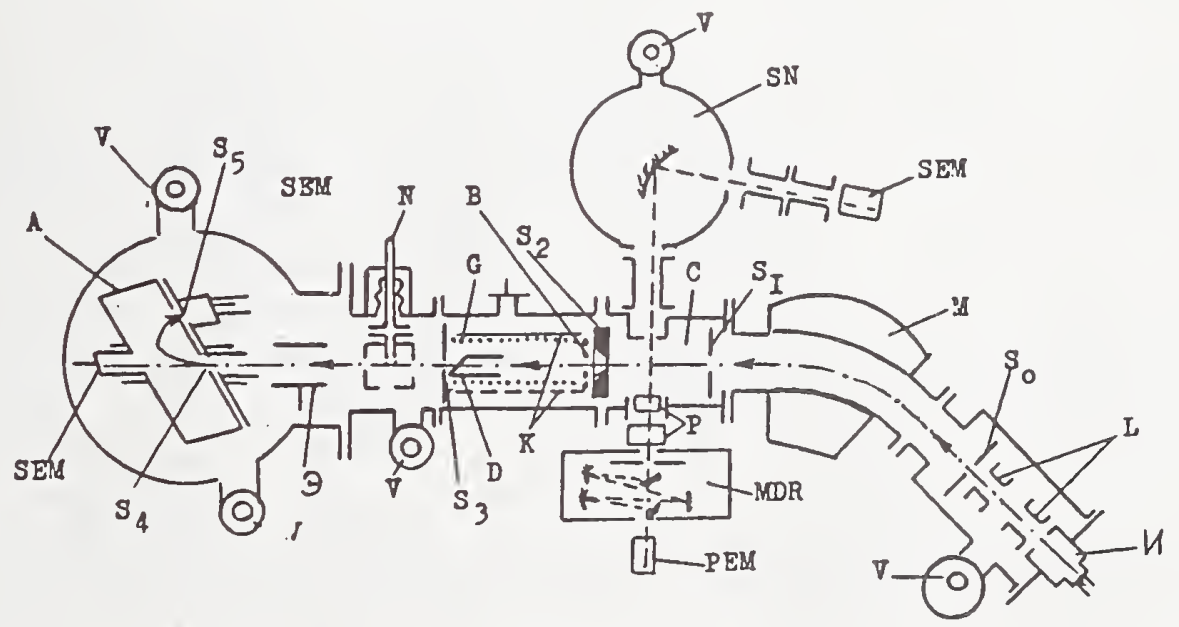

Figure 1 
The optimum regime for the operation of these sources has been established by special control experiments. For example, in the case of the $\mathrm{H}_{3}{ }^{+}-\mathrm{He}$ pair it was determined that the vibrational excitation of $\mathrm{H}_{3}+*$ molecules strongly influences the efficiency of the excitation of the $\mathrm{L}_{\alpha}$ line $(\lambda=120.0 \mathrm{~nm})$ in the dissociation processes. The control experiment showed that by increasing the hydrogen pressure in the gas discharge ion source this efficiency is decreasing and reaches the saturation region at a pressure of about $3 \times 10^{-3}$ torr. Under these conditions the deexcitation of vibrational excited $\mathrm{H}_{3}{ }^{+*}$ molecules takes place in the time of collisions with $\mathrm{H}_{2}$. The $\mathrm{H}_{3}{ }^{+}$ions are formed in the ground state only [1].

Particular attention was devoted to the reliable determination and control of the relative and absolute spectral sensitivity of the recording system for the emitted radiation. This was done by measuring the output signal of the molecular bands and atomic lines excited by electrons in the collisions e+ $\mathrm{H}_{2}, \mathrm{~N}_{2}, \mathrm{O}_{2}$. The relative spectral sensitivity and hence the values of the absolute cross sections were obtained by comparisons with cross sections for the same lines and molecular bands reported by other authors [2-6]. The spectra for $\mathrm{e}+\mathrm{H}_{2}$ and $\mathrm{e}+\mathrm{N}_{2}$ collisions at an electron energy $\mathrm{E}=200 \mathrm{eV}$ are presented in Fig. 2.

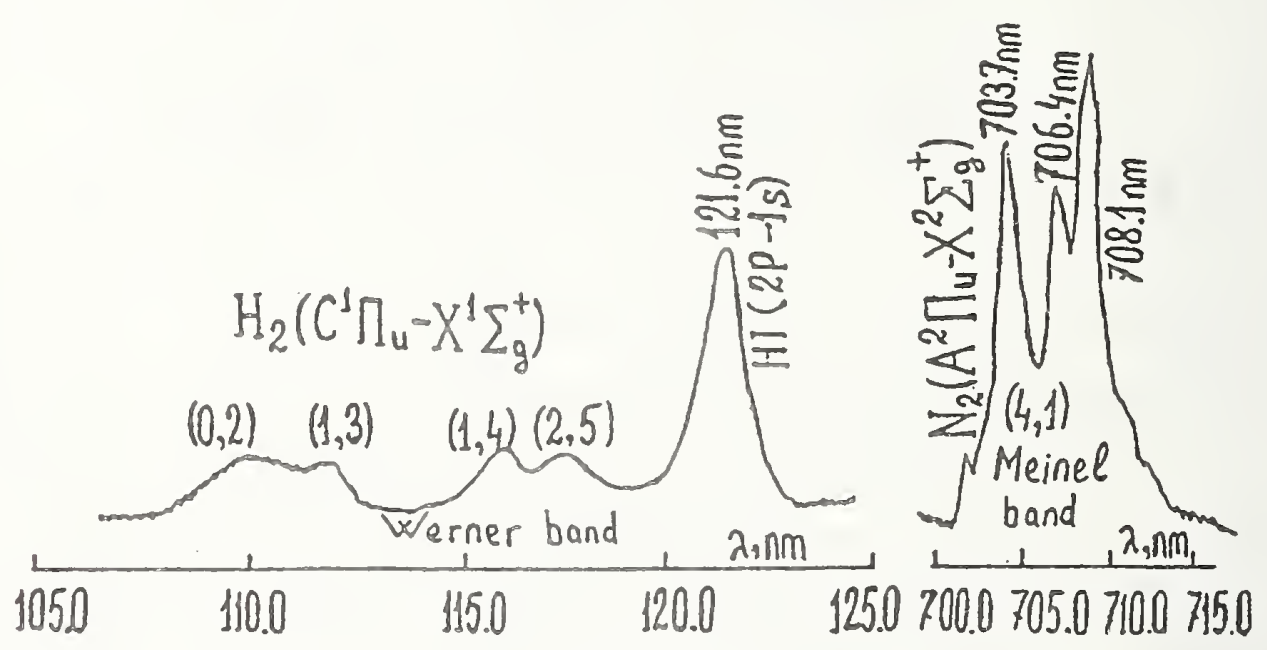

Figure 2

The transfer electrical field method (see for example [7]) is modified to measure the absolute values of total cross sections of ionization and charge transfer processes. The target gas ions and free electrons (secondary particles), arising as a result of collisions, are collected by the homogeneous electrical field of a plane capacitor $K$ (Fig. 1). Absolute cross sections for the production of these particles, $\sigma_{+}$and $\sigma$, are connected with cross sections $\sigma_{\mathrm{i}}, \sigma_{\mathrm{c}}, \sigma_{\mathrm{s}}$ by the following relations: $\sigma_{i} \cong \sigma_{i}+\sigma_{c}, \sigma \cong \cong \sigma_{i}+\sigma_{s}$. For the fixed homogeneous part of the field ("collision length") the capacitor electrode was divided by small plane parallel sections (1-10) and the charge distribution was studied with them. In front of the electrodes a grid G is placed to suppress the secondary electron emission from the surface of the sections. This grid is used to receive information about the energy spectrum of scattering particles by a "stopping potential," too. In Fig. 3 the ion current, scattering on the section closest to the entrance slit of chamber $\mathrm{C}$ (section 1, curves 3,4 ) and on the middle section (section 5, curves 1,2 ), is plotted as a function of the stopping potential for a $\mathrm{K}^{+}$-Ar pair at energies $\mathrm{E}=1 \mathrm{keV}$ and $\mathrm{E}=5 \mathrm{keV}$ (curves 1,3 and 3,4 respectively). Considerable differences between these currents are caused by the strong possibility that the scattering primary ions are hitting the middle section. Therefore, section 1 was chosen for the measurements 


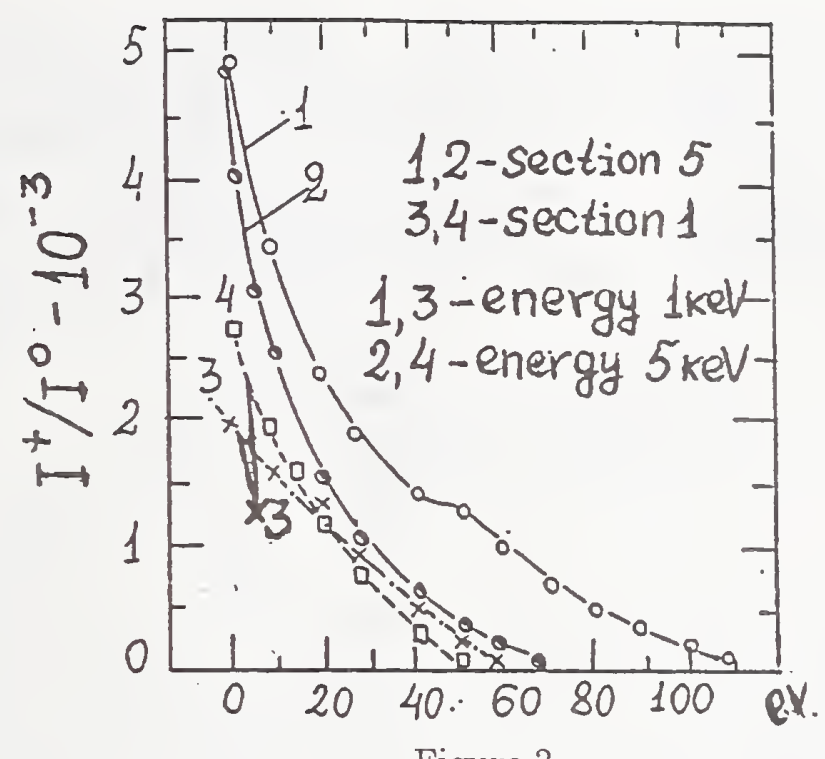

Figure 3

Absolute cross sections for the stripping processes $\sigma_{S}$ were measured in separate experiments by mass spectrometer analysis of the primary beam after passing through the collision chamber.

To determine the energetic and angular dependences of differential cross sections a "Box" type electrostatic analyzer was used [8]. The primary ion beam crossed a narrow thermal energy beam of targeted particles, formed in the neutral particle source $N$ (Fig. 1). The ion beam is then separated (slit $\mathrm{S}_{4}$ ) and directed into analyzer $\mathrm{A}$. The automatic regulation of the analyzer potential allows that ions with energy loss in the $0-100 \mathrm{eV}$ region pass through outlet slit $\mathrm{S}_{5}$ $\left(1 \times 10^{-4} \mathrm{~mm}^{2}\right)$. The energy resolution of the analyzer is about 500 . The whole analyzer system may be revolved in a horizontal plane around the center of the collision region in the angle interval of $\pm 15^{\circ}$, with a resolution of about $0.5^{\circ}$. During the time of measuring the charge transfer cross sections $\sigma \mathrm{c}$, the charged component of scattered primary beam is removed by a plane capacitor and the neutral component is detected by SEM.

\section{References}

[1] B. Peart, K. T. Dolder, J. Phys. B 7, 1567 (1974)

[2] E. J. Stone and E. C. Zipf, J. Chem. Phys. 56, 4646 (1972)

[3] M. J. Mumma and E. C. Zipf, J. Chem. Phys. 55, 1661, (1971)

[4] J. M. Ajello and B. J. Franklin, Chem. Phys. 82, 2519, (1985)

[5] K. H. Tan and J. W. McConkey, Phys. Rev. A 10, 1212 (1974)

[6] K. H. Tan et al., Can. J. Phys. 52, 786 (1971)

[7] N. V. Fedorenko et al., Sov. Zh. Tekh. Fiz. 26, 1929 (1956)

[8] V. P. Afanasev, S. Y. Yavor, "Electrostatic Analyzer for Charged Particles Beam", Moscow 1978 


\title{
Ionization, Charge Transfer, and Stripping Cross Sections for Alkali Metal Ion Collisions with Inert Gas Atoms and $\mathrm{H}_{2}$ and $\mathrm{N}_{2}$ Molecules in the 0.5-7.0 keV Energy Range
}

\author{
B. I. Kikiani, R. A. Lomsadze \\ Physics Department, Tbilisi State University \\ Tbilisi, 380028 Georgia
}

The absolute values of the total and differential cross sections for various inelastic processes in slow collisions of species with closed electron shells are measured. Ionization and charge transfer cross sections, $\sigma \mathrm{I}$ and $\sigma \mathrm{c}$, are obtained by an improved transfer electric field (condenser) method; stripping (primary ion electron loss) cross sections $\sigma \mathrm{s}$ by recording doubly charged primary ions, produced as a result of collisions; and differential cross sections do/d $\Omega$ by a "BOX" type electrostatic analyzer [1]. Because of the realization of these processes at small internuclear distances of colliding particles $\mathrm{R}$, primary ions are scattered through relatively large angles and this is accompanied by the formation of much more energetic secondary particles (target gas ions and free electrons) than is usual. This circumstance has not been considered in earlier papers, which must raise doubts about the reliability of the measurements themselves and of the conclusions drawn from them.

An estimate for the uncertainty in the absolute values of cross sections given below is about $15 \%$.

The energy dependences of the absolute cross sections $\sigma_{\mathrm{I}}$ for $\mathrm{Na}^{+} \mathrm{He}_{\mathrm{H}} \mathrm{Ne}, \mathrm{Ar}$ and $\sigma \mathrm{c}$ for $\mathrm{Li}^{+} \mathrm{He}_{\mathrm{H}}$ Ne, and Ar collisions are plotted, as an example, in Figs. 1 and 2. It appears that the discrepancies between our results and those of other authors [2,3] are considerable. The reasons for this are the experimental difficulties mentioned above. For example, we determined that in $\mathrm{Na}^{+}-\mathrm{Ne}$ collisions about $25 \%$ of the target gas ions have energies higher than $40 \mathrm{eV}$, and about $15 \%$ of the free electrons have energies above $30-35 \mathrm{eV}$.

The interpretation of our results by plotting schematic correlation diagrams of diabatic terms of the system of colliding particles [4] and the use of model representations [5,6] has made it possible to express certain conclusions about the mechanisms of the relevant processes. It was found that in $\mathrm{Li}^{+}$-Ar collisions, an electron is captured mainly into ground state of the lithium atom $\mathrm{Li}\left({ }^{2} \mathrm{~S}\right)$, at an internuclear distance $\mathrm{R} \sim 1.5$ a.u. For the $\mathrm{Li}^{+} \cdot \mathrm{Ne}$ pair, the charge transfer process is realized through an intermediate state $\mathrm{Li}^{+}-\mathrm{Ne}\left(2 \mathrm{p}^{5}{ }^{3} \mathrm{~S}\right)$ at relatively large internuclear distances $R$.

A comparison of our data for $\mathrm{Li}^{+}$- $\mathrm{He}$ collisions with the cross sections for emission of the resonance line of lithium atoms (transition ${ }^{2} \mathrm{P} \rightarrow 2 \mathrm{~S}, \lambda=670.8 \mathrm{~nm}$ ), reported in [7], shows that $\sigma \mathrm{C}$ includes a considerable $(\sim 50 \%)$ contribution from the cross section for electron capture by an excited state of the lithium atom, $\mathrm{Li}(2 \mathrm{p})$.

The contributions of the various channels to the total cross sections $\sigma_{I}$ were estimated to define the mechanism of the ionization process. For direct ionization channels such contribution was estimated by calculations of partial cross sections using a procedure described in ref. [6]. For an estimate of the contributions of molecular and atomic autoionization channels [8] experimental data for the spectrum of free electrons were used. We found that for the $\mathrm{Na}^{+}$-Ar pair the direct 
ionization channel is dominant, whereas for the $\mathrm{Na}^{+}-\mathrm{Ne}$ pair the process of atomic autoionization of neon is about $30 \%$ and direct ionization about $10 \%$. In the case of $\mathrm{Na}^{+}-\mathrm{He}$ pair the atomic autoionization channel of helium is the main one.

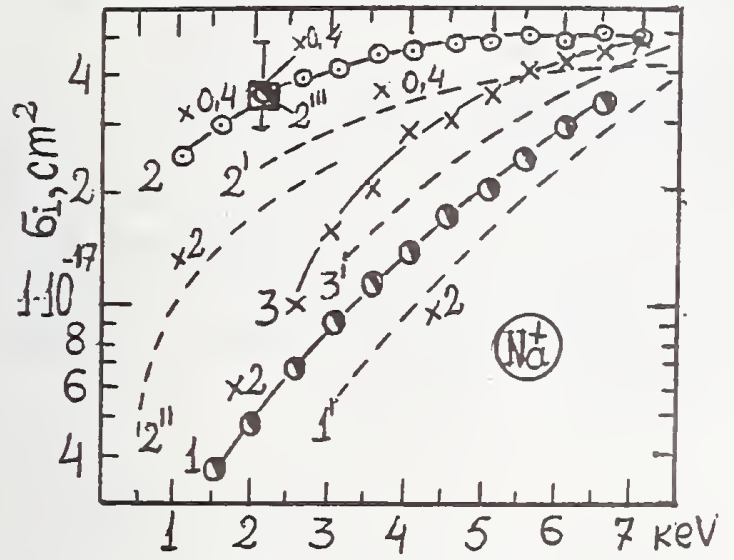

Figure 1: Ionization $\left(\mathrm{Na}^{+}-\mathrm{He}\right)-1$ ) our results, 1') [2]; $\left.\left(\mathrm{Na}^{+}-\mathrm{Ne}\right)-2\right)$ our results, 2') $\left.[2], 2^{\prime \prime}\right)$ [3] 2"') [10]; ( Na $\left.^{+}-\mathrm{Ar}\right)$ - 3) our results, $\left.3^{\prime}\right)[2]$.

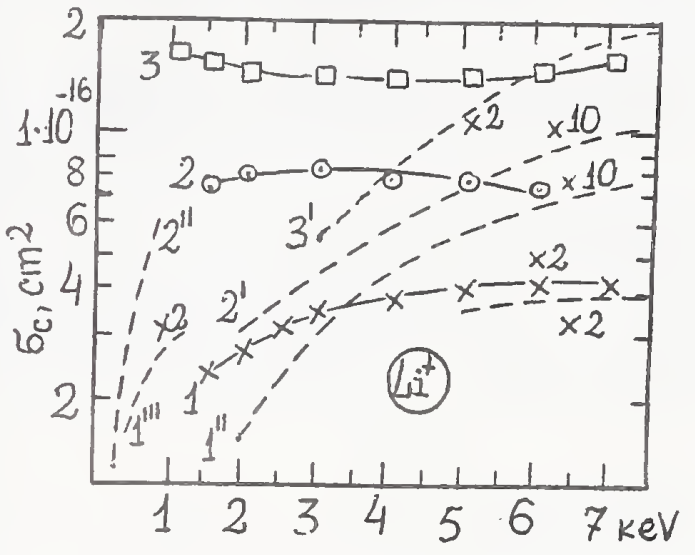

Figure 2: Charge Transfer: $\left.\left(\mathrm{Li}^{+}-\mathrm{He}\right)-1\right)$ our results, 1') [11], 1') [2], $\left.\left.1^{\prime \prime \prime}\right)[3] ;\left(\mathrm{Li}^{+}-\mathrm{Ne}\right)-2\right)$ our results, 2') [2], 2" [3]; (Li+-Ar) - 3) our results, $\left.3^{\prime}\right)[2]$.

In Fig. 3, our experimental (curves 1,4) and calculated data (curve 3) of stripping cross sections for the collisions $\mathrm{K}^{+}-\mathrm{He}$, Ne are compared with experimental data for the excitation cross sections of ten autoionization states of $\mathrm{K}^{+}$ions for the collision $\mathrm{K}^{+}$-He reported in ref. [9] (curve 2) in arbitrary units. The comparison shows that the main realization of the stripping process, at least for the $\mathrm{K}^{+}$-He pair in the investigated energy region, is connected with the direct ionization mechanism.

The energy and angular dependences of the differential cross sections of elastic scattering were defined for various pairs of particles. As an example, in Fig. 4 our results for $\mathrm{K}^{+}-\mathrm{H}_{2}, \mathrm{~N}_{2}, \mathrm{Ar}$ pairs are presented at an energy $E=2 \mathrm{keV}$. We use reduced coordinates: $\rho(\tau)=\theta \sin \theta \mathrm{d} \sigma / \mathrm{d} \Omega$

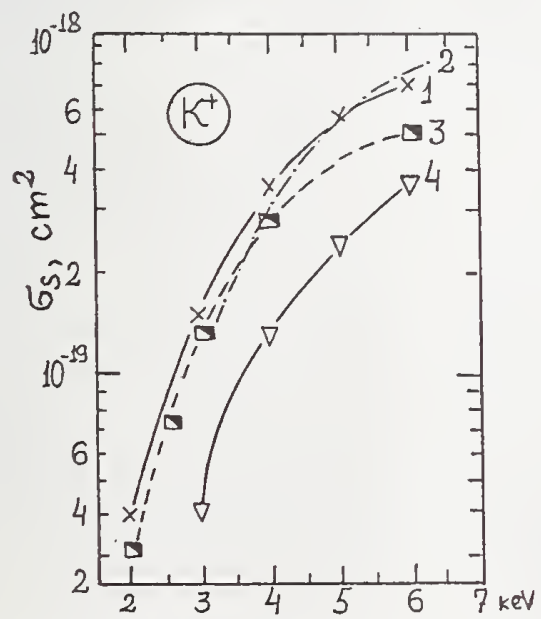

Figure 3: Stripping: $\left(\mathrm{K}^{+}-\mathrm{He}\right)$ - 1) our results, 2) [9], 3) [6]; $\left(\mathbf{K}^{+}-\mathrm{Ne}\right)$ - 4) our results.

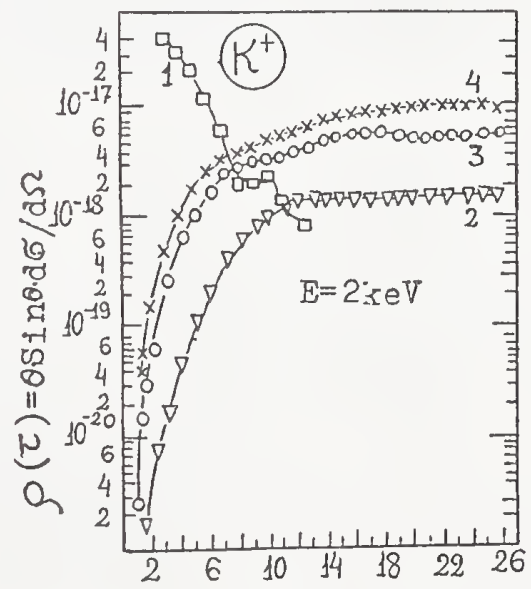

Figure 4: Differential Cross Sections: $\left.\left(\mathrm{K}^{+}-\mathrm{Ar}\right)-1\right)$ elastic scattering; $\left(\mathrm{K}^{+}-\mathrm{H}_{2}\right) \cdot 2$; $\left(\mathrm{K}^{+}-\mathrm{N}_{2}\right)-3$; $\left(\mathrm{K}^{+}-\mathrm{Ar}\right)$ - 4) charge transfer. 
(impact parameter), and $\tau=\mathrm{E} \theta$ (reduced angle), where $\mathrm{E}, \theta$ are the collision energy and scattering angle in the mass-center system. For definite absolute values, the total charge transfer cross sections (restored from differential cross sections) were normalized to the one for the $\mathrm{K}^{+}$-Ar collision, which was measured by the condenser method. It appears that the angular dependence of the charge transfer cross sections has a threshold behavior (curves $2,3,4$ ). The abrupt decrease of the elastic scattering cross section at $\tau \sim 5-7 \mathrm{keV}$ is due to reaching the internuclear distance $R$ at which the inelastic processes get excited. Our results indicate that the charge transfer processes in these collisions are connected with mechanisms of the pseudo-crossing of the quasimolecular potential energy terms of corresponding states. Because of the significance of the mass-ratio, the internuclear distance in the region of nonadiabatic collisions for $\mathrm{K}^{+}-\mathrm{H}_{2}$ is smaller. That is why for this pair the absolute cross sections $\sigma_{\mathrm{C}}$ are smaller for $\mathrm{K}^{+}-\mathrm{N}_{2}$, Ar pairs, as shown by our results.

\section{References}

[1] B. I. Kikiani, R. A. Lomsadze, et al., Sov. Zh. Tekh. Fiz. 55, 1612 (1985); See also the contributed paper by M. R. Gochitashvili, B. I. Kikiani, R. A. Lomsadze, "Measurements of the Absolute Cross Sections of Inelastic Processes in Slow Collisions", in these proceedings

[2] I. P. Flaks, B. I. Kikiani, and G. N. Ogurtsov, Sov. Zh. Tekh. Fiz. 35, 2076 (1965); Sov. Zh. Tekh. Fiz. 36, 491 (1966)

[3] Z. Z. Latypov and A. A. Shaporenko, Sov. Zh. Eksp. Teor. Fiz. 69 (1944) (1975); Zh. Eksp. Teor. Fiz. 18, 4303 (1973); Zh. Tekh. Fiz. 43, 1300 (1973)

[4] M. Barat and Litchen, Phys. Rev. A 6, 211, (1972)

[5] B. M. Smirnov, "Asymptotic Methods in the Theory of Atomic Collisions", Chap. 6 \& 6.1, Moscow 1973

[6] E. A. Solov'ev, Sov. Zh. Eksp. Teor. Fiz. 81 (1681)

[7] S. Tsurubuchi and T. J. Iwai, Phys. B 14, 243 (1981)

[8] W. Weizel and V. O. Beeck, Z. Phys. 76, 250 (1932)

[9] H. Aizawa, K. Wakiya, et al., J. Phys. B 18, 289 (1985)

[10] J. O. Olsen, T. Andersen, M. Barat, et al., Phys. Rev. A 19, 1457 (1979)

[11] J. van Eck, F. J. de Heer, J. and Kistemaker, Physica 26, 629 (1960) 


\title{
Atomic Data for Recombination Calculations
}

\author{
R. Kisielius and P. J. Storey \\ Department of Physics and Astronomy, University College London, \\ Gower St., London, WC1E 6BT, UK \\ A. R. Davey \\ Mullard Space Science Laboratory, University College London, \\ Holmbury St Mary, Dorking, Surrey, RH5 6NT, UK
}

\section{Introduction}

There exists a significant discrepancy in the abundances of carbon, nitrogen, oxygen, and neon ions that are derived for photoionised nebulae from recombination lines and from collisionally excited forbidden lines. This discrepancy emphasises the need for accurate theoretical work on bound-bound and bound-free radiative data for atomic ions. Such data were generated by the Opacity Project, using the $R$-matrix formulation of the close-coupling approximation, but are often inadequate for the calculation of recombination coefficients due to the presence of narrow, but important near-threshold resonances which are inadequately resolved and inaccurately positioned. We report on a programme of systematic new calculations of radiative data and line recombination coefficients for singly-ionised atoms of the astronomically most abundant elements $(\mathrm{C}, \mathrm{N}, \mathrm{O}, \mathrm{Ne})$ which uses quantum defect techniques to accurately map resonances.

\section{Photoionisation Cross-sections}

We treat the $(N+1)$-electron scattering problem in the close-coupling $(\mathrm{CC})$ approximation. Bound states and continuum states of the system are determined using the $R$-matrix method (see Berrington et al [1]) and from the resulting wave functions, radiative data for $g f$-values (bound-bound transitions) and photoionisation cross-sections (bound-free transitions) are calculated.

Configuration-interaction (CI) wave functions for the $N$-electron target systems are computed with the SUPERSTRUCTURE code of Nussbaumer and Storey [2]. In the Opacity Project calculations, the CI expansion of the target wave functions consists of $1 s, 2 s, 2 p$ spectroscopic orbitals for $\mathrm{C}, \mathrm{N}, \mathrm{O}, \mathrm{Ne}$ ions. We expand the configuration basis by adding $\overline{3} s, \overline{3} p, \overline{3} d, \overline{4} f$ pseudo orbitals for $\mathrm{C}$ III target and all $\overline{3} l$ and $\overline{4} l$ pseudo orbitals for $\mathrm{N}$ III target. In photoionised nebulae, the electron temperature is generally below $20000 \mathrm{~K}$, so only the near threshold resonances are important in determining recombination coefficients. The non-physical resonances that arise from the use of non-physical correlation orbitals are not a problem at low energies.

The photoionisation cross-sections typically exhibit resonances due to photoexcitation of the core electrons followed by autoionisation. A simple example for C II ions is

$$
2 s^{2} n l+h \nu \rightarrow 2 s 2 p n l^{\prime} \rightarrow 2 s^{2}+\varepsilon l^{\prime \prime} .
$$

We use quantum defect theory to determine resonance positions and widths. Resonances are due to poles in the scattering matrix $\mathcal{S}$ described by a matrix $\chi$ which varies slowly with energy 
and contains functions pertaining to both open $(o)$ and closed $(c)$ channels:

$$
\mathcal{S}=\chi_{o o}-\chi_{o c}\left[\chi_{c c}-\exp (-2 \pi i \nu]^{-1} \chi_{c o} .\right.
$$

Diagonalisation of the matrix $\chi_{c c}$ yields the effective quantum numbers, $\nu$ related to the complex quantum defect $\mu_{c}$

$$
\mu_{c}=\alpha_{c}+i \beta_{c}
$$

by $\nu=n-\mu_{c}$. The energy of the pole $\varepsilon($ pole $)$ is given by

$$
\varepsilon(\text { pole })=\varepsilon_{c}-(n-\alpha)^{-2}-\frac{i}{2} \gamma
$$

where $\gamma=4 \beta(n-\alpha)^{-3}$ is the autoionisation width of resonance and $\varepsilon_{c}$ is the appropriate target energy.

In contrast to the OP data, we use a variable step energy mesh for photoionisation crosssections that delineates all resonances to a prescribed accuracy. In the first step of the calculation, we determine resonance positions and widths using a coarse energy mesh. In the second step, we determine a new fine energy mesh of variable step length $\Delta \varepsilon$ assuming a Lorentzian profile for the resonance:

$$
F(\varepsilon, \gamma)=\frac{\left(\frac{\gamma}{(2}\right)^{2}}{(\varepsilon-\varepsilon(\text { pole }))^{2}+\left(\frac{\gamma}{2}\right)^{2}} .
$$

To calculate recombination coefficients, we integrate the photoionisation cross-sections using a 3-point Simpson's rule. If we specify a required accuracy as $\Delta F$, we can find the necessary energy interval for a given resonance:

$$
\Delta \varepsilon=\frac{90 \times \Delta F}{F^{(4)}(\xi)}
$$

where $F^{(4)}(\xi)$ is the 4 th derivative of the Lorentzian function. The adopted step length is the smallest value of $\Delta \varepsilon$ obtained for any resonance. In the third step we calculate photoionisation cross-sections using the new variable step mesh.

The Opacity Project photoionisation data were generated using a relatively coarse energy mesh. These data sometimes have poor resolution in resonance areas. In Fig.1, we show the photoionisation cross-sections for the $2 s 2 p^{2}{ }^{2} \mathrm{D}$ state of the $\mathrm{C}$ II ion. One can notice a difference in the number of energy points and in the resonance resolution. As a result, the area under the cross-section for the $2 s 2 p^{2}{ }^{2} \mathrm{D}$ state (Fig.1) is $1.585 \mathrm{Mb} \cdot \mathrm{Ry}$ from the Opacity Project data and $4.573 \mathrm{Mb} \cdot \mathrm{Ry}$ from our data. These differences are reflected directly in the recombination coefficients.

All ab initio calculations of the photoionisation cross-sections position the resonances incorrectly. An error of a few thousand inverse centimetres in a resonance position is typical and may significantly affect the resulting recombination coefficients, particularly at low temperatures $(10000 \mathrm{~K}$ or less). It is worth noting that earlier work on low-temperature dielectronic recombination (e.g. Storey, [3]), which treats the resonances as bound states, uses the experimental resonance positions and is not vulnerable to this uncertainty, although the quality of the wave functions and hence the radiative data, was certainly inferior. Therefore for $\mathrm{C}$ II ions, where near-threshold resonances $2 s 2 p\left({ }^{3} P^{o}\right) 3 d{ }^{2} \mathrm{~F}^{\circ}$ and ${ }^{2} \mathrm{P}^{\circ}$ make important contributions to the recombination coefficient, and the resonance positions are experimentally known, we adjust the resonance positions to their experimental values: $3301 \mathrm{~cm}^{-1}$ and $5523 \mathrm{~cm}^{-1}$ above the $2 \mathrm{~s}^{2}{ }^{1} \mathrm{~S}$ threshold instead of theoretical values of $3778 \mathrm{~cm}^{-1}$ and $6127 \mathrm{~cm}^{-1}$. This is done by calculating the resonance contribution to the recombination coefficient from a Fano profile fit to the cross-section near the resonances rather than from the calculated cross-sections themselves. The resonance positions can then be adjusted within the fit. These adjustments bring about an increase of $20 \%$ in the total recombination coefficient for the $2 s 2 p^{2}{ }^{2} \mathrm{D}$ state at temperatures below $5000 \mathrm{~K}$. 

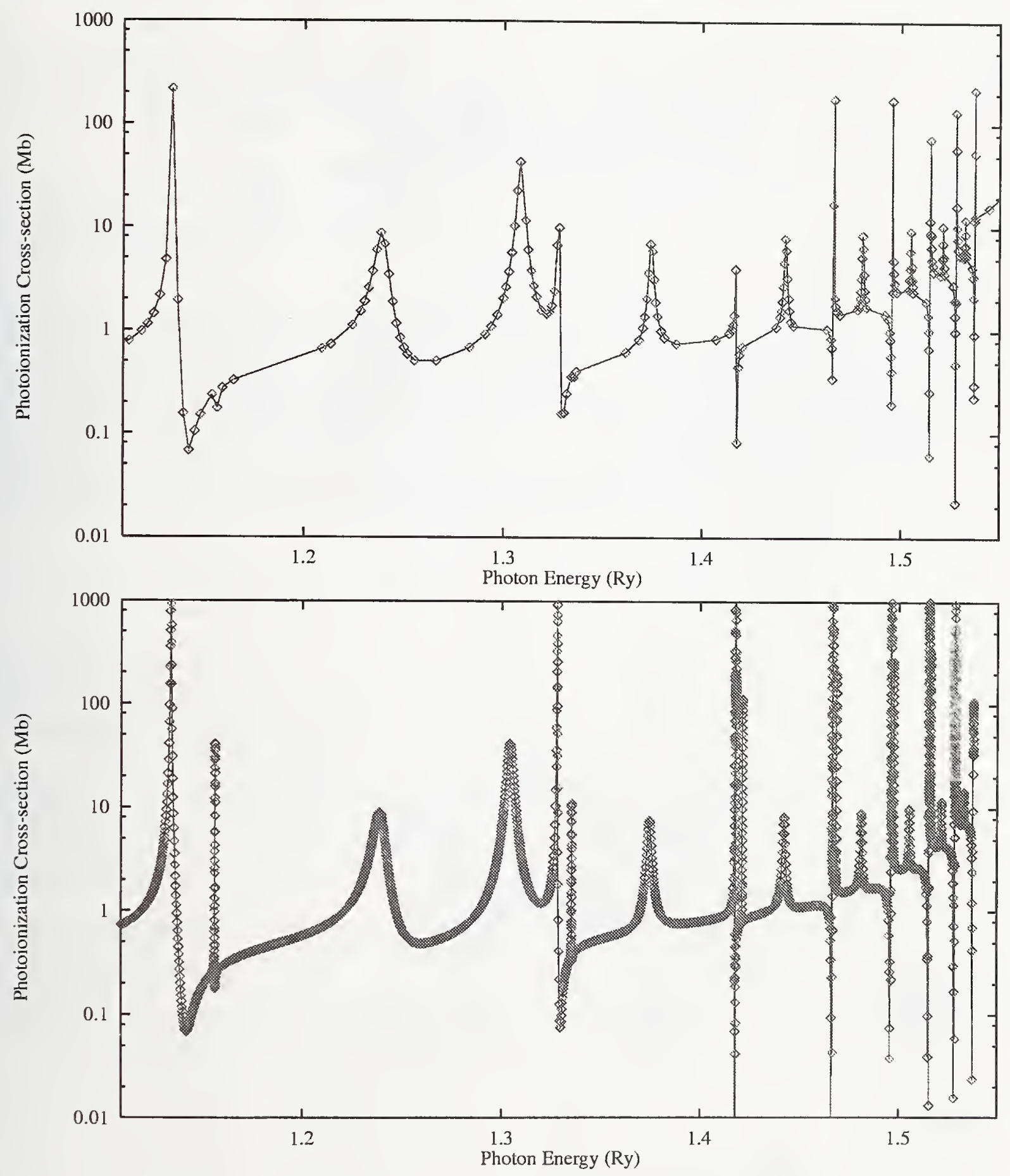

Figure 1: Comparison of the $\mathrm{C}$ II photoionisation cross-section for $2 \mathrm{~s} 2 \mathrm{p}^{2}{ }^{2} \mathrm{D}$ calculated by the Opacity Project (top) and this work. The OP cross-section consists of 199 points, based on a quantum defect mesh, whilst the latter is based on an energy mesh of 3281 points. 


\section{The Level Population Problem}

We calculate recombination coefficients for C II, N II, O II and Ne II ions. We determine both the total recombination coefficients and the effective recombination coefficients for particular spectral lines as functions of electron temperature $T_{e}$ and electron density $N_{e}$. In our model, recombination rates are determined using the $L S$-coupling scheme for ion states and, therefore, assuming no radiative transitions between states of different multiplicity.

Because of the very large number of levels included into the model, we have to partition the calculation of level populations according to the principal quantum number $n$. We define a principal quantum number $n=n_{d}$, such that for $n<n_{d}$, the population structure is determined solely by radiative processes. Collisionally induced transitions for these levels can be neglected. The value of $n_{d}$ depends on plasma density and temperature.

For $n>n_{d}$, the population depends on $n$ and $l$-changing collisions and populations are taken from purely hydrogenic calculation of departure coefficients, $b_{n l}$ by Hummer and Storey [4]. For $10<n \leq n_{d}$, populations are still determined only by radiative transitions but no accurate atomic data are available. Various approximate methods (quantum defect method, Coulomb approximation, hydrogenic approximation (when $l>3$ )) are used to obtain the necessary rate coefficients. For $n<10$, all atomic terms are included in the calculation of populations, irrespective of their parentage. It is assumed that the population is determined solely by recombination and radiative cascading from higher states.

\section{Theoretical Emission Spectra and Ion Abundances}

The calculated level populations together with $g f$-values are used to calculate theoretical emission spectra. Lines arising from $3 d-3 p$ and $4 f-3 d$ transitions in $\mathrm{O}$ II ions are prominent in observed spectra of some astrophysical objects. Comparing the measured intensities of these lines with those for hydrogen lines, we are able to determine abundances of $\mathrm{O}^{2+}$ ions in observed objects.

Theoretical spectra were determined using intermediate coupling (IC) and $L S J$-coupling schemes. For intermediate coupling (IC) spectra, we use level populations determined in $L S$-coupling and $g f$-values calculated using the Breit-Pauli Hamiltonian with spin-orbit interaction included. Alternatively, LSJ-coupling spectra are computed using the same $L S$-coupling level populations and splitting multiplet intensities using angular algebra only.

Relative intensities of various multiplets are used to determine the $\mathrm{O}^{2+}$ ion abundances in the planetary nebulae NGC 7009 and NGC 3242. The need for IC calculations was demonstrated by the comparison of the theoretical spectra with observed data by Liu et al [5]. Use of IC reduced significantly the scatter of the derived abundances demonstrating a breakdown of $L S J$-coupling. Using IC yields considerable improvement in the consistency of the derived abundances.

\section{References}

[1] J. Phys. B. 20, 6379 (1987)

[2] $A \& A$ 69, $139(1978)$

[3] MNRAS 195, 27 (1981)

[4] MNRAS 224, 801 (1987)

[5] MNRAS 272, 369 (1995) 


\title{
Unified Electron-Ion Recombination Cross Sections and Rates
}

\author{
Sultana N. Nahar, Honglin Zhang, and Anil K. Pradhan \\ Department of Astronomy, The Ohio State University \\ Columbus, Ohio 48210, U.S.A.
}

\section{Introduction}

Calculations of unified electron-ion recombination cross sections are described within the framework of the close coupling (CC) approximation using the R-matrix method. It is emphasized that the unified treatment subsumes and computationally unifies "radiative" recombination (RR) and "dielectronic" recombination (DR) processes. Calculations are carried out in non-relativistic and relativistic approximations for C V, C VI, O VIII and are compared with available accurate experimental measurements on ion storage rings and electron beam traps with very good agreement. Radiation damping of autoionizing resonances is included for these ions and found to be significant for H-like and He-like ions, although unlikely to be of importance in other ionic species. A comprehensive and self-consistent set of new atomic data of photoionization and recombination rate coefficients for oxygen ions is also obtained. These are for direct applications to astrophysical models. Sample results are presented. Complete datasets will be available electronically.

\section{Theoretical Summary}

Extending the $\mathrm{CC}$ approximation and the $\mathrm{R}$-matrix method [1] to electron-ion recombination, and using the DR theory by Bell and Seaton (BS) [2], Nahar and Pradhan [3] have developed a computationally unified approach to obtain total e+ion recombination cross sections, $\sigma_{R C}$, and rate coefficients, $\alpha_{\mathrm{R}}(T)$. The approach entails: (a) computation of photo-recombination cross sections from photoionization cross sections, $\sigma_{P}$, for all bound states with $v \leq v_{0} \approx 10$ ( $v$ is the effective quantum number) via the Milne detailed-balance relation and (b) DR cross sections from radiatively damped high- $n$ resonances, $v_{0}<v \leq \infty$, using analytic expressions for both the detailed and the resonance averaged cross sections derived from the BS theory. The computations are highly involved and, for example, include in (a) $\sigma_{P I}$ of several hundred bound states of the (e+ion) system with low- $n$ autoionizing resonances (up to $v \approx v_{0}$ ) delineated at several thousand photon energies. This method yields total $\sigma_{R C}$ in an $a b$ initio and unified manner, including the background RR and the resonant DR. Physically, there is no separation between the two processes, therefore the present total (unified) cross sections can be compared directly with absolute experimental measurements in the entire energy range. The $\alpha_{\mathrm{R}}(T)$ are obtained through maxwellian averaging over the recombination cross sections.

Experimental measurements of $\sigma_{R C}$ for carbon and oxygen ions, C V, C VI, O VIII, using the Test Storage Ring (TSR) at Heidelberg, exhibit detailed resonance structures observed at very high resolution in beam energy [5]. The experiments measure absolute cross sections and therefore provide ideal tests for theoretical methods, as well as the physical effects included in the 
calculations. The present work aims to demonstrate the accuracy of the unified method for electron-ion recombination, on par with the CC treatment of photoionization and electron impact excitation, by comparing $\sigma_{R C}$ with the measured values.

One additional consideration in the present work is the inclusion of radiation damping of low- $n$ resonances of $\mathrm{C} \mathrm{IV}, \mathrm{C} \mathrm{V}$, and O VIII since damping of resonances is known to be important for highly charged $\mathrm{H}$-like and He-like ions. The reason is that for the dipole allowed transitions, $2 p \rightarrow 1 s$ and $1 s 2 p^{1} \mathrm{P}^{0} \rightarrow 1 s^{2}$ iS the radiative decay rates, $\Gamma^{r}$, of the order of $10^{13}-10^{14} \mathrm{~s}^{-1}$, approach typical autoionization rates, $\Gamma^{a}$, of $10^{14} \mathrm{~S}^{-1}$. However, damping is negligible for all other ionization stages where for the outer core transitions such as $n=3 \rightarrow 2$, or $n=4 \rightarrow 3, \Gamma^{r}$ is generally orders of magnitude smaller than $\Gamma^{a}$. Recently, employing the Breit-Pauli (BP) R-matrix method (BPRM) [4], the calculations of recombination have been extended to highly charged ions including the $\mathrm{BP}$ relativistic effects and radiation damping [6]. We implement this new extension to C V, C VI, and O VIII.

\section{Results and Discussions}

We have calculated the unified recombination cross sections of $\mathrm{C} \mathrm{V,C} \mathrm{VI,} \mathrm{and} \mathrm{O} \mathrm{VIII.} \mathrm{Fig.} 1$ presents the detailed $\sigma_{R C}$ for e $+\mathrm{C} \mathrm{V} \rightarrow \mathrm{C}$ IV, obtained in three approximations, (a) in LS coupling and (b) in BP approximations, and (c) in the BP approximation with damping of low- $n$ autoionizing states included. The (a) LS and the (b) BP(NRD) results are almost identical (except that the resonances are better resolved due to a much finer energy mesh in the $\mathrm{BP}$ case), indicating that the relativistic effects are negligible. The BP calculations involve considerably more recombination channels than the LS calculations because of nearly twice as many fine structure levels as LS terms, but the final results do not show a significant difference between the two. The difference is less between LS coupling and BP approximation when damping is included. The convoluted average of the two sets of $\mathrm{BP}$ results, with and without radiation damping, are shown in Fig. 1(d) - solid and dashed lines respectively. The BP(RD) results agree very well with experiment, Fig. 1(e).

The total $\alpha R(T)$ is obtained for all ions in the isonuclear sequence of oxygen, O I - VIII. Calculations, such as abundances and ionization fractions, require the rates for all ionization stages. The typical feature of $\alpha R(T)$ is similar to that of O IV as shown in Fig. 2 (solid curve) where, starting with high recombination at very low temperatures, $\alpha R(T)$ decreases to a minimum at $\log _{10}(T)=4.4 \mathrm{~K}$ for $\mathrm{O} \mathrm{IV}$, above which the DR dominates giving a "high $\mathrm{T}$ bump", such as the bump around $\log _{10}(T)=5.2 \mathrm{~K}$ for $\mathrm{O} \mathrm{VI}$, beyond which the rate falls smoothly with $\mathrm{T}$. Due to near-threshold autoionization, a small bump may be observed in the low-T region. There is often considerable difference between the present and previously calculated values in the temperature region of interest such as shown in Fig. 2(b) where the sum of RR (dashed), low-T DR (dotted), and high-T DR (dot-dashed) rate curves [7] of Fig. 2(a) is compared with the present total recombination rate coefficients (solid) curve.

\section{Conclusion}

Unified electron-ion recombination cross sections, $\sigma_{R C}$, are obtained in the close coupling (CC) approximation. We find (i) very good agreement with the experiments, (ii) relativistic effects are small for the second row of elements, and (iii) radiation damping is significant for $\mathrm{H}$-like and He-like ions only. 

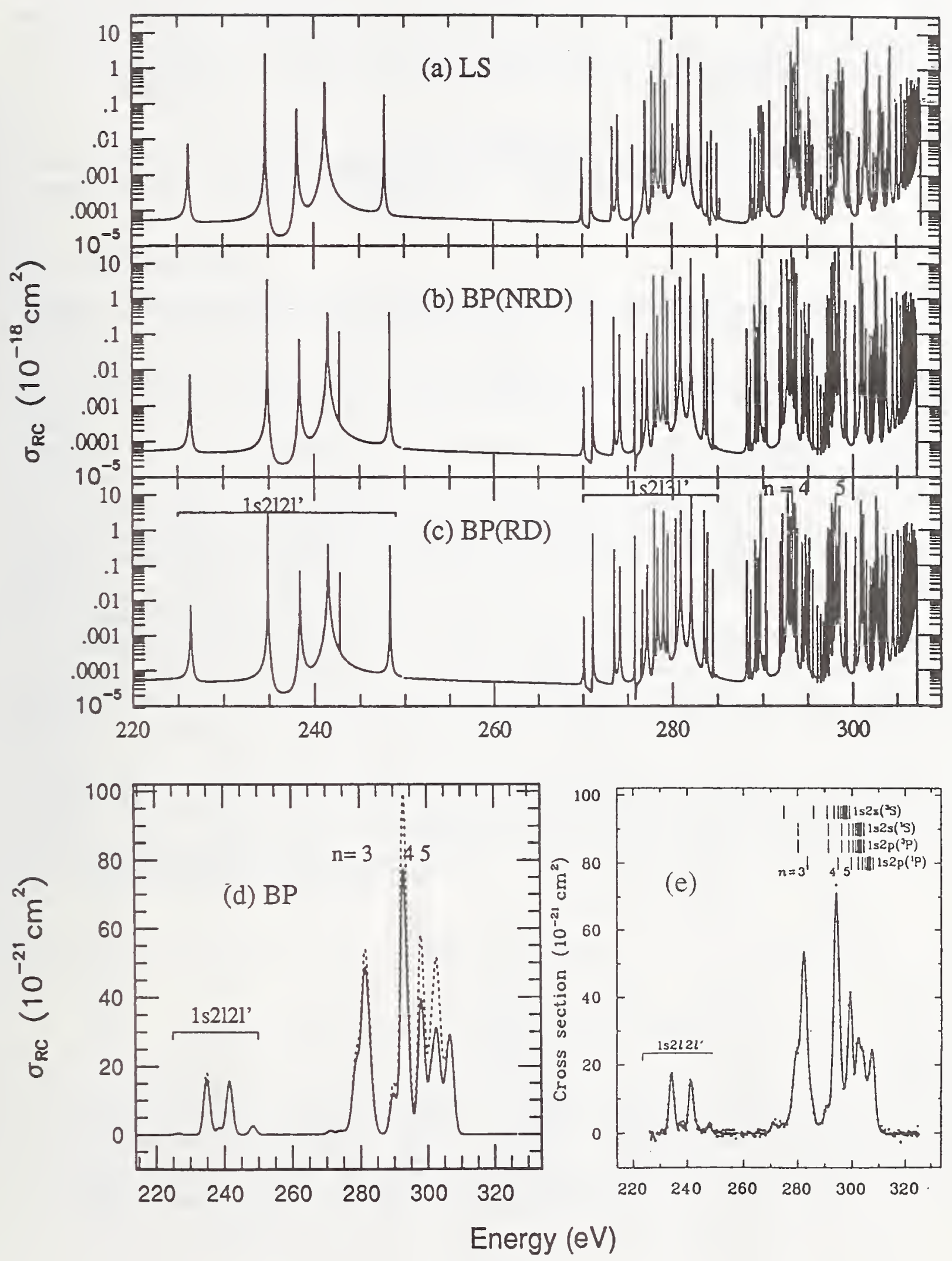

Figure 1 
The total unified recombination rate coefficients, $\alpha R(T)$, for all oxygen ions, O I - O VIII, are obtained. So far $\alpha R(T)$ have been calculated for 30 other ions including all carbon ions, nitrogen ions, and carbon sequence ions C I, N II, O III, F IV, Ne V, Na VI, Mg VII, Al VIII, Si IX, and S XI, and Si I, Si II, S II, S III, and iron ions, Fe I - Fe V.

\section{Acknowledgements}

The work was partially supported by grants from NSF (Iron Project at OSU, PHY-9421898) and NASA. The computations were carried out on the Cray Y-MP at the Ohio Supercomputer center.

\section{References}

[1] M. J. Seaton, J. Phys. B 20, 6363 (1987); K.A. Berrington, P.G. Burke, K. Butler, M.J. Seaton, P.J. Storey, K.T. Taylor, and Yu. Yan, J. Phys. B 20, 6379 (1987)

[2] R.H. Bell and M.J. Seaton, J. Phys. B 18, 1589 (1985)

[3] S.N. Nahar and A.K. Pradhan, Phys. Rev. A 49, 1816 (1994); S.N. Nahar and A.K. Pradhan, Astrophys. J. 447, 966 (1995)

[4] N.S. Scott and K.T. Taylor, Comput. Phys. Commun. 25, 347 (1982); D.G. Hummer, K.A. Berrington, W. Eissner, A.K. Pradhan, H.E. Saraph and J.A. Tully, Astron. Astrophys. 279, 298 (1993)

[5] Wolf et al, Z. Phys. D Suppl. 21, 569 (1991); Kilgus et al, Phys. Rev. Lett. 64, 737 (1990); Kilgus et al, Phys. Rev. A 47, 4859 (1993)

[6] H.L. Zhang and A.K. Pradhan, J. Phys. B 30, L571 (1997)

[7] Pequignot, D., Petitjean, P., \& Boisson, C., Astron. Astrophys. 251, 680 (1991); Nussbaumer, H. \& Storey, P.J., Astron. Astrophys. 126, 75 (1983); Shull, J.M. \& van Steenberg, M., ApJS 48, 95 (fitted from the works of Jacobs et al.) (1982a)
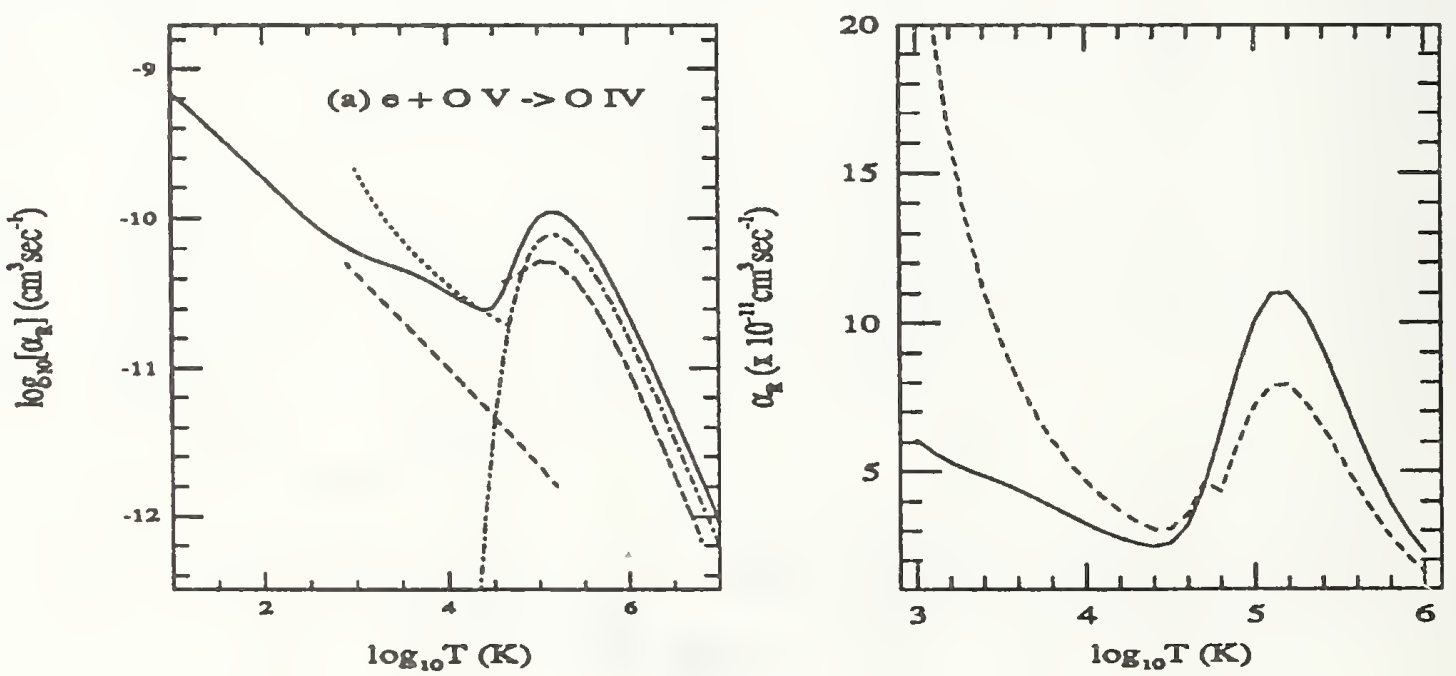

Figure 2 


\title{
K-matrix Correction of B, CB Cross-Sections
}

\author{
L. A. Vainshtein \\ P. N. Lebedev Physical Institute, Moscow, Russia
}

\section{Introduction}

There are two approaches in the calculations of excitation and ionization of atoms and ions by electrons.

$A$. Sophisticated $C C$-type methods, such as $R$-matrix [1] and $C C C$ [2]. They usually provide sufficiently accurate results. However, there are some disagreements and questions in particular cases.

$B$. Rather simple Born-type (1. order) methods $-B$ (Born), $C B$ (Coulomb-Born) with possible inclusion of exchange and normalization.

Generally, cross sections obtained by $A$-methods are preferable, but they are available for a very limited number of transitions and atoms. Fast calculations for many transitions required for atomic data in diagnostic code are in fact unreliable. Therefore, use of the $B$-methods is necessary. For example, using the code ATOM one can calculate as many cross sections and rate coefficients as necessary with minimal preparation.

Unfortunately, the accuracy of calculations by simple $C B$ methods is often insufficient. To create real databases it is important to understand the physical reasons for errors and to develop sufficiently simple ways of correcting them. One very efficient way is the $K$-matrix method. It is based on a 1 . order $B$ ( $C B$ for ions) calculation of the matrix $\mathbb{K}$ for a given set of transitions

$$
K_{\Gamma \Gamma^{\prime}}=\left\langle\Gamma|U| \Gamma^{\prime}\right\rangle, \quad \Gamma=a \epsilon l S_{T} L_{T}
$$

where $a$ is a set of target quantum numbers, $\epsilon l$ are incident electron energy and orbital momentum, and $S_{T} L_{T}$ are total angular momenta of the system. The Born $K$-matrix is used for the calculation of S-matrix and cross section $\sigma\left(a_{0}-a_{1}\right)$ :

$$
\begin{gathered}
\mathbf{S}(K)=\frac{\mathbb{I}+i \mathbb{K}(B)}{\mathbb{I}-i \mathbb{K}(B)} \\
\sigma\left(a_{0}-a_{1} \mid K\right)=\sum_{l_{0} l_{1} S_{T} L_{T}} \frac{\left[S_{T} L_{T}\right]^{2}}{\left[S_{0} L_{0}\right]^{2}}\left|S\left(\Gamma_{0}, \Gamma_{1} \mid K\right)-I\left(\Gamma_{0}, \Gamma_{1}\right)\right|^{2}
\end{gathered}
$$

where $K, B$ in the argument denote $K$-matrix and $B$ approximations. $[S L]^{2} \equiv(2 S+1)(2 L+1)$.

The full $K$-matrix approach is released in the coupled programs ATOM-AKM. They can be run on PC-486 and require about 10 minutes for 10 channels (transitions) in 10-20 energy points.

In the present report, we demonstrate two effects - normalization and channel interaction which can be readily described by the $K$-matrix method. 


\section{Normalization}

The effect of normalization is related to the unitarity of the $S$-matrix (conservation of incident particle flux). It can considerably decrease cross sections of strong transitions, such as $n l_{0}-n l_{1}$. Less evident is the similar decreasing effect for all other $n l_{0}-n^{\prime} l^{\prime}$ transitions, in particular weak ones.

The pure normalization effect can be described by an approximate $K$-matrix in the one-row one-column form :

$$
\mathbf{K}=\left\{\begin{array}{cccc}
0 & \Gamma_{0} \Gamma^{\prime} & \Gamma_{0} \Gamma^{\prime \prime} & \ldots \\
\Gamma^{\prime} \Gamma_{0} & 0 & 0 & 0 \\
\Gamma^{\prime \prime} \Gamma_{0} & 0 & 0 & 0 \\
\cdots & 0 & 0 & 0
\end{array}\right\}
$$

In this case, Eq. 2 can be solved and we obtain:

$$
\sigma\left(a_{0}-a_{1} \mid K\right)=\sum_{l_{0} l_{1} S_{T} L_{T}} \frac{\sigma\left(\Gamma_{0} \Gamma_{1} \mid B\right)}{\left[1+D\left(\Gamma_{0}\right)\right]^{2}}, \quad D\left(\Gamma_{0}\right)=\sum_{a^{\prime} l^{\prime}} K^{2}\left(\Gamma_{0} \Gamma^{\prime}\right)^{2} .
$$

One can see from Eq. 4 the influence of strong transitions on the weak ones due to the sum in $D$. Moreover, for a given initial state, $a_{0}$, the normalizing factor $(1+D)^{2}$ is the same for all final states. From now on we call the approximation given by Eqs. 3-4 normalized Born BNm, where $m$ is the number of states $a^{\prime}$ in the sum for $D$.

One important consequence is the normalization effect for all transitions from the excited states. As an illustration, we show in Fig. 1-2 the excitation and ionization cross sections in He from the $2 s^{1} S$ state. $B$ and $B N 8$ (all $n l, n=2,3,4$ ) cross sections are obtained by the code ATOM. $C C C$ data are from [2]. $B N 8$ results are considerably closer to $C C C$ as compared to $B$. One can see in Fig. 3 that the normalization effect for ionization is similar to that of excitation.

There is a large difference from experimental data for transitions from He metastables. As a rule, measured cross sections (for example see [3]) are a few times (up to an order) larger than calculated by any method. A typical example is shown in Fig. 4. The reason for such a discrepancy is not clear. Maybe it is worthwhile noting that the presence of a rather small number of atoms in the $2 p^{3} P$ state $\left(\sim 10 \%\right.$ of $\left.2 s^{3} S\right)$ might explain this disagreement (Fig. 4).

\section{Interaction of Channels}

The normalization effect decreases cross sections. Use of the full $K$-matrix in Eq. 2a yields more complicated results. Besides normalization it provides channel interaction, which manifests itself by:

i. Transitions through intermediate levels; for example $1 s-2 p-3 d$;

ii. Change of $\sigma(E)$ due to the interaction of many levels.

$1 s-2 p-3 d$ is a rather pure example of transitions through intermediate levels. Due to the dipole interaction in both stages, $\sigma(1 s-3 d)$ is considerably larger than the one obtained by the $B$ method. The case of the $2 s-3 d$ transition is more complicated: the effect of the two-stage dipole transition is partly compensated by the normalization effect (which is absent for transitions from $1 s$ state). This is manifested by the $K 36$ curve in Fig. 3. Channel interactions of many levels leads to a further decrease of the cross section (curve $K 74$ ).

All these effects are even more pronounced in the case of the transition $2 s-4 f$, see Fig 4 . 


\section{Conclusion}

Born-type methods ( $C B$ for ions) together with the many-channel $K$-matrix take into account effects of normalization and channel interactions and provide reasonable cross sections. Use of these methods in the code ATOM and complex ATOM-AKM give the possibility of a fast and comparatively simple calculation of cross sections and rate coefficients for as many transitions as necessary.

\section{References}

[1] K. A. Berrington, P. G. Burke, J. J. Chang, A. T. Chivers, W. D. Robb, K. T. Taylor, Comp. Phys. Commun. 8, 149 (1974)

K. A. Berrington, W. B. Eissner, P. H. Norrington, Comp. Phys. Commun. 92, 290 (1995)

[2] D. V. Fursa, I. Bray, Topical Rev. J.Phys B30, 757 (1997) and private communications

[3] M. E. Lagus, L. B. Boffard, L. W. Anderson, Ch. C. Lin, Ph. Rev. A53, 1505 (1996)

G. A. Piech, M. E. Lagus, L. W. Anderson, Ch. C. Lin, M. R. Flannery, Ph. Rev. A55, 2842 (1997) 


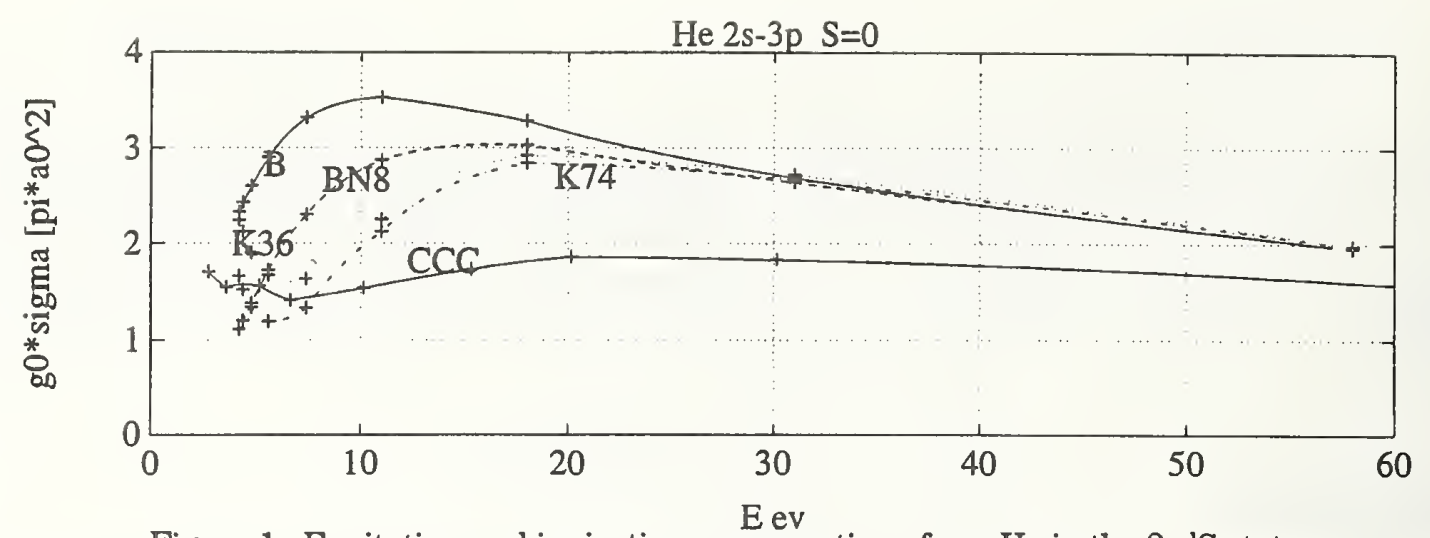

Figure 1: Excitation and ionization cross sections from $\mathrm{He}$ in the $2 \mathrm{~s}{ }^{1} \mathrm{~S}$ state.

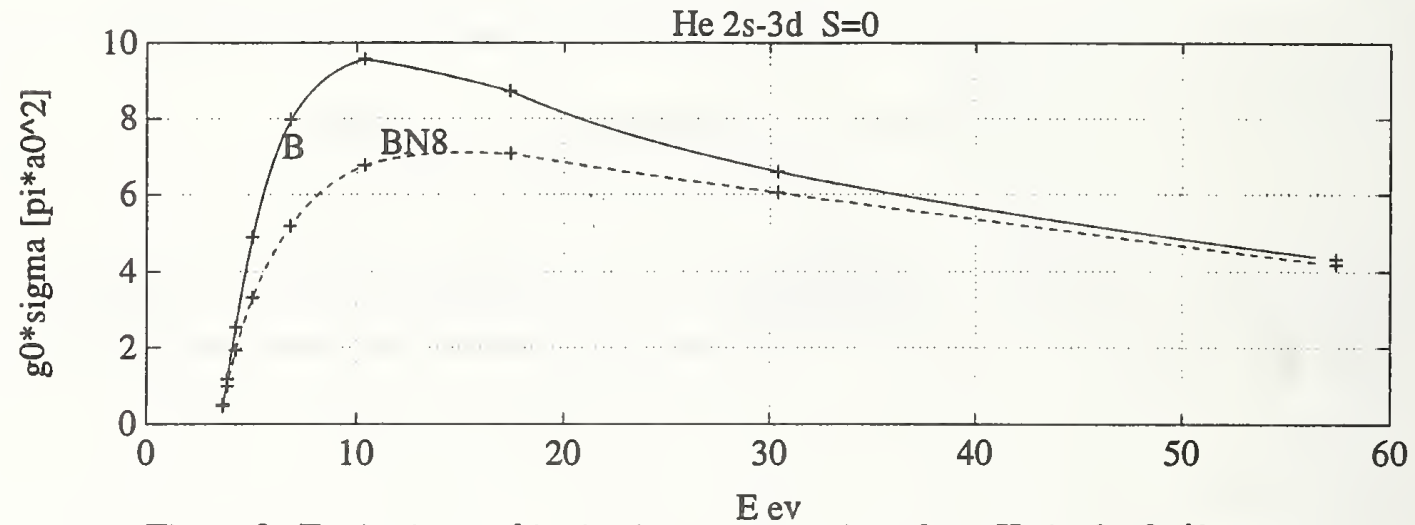

Figure 2: Excitation and ionization cross sections from $\mathrm{He}$ in the $2 \mathrm{~s}{ }^{1} \mathrm{~S}$ state.

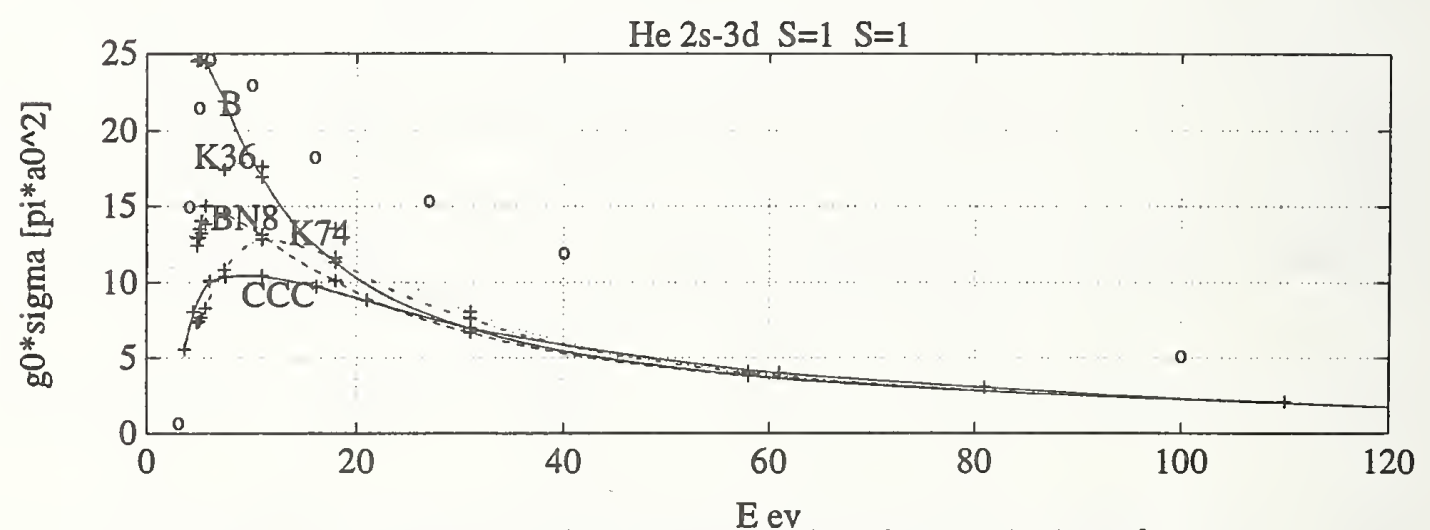

Figure 3: Excitation and ionization cross sections from $\mathrm{He}$ in the $2 \mathrm{~s}^{3} \mathrm{~S}$ state.

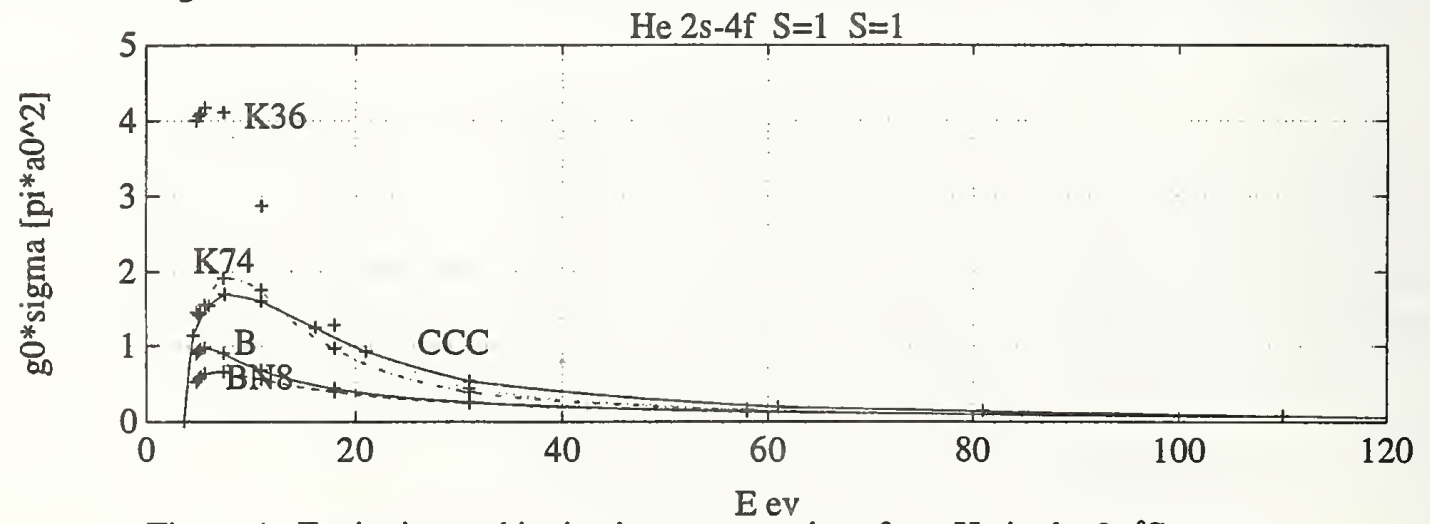

Figure 4: Excitation and ionization cross sections from $\mathrm{He}$ in the $2 \mathrm{~s}^{3} \mathrm{~S}$ state. 


\title{
The Iron Project (OSU): Large-Scale Computations of Atomic Data
}

\author{
H. L. Zhang, M. A. Bautista, S. N. Nahar, P. Romano, A. K. Pradhan \\ Department of Astronomy, The Ohio State University \\ Columbus, Ohio 43210, U.S.A.
}

\section{Introduction}

The Iron Project (IP) [1] is devoted to the study of radiative and collisional atomic processes and the calculation of large-scale accurate atomic data, primarily for the iron-group elements for applications in astrophysical and laboratory plasmas. Among the processes of particular interest are: electron impact excitation (EIE), photoionization, unified electron-ion recombination, and dipole allowed and forbidden transition probabilities. Calculations are carried out in the close-coupling (CC) approximation using the R-matrix method. The computer codes developed under the Opacity Project (OP) [2] were extended to include relativistic effects in the Breit-Pauli (BP) approximation. The codes have been also adapted to massively parallel processors, the Cray T3E and the IBM SP2. We report some sample results for one single astrophysically important ion, Fe IV, calculated recently by the Ohio-State Atomic Astrophysics group.

\section{Photoionization cross sections and oscillator strengths}

Computations have been carried out for photoionization cross sections, $\sigma_{P I}$, and oscillator strengths (f-values) for dipole allowed transitions for Fe IV [3]. A 31-state eigenfunction expansion formed from the configurations $3 d^{4}, 3 d^{3} 4 s$, and $3 d^{3} 4 p$ of $\mathrm{Fe} \mathrm{V}$ is used. The results include 746 LS bound states of sextet and quartet symmetries with $n \leq 10$, f-values for 34,635 LS transitions, and detailed $\sigma_{P I}$ with extensive resonance structures for all bound states. The new $\sigma_{P I}$ for the ground ${ }^{6} S$ state of Fe IV exhibits a large resonance near the threshold (Fig. 1) not found in previous calculations for the OP and is likely to affect quantities such as the effective photoionization rate, the opacity, and the recombination rate significantly. The resonance has been identified as the $3 s^{2} 3 p^{5} 3 d^{6}\left({ }^{6} P^{o}\right)$ state [3].

\section{Excitation collision strengths and rate coefficients}

Excitation collision strengths, $\Omega(E)$, and maxwellian averaged rate coefficients, $\Upsilon(T)$, of Fe IV have been calculated for 8,771 non-vanishing transitions among 140 fine structure levels, dominated by the ground and excited configurations $3 d^{5}, 3 d^{4} 4 s$, and $3 d^{4} 4 p$ [4]. Calculations are carried out using a 49 term expansion in the CC approximation employing the R-matrx method. $\Omega(E)$ is obtained for electron energies up to 15 rydbergs, and $\Upsilon(T)$ is obtained at a wide range of temperatures. Fig. 2 presents $\Omega(E)$ for transitions between levels within the ground configuration $3 d^{5}$. 


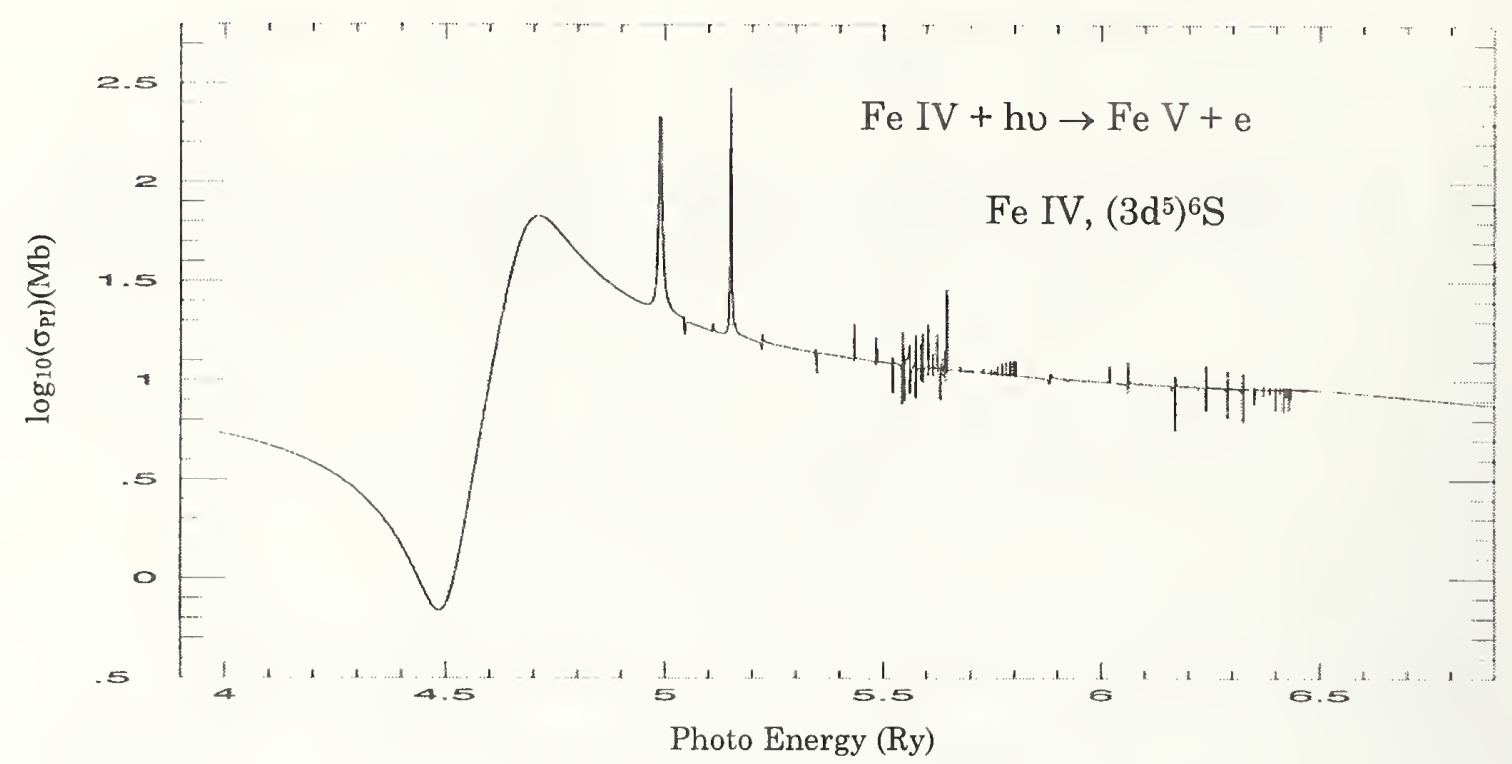

Figure 1

\section{Electron-ion recombination}

Calculations of the total electron-ion recombination rate coefficient, $\alpha_{R}(T)$, of low charged iron ions, such as Fe I - V, are being carried out [5] through large-scale computations employing the unified treatment of Nahar and Pradhan [6]. The treatment subsumes the radiative recombination (RR) and dielectronic recombination (DR) in a unified, self-consistent manner. This work represents the first detailed study of electron-ion recombination for these iron ions. Previous results are from much simpler approximations, such as central-field and hydrogenic approximations for $R R$ at lower temperatures and the Burgess general formula for DR at higher temperatures. Fig. 3(a) shows the general shape of $\alpha_{R}(T)$ for Fe IV in a wide temperature region. Starting with a high recombination rate, $\alpha_{R}(T)$ decreases to a minimum at $\log _{10}(T)=4.8 \mathrm{~K}$; above that the rate increases due to DR peaking at $\log _{10}(T)=5.3 \mathrm{~K}$, beyond which it falls monotonically. Fig. $3($ b) presents a detailed comparison of our total rate (solid curve) with the currently used ones [9] for the temperature range of significant $\mathrm{Fe}$ IV abundance. The currently used rates are underestimated at lower $\mathrm{T}$ because autoionizing resonances are not included in the $\mathrm{RR}$ rates and are overestimated at higher $\mathrm{T}$ because interference effects with continuum and autoionization into lower states are not included in the Burgess formula for DR.

\section{Resonance Averaged Photoionization Cross Sections for Astro- physical Modeling}

Under the OP and the IP, accurate photoionization cross sections, $\sigma_{P I}$, have been calculated incorporating the complex autoionizing resonance structures. Owing to the complexity in the structures thousands of points are normally calculated to represent the detailed features for each bound state of the ion. While this is a great advance in terms of atomic physics and accuracy, the huge amount and the inherent details of the data do present a serious practical problem for numerical modeling. We present the resonance averaged $\sigma_{P I}$ for the ground state of atoms and ions, available from the electronic OP database TOPbase [7] and the new data for the low ionization stages of iron Fe I - V. The average is obtained by convolving the detailed $\sigma_{P I}$ with a Gaussian distribution over the autoionizing resonances. This preserves the overall resonant contribution to the cross sections in 


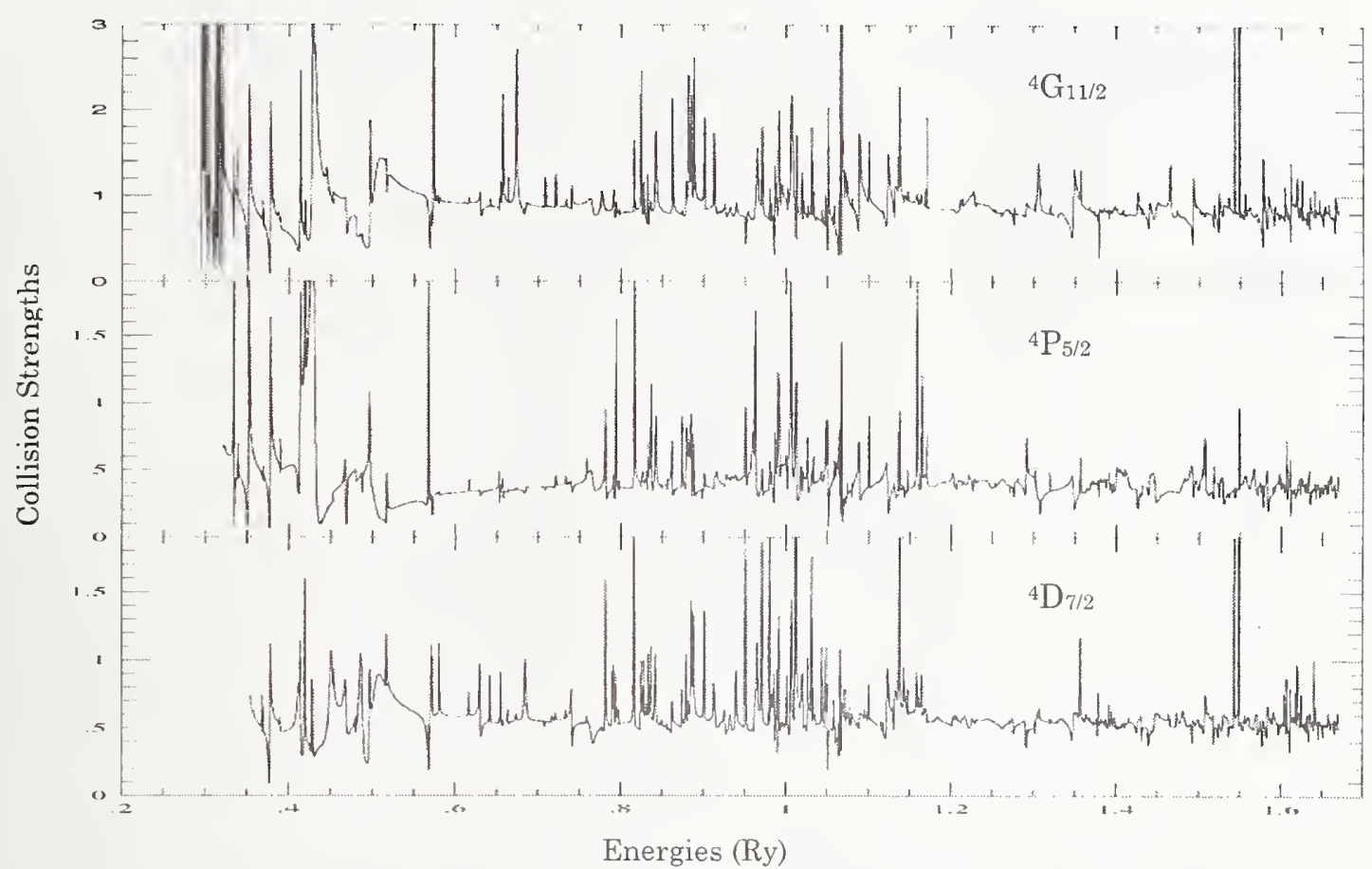

Figure 2

the important near-threshold regions. The effective cross sections are then represented by a small number of points that can be readily interpolated linearly for practical applications. A Fortran subroutine and data will be available electronically. Sample averaged cross sections along with the detailed ones are presented in Fig. 4.

\section{Conclusion}

Large-scale $a b$ initio calculations are carried out for atomic quantities of photoionization, oscillator strengths, excitation collision strengths and total electron-ion recombination rate coefficients. Resonance averaged photoionization cross sections are also presented for astrophysical models.

Acknowledgements: The work was partially supported by grants from NSF (Iron Project at OSU, PHY-9421898), and NASA. The computations were carried out on the Cray Y-MP at the Ohio Supercomputer center.

\section{References}

[1] D. G. Hummer, K. A. Berrington, W. Eissner, A. K. Pradhan, H. E. Saraph, and J. A. Tully, Astron. Astrophys. 279, 298 (1993)

[2] M. J. Seaton, J. Phys. B 20, 6363 (1987); K. A. Berrington, P. G. Burke, K. Butler, M. J. Seaton, P. J. Storey, K. T. Taylor, and Yu. Yan, J. Phys. B 20, 6379 (1987)

[3] M. A. Bautista and A. K. Pradhan, Astron. Astrophys. Suppl. (in press, 1997)

[4] H. L. Zhang and A. K. Pradhan, Astron. Astrophys. Suppl. (in press, 1997)

[5] S. N. Nahar, M. A. Bautista, and A. K. Pradhan, Astrophys. J. 479, 497 (1997) (Fe I); S. N. Nahar, Phys. Rev. A 55, 1980 (1997) (Fe II); S. N. Nahar, Phys. Rev. A 53, 2417 (1996) (Fe III); S. N. Nahar, M. A. Bautista, and A. K. Pradhan (Fe IV) (submitted, 1997) 

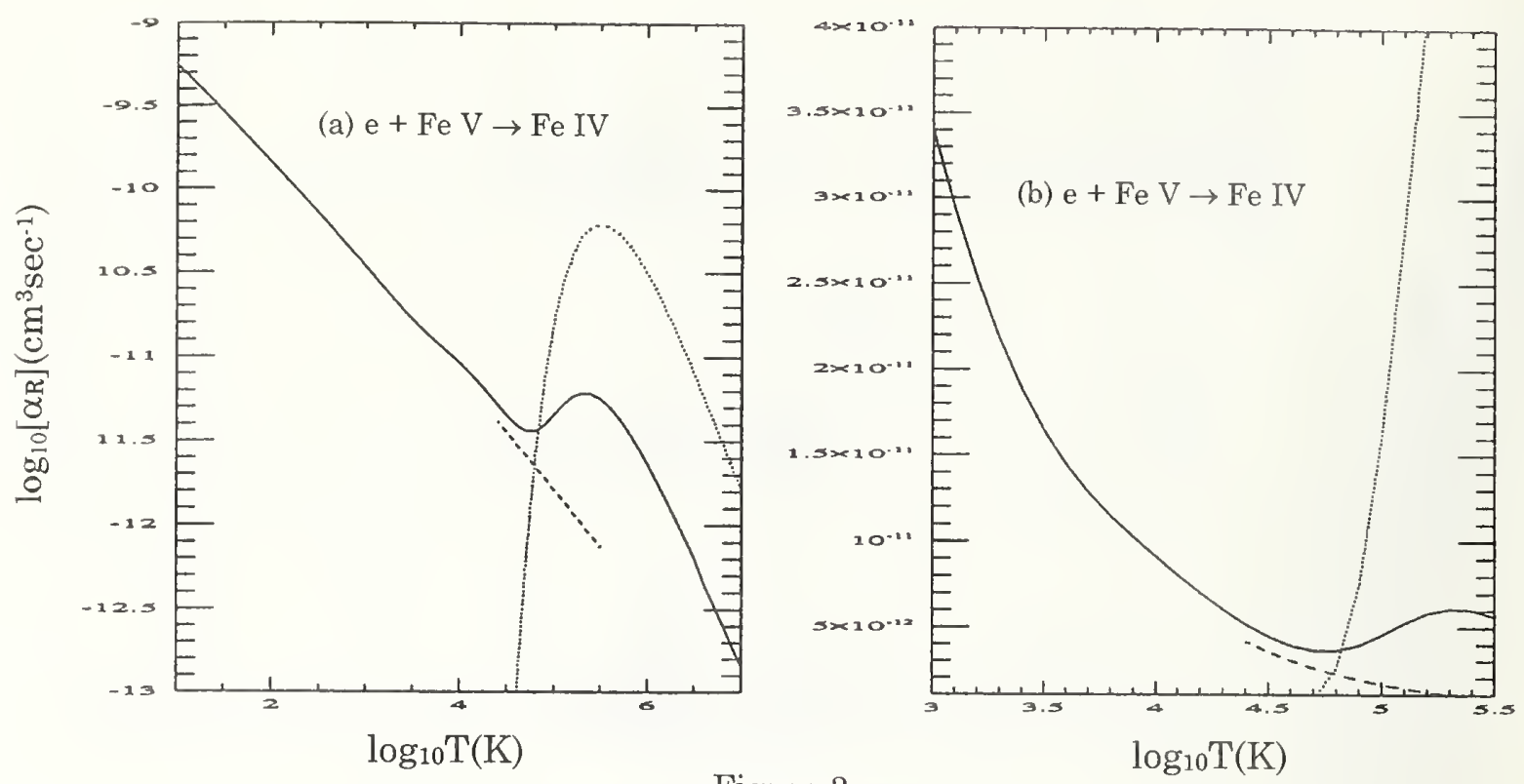

Figure 3

[6] S. N. Nahar and A. K. Pradhan, Phys. Rev. A 49, 1816 (1994); S. N. Nahar and A. K. Pradhan, Astrophys. J. 447, 966 (1995)

[7] W. Cunto, C. Mendoza, F. Ochsenbein, and C.J. Zeippen, Astron. Astrophys. 275, L5 (1993)

[8] N. S. Scott and K. T. Taylor, Comput. Phys. Commun. 25, 347 (1982); D. G. Hummer, K. A. Berrington, W. Eissner, A. K. Pradhan, H. E. Saraph, and J.A. Tully, Astron. Astrophys. 279, 298 (1993)

[9] D. T. Woods, J. M. Shull and C. L. Sarazin, Astrophys. J. 249, 399 (1981)

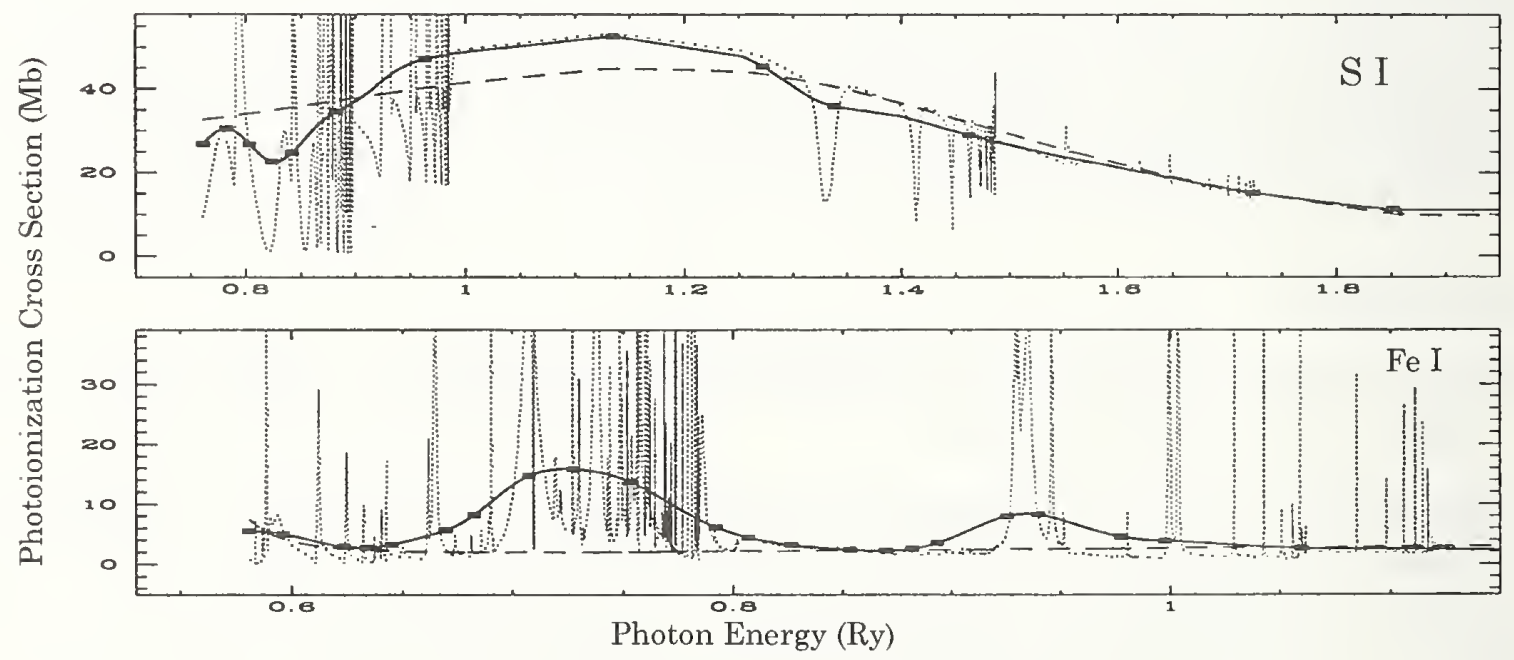

Figure 4 
\begin{tabular}{l|l}
3 & Data \\
Production
\end{tabular}

3.3 Molecules 



\title{
Ultraviolet Emission in the Dissociation of $\mathrm{N}_{2}$ and $\mathrm{O}_{2}$ by $\mathrm{K}^{+}$Impact
}

\author{
M. R. Gochitashvili, B. I. Kikiani, R. V. Kvizhinadze, and N. R. Jaliashvili \\ Physics Department, Tbilisi State University, \\ Tbilisi, 380028 Georgia
}

Ion-impact processes on molecular nitrogen and oxygen, $\mathrm{N}_{2}$ and $\mathrm{O}_{2}$, have been studied extensively because of their importance in upper-atmosphere reactions and gas discharges. The excitation function and absolute emission cross section of atomic lines of nitrogen and oxygen have been measured in the vacuum ultraviolet (VUV) spectral region from $50 \mathrm{~nm}$ to $130 \mathrm{~nm}$ by $\mathrm{K}^{+}$ionimpact dissociative excitation of $\mathrm{N}_{2}$ and $\mathrm{O}_{2}$ molecules in the $\mathrm{K}^{+}$projectile energy range $3-8 \mathrm{KeV}$. The experimental arrangement and calibration procedures of the light-recording system have been described in more detail previously [1]. The uncertainties in the absolute values of the cross sections given here are estimated to be about $20 \%$. Polarization of the radiation emitted in the investigated processes is assumed to be negligible.

Fig. 1 shows examples of the emission spectrum obtained for the collision of $\mathrm{K}^{+}$. ions with $\mathrm{N}_{2}$ and $\mathrm{O}_{2}$ at a laboratory energy of $4.0 \mathrm{keV}$ for the $\mathrm{K}^{+}$ion. Only the atomic lines of nitrogen and oxygen are observed. These results indicate that direct dissociation plays an important role in the formation of $\mathrm{N}^{*}$ and $\mathrm{O}^{*}$. There are many dissociative highly excited (Rydberg) molecular states that can produce a contribution to this inelastic channel. It is necessary to mention that the direct excitation of dissociative molecular states by alkali metal ions is favored energetically. For example, the minimum energies which are needed to produce the emission of dissociative products of $\mathrm{N} \mathrm{I}$ (at $\lambda=120.0 \mathrm{~nm}$ ) are $\Delta \mathrm{E}=20.1 \mathrm{eV}, 30.2 \mathrm{eV}, 34.6 \mathrm{eV}$ in direct-dissociative, charge-exchange dissociative and ionization-dissociative processes, respectively.

The energy dependence of the emission cross-sections of excited atomic products of $\mathrm{N}$ and $\mathrm{O}$ is presented in Fig. 2. It seems that the cross sections are independent of the energy of the $\mathrm{K}^{+}$ion in the investigated energy range. It appears that the "core" ion model [2] is adequate to interpret the experimental results. This model predicts that dissociation is governed entirely by the core of the ion and strongly depends on the form of the potential curves. Potential curves of the highly excited molecule will be parallel to those of the core ion and lie lower in energy (electron binding energy). The excited molecular states whose cores decay spontaneously in the Frank-Condon region give rise to a major contribution of the formation of excited atomic products. After the decay of the molecular ion core the weakly bound electron is moving to the atomic ions $\mathrm{N}^{+}$or $\mathrm{O}^{+}$and captured by them in excited states.

In beginning to explain the dissociation mechanism we should remember that the spin conservation rule is valid, because the spin-orbital interaction is ignored in our case. This implies that during $\mathrm{K}^{+}-\mathrm{N}_{2}$ collisions the intermediate molecular state which is excited is a singlet. Therefore, both fragments of atoms are left in the same state multiplicities. In the case of the excitation of the more intense nitrogen atomic line at $\lambda=120.0 \mathrm{~nm}$ (transition $3 \mathrm{~s}{ }^{4} \mathrm{P}-2 \mathrm{p}^{3}{ }^{4} \mathrm{~S}$ ) both fragments of the dissociation must be formed in quartet states in the following way:

$$
\mathrm{K}^{+}\left(3 \mathrm{p}^{6}{ }^{1} \mathrm{~S}\right)+\mathrm{N}_{2}\left(\mathrm{X}^{1} \sum_{\mathrm{g}}{ }^{+}\right) \rightarrow \mathrm{K}^{+}+\mathrm{N}\left(3 \mathrm{~s}^{4} \mathrm{P}\right)+\mathrm{N}\left(2 \mathrm{p}^{3}{ }^{4} \mathrm{~S}\right)
$$


Similarly, the atomic line at $\lambda=124.3 \AA$ (transition $3 \mathrm{~s}^{\prime}{ }^{2} \mathrm{D} \rightarrow 2 \mathrm{p}^{3}{ }^{2} \mathrm{D}$ ) can be emitted as a direct result of the dissociation process of $\mathrm{N}_{2}$ when both fragments are formed in doublet states:

$$
\mathrm{K}^{++} \mathrm{N}_{2} \rightarrow \mathrm{K}^{+}+\mathrm{N}\left(3 \mathrm{~s}^{\prime}{ }^{2} \mathrm{D}\right)+\mathrm{N}\left(2 \mathrm{p}^{3}{ }^{2 \mathrm{D}}\right)
$$

In the framework of the core ion model the emission of the more intense nitrogen atomic line at $\lambda=120.0 \mathrm{~nm}$ may be caused by the excitation of an intermediate molecular singlet state of $\mathrm{N}_{2}$ * in which the ion core $\mathrm{N}_{2}{ }^{+}$is in the states $\mathrm{C}^{2} \Sigma_{\mathrm{u}^{+}}$or $\mathrm{D}^{2} \pi_{\mathrm{g}}$ (Fig. 3). The dissociation limit for these cores is $\mathrm{N}\left(2 \mathrm{p}^{3}{ }^{4} \mathrm{~S}\right)-\mathrm{N}^{+}\left(2 \mathrm{p}^{2}{ }^{3} \mathrm{P}\right)$. For the $\mathrm{C}^{2} \sum_{\mathrm{u}}$ state this dissociation limit is reached by predissociation through the ${ }^{4} \Pi_{\mathrm{u}}$ state. Formation of the nitrogen atomic line at $\lambda=123.4 \mathrm{~nm}$ can be due to the decay of an excited intermediate molecular state of $\mathrm{N}_{2}{ }^{*}$ with core $\mathrm{N}_{2}+\left(2 \Pi_{\mathrm{u}}\right)$. The dissociation limit for this core is $\mathrm{N}\left(2 \mathrm{p}^{3}{ }^{2} \mathrm{D}\right)+\mathrm{N}^{+}\left(2 \mathrm{p}^{2}{ }^{3} \mathrm{P}\right)$. In both cases the excited atomic products are formed when the weakly bonding molecular electron is attached to the $\mathrm{N}^{+}\left(2 \mathrm{p}^{2}{ }^{3} \mathrm{P}\right)$ ion. A high value of the cross section in the first case may be connected with a comparably small value of energy defects for the inelastic process in this case.
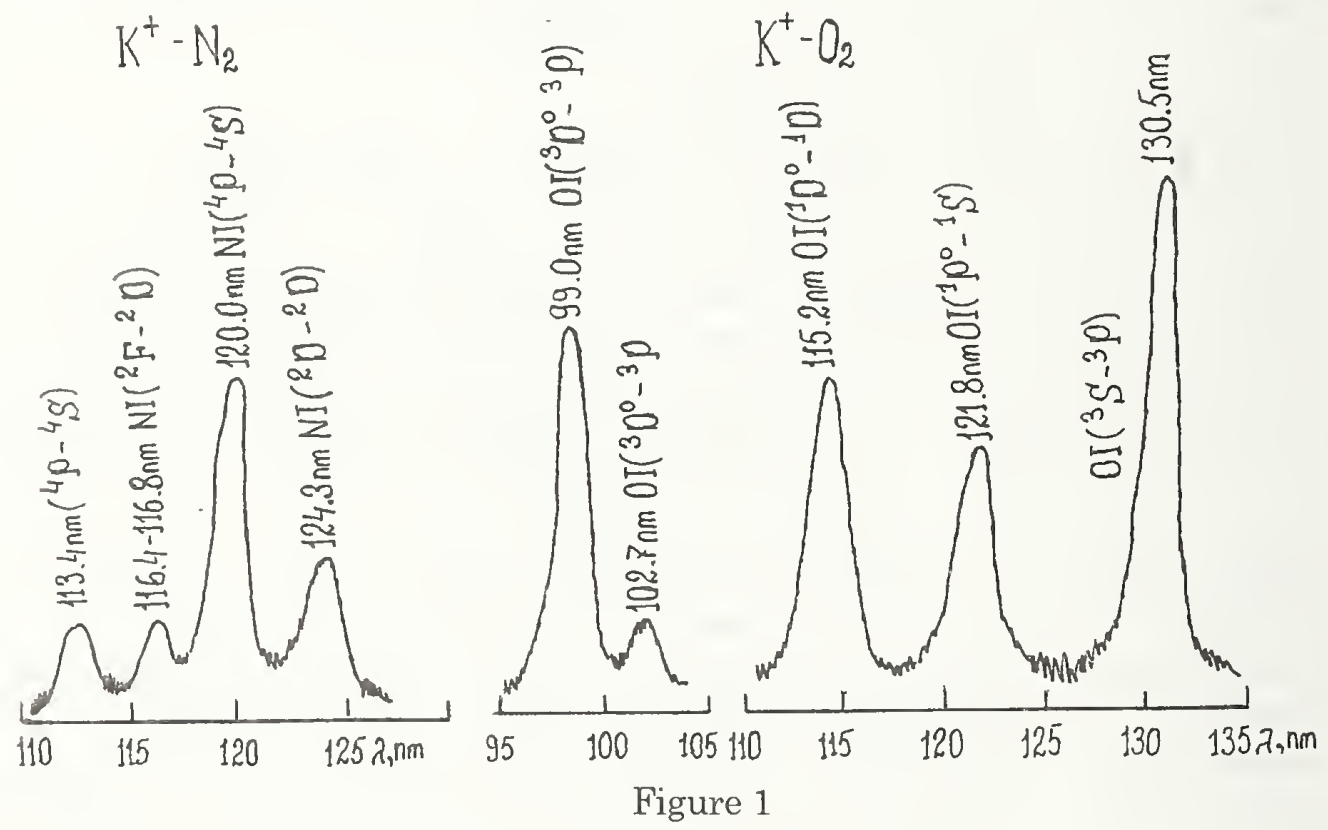

In the $\mathrm{K}^{+}-\mathrm{O}_{2}$ collision the intermediate highly excited state of molecular oxygen must be a triplet because the oxygen molecule in the ground state is in the triplet state, too. Following the spin conservation rule the summed spin of the dissociative fragments should be equal to one. In the framework of the core ion model the observation of the more intense oxygen atomic lines at $\lambda=99.0$ $\mathrm{nm}$ (transition $3 \mathrm{~s}^{1}{ }^{3 \mathrm{D}} \rightarrow 2 \mathrm{p}^{4}{ }^{3} \mathrm{P}$ ) and at $\lambda=130.5 \mathrm{~nm}$ (transition $3 \mathrm{~s}^{3} \mathrm{~S} \rightarrow 2 \mathrm{p}^{4}{ }^{3} \mathrm{P}$ ) may be due to the excitation of an intermediate molecular triplet state $\mathrm{O}_{2}{ }^{*}$ with an ion core $\mathrm{O}_{2}{ }^{+}$in the $\mathrm{A}^{2} \Pi_{\mathrm{u}}$ or $\mathrm{B}^{4} \Sigma_{\mathrm{g}}$ states. The dissociation limit for these cores is $\mathrm{O}^{+}\left({ }^{4} \mathrm{~S}\right)-\mathrm{O}\left({ }^{3} \mathrm{P}\right)$. The triplet $3 \mathrm{~s}^{\prime}{ }^{3} \mathrm{D}$ and $3 \mathrm{~s}{ }^{3} \mathrm{~S}$ states of the oxygen atom can be formed as a result of the capture of an electron by the $\mathrm{O}^{+}\left({ }^{4} \mathrm{~S}\right)$ ion and hence these dissociative fragments must be correlated with the dissociative fragment $\mathrm{O}\left({ }^{3} \mathrm{P}\right)$. Similarly, the formation of the oxygen atomic lines at $\lambda=115.2 \mathrm{~nm}$ (transition $3 \mathrm{~s}^{\prime}{ }^{1} \mathrm{D}^{0} \rightarrow \mathrm{p}^{4}{ }^{1} \mathrm{D}$ ) and at $\lambda=121.8 \mathrm{~nm}$ (transition $3 \mathrm{~s}^{\prime 1}{ }^{1} \mathrm{P}^{0} \rightarrow 2 \mathrm{p}^{4}{ }^{1} \mathrm{~S}$ ) may be caused by the decay of the intermediate molecular triplet state with the core ${ }^{2} \Pi_{\mathrm{u}}(\mathrm{III})$. The dissociation limit for them is $\mathrm{O}^{+}\left({ }^{2} \mathrm{D}\right)-\mathrm{O}\left({ }^{3} \mathrm{P}\right)$ when a weakly bonded electron is attracted by $\mathrm{O}^{+}(2 \mathrm{D})$ ions and captured by them. The formation of 
singlet states $3 s^{1}{ }^{1 D^{0}}$ or $3 s^{\prime \prime}{ }^{1 P^{0}}$ takes place and hence these dissociative fragments should be correlated with the fragment $\mathrm{O}\left({ }^{3} \mathrm{P}\right)$, too.

In summary, it is necessary to mention that all core states $\mathrm{N}_{2}+\left(\mathrm{C}^{2} \Sigma_{\mathrm{u}^{+}}, \mathrm{D}^{2} \Pi_{\mathrm{g}},{ }^{2} \Pi_{\mathrm{u}}\right)$ and $\mathrm{O}_{2}{ }^{+}\left(\mathrm{A}^{2} \Pi_{\mathrm{u}}\right.$, $\left.\mathrm{B}^{4} \sum_{\mathrm{g}^{*}},{ }^{2} \Pi_{\mathrm{u}}(\mathrm{III})\right)$ decay spontaneously in the Frank-Condon region.
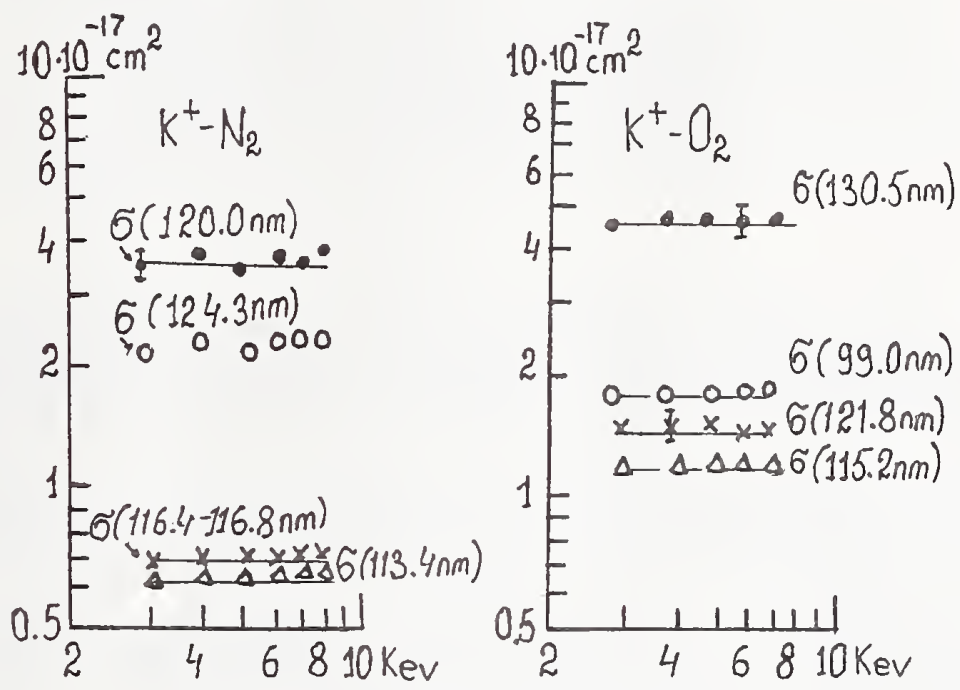

Figure 2

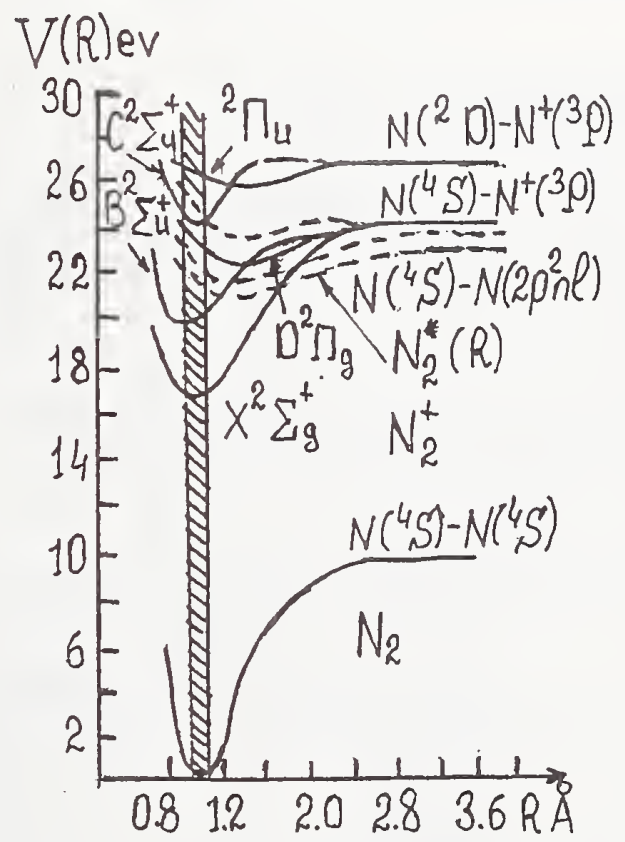

Figure 3

\section{References}

[1] M. R. Gochitashvili, R. V. Kvizhinadze, N. R. Jaliashvili, and B. I. Kikiani, Zh. Tech. Fiz. 49 2338-43 (1993); see also the contributed paper by M. R. Gochitashvili, B. I. Kikiani, and R. A. Lomsadze "Measurement of the absolute cross section of inelastic processes in slow atomic collisions", in these proceedings

[2] K. C. Singlet, J. A. Schiavone, and R. S. Freund, J. Chem. Phys. 59, 5225-40 (1973) 


\title{
Electron Collision Cross Sections for the $\mathrm{CF}_{4}$ Molecule by Electron Swarm Study
}

\author{
Yuichi Hayashi and Yoshiharu Nakamura \\ Faculty of Science and Technology, Keio University, \\ 3-14-1 Hiyoshi, Yokohama 223, Japan
}

\section{Introduction}

Tetrafluoromethane $\left(\mathrm{CF}_{4}\right)$ is widely used as working gas for gas discharge opening switches and in etching plasmas for material processing [1]. Thus it is important to know an accurate electron collision cross section set for the $\mathrm{CF}_{4}$ molecule in order to quantitatively understand the plasma phenomena occurring in the above mentioned area. But the cross section data for $\mathrm{CF}_{4}$ in the low energy region is not sufficiently reliable at present. Magnitudes and energy dependence of vibrational excitation cross sections and the momentum transfer cross section, in particular, are considerably different according to various researchers [1,2]. We therefore have tried to determine low energy electron collision cross sections for the $\mathrm{CF}_{4}$ molecule mainly from our own experimental results for the electron transport coefficients.

\section{Experimental Description}

We used a double shutter drift tube with variable drift distance $(1-10 \mathrm{~cm})$ and measured the arrival time spectra of electrons at each $\mathrm{E} / \mathrm{N}$ value. From those spectra, the electron drift velocity, $\mathrm{W}$, and the product of the gas number density and the longitudinal diffusion coefficient, $\mathrm{ND}_{\mathrm{L}}$, were obtained [3]. The electron transport coefficients were measured in pure $\mathrm{CF}_{4}$ and two $\mathrm{CF}_{4}-\mathrm{Ar}$ mixtures. All the measurements were carried out at room temperature, and at each $\mathrm{E} / \mathrm{N}$, the measurements were repeated at three different pressures, at least. The advantages of using both dilute molecular-rare gas mixtures and a pure molecular gas to determine the set of cross sections for the molecular gas are mentioned in Ref. [4].

The mixing ratios of the gases and the range of the measurements are shown in Table 1.

Table 1: Mixing Ratios and the Range of the Measurements

\begin{tabular}{|l|l|l|}
\hline & $\mathrm{E} / \mathrm{N}(\mathrm{Td})$ & Gas Pressure (Torr) \\
\hline pure $\mathrm{CF}_{4}(99.99 \%)$ & $0.04-300$ & $0.03-700$ \\
$5.08 \% \mathrm{CF}_{4}-\mathrm{Ar}$ & $0.14-100$ & $1.45-300$ \\
$0.495 \mathrm{CF}_{4} \mathrm{Ar}$ & $0.04-100$ & $1.5-700$ \\
\hline
\end{tabular}

\section{Results of the Measurements}

Fig. 2 shows the electron drift velocity, $W$ (solid squares), and $\mathrm{ND}_{\mathrm{L}}$ (solid circles) measured in pure $\mathrm{CF}_{4}$. In this figure, the measured drift velocity (crosses) by Hunter [5] is also shown for 
comparison. Figs. 3 and 4 show W and NDL, with the same symbols as in Fig. 2 measured in the $5.08 \%$ and $0.495 \% \mathrm{CF}_{4}$-Ar mixtures, respectively.

\section{Boltzmann Equation Analysis}

A multi-term code of the Boltzmann equation analysis [6] and a Monte Carlo simulation (MCS) were used, and an initial set of the cross sections for the $\mathrm{CF}_{4}$ molecule was modified by trial and error until consistency between the electron transport coefficients and the experimental results was obtained while applying the following steps:

Step 1 Modify inelastic cross sections (mainly vibrational excitation cross sections) until the calculated $W$ and NDL in the two mixtures agree with the experimental results in the $\mathrm{CF}_{4}$-Ar-mixtures.

Step 2 Modify elastic momentum transfer cross sections so that the calculated W and NDL for the pure $\mathrm{CF}_{4}$ molecule agree with the experimental results in pure $\mathrm{CF}_{4}$.

Step 3 Modify inelastic cross sections except vibrational excitation cross sections until the calculated attachment and ionization coefficients agree with the experimental results for pure $\mathrm{CF}_{4}$.

The cross section set for the Ar atom determined by Nakamura and Kurachi [7] was used throughout the study. The W and $\mathrm{ND}_{\mathrm{L}}$ calculated using the latest cross sections (Fig. 1) are shown in Figs. 2-4 by solid lines. The calculated attachment and ionization coefficients are shown by solid lines in Fig. 5, with the experimental results from [8-13].

As is seen in Figs. 2-5, the agreement between measurements and calculations is fairly good.

\section{A Set of Cross Sections for the $\mathrm{CF}_{4}$ Molecule}

The latest cross section set for the $\mathrm{CF}_{4}$ molecule is summarized in Fig. 1. This cross section set includes momentum transfer $\left(\mathrm{Q}_{\mathrm{m}}\right)$, vibrational excitation $\left(\mathrm{Q}_{\mathrm{v}}\right)$, electron attachment $\left(\mathrm{Q}_{\mathrm{a}}\right)$, neutral dissociation $\left(\mathrm{Q}_{\mathrm{dn}}\right)$, and ionization $\left(\mathrm{Q}_{\mathrm{i}}\right)$. Also shown in this figure is the total scattering cross section by L. G. Christophorou, J. K. Olthoff, and M. V. V. S. Rao [7].

In this cross section set, four vibrational excitation cross sections, $\mathrm{v} 4$ (degenerate deformation, $78 \mathrm{meV}$ ), v1 (symmetric stretch, $112 \mathrm{meV}$ ), v3 (asymmetric stretch, 157meV), and 2v3 (the second harmonics of v3) were considered in accordance with the energy loss spectra measured by an electron beam experiment [14] at a resonance peak centered at $8 \mathrm{eV}$, and the relative magnitude was also maintained in the present vibrational cross sections.

The Ramsauer-Townsend minimum of the momentum transfer cross section is similar to that of L. G. Christophorou, J. K. Olthoff, and M. V. V. S. Rao [15]. The present attachment cross section is about $20 \%$ smaller than given by them, but the neutral dissociation cross section is much larger. 


\section{Conclusion}

The drift velocity, $\mathrm{W}$, and the product of the gas number density and the longitudinal diffusion coefficient, $\mathrm{ND}_{\mathrm{L}}$, were measured in pure $\mathrm{CF}_{4}$ and $\mathrm{CF}_{4}$ - $\mathrm{Ar}$ mixtures over a wide $\mathrm{E} / \mathrm{N}$ range.

A cross section set for the $\mathrm{CF}_{4}$ molecule being consistent with the measured electron transport coefficients (drift velocity, longitudinal diffusion coefficient, attachment coefficients, ionization coefficients) was obtained.

\section{References}

[1] A C.Bordage, P. Segur and A. Chouki, J. AppL Phys. 801325 (1996)

[2] H. Itoh et al., Tech. Papers of Gas Disch. Committee IEE Japan ED-93-93 (1993)

[3] A Kurachi and Y. Nakamura, J. Phys. D 21602 (1988)

[4] Y. Shishikura, K. Asano and Y. Nakamura, J. Phys. D 301610 (1997)

[5] S. R. Hunter, J. G. Carter, and L. G. Christophorou, Phys. Rev. A 3858 (1988)

[6] R. E. Robson and K. F. Ness, Phys. Rev. A 332068 (1986)

[7] Y. Nakamura and M. Kurachi, J. Phys. D 21718 (1988)

[8] I. M.Bortnik, and A. A. Panov, Sov. Phys. Tech. Phys. 16571 (1986)

[9] M. S.Naidu,and A. N.Prasad, J. Phys. D 5983 (1972)

[10] C. S. Lakshminarasimha, J. Lucas, and D. A. Price, Proc. IEE 1201044 (1973)

[11] S. E. Bozin, and C. C. Goodyear, J. Phys. D 1327 (1968)

[12] C. S. Lakshminarasimha, J. Lucas, and R. A. Snrlson, Proc. IEE 1221162 (1975)

[13] S. R. Hunter, I G. Carter, and L. G. Christophorou, J. Chem. Phys. 86693 (1986)

[14] L. Boesten, H. Tanaka, A. Kobayashi, A A. Dillon, and M. Kimura, J. Phys. B 251607 (1992)

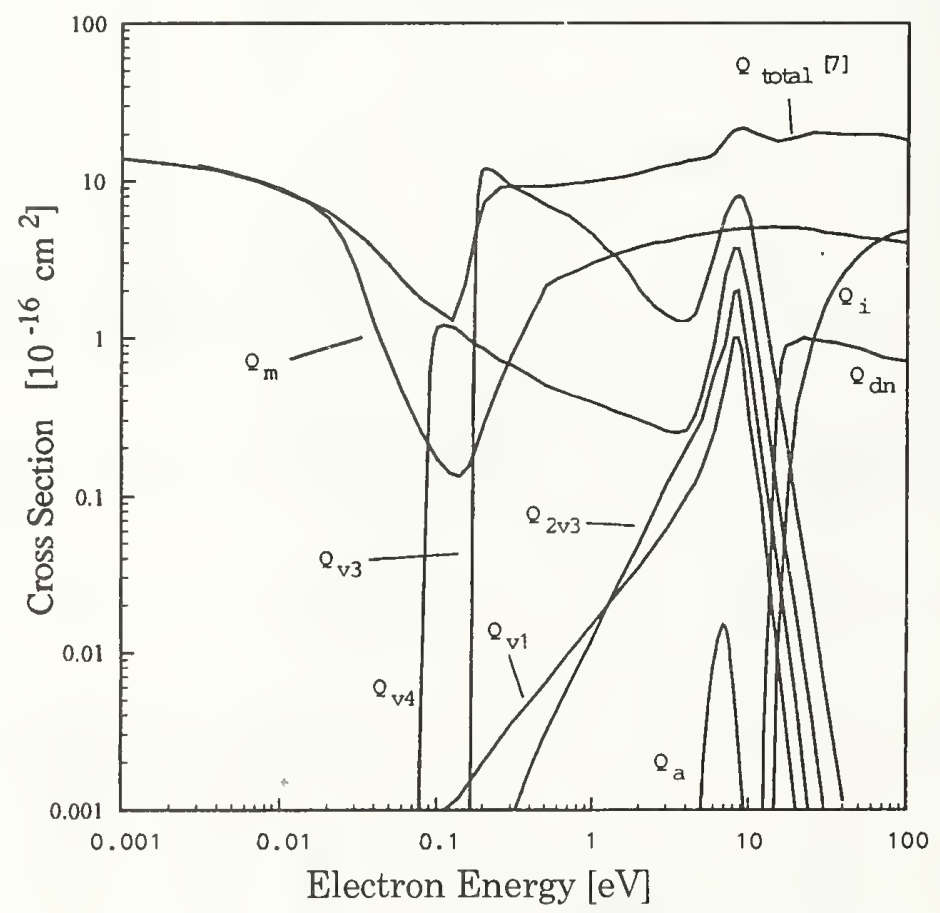

Figure 1: A set of electron collision cross sections for the $\mathrm{CF}_{4}$ molecule. 


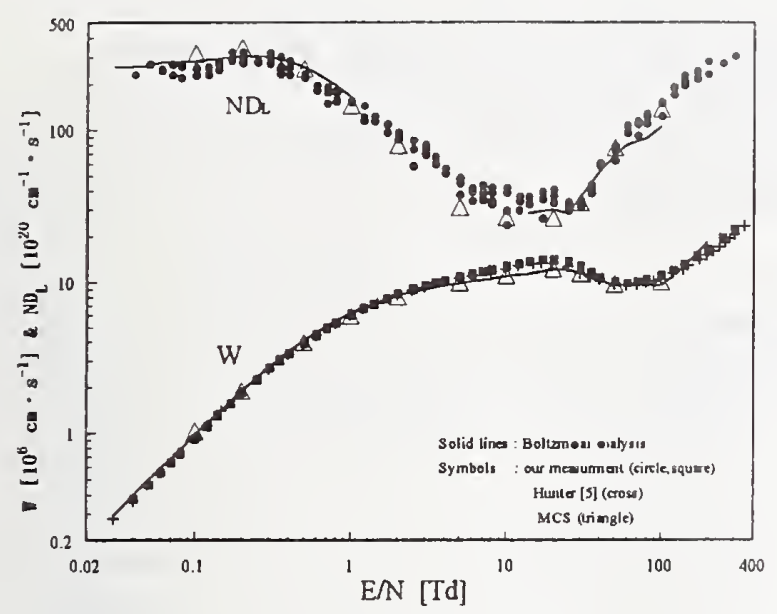

Figure 2: $\mathrm{W}$ and $\mathrm{ND}_{\mathrm{L}}$ in pure $\mathrm{CF}_{4}$.

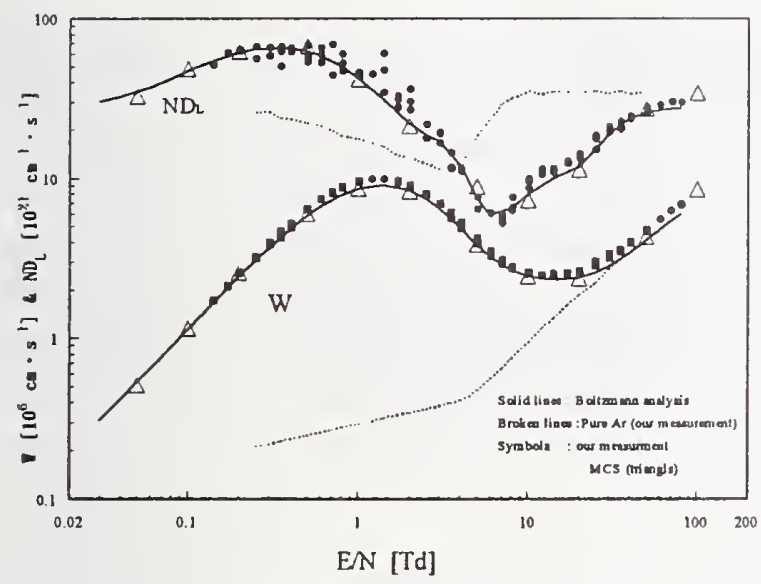

Figure 3: $\mathrm{W}$ and $\mathrm{ND}_{\mathrm{L}}$ in the $5.08 \% \mathrm{CF}_{4}$ - $\mathrm{Ar}$ mixture.

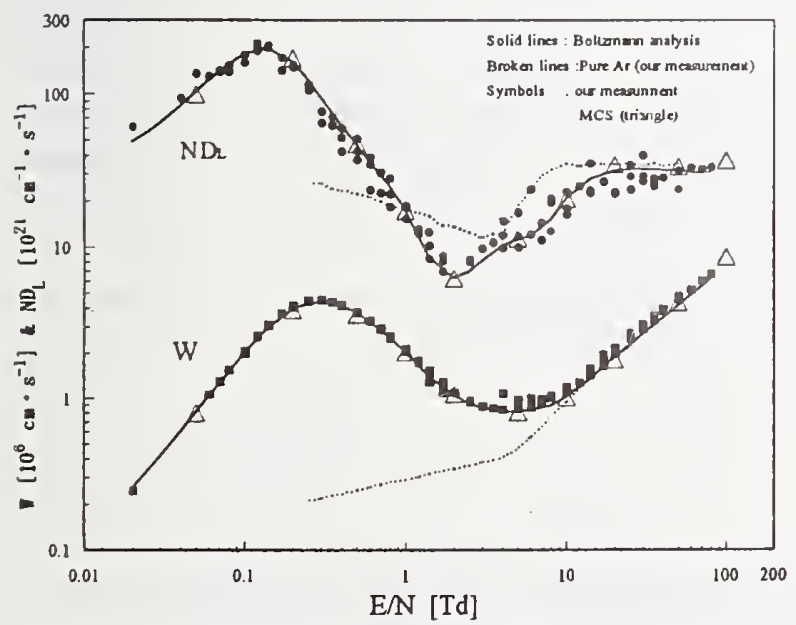

Figure 4: $\mathrm{W}$ and NDL in the $0.495 \%$ CF4-Ar mixture.

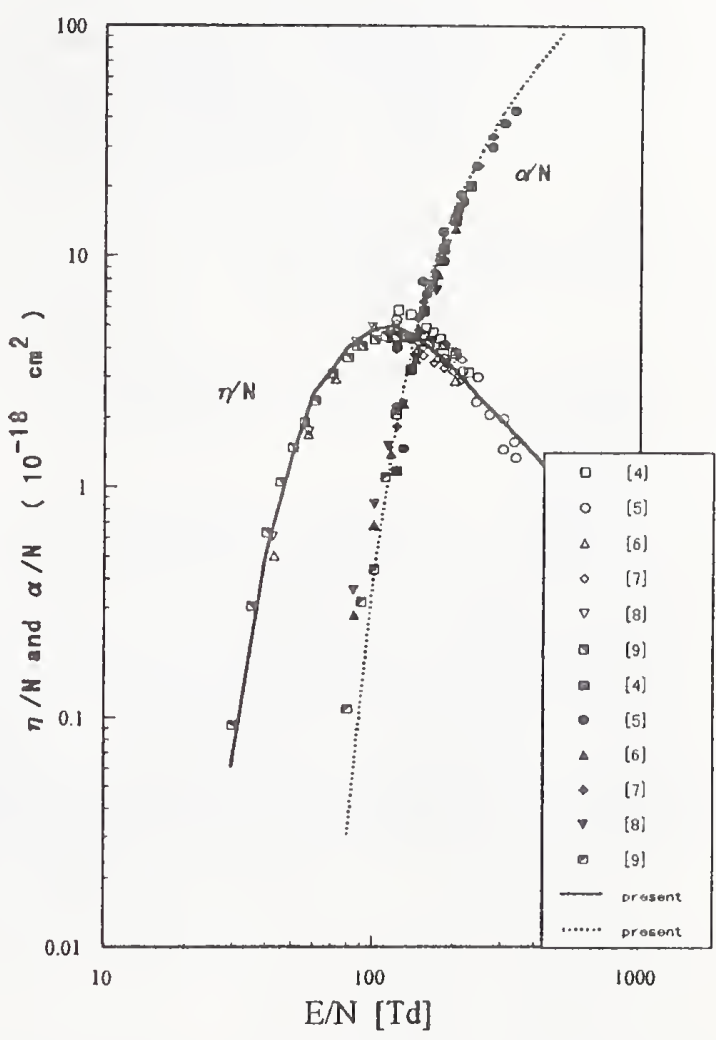

Figure 5: Attachment and ionization coefficients in pure $\mathrm{CF}_{4}$. 


\title{
Inelastic Cross Sections for the $\mathrm{C}_{3} \mathrm{~F}_{8}$ Molecule from Electron Transport Coefficients in $\mathrm{C}_{3} \mathrm{~F}_{8}$-Ar Mixtures
}

\author{
Byoung-Hoon Jeon and Yoshiharu Nakamura \\ Department of Electrical Engineering, Faculty of Science and Technology, \\ Keio University, 3-14-1 Hiyoshi, Yokohama 223, Japan
}

\section{Introduction}

Perfluoropropane $\left(\mathrm{C}_{3} \mathrm{~F}_{8}\right)$ is frequently used as an effective etchant in semiconductor etching processes, and it is also used as a gaseous medium in gas discharge switches and radiation detectors [1].

Pirgov and Stefanov [2] derived a momentum transfer cross section and a total vibrationally inelastic cross section for the molecule from electron swarm data measured by Hunter et al. [3] by using a two-term approximation of the Boltzmann analysis, which may not be valid for molecules with large vibrationally inelastic cross sections. There are also new measurements of the total ionization cross sections including various positive ions of the molecules by Chantry and Chen [4].

In the present study, we measured the drift velocity and the longitudinal diffusion coefficient of electrons in $\mathrm{C}_{3} \mathrm{~F}_{8}$-Ar mixtures and derived a new set of electron collision cross sections for the $\mathrm{C}_{3} \mathrm{~F}_{8}$ molecule for applications in quantitative modeling of related plasmas.

\section{Experiments}

The apparatus and the data processing procedure used in the present measurement of the drift velocity and the longitudinal diffusion coefficient were the same as those used in the works of Nakamura [5] and Kurachi and Nakamura [6]. The special feature of the apparatus is that the drift distance is variable $(1-10 \mathrm{~cm})$. This allows the application of the so-called differential method which is very effective in reducing the effects of the electron energy relaxation, of the finite width of the starting electron swarm, and of the non-uniform electric field around the electron shutters.

Measurements were repeated at least at three different gas densities at each $\mathrm{E} / \mathrm{N}$ value. All measurements were carried out at room temperature, $300 \pm 2 \mathrm{~K}$.

The mixtures were composed of pure $\mathrm{C}_{3} \mathrm{~F}_{8}$ gas (purity $99.99 \%$ ) and argon $(99.9999 \%$ ), and the actual mix ratio was determined by using a gas chromatography test.

\section{$3 \quad$ Experimental Results}

Electron transport coefficients, the drift velocity, W, and the product of the gas number density and the longitudinal diffusion coefficient, $\mathrm{ND}_{\mathrm{L}}$, of electrons, were measured in $0.526 \%$ and $5.05 \%$ $\mathrm{C}_{3} \mathrm{~F}_{8}$-Ar mixtures over the $\mathrm{E} / \mathrm{N}$ range 0.03 to $100 \mathrm{Td}$ and over the gas pressure range from 0.133 to $122 \mathrm{kPa}$ ( 1 to 900 torr, 1 torr $=133.322 \mathrm{~Pa}$ ). Typical scatter of the measured values at different pressures at each $\mathrm{E} / \mathrm{N}$ was less than $2 \%$ for the drift velocity and less than $10 \%$ for the NDL. 
$122 \mathrm{kPa}(1$ to 900 torr, 1 torr $=133.322 \mathrm{~Pa}$ ). Typical scatter of the measured values at different pressures at each $\mathrm{E} / \mathrm{N}$ was less than $2 \%$ for the drift velocity and less than $10 \%$ for the $\mathrm{ND}_{\mathrm{L}}$.

Fig. 1 shows the electron transport coefficients ( $W$ and $\mathrm{ND}_{\mathrm{L}}$ ) measured in the $0.526 \% \mathrm{C}_{3} \mathrm{~F}_{8}-\mathrm{Ar}$ mixture (solid squares), and Fig. 2 shows those in the $5.05 \% \mathrm{C}_{3} \mathrm{~F}_{8}$-Ar mixture with the same symbols as in Fig. 1. Both figures also show the respective transport coefficients in pure argon [7], by solid triangles, for comparison.

The drift velocity measured in each mixture was enhanced by more than an order of magnitude from that in pure argon by adding only a small fraction of the $\mathrm{C}_{3} \mathrm{~F}_{8}$ molecule and showed clearly the so-called negative differential conductivity (NDC) over the E/N range from 0.4 to $10 \mathrm{Td}$ in the $0.526 \%$ mixture and from 2 to $35 \mathrm{Td}$ in the $5.05 \%$ mixture. The NDL in each mixture showed a similar but pronounced structure over the measured $\mathrm{E} / \mathrm{N}$ range.

For higher $\mathrm{E} / \mathrm{N}$ values each transport coefficient approached that of pure argon. This can be explained by the shift of the dominant inelastic collision processes in the swarm from those with $\mathrm{C}_{3} \mathrm{~F}_{8}$ molecules to electronic excitations of the majority of argon atoms.

\section{Boltzmann equation analysis}

The electron transport coefficients (W and NDL) were calculated using a multi-term approximation of the Boltzmann equation analysis [8].

The cross section set for Ar atoms determined by Nakamura and Kurachi [7] was used throughout the present study. The initial cross section set for the $\mathrm{C}_{3} \mathrm{~F}_{8}$ molecule consisted of those for elastic momentum transfer $\left(\mathrm{Q}_{\mathrm{m}}\right)$ cross sections by Pirgov and Stefanov [2], total attachment including various negative ions (Qatt) by Hunter and Christophorou [9], total ionization including various positive ions $\left(\mathrm{Q}_{\mathrm{i}}\right)$ by Chantry and Chen [4], and vibrational excitations ( $\left.\mathrm{Q}_{\mathrm{v}}\right)$ by Pirgov and Stefanov [2]. A trial calculation showed the need for inclusion of additional vibrational cross sections, and we also tentatively included the set of vibrational cross sections for $\mathrm{C}_{2} \mathrm{~F}_{6}$ proposed by Hayashi and Niwa [10] because of insufficient information on the vibrational threshold energies of the $\mathrm{C}_{3} \mathrm{~F}_{8}$ molecule.

According to a comparison of the present transport coefficients in the $\mathrm{C}_{3} \mathrm{~F}_{8}$-Ar mixtures with those in $\mathrm{C}_{2} \mathrm{~F}_{6}$-Ar mixtures [11], the E/N dependence of the measured transport coefficients for the two mixtures are similar, and we may assume the threshold and the energy dependence of vibrational cross sections of the $\mathrm{C}_{3} \mathrm{~F}_{8}$ molecule to be similar to those of the $\mathrm{C}_{2} \mathrm{~F}_{6}$ molecule, possibly with lower resonance energy.

Electron transport coefficients, W and NDL, calculated using this initial cross section set, are shown by solid and dashed lines in Figs. 1 and 2.

In Fig. 1, the maximum differences in $\mathrm{W}$ and NDL between the measurements and calculations, shown by solid lines, are $57 \%$ and $83 \%$, and those by dashed lines are $21 \%$ and $48 \%$, respectively, over the $\mathrm{E} / \mathrm{N}$ range from 0.03 to $0.2 \mathrm{Td}$. In Figs. 2 and 3, those differences are $47.6 \%$ and $86 \%$, and $16.7 \%$ and $56 \%$, respectively, over the $\mathrm{E} / \mathrm{N}$ range from 0.03 to $1.2 \mathrm{Td}$. The corresponding mean electron energies for these $\mathrm{E} / \mathrm{N}$ ranges were $0.09-0.3 \mathrm{eV}$, and the magnitudes of the vibrational excitation cross sections in this electron energy range were amended so that the calculated results agreed with the experimental results.

The magnitude of the vibrational excitation cross section of about $0.09 \mathrm{eV}$ threshold energy was multiplied by a factor of 5 in the energy range 0.09-0.2 eV. That of about $0.13 \mathrm{eV}$ threshold energy was multiplied by a factor of 3.4 in the energy range $0.13-0.3 \mathrm{eV}$, and the threshold energy of this cross section was changed by $0.113 \mathrm{eV}$ after numerous trials.

The new set of electron collision cross sections for the $\mathrm{C}_{3} \mathrm{~F}_{8}$ molecule is shown in Fig. 3 . 


\section{Conclusion}

We measured electron transport coefficients for $0.526 \%$ and $5.05 \% \mathrm{C}_{3} \mathrm{~F}_{8}$-Ar mixtures over the E/N range from 0.03 to $100 \mathrm{Td}$. The measured transport coefficients were compared with those measured in the $\mathrm{C}_{\mathrm{n}} \mathrm{F}_{2 \mathrm{n}+2(\mathrm{n}=1,2)}$ - $\mathrm{Ar}$ mixtures with similar mixing ratios.

The $\mathrm{E} / \mathrm{N}$ dependence of the measured transport coefficients clearly indicates possible directions for changes of the vibrational excitation cross sections. We have determined the inelastic cross sections for the $\mathrm{C}_{3} \mathrm{~F}_{8}$ molecule. We started the measurement of transport coefficients in pure $\mathrm{C}_{3} \mathrm{~F}_{8}$ and are going to determine the elastic momentum cross section for the $\mathrm{C}_{3} \mathrm{~F}_{8}$ molecule, after completion of the measurements, by using the present vibrational cross sections.

\section{References}

[1] M. Hayashi, Proc. "4th Symp. Inelastic Electron-Molecule Collisions", Springer, New York 1985

[2] P. Pirgov and B. Stefanov, J. Phys. B 23, 2879 (1990)

[3] S. R. Hunter, J. Carter and L. J. Christophorou, J. Appl. Phys. 58, 3001 (1985)

[4] P. J. Chantry and C. L. Chen, J. Chem. Phys. 90, 2585 (1989)

[5] Y. Nakamura, J. Phys. D 20, 933 (1987)

[6] M. Kurachi and Y. Nakamura, J. Phys. D 21, 602 (1988)

[7] Y. Nakamura and M. Kurachi, J. Phys. D 21, 718 (1988)

[8] K. F. Ness and R. E. Robson, Phys. Rev. A 33, 2068 (1986)

[9] S. R. Hunter and L. J. Christophorou, J. Chem. Phys. 80, 3001 (1984)

[10] M. Hayashi and A. Niwa, in Gaseous Dielectrics V, Proc. of the 5th International Symposium on Gaseous Dielectrics (1987)

[11] H. Okumo and Y. Nakamura, in these proceedings

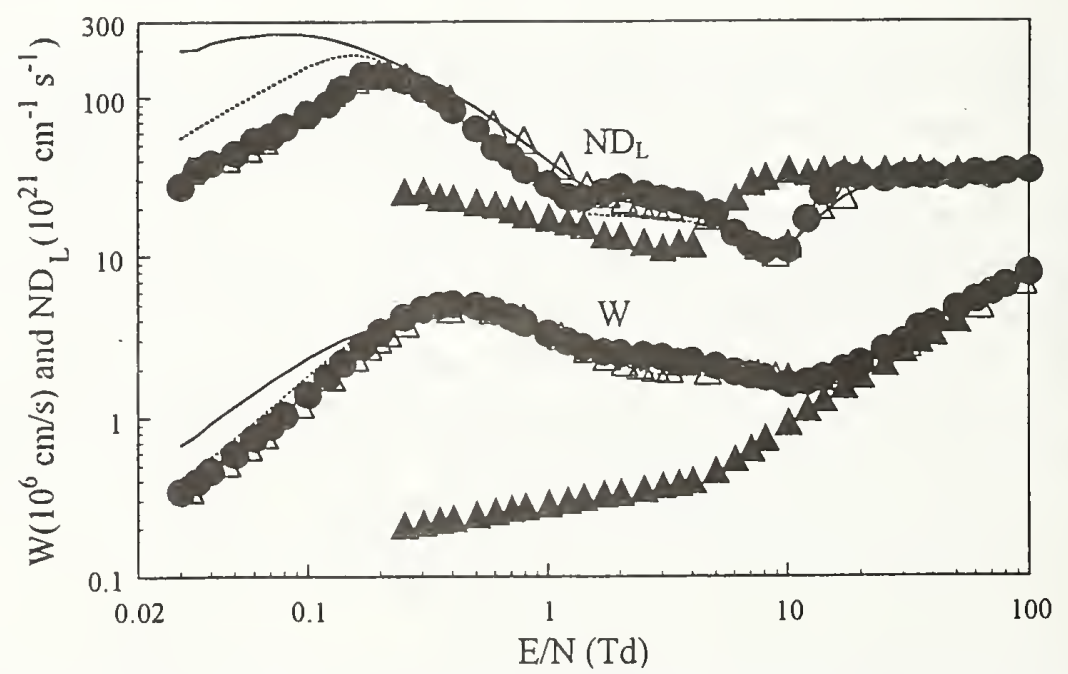

Figure 1: The electron drift velocity, $W$, and the product of the longitudinal diffusion coefficient and the gas number density, $\mathrm{NDL}$, as a function of $\mathrm{E} / \mathrm{N}$ in a $0.526 \% \mathrm{C}_{3} \mathrm{~F}$ - Ar mixture. The broken curves show the analysis using the initial cross section for $\mathrm{C}_{3} \mathrm{~F}_{8}$ molecule. - Experimental results; $\Delta$ - Calculated values using the present cross section set; $\mathbf{\Delta}$ - Ar. (Nakamura and Kurachi 1988) 


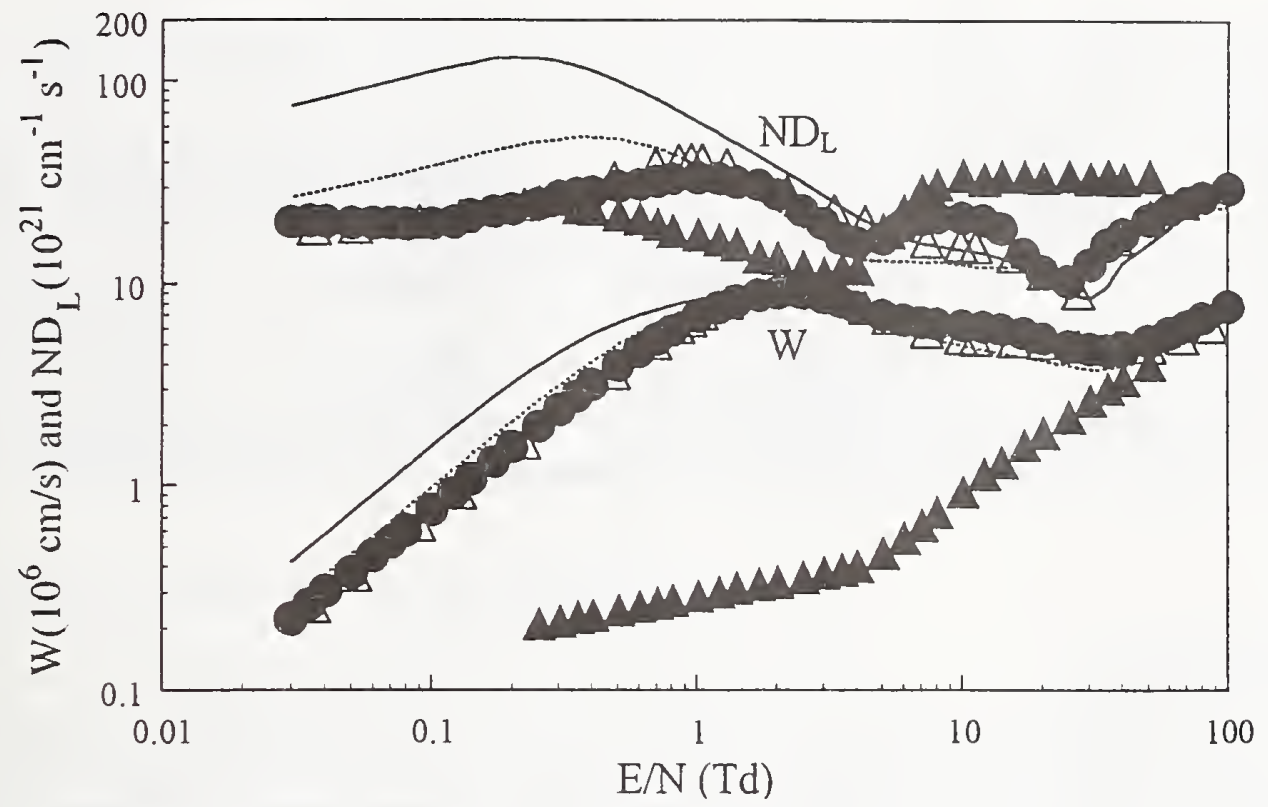

Figure 2: The electron drift velocity, $W$, and the product of the longitudinal diffusion coefficient and the gas number density, $\mathrm{ND}_{\mathrm{L}}$, as a function of $\mathrm{E} / \mathrm{N}$ in a $5.05 \% \mathrm{C}_{3} \mathrm{~F}_{8}$-Ar mixture. The broken curves show the analysis using the initial cross section for $\mathrm{C}_{3} \mathrm{~F}_{8}$ molecule. - Experimental results; $\Delta$ - Calculated values using the present cross section set; $\boldsymbol{\Delta}$ - Ar. (Nakamura and Kurachi 1988).

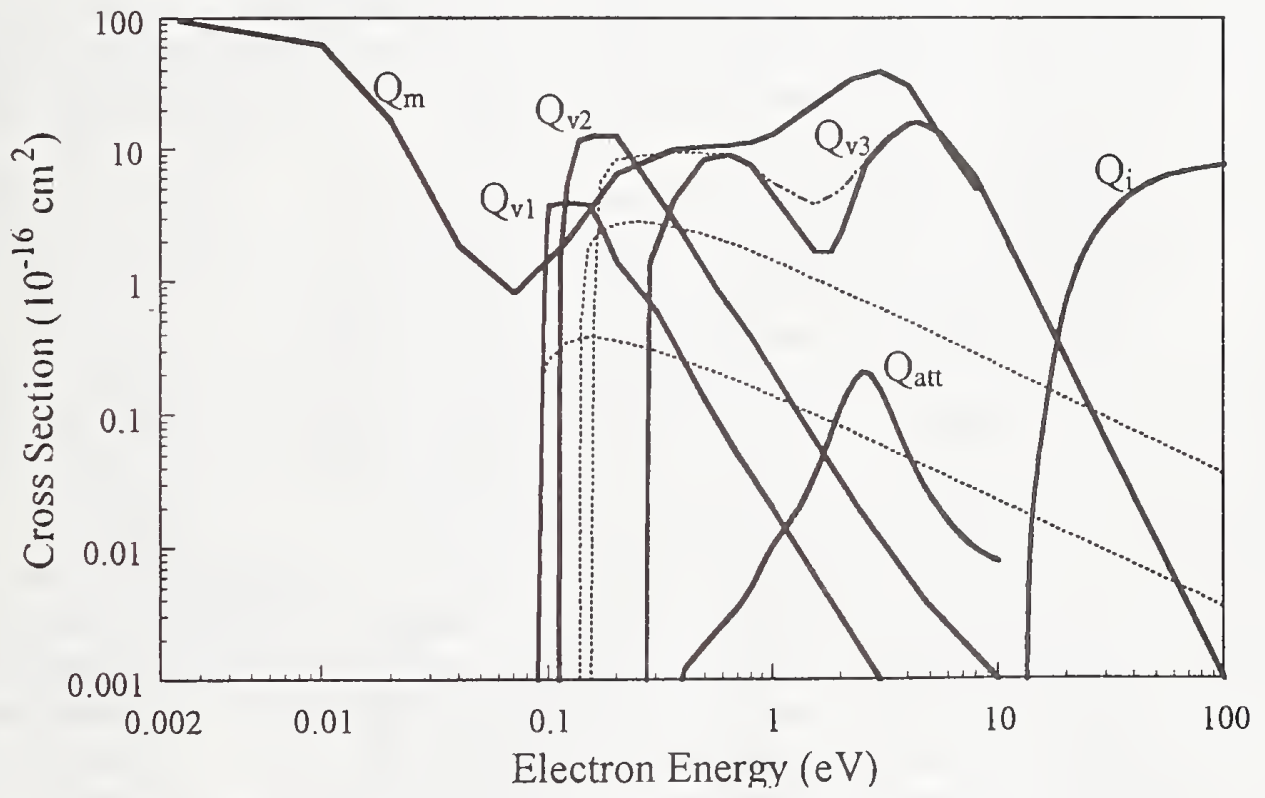

Figure 3: The present set of electron collision cross sections for the $\mathrm{C}_{3} \mathrm{~F}_{8}$ molecule. The solid curves show the present cross section set for the $\mathrm{C}_{3} \mathrm{~F}_{8}$ molecule. The dotted curves show initial cross section for the $\mathrm{C}_{3} \mathrm{~F}_{8}$ molecule. 


\title{
Accurate Ab Initio Calculation of Molecular Constants*
}

\author{
S. Kotochigova and I. Tupitsyn ${ }^{1}$ \\ National Institute of Standards and Technology, Gaithersburg, MD 20899 \\ ${ }^{1}$ Chemistry Institute, St. Petersburg University, Russia, 198904
}

\section{Introduction}

We present a new version of the valence-bond (VB) method, which is able to calculate the electronic structure of diatomic molecules. The first version of this $a b$ initio method was developed by Heitler and London [1] and applied to the $\mathrm{H}_{2}$ molecule. The main idea of the method is to describe the molecular wave function as a linear combination of localized atomic wave functions. The new version of the method is oriented toward the calculation of many-electron molecules [2]. The self-consistent wave functions of the constituent atoms are constructed from determinants by using the HartreeFock (HF) method for non-relativistic atoms or the Dirac-Fock (DF) method for relativistic atoms. The construction of molecular wave functions from the many-electron atomic wave functions leads to the proper dissociation behavior. The total energy of the molecule as a function of inter-nuclear separation is presented as a power series in overlap integrals. This series converges quickly with atomic $\mathrm{HF}$ and DF functions.

The molecular basis set contains both occupied and unoccupied atomic HF or DF orbitals. Since the excited orbitals have a large radius and are not effective in describing the correlation and polarization in the molecule, we create additional basis functions made of localized orbitals, i.e., the Sturm's wave functions [3]. This way of constructing the molecular wave functions avoids the need for large basis sets and ensures much shorter computation times as well as smaller errors in the calculation of the correlation expansion. The configuration interaction (CI) procedure, which takes into account the most important inner and outer shell correlation, simplifies and converges quickly as well, due to presence of HF or DF occupied orbitals.

The important advantage of this method is that the atomic core orbitals are not introduced by a pseudo-potential but calculated exactly. Although the inner-core orbitals do not affect significantly the molecular formation, the exact representation of the core orbitals is important for properties of molecules that depend on the inner core wave functions such as, for example, the hyperfine interactions. These core effects are especially pronounced for molecules with heavy atoms. In principle the Coulomb and exchange interactions can be calculated between all atomic orbitals, but this procedure can be limited to the most important orbitals to simplify the diagonalization procedure and save computer memory for the CI. The remaining orbitals will then form the core.

A crucial step in the method is the calculation of many-center integrals using the sophisticated $\mathrm{HF}$ and $\mathrm{DF}$ functions. For the dimers the evaluation of the two-center integrals is simplified by using a reexpansion procedure [4]. In this procedure each of the two-center integrals is expanded

${ }^{*}$ This paper was inadvertently published in the NIST Journal of Research. In order to have a complete set of poster papers of ICAMDATA 97 in this Special Publication, it is reprinted here again. 
in terms of one-center integrals located on the atomic centers. We have found that convergence of this expansion is accelerated by dividing the range of integration in two and considering only a reexpansion of the slowly varying part of the atomic wave function away from its nuclear center.

The formalism of the VB method is described in more detail in Ref. [2]. Here we discuss the computational details of a calculation of the molecular constants of the monohydrides $\mathrm{OH}$ and $\mathrm{AgH}^{+}$. The $\mathrm{OH}$ molecule is chosen because precise experimental data $[5,6,7]$ as well as theoretical data [8] are available. To our knowledge no experimental data exist for the $\mathrm{AgH}^{+}$molecule. However due to the fact that the hydrogen atom is slightly polarized by the $\mathrm{Ag}^{+}$core, the bond is weak and an accurate determination of binding energy and bond length requires high precision of the theoretical method. Our $\mathrm{AgH}^{+}$result is compared to the pseudo-potential calculation of Ref. [10].

\section{Computational Details and Results}

The first step of the calculation is related to computing the atomic wave functions. The HF functions are obtained with the one-electron center of gravity approximation and calculated for each atom of the molecule. Instead of taking large-radius unoccupied orbitals in building CI wave functions, we have added the Sturm's orbitals. They are localized and create a nearly complete basis set. A multi-configuration (CI) technique is employed to calculate correlation corrections. For the $\mathrm{OH}$ molecule two CI basis sets were considered. The first has 38 configurations. The configurations are built from the $2 \mathrm{p}^{4}$ valence shell of oxygen, with additional single and double excitations of the $2 \mathrm{p}$ electrons into the $3 \mathrm{~s}, 3 \mathrm{p}, 3 \mathrm{~d}, 4 \mathrm{~s}, 4 \mathrm{p}$, and $4 \mathrm{~d}$ orbitals, and the $1 \mathrm{~s}$ orbital of hydrogen with excitation in the $2 \mathrm{~s}, 2 \mathrm{p}, 3 \mathrm{~s}, 3 \mathrm{p}$, and $3 \mathrm{~d}$ orbitals. The excited states in both atoms are described by Sturm's wave functions. The $1 s^{2} 2 s^{2}$ shells of the $\mathrm{O}$ atom are treated as a core. The second basis has 262 configurations. The CI configurations include single-excitations of $1 \mathrm{~s}^{2}, 2 \mathrm{~s}^{2}$, and $2 \mathrm{p}^{4}$ shells as well as double-excitation of the $2 \mathrm{p}^{4}$ shell where again these excitations are represented in terms of Sturm's orbitals. Moreover both basis sets have configurations that represent the ionic states $\mathrm{O}^{+} \mathrm{H}^{-}$, and $\mathrm{O}^{-} \mathrm{H}^{+}$. The inclusion of excitation from the $1 \mathrm{~s}$ and $2 \mathrm{~s}$ orbitals of the oxygen atom in the second basis set leads to a better treatment of the core polarization. Table 1 shows that this substantially improves the agreement with experiment. The table includes the spectroscopical constants of the ground state of $\mathrm{OH}$. Our best results disagree with experiment by $0.2 \%$ for the equilibrium internuclear distance $R_{\mathrm{e}}, 2.7 \%$ for the vibrational constant $\omega_{\mathrm{e}}, 0.4 \%$ for the rotational constant $B_{\mathrm{e}}$, and $4 \%$ for the dissociation energy $D_{\mathrm{e}}$. We want to point out that even with a very small basis set (38 configurations) we reached good agreement with experiment for the $R_{\mathrm{e}}$ and $B_{\mathrm{e}}$ parameters. Another theoretical result [8] obtained by a Hartree-Fock method using a $K$-functional treatment of correlation effects has a larger difference from the experimental values than ours. The origin of the disagreement in the dissociation energy of the VB and HF method seems to be related to the fundamental difference in constructing the molecular wave function. In the VB method the molecular wave function is constructed directly from many-electron atomic determinants with singly occupied atomic orbitals. For large internuclear separation this molecular wave function has the correct asymptotic behaviour and reduces to a product of the wave functions of the individual atoms. The HF method makes use of molecular orbital (MO) wave functions, which contain two electrons at all internuclear separations. In general, the one-determinant form of the HF method is a very poor approximation for large internuclear distances. As it is described in Ref. [9] the molecular wave function in Ref. [8] is introduced in a two-determinant form, which enables them to improve the asymptotic behavior of the energy. However, this modification is still insufficient to get a fair agreement of $D_{\mathrm{e}}$ with the experimental value [7].

The molecular constant, $R_{\mathrm{e}}$, from Ref. [8] and our value (Table 1), lie on the opposite sides 
Table 1: Constants of the $\mathrm{X}^{2} \Pi \mathrm{OH}$ molecule $\left(1 \AA=0.1 \mathrm{~nm}\right.$; the energy equivalent $1 \mathrm{~cm}^{-1}$ is $29.9792458 \mathrm{GHz}$; HF: the Hartree-Fock method).

\begin{tabular}{|c|c|c|c|c|c|}
\hline Ref. & $\begin{array}{c}\text { Basis set } \\
\text { (number of conf.) }\end{array}$ & $R_{\mathrm{e}}(\AA)$ & $\omega_{\mathrm{e}}\left(\mathrm{cm}^{-1}\right)$ & $B\left(\mathrm{~cm}^{-1}\right)$ & $D_{\mathrm{e}}(\mathrm{eV})$ \\
\hline This work & $\begin{array}{c}38 \\
262\end{array}$ & $\begin{array}{l}0.972 \\
0.967\end{array}$ & $\begin{array}{l}7297.25 \\
3638.39\end{array}$ & $\begin{array}{l}18.782 \\
18.834\end{array}$ & $\begin{array}{l}-3.1701 \\
-4.4302\end{array}$ \\
\hline $\mathrm{HF}+\mathrm{K}$-functional [8] & & 0.947 & & & -4.4082 \\
\hline Experiment[7] & & 0.969 & 3737.76 & 18.910 & -4.6259 \\
\hline
\end{tabular}

of the experimental value [7]. This can be related to the difference in the treatment of correlation in the two approaches. In our study we apply Sturm's wave functions in the CI expansion. This is a very efficient treatment of correlation effects in atoms and molecules, because the purely discrete spectrum of Sturm's eigenvalues contains the continuum spectrum as well and hence the CI expansion converges quickly. On the other hand, the $K$-functional technique of Ref. [8], makes use of an effective potential obtained by modifying the exchange operator in the Hamiltonian. This modification does not effect the closed shells of the interacting atoms, therefore the polarization and correlation effects of the core are not taken into account. Finaly, we conclude that the use of Sturm's CI expansions for all orbitals of the $\mathrm{OH}$ molecule gives us an advantage in calculating the equilibrium internuclear distance $R_{\mathrm{e}}$.

The CI treatment of the $\mathrm{AgH}^{+}$molecule excludes all closed orbitals of the $\mathrm{Ag}^{+}$ion with the exception of the $4 \mathrm{p}^{6} 4 \mathrm{~d}^{10}$ shells. Single- and double-excitations of the $4 \mathrm{p}^{6}$ and $4 \mathrm{~d}^{10}$ shells are considered. Additional Sturm's functions for $5 \mathrm{~s}, 5 \mathrm{p}, 5 \mathrm{~d}, 6 \mathrm{~s}$, and $6 \mathrm{p}$ orbitals were constructed for the CI expansion. The number of configurations is 150 . Our calculated values for the molecular constants of $\mathrm{AgH}^{+}$are given in Table 2. The other theoretical results [10] are obtained by using a DF pseudopotential and a density functional approach to describe the valence correlation and polarization energies of the molecule. Due to the fact that $\mathrm{AgH}^{+}$is a "single" electron molecule, the correlation effects are not very important. However, the presence of the hydrogen atom polarizes the $\mathrm{Ag}^{+}$ion orbitals and the CI treatment is still required. The comparison with other theoretical results [10] shows that we agree very well in the calculation of dissociation energies and mostly disagree in the computation of the rotational constant $\omega_{e}$.

\section{Conclusions}

In this paper we have shown that a quite accurate determination of molecular constants of molecules with open shells using our version of the valence-bond method is possible. We treated $\mathrm{O}$ and $\mathrm{Ag}$ as many-electron atoms, where core polarization must be properly accounted for. The disagreement with experiment varies from $0.2 \%$ to $4 \%$ for the molecular parameters of $\mathrm{OH}$. In addition the calculation of $\mathrm{OH}$ with a small number of configurations (38), which requires a few seconds of 
Table 2: Constants of the $\mathrm{X}^{2} \Sigma \mathrm{AgH}^{+}$molecule (DF: the Dirac-Fock method).

\begin{tabular}{|c|c|c|c|c|c|}
\hline Ref. & $\begin{array}{c}\text { Basis set } \\
\text { (number of conf.) }\end{array}$ & $R_{\mathrm{e}}(\AA)$ & $\omega_{\mathrm{e}}\left(\mathrm{cm}^{-1}\right)$ & $B\left(\mathrm{~cm}^{-1}\right)$ & $D_{\mathrm{e}}(\mathrm{eV})$ \\
\hline This work & 150 & 2.19 & 366.14 & 3.5172 & -0.145 \\
\hline DF [10] & & 2.37 & 515 & & -0.15 \\
DF+core [10] & & 2.41 & 519 & & -0.14 \\
\hline
\end{tabular}

computer time for each internuclear separation, has less than a $1 \%$ discrepancy for the $R_{\mathrm{e}}$ and $B_{\mathrm{e}}$ parameters. The difference between our values and other theoretical values for the $\mathrm{AgH}^{+}$molecule does not exceed $7 \%$, except for $\omega_{\mathrm{e}}$, which also serves as a further test of our method.

\section{References}

[1] W. Heitler and F. London, Z. Phys. 44, 455 (1927)

[2] S. Kotochigova and I. Tupitsyn, Int. J. Quant. Chem. 29, 307 (1995)

[3] A. I. Sherstyk and P. F. Gruzdev, Opt. Spectrosc. (USSR) 42, 690 (1977)

[4] P. O. Lövdin, Advances in Physics 5, 1 (1956)

[5] J. A. Coxon and S. C. Foster, Can. J. Phys. 60, 41 (1982)

[6] J. A. Coxon and S. C. Foster, J. Mol. Spectrosc. 93, 117 (1982)

[7] K. Huber and G. Herzberg, "Molecular Spectra and Molecular Structure 4. Constants of Diatomic Molecules", Van Nostrand, Princeton 1979

[8] L. Pisani and E. Clementi, J.Chem. Phys. 103, 9321 (1995)

[9] L. Pizani, L. de Windt, and E. Clementi, Int. J. Quant. Chem. 58, 219 (1996)

[10] H. Stoll, P. Fuentealba, M. Dolg, J. Flad, L. V. Szentpaly, and H. Preuss, J. Chem. Phys. 79, 5532 (1983) 


\title{
Electron Collisions with Small Molecules
}

\author{
B. M. McLaughlin, C. P. Ballance, D. G. Thompson, K. A. Berrington, and P. G. Burke \\ Department of Applied Mathematics \& Theoretical Physics \\ The Queen's University of Belfast, Belfast BT7 1NN, UK
}

\section{Introduction}

Ab initio methods in electron collisions with molecules provide accurate data for use in the modelling of many astrophysical phenomena. Molecules are found in shock waves, dense interstellar clouds, circumstellar outflows, supernova remnants and accretion disks. Molecular species such a water, hydrogen, oxygen, and carbon monoxide and triatomic hydrogen ions are of interest at temperatures below $4000 \mathrm{~K}$. Developments in techniques using R-matrix methodology [1] by the UK group over many years, based around the ALCHEMY [2] and more recently the SWEDEN-MOLECULE [3] quantum chemistry programs are providing information on a variety of systems [4]. Species such as molecular oxygen and nitrogen and their ions are of great interest in understanding the ionospheric and auroral phenomena and in many gaseous discharges. A vast amount of R-matrix work has been done on the molecular oxygen complex, providing electronic and vibrational excitation cross sections together with dissociation [4]. Hydrocarbons and many impurities such as water and carbon monoxide are of interest in tokamak plasmas. Many of the sought after observables, cross sections and rates, for electronic and vibrationally elastic and inelastic processes are of great importance for applications.

\section{Diatomics}

Electron and photon collisions with nitrogen molecules are of fundamental interest in understanding ionospheric and auroral phenomena in the Earth's upper atmosphere, in the modelling of re-entry flow fields around high velocity projectiles and in many gaseous discharge processes. Quite a number of calculations have been reported on electronically elastic, low energy, electron collisions with $\mathrm{N}_{2}$ molecules but there are relatively fewer studies in which the target is itself excited $[5,6,8]$. Although, experiments exist they are frequently limited in scope, in energy range and in the range of angles for which differential cross sections are reported. Recently, R-matrix theoretical studies on $\mathrm{e}^{-}$ - $\mathrm{N}_{2}$ collisions [7] have been extended to study electronic excitation of the target, with a comparison of the integrated and differential cross sections being made with the available experimental data [8]. Extensive calculations of angular distributions in $\mathrm{e}^{-}-\mathrm{N}_{2}$ collisions were then performed to provide a comprehensive set of differential cross section data on several low lying optically forbidden transitions, from each of their thresholds to $17 \mathrm{eV}$ [9].

The applications of cross sections and rates obtained from ab initio studies on electron impact excitation of $\mathrm{CH}$ molecules are multi-fold. $\mathrm{CH}$ molecules are of particular interest due to recent speculation of their presence in the Red Rectangle nebula at wavelengths longer than $3000 \AA[10]$. The use of carbon fibre composites as a plasma facing material give rise to a range of $\mathrm{C}_{n} \mathrm{H}_{n}$ $(n \geq 1)$ hydrocarbon impurities in tokamak plasmas and molecular constituents such as $\mathrm{BeD}$, $\mathrm{CD}, \mathrm{H}_{2} \mathrm{O}$ and $\mathrm{CO}$ are additional species of interest. Therefore electronic and vibronic excitation 
of the $\mathrm{CH}$ molecule by electrons are of fundamental importance in fusion plasmas edges and for astrophysical applications. Furthermore, the excited Rydberg states of the $\mathrm{CH}$ molecule are thought to play a vital role in the dissociative recombination process, that occurs in interstellar shocks and diffuse molecular clouds. The chemistry of the interstellar medium is influenced by hydrocarbon molecules and their cyclic partners which are important constituents that play an active role in the astrochemistry. Cross section calculations for electronic excitation were performed on the $\mathrm{e}^{-}$$\mathrm{CH}$ complex, for a range of low electron impact energies, using the $\mathrm{R}$-matrix technique. The $\mathrm{X}^{2} \Pi$ ground state and the lowest four valence excited states $\left(\mathrm{A}^{2} \Delta, \mathrm{B}^{2} \Sigma^{-}, \mathrm{C}^{2} \Sigma^{+}\right.$and a $\left.{ }^{4} \Sigma^{-}\right)$, of $\mathrm{CH}$ are included in the close coupling expansion with each state being represented by a multi-configuration interaction wave function. Cross section calculations for electronic excitation of the CH molecule by electron impact were then made for the, $A^{2} \Delta-X^{2} \Pi, B^{2} \Sigma^{-}-X^{2} \Pi$ and $C^{2} \Sigma^{+}-X^{2} \Pi$ transitions. A Morse potential was fitted to the ab initio values and the adiabatic approximation invoked to determine the vibronic excitation cross sections for the X-A transition in both the $\mathrm{CH}$ and $\mathrm{CD}$ molecules $[11,12]$.

For R-matrix studies on the molecular nitrogen cation the lowest three electronic target states; $X^{2} \Sigma_{g}^{+}: 1 \sigma_{g}^{2} 1 \sigma_{u}^{2} 2 \sigma_{g}^{2} 2 \sigma_{u}^{2} 3 \sigma_{g} 1 \pi_{u}^{4}, A^{2} \Pi_{u}: 1 \sigma_{g}^{2} 1 \sigma_{u}^{2} 2 \sigma_{g}^{2} 2 \sigma_{u}^{2} 3 \sigma_{g}^{2} 1 \pi_{u}^{3}$ and the $B^{2} \Sigma_{u}^{+}: 1 \sigma_{g}^{2} 1 \sigma_{u}^{2} 2 \sigma_{g}^{2} 2 \sigma_{u} 3 \sigma_{g}^{2} 1 \pi_{u}^{4}$, were included in the close coupling expansion of the inner region wave-function with each state represented by the valence CI model. The $\mathrm{N}_{2}^{+}$molecular cation belongs to the $\mathrm{D}_{\infty h}$ Abelian point group, so the Slater basis from the work of Ermler and co-workers [13] is a natural choice to use to represent the target states. Initially an SCF calculation was performed on the ground state of $\mathrm{N}_{2}^{+}$. In order to provide a suitable representation of the excited states of the molecular cation at the equilibrium separation, this was extended to a valence CI and an elaborate CI model. Closed channel bound state calculations were performed for several symmetries of the neutral nitrogen molecule within both approximations. In Table 2, the Rydberg bound state results for the $\mathrm{N}_{2}$ molecule close to equilibrium separation for the ${ }^{1,3} \Sigma_{g}^{+}$symmetries in both approximations are given. Electronic excitation cross sections for the X-A and X-B states of the $\mathrm{N}_{2}^{+}$cation were investigated and vibronic excitation calculated for the same transitions in both the adiabatic and non-adiabatic approximations [14].

In our $\mathrm{R}$-matrix work on the molecular oxygen cation, the lowest five electronic target states; $X{ }^{2} \Pi_{g}, a{ }^{4} \Pi_{u}, A^{2} \Pi_{u}, b^{4} \Sigma_{u}^{-}$and the $B^{2} \Sigma_{u}^{-}$were included in the close coupling expansion with each target state represented by the valence CI model. There has been recent photon impact experimental studies on the oxygen molecule [15] and many of the resonant characteristics evident in the corresponding photoabsorption cross sections may be identified from electron scattering by the molecular oxygen cation. The possible channels open within our five-state valence CI model would therefore feature the following;

$$
h \nu+O_{2} \rightarrow O_{2}^{* *} \rightarrow O_{2}^{+}\left(X^{2} \Pi_{g}, a{ }^{4} \Pi_{u}, A^{2} \Pi_{u}, b^{4} \Sigma_{g}^{-}, B^{2} \Sigma_{g}^{-}\right)+e^{-} .
$$

We note that the lowest five states of the molecular cation are denoted by; $X{ }^{2} \Pi_{g}, A^{4} \Pi_{u}, a{ }^{2} \Pi_{u}$, $b^{4} \Sigma_{g}^{-}$and $B^{2} \Sigma_{g}^{-}$. In the SCF representation these are; $X{ }^{2} \Pi_{g}: 1 \sigma_{g}^{2} 1 \sigma_{u}^{2} 2 \sigma_{g}^{2} 2 \sigma_{u}^{2} 3 \sigma_{g}^{2} 1 \pi_{u}^{4} 1 \pi_{g}, a{ }^{4} \Pi_{u}$ and $A^{2} \Pi_{u}: 1 \sigma_{g}^{2} 1 \sigma_{u}^{2} 2 \sigma_{g}^{2} 2 \sigma_{u}^{2} 3 \sigma_{g}^{2} 1 \pi_{u}^{3} 1 \pi_{g}^{2}, b^{4} \Sigma_{g}^{-}$and $B{ }^{2} \Sigma_{g}^{-}: 1 \sigma_{g}^{2} 1 \sigma_{u}^{2} 2 \sigma_{g}^{2} 2 \sigma_{u}^{2} 3 \sigma_{g}^{1} 1 \pi_{u}^{4} 1 \pi_{g}^{2}$. In order to improve upon the SCF representation of the target states one has either to use, the valence CI, an elaborate CI, the MCSCF approach or a combination of them. In order to highlight the resonant features we have chosen the ${ }^{3} \Sigma_{u}^{-}$scattering symmetry. Table 3 gives a comparison of our 5 -state VCI results for the $n d \pi_{g}$ resonances in the ${ }^{3} \Sigma_{u}^{-}$scattering symmetry with recent 3-state VCI work [17]. The $n d \pi_{g}$ resonances in this scattering symmetry represent a transitory state of a Rydberg electron interacting with the $\mathrm{O}_{2}^{+}$core on the fringes of the molecular charge cloud. This activity manifests itself in the form of Rydberg resonance series in the ${ }^{3} \Sigma_{u}^{-}$symmetry denoted by $n d \pi_{g}$ and 
$n g \pi_{g}$ which approach the $a^{4} \Pi_{u}$ and $A^{2} \Pi_{u}$ thresholds of the molecular oxygen cation. In this half collision process for the ${ }^{3} \Sigma_{u}^{-}$scattering symmetry, we note that the following resonant processes may occur;

$$
\begin{aligned}
& \left.h \nu+O_{2}\left(X^{3} \Sigma_{g}^{-}\right) \rightarrow O_{2}^{*}\left(1 \sigma_{g}^{2} 1 \sigma_{u}^{2} \ldots 3 \sigma_{g}^{2} 1 \pi_{u}^{3} 1 \pi_{g}^{2}: \quad a^{4} \Pi_{u}, A^{2} \Pi_{u}\right] n d \pi_{g}, n g \pi_{g}{ }^{3} \Sigma_{u}^{-}\right) \\
& O_{2}^{+}\left(1 \sigma_{g}^{2} 1 \sigma_{u}^{2} \ldots 3 \sigma_{g}^{2} 1 \pi_{u}^{3} 1 \pi_{g}^{2}: \quad \quad a{ }^{4} \Pi_{u}, A^{2} \Pi_{u}\right)+e^{-}
\end{aligned}
$$

As noted by Noble [17] the $n g \pi_{g}$ resonances are three orders of magnitude narrower than those of the corresponding $n d \pi_{g}$ series. The lower members of the $n d \pi_{g}$ series are sufficiently broad and isolated that the resonance parameters may be obtained by fitting the eigenphase sum. Higher members of the $n d \pi_{g}$ series become narrow and so reliable results require the use of the complex energy technique or variant theories that calculate the parameters directly. For the ${ }^{3} \Pi_{u}$ scattering symmetry there is more elaborate resonance structure, due to the target states being able to couple to both $\sigma$ and $\delta$ continuum. Rydberg series of the form $n s \sigma, n d \sigma, n s \delta$ and $n d \delta$ will therefore be associated with the respective $a^{4} \Pi_{u}$ and $A^{2} \Pi_{u}$ thresholds of the molecular oxygen cation.

\section{Polyatomics}

Electron collisions with polyatomic systems within the R-matrix methodology [4] is currently being undertaken as a part of a UK wide collaborative project based around the SWEDEN-MOLECULE Gaussian code [3]. Integral and differential cross sections results have been performed on $\mathrm{N}_{2} \mathrm{O}$ [18] at the static-exchange plus polarization (SEP) level and compared with experiment and previous theoretical work [19]. Presently the UK group are working on several systems such as; $\mathrm{H}_{2} \mathrm{O}, \mathrm{O}_{3}$, $\mathrm{CO}_{2}, \mathrm{CH}_{4}$ and $\mathrm{CH}_{2}$. Table 1 gives the SCF energies of the lowest ${ }^{1} A_{1}$ state for the three polyatomic systems; $\mathrm{CH}_{2}, \mathrm{CH}_{4}$ and $\mathrm{H}_{2} \mathrm{O}$ obtained using the SWEDEN-MOLECULE code with their respective contracted Gaussian type orbital (CGTO) basis. Detailed scattering results for these and several other systems will be forthcoming in the near future.

Table 1: Electronic energies of the polyatomic molecular systems; $\mathrm{CH}_{2}, \mathrm{CH}_{4}$ and $\mathrm{H}_{2} \mathrm{O}$ at the SCF level in the Abelian point group $\mathrm{C}_{2 \mathrm{v}}$ from the SWEDEN-MOLECULE Gaussian code. The bond

\begin{tabular}{|c|c|c|c|c|c|c|}
\hline CGTO Basis & Species & $\mathrm{E}_{\mathrm{SCF}}\left(\mathrm{a}_{0}\right)$ & $r\left(a_{0}\right)$ & $r(\AA)$ & $\Theta(\mathrm{Deg})$ & Molecular State \\
\hline$\left[\begin{array}{llll}4 s & 2 p & 1 d / 2 s & 1 p\end{array}\right]$ & $\mathrm{CH}_{2}$ & -38.8677980686 & 2.110 & 1.11656 & $102.4^{\circ}$ & $1 a_{1}^{2} 2 a_{1}^{2} 1 b_{2}^{2} 3 a_{1}^{2}{ }^{1} A_{1}$ \\
\hline$\left[\begin{array}{llll}8 s & 5 p & 2 d / 5 s & 2 p\end{array}\right]$ & $\mathrm{CH}_{4}$ & -40.2148093594 & 2.066 & 1.09328 & $109.47^{\circ}$ & $1 a_{1}^{2} 2 a_{1}^{2} 1 b_{2}^{2} 3 a_{1}^{2} 1 b_{1}^{2}{ }^{1} A_{1}$ \\
\hline$\left[\begin{array}{llll}5 s & 3 p & 2 d / 2 s & 1 p\end{array}\right]$ & $\mathrm{H}_{2} \mathrm{O}$ & -76.0440308704 & 1.800 & 0.95252 & $105.0^{\circ}$ & $1 a_{1}^{2} 2 a_{1}^{2} 1 b_{2}^{2} 3 a_{1}^{2} 1 b_{2}^{2}{ }^{1} A_{1}$ \\
\hline
\end{tabular}
angle $\Theta$ and the bond distance $r$ are given in their appropriate units.

\section{Acknowledgments}

This work was supported by the UK Engineering and Physical Sciences Research Council (EPSRC), the Collaborative Computational Project Two (CCP2), the US National Science Foundation and NIDevR/ESF. Mr. C P Ballance thanks NIDevR/ESF for financial support during the course of this work. 
Table 2: The lowest ten ${ }^{1} \Sigma_{g}^{+}$states of the $\mathrm{N}_{2}$ molecule at the fixed internuclear distance of $2.1 a_{0}$ from the basic valence and the extended CI scattering models. All energies are given in Hartrees. The effective quantum numbers and defects, respectively $\nu$ and $\mu$ of these states are presented for both scattering approximations.

\begin{tabular}{|c|c|c|c|c|c|c|}
\hline & VALENCE - CI (VCI) & & & EXTENDED - CI (EX-CI) & & \\
\hline State & Energy (Ry) & $\nu$ & $\mu$ & Energy (Ry) & $\nu$ & $\mu$ \\
\hline $\mathrm{X}^{1} \Sigma_{g}^{+}$ & -109.083784 & 0.950793 & 0.049207 & -109.087911 & 0.949250 & 0.050750 \\
\hline 2 & -108.644130 & 2.099451 & -0.099451 & -108.648555 & 2.080286 & -0.080286 \\
\hline 3 & -108.589836 & 2.907556 & 0.092444 & -108.592441 & 2.900719 & 0.099281 \\
\hline 4 & -108.582016 & 3.121228 & -0.121228 & -108.584833 & 3.106394 & -0.106394 \\
\hline 5 & -108.570430 & 3.547164 & 0.452836 & -108.570982 & 3.629105 & 0.370895 \\
\hline 6 & -108.563275 & 3.917315 & 0.082685 & -108.565722 & 3.910057 & 0.089943 \\
\hline 7 & -108.559335 & 4.178071 & -0.178071 & -108.561758 & 4.170959 & -0.170959 \\
\hline 8 & -108.551443 & 4.908630 & 0.091370 & -108.553849 & 4.899187 & 0.100813 \\
\hline 9 & -108.550750 & 4.992768 & 0.007232 & -108.553073 & 4.993064 & 0.006936 \\
\hline 10 & -108.549396 & 5.170251 & -0.170251 & -108.551823 & 5.156397 & -0.156397 \\
\hline
\end{tabular}

Table 3: The $n d \pi_{g}$ resonance positions $\left(\epsilon_{r}\right)$ and autoionization widths $(\Gamma)$ for $\mathrm{e}^{-}-\mathrm{O}_{2}^{+}$scattering in the ${ }^{3} \Sigma_{u}^{-}$symmetry at the fixed bond length of $2.30 a_{0}$. The effective quantum number $\nu$, the real and imaginary parts, $\alpha$ and $\beta$ respectively of the complex quantum defect $\mu=\alpha+i \beta$ for several members of the $n d \pi_{g}$ series are included for completeness. The $n d \pi_{g}$ resonance parameters from our work for the ${ }^{3} \Sigma_{u}^{-}$symmetry were obtained by fitting the eigenphase sum of the 5 -state valence configuration interaction (VCI) scattering model to a Breit-Wigner form. The 3-state valence CI results obtained using a similar approach for the molecular oxygen Rydberg resonance states; $\mathrm{O}_{2}$ $\left(1 \sigma_{g}^{2} 1 \sigma_{u}^{2} \ldots\left(a^{4} \Pi_{u}\right) n d \pi_{g}{ }^{3} \Sigma_{u}^{-}\right)$are included for comparison purposes.

\begin{tabular}{ccccccccc}
\hline Resonance & $\epsilon_{r}^{a}(\mathrm{Ry})$ & $\epsilon_{r}^{b}(\mathrm{Ry})$ & $\Gamma^{a}(\mathrm{Ry})$ & $\Gamma^{b}(\mathrm{Ry})$ & $\nu$ & $R e(\mu)$ & $\beta$ & $\Gamma^{b} \nu^{3}$ \\
\hline & & & & & & & & \\
$3 d \pi_{g}$ & 0.2151 & 0.2103 & $0.1060(-2)$ & $0.1085(-2)$ & 3.0088 & 0.0088 & $0.7390(-2)$ & 0.029 \\
$4 d \pi_{g}$ & 0.2610 & 0.2583 & $0.4270(-3)$ & $0.2102(-3)$ & 4.0019 & 0.0019 & $0.3367(-2)$ & 0.048 \\
$5 d \pi_{g}$ & 0.2822 & 0.2796 & $0.1990(-3)$ & $0.2185(-3)$ & 4.9264 & 0.0736 & $0.6531(-2)$ & 0.026 \\
$6 d \pi_{g}$ & 0.2937 & 0.2918 & $0.0822(-3)$ & $0.1172(-3)$ & 5.8724 & 0.1736 & $0.3811(-2)$ & 0.015 \\
$7 d \pi_{g}$ & - & 0.2998 & - & $0.1414(-3)$ & 6.9176 & 0.0824 & $0.1170(-1)$ & 0.039 \\
$8 d \pi_{g}$ & - & 0.3047 & - & $0.7934(-4)$ & 7.8934 & 0.1066 & $0.9756(-2)$ & 0.037 \\
$9 d \pi_{g}$ & - & 0.3081 & - & $0.5237(-4)$ & 8.8840 & 0.1160 & $0.9182(-2)$ & 0.036 \\
$10 d \pi_{g}$ & - & 0.3105 & - & $0.3688(-4)$ & 9.8778 & 0.1222 & $0.8883(-2)$ & 0.035 \\
$11 d \pi_{g}$ & - & 0.3123 & - & $0.2704(-4)$ & 10.8744 & 0.1256 & $0.8667(-2)$ & 0.034 \\
$12 d \pi_{g}$ & - & 0.3137 & - & $0.2044(-4)$ & 11.8717 & 0.1283 & $0.8551(-2)$ & 0.034 \\
& & & & & & & & \\
\hline
\end{tabular}

\footnotetext{
a 3-state valence CI

${ }^{b}$ jo-state valence CI
} 


\section{References}

[1] C. J. Gillan, J. Tennyson and P. G. Burke, Computational Methods in Electron-Molecule Collisions, Edited by W. M. Huo and F. Gianturco, (Plenum: New York and London, 1995) 239 and references therein

[2] The ALCHEMY I and II suite of programs were originally developed by P. S. Bagus, B. Liu, A. D. McLean, M. Yoshimine and B. Lengsfield III

[3] J. Almlöf and P. R. Taylor, Advanced Theories and Computational Approaches to the Electronic Structure of Molecules, Edited by C. E. Dykstra (Dordrecht: Reidel, 1984)

[4] L. A. Morgan, Photon and Electron Collisions with Atoms and Molecules, Edited by P. G. Burke and C. J. Joachain, (Plenum: New York and London, 1997) 57 and references therein

[5] C. J. Gillan, C. J. Noble and P. G. Burke, J. Phys. B: At. Mol. Phys. 23, L407 (1990)

[6] W. M. Huo, Nonequilibrium Processes in Partially Ionized Gases, Edited by M Capitelli and J N Bardsley, NATO ASI Series (Plenum: New York and London) 341 (1990)

[7] C. J. Gillan, O. Nagy, P. G. Burke, L. A. Morgan and C. J. Noble, J. Phys. B: At. Mol. Phys. 20, 4585 (1987)

[8] C. J. Gillan, J. Tennyson, B. M. McLaughlin, and P. G. Burke, J. Phys. B: At. Mol. \& Opt. Phys. 29, 1531 (1996)

[9] C. J. Gillan, B. M. McLaughlin and J. Tennyson, At. Data and Nucl. Data Tables, submitted for publication

[10] R. J. Glinski, J. A. Nuth III, M. D. Reese and M. L. Sitko, Astrophys. J. 467, L109 (1996)

[11] K. A. Berrington, P. G. Burke, W. G. V. Darlington, W. C. Fon, C. J. Gillan, B. M. McLaughlin, J. Pelan, R. H. G. Reid and J. H. Tait, Atomic Data for Spectroscopic Studies on JET, JET Report JP5/10061 (1997)

[12] B. M. McLaughlin, C. J. Gillan and P. G. Burke, J. Phys. B: At. Mol. \& Opt. Phys., in preparation

[13] W. C. Ermler, A. D. McLean and P. S. Bagus, IBM Res. Rep. RJ3243, 1 (1981)

[14] O. Nagy, B. M. McLaughlin, C. P. Ballance and P. G. Burke, J. Phys. B: At. Mol. \& Opt. Phys. in preparation

[15] D. M. P. Holland, private communication.

[16] C. P. Ballance, B. M. McLaughlin and K. A. Berington, J. Phys. B: At. Mol. \& Opt. Phys. in preparation

[17] C. J. Noble, in Computational Methods for Electron Molecule Collisions, Edited by W. M. Huo and F. Gianturco (Plenum: New York and London, 1995) 324

[18] L. A. Morgan, C. J. Gillan, J. Tennyson and X. Chen, J. Phys. B: At. Mol. E Opt. Phys. 30, 4087 (1997)

[19] B. K. Sarpal, K. P. Pfingst, B. M. Nestmann and S. D. Peyerimhoff J. Phys. B: At. Mol. \& Opt. Phys. 28, 3045 (1996) 


\title{
Electron Transport Coefficients in $\mathrm{C}_{2} \mathrm{~F}_{6}$-Ar Mixtures and Inelastic Cross Sections for the $\mathrm{C}_{2} \mathrm{~F}_{6}$ Molecule
}

\author{
Hiromichi Okumo and Yoshiharu Nakamura \\ Faculty of Science and Technology, Keio University, \\ 3-14-1 Hiyoshi, Yokohama 223, Japan
}

\section{Introduction}

Hexafluoroethane $\left(\mathrm{C}_{2} \mathrm{~F}_{6}\right)$ is used as a working gas for diffuse discharge switches and etching plasmas for materials processing and also as a gaseous insulator in high voltage equipment. But the present state of cross section data for the molecule is not reliable enough for numerical simulations. We have been studying electron collision cross sections of molecules by the measurement of electron swarm parameters in dilute molecular gas-rare gas mixtures and in the pure molecular gas. Electron swarm parameters in the mixtures are very sensitive to the vibrational cross sections of the molecule and the elastic cross section of the rare gas atom and are nearly insensitive to the elastic cross section of the molecule. We, therefore, were able to derive detailed information on the vibrational cross sections of the molecule from the electron swarm parameters, measured in the dilute molecular gas-rare gas mixtures exclusively. The swarm parameters in the pure molecular gas, on the other hand, depend on the elastic and inelastic cross sections of the molecule, and, once the inelastic cross sections were known, we were able to derive the elastic cross section for the molecule from the measured swarm parameters measured in the pure molecular gas.

Presented in this report are the experimental results for the drift velocity and longitudinal diffusion coefficient of electrons in dilute $\mathrm{C}_{2} \mathrm{~F}_{6}$-Ar mixtures and the derived vibrational excitation cross sections which are consistent with the measured swarm parameters.

We are now measuring the swarm parameters in the pure $\mathrm{C}_{2} \mathrm{~F}_{6}$ gas and we are going to derive the elastic cross section for $\mathrm{C}_{2} \mathrm{~F}_{6}$ using the present vibrational cross sections after completion of the measurements.

\section{Measurements}

We used the double shutter drift tube [1] with variable drift distances $(1-10 \mathrm{~cm})$ for the measurements of the electron drift velocity, W, and the product of the gas number density and the longitudinal diffusion coefficient, $\mathrm{ND}_{L}$, in $0.524 \%$ and $5.47 \% \mathrm{C}_{2} \mathrm{~F}_{6}$-Ar mixtures. The ranges of the measurements are shown in Table 1.

The measured electron drift velocities in the mixtures show the Negative Differential Conductivity (NDC) indicating a strong contribution from the vibrational inelastic processes of the $\mathrm{C}_{2} \mathrm{~F}_{6}$ molecule over the $\mathrm{E} / \mathrm{N}$ range $2-25 \mathrm{Td}$ in the $5.47 \%$ mixture, and $0.35-20 \mathrm{Td}$ in the $0.524 \%$ mixture.

The measured products $\mathrm{ND}_{L}$ in mixtures also show clearly the contributions of the molecular additive in argon. The peaks of the measured $\mathrm{ND}_{L}$ over the $\mathrm{E} / \mathrm{N}$ range $5-20 \mathrm{Td}$ in the $5.47 \%$ 
Table 1: The ranges of the measurements

\begin{tabular}{|c|l|l|}
\hline Mix Ratio (\%) & E/N Range (Td) & Gas Pressure Range (Torr) \\
\hline 5.47 & $0.04-100$ & $1-560$ \\
0.524 & $0.04-70$ & $1-700$ \\
\hline
\end{tabular}

mixture and $1.4-7 \mathrm{Td}$ in the $0.524 \%$ mixture show the characteristic of perfluoroalkanes, $\mathrm{C}_{n} \mathrm{~F}_{2 n+2}$ $(\mathrm{n}=1,2,3)$.

Fig. 1 compares the results of the present measurements of $\mathrm{W}$ and $\mathrm{ND}_{L}$ in the $5 \%$ and $0.5 \%$ $\mathrm{C}_{2} \mathrm{~F}_{6}$-Ar mixtures [2], and of the electron drift velocity $\mathrm{W}$ in pure $\mathrm{Ar}$ [3].

\section{Analysis}

The cross sections are shown in Fig. 2. The initial set of cross sections for the $\mathrm{C}_{2} \mathrm{~F}_{6}$ molecule by Hayashi [4] was modified by using a multi-term Boltzmann analysis [5].

We modified the vibrational excitation cross sections for the $\mathrm{C}_{2} \mathrm{~F}_{6}$ molecule until consistent values for $\mathrm{W}$ and $\mathrm{ND}_{L}$ were obtained from the measurements. The thresholds of the vibrational excitation cross sections for the $\mathrm{C}_{2} \mathrm{~F}_{6}$ molecule by Hayashi are shown in Table 2.

Table 2: Thresholds of the vibrational excitation cross sections for the $\mathrm{C}_{2} \mathrm{~F}_{6}$ molecule

\begin{tabular}{|c|l|}
\hline vibrational cross sections & thresholds (eV) \\
\hline qv6 & 0.089 \\
qv5 & 0.138 \\
qv7 & 0.155 \\
\hline
\end{tabular}

The results are shown in Fig. 3 and Fig. 4. The measured drift velocities in the two mixtures agree with the calculated ones over the $\mathrm{E} / \mathrm{N}$ range $0.04-10 \mathrm{Td}$ in the $5.47 \%$ mixture, and $0.04-4$ $\mathrm{Td}$ in the $0.524 \%$ mixture.

\section{Conclusion}

We measured two electron swarm parameters, drift velocities and longitudinal diffusion coefficients in $0.524 \%$ and $5.47 \% \mathrm{C}_{2} \mathrm{~F}_{6}$-Ar mixtures over the $\mathrm{E} / \mathrm{N}$ range from 0.04 to $100 \mathrm{Td}$. The measured swarm parameters were compared with those measured in $\mathrm{CF}_{4}-\mathrm{Ar}$ and $\mathrm{C}_{3} \mathrm{~F}_{8}$ - $\mathrm{Ar}$ mixtures with similar mixing ratios. The $\mathrm{E} / \mathrm{N}$ dependence of the measured swarm parameters clearly indicates possible direction for refinement of the vibrational cross sections.

\section{$5 \quad$ Future work}

We will determine the best consistent vibrational cross sections for $\mathrm{C}_{2} \mathrm{~F}_{6}$ from the present measurement in the mixtures. And after that, we will determine the momentum transfer cross section for $\mathrm{C}_{2} \mathrm{~F}_{6}$ from our new measurement of swarm parameters in pure $\mathrm{C}_{2} \mathrm{~F}_{6}$, which has just been started. 


\section{References}

[1] Y. Nakamura, J. Phys. D 20, 933 (1987)

[2] S.R. Hunter, J.G. Carter, and L.G. Christophorou, J. Appl. Phys. 58, 3001 (1985)

[3] Y. Nakamura and M. Kurachi, J. Phys. D 21, 718 (1988)

[4] M. Hayashi and A. Niwa, in Gaseous dielectrics V, Proceedings of the 5th international symposium on gaseous dielectrics (1987)

[5] R.E. Robson and K.F. Ness, Phys. Rev A 33, 2068 (1986) : James Cook University multi-term Boltzmann code

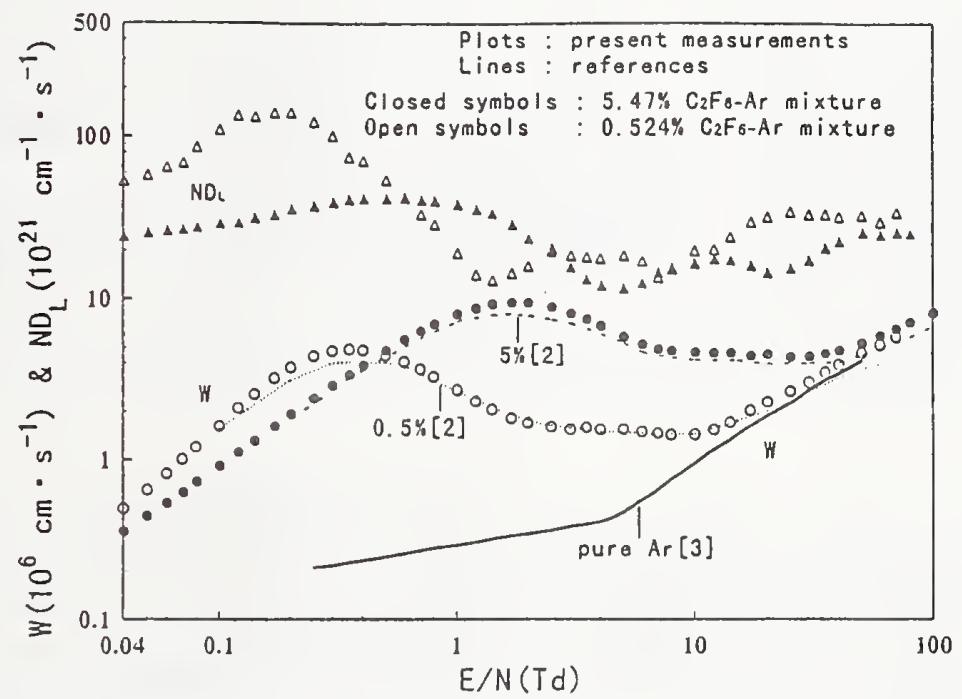

Fig.1 W and NDL as a function of $\mathrm{E} / \mathrm{N}$ in $\mathrm{C}_{2} \mathrm{~F}_{6}$-Ar mixtures

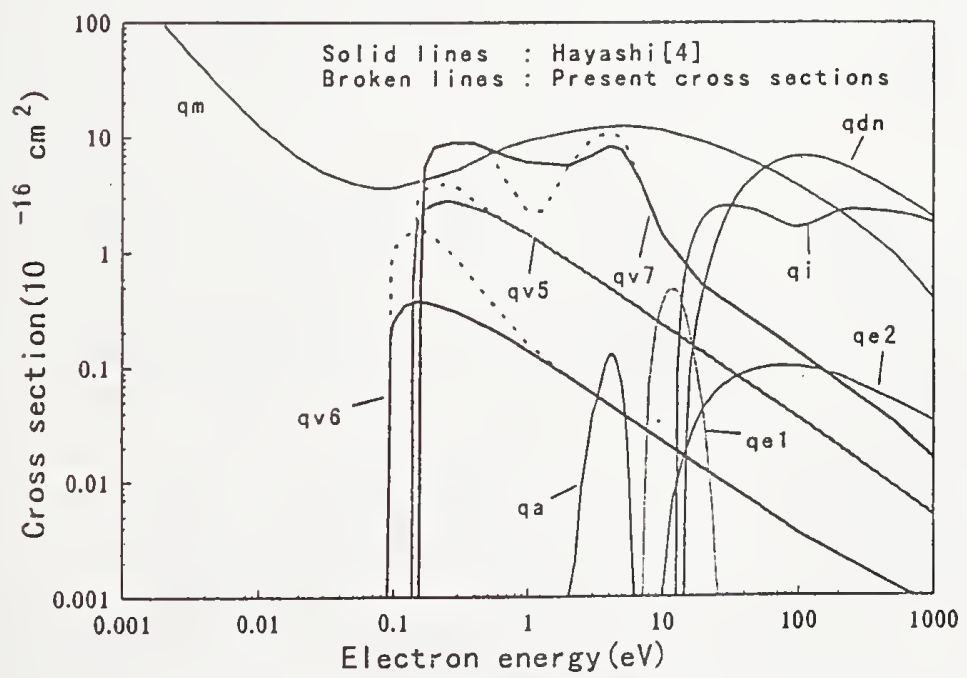

Fig.2 Electron collision cross sections for $\mathrm{C}_{2} \mathrm{~F}_{6}$ 


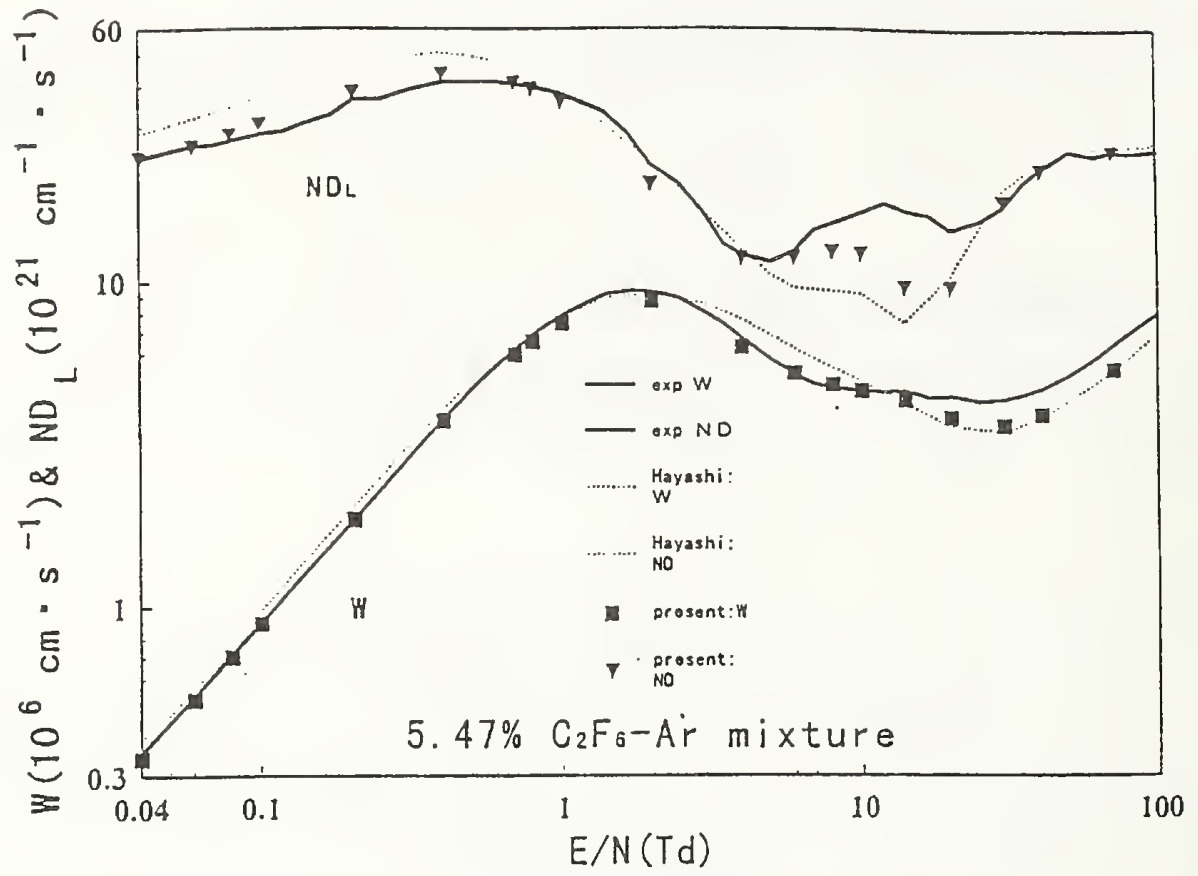

Fig. 3: $\mathrm{W}$ and $\mathrm{ND}_{\mathrm{L}}$ in the $5.47 \% \mathrm{C}_{2} \mathrm{~F}_{6}$-Ar Mixture

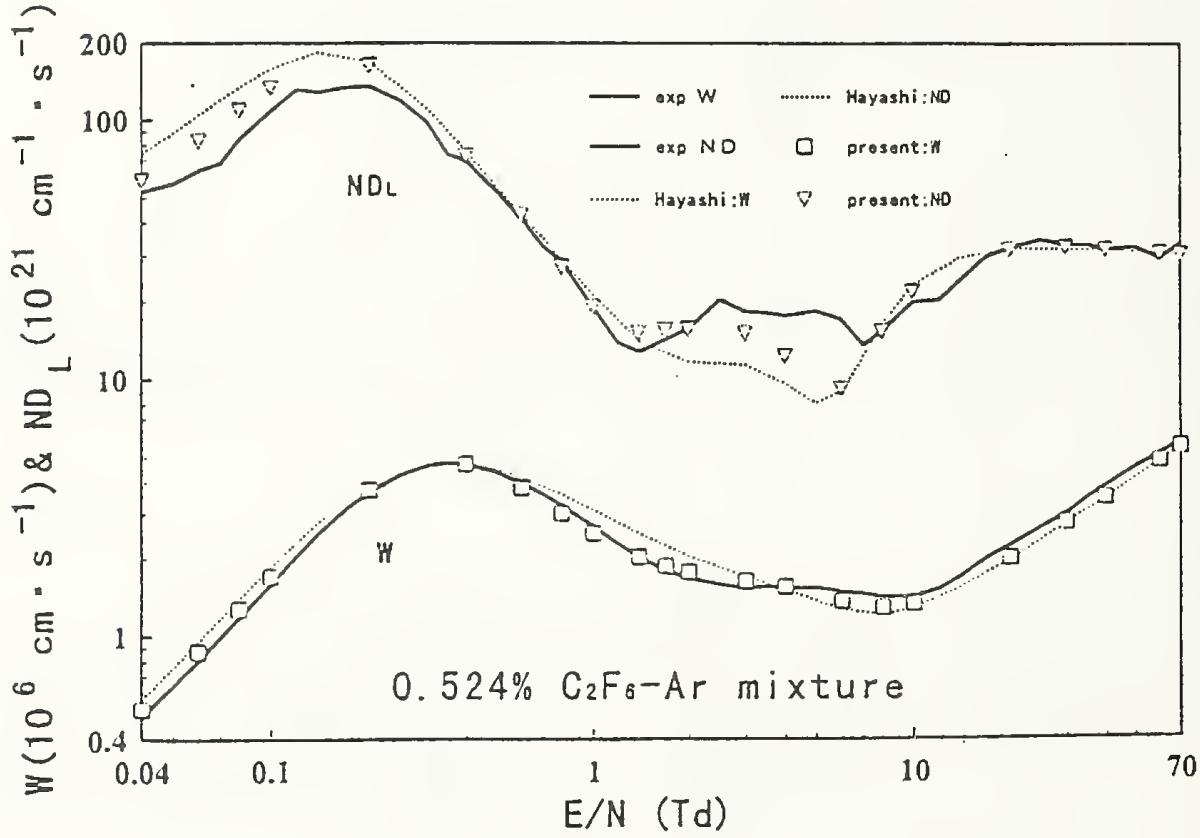

Fig. 4: $\mathrm{W}$ and $\mathrm{ND}_{\mathrm{L}}$ in the $0.524 \% \mathrm{C}_{2} \mathrm{~F}_{6}$-Ar Mixture 


\title{
Validation of Ozone Infrared Line Intensities
}

\author{
M. A. H. Smith \\ NASA Langley Research Center \\ Hampton, Virginia
}

\section{Introduction}

Measurements of infrared absorption or emission are used to determine the concentrations and distributions of atmospheric gases on global, regional, and local scales. The accuracy of these remote measurements depends significantly on the accuracy of our knowledge of the molecular spectra of ozone and other infrared-active atmospheric species. Several recent high-resolution spectroscopic studies $[1,2,3,4]$ have determined absolute intensities of ozone infrared absorption lines in the 9-11 $\mu \mathrm{m}$ region, using as a reference standard the UV absorption cross-section of ozone at $254 \mathrm{~nm}$. This paper reviews the various measurements and compares them to each other and to the current HITRAN line parameters compilation [5].

\section{Review of Measurements}

The first study reporting measurements of ozone 10- $\mu \mathrm{m}$ line intensities referenced to a 254-nm UV absorption standard was that of Pickett et al. [1]. They used a high-resolution Fourier transform spectrometer (FTS) to measure intensities of 47 lines in the P branch of the $\nu_{3}$ fundamental band. Ozone partial pressures in the sample cell were determined through measurements of UV absorption at $254 \mathrm{~nm}$, using as a reference standard the cross-section measurements of Hearn [6]. The results are based on individual measurements in two spectra fitted separately. Taking an arithmetic average of all the intensity measurements, the mean difference from HITRAN is $+8.3 \%$. However, if the most well-determined intensities are given greater weight, the mean difference from HITRAN is $+5.1 \%$. The reported absolute accuracy of these measurements is $1 \%$; however, we note that the reference UV standard [6] has an accuracy of only $2 \%$.

Measurements of the intensity of the single $(1056) \leftarrow(955) \nu_{3}$ line at $1048.6736 \mathrm{~cm}^{-1}$, made using both tunable diode laser (TDL) and laser heterodyne spectrometers, were reported by DeBacker et al. [2]. Ozone concentrations in these experiments were determined by continuously monitoring the total pressure in a closed system. The accuracy of this technique has been verified [7] by measuring the UV absorption at $254 \mathrm{~nm}$, using the more recent reference standard of Mauersberger et al. [8]. The line intensity resulting from the TDL measurements agrees exactly with the HITRAN value, while the laser heterodyne result is approximately $1 \%$ larger. These small differences are consistent with the $1 \%$ precision reported for the measurements.

In addition to the $\left(\begin{array}{lll}10 & 5 & 6\end{array}\right) \leftarrow\left(\begin{array}{l}9 \\ 5\end{array} 5\right)$ line, DeBacker-Barilly and Courtois [3] used their TDL system to measure intensities of 46 other lines in the $\mathrm{R}$ branch of the $\nu_{3}$ band. The reported accuracy of the intensities for these lines is $4 \%$ and their weighted-mean difference from HITRAN is $-5 \%$. However, there is considerable scatter among the individual differences from HITRAN, and their arithmetic average is $-7.5 \%$. 
Recently Smith et al. [4] have determined absolute intensities of numerous ${ }^{16} \mathrm{O}_{3}$ lines in the 9-11 $\mu \mathrm{m}$ region from spectra recorded using the 1-m FTS at Kitt Peak. Ozone concentrations in the sample cell were determined using a $254 \mathrm{~nm}$ UV-absorption monitor of the same design as Pickett et al. [1]. However, the more accurate $( \pm 0.5 \%)$ UV cross-section value of Mauersberger et al. [8] was used as the reference standard for these measurements. Four spectra for which the ozone partial pressure varied by $<1.0 \%$ during the recording were fit simultaneously using a multispectrum nonlinear least-squares procedure [9]. Line positions and intensities were determined for over 270 lines in the $P, Q$, and $R$ branches of the $\nu_{3}$ fundamental band and 10 lines in the $R$ branch of the $\nu_{1}$ band. Absolute accuracies of these intensity values range from $2 \%$ for the strongest, most well-isolated lines to over $4 \%$ for the weakest lines measured. For both arithmetic and weighted averages, the mean difference from HITRAN is $+1 \%$ in the $\nu_{3}$ band and $-0.2 \%$ in the $\nu_{1}$ band.

\section{Intercomparison}

On average, taking all the measurements $[1,2,3 ; 4]$ together, the line intensities are about $1 \%$ larger than the values on the current HITRAN compilation [5]. However, a few individual measured intensities differ from the HITRAN values by as much as 9 to $15 \%$. Only 44 lines were measured in more than one experimental study, and only one of these lines has more than two independent measurements. Comparison of the various intensity measurements shows excellent agreement for a few lines but considerable discrepancies (greater than the stated uncertainties) for others.

The $(1056) \leftarrow(955)$ line at $1048.6736 \mathrm{~cm}^{-1}$ was measured by three different techniques: TDL $[2,3]$, laser heterodyne spectrometer [2], and FTS [4]; all three intensities are within $1.1 \%$ of the HITRAN value. For the $44 \nu_{3}$ band transitions with at least two measurements, the intensities of Smith et al. [4], obtained for both $P$ - and $R$-branch transitions, average $1.4 \%$ larger than the values on the HITRAN compilation [5]. This result appears to split the difference between the FTS measurements of Pickett et al. [1] in the $P$ branch and the tunable diode laser (TDL) results reported by DeBacker-Barilly and Courtois [3] in the $R$ branch. However, for these two earlier sets of measurements $[1,3]$ the intensity differences from HITRAN appear to become larger for increasing rotational quantum number $J^{\prime \prime}$. There may be a contribution to these trends from experimental temperature uncertainties; further investigation is needed to determine the exact cause. The study of Smith et al. [4], which covered a wide range of rotational quantum numbers in the $P, Q$, and $R$ branches of the $\nu_{3}$ band, found no obvious $J^{\prime \prime}$-dependence of the intensity differences from the HITRAN compilation.

In summary, the recent experimental studies in the 9-11 $\mu \mathrm{m}$ region appear to have verified that the intensities of ${ }^{16} \mathrm{O}_{3}$ transitions given in the HITRAN line parameters compilation [5] in this region have an absolute accuracy of $2 \%$ at best. The HITRAN ozone intensities in the infrared also are quite consistent ( $1 \%$ precision) with the ultraviolet absorption cross-section standard at $254 \mathrm{~nm}[8]$.

\section{References}

[1] H. M. Pickett, D. B. Peterson, and J. S. Margolis, J. Geophys. Res. 97, 20,787-20,793 (1992)

[2] M. R. DeBacker, B. Parvitte, V. Zeninari, and D. Courtois, J. Quant. Spectrosc. Radiat. Transfer 54, 1009-1018 (1995)

[3] M. R. DeBacker-Barilly and D. Courtois, Appl. Phys. B 64, 607-611 (1997) 
[4] M. A. H. Smith, C. P. Rinsland, V. Malathy Devi, and D. Chris Benner, 52nd International Symposium on Molecular Spectroscopy, Ohio State University, Paper RF03 (1997); manuscript in preparation (1997)

[5] L. S. Rothman, R. R. Gamache, R. H. Tipping, C. P. Rinsland, M. A. H. Smith, D. C. Benner, V. Malathy Devi, J.-M. Flaud, C. Camy-Peyret, A. Perrin, A. Goldman, S. T. Massie, L. R. Brown, and R. A. Toth, J. Quant. Spectrosc. Radiat. Transfer 48, 469-507 (1992); L. S. Rothman et al., manuscript in preparation, (1997)

[6] A. G. Hearn, Proc. Phys. Soc. 78, 932-940 (1961)

[7] M. R. DeBacker, Ph.D. Thesis, Université de Reims Champagne-Ardenne, 1995

[8] K. Mauersberger, D. Hanson, J. Barnes, and J. Morton, J. Geophys. Res. 92, 8480-8482 (1987)

[9] D. Chris Benner, C. P. Rinsland, V. Malathy Devi, M. A. H. Smith, and D. Atkins, J. Quant. Spectrosc. Radiat. Transfer 53, 705-721 (1995)

3.3 Molecules 271 


\title{
Hyperfine Structure Constants for Diatomic Molecules*
}

\author{
I. Tupitsyn and S. Kotochigova ${ }^{1}$ \\ Physics Department, St. Petersburg University, Russia, 198904 \\ ${ }^{1}$ National Institute of Standards and Technology, Gaithersburg, MD 20899
}

\section{Introduction}

The hyperfine Fermi contact term as a function of internuclear distance $R$ is computed for the $\mathrm{X}^{2} \Pi$ state of $\mathrm{OH}$ and the $\mathrm{X}^{2} \Sigma$ state of $\mathrm{AgH}^{+}$. The all electron $a b$ initio valence-bond method [1] with Hartree-Fock (HF) and Sturm's [2] basis sets is used to calculate the electron spin density near the nuclei correctly. This leads to an accurate determination of the molecular magnetic dipole coupling constants and in particular the molecular Fermi contact term, $A_{c}$. The method uses many-electron atomic wave functions to construct the molecular wave function and yields the correct asymptotic properties for the molecule, one of which is the convergence of the molecular hyperfine interaction parameters to the atomic values for large internuclear separations.

Magnetic hyperfine parameters are very sensitive to the quality of the molecular wave function in general and to the spin polarization of atomic cores in particular. One of these parameters, the Fermi contact term, is proportional to the electron spin density at the position of the nuclei that have nonzero spin. When a molecule has electrons in open shells, it leads to one or more unpaired spins in valence orbitals and gives an unbalanced spin density at the nuclei. Computationally it implies different exchange potentials for electrons with spin up and spin down. The contact term, $A_{\mathrm{c}}$, is proportional to the difference in the spin densities of electrons with the opposite direction of spin. The accurate determination of this parameter requires both correlation and polarization interactions in the model. The configuration interaction (CI) approach is used to treat these effects. We build the CI on the basis of the non-orthogonal many-electron atomic HF and Sturm's functions.

Hyperfine splittings have been observed in the optical spectra of diatomic molecules with very high accuracy. Very precise theoretical calculations are required for comparison. One of these calculations was performed by Kristiansen and Veseth [3] for the $\mathrm{OH}$ molecule. A many-body perturbation theory was used to compute magnetic hyperfine parameters for the lowest vibrational levels of the ${ }^{2} \Pi$ ground state. Their disagreement with experiment does not exceed $2 \%$ near the equilibrium distance $R_{\mathrm{e}}=1.8342$ a.u. ( 1 a.u. $=0.0529177 \mathrm{~nm}$ ).

In our calculations the Fermi contact term is determined over a wide range of $R$ (1.5 a.u. to 8 a.u.). The asymptotic value of $A_{c}$ can be compared with our value at the largest internuclear separations.

The only previous study of the molecular potentials of the $\mathrm{AgH}^{+}$molecule [4] was performed using a pseudopotential approach with its inherent integrated treatment of the core orbitals, and therefore does not give theoretical information about the $\mathrm{AgH}^{+}$hyperfine coupling constants. Our $A_{\mathrm{c}}(\mathrm{R})$ values were obtained for $R$ between 2 a.u. and 18 a.u. and converge for large $R$ to the atomic values.

*This paper was inadvertently published in the NIST Journal of Research. In order to have a complete set of poster papers of ICAMDATA 97 in this Special Publication, it is reprinted here again. 


\section{Theoretical and computational details.}

The total electronic wave function $\Psi_{\mathrm{AB}}$ of the molecule is introduced as a linear combination of Slater's determinants $\operatorname{det}_{\alpha}$, corresponding to various configurations $\alpha$ of atoms $A$ and $B$. That is

$$
\psi_{\mathrm{AB}}=\sum_{\alpha} C_{\alpha} \operatorname{det}_{\alpha}
$$

where the $C_{\alpha}$ are obtained by solving a generalized eigenvalue matrix problem, described by the equation

$$
\hat{H}_{\mathrm{AB}} \vec{C}=\hat{S}_{\mathrm{AB}} \vec{C},
$$

where $\hat{H}_{A B}$ is the Hamiltonian of the molecule AB. The right-hand-side of (2) includes the nonorthogonality matrix $\hat{S}_{A B}$, which describes an overlap between determinants $<\operatorname{det}_{\alpha} \mid \operatorname{det}_{\beta}>$ and it is given as

$$
\left(\hat{S}_{A B}\right)_{\alpha \beta}=<\operatorname{det}_{\alpha} \mid \operatorname{det}_{\beta}>=\left(D_{\alpha \alpha} D_{\beta \beta}\right)^{-1 / 2} D_{\alpha \beta}
$$

$D_{\alpha \beta}=\operatorname{det}\left|<\alpha_{1}\right| \beta_{1}>\ldots<\alpha_{i}\left|\beta_{j}>\ldots<\alpha_{N}\right| \beta_{N}>\mid$, and $N$ is the total number of electrons in the molecule. For the particular one-electron orbitals $\alpha_{i}$ and $\beta_{j}$, the overlap matrix elements $S_{i, j}^{\alpha \beta}$ are used to describe the one-electron density matrix $\rho_{1}^{\alpha, \beta}\left(x, x^{\prime}\right)$ of the molecule as

$$
\rho_{1}^{\alpha, \beta}\left(x, x^{\prime}\right)=\left(D_{\alpha \alpha} D_{\beta \beta}\right)^{-1 / 2} D_{\alpha \beta} \sum_{i, j}^{N}\left(S^{-1}\right)_{i, j}^{\alpha, \beta} \cdot \phi_{i}(x) \cdot \phi_{j}^{*}\left(x^{\prime}\right),
$$

where the $\phi(x)$ are the one-electron wave functions and $x$ denotes both coordinates and spin of the electron.

Moreover, the two-electron density matrix is

$$
\begin{aligned}
\rho_{2}^{\alpha, \beta}\left(x_{1}, x_{2} \mid x_{1}^{\prime}, x_{2}^{\prime}\right)= & \left(D_{\alpha \alpha} D_{\beta \beta}\right)^{-1 / 2} \cdot D_{\alpha \beta} \sum_{i, j}^{N} \sum_{k, l}^{N} D_{i, j, k, l}^{\alpha \beta} . \\
& \phi_{i}\left(x_{1}\right) \phi_{j}^{*}\left(x_{1}^{\prime}\right) \phi_{k}\left(x_{2}\right) \phi_{l}^{*}\left(x_{2}^{\prime}\right),
\end{aligned}
$$

where

$$
D_{i, j, k, l}^{\alpha \beta}=D_{\alpha \beta} \cdot\left[\left(S^{-1}\right)_{i, j}^{\alpha, \beta} \cdot\left(S^{-1}\right)_{k, l}^{\alpha, \beta}\left(S^{-1}\right)_{i, l}^{\alpha, \beta} \cdot\left(S^{-1}\right)_{k, j}^{\alpha, \beta}\right]
$$

Finally, the Fermi contact term can be expressed in terms of the total spin densities $\rho^{\alpha, \beta}(\uparrow)$ and $\rho^{\alpha, \beta}(\downarrow)$ at each nuclear site for the electrons with spin pointed up ( $\left.\uparrow\right)$ and down $(\downarrow)$. The (CI) form of $A_{\mathrm{c}}$ is introduced as

$$
A_{\mathrm{c}}=2 \mu_{0} g_{I} \mu_{N} \sum_{\alpha, \beta} \frac{8 \pi}{3} c_{\alpha} c_{\beta}\left(\rho^{\alpha, \beta}(\uparrow)-\rho^{\alpha, \beta}(\downarrow)\right),
$$

where $\mathrm{g}_{I}$ denotes the nuclear g-factor, $\mu_{N}$ is the nuclear magneton, and $\mu_{0}$ is the Bohr magneton. 


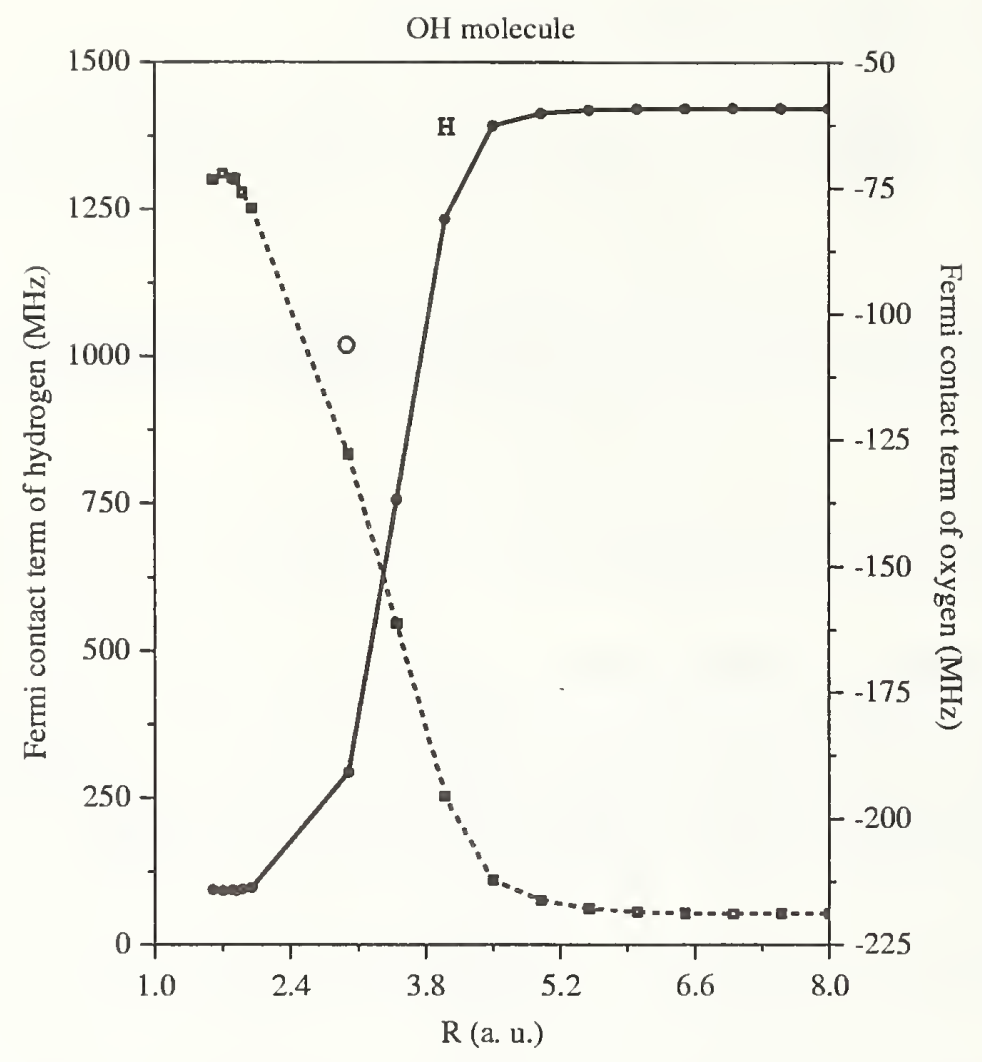

Figure 1: Fermi contact term $\mathrm{A}_{c}$ of the $\mathrm{OH}$ molecule as a function of the internuclear separation.

In our model the CI expansion for $\mathrm{OH}$ includes configurations obtained from the occupied $1 \mathrm{~s}^{2}$, $2 \mathrm{~s}^{2}$, and $2 \mathrm{p}^{4}$ shells of oxygen and the $1 \mathrm{~s}$ shell of hydrogen, as well as configurations created by excitations of these electrons into the $3 \mathrm{~s}, 3 \mathrm{p}, 3 \mathrm{~d}$ and $4 \mathrm{~s}$ states of $\mathrm{O}$ and the $2 \mathrm{~s}, 2 \mathrm{p}, 3 \mathrm{~s}, 3 \mathrm{p}$ and $3 \mathrm{~d}$ states of $\mathrm{H}$. The total number of configurations was equal to 238 . To describe the virtual excited states we use localized Sturm's functions. The result of our computation of the $A_{c}(R)$ for the oxygen and hydrogen atom in the ${ }^{17} \mathrm{OH}$ molecule is shown in Fig. 1. The agreement of the calculation over the wide range of internuclear separations $R$ (1.5 a.u. to 8 a.u.) is better than $1 \%$ in comparison with precise experimental values, which are for the equilibrium distance $R_{\mathrm{e}}=1.8342$ a.u., $A_{\mathrm{c}}=$ $-73.1258 \mathrm{MHz}[5]$, and for the asymptotic values $A(\mathrm{O})=-219.6 \mathrm{MHz}$ and $A(\mathrm{H})=1420.40575 \mathrm{MHz}$ $[6,7]$.

The CI treatment of the $\mathrm{AgH}^{+}$molecule was based on the occupied $4 \mathrm{p}^{6} 4 \mathrm{~d}^{10}$ shells for the $\mathrm{Ag}^{+}$ ion and the $1 \mathrm{~s}$ shell for the $\mathrm{H}$ atom as well as the $5 \mathrm{~s}, 5 \mathrm{p}, 5 \mathrm{~d}, 6 \mathrm{~s}$ and $2 \mathrm{~s}, 2 \mathrm{p}, 3 \mathrm{~s}, 3 \mathrm{p}, 3 \mathrm{~d}$ virtual Sturm's orbitals for $\mathrm{Ag}^{+}$and $\mathrm{H}$, respectively. Computed values of the Fermi contact term of the ${ }^{107} \mathrm{AgH}^{+}$molecule are presented in Fig.2. The asymptotic values of $A_{\mathrm{c}}$ for $\mathrm{H}$ and $\mathrm{Ag}^{+}$fit, within about $1 \%$, the well known experimental value of the hyperfine coupling constant for the $\mathrm{H}$ atom, and the expected zero value for the closed shell ion $\mathrm{Ag}^{+}$. 


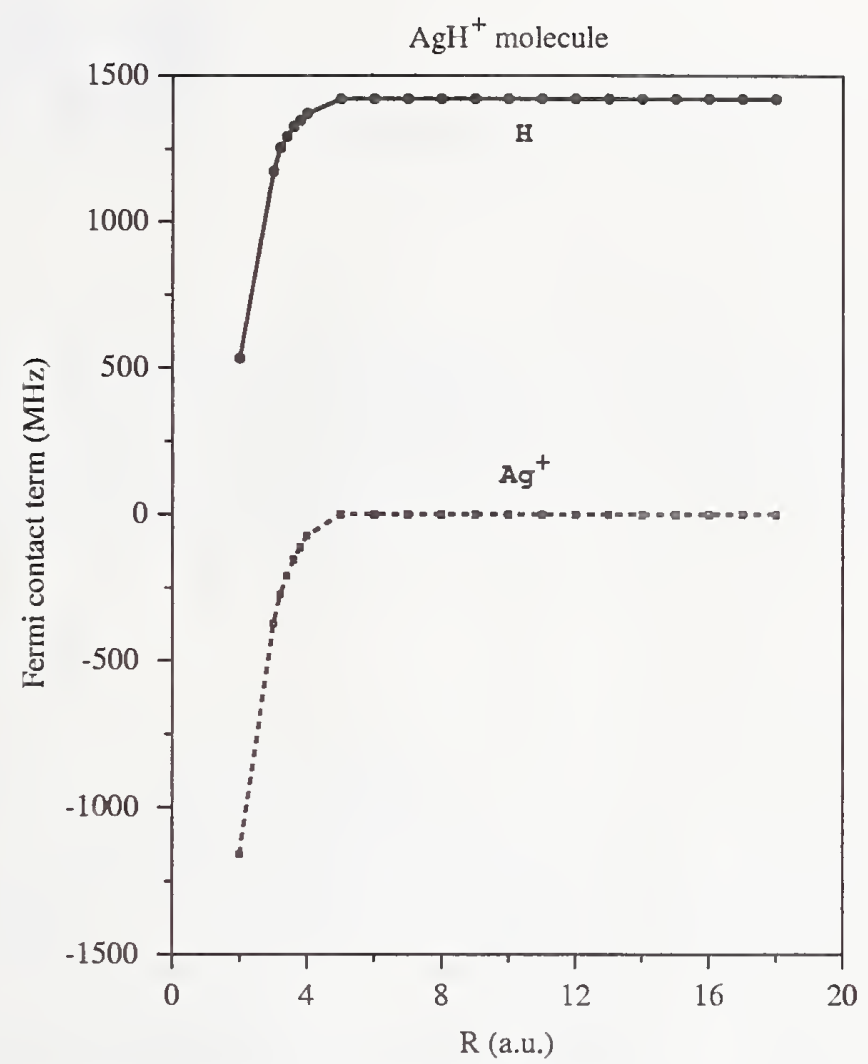

Figure 2: Fermi contact term $\mathrm{A}_{c}$ of the $\mathrm{AgH}^{+}$molecule as a function of the internuclear separation.

\section{References}

[1] S. Kotochigova and I. Tupitsyn, Int. J. Quant. Chem. 29, 307 (1995)

[2] A. I. Sherstyk and P. F. Gruzdev, Opt. Spectrosc. (USSR) 42, 690 (1977)

[3] P. Kristiansen and L. Veseth, J. Chem. Phys. 84, 2711 (1986).

[4] H. Stoll, P. Fuentealba, M. Dolg, J. Flad, L. V. Szentpaly, and H. Preuss, J. Chem. Phys. 79, $5532(1983)$

[5] J. A. Coxon, K. V. L. N. Sastry, J. A. Austin, and D. H. Levy, Can. J. Phys. 57, 619 (1964)

[6] G. H. Fuller, J. Phys. Chem. Ref. Data. 5, 835 (1976)

[7] E. Arimondo, M. Inguscio, and P. Violino, Rev. Mod. Phys. 49, 31 (1977) 


\section{NTSTT Technical Publications}

\section{Periodical}

Journal of Research of the National Institute of Standards and Technology-Reports NIST research and development in those disciplines of the physical and engineering sciences in which the Institute is active. These include physics, chemistry, engineering, mathematics, and computer sciences. Papers cover a broad range of subjects, with major emphasis on measurement methodology and the basic technology underlying standardization. Also included from time to time are survey articles on topics closely related to the Institute's technical and scientific programs. Issued six times a year.

\section{Nonperiodicals}

Monographs-Major contributions to the technical literature on various subjects related to the Institute's scientific and technical activities.

Handbooks-Recommended codes of engineering and industrial practice (including safety codes) developed in cooperation with interested industries, professional organizations, and regulatory bodies.

Special Publications-Include proceedings of conferences sponsored by NIST, NIST annual reports, and other special publications appropriate to this grouping such as wall charts, pocket cards, and bibliographies.

National Standard Reference Data Series-Provides quantitative data on the physical and chemical properties of materials, compiled from the world's literature and critically evaluated. Developed under a worldwide program coordinated by NIST under the authority of the National Standard Data Act (Public Law 90-396). NOTE: The Journal of Physical and Chemical Reference Data (JPCRD) is published bimonthly for NIST by the American Chemical Society (ACS) and the American Institute of Physics (AIP). Subscriptions, reprints, and supplements are available from ACS, 1155 Sixteenth St., NW, Washington, DC 20056.

Building Science Series-Disseminates technical information developed at the Institute on building materials, components, systems, and whole structures. The series presents research results, test methods, and performance criteria related to the structural and environmental functions and the durability and safety characteristics of building elements and systems.

Technical Notes-Studies or reports which are complete in themselves but restrictive in their treatment of a subject. Analogous to monographs but not so comprehensive in scope or definitive in treatment of the subject area. Often serve as a vehicle for final reports of work performed at NIST under the sponsorship of other government agencies.

Voluntary Product Standards-Developed under procedures published by the Department of Commerce in Part 10, Title 15, of the Code of Federal Regulations. The standards establish nationally recognized requirements for products, and provide all concerned interests with a basis for common understanding of the characteristics of the products. NIST administers this program in support of the efforts of private-sector standardizing organizations.

Order the following NIST publications_FIPS and NISTIRs-from the National Technical Information Service, Springfield, VA 22161.

Federal Information Processing Standards Publications (FIPS PUB)-Publications in this series collectively constitute the Federal Information Processing Standards Register. The Register serves as the official source of information in the Federal Government regarding standards issued by NIST pursuant to the Federal Property and Administrative Services Act of 1949 as amended, Public Law 89-306 (79 Stat. 1127), and as implemented by Executive Order 11717 (38 FR 12315, dated May 11, 1973) and Part 6 of Title 15 CFR (Code of Federal Regulations).

NIST Interagency or Internal Reports (NISTIR)-The series includes interim or final reports on work performed by NIST for outside sponsors (both government and nongovernment). In general, initial distribution is handled by the sponsor, public distribution is handled by sales through the National Technical Information Service, Springfield, VA 22161, in hard copy, electronic media, or microfiche form. NISTIR's may also report results of NIST projects of transitory or limited interest, including those that will be published subsequently in more comprehensive form. 


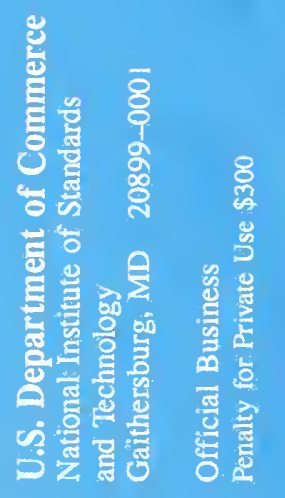

